\title{
Symmetrical 2-extensions of the 3-dimensional grid. I
}

\author{
Kirill V. Kostousov* \\ Krasovskii Institute of Mathematics and Mechanics, \\ S. Kovalevskaya Str, 16, 620108, Yekaterinburg, Russia
}

Received 24 January 2020, accepted 30 May 2020, published online 10 March 2021

\begin{abstract}
For a positive integer $d$, a connected graph $\Gamma$ is a symmetrical 2-extension of the $d$ dimensional grid $\Lambda^{d}$ if there exists a vertex-transitive group $G$ of automorphisms of $\Gamma$ and its imprimitivity system $\sigma$ with blocks of size 2 such that there exists an isomorphism $\varphi$ of the quotient graph $\Gamma / \sigma$ onto $\Lambda^{d}$. The tuple $(\Gamma, G, \sigma, \varphi)$ with specified components is called a realization of the symmetrical 2-extension $\Gamma$ of the grid $\Lambda^{d}$. Two realizations $\left(\Gamma_{1}, G_{1}\right.$, $\left.\sigma_{1}, \varphi_{1}\right)$ and $\left(\Gamma_{2}, G_{2}, \sigma_{2}, \varphi_{2}\right)$ are called equivalent if there exists an isomorphism of the graph $\Gamma_{1}$ onto $\Gamma_{2}$ which maps $\sigma_{1}$ onto $\sigma_{2}$. V.I. Trofimov proved that, up to equivalence, there are only finitely many realizations of symmetrical 2-extensions of $\Lambda^{d}$ for each positive integer $d$. E.A. Konovalchik and K.V. Kostousov found all, up to equivalence, realizations of symmetrical 2-extensions of the grid $\Lambda^{2}$. In this work we found all, up to equivalence, realizations $(\Gamma, G, \sigma, \varphi)$ of symmetrical 2-extensions of the grid $\Lambda^{3}$ for which only the trivial automorphism of $\Gamma$ preserves all blocks of $\sigma$. Namely we prove that there are 5573 such realizations, and that among corresponding graphs $\Gamma$ there are 5350 pairwise nonisomorphic.
\end{abstract}

Keywords: Symmetrical extensions of a graph, d-dimensional grid.

Math. Subj. Class.: $20 H 15$

\section{Introduction}

Recall that, for a positive integer $d$, a $d$-dimensional grid $\Lambda^{d}$ is a graph whose vertices are integer tuples $\left(a_{1}, \ldots a_{d}\right)$ and two vertices $\left(a_{1}^{\prime}, \ldots, a_{d}^{\prime}\right)$ and $\left(a_{1}^{\prime \prime}, \ldots, a_{d}^{\prime \prime}\right)$ are adjacent if and only if $\left|a_{1}^{\prime}-a_{1}^{\prime \prime}\right|+\ldots+\left|a_{d}^{\prime}-a_{d}^{\prime \prime}\right|=1$. According to [6] for a finite graph $\Delta$, define

\footnotetext{
* Our work was performed using 'Uran' supercomputer of IMM UB RAS, Yekaterinburg, Russia.
}

E-mail address: kkostousov@gmail.com (Kirill V. Kostousov) 
a connected graph $\Gamma$ to be a symmetrical extension of $\Lambda^{d}$ by $\Delta$ if there exists a vertextransitive group $G$ of automorphisms of $\Gamma$ and an imprimitivity system $\sigma$ of $G$ on $V(\Gamma)$ such that subgraphs of $\Gamma$ generated by blocks of $\sigma$ are isomorphic to $\Delta$ and there exists an isomorphism of $\Gamma / \sigma$ (i.e., of factor-graph of $\Gamma$ by partition $\sigma$ of its vertex set) onto $\Lambda^{d}$. A tuple $(\Gamma, G, \sigma, \varphi)$ with specified components is called a realization of symmetrical extension $\Gamma$ of the grid $\Lambda^{d}$ by the graph $\Delta$. For a positive integer $q$, a graph $\Gamma$ is called a symmetrical q-extension of the grid $\Lambda^{d}$, if $\Gamma$ is a symmetrical extension of the grid $\Lambda^{d}$ by some graph $\Delta$ such that $|V(\Delta)|=q$. In this situation the tuple $(\Gamma, G, \sigma, \varphi)$ with specified components is called a realization of the symmetrical q-extension $\Gamma$ of the grid $\Lambda^{d}$, and we say that $\Gamma$ is a graph of this realization. Along with purely mathematical interest, symmetrical $q$-extensions of the grid $\Lambda^{d}$ for small $d \geq 1$ and $q>1$ are iteresting for crystallography and some physical theories (see [5]). For crystallography, symmetrical 2-extensions of grids $\Lambda^{d}$ are of the most interest. They naturally arise when considering "molecular" crystals whose "molecules" consist of two "atoms" or, more generally, have a distinguished axis.

It is natural to consider realizations of symmetric $q$-extensions of the grid $\Lambda^{d}$ up to equivalence defined as follows (see [5]). We call two such realizations $R_{1}=\left(\Gamma_{1}, G_{1}\right.$, $\left.\sigma_{1}, \varphi_{1}\right)$ and $R_{2}=\left(\Gamma_{2}, G_{2}, \sigma_{2}, \varphi_{2}\right)$ equivalent and write $R_{1} \sim R_{2}$ if there exists an isomorphism of the graph $\Gamma_{1}$ to the graph $\Gamma_{2}$ which maps $\sigma_{1}$ onto $\sigma_{2}$. We say that the realization $(\Gamma, G, \sigma, \varphi)$ of the symmetrical $q$-extension of the grid $\Lambda^{d}$ is maximal if $G=\operatorname{Aut}_{\sigma}(\Gamma)$ is the group of all automorphisms of the graph $\Gamma$ which preserve the partition $\sigma$. It is clear that each realization of the symmetrical $q$-extension of the grid $\Lambda^{d}$ has an equivalent maximal realization (unique up to equivalence). V.I. Trofimov proved that, up to equivalence, for an arbitrary positive integer $d$, there is only a finite number of realizations of symmetrical 2-extensions of $d$-dimensional grid (see [7, Theorem 2]). An algorithm for constructing these extensions is also proposed in [7].

Using this algorithm, in [3] and [4] were found all, up to equivalence, realizations of symmetrical 2-extensions of the grid $\Lambda^{2}$ (162 realizations). Among the graphs of these realizations, there are exactly 152 pairwise nonisomorphic graphs.

For an arbitrary realization $(\Gamma, G, \sigma, \varphi)$ of the symmetrical 2-extension of the grid $\Lambda^{d}$ and an arbitrary pair of adjacent vertices $B_{1}, B_{2}$ of the graph $\Gamma / \sigma$, the set of edges of the graph $\Gamma$, one end of which lies in $B_{1}$ and the other in $B_{2}$, will be called a connection. The following types of connections are possible: type 1 means a single edge; type $2_{\|}$means two non-adjacent edges; type $2_{V}$ means two adjacent edges; type 3 means three edges; type 4 means full connection (4 edges). A realization that necessarily has connections of types not equal to $2_{\|}$and 4 will be called a realization of class I. A realization that have connections only of types $2_{||}$and 4 (maybe only of one type) will called a realizations of class II. By Proposition 4 from [7], realizations of class I are exactly the realizations of symmetrical 2-extensions of the grid $\Lambda^{d}$ such that only a trivial automorphism of their graph fixes all blocks.

All 162 realizations of symmetrical 2-extensions of the grid $\Lambda^{2}$ are distributed in classes I and II as follows: 87 realizations of class I (see [3]) and 75 realizations of class II (see [4]). This paper is devoted to the description of all, up to equivalence, realizations of symmetrical 2-extensions of the grid $\Lambda^{3}$ of class I.

A realization of symmetrical extension of the grid $\Lambda^{d}$ by the graph $K_{2}$ (full graph on two vertices) will be called a it saturated realization of the symmetrical 2-extension of the grid $\Lambda^{d}$. Accordingly, a realization of symmetrical extension of the grid $\Lambda^{d}$ by the 
graph complemented to $K_{2}$ will be call a it non-saturated realization of the symmetrical 2-extension of the grid $\Lambda^{d}$.

In this paper, we have proved that, up to equivalence, there are 5573 realizations of symmetrical 2-extensions of the grid $\Lambda^{3}$ of class I, among which 2872 are saturated and 2701 are non-saturated (see Theorem 1 and Corollary 1). Among the graphs of saturated realizations of symmetrical 2-extensions of the grid $\Lambda^{3}$ of class I there are exactly 2792 pairwise nonisomorphic; among the graphs of non-saturated realizations of class I there are exactly 2594 pairwise nonisomorphic; and among all graphs of realizations of class I there are 5350 pairwise nonisomorphic (see Corollary 2).

In Sec. 3, we give the description of all, up to equivalence, realizations of symmetrical 2-extensions of the grid $\Lambda^{3}$ of class I (Theorem 1 and Corollary 1). This is obtained using the approach from [7] implemented in GAP [2] (Algorithms 1 and 2 from [3]). Sec. 2 contains preliminary results.

\section{Preliminaries}

Using GAP [2], we have listed all conjugacy classes of vertex-transitive subgroups of the group $\operatorname{Aut}\left(\Lambda^{3}\right)$. It turned out that there are 786 such classes.

The details are as follows. Each vertex-transitive group of automorphisms of $\Lambda^{3}$ is generated by the stabilizer in this group of the vertex $(0,0,0)$ and six elements of this group that translate the vertex $(0,0,0)$ to the vertices adjacent to it. Let $S_{0}$ be a stabilizer of $(0,0,0)$ in $\operatorname{Aut}\left(\Lambda^{3}\right), N_{1}=S_{0} t_{x}, N_{2}=t_{x}^{-1}, N_{3}=S_{0} t_{y}, N_{4}=t_{x}^{-1}, N_{5}=S_{0} t_{z}, N_{6}=$ $t_{z}^{-1}$, and $N=N_{1} \cup N_{2} \cup \ldots \cup N_{6}$. We look over all subgroups $S$ of $S_{0}$, up to conjugation in $S_{0}$. For every $S$, using backtracking, we search for all minimal subsets $N^{\prime}$ of $N$ such that $\left|N^{\prime} \cap N_{i}\right| \leq 1$ for all $i=1, \ldots, 6$ and $\left\langle S, N^{\prime}\right\rangle \cap N_{i} \neq \emptyset$ for all $i=1, \ldots, 6$. For every found $N^{\prime}$, if $\left\langle S, N^{\prime}\right\rangle$ is a proper subgroup of $\operatorname{Aut}\left(\Lambda^{3}\right)$, then we put this group into the resulting list $\mathbf{H}$. At the end, we thin out the list $L$ up to conjugation in $\operatorname{Aut}\left(\Lambda^{3}\right)$.

It turned out that $|\mathbf{H}|=786, \mathbf{H}=\left\{H_{1}, \ldots, H_{786}\right\}$. These groups are given in Table 2 below by their generating systems. The following notation is used for certain automorphisms of the grid $\Lambda^{3}$ :

$$
\begin{aligned}
& r_{x}:(x, y, z) \mapsto(x,-z, y), \quad r_{y}:(x, y, z) \mapsto(z, y,-x), \\
& r_{z}:(x, y, z) \mapsto(y,-x, z), \quad m_{x}:(x, y, z) \mapsto(-x, y, z), \\
& m_{y}:(x, y, z) \mapsto(x,-y, z), \quad m_{z}:(x, y, z) \mapsto(x, y,-z), \\
& i:(x, y, z) \mapsto(-x,-y,-z), \quad t_{x}:(x, y, z) \mapsto(x+1, y, z) \text {, } \\
& t_{y}:(x, y, z) \mapsto(x, y+1, z), \quad t_{z}:(x, y, z) \mapsto(x, y, z+1),
\end{aligned}
$$

where $x, y, z \in \mathbb{Z}$.

Remark 2.1. In the natural embedding of the grid $\Lambda^{3}$ in the Euclidean space $\mathbb{R}^{3}$, each automorphism $g \in \operatorname{Aut}\left(\Lambda^{3}\right)$ is induced by the only isometry $\tilde{g}$ of this space. The isometries that induce the above automorphisms of $\Lambda^{3}$ have the following geometric meaning: $\tilde{r_{x}}, \tilde{r_{y}}, \tilde{r_{z}}$ are rotations by the angle $\frac{\pi}{2}$ around coordinate axes $x, y$, and $z$ respectively, $\tilde{m}_{x}, \tilde{m}_{y}, \tilde{m}_{z}$ are reflections relative coordinate planes, $\tilde{i}$ - central symmetry about the origin, $\tilde{t_{x}}, \tilde{t_{y}}, \tilde{t_{z}}$ are translations by 1 along axes $x, y$, and $z$ respectively.

Using GAP, we constructed and tested 786 stabilizers $\left\{H_{(0,0,0)}: H \in \mathbf{H}\right\}$ for conjugacy in $\operatorname{Aut}\left(\Lambda^{3}\right)_{(0,0,0)}$. It turned out that, up to conjugation, there are only 33 such 
stabilizers. We give them in Table 1 by their generators indicated in column 3. For each of the 33 groups, the abstract group structure is given in column 2 .

Stabilizers of vertex $(0,0,0)$ in vertex-transitive subgroups of $\operatorname{Aut}\left(\Lambda^{3}\right)$

up to conjugation in $\operatorname{Aut}\left(\Lambda^{3}\right)_{(0,0,0)}$

\begin{tabular}{|c|c|c|}
\hline № & Group structure & Generators \\
\hline 1 & 1 & 1 \\
\hline 2 & $C_{2}$ & $\langle i\rangle$ \\
\hline 3 & $C_{2}$ & $\left\langle m_{z}\right\rangle$ \\
\hline 4 & $C_{2}$ & $\left\langle r_{z}^{2} r_{x}\right\rangle$ \\
\hline 5 & $C_{2}$ & $\left\langle r_{z}^{2}\right\rangle$ \\
\hline 6 & $C_{2}$ & $\left\langle m_{z} r_{x}\right\rangle$ \\
\hline 7 & $C_{3}$ & $\left\langle r_{y}^{-1} r_{z}^{-1}\right\rangle$ \\
\hline 8 & $C_{2} \times C_{2}$ & $\left\langle r_{y}^{2}, r_{z}^{2}\right\rangle$ \\
\hline 9 & $C_{2} \times C_{2}$ & $\left\langle i, r_{z}^{2}\right\rangle$ \\
\hline 10 & $C_{2} \times C_{2}$ & $\left\langle m_{z} r_{x}^{-1}, r_{x}^{2}\right\rangle$ \\
\hline 11 & $C_{2} \times C_{2}$ & $\left\langle m_{x}, r_{y}^{2} r_{x}\right\rangle$ \\
\hline 12 & $C_{2} \times C_{2}$ & $\left\langle i, r_{z}^{2} r_{x}\right\rangle$ \\
\hline 13 & $C_{2} \times C_{2}$ & $\left\langle r_{y}^{2} r_{x}, r_{z}^{2} r_{x}\right\rangle$ \\
\hline 14 & $C_{2} \times C_{2}$ & $\left\langle m_{x}, r_{z}^{2}\right\rangle$ \\
\hline 15 & $C_{4}$ & $\left\langle r_{x}^{-1}, r_{x}^{2}\right\rangle$ \\
\hline 16 & $C_{4}$ & $\left\langle m_{x} r_{x}^{-1}\right\rangle$ \\
\hline 17 & $C_{6}$ & $\left\langle i, m_{x} r_{y}^{-1} r_{x}^{-1}\right\rangle$ \\
\hline 18 & $S_{3}$ & $\left\langle m_{x} r_{y}, r_{y}^{-1} r_{z}^{-1}\right\rangle$ \\
\hline 19 & $S_{3}$ & $\left\langle r_{y}^{2} r_{z}, r_{z}^{2} r_{x}\right\rangle$ \\
\hline 20 & $C_{2} \times C_{2} \times C_{2}$ & $\left\langle i, r_{y}^{2} r_{x}, r_{z}^{2} r_{x}\right\rangle$ \\
\hline 21 & $C_{2} \times C_{2} \times C_{2}$ & $\left\langle i, r_{y}^{2}, r_{z}^{2}\right\rangle$ \\
\hline 22 & $C_{4} \times C_{2}$ & $\left\langle i, m_{x} r_{x}^{-1}\right\rangle$ \\
\hline 23 & $D_{8}$ & $\left\langle m_{x} r_{x}^{-1}, r_{z}^{2} r_{x}\right\rangle$ \\
\hline 24 & $D_{8}$ & $\left\langle r_{z}^{2}, r_{z}^{2} r_{x}\right\rangle$ \\
\hline 25 & $D_{8}$ & $\left\langle m_{y}, m_{z} r_{x}^{-1}, r_{x}^{2}\right\rangle$ \\
\hline 26 & $D_{8}$ & $\left\langle m_{x} r_{x}^{-1}, r_{z}^{2}\right\rangle$ \\
\hline 27 & $A_{4}$ & $\left\langle r_{y} r_{x}^{-1}, r_{y}^{2}, r_{z}^{2}\right\rangle$ \\
\hline 28 & $D_{12}$ & $\left\langle i, r_{y}^{2} r_{z}, r_{z}^{2} r_{x}\right\rangle$ \\
\hline 29 & $C_{2} \times D_{8}$ & $\left\langle i, r_{z}^{2}, r_{z}^{2} r_{x}\right\rangle$ \\
\hline 30 & $C_{2} \times A_{4}$ & $\left\langle i, m_{x} r_{y}^{-1} r_{x}^{-1}, r_{y}^{2}, r_{z}^{2}\right\rangle$ \\
\hline 31 & $S_{4}$ & $\left\langle m_{x} r_{x}^{-1}, m_{x} r_{z}, r_{z}^{2}\right\rangle$ \\
\hline 32 & $S_{4}$ & $\left\langle r_{y}^{2} r_{z}, r_{z}^{2}, r_{z}^{2} r_{x}\right\rangle$ \\
\hline 33 & $C_{2} \times S_{4}$ & $\left\langle i, r_{y}^{2} r_{z}, r_{z}^{2}, r_{z}^{2} r_{x}\right\rangle$ \\
\hline
\end{tabular}

Each group $H \in \mathbf{H}$ is identified with some space group and, therefore, has a point group $\mathrm{P}(H)$ and a translation basis (see [1]). Using GAP, we verified that the set of point groups $\{\mathrm{P}(H): H \in \mathbf{H}\}$ is equal, up to conjugation in $\mathrm{P}\left(\operatorname{Aut}\left(\Lambda^{3}\right)\right)$, to the set of 33 stabilizers given in Table 1 . In column 1 of Table 2 below, we give the set of groups $\mathbf{H}$ defined by their generators. In column 2, for each group $H \in \mathbf{H}$, we give the № of the group from Table 1 conjugate to the stabilizer $H_{(0,0,0)}$ in $\operatorname{Aut}\left(\Lambda^{3}\right)_{(0,0,0)}$. In column 3, for 
each group $H \in \mathbf{H}$, we give the № of the group from Table 1 conjugate to the point group $\mathrm{P}(H)$ in $\mathrm{P}\left(\operatorname{Aut}\left(\Lambda^{3}\right)\right)$. In column 4 , for each group $H \in \mathbf{H}$, we give its translation basis. The groups in Table 2 are sorted lexicographically first by № in column 2 and then by № in column 3.

Representatives of conjugacy classes for

Table 2

\section{vertex-transitive subgroups of $\operatorname{Aut}\left(\Lambda^{3}\right)$}

\begin{tabular}{|c|c|c|c|}
\hline$H$ & $H_{0,0,0}$ & $P(H)$ & Translation basis of $H$ \\
\hline$H_{1}=\left\langle t_{x}, t_{y}^{-1}, t_{z}^{-1}\right\rangle$ & 1 & 1 & {$[[1,0,0],[0,1,0],[0,0,1]]$} \\
\hline$H_{2}=\left\langle i t_{z}, i t_{z}^{-1}, t_{x}, t_{y}^{-1}\right\rangle$ & 1 & 2 & {$[[1,0,0],[0,1,0],[0,0,2]]$} \\
\hline$H_{3}=\left\langle i t_{y}, i t_{y}^{-1}, i t_{z}, i t_{z}^{-1}, t_{x}\right\rangle$ & 1 & 2 & {$[[1,0,0],[0,1,1],[0,0,2]]$} \\
\hline$H_{4}=\left\langle i t_{x}, i t_{x}^{-1}, i t_{y}, i t_{y}^{-1}, i t_{z}, i t_{z}^{-1}\right\rangle$ & 1 & 2 & {$[[1,0,1],[0,1,1],[0,0,2]]$} \\
\hline$H_{5}=\left\langle m_{x} t_{y}^{-1}, t_{x}, t_{z}^{-1}\right\rangle$ & 1 & 3 & {$[[1,0,0],[0,2,0],[0,0,1]]$} \\
\hline$H_{6}=\left\langle m_{y}{ }^{t} y, m_{y} t_{y}^{-1}, t_{x}, t_{z}^{-1}\right\rangle$ & 1 & 3 & {$[[1,0,0],[0,2,0],[0,0,1]]$} \\
\hline$H_{7}=\left\langle m_{y} t_{y}, m_{y} t_{y}^{-1}, m_{y} t_{z}^{-1}, t_{x}\right\rangle$ & 1 & 3 & {$[[1,0,0],[0,1,1],[0,0,2]]$} \\
\hline$H_{8}=\left\langle m_{y} t_{x}, m_{y} t_{z}^{-1}, t_{y}^{-1}\right\rangle$ & 1 & 3 & {$[[1,0,1],[0,1,0],[0,0,2]]$} \\
\hline$H_{9}=\left\langle m_{y} t_{x}, m_{y} t_{y}, m_{y} t_{y}^{-1}, m_{y} t_{z}^{-1}\right\rangle$ & 1 & 3 & {$[[1,0,1],[0,1,1],[0,0,2]]$} \\
\hline$H_{10}=\left\langle r_{x}^{2} r_{y} t_{y}, r_{x}^{2} r_{y} t_{y}^{-1}, t_{x}, t_{z}^{-1}\right\rangle$ & 1 & 4 & {$[[1,0,0],[0,2,0],[0,0,1]]$} \\
\hline$H_{11}=\left\langle r_{z}^{2} r_{x} t_{y}^{-1}, r_{z}^{2} r_{x} t_{z}, t_{x}\right\rangle$ & 1 & 4 & {$[[1,0,0],[0,1,1],[0,0,2]]$} \\
\hline$H_{12}=\left\langle r_{y} r_{x}^{2} t_{x}, r_{y} r_{x}^{2} t_{x}^{-1}, r_{y} r_{x}^{2} t_{y}, r_{y} r_{x}^{2} t_{y}^{-1}\right\rangle$ & 1 & 4 & {$[[1,0,1],[0,1,1],[0,0,2]]$} \\
\hline$H_{13}=\left\langle r_{x}^{2} t_{y}, r_{x}^{2} t_{y}^{-1}, t_{x}, t_{z}^{-1}\right\rangle$ & 1 & 5 & {$[[1,0,0],[0,2,0],[0,0,1]]$} \\
\hline$H_{14}=\left\langle r_{z}^{2} t_{z}^{-1}, t_{x}, t_{y}^{-1}\right\rangle$ & 1 & 5 & {$[[1,0,0],[0,1,0],[0,0,2]]$} \\
\hline$H_{15}=\left\langle r_{x}^{2} t_{y}, r_{x}^{2} t_{y}^{-1}, r_{x}^{2} t_{z}, r_{x}^{2} t_{z}^{-1}, t_{x}\right\rangle$ & 1 & 5 & {$[[1,0,0],[0,1,1],[0,0,2]]$} \\
\hline$H_{16}=\left\langle r_{z}^{2} t_{y}, r_{z}^{2} t_{y}^{-1}, r_{z}^{2} t_{z}^{-1}, t_{x}\right\rangle$ & 1 & 5 & {$[[1,0,0],[0,1,1],[0,0,2]]$} \\
\hline$H_{17}=\left\langle r_{y}^{2} t_{x}, r_{y}^{2} t_{x}^{-1}, r_{y}^{2} t_{y}^{-1}, r_{y}^{2} t_{z}, r_{y}^{2} t_{z}^{-1}\right\rangle$ & 1 & 5 & {$[[1,0,1],[0,1,1],[0,0,2]]$} \\
\hline$H_{18}=\left\langle m_{x} r_{y}^{-1} t_{y}^{-1}, t_{x}, t_{z}^{-1}\right\rangle$ & 1 & 6 & {$[[1,0,0],[0,2,0],[0,0,1]]$} \\
\hline$H_{19}=\left\langle m_{z} r_{x}^{-1} t_{y}^{\frac{9}{y}}, m_{z} r_{x}^{-1} t_{z}, t_{x}\right\rangle$ & 1 & 6 & {$[[1,0,0],[0,1,1],[0,0,2]]$} \\
\hline$H_{20}=\left\langle m_{z} r_{x}^{-1} t_{x}, m_{z} r_{x}^{-1} t_{y}^{-1}, m_{z} r_{x}^{-1} t_{z}\right\rangle$ & 1 & 6 & {$[[1,0,1],[0,1,1],[0,0,2]]$} \\
\hline$H_{21}=\left\langle r_{y} r_{x} t_{x}^{-1}, r_{y} r_{x} t_{z}^{-1}, r_{y}^{-1} r_{z}^{-1} t_{x}\right\rangle$ & 1 & 7 & {$[[1,0,2],[0,1,2],[0,0,3]]$} \\
\hline$H_{22}=\left\langle r_{x}^{2} t_{y}^{-1}, r_{z}^{2} t_{y}, t_{x}, t_{z}^{-1}\right\rangle$ & 1 & 8 & {$[[1,0,0],[0,4,0],[0,0,1]]$} \\
\hline$H_{23}=\left\langle r_{x}^{2} t_{y}, r_{x}^{2} t_{y}^{-1}, r_{z}^{2} t_{z}^{-1}, t_{x}\right\rangle$ & 1 & 8 & {$[[1,0,0],[0,2,0],[0,0,2]]$} \\
\hline$H_{24}=\left\langle r_{x}^{2} t_{y}, r_{x}^{2} t_{y}^{-1}, r_{y}^{2} t_{z}, r_{y}^{2} t_{z}^{-1}, t_{x}\right\rangle$ & 1 & 8 & {$[[1,0,0],[0,2,0],[0,0,2]]$} \\
\hline$H_{25}=\left\langle r_{x}^{2} t_{y}, r_{x}^{2} t_{y}^{-1}, r_{y}^{2} t_{x}, r_{y}^{2} t_{x}^{-1}, t_{z}^{-1}\right\rangle$ & 1 & 8 & {$[[2,0,0],[0,2,0],[0,0,1]]$} \\
\hline$H_{26}=\left\langle r_{y}^{2} t_{y}^{-1}, r_{z}^{2} t_{z}^{-1}, t_{x}\right\rangle$ & 1 & 8 & {$[[1,0,0],[0,2,0],[0,0,2]]$} \\
\hline$H_{27}=\left\langle r_{x}^{2} t_{y}^{-1}, r_{y}^{2} t_{z}, r_{y}^{2} t_{z}^{-1}, r_{z}^{2} t_{y}, t_{x}\right\rangle$ & 1 & 8 & {$[[1,0,0],[0,2,1],[0,0,2]]$} \\
\hline$H_{28}=\left\langle r_{x}^{2} t_{y}, r_{x}^{2} t_{y}^{-1}, r_{x}^{2} t_{z}, r_{x}^{2} t_{z}^{-1}, r_{y}^{2} t_{x}, r_{y}^{2} t_{x}^{-1}\right\rangle$ & 1 & 8 & {$[[2,0,0],[0,1,1],[0,0,2]]$} \\
\hline$H_{29}=\left\langle r_{y}^{2} t_{x}, r_{y}^{2} t_{x}^{-1}, r_{z}^{2} t_{y}, r_{z}^{2} t_{y}^{-1}, r_{z}^{2} t_{z}^{-1}\right\rangle$ & 1 & 8 & {$[[2,0,0],[0,1,1],[0,0,2]]$} \\
\hline$H_{30}=\left\langle r_{y}^{2} t_{x}, r_{y}^{2} t_{x}^{-1}, r_{y}^{2} t_{y}^{-1}, r_{z}^{2} t_{z}^{-1}\right\rangle$ & 1 & 8 & {$[[1,1,0],[0,2,0],[0,0,2]]$} \\
\hline$H_{31}=\left\langle r_{x}^{2} t_{y}, r_{x}^{2} t_{y}^{-1}, r_{x}^{2} t_{z}, r_{x}^{2} t_{z}^{-1}, r_{y}^{2} t_{x}, r_{z}^{2} t_{x}^{-1}\right\rangle$ & 1 & 8 & {$[[2,0,1],[0,1,1],[0,0,2]]$} \\
\hline$H_{32}=\left\langle r_{x}^{2} t_{z}, r_{x}^{2} t_{z}^{-1}, r_{y}^{2} t_{x}, r_{y}^{2} t_{x}^{-1}, r_{z}^{2} t_{y}, r_{z}^{2} t_{y}^{-1}\right\rangle$ & 1 & 8 & {$[[1,1,1],[0,2,0],[0,0,2]]$} \\
\hline$H_{33}=\left\langle r_{x}^{2} t_{y}, r_{x}^{2} t_{y}^{-1}, r_{y}^{2} t_{x}, r_{y}^{2} t_{x}^{-1}, r_{z}^{2} t_{z}^{-1}\right\rangle$ & 1 & 8 & {$[[1,1,1],[0,2,0],[0,0,2]]$} \\
\hline$H_{34}=\left\langle r_{x}^{2} t_{x}, r_{y}^{2} t_{y}^{-1}, r_{z}^{2} t_{z}^{-1}\right\rangle$ & 1 & 8 & {$[[1,1,1],[0,2,0],[0,0,2]]$} \\
\hline$H_{35}=\left\langle i t_{y}, r_{x}^{2} t_{y}^{-1}, t_{x}, t_{z}^{-1}\right\rangle$ & 1 & 9 & {$[[1,0,0],[0,4,0],[0,0,1]]$} \\
\hline$H_{36}=\left\langle i t_{y}, m_{y} t_{y}^{-1}, t_{x}, t_{z}^{-1}\right\rangle$ & 1 & 9 & {$[[1,0,0],[0,4,0],[0,0,1]]$} \\
\hline$H_{37}=\left\langle i t_{y}, i t \bar{z}^{-1}, r_{x}^{2} t_{y}^{-1}, r_{x}^{2} t_{z}, t_{x}\right\rangle$ & 1 & 9 & {$[[1,0,0],[0,1,1],[0,0,4]]$} \\
\hline$H_{38}=\left\langle i t_{z}^{-1}, m_{y} t_{y}, m_{y} t_{y}^{-1}, r_{y}^{2} t_{z}, t_{x}\right\rangle$ & 1 & 9 & {$[[1,0,0],[0,1,2],[0,0,4]]$} \\
\hline$H_{39}=\left\langle m_{x} t_{y}^{-1}, r_{x}^{2} t_{z}, r_{x}^{2} t_{z}^{-1}, t_{x}\right\rangle$ & 1 & 9 & {$[[1,0,0],[0,2,0],[0,0,2]]$} \\
\hline$H_{40}=\left\langle i t_{z}, i t_{z}^{-1}, r_{x}^{2} t_{y}, r_{x}^{2} t_{y}^{-1}, t_{x}\right\rangle$ & 1 & 9 & {$[[1,0,0],[0,2,0],[0,0,2]]$} \\
\hline$H_{41}=\left\langle i t_{z}, i t_{z}^{-1}, m_{y} t_{y}, m_{y} t^{-1}, t_{x}\right\rangle$ & 1 & 9 & {$[[1,0,0],[0,2,0],[0,0,2]]$} \\
\hline$H_{42}=\left\langle m_{y} t_{y}, m_{y} t_{y}^{-1}, r_{y}^{2} t_{z}, r_{y}^{2} t_{z}^{-1}, t_{x}\right\rangle$ & 1 & 9 & {$[[1,0,0],[0,2,0],[0,0,2]]$} \\
\hline$H_{43}=\left\langle i t_{z}, i t_{z}^{-1}, m_{z} t_{y}^{-1}, t_{x}\right\rangle$ & 1 & 9 & {$[[1,0,0],[0,2,0],[0,0,2]]$} \\
\hline$H_{44}=\left\langle m_{z} t_{y}^{-1}, r_{z}^{2} t_{z}^{\left.-1^{g}, t_{x}\right\rangle}\right.$ & 1 & 9 & {$[[1,0,0],[0,2,0],[0,0,2]]$} \\
\hline$H_{45}=\left\langle i t_{z}, i t_{z}^{-1}, m_{y} t_{x}, t_{y}^{-1}\right\rangle$ & 1 & 9 & {$[[2,0,0],[0,1,0],[0,0,2]]$} \\
\hline$H_{46}=\left\langle i t_{y}, i t_{y}^{-1}, r_{z}^{2} t_{z}^{-1}, t_{x}\right\rangle$ & 1 & 9 & {$[[1,0,0],[0,2,0],[0,0,2]]$} \\
\hline$H_{47}=\left\langle i t_{y}, i t_{y}^{-1}, r_{y}^{2} t_{z}, r_{y}^{2} t_{z}^{-1}, t_{x}\right\rangle$ & 1 & 9 & {$[[1,0,0],[0,2,0],[0,0,2]]$} \\
\hline$H_{48}=\left\langle i t_{y}, m_{x} t_{z}^{-1}, r_{x}^{2} t_{y}^{-1}, t_{x}\right\rangle$ & 1 & 9 & {$[[1,0,0],[0,2,1],[0,0,2]]$} \\
\hline$H_{49}=\left\langle i t_{y}, m_{y} t_{y}^{-1}, r_{y}^{2} t_{z}, r_{y}^{2} t_{z}^{-1}, t_{x}\right\rangle$ & 1 & 9 & {$[[1,0,0],[0,2,1],[0,0,2]]$} \\
\hline$H_{50}=\left\langle m_{y} t_{y}, m_{y} t_{y}^{-1}, r_{y}^{2} t_{x}, r_{y}^{2} t_{x}^{-1}, r_{y}^{2} t_{z}, r_{y}^{2} t_{z}^{-1}\right\rangle$ & 1 & 9 & {$[[1,0,1],[0,2,0],[0,0,2]]$} \\
\hline$H_{51}=\left\langle m_{y} t_{y}, m_{y} t_{y}^{-1}, m_{y} t_{z}^{-1}, r_{y}^{2} t_{x}, r_{y}^{2} t_{x}^{-1}\right\rangle$ & 1 & 9 & {$[[2,0,0],[0,1,1],[0,0,2]]$} \\
\hline$H_{52}=\left\langle i t_{z}, i t_{z}^{-1}, m_{y} t_{x}, m_{y} t_{y}, m_{y} t_{y}^{-1}\right\rangle$ & 1 & 9 & {$[[1,1,0],[0,2,0],[0,0,2]]$} \\
\hline$H_{53}=\left\langle i t_{y}, i t_{y}^{-1}, i t_{z}, i t_{z}^{-1}, m_{z} t_{x}\right\rangle$ & 1 & 9 & {$[[2,0,0],[0,1,1],[0,0,2]]$} \\
\hline$H_{54}=\left\langle m_{z} t_{x}, r_{z}^{2} t_{y}, r_{z}^{2} t_{y}^{-1}, r_{z}^{2} t_{z}^{-1}\right\rangle$ & 1 & 9 & {$[[2,0,0],[0,1,1],[0,0,2]]$} \\
\hline$H_{55}=\left\langle i t_{z}, i t_{z}^{-1}, m_{z} t_{x}, m_{z} t_{y}^{-1}\right\rangle$ & 1 & 9 & {$[[1,1,0],[0,2,0],[0,0,2]]$} \\
\hline$H_{56}=\left\langle m_{z} t_{x}, m_{z} t_{y}^{-1}, r_{z}^{2} t_{z}^{-1}\right\rangle$ & 1 & 9 & {$[[1,1,0],[0,2,0],[0,0,2]]$} \\
\hline$H_{57}=\left\langle i t_{x}, i t_{x}^{-1}, i t_{y}, i t_{y}^{-1}, r_{z}^{2} t_{z}^{-1}\right\rangle$ & 1 & 9 & {$[[1,1,0],[0,2,0],[0,0,2]]$} \\
\hline
\end{tabular}




\begin{tabular}{|c|c|c|c|}
\hline$H$ & $H_{0,0,0}$ & $P(H)$ & Translation basis of $H$ \\
\hline$H_{58}=\left\langle i t_{x}, i t_{x}^{-1}, i t_{y}, i t_{y}^{-1}, r_{y}^{2} t_{z}, r_{y}^{2} t_{z}^{-1}\right\rangle$ & 1 & 9 & {$[[1,1,0],[0,2,0],[0,0,2]]$} \\
\hline$H_{59}=\left\langle i t_{x}, i t_{x}^{-1}, i t_{y}, i t_{y}^{-1}, m_{z} t_{z}, m_{z} t_{z}^{-1}\right\rangle$ & 1 & 9 & {$[[1,1,0],[0,2,0],[0,0,2]]$} \\
\hline$H_{60}=\left\langle i t_{x}, i t_{x}^{-1}, r_{z}^{2} t_{y}, r_{z}^{2} t_{y}^{-1}, r_{z}^{2} t_{z}^{-1}\right\rangle$ & 1 & 9 & {$[[2,0,0],[0,1,1],[0,0,2]]$} \\
\hline$H_{61}=\left\langle i t_{x}, i t_{x}^{-1}, r_{x}^{2} t_{y}, r_{x}^{2} t_{y}^{-1}, r_{x}^{2} t_{z}, r_{x}^{2} t_{z}^{-1}\right\rangle$ & 1 & 9 & {$[[2,0,0],[0,1,1],[0,0,2]]$} \\
\hline$H_{62}=\left\langle i t_{x}, i t_{y}^{-1}, m_{z} t_{z}, m\right.$ & 1 & 9 & {$[[1,1,0],[0,2,1],[0,0,2]]$} \\
\hline$H_{63}=\left\langle i t_{y}, m_{y} t_{y}^{-1}, r_{y}^{2} t_{x}\right.$ & 1 & 9 & {$[[1,0,1],[0,2,1],[0,0,2]]$} \\
\hline$H_{64}=\left\langle i t_{z}^{-1}, m_{y} t_{x}, m_{y} t_{y}\right.$ & 1 & 9 & {$[[1,0,2],[0,1,2],[0,0,4]]$} \\
\hline$H_{65}=\left\langle i t_{z}, i t_{z}^{-1}, m_{y} t_{y}, m_{y} t_{y}^{-1}, r_{y}^{2} t_{x}, r_{y}^{2} t_{x}^{-1}\right\rangle$ & 1 & 9 & {$[[1,1,1],[0,2,0],[0,0,2]]$} \\
\hline$H_{66}=\left\langle i t_{y}, i t_{y}^{-1}, m_{z} t_{x}, r_{z}^{2} t_{z}^{-1}\right\rangle$ & 1 & 9 & {$[[1,1,1],[0,2,0],[0,0,2]]$} \\
\hline$H_{67}=\left\langle i t_{z}, i t_{z}^{-1}, m_{z} t_{x}, r_{z}^{2} t_{y}, r_{z}^{2} t_{y}^{-1}\right\rangle$ & 1 & 9 & {$[[1,1,1],[0,2,0],[0,0,2]]$} \\
\hline$H_{68}=\left\langle m_{z} r_{x}^{-1} t_{z}, r_{x}^{2} t_{y}^{-1}, r_{x}^{2}\right.$ & 1 & 10 & $[[1,0,0],[0,1,3],[0,0,4]]]$ \\
\hline$H_{69}=\left\langle m_{z} r_{x}^{-1} t_{x}, r_{x}^{2} t_{y}, r_{x}^{2} t_{y}^{-1}, r_{x}^{2} t_{z}, r_{x}^{2} t_{z}^{-1}\right\rangle$ & 1 & 10 & {$[[2,0,0],[0,1,1],[0,0,2]]$} \\
\hline$H_{70}=\left\langle m_{z} r_{x} t_{y}^{-1}, m_{z} r_{x} t_{z}^{-1}, m_{z} r_{x}^{-1} t_{x}\right\rangle$ & 1 & 10 & $[[2,0,0],[0,1,1],[0,0,2]]]$ \\
\hline$H_{71}=\left\langle m_{x} r_{y} t_{x}, m_{x} r_{y} t_{x}^{-1}, r_{y}^{2} t_{y}^{-1}\right\rangle$ & 1 & 10 & {$[[1,0,1],[0,2,0],[0,0,2]]$} \\
\hline$H_{72}=\left\langle m_{x} r_{y} t_{y}^{-1}, m_{x} r_{y}^{-1} t_{x}^{-1}, r_{y}^{2} t_{x}, r_{y}^{2} t_{z}\right\rangle$ & 1 & 10 & $[[1,0,3],[0,1,2],[0,0,4]]]$ \\
\hline$H_{73}=\left\langle m_{y} t_{y}, r_{x}^{2} r_{y} t_{y}^{-1}, t_{x}, t_{z}^{-1}\right\rangle$ & 1 & 11 & {$[[1,0,0],[0,4,0],[0,0,1]]$} \\
\hline$H_{74}=\left\langle m_{z} r_{x}^{-1} t_{y}^{-1}, r_{z}^{2} r_{x} t_{z}\right.$ & 1 & 11 & {$[[1,0,0],[0,1,3],[0,0,4]]$} \\
\hline$H_{75}=\left\langle m_{z} r_{x}^{-1} t_{x}, r_{z}^{2} r_{x} t_{y}^{-1}\right.$ & 1 & 11 & {$[[2,0,0],[0,1,1],[0,0,2]]$} \\
\hline$H_{76}=\left\langle m_{x} t_{y}^{-1}, m_{x} t_{z}^{-1}, m_{z} r_{x}^{-1} t_{x}\right\rangle$ & 1 & 11 & {$[[2,0,0],[0,1,1],[0,0,2]]$} \\
\hline$H_{77}=\left\langle m_{z} t_{x}, m_{z} t_{y}^{-1}, r_{y}^{2} r_{z} t_{z}, r_{y}^{2} r_{z} t_{z}^{-1}\right\rangle$ & 1 & 11 & {$[[1,1,0],[0,2,0],[0,0,2]]$} \\
\hline$H_{78}=\left\langle m_{x} t_{x}, m_{x} t_{x}^{-1}, r_{z}^{2} r\right.$ & 1 & 11 & {$[[2,0,0],[0,1,1],[0,0,2]]$} \\
\hline$H_{79}=\left\langle m_{x} r_{y} t_{x}, m_{x} r_{y} t_{x}^{-1}, r_{y} r_{x}^{2} t_{y}, r_{y} r_{x}^{2} t_{y}^{-1}\right\rangle$ & 1 & 11 & {$[[1,0,1],[0,2,0],[0,0,2]]$} \\
\hline$H_{80}=\left\langle m_{x} r_{y} t_{x}, m_{x} r_{y} t_{x}^{-1}, m_{y} t_{y}, m_{y} t_{y}^{-1}\right\rangle$ & 1 & 11 & {$[[1,0,1],[0,2,0],[0,0,2]]$} \\
\hline$H_{81}=\left\langle m_{x} t_{x}, m_{z} r_{x}^{-1} t_{y}^{-1}, m_{z} r_{x}^{-1} t_{z}, r_{z}^{2} r_{x} t_{x}^{-1}\right\rangle$ & 1 & 11 & $[[2,0,1],[0,1,1],[0,0,2]]]$ \\
\hline$H_{82}=\left\langle m_{x} t_{x}, m_{x} t_{x}^{-1}, m_{z} r_{x}^{-1} t_{z}, r_{z}^{2} r_{x} t_{y}^{-1}\right\rangle$ & 1 & 11 & {$[[1,0,2],[0,1,3],[0,0,4]]$} \\
\hline$H_{83}=\left\langle i t_{y}, r_{x}^{2} r_{y} t_{y}^{-1}, t_{x}, t_{z}^{-1}\right\rangle$ & 1 & 12 & {$[[1,0,0],[0,4,0],[0,0,1]]$} \\
\hline$H_{84}=\left\langle i t_{y}, i t_{z}, m_{z} r_{x}^{-1} t_{y}^{-1}, t_{x}\right\rangle$ & 1 & 12 & {$[[1,0,0],[0,1,3],[0,0,4]]$} \\
\hline$H_{85}=\left\langle i t_{y}^{-1}, i t_{z}^{-1}, r_{z}^{2} r_{x} t_{z}, t_{x}\right\rangle$ & 1 & 12 & {$[[1,0,0],[0,1,3],[0,0,4]]$} \\
\hline$H_{86}=\left\langle i t_{y}^{-1}, i t_{z}, m_{z} r_{x}^{-1} t_{x}, r_{y}^{2} r_{x} t_{z}^{-1}\right\rangle$ & 1 & 12 & {$[[1,0,2],[0,1,1],[0,0,4]]$} \\
\hline$H_{87}=\left\langle i t_{x}, i t_{y}^{-1}, i t_{z}^{-1}, r_{z}^{2} r_{x}\right.$ & 1 & 12 & {$[[1,0,1],[0,1,3],[0,0,4]]$} \\
\hline$H_{88}=\left\langle i t_{y}, i t_{y}^{-1}, i t_{z}, i t_{z}^{-1}, r\right.$ & 1 & 12 & {$[[2,0,0],[0,1,1],[0,0,2]]$} \\
\hline$H_{89}=\left\langle m_{z} r_{x}^{-1} t_{x}, r_{y}^{2} r_{x} t_{y}^{-1}, r_{y}^{2} r_{x} t_{z}^{-1}\right\rangle$ & 1 & 12 & {$[[2,0,0],[0,1,1],[0,0,2]]$} \\
\hline$H_{90}=\left\langle i t_{x}, i t_{x}^{-1}, i t_{y}, i t \frac{y}{y}, r_{y}^{2} r_{z} t_{z}, r_{y}^{2} r_{z} t_{z}^{-1}\right\rangle$ & 1 & 12 & {$[[1,1,0],[0,2,0],[0,0,2]]$} \\
\hline$H_{91}=\left\langle i t_{x}, i t_{x}^{-1}, r_{z}^{2} r_{x} t_{y}^{-1}, r_{z}^{2} r\right.$ & 1 & 12 & {$[[2,0,0],[0,1,1],[0,0,2]]$} \\
\hline$H_{92}=\left\langle i t_{x}, i t_{x}^{-1}, m_{z} r_{x}^{-1} t_{y}^{-1}, m_{z} r_{x}^{-1} t_{z}\right\rangle$ & 1 & 12 & {$[[2,0,0],[0,1,1],[0,0,2]]$} \\
\hline$H_{93}=\left\langle m_{x} r_{y} t_{x}, m_{x} r_{y} t_{x}^{-1}, r_{x}^{2} r_{y} t_{y}, r_{x}^{2} r_{y} t_{y}^{-1}\right\rangle$ & 1 & 12 & {$[[1,0,1],[0,2,0],[0,0,2]]$} \\
\hline$H_{94}=\left\langle i t_{x}, m_{z} r_{x} t_{y}^{-1}, m_{z} r_{x} t_{z}^{-1}, r_{z}^{2} r_{x} t_{x}^{-1}\right\rangle$ & 1 & 12 & {$[[2,0,1],[0,1,1],[0,0,2]]$} \\
\hline$H_{95}=\left\langle i t_{x}, i t_{y}, m_{x} r_{z} t_{x}^{-1}, r_{x}^{2} r_{z} t_{z}, r_{x}^{2} r_{z} t_{z}^{-1}\right\rangle$ & 1 & 12 & {$[[1,1,1],[0,2,1],[0,0,2]]$} \\
\hline$H_{96}=\left\langle r_{x}^{2} r_{y} t_{y}^{-1}, r_{y} r_{x}^{2} t_{y}, t_{x}, t_{z}^{-1}\right\rangle$ & 1 & 13 & {$[[1,0,0],[0,4,0],[0,0,1]]$} \\
\hline$H_{97}=\left\langle r_{x}^{2} t_{y}^{-1}, r_{x}^{2} t_{z}, r_{y}^{2} r_{x} t_{z}^{-1}, t_{x}\right\rangle$ & 1 & 13 & {$[[1,0,0],[0,1,1],[0,0,4]]$} \\
\hline$H_{98}=\left\langle r_{y} r_{x}^{2} t_{x}, r_{y} r_{x}^{2} t_{x}^{-1}, r_{y}^{2} t_{y}^{-1}\right\rangle$ & 1 & 13 & {$[[1,0,1],[0,2,0],[0,0,2]]$} \\
\hline$H_{99}=\left\langle r_{x}^{2} r_{y} t_{y}, r_{x}^{2} r_{y} t_{y}^{-1}, r_{y} r_{x}^{2} t_{x}, r_{y} r_{x}^{2} t_{x}^{-1}\right\rangle$ & 1 & 13 & {$[[1,0,1],[0,2,0],[0,0,2]]$} \\
\hline$H_{100}=\left\langle r_{x}^{2} t_{y}, r_{x}^{2} t_{y}^{-1}, r_{x}^{2} t_{z}, r_{x}^{2} t_{z}^{-1}, r_{y}^{2} r_{x} t_{x}, r_{y}^{2} r_{x} t_{x}^{-1}\right\rangle$ & 1 & 13 & {$[[2,0,0],[0,1,1],[0,0,2]]$} \\
\hline$H_{101}=\left\langle r_{x}^{2} r_{y} t_{y}^{-1}, r_{y} r_{x}^{2} t_{y}, r_{y}^{2} t_{x}, r_{y}^{2} t_{x}^{-1}, r_{y}^{2} t_{z}, r_{y}^{2} t_{z}^{-1}\right\rangle$ & 1 & 13 & {$[[1,0,1],[0,2,1],[0,0,2]]$} \\
\hline$H_{102}=\left\langle r_{x}^{2} r_{y} t_{x}^{-1}, r_{y} r_{x}^{2} t_{y}, r_{y} r_{x}^{2} t_{y}^{-1}, r_{y}^{2} t_{x}, r_{y}^{2} t_{z}\right\rangle$ & 1 & 13 & {$[[1,0,3],[0,1,2],[0,0,4]]$} \\
\hline$H_{103}=\left\langle m_{y} t_{y}, r_{x}^{2} t_{y}^{-1}, t_{x}, t_{z}^{-1}\right\rangle$ & 1 & 14 & {$[[1,0,0],[0,4,0],[0,0,1]]$} \\
\hline$H_{104}=\left\langle m_{x} t_{y}^{-1}, m_{z} t_{z}^{-1}, r_{y}^{2} t_{z}, t_{x}\right\rangle$ & 1 & 14 & {$[[1,0,0],[0,1,2],[0,0,4]]$} \\
\hline$H_{105}=\left\langle m_{x} t_{y}^{-1}, m_{y} t_{z}^{-1}, t_{x}\right\rangle$ & 1 & 14 & {$[[1,0,0],[0,2,0],[0,0,2]]$} \\
\hline$H_{106}=\left\langle m_{x} t^{-1}, m_{z} t_{z}, m_{z} t^{-1}, t_{x}\right\rangle$ & 1 & 14 & {$[[1,0,0],[0,2,0],[0,0,2]]$} \\
\hline$H_{107}=\left\langle m_{y} t_{z}^{-1}, r_{x}^{2} t_{y}, r_{x}^{2} t_{y}^{-1}, t_{x}\right\rangle$ & 1 & 14 & {$[[1,0,0],[0,2,0],[0,0,2]]$} \\
\hline$H_{108}=\left\langle m_{z} t_{z}, m_{z} t_{z}^{-1}, r_{x}^{2} t_{y}, r_{x}^{2} t_{y}^{-1}, t_{x}\right\rangle$ & 1 & 14 & {$[[1,0,0],[0,2,0],[0,0,2]]$} \\
\hline$H_{109}=\left\langle m_{y} t_{y}, m_{y} t_{y}^{-1}, r_{z}^{2} t_{z}^{-1}, t_{x}\right\rangle$ & 1 & 14 & {$[[1,0,0],[0,2,0],[0,0,2]]$} \\
\hline$H_{110}=\left\langle m_{y} t_{y}, m_{y} t_{y}^{-1}, m_{z} t_{z}, m_{z} t_{z}^{-1}, t_{x}\right\rangle$ & 1 & 14 & {$[[1,0,0],[0,2,0],[0,0,2]]$} \\
\hline$H_{111}=\left\langle m_{z} t_{y}^{-1}, r_{y}^{2} t_{z}, r_{y}^{2} t_{z}^{-1}, t_{x}\right\rangle$ & 1 & 14 & {$[[1,0,0],[0,2,0],[0,0,2]]$} \\
\hline$H_{112}=\left\langle m_{y} t_{z}^{-1}, m_{z} t_{y}^{-1}, t_{x}\right\rangle$ & 1 & 14 & {$[[1,0,0],[0,2,0],[0,0,2]]$} \\
\hline$H_{113}=\left\langle m_{z} t_{y}^{-1}, r_{y}^{2} t_{x}, r_{y}^{2} t_{x}^{-1}, t_{z}^{-1}\right\rangle$ & 1 & 14 & {$[[2,0,0],[0,2,0],[0,0,1]]$} \\
\hline$H_{114}=\left\langle m_{y} t_{x}, r_{z}^{2} t_{z}^{-1}, t_{y}^{-1}\right\rangle$ & 1 & 14 & {$[[2,0,0],[0,1,0],[0,0,2]]$} \\
\hline$H_{115}=\left\langle m_{y} t_{y}, m_{z} t_{z}, m_{z} t_{z}^{-1}, r_{x}^{2} t_{y}^{-1}, t_{x}\right\rangle$ & 1 & 14 & {$[[1,0,0],[0,2,1],[0,0,2]]$} \\
\hline$H_{116}=\left\langle m_{z} t_{y}^{-1}, r_{y}^{2} t_{x}, r_{y}^{2} t_{x}^{-1}, r_{y}^{2} t_{z}, r_{y}^{2} t_{z}^{-1}\right\rangle$ & 1 & 14 & {$[[1,0,1],[0,2,0],[0,0,2]]$} \\
\hline$H_{117}=\left\langle m_{z} t_{y}^{-1}, m_{z} t_{z}, m_{z} t_{z}^{-1}, r_{y}^{2} t_{x}, r_{y}^{2} t_{x}^{-1}\right\rangle$ & 1 & 14 & {$[[2,0,0],[0,1,1],[0,0,2]]$} \\
\hline$H_{118}=\left\langle m_{y} t_{x}, m_{y} t_{y}, m_{y} t_{y}^{-1}, r_{z}^{2} t_{z}^{-1}\right\rangle$ & 1 & 14 & {$[[1,1,0],[0,2,0],[0,0,2]]$} \\
\hline$H_{119}=\left\langle m_{x} t_{z}^{-1}, m_{y} t_{x}, m_{y} t_{y}, m_{y} t_{y}^{-1}\right\rangle$ & 1 & 14 & {$[[1,1,0],[0,2,0],[0,0,2]]$} \\
\hline$H_{120}=\left\langle m_{y} t_{x}, m_{y} t_{y}, m_{y} t_{y}^{-1}, m_{z} t_{z}, m_{z} t_{z}^{-1}\right\rangle$ & 1 & 14 & {$[[1,1,0],[0,2,0],[0,0,2]]$} \\
\hline$H_{121}=\left\langle m_{y} t_{x}, m_{y} t_{z}^{-1}, m_{z} t_{y}^{-1}\right\rangle$ & 1 & 14 & {$[[1,0,1],[0,2,0],[0,0,2]]$} \\
\hline$H_{122}=\left\langle m_{z} t_{x}, r_{y}^{2} t_{y}^{-1}, r_{y}^{2} t_{z}, r_{y}^{2} t_{z}^{-1}\right\rangle$ & 1 & 14 & {$[[2,0,0],[0,1,1],[0,0,2]]$} \\
\hline$H_{123}=\left\langle m_{z} t_{x}, m_{z} t_{y}^{-1}, r_{y}^{2} t_{z}, r_{y}^{2} t_{z}^{-1}\right\rangle$ & 1 & 14 & {$[[1,1,0],[0,2,0],[0,0,2]]$} \\
\hline$H_{124}=\left\langle m_{x} t_{x}, m_{x} t_{x}^{-1}, r_{z}^{2} t_{y}, r_{z}^{2} t_{y}^{-1}, r_{z}^{2} t_{z}^{-1}\right\rangle$ & 1 & 14 & {$[[2,0,0],[0,1,1],[0,0,2]]$} \\
\hline
\end{tabular}




\begin{tabular}{|c|c|c|c|}
\hline$H$ & $H_{0,0,0}$ & $P(H)$ & Translation basis of $H$ \\
\hline$H_{125}=\left\langle m_{x} t_{x}^{-1}, m_{z} t_{y}^{-1}, m_{z} t_{z}, m_{z} t_{z}^{-1}, r_{y}^{2} t_{x}\right\rangle$ & 1 & 14 & {$[[2,0,1],[0,1,1],[0,0,2]]$} \\
\hline$H_{126}=\left\langle m_{x} t_{z}^{-1}, m_{z} t_{y}^{-1}, r_{y}^{2} t_{x}, r_{y}^{2} t_{x}^{-1}\right\rangle$ & 1 & 14 & {$[[1,1,1],[0,2,0],[0,0,2]]$} \\
\hline$H_{127}=\left\langle m_{x} t_{z}^{-1}, m_{z} t_{x}, r_{y}^{2} t_{y}^{-1}\right\rangle$ & 1 & 14 & {$[[1,1,1],[0,2,0],[0,0,2]]$} \\
\hline$H_{128}=\left\langle m_{y} t_{y}, m_{y} t_{y}^{-1}, m_{z} t_{x}, r_{x}^{2} t\right.$ & 1 & 14 & {$[[1,1,1],[0,2,0],[0,0,2]]$} \\
\hline$H_{129}=\left\langle m_{x} t_{x}, m_{x} t_{x}^{-1}, m_{z} t_{z}\right.$ & 1 & 14 & {$[[1,1,1],[0,2,0],[0,0,2]]$} \\
\hline$H_{130}=\left\langle r_{y} t_{y}^{-1}, t_{x}, t_{z}^{-1}\right\rangle$ & 1 & 15 & {$[[1,0,0],[0,4,0],[0,0,1]]$} \\
\hline$H_{131}=\left\langle r_{x} t_{z}^{-1}, r_{x}^{-1} t_{y}^{-1}, t_{x}\right\rangle$ & 1 & 15 & {$[[1,0,0],[0,2,0],[0,0,2]]$} \\
\hline$H_{132}=\left\langle r_{x}^{-1} t_{x}, r_{x}^{2} t_{y}, r_{x}^{2} t_{y}^{-1}, r_{x}^{2} t_{z}, r_{x}^{2} t_{z}^{-1}\right\rangle$ & 1 & 15 & {$[[2,0,1],[0,1,1],[0,0,2]]$} \\
\hline$H_{133}=\left\langle r_{y}^{-1} t_{x}, r_{y}^{-1} t_{x}^{-1}, r_{y}^{2} t_{y}^{-1}\right\rangle$ & 1 & 15 & {$[[1,1,1],[0,2,0],[0,0,2]]$} \\
\hline$H_{134}=\left\langle m_{x} r_{x} t_{z}^{-1}, m_{x} r_{x}^{-}\right.$ & 1 & 16 & {$[[1,0,0],[0,2,0],[0,0,2]]$} \\
\hline$H_{135}=\left\langle m_{z} r_{z} t_{x}, m_{z} r_{z} t_{x}^{-}\right.$ & 1 & 16 & {$[[1,1,1],[0,2,0],[0,0,2]]$} \\
\hline$H_{136}=\left\langle r_{y} r_{x}^{2} t_{x}, r_{y} r_{x}^{2} t_{y}, r\right.$ & 1 & 19 & {$[[1,0,1],[0,1,1],[0,0,6]]$} \\
\hline$H_{137}=\left\langle i t_{y}^{-1}, i t_{z}, m_{z} r_{x}^{-1}\right.$ & 1 & 20 & {$[[2,0,0],[0,1,1],[0,0,4]]$} \\
\hline$H_{138}=\left\langle i t_{y}^{\frac{y}{y}}, i t_{z}, m_{z} r_{x} t_{z}^{-}\right.$ & 1 & 20 & {$[[2,0,0],[0,1,1],[0,0,4]]$} \\
\hline$H_{139}=\left\langle m_{z} r_{x} t_{z}^{-1}, m_{z} r_{x}^{-1} t_{x}\right.$ & 1 & 20 & {$[[2,0,0],[0,1,1],[0,0,4]]$} \\
\hline$H_{140}=\left\langle i t_{x}, i t_{y}^{-1}, r_{y}^{2} r_{z} t_{z}, r_{y}^{2} r_{z}\right.$ & 1 & 20 & {$[[1,1,0],[0,4,0],[0,0,2]]$} \\
\hline$H_{141}=\left\langle m_{x} r_{y} t_{x}^{-1}, r_{x}^{2} r_{y} t_{z}\right.$ & 1 & 20 & {$[[1,0,1],[0,2,0],[0,0,4]]$} \\
\hline$H_{142}=\left\langle m_{y} t_{y}^{-1}, r_{x}^{2} r_{y} t_{y}, r_{y}^{2} t_{x}\right.$ & 1 & 20 & {$[[1,0,1],[0,4,0],[0,0,2]]$} \\
\hline$H_{143}=\left\langle i t_{x}^{-1}, m_{x} t_{x}, m_{z} r\right.$ & 1 & 20 & {$[[4,0,0],[0,1,1],[0,0,2]]$} \\
\hline$H_{144}=\left\langle m_{x} t_{x}, m_{z} r_{x} t_{y}^{-1}\right.$, & 1 & 20 & {$[[4,0,0],[0,1,1],[0,0,2]]$} \\
\hline$H_{145}=\left\langle i t_{x}, r_{x}^{2} t_{y}, r_{x}^{2} t_{y}^{y}-1\right.$ & 1 & 20 & {$[[4,0,0],[0,1,1],[0,0,2]]$} \\
\hline$H_{146}=\left\langle i t_{x}, m_{z} r_{x}^{-1} t_{y}^{-1}\right.$, & 1 & 20 & {$[[4,0,0],[0,1,1],[0,0,2]]$} \\
\hline$H_{147}=\left\langle m_{x} r_{y} t_{x}, m_{x} r_{y} t_{x}\right.$ & 1 & 20 & {$[[1,0,1],[0,4,0],[0,0,2]]$} \\
\hline$H_{148}=\left\langle m_{x} r_{y} t_{y}^{-1}, r_{x}^{2} r_{y} t_{x}^{-1}\right.$, & 1 & 20 & {$[[1,0,3],[0,2,0],[0,0,4]]$} \\
\hline$H_{149}=\left\langle m_{y} t_{y}, m_{y} t_{y}^{-1}, r_{x}^{2} r_{y}\right.$ & 1 & 20 & {$[[1,0,3],[0,2,0],[0,0,4]]$} \\
\hline$H_{150}=\left\langle m_{x} r_{y}^{-1} t_{x}^{-1}{ }^{y}, r_{y} r_{x}^{2} t\right.$ & 1 & 20 & {$[[1,0,3],[0,2,0],[0,0,4]]$} \\
\hline$H_{151}=\left\langle m_{x} r_{y}^{-} 1_{t} t_{x}^{-1}, m_{y} t_{x}\right.$ & 1 & 20 & {$[[1,0,3],[0,2,0],[0,0,4]]$} \\
\hline$H_{152}=\left\langle i t_{x}, i t_{y}, r_{x}^{2} r_{z} t_{z}, r\right.$ & 1 & 20 & {$[[1,3,0],[0,4,0],[0,0,2]]$} \\
\hline$H_{153}=\left\langle i t_{x}, i t_{y}, m_{z} t_{z}, m_{z} t_{z}^{-1}, r_{y}^{2} r\right.$ & 1 & 20 & {$[[1,3,0],[0,4,0],[0,0,2]]$} \\
\hline$H_{154}=\left\langle i t_{x}, i t_{y}, m_{x} r_{z} t_{x}^{-1}, m_{z}\right.$ & 1 & 20 & {$[[1,3,0],[0,4,0],[0,0,2]]$} \\
\hline$H_{155}=\left\langle m_{x} r_{y}^{-1} t_{x}^{-1}, m_{y} t_{y}\right.$ & 1 & 20 & {$[[1,0,3],[0,2,2],[0,0,4]]$} \\
\hline$H_{156}=\left\langle i t_{y}, i t_{y}^{-1}, m_{z} t_{x}, r_{x}^{2} r_{z}\right.$ & 1 & 20 & {$[[1,1,2],[0,2,0],[0,0,4]]$} \\
\hline$H_{157}=\left\langle m_{x} t_{y}^{-1}, m_{z} t_{z}, r_{x}^{2} t_{z}^{-1}, t_{x}\right\rangle$ & 1 & 21 & {$[[1,0,0],[0,2,0],[0,0,4]]$} \\
\hline$H_{158}=\left\langle i t_{z}, m_{x} t_{y}^{-1}, m_{z} t_{z}^{-1}, t_{x}\right.$ & 1 & 21 & {$[[1,0,0],[0,2,0],[0,0,4]]$} \\
\hline$H_{159}=\left\langle i t_{y}, r_{x}^{2} t_{y}^{-1}, r_{y}^{2} t_{z}, r_{y}^{2} t_{z}^{-}\right.$ & 1 & 21 & {$[[1,0,0],[0,4,0],[0,0,2]]$} \\
\hline$H_{160}=\left\langle m_{y} t_{y}, r_{x}^{2} t_{y}^{-1}, r_{y}^{2} t_{z}, r_{y}^{2}\right.$ & 1 & 21 & {$[[1,0,0],[0,4,0],[0,0,2]]$} \\
\hline$H_{161}=\left\langle m_{x} t_{z}^{-1}, r_{x}^{2} t_{y}^{-1}, r_{z}^{2} t_{y}, t\right.$ & 1 & 21 & {$[[1,0,0],[0,4,0],[0,0,2]]$} \\
\hline$H_{162}=\left\langle i t_{y}, m_{z} t_{z}, m_{z} t_{z}^{-1}, r_{x}^{2} t\right.$ & 1 & 21 & {$[[1,0,0],[0,4,0],[0,0,2]]$} \\
\hline$H_{163}=\left\langle m_{z} t_{z}, m_{z} t_{z}^{-1}, r_{x}^{2} t_{y}^{-1}, v\right.$ & 1 & 21 & {$[[1,0,0],[0,4,0],[0,0,2]]$} \\
\hline$H_{164}=\left\langle i t_{z}^{-1}, m_{y} t_{y}, m_{y} t_{y}^{-1}, m_{z}\right.$ & 1 & 21 & {$[[1,0,0],[0,2,0],[0,0,4]]$} \\
\hline$H_{165}=\left\langle m_{y} t_{y}^{-1}, r_{y}^{2} t_{z}, r_{y}^{2} t_{z}^{y}, r_{z}^{2} t_{y}, t_{x}\right\rangle$ & 1 & 21 & {$[[1,0,0],[0,4,0],[0,0,2]]$} \\
\hline$H_{166}=\left\langle m_{y} t_{y}, m_{y} t_{y}^{-1}, m_{z} t_{z}, r_{y}^{2} t_{z}^{-1}, t_{x}\right\rangle$ & 1 & 21 & {$[[1,0,0],[0,2,0],[0,0,4]]$} \\
\hline$H_{167}=\left\langle i t_{y}, r_{x}^{2} t_{y}^{-1}, r_{y}^{2} t_{x}, r_{y}^{2} t_{x}^{-1}\right.$ & 1 & 21 & {$[[2,0,0],[0,4,0],[0,0,1]]$} \\
\hline$H_{168}=\left\langle i t_{z}^{-1}, m_{y} t_{x}, r_{x}^{2} t_{z}, t_{y}^{-1}\right\rangle$ & 1 & 21 & {$[[2,0,0],[0,1,0],[0,0,4]]$} \\
\hline$H_{169}=\left\langle m_{y} t_{y}^{-1}, m_{z} t_{z}, r_{y}^{2} t_{z}^{-1}, r\right.$ & 1 & 21 & {$[[1,0,0],[0,2,2],[0,0,4]]$} \\
\hline$H_{170}=\left\langle m_{y} t_{y}^{\frac{9}{y}}, r_{y}^{2} t_{x}, r_{y}^{2} t_{x}^{-1}, r_{y}^{2} t_{z}\right.$, & 1 & 21 & {$[[1,0,1],[0,4,0],[0,0,2]]$} \\
\hline$H_{171}=\left\langle m_{x} t_{x}^{-1}, m_{y} t_{y}, m_{y} t_{y}^{-1}, m_{y}\right.$ & 1 & 21 & {$[[4,0,0],[0,1,1],[0,0,2]]$} \\
\hline$H_{172}=\left\langle i t_{x}^{-1}, m_{x} t_{x}, m_{y} t_{y}, m_{y}\right.$ & 1 & 21 & {$[[4,0,0],[0,1,1],[0,0,2]]$} \\
\hline$H_{173}=\left\langle i t_{z}^{-1}, m_{y} t_{x}, m_{y} t_{y}, m_{y}\right.$ & 1 & 21 & {$[[1,1,0],[0,2,0],[0,0,4]]$} \\
\hline$H_{174}=\left\langle m_{y} t_{x}, m_{y} t_{y}, m_{y} t_{y}^{-1}\right.$, & 1 & 21 & {$[[1,1,0],[0,2,0],[0,0,4]]$} \\
\hline$H_{175}=\left\langle i t_{x}, r_{x}^{2} t_{y}, r_{x}^{2} t_{y}^{-1}, r_{x}^{2} t_{z}\right.$ & 1 & 21 & {$[[4,0,0],[0,1,1],[0,0,2]]$} \\
\hline$H_{176}=\left\langle m_{z} t_{z}, m_{z} t_{z}^{-1}, r_{x}^{2} t_{y}^{-1}, r_{y}^{2} t_{x}, r_{y}^{2} t_{x}^{-1}, r_{z}^{2} t_{y}\right\rangle$ & 1 & 21 & {$[[1,2,0],[0,4,0],[0,0,2]]$} \\
\hline$H_{177}=\left\langle i t_{y}, m_{x} t_{z}^{-1}, m_{y} t_{y}^{-1}, r_{y}^{2} t_{x}, r_{y}^{2} t_{x}^{-1}\right\rangle$ & 1 & 21 & {$[[1,2,0],[0,4,0],[0,0,2]]$} \\
\hline$H_{178}=\left\langle i t_{y}, m_{y} t_{y}^{-1}, m_{z} t_{z}, m_{z} t\right.$ & 1 & 21 & {$[[1,2,0],[0,4,0],[0,0,2]]$} \\
\hline$H_{179}=\left\langle i t_{y}^{-1}, m_{z} t_{x}, r_{y}^{2} t_{z}\right.$ & 1 & 21 & {$[[1,2,0],[0,4,0],[0,0,2]]$} \\
\hline$H_{180}=\left\langle i t_{y}^{-1}, m_{x} t_{z}^{-1}, m_{z} t_{x}, r_{z}^{2}\right.$ & 1 & 21 & {$[[1,2,0],[0,4,0],[0,0,2]]$} \\
\hline$H_{181}=\left\langle i t_{y}, m_{z} t_{z}, m_{z} t_{z}^{-1}, r_{x}^{2} t\right.$ & 1 & 21 & {$[[1,2,1],[0,4,0],[0,0,2]]$} \\
\hline$H_{182}=\left\langle m_{x} t_{z}^{-1}, m_{y} t_{y}^{-1}, r_{x}^{2} t_{y}\right.$, & 1 & 21 & {$[[1,2,1],[0,4,0],[0,0,2]]$} \\
\hline$H_{183}=\left\langle m_{y} t_{y}^{-1}, m_{z} t_{z}, m_{z} t_{z}^{-1}, r_{y}^{2} t_{x}, r_{y}^{2} t_{x}^{-1}, r_{z}^{2} t_{y}\right\rangle$ & 1 & 21 & {$[[1,2,1],[0,4,0],[0,0,2]]$} \\
\hline$H_{184}=\left\langle i t_{y}^{-1}, m_{z} t_{x}, r_{x}^{2} t_{y}, r_{y}^{2} t_{z}, r_{y}^{2} t_{z}^{-1}\right\rangle$ & 1 & 21 & {$[[1,2,1],[0,4,0],[0,0,2]]$} \\
\hline$H_{185}=\left\langle m_{x} t_{z}^{-1}, m_{z} t_{x}, r_{x}^{2} t_{y}, r_{z}^{2} t_{y}^{-1}\right\rangle$ & 1 & 21 & {$[[1,2,1],[0,4,0],[0,0,2]]$} \\
\hline$H_{186}=\left\langle m_{x} t_{x}, m_{x} t_{x}^{-1}, m_{z} t_{z}, m_{z} t_{z}^{-1}, r_{x}^{2} t_{y}, r_{z}^{2} t_{y}^{-1}\right\rangle$ & 1 & 21 & {$[[1,2,1],[0,4,0],[0,0,2]]$} \\
\hline$H_{187}=\left\langle m_{y} t_{z}^{-1}, r_{x}^{2} t_{y}, r_{x}^{2} t_{y}^{-1}, r_{y}^{2} t_{x}, r_{y}^{2} t_{x}^{-1}\right\rangle$ & 1 & 21 & {$[[2,0,0],[0,2,0],[0,0,2]]$} \\
\hline$H_{188}=\left\langle m_{z} t_{z}, m_{z} t_{z}^{-1}, r_{x}^{2} t_{y}, r_{x}^{2} t_{y}^{-1}, r_{y}^{2} t_{x}, r_{y}^{2} t_{x}^{-1}\right\rangle$ & 1 & 21 & {$[[2,0,0],[0,2,0],[0,0,2]]$} \\
\hline$H_{189}=\left\langle m_{y} t_{y}, m_{y} t_{y}^{-1}, r_{y}^{2} t_{x}, r_{y}^{2} t_{x}^{-1}, r_{z}^{2} t_{z}^{-1}\right\rangle^{y}$ & 1 & 21 & {$[[2,0,0],[0,2,0],[0,0,2]]$} \\
\hline$H_{190}=\left\langle m_{x} t_{z}^{-1}, m_{y} t_{y}, m_{y} t_{y}^{-1}, r_{y}^{2} t_{x}, r_{y}^{2} t_{x}^{-1}\right\rangle$ & 1 & 21 & {$[[2,0,0],[0,2,0],[0,0,2]]$} \\
\hline$H_{191}=\left\langle m_{y} t_{y}, m_{y} t_{y}^{-1}, r_{x}^{2} t_{z}, r_{x}^{2} t_{z}^{\frac{y}{1}}, r_{y}^{2} t_{x}, r_{y}^{2} t_{x}^{-1}\right\rangle$ & 1 & 21 & {$[[2,0,0],[0,2,0],[0,0,2]]$} \\
\hline
\end{tabular}




\begin{tabular}{|c|c|c|c|}
\hline$H$ & $H_{0,0,0}$ & $P(H)$ & Translation basis of $H$ \\
\hline$H_{192}=\left\langle m_{y} t_{y}, m_{y} t_{y}^{-1}, m_{z} t_{z}, m_{z} t_{z}^{-1}, r_{y}^{2} t_{x}, r_{y}^{2} t_{x}^{-1}\right\rangle$ & 1 & 21 & {$[[2,0,0],[0,2,0],[0,0,2]]$} \\
\hline$H_{193}=\left\langle i t_{z}, i t_{z}^{-1}, m_{z} t_{y}^{-1}, r_{y}^{2} t_{x}, r_{y}^{2} t_{x}^{-1}\right\rangle^{y}$ & 1 & 21 & {$[[2,0,0],[0,2,0],[0,0,2]]$} \\
\hline$H_{194}=\left\langle m_{z} t_{y}^{-1}, r_{y}^{2} t_{x}, r_{y}^{2} t_{x}^{-1}, r_{z}^{2} t_{z}^{-1}\right\rangle$ & 1 & 21 & {$[[2,0,0],[0,2,0],[0,0,2]]$} \\
\hline$H_{195}=\left\langle m_{z} t_{y}^{-1}, r_{x}^{2}\right.$ & 1 & 21 & {$[[2,0,0],[0,2,0],[0,0,2]]$} \\
\hline$H_{196}=\left\langle m_{y} t_{z}^{\frac{9}{z}}, m\right.$ & 1 & 21 & $[[2,0,0],[0,2,0],[0,0,2]]]$ \\
\hline$H_{197}=\left\langle i t_{z}, i t_{z}^{-1}, m_{y} t_{x}\right.$ & 1 & 21 & {$[[2,0,0],[0,2,0],[0,0,2]]$} \\
\hline$H_{198}=\left\langle m_{y} t_{x}, m_{z}\right.$ & 1 & 21 & {$[[2,0,0],[0,2,0],[0,0,2]]$} \\
\hline$H_{199}=\left\langle m_{y} t_{x}, m_{z} t_{y}^{-1}, r_{y}^{2} t_{z}, r_{y}^{2} t_{z}^{-1}\right\rangle$ & 1 & 21 & {$[[2,0,0],[0,2,0],[0,0,2]]$} \\
\hline$H_{200}=\left\langle m_{x} t_{z}^{-1}, m_{y} t_{x}, m_{z} t_{y}^{-1}\right\rangle$ & 1 & 21 & {$[[2,0,0],[0,2,0],[0,0,2]]$} \\
\hline$H_{201}=\left\langle i t_{y}, i t_{y}^{-1}, m_{z} t_{x}\right.$ & 1 & 21 & {$[[2,0,0],[0,2,0],[0,0,2]]$} \\
\hline$H_{202}=\left\langle i t_{y}, i t_{y}^{-1}\right.$, & 1 & 21 & {$[[2,0,0],[0,2,0],[0,0,2]]$} \\
\hline$H_{203}=\left\langle i t_{y}, i t_{y}^{-1}\right.$, & 1 & 21 & {$[[2,0,0],[0,2,0],[0,0,2]]$} \\
\hline$H_{204}=\left\langle m_{z} t_{x}, r_{x}^{2} t\right.$ & 1 & 21 & $[[2,0,0],[0,2,0],[0,0,2]]]$ \\
\hline$H_{205}=\left\langle i t_{z}, i t_{z}^{-1}\right.$ & 1 & 21 & {$[[2,0,0],[0,2,0],[0,0,2]]$} \\
\hline$H_{206}=\left\langle m_{z} t_{x}, r_{y}^{2} t\right.$ & 1 & 21 & {$[[2,0,0],[0,2,0],[0,0,2]]$} \\
\hline$H_{207}=\left\langle m_{z} t_{x}, r_{x}^{2} t_{z}, r_{x}^{2} t_{z}^{-1}, r_{y}^{2} t_{y}^{-1}\right\rangle$ & 1 & 21 & {$[[2,0,0],[0,2,0],[0,0,2]]$} \\
\hline$H_{208}=\left\langle i t_{z}, i t_{z}^{-1}, m_{y} t_{y}, m_{y} t_{y}^{-1}, m_{z} t_{x}\right\rangle$ & 1 & 21 & {$[[2,0,0],[0,2,0],[0,0,2]]$} \\
\hline$H_{209}=\left\langle m_{y} t_{y}, m_{y} t_{y}^{-1}\right.$, & 1 & 21 & {$[[2,0,0],[0,2,0],[0,0,2]]$} \\
\hline$H_{210}=\left\langle m_{x} t_{z}^{-1}, m_{y} t_{y}\right.$, & 1 & 21 & {$[[2,0,0],[0,2,0],[0,0,2]]$} \\
\hline$H_{211}=\left\langle i t_{x}, i t_{x}^{-1}\right.$, & 1 & 21 & {$[[2,0,0],[0,2,0],[0,0,2]]$} \\
\hline$H_{212}=\left\langle i t_{x}, i t_{x}^{-1}\right.$, & 1 & 21 & {$[[2,0,0],[0,2,0],[0,0,2]]$} \\
\hline$H_{213}=\left\langle i t_{x}, i t_{x}^{-1}\right.$, & 1 & 21 & {$[[2,0,0],[0,2,0],[0,0,2]]$} \\
\hline$H_{214}=\left\langle i t_{x}, i t_{x}^{-1}\right.$, & 1 & 21 & $[[2,0,0],[0,2,0],[0,0,2]]]$ \\
\hline$H_{215}=\left\langle i t_{x}, i t_{x}^{-1}\right.$, & 1 & 21 & {$[[2,0,0],[0,2,0],[0,0,2]]$} \\
\hline$H_{216}=\left\langle i t_{x}, i t_{x}^{-1}\right.$, & 1 & 21 & $[[2,0,0],[0,2,0],[0,0,2]]]$ \\
\hline$H_{217}=\left\langle i t_{x}, i t_{x}^{-1}\right.$, & 1 & 21 & {$[[2,0,0],[0,2,0],[0,0,2]]$} \\
\hline$H_{218}=\left\langle m_{x} t_{x}, m_{x}\right.$ & 1 & 21 & {$[[2,0,0],[0,2,0],[0,0,2]]$} \\
\hline$\left.y_{y} t_{y}, m_{y} t_{y}^{-1}, m_{z} t_{z}, m_{z} t_{z}^{-1}\right\rangle$ & 1 & 21 & $[[2,0,0],[0,2,0],[0,0,2]]]$ \\
\hline$\left.z^{-1}, r_{x}^{2} t_{y}^{-1}, r_{y}^{2} t_{x}, r_{y}^{2} t_{x}^{-1}\right\rangle$ & 1 & 21 & {$[[2,0,0],[0,2,1],[0,0,2]]$} \\
\hline$H_{221}=\left\langle m_{x} t_{z}^{-1}, n\right.$ & 1 & 21 & $[[2,0,0],[0,2,1],[0,0,2]]]$ \\
\hline$H_{222}=\left\langle m_{x} t_{x}^{-1}, m_{y} t_{y}, m_{y} t_{y}^{-1}, m_{z} t_{z}, m_{z} t_{z}^{-1}, r_{y}^{2} t_{x}\right\rangle$ & 1 & 21 & {$[[2,0,1],[0,2,0],[0,0,2]]$} \\
\hline$H_{223}=\left\langle m_{x} t_{x}^{-1}, m_{y}\right.$ & 1 & 21 & {$[[2,1,0],[0,2,0],[0,0,2]]$} \\
\hline$H_{224}=\left\langle m_{z} t_{x}, r_{x}^{2} t_{y}\right.$, & 1 & 21 & $[[2,0,0],[0,2,1],[0,0,2]]]$ \\
\hline$H_{225}=\left\langle i t_{x}, m_{z} t_{z}, m_{z} t\right.$ & 1 & 21 & {$[[2,0,1],[0,2,0],[0,0,2]]$} \\
\hline$H_{226}=\left\langle i t_{x}, m_{y} t_{y}, m_{y} t_{y}^{-1}\right.$ & 1 & 21 & {$[[2,0,1],[0,2,0],[0,0,2]]$} \\
\hline$H_{227}=\left\langle i t_{x}^{-1}, m_{x} t_{x}, m_{y} t_{y}\right.$ & 1 & 21 & {$[[2,1,1],[0,2,0],[0,0,2]]$} \\
\hline$H_{228}=\left\langle i t_{x}^{-1}, m_{x} t_{x}, m_{y} t_{z}^{-1}, m_{z} t_{y}^{-1}\right\rangle$ & 1 & 21 & {$[[2,1,1],[0,2,0],[0,0,2]]$} \\
\hline$H_{229}=\left\langle m_{x} t_{x}^{-1}, m_{y} t_{y}, m_{z} t_{z}, m_{z} t_{z}^{-1}, r_{x}^{2} t_{y}^{-1}, r_{y}^{2} t_{x}\right\rangle$ & 1 & 21 & $[[2,0,1],[0,2,1],[0,0,2]]]$ \\
\hline$H_{230}=\left\langle m_{x} r_{x} t_{z}^{-1}, r_{x}^{-1} t_{y}^{-1}, t_{x}\right\rangle$ & 1 & 22 & {$[[1,0,0],[0,2,2],[0,0,4]]$} \\
\hline$H_{231}=\left\langle m_{x} r_{x}^{-1} t_{y}^{-1}, m_{x} t_{x}, m_{x} t_{x}^{-1}\right.$, & 1 & 22 & {$[[1,0,2],[0,2,2],[0,0,4]]$} \\
\hline$H_{232}=\left\langle i t_{x}, i t_{x}^{-1}, m_{x} r_{x} t_{z}^{-1}, m_{x} r_{x}^{-1} t_{y}^{-1}\right\rangle$ & 1 & 22 & {$[[2,0,0],[0,2,0],[0,0,2]]$} \\
\hline$H_{233}=\left\langle i t_{x}, i t_{x}^{-1}, r_{x} t_{z}^{-}\right.$ & 1 & 22 & {$[[2,0,0],[0,2,0],[0,0,2]]$} \\
\hline$H_{234}=\left\langle m_{x} r_{x} t_{z}^{-1}\right.$ & 1 & 22 & {$[[2,0,0],[0,2,0],[0,0,2]]$} \\
\hline$H_{235}=\left\langle m_{y} t_{y}, m_{y} t_{y}^{-1}\right.$ & 1 & 22 & {$[[2,0,0],[0,2,0],[0,0,2]]$} \\
\hline$H_{236}=\left\langle i t_{x}^{-1}, m_{x} t\right.$ & 1 & 22 & {$[[2,1,1],[0,2,0],[0,0,2]]$} \\
\hline$H_{237}=\left\langle i t_{y}, i t_{y}^{-1}\right.$, & 1 & 22 & {$[[2,1,1],[0,2,0],[0,0,2]]$} \\
\hline$H_{238}=\left\langle m_{x} r_{x} t_{z}^{-1}, m_{x} r_{x}^{-1} t_{y}^{-1}, r_{x}^{-1} t_{x}\right\rangle$ & 1 & 22 & {$[[2,1,1],[0,2,0],[0,0,2]]$} \\
\hline$H_{239}=\left\langle m_{x} r_{x} t_{z}^{-1}, m_{y} t_{y}^{-1}, m_{z} t_{z}, t_{x}\right\rangle$ & 1 & 23 & {$[[1,0,0],[0,2,2],[0,0,4]]$} \\
\hline$H_{240}=\left\langle m_{x} t_{z}^{-1}, m_{z} t_{x}, r_{x}^{2} r_{y} t_{y}^{-1}, r_{y} r_{x}^{2} t_{y}\right\rangle$ & 1 & 23 & {$[[1,2,1],[0,4,0],[0,0,2]]$} \\
\hline$H_{241}=\left\langle m_{x} t_{x}, m_{x} t_{x}^{-1}, m_{z} t_{z}, m_{z} t_{z}^{-1}, r_{x}^{2} r_{y} t_{y}^{-1}, r_{y} r_{x}^{2} t_{y}\right\rangle$ & 1 & 23 & {$[[1,2,1],[0,4,0],[0,0,2]]$} \\
\hline$H_{242}=\left\langle m_{x} t_{z}^{-1}, m_{z} t_{x}, r_{x}^{2} r_{y} t_{y}, r_{x}^{2} r_{y} t_{y}^{-1}\right\rangle$ & 1 & 23 & {$[[2,0,0],[0,2,0],[0,0,2]]$} \\
\hline$H_{243}=\left\langle m_{x} t_{x}, m_{x} t_{x}^{-1}, m_{z} t_{z}, m_{z} t_{z}^{-1}, r_{x}^{2} r_{y} t_{y}, r_{x}^{2} r_{y} t_{y}^{-1}\right\rangle$ & 1 & 23 & {$[[2,0,0],[0,2,0],[0,0,2]]$} \\
\hline$H_{244}=\left\langle m_{z} r_{z} t_{x}, m_{z} r_{z} t_{x}^{-}\right.$ & 1 & 23 & {$[[2,0,0],[0,2,0],[0,0,2]]$} \\
\hline$H_{245}=\left\langle m_{z} r_{z} t_{x}, m_{z} r_{z} t_{x}^{\frac{\alpha}{x}}\right.$ & 1 & 23 & {$[[2,0,0],[0,2,0],[0,0,2]]$} \\
\hline$H_{246}=\left\langle m_{x} t_{x}^{-1}, m_{y} t_{y}^{-1}, m_{z} r_{z} t_{x}, r_{x}^{2} r_{z} t_{z}, r_{x}^{2} r_{z} t_{z}^{-1}\right\rangle$ & 1 & 23 & {$[[2,0,1],[0,2,1],[0,0,2]]$} \\
\hline$H_{247}=\left\langle r_{x}^{2} r_{y} t_{y}^{-1}, r_{z}^{2} t_{y}, t_{x}, t_{z}^{-1}\right\rangle$ & 1 & 24 & {$[[1,0,0],[0,8,0],[0,0,1]]$} \\
\hline$H_{248}=\left\langle r_{x}^{-1} t_{y}^{-1}, r_{y}^{2} t_{z}^{-1}, r_{z}^{2} t_{y}, t_{x}\right\rangle$ & 1 & 24 & {$[[1,0,0],[0,2,2],[0,0,4]]$} \\
\hline$H_{249}=\left\langle r_{x}^{-1} t_{x}, r_{z}^{2} t_{y}, r_{z}^{2} t_{y}^{-1}, r_{z}^{2} t_{z}^{-1}\right\rangle$ & 1 & 24 & {$[[4,0,0],[0,1,1],[0,0,2]]$} \\
\hline$\left.{ }^{1} t_{x}, r_{z}^{2} r_{x} t_{y}^{-1}, r_{z}^{2} r_{x} t_{z}\right\rangle$ & 1 & 24 & {$[[4,0,0],[0,1,1],[0,0,2]]$} \\
\hline$H_{251}=\left\langle r_{x}^{2} t_{y}, r_{y} r_{x}^{2} t_{x}\right.$ & 1 & 24 & {$[[1,0,1],[0,4,0],[0,0,2]]$} \\
\hline$\left.z^{-1}, r_{y}^{2} t_{x}, r_{y}^{2} t_{x}^{\frac{9}{1}}, r_{y}^{2} t_{y}^{-1}\right\rangle$ & 1 & 24 & {$[[1,1,0],[0,2,0],[0,0,4]]$} \\
\hline$H_{253}=\left\langle r_{x}^{2} t_{y}, r_{x}^{2} t_{y}^{-1}, r_{x}^{2} t_{z}, r_{x}^{2} t_{z}^{-1}, r_{y}^{2} r_{x} t_{x}, r_{y}^{2} t_{x}^{-1}\right\rangle$ & 1 & 24 & {$[[4,0,1],[0,1,1],[0,0,2]]$} \\
\hline$H_{254}=\left\langle r_{x}^{2} r_{y} t_{y}^{-1}, r_{y} r_{x}^{2} t_{y}, r_{y}^{-1} t_{x}, r_{y}^{-1} t_{x}^{-1}\right\rangle$ & 1 & 24 & {$[[1,2,1],[0,4,0],[0,0,2]]$} \\
\hline$H_{255}=\left\langle r_{x}^{2} t_{y}, r_{x}^{2} t_{y}^{-1}, r_{y}^{2} r_{z} t_{z}, r_{y}^{2} r_{z} t_{z}^{-1}, r_{y}^{2} t_{x}, r_{y}^{2} t_{x}^{-1}\right\rangle$ & 1 & 24 & $[[2,0,0],[0,2,0],[0,0,2]]]$ \\
\hline$H_{256}=\left\langle r_{x}^{2} r_{y} t_{y}, r_{x}^{2} r_{y} t_{y}^{-1}, r_{y}^{-1} t_{x}, r_{y}^{-1} t_{x}^{-1}\right\rangle$ & 1 & 24 & {$[[2,0,0],[0,2,0],[0,0,2]]$} \\
\hline$H_{257}=\left\langle r_{y} r_{x}^{2} t_{y}, r_{y} r_{x}^{2} t_{y}^{-1}, r_{y}^{-1} t_{x}, r_{y}^{-1} t_{x}^{-1}\right\rangle$ & 1 & 24 & {$[[2,0,0],[0,2,0],[0,0,2]]$} \\
\hline$H_{258}=\left\langle r_{y}^{2} r_{x} t_{x}, r_{y}^{2} r_{x} t_{x}^{-1}, r_{y}^{2} t_{y}^{-1}, r_{z}^{2} t_{z}^{-1}\right\rangle$ & 1 & 24 & {$[[2,0,0],[0,2,0],[0,0,2]]$} \\
\hline
\end{tabular}




\begin{tabular}{|c|c|c|c|}
\hline$H$ & $H_{0,0,0}$ & $P(H)$ & Translation basis of $H$ \\
\hline$H_{259}=\left\langle r_{x}^{2} t_{y}^{-1}, r_{y}^{2} r_{z} t_{z}, r_{y}^{2} r_{z} t_{z}^{-1}, r_{y}^{2} t_{x}, r_{z}^{-1} t_{x}^{-1}\right\rangle$ & 1 & 24 & {$[[2,0,1],[0,2,1],[0,0,2]]$} \\
\hline$H_{260}=\left\langle m_{y} t_{y}^{-1}, m_{z} t_{z}, r_{x} t_{z}^{-1}, t_{x}\right\rangle$ & 1 & 25 & $[[1,0,0],[0,2,2],[0,0,4]]]$ \\
\hline$H_{261}=\left\langle m_{y} t_{y}^{-1}, m_{z} r_{x}^{-1} t_{x}, m_{z} t_{z}, r_{x} t_{z}^{-1}\right\rangle$ & 1 & 25 & $[[1,0,2],[0,2,2],[0,0,4]]]$ \\
\hline$H_{262}=\left\langle m_{y} t_{y}, m_{y} t_{y}^{-1}, m_{z} r_{x}^{-1} t_{x}, m_{z} t_{z}, m_{z} t_{z}^{-1}\right\rangle$ & 1 & 25 & {$[[2,0,0],[0,2,0],[0,0,2]]$} \\
\hline$H_{263}=\left\langle m_{z} r_{x}^{-1} t_{x}, r_{x}\right.$ & 1 & 25 & {$[[2,0,0],[0,2,0],[0,0,2]]$} \\
\hline$H_{264}=\left\langle m_{z} r_{x}^{-1} t_{x}\right.$ & 1 & 25 & $[[2,0,0],[0,2,0],[0,0,2]]]$ \\
\hline$H_{265}=\left\langle m_{y} t_{z}^{-1}, m_{z} r_{x}^{-1} t_{x}, m_{z} t_{y}^{-1}\right\rangle$ & 1 & 25 & {$[[2,0,0],[0,2,0],[0,0,2]]$} \\
\hline$H_{266}=\left\langle m_{y} t_{y}, m_{y} t_{y}^{-1}, m_{z} t_{z}, m_{z} t_{z}^{-1}, r_{x}^{-1} t_{x}\right\rangle$ & 1 & 25 & $[[2,1,1],[0,2,0],[0,0,2]]]$ \\
\hline$H_{267}=\left\langle m_{y} t_{z}^{-1}, m_{z} t\right.$ & 1 & 25 & {$[[2,1,1],[0,2,0],[0,0,2]]$} \\
\hline$H_{268}=\left\langle m_{z} r_{z}^{-1} t_{x}^{-1}\right.$ & 1 & 26 & {$[[2,2,0],[0,4,0],[0,0,1]]$} \\
\hline$H_{269}=\left\langle m_{z} r_{z} t_{x}, m_{z}\right.$ & 1 & 26 & {$[[1,1,2],[0,2,0],[0,0,4]]$} \\
\hline$H_{270}=\left\langle m_{x} r_{z} t_{z}^{-1}, r_{x}^{2} t_{y}, r_{x}^{2} t_{y}^{-1}, r_{y}^{2} t_{x}, r_{y}^{2} t_{x}^{-1}\right\rangle$ & 1 & 26 & {$[[2,0,0],[0,2,0],[0,0,2]]$} \\
\hline$H_{271}=\left\langle m_{x} r_{x} t \frac{-1}{z}, m_{x}\right.$ & 1 & 26 & $[[2,0,0],[0,2,0],[0,0,2]]]$ \\
\hline$H_{272}=\left\langle m_{x} r_{x} t_{y}^{-1}\right.$, & 1 & 26 & $[[2,0,0],[0,2,0],[0,0,2]]]$ \\
\hline$H_{273}=\left\langle m_{z} r_{x}^{-1} t_{x}\right.$ & 1 & 26 & $[[2,0,0],[0,2,0],[0,0,2]]]$ \\
\hline$\left.{ }^{1} t_{x}^{-1}, r_{x}^{2} t_{y}^{-1}, r_{y}^{2} t_{x}\right\rangle$ & 1 & 26 & $[[2,0,1],[0,2,1],[0,0,2]]]$ \\
\hline$H_{275}=\left\langle m_{x} t_{z}^{-1}, m\right.$ & 1 & 29 & {$[[1,4,1],[0,8,0],[0,0,2]]$} \\
\hline$\left.z t_{z}, m_{z} t_{z}^{-1}, r_{x}^{2} r_{y} t_{y}, r_{z}^{2} t_{y}^{-1}\right\rangle$ & 1 & 29 & {$[[1,4,1],[0,8,0],[0,0,2]]$} \\
\hline$\left.{ }^{t}{ }_{y}^{-1}, m_{z} t_{z}, m_{z} t_{z}^{-1}, r_{z}^{2} r_{x} t_{x}^{-1}\right\rangle$ & 1 & 29 & $[[4,0,0],[0,2,0],[0,0,2]]]$ \\
\hline$H_{278}=\left\langle m_{x} t_{x}, r_{x} t_{z} \bar{z}\right.$ & 1 & 29 & {$[[4,0,0],[0,2,0],[0,0,2]]$} \\
\hline$H_{279}=\left\langle m_{x} t_{x}, r_{x} t_{y}^{-}\right.$ & 1 & 29 & {$[[4,0,0],[0,2,0],[0,0,2]]$} \\
\hline$H_{280}=\left\langle m_{x} t_{x}, m_{y} t_{z}^{-1}\right.$ & 1 & 29 & {$[[4,0,0],[0,2,0],[0,0,2]]$} \\
\hline$H_{281}=\left\langle i t y_{y}^{-1}, m_{x} t_{z}^{-1}, r\right.$ & 1 & 29 & {$[[2,0,0],[0,4,0],[0,0,2]]$} \\
\hline$H_{282}=\left\langle i t_{x}, m_{y} t_{y}, m_{y} t_{y}^{-1}, m_{z} t_{z}, m_{z} t_{z}^{-1}, r_{z}^{2} r_{x} t_{x}^{-1}\right\rangle$ & 1 & 29 & $[[4,0,0],[0,2,0],[0,0,2]]]$ \\
\hline$H_{283}=\left\langle i t_{x}, r_{x} t_{z}^{-1}\right.$, & 1 & 29 & $[[4,0,0],[0,2,0],[0,0,2]]]$ \\
\hline$H_{284}=\left\langle i t_{x}, r_{x} t \frac{1}{y}\right.$ & 1 & 29 & $[[4,0,0],[0,2,0],[0,0,2]]]$ \\
\hline$\left.{ }_{x}^{2} t_{y}^{-1}, r_{y}^{2} r_{z} t_{z}, r_{y}^{2} r_{z} t_{z}^{-1}, r_{y}^{2} t_{x}\right\rangle$ & 1 & 29 & {$[[2,2,0],[0,4,0],[0,0,2]]$} \\
\hline $\left.1, m_{y} t_{y}, r_{x}^{2} t_{y}^{-1}, r_{y}^{2} t_{x}\right\rangle$ & 1 & 29 & $[[2,2,0],[0,4,0],[0,0,2]]]$ \\
\hline $\left.1, r_{y}^{2} r_{z} t_{z}, r_{y}^{2} r_{z} t_{z}^{-1}, r_{y}^{2} t_{x}\right\rangle$ & 1 & 29 & {$[[2,2,0],[0,4,0],[0,0,2]]$} \\
\hline$\left.z, m_{z} t_{z}^{-1}, r_{x}^{2} t_{y}^{-1}, r_{y}^{2} t_{x}\right\rangle$ & 1 & 29 & {$[[2,2,0],[0,4,0],[0,0,2]]$} \\
\hline$H_{289}=\left\langle m_{x} r_{z} t_{z}^{-1}, r_{x}^{2}\right.$ & 1 & 29 & {$[[2,2,0],[0,4,0],[0,0,2]]$} \\
\hline$\left.{ }_{x}^{2} t_{y}^{-1}, r_{y}^{2} t_{x}, r_{z}^{-1} t_{x}^{-1}\right\rangle$ & 1 & 29 & {$[[2,2,0],[0,4,0],[0,0,2]]$} \\
\hline$H_{291}=\left\langle m_{x} r_{x}^{-1} t_{y}^{-1}\right.$, & 1 & 29 & {$[[2,0,0],[0,2,2],[0,0,4]]$} \\
\hline$\left.t_{y}, m_{z} r_{x}^{-1} t_{x}, m_{z} t_{z}^{-1}\right\rangle$ & 1 & 29 & {$[[2,0,0],[0,2,2],[0,0,4]]$} \\
\hline$H_{293}=\left\langle m_{x} r_{x}^{-1} t_{y}^{\frac{y}{1}}, m_{x} t_{x}, m_{x} t_{x}^{-1}, m_{y} t_{y}, m_{z} t_{z}^{-1}\right\rangle$ & 1 & 29 & {$[[2,0,0],[0,2,2],[0,0,4]]$} \\
\hline$\left.r_{y} r_{x}^{2} t_{y}, r_{y} r_{x}^{2} t_{y}^{-1}, r_{y}^{-1} t_{x}\right\rangle$ & 1 & 29 & {$[[2,0,2],[0,2,0],[0,0,4]]$} \\
\hline$\left.{ }_{y} t_{y}^{-1}, m_{z} t_{z}^{-1}, r_{y}^{-1} t_{x}\right\rangle$ & 1 & 29 & {$[[2,0,2],[0,2,0],[0,0,4]]$} \\
\hline $\left.1, r_{y}^{-1} m_{y} t_{x}^{-1}, r_{y}^{-1} t_{x}\right\rangle$ & 1 & 29 & $[[2,0,2],[0,2,0],[0,0,4]]]$ \\
\hline$H_{297}=\left\langle i t_{z}^{-1}, m_{z} r_{z}^{-1} t_{x}^{-1}, r_{x}^{2} r_{z} t_{z}, r_{x}^{2} t_{y}^{-1}, r_{y}^{2} t_{x}\right\rangle$ & 1 & 29 & {$[[2,0,2],[0,2,2],[0,0,4]]$} \\
\hline$H_{298}=\left\langle m_{x} t_{x}, m_{y} t_{y}^{-1}, m_{z} t_{z}, r_{x} t_{z}^{-1}, r_{z}^{2} r_{x} t_{x}^{-1}\right\rangle$ & 1 & 29 & {$[[2,0,2],[0,2,2],[0,0,4]]$} \\
\hline$H_{299}=\left\langle i, i t_{x}, i t_{y}^{-1}, i t\right.$ & 2 & 2 & {$[[1,0,0],[0,1,0],[0,0,1]]$} \\
\hline$H_{300}=\left\langle i, i t_{x}, i t_{y}^{-1}\right.$, & 2 & 9 & {$[[1,0,0],[0,1,0],[0,0,2]]$} \\
\hline$H_{301}=\left\langle i, i t_{x}, i t_{y}^{-1}\right.$ & 2 & 9 & {$[[1,0,0],[0,1,0],[0,0,2]]$} \\
\hline$H_{302}=\left\langle i, i t_{x}, r_{z}^{2} t_{y}^{-1}, r\right.$ & 2 & 9 & {$[[1,0,0],[0,1,1],[0,0,2]]$} \\
\hline$H_{303}=\left\langle i, i t_{x}, m_{x} t_{y}^{-1}, m_{x} t_{z}^{-1}\right\rangle$ & 2 & 9 & {$[[1,0,0],[0,1,1],[0,0,2]]$} \\
\hline$H_{304}=\left\langle i, r_{z}^{2} t_{x}, r_{z}^{2} t_{y}^{-1}\right.$ & 2 & 9 & {$[[1,0,1],[0,1,1],[0,0,2]]$} \\
\hline$H_{305}=\left\langle i, i t_{x}, i t_{y}^{-1}, r_{z}\right.$ & 2 & 12 & {$[[1,0,0],[0,1,0],[0,0,2]]$} \\
\hline$H_{306}=\left\langle i, i t_{x}, r_{z}^{2} r_{x}\right.$ & 2 & 12 & {$[[1,0,0],[0,1,1],[0,0,2]]$} \\
\hline$H_{307}=\left\langle i, r_{z}^{2} r_{x} t_{x}, r_{z}^{2} r\right.$ & 2 & 12 & {$[[1,0,1],[0,1,1],[0,0,2]]$} \\
\hline$H_{308}=\left\langle i, r_{y}^{2} r_{z} t_{z}^{-1}, r_{z}^{2} t_{x}, r_{z}^{2} t_{y}^{-1}\right\rangle$ & 2 & 20 & {$[[1,1,0],[0,2,0],[0,0,2]]$} \\
\hline$H_{309}=\left\langle i, r_{y}^{2} r_{x} t_{y}^{-1}, r_{z}^{2} r_{x} t_{x}\right\rangle$ & 2 & 20 & $[[2,0,0],[0,1,1],[0,0,2]]]$ \\
\hline$H_{310}=\left\langle i, m_{x} t_{x}, r_{z}^{2} r_{x} t_{y}^{-1}\right\rangle$ & 2 & 20 & {$[[2,0,0],[0,1,1],[0,0,2]]$} \\
\hline$H_{311}=\left\langle i, i t_{x}, r_{y}^{2} t_{z}^{-1}, r_{z}^{2} t_{y}^{-1}\right\rangle$ & 2 & 21 & {$[[1,0,0],[0,2,0],[0,0,2]]$} \\
\hline$H_{312}=\left\langle i, i t_{x}, m_{x} t_{z}^{-1}, r_{z}^{2} t_{y}^{-1}\right\rangle$ & 2 & 21 & {$[[1,0,0],[0,2,0],[0,0,2]]$} \\
\hline$H_{313}=\left\langle i, i t_{x}, r_{y}^{2} t_{y}^{-1}, r_{z}^{2} t_{z}^{-1}\right\rangle$ & 2 & 21 & $[[1,0,0],[0,2,0],[0,0,2]]]$ \\
\hline$H_{314}=\left\langle i, i t_{x}, m_{x} t_{z}^{-1}, r_{y}^{2} t_{y}^{-1}\right\rangle$ & 2 & 21 & {$[[1,0,0],[0,2,0],[0,0,2]]$} \\
\hline$H_{315}=\left\langle i, r_{y}^{2} t z^{-1}, r_{z}^{2}\right.$ & 2 & 21 & {$[[1,1,0],[0,2,0],[0,0,2]]$} \\
\hline$H_{316}=\left\langle i, r_{y}^{2} t_{y}^{-1}, r^{2}\right.$ & 2 & 21 & {$[[1,0,1],[0,2,0],[0,0,2]]$} \\
\hline$H_{317}=\left\langle i, r_{y}^{2} t_{y}^{-1}, r_{y}^{2} t_{z}^{-1}, r_{z}^{2} t_{x}\right\rangle$ & 2 & 21 & {$[[2,0,0],[0,1,1],[0,0,2]]$} \\
\hline$H_{318}=\left\langle i, m_{x} t^{-1}, r_{y}^{2} t^{-1}, r_{z}^{2} t_{x}\right\rangle$ & 2 & 21 & {$[[1,1,1],[0,2,0],[0,0,2]]$} \\
\hline$H_{319}=\left\langle i, m_{x} t_{y}^{-1}, r_{y}^{2} t\right.$ & 2 & 21 & {$[[1,1,1],[0,2,0],[0,0,2]]$} \\
\hline$H_{320}=\left\langle i, m_{x} t_{x}, r_{y}^{2} t_{y}^{-1}, r_{z}^{2} t_{z}^{-1}\right\rangle$ & 2 & 21 & {$[[1,1,1],[0,2,0],[0,0,2]]$} \\
\hline$H_{321}=\left\langle i, i t_{x}, m_{x} r_{x}^{-1} t_{y}^{-1}\right\rangle$ & 2 & 22 & $[[1,0,0],[0,2,0],[0,0,2]]]$ \\
\hline$H_{322}=\left\langle i, m_{x} r_{x}^{-1} t_{y}^{-1}, m_{x} t_{x}\right\rangle$ & 2 & 22 & {$[[1,1,1],[0,2,0],[0,0,2]]$} \\
\hline$H_{323}=\left\langle i, m_{x} t_{z}^{-1}, r_{x}^{2} r_{y} t_{y}^{-1}, r_{z}^{2} t_{x}\right\rangle$ & 2 & 29 & {$[[2,0,0],[0,2,0],[0,0,2]]$} \\
\hline$H_{324}=\left\langle i, m_{x} r_{x}^{-1} t_{y}^{-1}, r_{z}^{2} r_{x} t_{x}\right\rangle$ & 2 & 29 & $[[2,0,0],[0,2,0],[0,0,2]]]$ \\
\hline$H_{325}=\left\langle i, m_{x} r_{x} t_{y}^{-1}, r_{z}^{2} r_{x} t_{x}\right\rangle$ & 2 & 29 & {$[[2,0,0],[0,2,0],[0,0,2]]$} \\
\hline
\end{tabular}




\begin{tabular}{|c|c|c|c|}
\hline$H$ & $H_{0,0,0}$ & $P(H)$ & Translation basis of $H$ \\
\hline$H_{326}=\left\langle i, r_{y}^{2} t_{y}^{-1}, r_{z}^{2} r_{x} t_{x}, r_{z}^{2} t_{z}^{-1}\right\rangle$ & 2 & 29 & {$[[2,0,0],[0,2,0],[0,0,2]]$} \\
\hline$H_{327}=\left\langle m_{z}, m_{z} t_{x}, m_{z} t_{y}^{-1}, m_{z} t_{z}^{-1}\right\rangle$ & 3 & 3 & {$[[1,0,0],[0,1,0],[0,0,1]]$} \\
\hline$H_{328}=\left\langle i t_{x}, i t_{x}^{-1}, m_{z}, m_{z} t_{y}^{-1}, m_{z} t_{z}^{-1}\right\rangle$ & 3 & 9 & {$[[2,0,0],[0,1,0],[0,0,1]]$} \\
\hline$H_{329}=\left\langle i t_{z}^{-1}, m_{z}, m_{z} t_{x}, m_{z} t_{y}^{-1}\right\rangle$ & 3 & 9 & {$[[1,0,0],[0,1,0],[0,0,2]]$} \\
\hline$H_{330}=\left\langle i t_{x}, i t_{x}^{-1}, i t_{y}, i t_{y}^{-1}, m_{z}, m_{z} t_{z}^{-1}\right\rangle$ & 3 & 9 & {$[[1,1,0],[0,2,0],[0,0,1]]$} \\
\hline$H_{331}=\left\langle i t_{x}, i t_{x}^{-1}, i t_{z}^{-1}, m_{z}, m_{z} t_{y}^{-1}\right\rangle$ & 3 & 9 & {$[[1,0,1],[0,1,0],[0,0,2]]$} \\
\hline$H_{332}=\left\langle i t_{x}, i t_{x}^{-1}, i t_{y}, i t_{y}^{-1}, i t_{z}^{-1}, m_{z}\right\rangle$ & 3 & 9 & {$[[1,0,1],[0,1,1],[0,0,2]]$} \\
\hline$H_{333}=\left\langle m_{z}, m_{z} t_{x}, m_{z} t_{y}^{-1}, r_{y}^{2} r_{z} t_{z}^{-1}\right\rangle$ & 3 & 11 & {$[[1,0,0],[0,1,0],[0,0,2]]$} \\
\hline$H_{334}=\left\langle m_{z}, m_{z} t_{z}^{-1}, r_{y}^{2} r_{z} t_{x}, r_{y}^{2} r_{z} t_{x}^{-1}\right\rangle$ & 3 & 11 & {$[[1,1,0],[0,2,0],[0,0,1]]$} \\
\hline$H_{335}=\left\langle m_{z}, r_{y}^{2} r_{z} t_{x}, r_{y}^{2} r_{z} t_{x}^{-1}, r_{y}^{2} r_{z} t_{z}^{-1}\right\rangle$ & 3 & 11 & {$[[1,0,1],[0,1,1],[0,0,2]]$} \\
\hline$H_{336}=\left\langle m_{z}, m_{z} t_{y}^{-1}, m_{z} t_{z}^{-1}, r_{y}^{2} t_{x}, r_{y}^{2} t_{x}^{-1}\right\rangle$ & 3 & 14 & {$[[2,0,0],[0,1,0],[0,0,1]]$} \\
\hline$H_{337}=\left\langle m_{z}, m_{z} t^{\frac{y}{y}} 1\right.$ & 3 & 14 & {$[[2,0,0],[0,1,0],[0,0,1]]$} \\
\hline$H_{338}=\left\langle m_{z}, m_{z} t_{x}\right.$, & 3 & 14 & {$[[1,0,0],[0,1,0],[0,0,2]]$} \\
\hline$H_{339}=\left\langle m_{z}, m_{z} t\right\rangle$ & 3 & 14 & {$[[1,1,0],[0,2,0],[0,0,1]]$} \\
\hline$H_{340}=\left\langle m_{z}, m_{z} t\right.$ & 3 & 14 & {$[[1,0,1],[0,1,0],[0,0,2]]$} \\
\hline$H_{341}=\left\langle m_{z}, m_{z} t^{\frac{9}{y}}\right.$ & 3 & 14 & {$[[1,0,1],[0,1,0],[0,0,2]]$} \\
\hline$H_{342}=\left\langle m_{z}, r_{y}^{2} t_{x}, r_{y}^{2} t_{x}^{-1}, r_{y}^{2} t_{y}^{-1}, r_{y}^{2} t_{z}^{-1}\right\rangle$ & 3 & 14 & {$[[1,0,1],[0,1,1],[0,0,2]]$} \\
\hline$H_{343}=\left\langle i t_{x}, i t_{y}, m\right.$ & 3 & 20 & {$[[1,3,0],[0,4,0],[0,0,1]]$} \\
\hline$H_{344}=\left\langle i t_{x}, i t_{x}^{-1}, i t_{y}, i t_{y}^{-1}, m_{z}, r_{y}^{2} r_{z} t_{z}^{-1}\right\rangle$ & 3 & 20 & {$[[1,1,0],[0,2,0],[0,0,2]]$} \\
\hline$H_{345}=\left\langle i t_{z}^{-1}, m_{z}, r_{y}^{2} r_{z} t_{x}^{9}\right.$ & 3 & 20 & {$[[1,1,0],[0,2,0],[0,0,2]]$} \\
\hline$H_{346}=\left\langle m_{z}, r_{x}^{2} r_{z} t_{z}^{-1}, r_{y}^{2} r\right.$ & 3 & 20 & {$[[1,1,0],[0,2,0],[0,0,2]]$} \\
\hline$H_{347}=\left\langle i t_{x}, i t_{y}, m\right.$ & 3 & 20 & {$[[1,1,1],[0,2,1],[0,0,2]]$} \\
\hline$H_{348}=\left\langle i t_{x}, m_{z}, m\right.$ & 3 & 21 & {$[[4,0,0],[0,1,0],[0,0,1]]$} \\
\hline$H_{349}=\left\langle i t_{x}, i t_{x}^{-1}, r\right.$ & 3 & 21 & {$[[2,0,0],[0,2,0],[0,0,1]]$} \\
\hline$H_{350}=\left\langle i t_{x}, i t_{x}^{-1}\right.$ & 3 & 21 & {$[[2,0,0],[0,2,0],[0,0,1]]$} \\
\hline$H_{351}=\left\langle i t_{x}, i t_{x}^{-1}\right.$, & 3 & 21 & {$[[2,0,0],[0,1,0],[0,0,2]]$} \\
\hline$H_{352}=\left\langle i t_{x}, i t_{x}^{-1}\right.$, & 3 & 21 & {$[[2,0,0],[0,1,0],[0,0,2]]$} \\
\hline$H_{353}=\left\langle m_{z}, m_{z} t_{z}^{-1}, r_{x}^{2} t_{y}, r_{x}^{2} t_{y}^{-1}, r_{y}^{2} t_{x}, r_{y}^{2} t_{x}^{-1}\right\rangle$ & 3 & 21 & {$[[2,0,0],[0,2,0],[0,0,1]]$} \\
\hline$H_{354}=\left\langle i t_{z}^{-1}, m_{z}, m_{z} t_{y}^{-1}, r_{y}^{2} t_{x}, r_{y}^{2} t_{x}^{-1}\right\rangle$ & 3 & 21 & {$[[2,0,0],[0,1,0],[0,0,2]]$} \\
\hline$H_{355}=\left\langle m_{z}, m_{z} t_{y}^{-1}\right.$ & 3 & 21 & {$[[2,0,0],[0,1,0],[0,0,2]]$} \\
\hline$H_{356}=\left\langle m_{z}, m_{z} t_{z}^{-1}\right.$ & 3 & 21 & {$[[2,0,0],[0,2,0],[0,0,1]]$} \\
\hline$H_{357}=\left\langle i t_{z}^{-1}, m_{z}\right.$, & 3 & 21 & {$[[2,0,0],[0,1,0],[0,0,2]]$} \\
\hline$H_{358}=\left\langle m_{z}, m_{z} t_{y}^{-1}, r_{x}^{2} t_{x}, r_{y}^{2} t_{z}^{-1}\right\rangle$ & 3 & 21 & {$[[2,0,0],[0,1,0],[0,0,2]]$} \\
\hline$H_{359}=\left\langle i t_{x}, m_{z}, m_{z} t_{z}^{-1}, r_{x}^{2} t_{y}, r_{x}^{2} t_{y}^{-1}, r_{y}^{2} t_{x}^{-1}\right\rangle$ & 3 & 21 & {$[[2,1,0],[0,2,0],[0,0,1]]$} \\
\hline$H_{360}=\left\langle i t_{x}, m_{z}, m_{z} t_{y}^{-1}, r_{x}^{2} t_{z}^{-1}, r_{y}^{2} t_{x}^{-1}\right\rangle^{y}$ & 3 & 21 & {$[[2,0,1],[0,1,0],[0,0,2]]$} \\
\hline$H_{361}=\left\langle i t_{x}, i t_{x}^{-1}, i\right.$ & 3 & 21 & {$[[1,1,0],[0,2,0],[0,0,2]]$} \\
\hline$H_{362}=\left\langle i t_{x}, i t_{x}^{-1}, i\right.$ & 3 & 21 & {$[[1,0,1],[0,2,0],[0,0,2]]$} \\
\hline$H_{363}=\left\langle i t_{x}, i t_{x}^{-1}, r\right.$ & 3 & 21 & {$[[2,0,0],[0,1,1],[0,0,2]]$} \\
\hline$H_{364}=\left\langle i t_{x}, i t_{x}^{-1}, i\right.$ & 3 & 21 & {$[[1,0,1],[0,2,0],[0,0,2]]$} \\
\hline$H_{365}=\left\langle i t_{x}, i t_{x}^{-1}, m_{z}, r_{x}^{2}\right.$ & 3 & 21 & {$[[2,0,0],[0,1,1],[0,0,2]]$} \\
\hline$H_{366}=\left\langle i t_{z}^{-1}, m_{z}, r_{y}^{2} t_{x}, r\right.$ & 3 & 21 & {$[[1,1,0],[0,2,0],[0,0,2]]$} \\
\hline$H_{367}=\left\langle m_{z}, r_{x}^{2} t_{z}^{-1}\right.$ & 3 & 21 & {$[[1,1,0],[0,2,0],[0,0,2]]$} \\
\hline$\left.r_{y}^{2} t_{x}, r_{y}^{2} t_{x}^{-1}, r_{y}^{2} t_{z}^{-1}\right\rangle$ & 3 & 21 & {$[[1,0,1],[0,2,0],[0,0,2]]$} \\
\hline$H_{369}=\left\langle m_{z}, r_{x}^{2} t_{x}, r_{y}^{2} t_{y}^{-1}, r_{y}^{2} t_{z}^{-1}\right\rangle$ & 3 & 21 & {$[[2,0,0],[0,1,1],[0,0,2]]$} \\
\hline$H_{370}=\left\langle i t_{x}, m_{z}, r_{x}^{2} t_{y}, r_{x}^{2} t_{y}^{-1}, r_{x}^{2} t_{z}^{-1}, r_{y}^{2} t_{x}^{-1}\right\rangle$ & 3 & 21 & {$[[2,0,1],[0,1,1],[0,0,2]]$} \\
\hline$H_{371}=\left\langle i t_{x}, i t_{x}^{-1}, m_{z}, r_{x}^{2} t_{z}^{-1}, r_{y}^{2} t_{y}^{-1}\right\rangle$ & 3 & 21 & {$[[1,1,1],[0,2,0],[0,0,2]]$} \\
\hline$H_{372}=\left\langle i t_{x}, i t_{x}^{-1}, m\right.$ & 3 & 21 & {$[[1,1,1],[0,2,0],[0,0,2]]$} \\
\hline$H_{373}=\left\langle i t_{z}^{-1}, m_{z}, r_{x}^{2} t_{y}, r_{x}^{2} t_{y}^{-1}, r_{y}^{2} t_{x}, r_{y}^{2} t_{x}^{-1}\right\rangle$ & 3 & 21 & {$[[1,1,1],[0,2,0],[0,0,2]]$} \\
\hline$H_{374}=\left\langle i t_{z}^{-1}, m_{z}, r_{x}^{2} t_{x}, r_{y}^{2} t_{y}^{-1}\right\rangle$ & 3 & 21 & {$[[1,1,1],[0,2,0],[0,0,2]]$} \\
\hline$H_{375}=\left\langle m_{z}, m_{z} r_{z}^{-1} t_{x}, m_{z} r_{z}^{-1} t_{x}^{-1}, m_{z} t_{z}^{-1}\right\rangle$ & 3 & 22 & {$[[2,0,0],[0,2,0],[0,0,1]]$} \\
\hline$H_{376}=\left\langle i t_{z}^{-1}, m_{z}, m_{z} r_{z}^{-1} t_{x}, m_{z} r_{z_{0}}^{-1} t_{x}^{-1}\right\rangle$ & 3 & 22 & {$[[1,1,1],[0,2,0],[0,0,2]]$} \\
\hline$H_{377}=\left\langle m_{z}, m_{z} r_{z}^{-1} t_{x}^{-1}\right.$ & 3 & 29 & {$[[2,2,0],[0,4,0],[0,0,1]]$} \\
\hline$H_{378}=\left\langle m_{z}, r_{x}^{2} t_{y}, r_{x}^{2} t_{y}^{-1}, r_{y}^{2} r_{z} t_{z}^{-1}, r_{y}^{2} t_{x}, r_{y}^{2} t_{x}^{-1}\right\rangle$ & 3 & 29 & {$[[2,0,0],[0,2,0],[0,0,2]]$} \\
\hline$H_{379}=\left\langle m_{z}, m_{z} r_{z}^{-1} t_{x}, m_{z} r_{z}^{-1} t_{x}^{-1}, r_{y}^{2} r_{z} t_{z}^{-1}\right\rangle$ & 3 & 29 & {$[[2,0,0],[0,2,0],[0,0,2]]$} \\
\hline$H_{380}=\left\langle m_{z}, m_{z} r_{z}^{-1} t_{x}, m_{z} r_{z}^{-1} t_{x}^{-1}, r_{x}^{2} r_{z} t_{z}^{-1}\right\rangle$ & 3 & 29 & {$[[2,0,0],[0,2,0],[0,0,2]]$} \\
\hline$H_{381}=\left\langle m_{z}, r_{x}^{2} t_{x}, r_{y}^{2} r_{z} t_{z}^{-1}, r_{y}^{2} t_{y}^{-1}\right\rangle$ & 3 & 29 & {$[[2,0,0],[0,2,0],[0,0,2]]$} \\
\hline$H_{382}=\left\langle m_{z}, m_{z} r_{z}^{-1} t_{x}^{-1}, r_{x}^{2} t_{y}^{-1}, r_{y}^{2} r_{z} t_{z}^{-1}, r_{y}^{2} t_{x}\right\rangle$ & 3 & 29 & {$[[2,0,1],[0,2,1],[0,0,2]]$} \\
\hline$H_{383}=\left\langle r_{z}^{2} r_{x}, r_{z}^{2} r_{x} t_{x}, r_{z}^{2} r_{x} t_{y}^{-1}\right\rangle$ & 4 & 4 & {$[[1,0,0],[0,1,0],[0,0,1]]$} \\
\hline$H_{384}=\left\langle m_{x} t_{x}, r_{z}^{2} r_{x}, r_{z}^{2} r_{x} t_{y}^{-1}\right\rangle$ & 4 & 11 & {$[[2,0,0],[0,1,0],[0,0,1]]$} \\
\hline$H_{385}=\left\langle m_{x} t_{y}^{-1}, r_{z}^{2} r_{x}, r_{z}^{2} r_{x} t_{x}\right\rangle$ & 4 & 11 & {$[[1,0,0],[0,1,1],[0,0,2]]$} \\
\hline$H_{386}=\left\langle m_{x} t_{x}, m_{x} t_{y}^{-1}, r_{z}^{2} r_{x}\right\rangle$ & 4 & 11 & {$[[1,0,1],[0,1,1],[0,0,2]]$} \\
\hline$H_{387}=\left\langle i t_{x}, r_{z}^{2} r_{x}, r_{z}^{2} r_{x} t_{y}^{-1}\right\rangle$ & 4 & 12 & {$[[2,0,0],[0,1,0],[0,0,1]]$} \\
\hline$H_{388}=\left\langle i t_{y}^{-1}, i t_{z}^{-1}, r_{z}^{2} r_{x}, r_{z}^{2} r_{x} t_{x}\right\rangle$ & 4 & 12 & {$[[1,0,0],[0,1,1],[0,0,2]]$} \\
\hline$H_{389}=\left\langle i t_{x}, i t_{y}^{-1}, i t_{z}^{-1}, r_{z}^{2} r_{x}\right\rangle$ & 4 & 12 & {$[[1,0,1],[0,1,1],[0,0,2]]$} \\
\hline$H_{390}=\left\langle r_{y}^{2} r_{x} t_{x}, r_{z}^{2} r_{x}, r_{z}^{2} r_{x} t_{y}^{-1}\right\rangle$ & 4 & 13 & {$[[2,0,0],[0,1,0],[0,0,1]]$} \\
\hline$H_{391}=\left\langle r_{y}^{2} r_{x} t_{y}^{-1}, r_{y}^{2} r_{x} t_{z}^{-1}, r_{z}^{2} r_{x}, r_{z}^{2} r_{x} t_{x}\right\rangle$ & 4 & 13 & {$[[1,0,0],[0,1,1],[0,0,2]]$} \\
\hline$H_{392}=\left\langle r_{y}^{2} r_{x} t_{x}, r_{y}^{2} r_{x} t_{y}^{-1}, r_{y}^{2} r_{x} t_{z}^{-1}, r_{z}^{2} r_{x}\right\rangle$ & 4 & 13 & {$[[1,0,1],[0,1,1],[0,0,2]]$} \\
\hline
\end{tabular}




\begin{tabular}{|c|c|c|c|}
\hline$H$ & $H_{0,0,0}$ & $P(H)$ & Translation basis of $H$ \\
\hline$H_{393}=\left\langle r_{x}^{2} r_{y} t_{y}^{-1}, r_{y}^{2} r_{z} t_{x}, r_{z}^{2} r_{x}\right\rangle$ & 4 & 19 & {$[[1,0,2],[0,1,2],[0,0,3]]$} \\
\hline$H_{394}=\left\langle i t_{y}^{-1}, r_{y}^{2} r_{x} t_{z}^{-1}, r_{z}^{2} r_{x}, r_{z}^{2} r_{x} t_{x}\right\rangle$ & 4 & 20 & {$[[1,0,0],[0,1,1],[0,0,4]]$} \\
\hline$H_{395}=\left\langle i t_{y}^{-1}, m_{x} t_{x}, r_{y}^{2} r_{x} t_{z}^{-1}, r_{z}^{2} r_{x}\right\rangle$ & 4 & 20 & {$[[1,0,2],[0,1,1],[0,0,4]]$} \\
\hline$H_{396}=\left\langle i t_{x}, r_{y}^{2} r_{x} t_{y}^{-1}, r_{y}^{2} r_{x} t_{z}^{-1}, r_{z}^{2} r_{x}\right\rangle$ & 4 & 20 & {$[[2,0,0],[0,1,1],[0,0,2]]$} \\
\hline$H_{397}=\left\langle i t_{x}, m_{x} t_{y}^{-1}, r_{z}^{2} r_{x}\right\rangle$ & 4 & 20 & {$[[2,0,0],[0,1,1],[0,0,2]]$} \\
\hline$H_{398}=\left\langle i t_{y}^{-1}, i t_{z}^{-1}, r_{y}^{2} r_{x} t_{x}, r_{z}^{2} r_{x}\right\rangle$ & 4 & 20 & {$[[2,0,0],[0,1,1],[0,0,2]]$} \\
\hline$H_{399}=\left\langle m_{x} t_{y}^{-1}, r_{y}^{2} r_{x}\right.$ & 4 & 20 & {$[[2,0,0],[0,1,1],[0,0,2]]$} \\
\hline$H_{400}=\left\langle i t_{y}^{-1}, i t z^{-1}, r\right.$ & 4 & 20 & {$[[2,0,0],[0,1,1],[0,0,2]]$} \\
\hline$H_{401}=\left\langle m_{x} t_{x}, r_{y}^{2} r_{x} t_{y}^{-1}, r_{y}^{2} r_{x} t_{z}^{-1}, r_{z}^{2} r_{x}\right\rangle$ & 4 & 20 & {$[[2,0,0],[0,1,1],[0,0,2]]$} \\
\hline$H_{402}=\left\langle m_{x} r_{x} t_{z}^{-1}, m_{x} r_{x}^{-1} t_{y}^{-1}, r_{z}^{2} r_{x}, r_{z}^{2} r_{x} t_{x}\right\rangle$ & 4 & 23 & {$[[1,0,0],[0,2,0],[0,0,2]]$} \\
\hline$H_{403}=\left\langle m_{x} r_{x} t_{y}^{-1}, r_{z}^{2} r_{x}\right.$ & 4 & 23 & {$[[1,0,0],[0,2,0],[0,0,2]]$} \\
\hline$H_{404}=\left\langle m_{x} r_{x} t_{z}^{-1}, m_{x} r_{x}^{-1} t_{y}^{-1}, r_{y}^{2} r_{x} t_{x}, r_{z}^{2} r_{x}\right\rangle$ & 4 & 23 & {$[[1,1,1],[0,2,0],[0,0,2]]$} \\
\hline$H_{405}=\left\langle m_{x} r_{x} t_{y}^{-1}, r_{y}^{2} r_{x} t_{x}, r_{z}^{2} r_{x}\right\rangle$ & 4 & 23 & {$[[1,1,1],[0,2,0],[0,0,2]]$} \\
\hline$H_{406}=\left\langle r_{z}^{2} r_{x}, r_{z}^{2} r_{x} t_{y}^{-1}, r_{z}^{2} t_{x}\right\rangle$ & 4 & 24 & {$[[4,0,0],[0,1,0],[0,0,1]]$} \\
\hline$H_{407}=\left\langle r_{y}^{\tilde{2}} t_{z}^{-1}, r_{z}^{2} r_{x}, r_{z}^{2} r_{x} t_{x}, r_{z}^{2} t_{y}^{-1}\right\rangle$ & 4 & 24 & {$[[1,0,0],[0,2,0],[0,0,2]]$} \\
\hline$H_{408}=\left\langle r_{y}^{2} t_{y}^{-1}, r_{z}^{2} r_{x}, r_{z}^{2} r_{x} t_{x}\right\rangle$ & 4 & 24 & {$[[1,0,0],[0,2,0],[0,0,2]]$} \\
\hline$H_{409}=\left\langle r_{y}^{2} r_{x} t_{y}^{-1}, r_{y}^{2} r_{x}\right.$ & 4 & 24 & {$[[2,0,1],[0,1,1],[0,0,2]]$} \\
\hline$H_{410}=\left\langle r_{y}^{2} r_{x} t_{x}, r_{y}^{2} t_{z}^{-1}, r_{z}^{2} r_{x}, r_{z}^{2} t_{y}^{-1}\right\rangle$ & 4 & 24 & {$[[1,1,1],[0,2,0],[0,0,2]]$} \\
\hline$H_{411}=\left\langle r_{y}^{2} r_{x} t_{x}, r_{y}^{2} t_{y}^{-1}, r_{z}^{2} r_{x}\right\rangle$ & 4 & 24 & {$[[1,1,1],[0,2,0],[0,0,2]]$} \\
\hline$H_{412}=\left\langle m_{x} r_{x} t_{z}^{-1}, r_{z}^{2} r_{x}, r_{z}^{2} r_{x} t_{x}\right.$ & 4 & 29 & {$[[1,0,0],[0,2,2],[0,0,4]]$} \\
\hline$H_{413}=\left\langle m_{x} r_{x} t_{z}^{-1}, m_{x} t\right.$ & 4 & 29 & {$[[1,0,2],[0,2,2],[0,0,4]]$} \\
\hline$H_{414}=\left\langle i t_{x}, r_{y}^{2} t_{z}^{-1}, r_{z}^{2} r\right.$ & 4 & 29 & {$[[2,0,0],[0,2,0],[0,0,2]]$} \\
\hline$\left.r_{x} t_{z}^{-1}, m_{x} r_{x}^{-1} t_{y}^{-1}, r_{z}^{2} r_{x}\right\rangle$ & 4 & 29 & {$[[2,0,0],[0,2,0],[0,0,2]]$} \\
\hline$H_{416}=\left\langle i t_{x}, m_{x} r_{x} t_{y}^{-1}, r_{z}^{2} r_{x}\right\rangle$ & 4 & 29 & {$[[2,0,0],[0,2,0],[0,0,2]]$} \\
\hline$H_{417}=\left\langle i t_{x}, r_{y}^{2} t_{y}^{-1}, r_{z}^{2} r_{x}\right\rangle$ & 4 & 29 & {$[[2,0,0],[0,2,0],[0,0,2]]$} \\
\hline$H_{418}=\left\langle m_{x} t_{x}, r_{y}^{2} t_{z}^{-1}, r_{z}^{2} r_{x}, r_{z}^{2} t_{y}^{-1}\right\rangle$ & 4 & 29 & {$[[2,0,0],[0,2,0],[0,0,2]]$} \\
\hline$H_{419}=\left\langle m_{x} r_{x} t_{z}^{-1}, m_{x} r_{x}^{-1} t_{y}^{-1}, m_{x} t_{x}, r_{z}^{2} r_{x}\right\rangle$ & 4 & 29 & {$[[2,0,0],[0,2,0],[0,0,2]]$} \\
\hline$H_{420}=\left\langle m_{x} r_{x} t_{y}^{-1}, m_{x} t_{x}, r_{z}^{2} r_{x}\right\rangle$ & 4 & 29 & {$[[2,0,0],[0,2,0],[0,0,2]]$} \\
\hline$H_{421}=\left\langle m_{x} t_{x}, r_{y}^{2} t_{y}^{-1}, r_{z}^{2} r_{x}\right\rangle$ & 4 & 29 & {$[[2,0,0],[0,2,0],[0,0,2]]$} \\
\hline$H_{422}=\left\langle m_{x} r_{x} t_{z}^{-1}, m_{x} r_{x}^{-1} t_{y}^{-1}, r_{z}^{2} r_{x}, r_{z}^{2} t_{x}\right\rangle$ & 4 & 29 & {$[[2,1,1],[0,2,0],[0,0,2]]$} \\
\hline$H_{423}=\left\langle m_{x} r_{x} t_{y}^{-1}, r_{z}^{2} r_{x}, r_{z}^{2} t_{x}\right\rangle$ & 4 & 29 & {$[[2,1,1],[0,2,0],[0,0,2]]$} \\
\hline$H_{424}=\left\langle r_{z}^{2}, r_{z}^{2} t_{x}, r_{z}^{2} t_{y}^{-1}, r_{z}^{2} t_{z}^{-1}\right\rangle$ & 5 & 5 & {$[[1,0,0],[0,1,0],[0,0,1]]$} \\
\hline$H_{425}=\left\langle r_{y}^{2} t_{z}, r_{y}^{2} t_{z}^{-1}, r_{z}^{2}, r_{z}^{2} t_{x}, r_{z}^{2} t_{y}^{-1}\right\rangle$ & 5 & 8 & {$[[1,0,0],[0,1,0],[0,0,2]]$} \\
\hline$H_{426}=\left\langle r_{y}^{2} t_{y}^{-1}, r_{z}^{2}, r_{z}^{2} t_{x}, r_{z}^{2} t\right.$ & 5 & 8 & {$[[1,0,0],[0,2,0],[0,0,1]]$} \\
\hline$H_{427}=\left\langle r_{y}^{2} t_{y}^{\frac{9}{y}}\right.$ & 5 & 8 & {$[[1,0,0],[0,1,1],[0,0,2]]$} \\
\hline$H_{428}=\left\langle r_{y}^{2} t_{x}, r_{y}^{2}\right.$ & 5 & 8 & {$[[1,1,0],[0,2,0],[0,0,1]]$} \\
\hline$H_{429}=\left\langle r_{y}^{2} t_{x}, r_{y}^{2} t_{y}^{-1}, r_{y}^{2} t_{z}, r_{y}^{2} t_{z}^{-1}, r_{z}^{2}\right\rangle$ & 5 & 8 & {$[[1,0,1],[0,1,1],[0,0,2]]$} \\
\hline$H_{430}=\left\langle i t_{x}, r_{z}^{2}, r_{z}^{2} t_{y}^{-1}, r_{z}^{2} t_{z}^{-1}\right\rangle$ & 5 & 9 & {$[[2,0,0],[0,1,0],[0,0,1]]$} \\
\hline$H_{431}=\left\langle i t_{z}, i t_{z}^{-1}, r_{z}^{2}, r_{z}^{2} t_{x}, r_{z}^{2} t_{y}^{-1}\right\rangle$ & 5 & 9 & {$[[1,0,0],[0,1,0],[0,0,2]]$} \\
\hline$H_{432}=\left\langle i t_{x}, i t_{y}^{-1}, r_{z}^{2}, r_{z}^{2} t t_{z}\right.$ & 5 & 9 & {$[[1,1,0],[0,2,0],[0,0,1]]$} \\
\hline$H_{433}=\left\langle i t_{x}, i t_{z}, i t_{z}^{-1}, r_{z}^{2}\right.$, & 5 & 9 & {$[[1,0,1],[0,1,0],[0,0,2]]$} \\
\hline$H_{434}=\left\langle i t_{x}, i t_{y}^{-1}, i t_{z}, i t_{z}-\right.$ & 5 & 9 & {$[[1,0,1],[0,1,1],[0,0,2]]$} \\
\hline$H_{435}=\left\langle m_{x} r_{z} t_{z}^{-1}, r_{z}^{2}, r_{z}^{2} t_{x}, r_{z}^{2} t_{y}^{-1}\right\rangle$ & 5 & 10 & {$[[1,0,0],[0,1,0],[0,0,2]]$} \\
\hline$H_{436}=\left\langle m_{x} r_{z} t_{x}, r_{z}^{2}, r_{z}^{2} t_{z}^{-1}\right\rangle$ & 5 & 10 & {$[[1,1,0],[0,2,0],[0,0,1]]$} \\
\hline$H_{437}=\left\langle m_{x} r_{z} t_{x}, m_{x} r_{z} t_{z}^{-1}\right.$, & 5 & 10 & {$[[1,0,1],[0,1,1],[0,0,2]]$} \\
\hline$H_{438}=\left\langle r_{y}^{2} r_{z} t_{z}, r_{y}^{2} r_{z} t_{z}^{-1}, r_{z}^{2}, r_{z}^{2} t_{x}, r_{z}^{2} t_{y}^{-1}\right\rangle$ & 5 & 13 & {$[[1,0,0],[0,1,0],[0,0,2]]$} \\
\hline$H_{439}=\left\langle r_{y}^{2} r_{z} t_{x}, r_{z}^{2}, r_{z}^{2} t_{z}^{-1}\right\rangle$ & 5 & 13 & {$[[1,1,0],[0,2,0],[0,0,1]]$} \\
\hline$H_{440}=\left\langle r_{y}^{2} r_{z} t_{x}, r_{y}^{2} r_{z} t_{z}, r_{y}^{2}\right.$ & 5 & 13 & {$[[1,0,1],[0,1,1],[0,0,2]]$} \\
\hline$H_{441}=\left\langle m_{x} t_{z}^{-1}, r_{z}^{2}, r_{z}^{2} t_{x}\right.$, & 5 & 14 & {$[[1,0,0],[0,1,0],[0,0,2]]$} \\
\hline$H_{442}=\left\langle m_{x} t_{y}^{-1}\right.$, & 5 & 14 & {$[[1,0,0],[0,2,0],[0,0,1]]$} \\
\hline$H_{443}=\left\langle m_{x} t_{y}^{-1}, m_{x} t_{z}^{-1}, r_{z}^{2}, r_{z}^{2} t_{x}\right\rangle$ & 5 & 14 & {$[[1,0,0],[0,1,1],[0,0,2]]$} \\
\hline$H_{444}=\left\langle m_{x} t_{x}, m_{x} t_{y}^{-1}, r_{z}^{2}, r_{z}^{2} t_{z}^{-1}\right\rangle$ & 5 & 14 & {$[[1,1,0],[0,2,0],[0,0,1]]$} \\
\hline$H_{445}=\left\langle m_{x} t_{x}, m_{x} t_{y}^{-1}, m_{x} t \frac{-1}{z}, r_{z}^{2}\right\rangle$ & 5 & 14 & {$[[1,0,1],[0,1,1],[0,0,2]]$} \\
\hline$H_{446}=\left\langle r_{z}^{-1} t_{z}^{-1}, r_{z}^{2}, r_{z}^{2} t_{x}, r_{z}^{2} t_{y}^{-1}\right\rangle$ & 5 & 15 & {$[[1,0,0],[0,1,0],[0,0,2]]$} \\
\hline$H_{447}=\left\langle r_{z}^{-1} t_{x}, r_{z}^{2}, r_{z}^{2} t_{z}^{-1}\right\rangle$ & 5 & 15 & {$[[1,1,0],[0,2,0],[0,0,1]]$} \\
\hline$H_{448}=\left\langle r_{z}^{-1} t_{x}, r_{z}^{-1} t_{z}^{-1}, r_{z}^{2}\right\rangle$ & 5 & 15 & {$[[1,0,1],[0,1,1],[0,0,2]]$} \\
\hline$H_{449}=\left\langle m_{z} r_{z}^{-1} t_{z}, m_{z} r_{z}^{-1} t_{z}^{-1}, r_{z}^{2}, r_{z}^{2} t_{x}, r_{z}^{2} t_{y}^{-1}\right\rangle$ & 5 & 16 & {$[[1,0,0],[0,1,0],[0,0,2]]$} \\
\hline$H_{450}=\left\langle m_{z} r_{z}^{-1} t_{x}, r_{z}^{2}, r_{z}^{2} t_{z}^{-1}\right\rangle$ & 5 & 16 & {$[[1,1,0],[0,2,0],[0,0,1]]$} \\
\hline$H_{451}=\left\langle m_{z} r_{z}^{-1} t_{x}, m_{z} r_{z}^{-1} t_{z}, m_{z} r_{z}^{-1} t_{z}^{-1}, r_{z}^{2}\right\rangle$ & 5 & 16 & {$[[1,0,1],[0,1,1],[0,0,2]]$} \\
\hline$H_{452}=\left\langle i t_{z}^{-1}, r_{y}^{2} r_{z} t_{z}, r_{z}^{2}, r_{z}^{2} t_{x}, r_{z}^{2} t_{y}^{-1}\right\rangle^{z}$ & 5 & 20 & {$[[1,0,0],[0,1,0],[0,0,4]]$} \\
\hline$H_{453}=\left\langle i t_{x}, i t_{y}^{-1}, r_{y}^{2} r_{z} t_{z}, r_{y}^{2} r_{z} t_{z}^{-1}, r_{z}^{2}\right\rangle$ & 5 & 20 & {$[[1,1,0],[0,2,0],[0,0,2]]$} \\
\hline$H_{454}=\left\langle i t_{x}, i t_{y}^{-1}, m_{x} r_{z} t_{z}^{-1}, r_{z}^{2}\right\rangle$ & 5 & 20 & {$[[1,1,0],[0,2,0],[0,0,2]]$} \\
\hline$H_{455}=\left\langle i t_{z}, i t_{z}-1, r_{y}^{2} r_{z} t_{x}, r_{z}^{2}\right\rangle$ & 5 & 20 & {$[[1,1,0],[0,2,0],[0,0,2]]$} \\
\hline$H_{456}=\left\langle m_{x} r_{z} t_{z}^{-1}, r_{y}^{2} r_{z} t_{x}, r_{z}^{2}\right\rangle$ & 5 & 20 & {$[[1,1,0],[0,2,0],[0,0,2]]$} \\
\hline$H_{457}=\left\langle i t_{z}, i t_{z}^{-1}, m_{x} r_{z} t_{x}, r_{z}^{2}\right\rangle$ & 5 & 20 & {$[[1,1,0],[0,2,0],[0,0,2]]$} \\
\hline$H_{458}=\left\langle m_{x} r_{z} t_{x}, r_{y}^{2} r_{z} t_{z}, r_{y}^{2} r_{z} t_{z}^{-1}, r_{z}^{2}\right\rangle$ & 5 & 20 & {$[[1,1,0],[0,2,0],[0,0,2]]$} \\
\hline$H_{459}=\left\langle i t_{z}^{-1}, m_{x} r_{z} t_{x}, r_{y}^{2} r_{z} t_{z}, r_{z}^{2}\right\rangle$ & 5 & 20 & {$[[1,0,2],[0,1,2],[0,0,4]]$} \\
\hline$H_{460}=\left\langle i t_{z}^{-1}, r_{y}^{2} t_{z}, r_{z}^{2}, r_{z}^{2} t_{x}, r_{z}^{2} t_{y}^{-1}\right\rangle$ & 5 & 21 & {$[[1,0,0],[0,1,0],[0,0,4]]$} \\
\hline
\end{tabular}




\begin{tabular}{|c|c|c|c|}
\hline$H$ & $H_{0,0,0}$ & $P(H)$ & Translation basis of $H$ \\
\hline$H_{461}=\left\langle i t_{z}^{-1}, m_{x} t_{y}^{-1}, r_{y}^{2} t_{z}, r_{z}^{2}, r_{z}^{2} t_{x}\right\rangle$ & 5 & 21 & {$[[1,0,0],[0,1,2],[0,0,4]]$} \\
\hline$H_{462}=\left\langle i t_{x}, r_{y}^{2} t_{z}, r_{y}^{2} t_{z}^{-1}, r_{z}^{2}, r_{z}^{2} t_{y}^{-1}\right\rangle$ & 5 & 21 & {$[[2,0,0],[0,1,0],[0,0,2]]$} \\
\hline$H_{463}=\left\langle i t_{x}, m_{x} t_{z}^{-1}, r_{z}^{2}, r_{z}^{2} t_{y}^{-1}\right\rangle$ & 5 & 21 & {$[[2,0,0],[0,1,0],[0,0,2]]$} \\
\hline$H_{464}=\left\langle i t_{x}, r_{y}^{2} t_{y}^{-1}, r_{z}^{2}\right.$ & 5 & 21 & {$[[2,0,0],[0,2,0],[0,0,1]]$} \\
\hline$H_{465}=\left\langle i t_{x}, m_{x} t_{y}^{-1}, r_{z}^{2}, r_{z}^{2} t_{z}^{-1}\right\rangle$ & 5 & 21 & $[[2,0,0],[0,2,0],[0,0,1]]]$ \\
\hline$H_{466}=\left\langle i t_{z}, i t_{z}^{-1}, r_{y}^{2} t_{y}^{-1}, r_{z}^{2}, r_{z}^{2} t_{x}\right\rangle$ & 5 & 21 & {$[[1,0,0],[0,2,0],[0,0,2]]$} \\
\hline$H_{467}=\left\langle m_{x} t_{z}^{-1}, r_{y}^{2} t_{y}^{-1}, r_{z}^{2}, r_{z}^{2} t_{x}\right\rangle$ & 5 & 21 & $[[1,0,0],[0,2,0],[0,0,2]]]$ \\
\hline$H_{468}=\left\langle i t_{z}, i t_{z}^{-1}, m_{x} t\right.$ & 5 & 21 & $[[1,0,0],[0,2,0],[0,0,2]]]$ \\
\hline$H_{469}=\left\langle m_{x} t_{y}^{-1}, r_{y}^{2} t_{z}\right.$, & 5 & 21 & {$[[1,0,0],[0,2,0],[0,0,2]]$} \\
\hline$H_{470}=\left\langle m_{x} t_{y}^{-1}, r_{y}^{2} t_{x}\right.$ & 5 & 21 & $[[2,0,0],[0,2,0],[0,0,1]]]$ \\
\hline$H_{471}=\left\langle i t_{x}, i t_{y}^{-1}, r_{y}^{2} t\right.$ & 5 & 21 & $[[1,1,0],[0,2,0],[0,0,2]]]$ \\
\hline$H_{472}=\left\langle i t_{x}, i t_{y}^{-1}, m_{x}\right.$ & 5 & 21 & {$[[1,1,0],[0,2,0],[0,0,2]]$} \\
\hline$H_{473}=\left\langle i t_{x}, i t_{z}, i t_{z}^{-1}\right.$, & 5 & 21 & {$[[1,0,1],[0,2,0],[0,0,2]]$} \\
\hline$H_{474}=\left\langle i t_{x}, r_{y}^{2} t_{y}^{-1}, r_{y}^{2} t_{z}, r_{y}^{2} t_{z}^{-1}, r_{z}^{2}\right\rangle$ & 5 & 21 & {$[[2,0,0],[0,1,1],[0,0,2]]$} \\
\hline$H_{475}=\left\langle i t_{x}, i t_{z}, i t_{z}^{-1}, m_{x} t_{y}^{-1}, r_{z}^{2}\right\rangle$ & 5 & 21 & {$[[1,0,1],[0,2,0],[0,0,2]]$} \\
\hline$H_{476}=\left\langle i t_{x}, m_{x} t_{y}^{-\tilde{1}}\right.$, & 5 & 21 & {$[[2,0,0],[0,1,1],[0,0,2]]$} \\
\hline$H_{477}=\left\langle i t_{z}, i t_{z}^{-1}, r_{y}^{2}\right.$ & 5 & 21 & {$[[1,1,0],[0,2,0],[0,0,2]]$} \\
\hline$H_{478}=\left\langle m_{x} t_{z}^{-1}, r_{y}^{2} t_{x}\right.$, & 5 & 21 & $[[1,1,0],[0,2,0],[0,0,2]]]$ \\
\hline$H_{479}=\left\langle m_{x} t_{y}^{-1}, r_{y}^{2} t_{x}\right.$, & 5 & 21 & {$[[1,0,1],[0,2,0],[0,0,2]]$} \\
\hline$H_{480}=\left\langle m_{x} t_{y}^{-1}, m_{x} t_{\bar{z}}^{-}\right.$ & 5 & 21 & {$[[2,0,0],[0,1,1],[0,0,2]]$} \\
\hline$H_{481}=\left\langle i t_{z}, i t_{z}^{-1}, m_{x} t\right.$ & 5 & 21 & $[[1,1,0],[0,2,0],[0,0,2]]]$ \\
\hline$H_{482}=\left\langle m_{x} t_{x}, m_{x} t_{y}^{-1}\right.$ & 5 & 21 & {$[[1,1,0],[0,2,0],[0,0,2]]$} \\
\hline$H_{483}=\left\langle i t_{z}^{-1}, m_{x} t_{x}, r\right.$ & 5 & 21 & $[[1,0,2],[0,1,2],[0,0,4]]]$ \\
\hline$H_{484}=\left\langle i t_{x}, m_{x} t_{z}^{-1}\right.$ & 5 & 21 & {$[[1,1,1],[0,2,0],[0,0,2]]$} \\
\hline$H_{485}=\left\langle i t_{x}, m_{x} t_{y}^{-1}, r\right.$ & 5 & 21 & {$[[1,1,1],[0,2,0],[0,0,2]]$} \\
\hline$H_{486}=\left\langle i t_{z}, i t_{z}^{-1}, m_{x} t\right.$ & 5 & 21 & {$[[1,1,1],[0,2,0],[0,0,2]]$} \\
\hline$H_{487}=\left\langle i t_{z}^{-1}, m_{z} r_{z}^{-1} t\right.$ & 5 & 22 & {$[[1,0,0],[0,1,0],[0,0,4]]$} \\
\hline$H_{488}=\left\langle i t_{x}, i t_{y}^{-1}, m_{z} r_{z}^{-1} t_{z}, m_{z} r_{z}^{-1} t_{z}^{-1}, r_{z}^{2}\right\rangle$ & 5 & 22 & {$[[1,1,0],[0,2,0],[0,0,2]]$} \\
\hline$H_{489}=\left\langle i t_{x}, i t_{y}^{-1}, r_{z}^{-1} t_{z}^{-1}, r_{z}^{2}\right\rangle$ & 5 & 22 & {$[[1,1,0],[0,2,0],[0,0,2]]$} \\
\hline$H_{490}=\left\langle i t_{z}, i t \frac{y}{z} 1, m_{z}\right.$ & 5 & 22 & {$[[1,1,0],[0,2,0],[0,0,2]]$} \\
\hline$H_{491}=\left\langle m_{z} r_{z}^{-1} t_{x}, r\right.$ & 5 & 22 & {$[[1,1,0],[0,2,0],[0,0,2]]$} \\
\hline$H_{492}=\left\langle i t_{z}, i t_{z}^{-1}, r \tilde{z}\right.$ & 5 & 22 & {$[[1,1,0],[0,2,0],[0,0,2]]$} \\
\hline$H_{493}=\left\langle m_{z} r_{z}^{-1} t_{z}, m_{1}\right.$ & 5 & 22 & {$[[1,1,0],[0,2,0],[0,0,2]]$} \\
\hline$H_{494}=\left\langle i t_{z}^{-1}, m_{z} r_{z}^{-}\right.$ & 5 & 22 & {$[[1,0,2],[0,1,2],[0,0,4]]$} \\
\hline$H_{495}=\left\langle m_{z} r_{z}^{-1} t_{z}, r_{y}^{2}\right.$ & 5 & 23 & {$[[1,0,0],[0,1,0],[0,0,4]]$} \\
\hline$H_{496}=\left\langle m_{x} t_{x}, m_{x} t \frac{9}{y}\right.$ & 5 & 23 & {$[[1,1,0],[0,2,0],[0,0,2]]$} \\
\hline$H_{497}=\left\langle m_{x} t_{x}, m_{x} t_{y}^{-1}\right.$ & 5 & 23 & {$[[1,1,0],[0,2,0],[0,0,2]]$} \\
\hline$H_{498}=\left\langle m_{x} t_{z}^{-1}, r_{y}^{2} r_{z} t_{3}\right.$ & 5 & 23 & {$[[1,1,0],[0,2,0],[0,0,2]]$} \\
\hline$H_{499}=\left\langle m_{z} r_{z}^{-1} t_{z}, m_{z} r_{z}\right.$ & 5 & 23 & {$[[1,1,0],[0,2,0],[0,0,2]]$} \\
\hline$H_{500}=\left\langle m_{x} t_{z}^{-1}, m_{z} r \bar{z}\right.$ & 5 & 23 & {$[[1,1,0],[0,2,0],[0,0,2]]$} \\
\hline$H_{501}=\left\langle m_{z} r_{z}^{-1} t_{x}, r_{y}^{2}\right.$ & 5 & 23 & {$[[1,1,0],[0,2,0],[0,0,2]]$} \\
\hline$H_{502}=\left\langle m_{x} t_{x}, m_{x} t_{y}^{-1}\right.$ & 5 & 23 & {$[[1,0,2],[0,1,2],[0,0,4]]$} \\
\hline$H_{503}=\left\langle r_{y}^{2} r_{z} t_{z}, r_{y}^{2} t_{z} \bar{z}\right.$ & 5 & 24 & {$[[1,0,0],[0,1,0],[0,0,4]]$} \\
\hline$H_{504}=\left\langle r_{x}^{2} r_{y} t_{y}^{-1}, r_{z}^{2}\right.$, & 5 & 24 & {$[[1,0,0],[0,4,0],[0,0,1]]$} \\
\hline$H_{505}=\left\langle r_{y}^{2} r_{z} t_{z}, r_{y}^{2} r_{z}\right.$ & 5 & 24 & {$[[1,1,0],[0,2,0],[0,0,2]]$} \\
\hline$H_{506}=\left\langle r_{y}^{2} t_{x}, r_{y}^{2} t_{y}^{-1}\right.$, & 5 & 24 & {$[[1,1,0],[0,2,0],[0,0,2]]$} \\
\hline$H_{507}=\left\langle r_{y}^{2} r_{z} t_{x}, r_{y}^{2} t_{z}\right.$ & 5 & 24 & {$[[1,1,0],[0,2,0],[0,0,2]]$} \\
\hline$H_{508}=\left\langle r_{y}^{2} r_{z} t_{x}, r_{z}^{-1} t_{z}^{-}\right.$ & 5 & 24 & {$[[1,1,0],[0,2,0],[0,0,2]]$} \\
\hline$H_{509}=\left\langle r_{y}^{2} t_{z}, r_{y}^{2} t_{z}^{-1}, r_{z}^{-1} t_{x}, r_{z}^{2}\right\rangle$ & 5 & 24 & $[[1,1,0],[0,2,0],[0,0,2]]]$ \\
\hline$H_{510}=\left\langle r_{y}^{2} r_{z} t_{z}, r_{y}^{2} r_{z} t_{z}^{-1}, r_{z}^{-1} t_{x}, r_{z}^{2}\right\rangle$ & 5 & 24 & {$[[1,1,0],[0,2,0],[0,0,2]]$} \\
\hline$H_{511}=\left\langle r_{y}^{2} t_{y}^{-1}, r_{y}^{2} t_{z}, r_{y}^{2} t_{z}^{-1}, r_{z}^{2}, r_{z}^{2} r_{x} t_{x}\right\rangle$ & 5 & 24 & {$[[2,0,1],[0,1,1],[0,0,2]]$} \\
\hline$H_{512}=\left\langle r_{y}^{2} r_{z} t_{z}, r_{y}^{2} t_{z}^{-1}, r_{z}^{-1} t_{x}, r_{z}^{2}\right\rangle$ & 5 & 24 & {$[[1,0,2],[0,1,2],[0,0,4]]$} \\
\hline$H_{513}=\left\langle m_{x} r_{z} t_{z}^{-1}, m_{x} t_{x}, m_{x} t_{y}^{-1}, r_{z}^{2}\right\rangle$ & 5 & 25 & {$[[1,1,0],[0,2,0],[0,0,2]]$} \\
\hline$H_{514}=\left\langle m_{x} t_{x}, m_{x} t_{y}^{-1},{ }^{\infty}\right.$ & 5 & 25 & $[[1,1,0],[0,2,0],[0,0,2]]]$ \\
\hline$H_{515}=\left\langle m_{x} r_{z} t_{x}, m_{x} t_{z}^{-1}, r_{z}^{2}\right\rangle$ & 5 & 25 & {$[[1,1,0],[0,2,0],[0,0,2]]$} \\
\hline$H_{516}=\left\langle m_{x} r_{z} t_{x}, r_{z}^{-1} t_{z}^{-1}, r_{z}^{2}\right\rangle$ & 5 & 25 & {$[[1,1,0],[0,2,0],[0,0,2]]$} \\
\hline$H_{517}=\left\langle m_{x} t_{z}^{-1}, r_{z}^{-1} t_{x}, r_{z}^{2}\right\rangle$ & 5 & 25 & {$[[1,1,0],[0,2,0],[0,0,2]]$} \\
\hline$H_{518}=\left\langle m_{x} r_{z} t_{z}^{-1}, r_{z}^{-1} t_{x}, r_{z}^{2}\right\rangle$ & 5 & 25 & {$[[1,1,0],[0,2,0],[0,0,2]]$} \\
\hline$H_{519}=\left\langle m_{z} r_{z}^{-1} t_{z}, r_{y}^{2} t_{z}^{-1}, r_{z}^{2}, r_{z}^{2} t_{x}, r_{z}^{2} t_{y}^{-1}\right\rangle$ & 5 & 26 & {$[[1,0,0],[0,1,0],[0,0,4]]$} \\
\hline$H_{520}=\left\langle m_{x} r_{z} t_{z}^{-1}, r_{y}^{2} t_{x}, r_{y}^{2} t_{y}^{-1}, r_{z}^{2}\right\rangle$ & 5 & 26 & $[[1,1,0],[0,2,0],[0,0,2]]]$ \\
\hline$H_{521}=\left\langle m_{z} r_{z}^{-1} t_{z}, m_{z} r_{z}^{-1} t_{z}^{-1}, r_{y}^{2} t_{x}, r_{y}^{2} t_{y}^{-1}, r_{z}^{2}\right\rangle$ & 5 & 26 & {$[[1,1,0],[0,2,0],[0,0,2]]$} \\
\hline$H_{522}=\left\langle m_{x} r_{z} t_{x}, r_{y}^{2} t_{z}, r_{y}^{2} t_{z}^{-1}, r_{z}^{2}\right\rangle$ & 5 & 26 & $[[1,1,0],[0,2,0],[0,0,2]]]$ \\
\hline$H_{523}=\left\langle m_{x} r_{z} t_{x}, m_{z} r_{z}^{-1^{9}} t_{z}, m_{z} r_{z}^{-1} t_{z}^{-1}, r_{z}^{2}\right\rangle$ & 5 & 26 & {$[[1,1,0],[0,2,0],[0,0,2]]$} \\
\hline$H_{524}=\left\langle m_{z} r_{z}^{-1} t_{x}, r_{y}^{2} t_{z}, r_{y}^{2} t_{z}^{-1}, r_{z}^{2}\right\rangle$ & 5 & 26 & {$[[1,1,0],[0,2,0],[0,0,2]]$} \\
\hline$H_{525}=\left\langle m_{x} r_{z} t_{z}^{-1}, m_{z} r_{z}^{-1^{9}} t_{x}, r_{z}^{2}\right\rangle$ & 5 & 26 & {$[[1,1,0],[0,2,0],[0,0,2]]$} \\
\hline$H_{526}=\left\langle m_{x} r_{z} t_{x}, m_{z} r_{z}^{-1} t_{z}, r_{y}^{2} t_{z}^{-1}, r_{z}^{2}\right\rangle$ & 5 & 26 & {$[[1,0,2],[0,1,2],[0,0,4]]$} \\
\hline$H_{527}=\left\langle i t_{z}^{-1}, m_{x} t_{x}, m_{x} t_{y}^{-1}, r_{y}^{2} r_{z} t_{z}, r_{z}^{2}\right\rangle$ & 5 & 29 & {$[[1,1,0],[0,2,0],[0,0,4]]$} \\
\hline$H_{528}=\left\langle i t_{z}^{-1}, m_{x} t_{x}, m_{x} t_{y}^{-1}, m_{z} r_{z}^{-1} t_{z}, r_{z}^{2}\right\rangle$ & 5 & 29 & {$[[1,1,0],[0,2,0],[0,0,4]]$} \\
\hline
\end{tabular}




\begin{tabular}{|c|c|c|c|}
\hline$H$ & $H_{0,0,0}$ & $P(H)$ & Translation basis of $H$ \\
\hline$H_{529}=\left\langle m_{x} t_{x}, m_{x} t_{y}^{-1}, r_{y}^{2} r_{z} t_{z}, r_{y}^{2} t_{z}^{-1}, r_{z}^{2}\right\rangle$ & 5 & 29 & {$[[1,1,0],[0,2,0],[0,0,4]]$} \\
\hline$H_{530}=\left\langle m_{x} t_{x}, m_{x} t_{y}^{-1}, m_{z} r_{z}^{-1} t_{z}, r_{y}^{2} t_{z}^{-1}, r_{z}^{2}\right\rangle$ & 5 & 29 & {$[[1,1,0],[0,2,0],[0,0,4]]$} \\
\hline$H_{531}=\left\langle i t_{z}^{-1}, m_{x} r_{z} t_{x}, r_{y}^{2} t_{z}, r_{z}^{2}\right\rangle$ & 5 & 29 & {$[[1,1,0],[0,2,0],[0,0,4]]$} \\
\hline$H_{532}=\left\langle i t_{z}^{-1}, m_{x} r_{z} t_{x}, m_{z} r_{z}^{-1} t_{z}, r_{z}^{2}\right\rangle$ & 5 & 29 & {$[[1,1,0],[0,2,0],[0,0,4]]$} \\
\hline$H_{533}=\left\langle m_{x} r_{z} t_{x}, r_{y}^{2} r_{z} t_{z}, r_{y}^{2} t_{z}^{-1}, r_{z}^{2}\right\rangle$ & 5 & 29 & {$[[1,1,0],[0,2,0],[0,0,4]]$} \\
\hline$H_{534}=\left\langle m_{x} r_{z} t_{x}, m_{z} r_{z}^{-1} t_{z}, r_{y}^{2} r_{z} t_{z}^{-1}, r_{z}^{2}\right\rangle$ & 5 & 29 & {$[[1,1,0],[0,2,0],[0,0,4]]$} \\
\hline$H_{535}=\left\langle i t_{z}^{-1}, r_{y}^{2} t_{z}, r_{z}^{-1} t_{x}, r_{z}^{2}\right\rangle$ & 5 & 29 & {$[[1,1,0],[0,2,0],[0,0,4]]$} \\
\hline$H_{536}=\left\langle i t_{z}^{-1}, r_{y}^{2} r_{z} t_{z}, r\right.$ & 5 & 29 & {$[[1,1,0],[0,2,0],[0,0,4]]$} \\
\hline$H_{537}=\left\langle m_{z} r_{z}^{-1^{9}} t_{z}, r_{y}^{2} t_{z}^{-1}\right.$ & 5 & 29 & {$[[1,1,0],[0,2,0],[0,0,4]]$} \\
\hline$H_{538}=\left\langle m_{z} r_{z}^{-1} t_{z}, r_{y}^{2} r_{z} t_{z}^{-1}, r_{z}^{-1} t_{x}, r_{z}^{2}\right\rangle$ & 5 & 29 & {$[[1,1,0],[0,2,0],[0,0,4]]$} \\
\hline$H_{539}=\left\langle i t_{x}, m_{z} r_{z}^{-1} t_{z}, r_{y}^{2} r_{z} t_{z}^{-1}, r_{y}^{2} t_{y}^{-1}, r_{z}^{2}\right\rangle$ & 5 & 29 & {$[[1,1,2],[0,2,0],[0,0,4]]$} \\
\hline$H_{540}=\left\langle i t_{x}, m_{x} t_{z}^{-1}, r_{x}^{2} r_{3}\right.$ & 5 & 29 & {$[[1,2,1],[0,4,0],[0,0,2]]$} \\
\hline$H_{541}=\left\langle i t_{z}, i t_{z}^{-1}, m_{x} t_{y}^{-1}, r_{z}^{2}, r_{z}^{2} r_{x} t_{x}\right\rangle$ & 5 & 29 & {$[[2,1,1],[0,2,0],[0,0,2]]$} \\
\hline$H_{542}=\left\langle m_{z} r_{x}, m_{z} r_{x} t_{x}, m_{z} r_{x} t_{y}^{-1}\right\rangle$ & 6 & 6 & {$[[1,0,0],[0,1,0],[0,0,1]]$} \\
\hline$H_{543}=\left\langle m_{z} r_{x}, m_{z} r_{x} t_{y}^{-1}\right.$ & 6 & 10 & {$[[2,0,0],[0,1,0],[0,0,1]]$} \\
\hline$H_{544}=\left\langle m_{z} r_{x}, m_{z} r_{x} t_{x}, r_{x}^{2} t_{y}^{-1}, r_{x}^{2} t_{z}\right\rangle$ & 6 & 10 & {$[[1,0,0],[0,1,1],[0,0,2]]$} \\
\hline$H_{545}=\left\langle m_{z} r_{x}, r_{x}^{2} t_{x}, r_{x}^{2} t_{y}^{-1}, r_{x}^{2} t_{z}\right\rangle$ & 6 & 10 & {$[[1,0,1],[0,1,1],[0,0,2]]$} \\
\hline$H_{546}=\left\langle m_{z} r_{x}, m_{z} r_{x} t_{y}^{-1}, r_{y}^{2} r_{x} t_{x}, r_{y}^{2} r_{x} t_{x}^{-1}\right\rangle$ & 6 & 11 & {$[[2,0,0],[0,1,0],[0,0,1]]$} \\
\hline$H_{547}=\left\langle m_{z} r_{x}, m_{z} r_{x} t_{x}, r_{y}^{2} r_{x} t_{y}^{-1}\right\rangle$ & 6 & 11 & {$[[1,0,0],[0,1,1],[0,0,2]]$} \\
\hline$H_{548}=\left\langle m_{z} r_{x}, r_{y}^{2} r_{x} t_{x}, r_{y}^{2}\right.$ & 6 & 11 & {$[[1,0,1],[0,1,1],[0,0,2]]$} \\
\hline$H_{549}=\left\langle i t_{x}, i t_{x}^{-1}, m_{z} r_{x}, m_{z} r_{x} t_{y}^{-1}\right\rangle$ & 6 & 12 & {$[[2,0,0],[0,1,0],[0,0,1]]$} \\
\hline$H_{550}=\left\langle i t_{y}^{-1}, i t_{z}, m_{z} r_{x}, m_{z} r_{x} t_{x}\right\rangle$ & 6 & 12 & {$[[1,0,0],[0,1,1],[0,0,2]]$} \\
\hline$H_{551}=\left\langle i t_{x}, i t_{x}^{-1}, i t_{y}^{-1}, i t_{z}, m_{z} r_{x}\right\rangle$ & 6 & 12 & {$[[1,0,1],[0,1,1],[0,0,2]]$} \\
\hline$H_{552}=\left\langle i t_{x}, m_{z} r_{x}, m_{z} r_{x} t_{y}^{-1}, r_{y}^{2} r_{x} t_{x}^{-1}\right\rangle$ & 6 & 20 & {$[[4,0,0],[0,1,0],[0,0,1]]$} \\
\hline$H_{553}=\left\langle i t_{y}^{-1}, m_{z} r_{x}, m_{z} r_{x} t_{x}, r_{x}^{2} t_{z}\right\rangle$ & 6 & 20 & {$[[1,0,0],[0,1,3],[0,0,4]]$} \\
\hline$H_{554}=\left\langle i t_{x}, i t_{x}^{-1}, m_{z} r_{x}, r_{y}^{2} r_{x} t_{y}^{-1}\right\rangle$ & 6 & 20 & {$[[2,0,0],[0,1,1],[0,0,2]]$} \\
\hline$H_{555}=\left\langle i t_{x}, i t_{x}^{-1}, m_{z r}\right.$ & 6 & 20 & {$[[2,0,0],[0,1,1],[0,0,2]]$} \\
\hline$H_{556}=\left\langle i t_{y}^{-1}, i t_{z}, m_{z} r\right.$ & 6 & 20 & {$[[2,0,0],[0,1,1],[0,0,2]]$} \\
\hline$H_{557}=\left\langle m_{z} r_{x}, r_{x}^{2} t_{y}^{-1}, r_{x}^{2} t_{z}, r_{y}^{2} r_{x} t_{x}, r_{y}^{2} r_{x} t_{x}^{-1}\right\rangle$ & 6 & 20 & {$[[2,0,0],[0,1,1],[0,0,2]]$} \\
\hline$H_{558}=\left\langle i t_{y}^{-1}, i t_{z}, m_{z} r_{x}\right.$ & 6 & 20 & {$[[2,0,0],[0,1,1],[0,0,2]]$} \\
\hline$H_{559}=\left\langle m_{z} r_{x}, r_{x}^{2} t_{x}, r_{y}^{2}\right.$ & 6 & 20 & {$[[2,0,0],[0,1,1],[0,0,2]]$} \\
\hline$H_{560}=\left\langle i t_{x}, m_{z} r_{x}, r_{x}^{2} t_{y}^{g}\right.$ & 6 & 20 & {$[[2,0,1],[0,1,1],[0,0,2]]$} \\
\hline$H_{561}=\left\langle i t_{y}^{-1}, m_{z} r_{x}, r_{x}^{2} t_{z}, r_{y}^{2} r_{x} t_{x}, r_{y}^{2} r_{x} t_{x}^{-1}\right\rangle$ & 6 & 20 & {$[[1,0,2],[0,1,3],[0,0,4]]$} \\
\hline$H_{562}=\left\langle m_{y} t_{y}^{-1}, m_{z} r_{x}, m_{z} r_{x} t_{x}, r_{x}^{-1} t_{z}\right\rangle$ & 6 & 25 & {$[[1,0,0],[0,2,0],[0,0,2]]$} \\
\hline$H_{563}=\left\langle m_{z} r_{x}, m_{z} r_{x} t_{x}, r_{x}^{-1} t_{y}^{-1}\right\rangle$ & 6 & 25 & {$[[1,0,0],[0,2,0],[0,0,2]]$} \\
\hline$H_{564}=\left\langle m_{y} t_{y}^{-1}, m_{z} r_{x}, r_{x}^{-1} t_{z}, r_{x}^{2} t_{x}\right\rangle$ & 6 & 25 & {$[[1,1,1],[0,2,0],[0,0,2]]$} \\
\hline$H_{565}=\left\langle m_{z} r_{x}, r_{x}^{-1} t_{y}^{-1}, r_{x}^{2} t_{x}\right\rangle$ & 6 & 25 & {$[[1,1,1],[0,2,0],[0,0,2]]$} \\
\hline$H_{566}=\left\langle m_{x} r_{x}^{-1} t_{z}, m_{z} r_{x}, m_{z} r_{x} t_{x}, r_{z}^{2} t_{y}^{-1}\right\rangle$ & 6 & 26 & {$[[1,0,0],[0,2,0],[0,0,2]]$} \\
\hline$H_{567}=\left\langle m_{x} r_{x}^{-1} t_{y}^{-1}, m_{z} r_{x}, m_{z} r_{x} t_{x}\right\rangle$ & 6 & 26 & {$[[1,0,0],[0,2,0],[0,0,2]]$} \\
\hline$H_{568}=\left\langle m_{x} r_{x}^{-1} t_{z}, m_{z} r_{x}, r_{x}^{2} t_{x}, r_{z}^{2} t_{y}^{-1}\right\rangle$ & 6 & 26 & {$[[1,1,1],[0,2,0],[0,0,2]]$} \\
\hline$H_{569}=\left\langle m_{x} r_{x}^{-1} t_{y}^{-1}, m_{z} r_{x}, r_{x}^{2} t_{x}\right\rangle$ & 6 & 26 & {$[[1,1,1],[0,2,0],[0,0,2]]$} \\
\hline$H_{570}=\left\langle m_{z} r_{x}, m_{z} r_{x} t_{x}, r\right.$ & 6 & 29 & {$[[1,0,0],[0,2,2],[0,0,4]]$} \\
\hline$H_{571}=\left\langle m_{z} r_{x}, r_{x}^{-1} t_{z}, r_{y}^{2} r_{x} t_{x}, r_{y}^{2} r_{x} t_{x}^{-1}, r_{z}^{2} t_{y}^{-1}\right\rangle$ & 6 & 29 & {$[[1,0,2],[0,2,2],[0,0,4]]$} \\
\hline$H_{572}=\left\langle i t_{x}, i t_{x}^{-1}, m_{x} r_{x}^{-1} t_{z}, m_{z} r_{x}, r_{z}^{2} t_{y}^{-1}\right\rangle$ & 6 & 29 & {$[[2,0,0],[0,2,0],[0,0,2]]$} \\
\hline$H_{573}=\left\langle i t_{x}, i t_{x}^{-1}, m_{x} r_{x}^{-1} t_{y}^{-1}, m_{z} r_{x}\right\rangle$ & 6 & 29 & {$[[2,0,0],[0,2,0],[0,0,2]]$} \\
\hline$H_{574}=\left\langle i t_{x}, i t_{x}^{-1}, m_{y} t_{y}^{-1}, m_{z} r_{x}, r_{x}^{-1} t_{z}\right\rangle$ & 6 & 29 & {$[[2,0,0],[0,2,0],[0,0,2]]$} \\
\hline$H_{575}=\left\langle i t_{x}, i t_{x}^{-1}, m_{z} r_{x}, r_{x}^{-1} t_{y}^{-1}\right\rangle$ & 6 & 29 & {$[[2,0,0],[0,2,0],[0,0,2]]$} \\
\hline$H_{576}=\left\langle m_{x} r_{x}^{-1} t_{z}, m_{z} r_{x}, r_{y}^{2} r_{x} t_{x}, r_{y}^{2} r_{x} t_{x}^{-1}, r_{z}^{2} t_{y}^{-1}\right\rangle$ & 6 & 29 & {$[[2,0,0],[0,2,0],[0,0,2]]$} \\
\hline$H_{577}=\left\langle m_{x} r_{x}^{-1} t_{y}^{-1}, m_{z} r_{x}, r_{y}^{2} r_{x} t_{x}, r_{y}^{2} r_{x} t_{x}^{-1}\right\rangle$ & 6 & 29 & {$[[2,0,0],[0,2,0],[0,0,2]]$} \\
\hline$H_{578}=\left\langle m_{y} t_{y}^{-1}, m_{z} r_{x}, r_{x}^{-1} t_{z}, r_{y}^{2} r_{x} t_{x}, r_{y}^{2} r_{x} t_{x}^{-1}\right\rangle$ & 6 & 29 & {$[[2,0,0],[0,2,0],[0,0,2]]$} \\
\hline$H_{579}=\left\langle m_{z} r_{x}, r_{x}^{-1} t_{y}^{-1}, r_{y}^{2} r_{x} t_{x}, r_{y}^{2} r_{x} t_{x}^{-1}\right\rangle$ & 6 & 29 & {$[[2,0,0],[0,2,0],[0,0,2]]$} \\
\hline$H_{580}=\left\langle i t_{x}, m_{y} t_{y}^{-1}, m_{z} r_{x}, r_{x}^{-1} t_{z}, r_{y}^{2} r_{x} t_{x}^{-1}\right\rangle$ & 6 & 29 & {$[[2,1,1],[0,2,0],[0,0,2]]$} \\
\hline$H_{581}=\left\langle i t_{x}, m_{z} r_{x}, r_{x}^{-1} t_{y}^{-1}, r_{y}^{2} r_{x} t_{x}^{-1}\right\rangle$ & 6 & 29 & {$[[2,1,1],[0,2,0],[0,0,2]]$} \\
\hline$H_{582}=\left\langle r_{y} r_{x}, r_{y}^{-1} r_{z}^{-1} t_{x}\right\rangle$ & 7 & 7 & {$[[1,0,0],[0,1,0],[0,0,1]]$} \\
\hline $\begin{array}{l}H_{583}=\left\langle i t_{x}, i t_{x}^{-1}, r_{y} r_{x}\right\rangle \\
H_{584}=\left\langle m_{x} r_{y} t_{x}, r_{y} r_{x}\right\rangle\end{array}$ & $\begin{array}{l}7 \\
7\end{array}$ & $\begin{array}{l}17 \\
18\end{array}$ & $\begin{array}{l}{[[1,0,1],[0,1,1],[0,0,2]]} \\
{[[1,0,1],[0,1,1],[0,0,2]]}\end{array}$ \\
\hline$H_{585}=\left\langle r_{y} r_{x}, r_{z}^{2} r_{x} t_{x}, r_{z}^{2} r_{x} t_{x}^{-1}\right\rangle$ & 7 & 19 & {$[[1,0,1],[0,1,1],[0,0,2]]$} \\
\hline$H_{586}=\left\langle r_{y} r_{x}, r_{z}^{2} t_{x}, r_{z}^{2} t_{x}^{-1}\right\rangle$ & 7 & 27 & {$[[1,1,1],[0,2,0],[0,0,2]]$} \\
\hline$H_{587}=\left\langle r_{y} r_{x}, r_{y} r_{x}^{-1} t_{x}\right\rangle$ & 7 & 27 & {$[[1,1,1],[0,2,0],[0,0,2]]$} \\
\hline$H_{588}=\left\langle i t_{x}, r_{y} r_{x}, r_{z}^{2} r_{x} t_{x}^{-1}\right\rangle$ & 7 & 28 & {$[[1,0,3],[0,1,3],[0,0,4]]$} \\
\hline$H_{589}=\left\langle m_{x} t_{x}, m_{x} t_{x}^{-1}, r_{y} r_{x}\right\rangle$ & 7 & 30 & {$[[2,0,0],[0,2,0],[0,0,2]]$} \\
\hline$H_{590}=\left\langle m_{x} r_{y} r_{x} t_{x}, r_{y} r_{x}\right\rangle$ & 7 & 30 & {$[[2,0,0],[0,2,0],[0,0,2]]$} \\
\hline$H_{591}=\left\langle m_{z} r_{z}^{-1} t_{x}, r_{y} r_{x}\right\rangle$ & 7 & 31 & {$[[2,0,0],[0,2,0],[0,0,2]]$} \\
\hline$H_{592}=\left\langle r_{y} r_{x}, r_{y}^{2} r_{x} t_{x}, r_{y}^{2} r_{x} t_{x}^{-1}\right\rangle$ & 7 & 32 & {$[[2,0,0],[0,2,0],[0,0,2]]$} \\
\hline$H_{593}=\left\langle m_{x} t_{x}^{-1}, r_{y} r_{x}, r_{y}^{2} r_{x} t_{x}\right\rangle$ & 7 & 33 & {$[[2,0,2],[0,2,2],[0,0,4]]$} \\
\hline$H_{594}=\left\langle r_{x}^{2}, r_{z}^{2}, r_{z}^{2} t_{x}, r_{z}^{2} t_{y}^{9}-r_{z}^{2} t_{z}^{-1}\right\rangle$ & 8 & 8 & {$[[1,0,0],[0,1,0],[0,0,1]]$} \\
\hline$H_{595}=\left\langle i t_{x}, r_{x}^{2}, r_{z}^{2}, r_{z}^{2} t_{y}^{-1}, r_{z}^{2} t_{z}^{-1}\right\rangle$ & 8 & 21 & {$[[2,0,0],[0,1,0],[0,0,1]]$} \\
\hline$H_{596}=\left\langle i t_{x}, i t_{y}^{-1}, r_{x}^{2}, r_{z}^{2}, r_{z}^{2} t_{z}^{-1}\right\rangle$ & 8 & 21 & {$[[1,1,0],[0,2,0],[0,0,1]]$} \\
\hline
\end{tabular}




\begin{tabular}{|c|c|c|c|}
\hline$H$ & $H_{0,0,0}$ & $P(H)$ & Translation basis of $H$ \\
\hline$H_{597}=\left\langle i t_{x}, i t_{y}^{-1}, i t_{z}^{-1}, r_{x}^{2}, r_{z}^{2}\right\rangle$ & 8 & 21 & {$[[1,0,1],[0,1,1],[0,0,2]]$} \\
\hline$H_{598}=\left\langle r_{x}^{2}, r_{y}^{2} r_{z} t_{z}^{-1}, r_{z}^{2}, r_{z}^{2} t_{x}, r_{z}^{2} t_{y}^{-1}\right\rangle$ & 8 & 24 & {$[[1,0,0],[0,1,0],[0,0,2]]$} \\
\hline$H_{599}=\left\langle r_{x}^{2}, r_{z}^{2}, r_{z}^{2} r_{x} t_{y}^{-1}, r_{z}^{2} t_{x}\right\rangle$ & 8 & 24 & {$[[1,0,0],[0,1,1],[0,0,2]]$} \\
\hline$H_{600}=\left\langle r_{x}^{2}, r_{z}^{2}, r_{z}^{2} r_{x} t_{x}, r_{z}^{2} r_{x} t_{y}^{-1}\right\rangle$ & 8 & 24 & {$[[1,0,1],[0,1,1],[0,0,2]]$} \\
\hline$H_{601}=\left\langle m_{x} r_{z} t_{z}^{-1}, r_{x}^{2}, r_{z}^{2}, r_{z}^{2} t_{x}^{y}, r_{z}^{2} t_{y}^{-1}\right\rangle$ & 8 & 26 & {$[[1,0,0],[0,1,0],[0,0,2]]$} \\
\hline$H_{602}=\left\langle m_{x} r_{x}^{-1} t_{y}^{-1}, r_{x}^{2}, r_{z}^{2}, r_{z}^{2} t_{x}\right\rangle$ & 8 & 26 & {$[[1,0,0],[0,1,1],[0,0,2]]$} \\
\hline$H_{603}=\left\langle m_{x} r_{x}^{-1} t_{x}, m_{x} r_{x}^{-1} t_{y}^{-1}, r_{x}^{2}, r_{z}^{2}\right\rangle$ & 8 & 26 & {$[[1,0,1],[0,1,1],[0,0,2]]$} \\
\hline$H_{604}=\left\langle i t_{x}, i t_{y}^{-1}, r_{x}^{2}, r_{y}^{2} r_{z} t_{z}^{-1}, r_{z}^{2}\right\rangle$ & 8 & 29 & $[[1,1,0],[0,2,0],[0,0,2]]]$ \\
\hline$H_{605}=\left\langle i t_{x}, i t_{y}^{-1}, m_{x} r_{z} t_{z}^{-1}, r_{x}^{2}, r_{z}^{2}\right\rangle$ & 8 & 29 & {$[[1,1,0],[0,2,0],[0,0,2]]$} \\
\hline$H_{606}=\left\langle i t_{x}, r_{x}^{2}, r_{z}^{2}, r_{z}^{2} r_{x} t_{y}^{-1}\right\rangle$ & 8 & 29 & $[[2,0,0],[0,1,1],[0,0,2]]]$ \\
\hline$H_{607}=\left\langle i t_{x}, m_{x} r_{x}^{-1} t_{y}^{-1}, r_{x}^{2}, r_{z}^{2}\right\rangle$ & 8 & 29 & $[[2,0,0],[0,1,1],[0,0,2]]]$ \\
\hline$H_{608}=\left\langle m_{x} r_{x}^{-1} t_{y}^{-1}, r_{x}^{2}, r_{z}^{2}, r_{z}^{2} r_{x} t_{x}\right\rangle$ & 8 & 29 & {$[[2,0,0],[0,1,1],[0,0,2]]$} \\
\hline$H_{609}=\left\langle m_{x} r_{x}^{-1} t_{x}, r_{x}^{2}, r_{z}^{2}, r_{z}^{2} r_{x} t_{y}^{-1}\right\rangle$ & 8 & 29 & {$[[2,0,0],[0,1,1],[0,0,2]]$} \\
\hline$H_{610}=\left\langle i, i t_{x}, i t_{y}^{-1}\right.$, & 9 & 9 & {$[[1,0,0],[0,1,0],[0,0,1]]$} \\
\hline$H_{611}=\left\langle i, i t_{x}, i t_{y}^{\frac{9}{y}}-1\right.$ & 9 & 20 & {$[[1,0,0],[0,1,0],[0,0,2]]$} \\
\hline$H_{612}=\left\langle i, i t_{z}^{-1}, r_{y}^{2} r_{z} t_{x}, r_{z}^{2}\right\rangle$ & 9 & 20 & {$[[1,1,0],[0,2,0],[0,0,1]]$} \\
\hline$H_{613}=\left\langle i, r_{y}^{2} r_{z} t_{x}, r_{y}^{2} r_{z} t_{z}^{-1}, r_{z}^{2}\right\rangle$ & 9 & 20 & {$[[1,0,1],[0,1,1],[0,0,2]]$} \\
\hline$H_{614}=\left\langle i, i t_{x}, i t_{y}^{-1}\right.$ & 9 & 21 & {$[[1,0,0],[0,1,0],[0,0,2]]$} \\
\hline$H_{615}=\left\langle i, i t_{x}, i t_{z}-1\right.$ & 9 & 21 & $[[1,0,0],[0,2,0],[0,0,1]]]$ \\
\hline$H_{616}=\left\langle i, i t_{x}, r_{y}^{2} t_{y}\right.$ & 9 & 21 & $[[1,0,0],[0,1,1],[0,0,2]]]$ \\
\hline$H_{617}=\left\langle i, i t_{z}^{-1}, r_{y}^{2} t\right.$ & 9 & 21 & {$[[1,1,0],[0,2,0],[0,0,1]]$} \\
\hline$H_{618}=\left\langle i, r_{y}^{2} t_{x}, r_{y}^{2} t_{y}^{-1}, r_{y}^{2} t_{z}^{-1}, r_{z}^{2}\right\rangle$ & 9 & 21 & {$[[1,0,1],[0,1,1],[0,0,2]]$} \\
\hline$H_{619}=\left\langle i, i t_{x}, i t_{y}^{-1}, m_{z} r_{z}^{-1} t_{z}^{-1}, r_{z}^{2}\right\rangle$ & 9 & 22 & $[[1,0,0],[0,1,0],[0,0,2]]]$ \\
\hline$H_{620}=\left\langle i, i t_{z}^{-1}, m_{z}\right.$ & 9 & 22 & $[[1,1,0],[0,2,0],[0,0,1]]]$ \\
\hline$H_{621}=\left\langle i, m_{z}^{z} r_{z}^{-1} t_{x}\right.$ & 9 & 22 & {$[[1,0,1],[0,1,1],[0,0,2]]$} \\
\hline$H_{622}=\left\langle i, r_{y}^{2} r_{z} t_{z}^{-1}\right.$, & 9 & 29 & {$[[1,1,0],[0,2,0],[0,0,2]]$} \\
\hline$H_{623}=\left\langle i, m_{z} r_{z}^{-1} t_{z}^{-1}, r_{y}^{2} t_{x}, r_{y}^{2} t_{y}^{-1}, r_{z}^{2}\right\rangle$ & 9 & 29 & {$[[1,1,0],[0,2,0],[0,0,2]]$} \\
\hline$H_{624}=\left\langle i, r_{y}^{2} r_{z} t_{x}, r\right.$ & 9 & 29 & {$[[1,1,0],[0,2,0],[0,0,2]]$} \\
\hline$H_{625}=\left\langle i, m_{z} r_{z}^{-1} t_{z}^{-1}, r_{y}^{2} r_{z} t_{x}, r_{z}^{2}\right\rangle$ & 9 & 29 & {$[[1,1,0],[0,2,0],[0,0,2]]$} \\
\hline$H_{626}=\left\langle i, m_{z} r_{z}^{-1} t_{x}, r_{y}^{2} t_{z}^{-1}, r_{z}^{2}\right\rangle$ & 9 & 29 & {$[[1,1,0],[0,2,0],[0,0,2]]$} \\
\hline$H_{627}=\left\langle i, m_{z} r_{z}^{-1} t_{x}\right.$ & 9 & 29 & $[[1,1,0],[0,2,0],[0,0,2]]]$ \\
\hline$H_{628}=\left\langle m_{z} r_{x}, r_{x}^{2}, r_{x}^{2} t_{x}, r_{x}^{2} t_{y}^{-1}\right\rangle$ & 10 & 10 & $[[1,0,0],[0,1,0],[0,0,1]]]$ \\
\hline$H_{629}=\left\langle i t_{x}, i t_{x}^{-1}, m_{z} r_{x}, r_{x}^{2}, r_{x}^{2} t_{y}^{-1}\right\rangle$ & 10 & 20 & $[[2,0,0],[0,1,0],[0,0,1]]]$ \\
\hline$H_{630}=\left\langle i t_{y}^{-1}, m_{z} r_{x}\right.$ & 10 & 20 & {$[[1,0,0],[0,1,1],[0,0,2]]$} \\
\hline$H_{631}=\left\langle i t_{x}, i t_{x}^{-1}, i\right.$ & 10 & 20 & {$[[1,0,1],[0,1,1],[0,0,2]]$} \\
\hline$H_{632}=\left\langle m_{y} t_{x}, m_{z} r\right.$ & 10 & 25 & {$[[2,0,0],[0,1,0],[0,0,1]]$} \\
\hline$H_{633}=\left\langle m_{y} t_{y}^{-1}, m_{z} r_{x}, r_{x}^{2}, r_{x}^{2} t_{x}\right\rangle$ & 10 & 25 & {$[[1,0,0],[0,1,1],[0,0,2]]$} \\
\hline$H_{634}=\left\langle m_{y} t_{x}, m_{y} t_{y}^{-1}, m_{z} r_{x}, r_{x}^{2}\right\rangle$ & 10 & 25 & {$[[1,0,1],[0,1,1],[0,0,2]]$} \\
\hline$H_{635}=\left\langle m_{z} r_{x}, r_{x}^{2}, r_{x}^{2} t_{y}^{-1}, r_{z}^{2} t_{x}, r_{z}^{2} t_{x}^{-1}\right\rangle$ & 10 & 26 & {$[[2,0,0],[0,1,0],[0,0,1]]$} \\
\hline$H_{636}=\left\langle m_{z} r_{x}, r_{x}^{2}, r_{x}^{2} t_{x}, r_{z}^{2} t_{y}^{-1}\right\rangle$ & 10 & 26 & {$[[1,0,0],[0,1,1],[0,0,2]]$} \\
\hline$H_{637}=\left\langle m_{z} r_{x}, r_{x}^{\tilde{2}}, r_{z}^{2} t_{x}, r_{z}^{\tilde{2}} t_{x}^{-1}, r_{z}^{2} t_{y}^{-1}\right\rangle$ & 10 & 26 & {$[[1,0,1],[0,1,1],[0,0,2]]$} \\
\hline$H_{638}=\left\langle i t_{x}, m_{z} r_{x}, r_{x}^{2}, r_{x}^{2} t_{y}^{-1}, r_{z}^{2} t_{x}^{-1}\right\rangle$ & 10 & 29 & $[[4,0,0],[0,1,0],[0,0,1]]]$ \\
\hline$H_{639}=\left\langle i t_{x}, i t_{x}^{-1}, m_{z} r_{x}, r_{x}^{2}, r_{z}^{2} t_{y}^{-1}\right\rangle$ & 10 & 29 & $[[2,0,0],[0,1,1],[0,0,2]]]$ \\
\hline$H_{640}=\left\langle i t_{x}, i t_{x}^{-1}, m_{y} t_{y}^{-1}, m_{z} r_{x}, r_{x}^{2}\right\rangle$ & 10 & 29 & {$[[2,0,0],[0,1,1],[0,0,2]]$} \\
\hline$H_{641}=\left\langle i t_{y}^{-1}, m_{z} r_{x}, r_{x}^{2}, r_{z}^{2} t_{x}, r_{z}^{2} t_{x}^{-1}\right\rangle$ & 10 & 29 & {$[[2,0,0],[0,1,1],[0,0,2]]$} \\
\hline$H_{642}=\left\langle m_{y} t_{y}^{-1}, m_{z} r_{x}, r_{x}^{2}, r_{z}^{2} t_{x}, r_{z}^{2} t_{x}^{-1}\right\rangle$ & 10 & 29 & {$[[2,0,0],[0,1,1],[0,0,2]]$} \\
\hline$H_{643}=\left\langle i t_{y}^{-1}, m_{y} t_{x}, m_{z} r_{x}, r_{x}^{2}\right\rangle$ & 10 & 29 & {$[[2,0,0],[0,1,1],[0,0,2]]$} \\
\hline$H_{644}=\left\langle m_{y} t_{x}, m_{z} r_{x}, r_{x}^{2}, r_{z}^{2} t_{y}^{-1}\right\rangle$ & 10 & 29 & {$[[2,0,0],[0,1,1],[0,0,2]]$} \\
\hline$H_{645}=\left\langle i t_{x}, m_{y} t_{y}^{-1}, m_{z} r_{x}, r_{x}^{2}, r_{z}^{2} t_{x}^{-1}\right\rangle$ & 10 & 29 & {$[[2,0,1],[0,1,1],[0,0,2]]$} \\
\hline$H_{646}=\left\langle m_{x}, m_{z} r_{x}, r_{y}^{2} r_{x} t_{x}, r_{y}^{2} r_{x} t_{y}^{-1}\right\rangle$ & 11 & 11 & {$[[1,0,0],[0,1,0],[0,0,1]]$} \\
\hline$H_{647}=\left\langle i t_{x}, m_{x}, m_{z} r_{x}, r_{y}^{2} r_{x} t_{y}^{-1}\right\rangle$ & 11 & 20 & {$[[2,0,0],[0,1,0],[0,0,1]]$} \\
\hline$H_{648}=\left\langle i t_{y}^{-1}, i t_{z}, m_{x}, m_{z} r_{x}, r_{y}^{2} r_{x} t_{x}\right\rangle$ & 11 & 20 & {$[[1,0,0],[0,1,1],[0,0,2]]$} \\
\hline$H_{649}=\left\langle i t_{x}, i t_{y}^{-1}, i t_{z}, m_{x}, m_{z} r_{x}\right\rangle$ & 11 & 20 & {$[[1,0,1],[0,1,1],[0,0,2]]$} \\
\hline$H_{650}=\left\langle m_{x}, m_{x} r_{x}^{-1} t_{z}, m_{z} r_{x}, r_{y}^{2} r_{x} t_{x}, r_{z}^{2} t_{y}^{-1}\right\rangle$ & 11 & 29 & {$[[1,0,0],[0,2,0],[0,0,2]]$} \\
\hline$H_{651}=\left\langle m_{x}, m_{x} r_{x}^{-1} t_{y}^{-1}, m_{z} r_{x}, r_{y}^{2} r_{x} t_{x}\right\rangle$ & 11 & 29 & {$[[1,0,0],[0,2,0],[0,0,2]]$} \\
\hline$H_{652}=\left\langle i t_{x}, m_{x}, m_{x} r_{x}^{-1} t_{z}, m_{z} r_{x}, r_{z}^{2} t_{y}^{-1}\right\rangle$ & 11 & 29 & {$[[1,1,1],[0,2,0],[0,0,2]]$} \\
\hline$H_{653}=\left\langle i t_{x}, m_{x}, m_{x} r_{x}^{-1} t_{y}^{-1}, m_{z} r_{x}\right\rangle$ & 11 & 29 & {$[[1,1,1],[0,2,0],[0,0,2]]$} \\
\hline$H_{654}=\left\langle i, i t_{x}, i t_{y}^{-1}, m_{z} r_{x}\right\rangle$ & 12 & 12 & {$[[1,0,0],[0,1,0],[0,0,1]]$} \\
\hline$H_{655}=\left\langle i, i t_{y}^{-1},{ }_{m z} r_{x}, r_{y}^{2} r_{x} t_{x}\right\rangle$ & 12 & 20 & {$[[2,0,0],[0,1,0],[0,0,1]]$} \\
\hline$H_{656}=\left\langle i, i t_{x}, m_{z} r_{x}, r_{y}^{2} r_{x} t_{y}^{-1}\right\rangle$ & 12 & 20 & {$[[1,0,0],[0,1,1],[0,0,2]]$} \\
\hline$H_{657}=\left\langle i, m_{z} r_{x}, r_{y}^{2} r_{x} t_{x}, r_{y}^{2} r_{x} t_{y}^{-1}\right\rangle$ & 12 & 20 & {$[[1,0,1],[0,1,1],[0,0,2]]$} \\
\hline$H_{658}=\left\langle i, i t_{x}, m_{z} r_{x}, r_{z}^{2} t_{y}^{-1}\right\rangle$ & 12 & 29 & {$[[1,0,0],[0,2,0],[0,0,2]]$} \\
\hline$H_{659}=\left\langle i, i t_{x}, m_{x} r_{x}^{-1} t_{y}^{-1}, m_{z} r_{x}\right\rangle$ & 12 & 29 & {$[[1,0,0],[0,2,0],[0,0,2]]$} \\
\hline$H_{660}=\left\langle i, m_{z} r_{x}, r_{y}^{2} r_{x} t_{x}, r_{z}^{2} t_{y}^{-1}\right\rangle$ & 12 & 29 & {$[[1,1,1],[0,2,0],[0,0,2]]$} \\
\hline$H_{661}=\left\langle i, m_{x} r_{x}^{-1} t_{y}^{-1}, m_{z} r_{x}, r_{y}^{2} r_{x} t_{x}\right\rangle$ & 12 & 29 & {$[[1,1,1],[0,2,0],[0,0,2]]$} \\
\hline$H_{662}=\left\langle r_{x}^{2}, r_{z}^{2} r_{x}, r_{z}^{2} r_{x} t_{x}, r_{z}^{2} r_{x} t_{y}^{-1}\right\rangle$ & 13 & 13 & {$[[1,0,0],[0,1,0],[0,0,1]]$} \\
\hline$H_{663}=\left\langle i t_{x}, r_{x}^{2}, r_{z}^{2} r_{x}, r_{z}^{2} r_{x} t_{y}^{-1}\right\rangle^{9}$ & 13 & 20 & $[[2,0,0],[0,1,0],[0,0,1]]]$ \\
\hline
\end{tabular}




\begin{tabular}{|c|c|c|c|}
\hline$H$ & $H_{0,0,0}$ & $P(H)$ & Translation basis of $H$ \\
\hline$H_{664}=\left\langle i t_{y}^{-1}, r_{x}^{2}, r_{z}^{2} r_{x}, r_{z}^{2} r_{x} t_{x}\right\rangle$ & 13 & 20 & {$[[1,0,0],[0,1,1],[0,0,2]]$} \\
\hline$H_{665}=\left\langle i t_{x}, i t_{y}^{-1}, r_{x}^{2}, r_{z}^{2} r_{x}\right\rangle$ & 13 & 20 & {$[[1,0,1],[0,1,1],[0,0,2]]$} \\
\hline$H_{666}=\left\langle m_{x} r_{x}^{-1} t_{x}, r_{x}^{2}, r_{z}^{2} r_{x}\right.$ & 13 & 23 & {$[[2,0,0],[0,1,0],[0,0,1]]$} \\
\hline$H_{667}=\left\langle m_{x} r_{x}^{-1} t_{y}^{-1}, r_{x}^{2}, r_{z}^{2} r_{x}, r_{z}^{2} r_{x} t_{x}\right\rangle$ & 13 & 23 & {$[[1,0,0],[0,1,1],[0,0,2]]$} \\
\hline$H_{668}=\left\langle m_{x} r_{x}^{-1} t_{x}, m_{x} r_{x}^{-1} t_{y}^{-1}, r_{x}^{2}, r_{z}^{2} r_{x}\right\rangle$ & 13 & 23 & {$[[1,0,1],[0,1,1],[0,0,2]]$} \\
\hline$H_{669}=\left\langle r_{x}^{2}, r_{z}^{2} r_{x}, r_{z}^{2} r\right.$ & 13 & 24 & {$[[2,0,0],[0,1,0],[0,0,1]]$} \\
\hline$H_{670}=\left\langle r_{x}^{\tilde{2}}, r_{z}^{\tilde{2}} r_{x}, r_{z}^{\tilde{2}} r_{2}\right.$ & 13 & 24 & {$[[1,0,0],[0,1,1],[0,0,2]]$} \\
\hline$H_{671}=\left\langle r_{x}^{2}, r_{z}^{2} r_{x}, r_{z}^{2} t_{x}, r_{z}^{2} t_{y}^{-1}\right\rangle$ & 13 & 24 & {$[[1,0,1],[0,1,1],[0,0,2]]$} \\
\hline$H_{672}=\left\langle i t_{x}, r_{x}^{2}, r_{z}^{2} r_{x}, r_{z}^{2} t_{y}^{-1}\right\rangle$ & 13 & 29 & {$[[2,0,0],[0,1,1],[0,0,2]]$} \\
\hline$H_{673}=\left\langle i t_{x}, m_{x} r_{x}^{-1} t_{y}^{-1}, r_{x}^{2}, r_{z}^{2} r_{x}\right\rangle$ & 13 & 29 & {$[[2,0,0],[0,1,1],[0,0,2]]$} \\
\hline$H_{674}=\left\langle i t_{y}^{-1}, r_{x}^{2}, r_{z}^{2} r\right.$ & 13 & 29 & {$[[2,0,0],[0,1,1],[0,0,2]]$} \\
\hline$H_{675}=\left\langle m_{x} r_{x}^{-1} t_{y}^{-1}, r_{x}^{2}, r_{z}^{2} r_{x}, r_{z}^{2} t_{x}\right\rangle$ & 13 & 29 & {$[[2,0,0],[0,1,1],[0,0,2]]$} \\
\hline$H_{676}=\left\langle i t_{y}^{-1}, m_{x} r_{x}^{-1}\right.$ & 13 & 29 & {$[[2,0,0],[0,1,1],[0,0,2]]$} \\
\hline$H_{677}=\left\langle m_{x} r_{x}^{-1} t_{x}, r^{2}\right.$ & 13 & 29 & {$[[2,0,0],[0,1,1],[0,0,2]]$} \\
\hline$H_{678}=\left\langle m_{x}, r_{z}^{2}, r_{z}^{2} t_{x}, r_{z}^{2} t_{y}^{-1}, r_{z}^{2} t_{z}^{-1}\right\rangle$ & 14 & 14 & {$[[1,0,0],[0,1,0],[0,0,1]]$} \\
\hline$H_{679}=\left\langle i t_{x}, m_{x}, r_{z}^{2}, r_{z}^{2} t_{y}^{-1}, r_{z}^{2} t_{z}^{-1}\right\rangle$ & 14 & 21 & {$[[2,0,0],[0,1,0],[0,0,1]]$} \\
\hline$H_{680}=\left\langle i t_{z}, i t_{z}^{-1}, m_{x}\right.$ & 14 & 21 & {$[[1,0,0],[0,1,0],[0,0,2]]$} \\
\hline$H_{681}=\left\langle i t_{x}, i t_{y}^{-1}, m_{x}\right.$ & 14 & 21 & {$[[1,1,0],[0,2,0],[0,0,1]]$} \\
\hline$H_{682}=\left\langle i t_{x}, i t_{z}, i t_{z}^{-1}, m\right.$ & 14 & 21 & {$[[1,0,1],[0,1,0],[0,0,2]]$} \\
\hline$H_{683}=\left\langle i t_{x}, i t_{y}^{-1}, i t_{z}\right.$ & 14 & 21 & {$[[1,0,1],[0,1,1],[0,0,2]]$} \\
\hline$H_{684}=\left\langle m_{x}, r_{y}^{2} r_{z} t_{z}, r_{y}^{2} r_{z}\right.$ & 14 & 23 & {$[[1,0,0],[0,1,0],[0,0,2]]$} \\
\hline$H_{685}=\left\langle m_{x}, r_{y}^{2} r_{z} t_{x}, r_{z}^{2}, r\right.$ & 14 & 23 & {$[[1,1,0],[0,2,0],[0,0,1]]$} \\
\hline$H_{686}=\left\langle m_{x}, r_{y}^{2} r_{z} t_{x}, r_{y}^{2} r_{z} t_{z}, r_{y}^{2} r_{z} t_{z}^{-1}, r_{z}^{2}\right\rangle$ & 14 & 23 & {$[[1,0,1],[0,1,1],[0,0,2]]$} \\
\hline$H_{687}=\left\langle m_{x}, m_{x} r_{z} t_{z}^{-1}, r_{z}^{2}, r_{z}^{2} t_{x}, r_{z}^{2} t_{y}^{-1}\right\rangle$ & 14 & 25 & {$[[1,0,0],[0,1,0],[0,0,2]]$} \\
\hline$H_{688}=\left\langle m_{x}, m_{x} r_{z} t_{x}, r_{z}^{2}, r_{z}^{2} t_{z}^{-1}\right\rangle$ & 14 & 25 & {$[[1,1,0],[0,2,0],[0,0,1]]$} \\
\hline$H_{689}=\left\langle m_{x}, m_{x} r_{z} t_{x}\right.$ & 14 & 25 & {$[[1,0,1],[0,1,1],[0,0,2]]$} \\
\hline$H_{690}=\left\langle i t_{z}^{-1}, m_{x}, r_{y}^{2}\right.$ & 14 & 29 & {$[[1,0,0],[0,1,0],[0,0,4]]$} \\
\hline$H_{691}=\left\langle i t_{x}, i t_{y}^{-1}, m_{x}\right.$ & 14 & 29 & {$[[1,1,0],[0,2,0],[0,0,2]]$} \\
\hline$H_{692}=\left\langle i t_{x}, i t_{y}^{-1}, m_{2}\right.$ & 14 & 29 & {$[[1,1,0],[0,2,0],[0,0,2]]$} \\
\hline$H_{693}=\left\langle i t_{z}, i t_{z}^{-1}, m_{x}\right.$ & 14 & 29 & {$[[1,1,0],[0,2,0],[0,0,2]]$} \\
\hline$H_{694}=\left\langle m_{x}, m_{x} r_{z} t_{z}^{-1}\right.$, & 14 & 29 & {$[[1,1,0],[0,2,0],[0,0,2]]$} \\
\hline$H_{695}=\left\langle i t_{z}, i t_{z}^{-1}, m_{x}\right.$ & 14 & 29 & {$[[1,1,0],[0,2,0],[0,0,2]]$} \\
\hline$H_{696}=\left\langle m_{x}, m_{x} r_{z} t_{x}\right.$, & 14 & 29 & {$[[1,1,0],[0,2,0],[0,0,2]]$} \\
\hline$H_{697}=\left\langle i t_{z}^{-1}, m_{x}, m_{x}\right.$ & 14 & 29 & {$[[1,0,2],[0,1,2],[0,0,4]]$} \\
\hline$H_{698}=\left\langle r_{x}^{-1}, r_{x}^{2}, r_{x}^{2} t_{x}\right.$ & 15 & 15 & {$[[1,0,0],[0,1,0],[0,0,1]]$} \\
\hline$H_{699}=\left\langle i t_{x}, i t_{x}^{-1}, r_{x}^{-1}, r_{x}^{2}, r_{x}^{2} t_{y}^{-1}\right\rangle$ & 15 & 22 & {$[[2,0,0],[0,1,0],[0,0,1]]$} \\
\hline$H_{700}=\left\langle i t_{y}^{-1}, r_{x}^{-1}, r_{x}^{2}, r_{x}^{2} t_{x}\right\rangle$ & 15 & 22 & {$[[1,0,0],[0,1,1],[0,0,2]]$} \\
\hline$H_{701}=\left\langle i t_{x}, i t_{x}^{-1}, i t_{y}^{-}\right.$ & 15 & 22 & {$[[1,0,1],[0,1,1],[0,0,2]]$} \\
\hline$H_{702}=\left\langle r_{x}^{-1}, r_{x}^{2}, r_{x}^{2} t^{-1}, r_{z}^{2} t_{x}, r_{z}^{2} t_{x}^{-1}\right\rangle$ & 15 & 24 & {$[[2,0,0],[0,1,0],[0,0,1]]$} \\
\hline$H_{703}=\left\langle r_{x}^{-1}, r_{x}^{2}, r_{x}^{2} t_{x}, r_{z}^{2} t_{y}^{-1}\right\rangle$ & 15 & 24 & {$[[1,0,0],[0,1,1],[0,0,2]]$} \\
\hline$H_{704}=\left\langle r_{x}^{-1}, r_{x}^{2}, r_{z}^{2} t_{x}, r_{z}^{2} t_{x}^{\frac{9}{1}}, r_{z}^{2} t_{y}^{-1}\right\rangle$ & 15 & 24 & {$[[1,0,1],[0,1,1],[0,0,2]]$} \\
\hline$H_{705}=\left\langle m_{y} t_{x}, r_{x}^{-1}, r_{x}^{2}, r_{x}^{2} t_{y}^{-1}\right\rangle$ & 15 & 25 & {$[[2,0,0],[0,1,0],[0,0,1]]$} \\
\hline$H_{706}=\left\langle m_{y} t_{y}^{-1}, r_{x}^{-1}\right.$, & 15 & 25 & {$[[1,0,0],[0,1,1],[0,0,2]]$} \\
\hline$H_{707}=\left\langle m_{y} t_{x}, m_{y} t_{y}^{-}\right.$ & 15 & 25 & {$[[1,0,1],[0,1,1],[0,0,2]]$} \\
\hline$H_{708}=\left\langle i t_{x}, r_{x}^{-1}, r_{x}^{2}\right.$, & 15 & 29 & {$[[4,0,0],[0,1,0],[0,0,1]]$} \\
\hline$H_{709}=\left\langle i t_{x}, i t_{x}^{-1}, r_{x}^{-}\right.$ & 15 & 29 & {$[[2,0,0],[0,1,1],[0,0,2]]$} \\
\hline$H_{710}=\left\langle i t_{x}, i t_{x}^{-1}, m_{y} t_{y}^{-1}, r_{x}^{-1}, r_{x}^{2}\right\rangle$ & 15 & 29 & {$[[2,0,0],[0,1,1],[0,0,2]]$} \\
\hline$H_{711}=\left\langle i t_{y}^{-1}, r_{x}^{-1}, r_{x}^{2}, r_{z}^{2} t_{x}, r_{z}^{2} t_{x}^{-1}\right\rangle$ & 15 & 29 & {$[[2,0,0],[0,1,1],[0,0,2]]$} \\
\hline$H_{712}=\left\langle m_{y} t_{y}^{-1}, r_{x}^{-1}, r_{x}^{2}, r_{z}^{2} t_{x}, r_{z}^{2} t_{x}^{-1}\right\rangle$ & 15 & 29 & {$[[2,0,0],[0,1,1],[0,0,2]]$} \\
\hline$H_{713}=\left\langle i t_{y}^{-1}, m_{y} t_{x}, r_{x}^{-1}, r_{x}^{2}\right\rangle$ & 15 & 29 & {$[[2,0,0],[0,1,1],[0,0,2]]$} \\
\hline$H_{714}=\left\langle m_{y} t_{x}, r_{x}^{-1}, r_{x}^{2}, r_{z}^{2} t_{y}^{-1}\right\rangle$ & 15 & 29 & {$[[2,0,0],[0,1,1],[0,0,2]]$} \\
\hline$H_{715}=\left\langle i t_{x}, m_{y} t_{y}^{-1}, r_{x}^{-1}, r_{x}^{2}, r_{z}^{2} t_{x}^{-1}\right\rangle$ & 15 & 29 & {$[[2,0,1],[0,1,1],[0,0,2]]$} \\
\hline$H_{716}=\left\langle m_{x} r_{x}, m_{x} r_{x}^{-1} t_{x}, m_{x} r_{x}^{-1} t_{y}^{-1}, r_{x}^{2}\right\rangle$ & 16 & 16 & {$[[1,0,0],[0,1,0],[0,0,1]]$} \\
\hline$H_{717}=\left\langle i t_{x}, m_{x} r_{x}, m_{x} r_{x}^{-1} t_{y}^{-1}, r_{x}^{2}\right\rangle$ & 16 & 22 & {$[[2,0,0],[0,1,0],[0,0,1]]$} \\
\hline$H_{718}=\left\langle i t_{y}^{-1}, m_{x} r_{x}, m_{x} r_{x}^{-1^{3}} t_{x}, r_{x}^{2}\right\rangle$ & 16 & 22 & {$[[1,0,0],[0,1,1],[0,0,2]]$} \\
\hline$H_{719}=\left\langle i t_{x}, i t_{y}^{-1}, m_{x} r_{x}, r_{x}^{2}\right\rangle$ & 16 & 22 & {$[[1,0,1],[0,1,1],[0,0,2]]$} \\
\hline$H_{720}=\left\langle m_{x} r_{x}, m_{x} r_{x}^{-1} t_{y}^{-1}, r_{x}^{2}, r_{z}^{2} r_{x} t_{x}\right\rangle$ & 16 & 23 & {$[[2,0,0],[0,1,0],[0,0,1]]$} \\
\hline$H_{721}=\left\langle m_{x} r_{x}, m_{x} r_{x}^{-1} t_{x}, r_{x}^{2}, r_{z}^{2} r_{x} t_{y}^{-1}\right\rangle$ & 16 & 23 & {$[[1,0,0],[0,1,1],[0,0,2]]$} \\
\hline$H_{722}=\left\langle m_{x} r_{x}, r_{x}^{2}, r_{z}^{2} r_{x} t_{x}, r_{z}^{2} r_{x} t_{y}^{-1}\right\rangle$ & 16 & 23 & {$[[1,0,1],[0,1,1],[0,0,2]]$} \\
\hline$H_{723}=\left\langle m_{x} r_{x}, m_{x} r_{x}^{-1} t_{y}^{-1}, r_{x}^{2}, r_{z}^{2} t_{x}\right\rangle$ & 16 & 26 & {$[[2,0,0],[0,1,0],[0,0,1]]$} \\
\hline$H_{724}=\left\langle m_{x} r_{x}, m_{x} r_{x}^{-1} t_{x}, r_{x}^{2}, r_{z}^{2} t_{y}^{-1}\right\rangle$ & 16 & 26 & {$[[1,0,0],[0,1,1],[0,0,2]]$} \\
\hline$H_{725}=\left\langle m_{x} r_{x}, r_{x}^{2}, r_{z}^{2} t_{x}, r_{z}^{2} t_{y}^{-1}\right\rangle$ & 16 & 26 & {$[[1,0,1],[0,1,1],[0,0,2]]$} \\
\hline$H_{726}=\left\langle i t_{x}, m_{x} r_{x}, r_{x}^{2}, r_{z}^{2} t_{y}^{-1}\right\rangle$ & 16 & 29 & {$[[2,0,0],[0,1,1],[0,0,2]]$} \\
\hline$H_{727}=\left\langle i t_{x}, m_{x} r_{x}, r_{x}^{2}, r_{z}^{2} r_{x} t_{y}^{-1}\right\rangle$ & 16 & 29 & {$[[2,0,0],[0,1,1],[0,0,2]]$} \\
\hline$H_{728}=\left\langle i t_{y}^{-1}, m_{x} r_{x}, r_{x}^{2}, r_{z}^{2} t_{x}\right\rangle$ & 16 & 29 & {$[[2,0,0],[0,1,1],[0,0,2]]$} \\
\hline$H_{729}=\left\langle m_{x} r_{x}, r_{x}^{2}, r_{z}^{2} r_{x} t_{y}^{-1}, r_{z}^{2} t_{x}\right\rangle$ & 16 & 29 & {$[[2,0,0],[0,1,1],[0,0,2]]$} \\
\hline$H_{730}=\left\langle i t_{y}^{-1}, m_{x} r_{x}, r_{x}^{2}, r_{z}^{2} r_{x} t_{x}\right\rangle$ & 16 & 29 & {$[[2,0,0],[0,1,1],[0,0,2]]$} \\
\hline
\end{tabular}




\begin{tabular}{|c|c|c|c|}
\hline$H$ & $H_{0,0,0}$ & $P(H)$ & Translation basis of $H$ \\
\hline$H_{731}=\left\langle m_{x} r_{x}, r_{x}^{2}, r_{z}^{2} r_{x} t_{x}, r_{z}^{2} t_{y}^{-1}\right\rangle$ & 16 & 29 & {$[[2,0,0],[0,1,1],[0,0,2]]$} \\
\hline$H_{732}=\left\langle i, i t_{x}, r_{y} r_{x}\right\rangle$ & 17 & 17 & {$[[1,0,0],[0,1,0],[0,0,1]]$} \\
\hline$H_{733}=\left\langle i, r_{y} r_{x}, r_{z}^{2} r_{x} t_{x}\right\rangle$ & 17 & 28 & {$[[1,0,1],[0,1,1],[0,0,2]]$} \\
\hline$H_{734}=\left\langle i, r_{y} r_{x}, r_{z}^{2} t_{x}\right\rangle$ & 17 & 30 & {$[[1,1,1],[0,2,0],[0,0,2]]$} \\
\hline$H_{735}=\left\langle i, m_{x} t_{x}, r_{y} r_{x}\right\rangle$ & 17 & 30 & {$[[1,1,1],[0,2,0],[0,0,2]]$} \\
\hline$H_{736}=\left\langle i, r_{y} r_{x}, r_{y}^{2} r_{x} t_{x}\right\rangle$ & 17 & 33 & {$[[2,0,0],[0,2,0],[0,0,2]]$} \\
\hline$H_{737}=\left\langle m_{x} r_{y} t_{x}, m_{z} r_{x}, r_{y} r_{x}\right\rangle$ & 18 & 18 & {$[[1,0,0],[0,1,0],[0,0,1]]$} \\
\hline$H_{738}=\left\langle i t_{x}, i t_{x}^{-1}, m_{z} r_{x}, r_{y} r_{x}\right\rangle$ & 18 & 28 & {$[[1,0,1],[0,1,1],[0,0,2]]$} \\
\hline$H_{739}=\left\langle m_{z} r_{x}, r_{y} r_{x}, r_{y} r_{x}^{-1} t_{x}\right\rangle$ & 18 & 31 & {$[[1,1,1],[0,2,0],[0,0,2]]$} \\
\hline$H_{740}=\left\langle m_{z} r_{x}, r_{y} r_{x}, r_{y}^{2} r_{x} t_{x}, r_{y}^{2} r_{x} t_{x}^{-1}\right\rangle$ & 18 & 33 & {$[[2,0,0],[0,2,0],[0,0,2]]$} \\
\hline$H_{741}=\left\langle r_{y} r_{x}, r_{z}^{2} r_{x}, r_{z}^{2} r_{x} t_{x}\right\rangle$ & 19 & 19 & {$[[1,0,0],[0,1,0],[0,0,1]]$} \\
\hline$H_{742}=\left\langle i t_{x}, r_{y} r_{x}, r_{z}^{2} r_{x}\right\rangle$ & 19 & 28 & {$[[1,0,1],[0,1,1],[0,0,2]]$} \\
\hline$H_{743}=\left\langle r_{y} r_{x}, r_{y}^{2} r_{x} t_{x}, r_{z}^{2} r_{x}\right\rangle$ & 19 & 32 & {$[[1,1,1],[0,2,0],[0,0,2]]$} \\
\hline$H_{744}=\left\langle m_{x} t_{x}, r_{y} r_{x}, r_{z}^{2} r_{x}\right\rangle$ & 19 & 33 & {$[[2,0,0],[0,2,0],[0,0,2]]$} \\
\hline$H_{745}=\left\langle i, i t_{x}, i t_{y}^{-1}, m_{z} r_{x}, r_{x}^{2}\right\rangle$ & 20 & 20 & {$[[1,0,0],[0,1,0],[0,0,1]]$} \\
\hline$H_{746}=\left\langle i, i t_{y}^{-1}, m_{z} r_{x}, r_{x}^{2}, r_{z}^{2} t_{x}\right\rangle$ & 20 & 29 & {$[[2,0,0],[0,1,0],[0,0,1]]$} \\
\hline$H_{747}=\left\langle i, i t_{x}, m_{z} r_{x}, r_{x}^{2}, r_{z}^{2} t_{y}^{-1}\right\rangle$ & 20 & 29 & {$[[1,0,0],[0,1,1],[0,0,2]]$} \\
\hline$H_{748}=\left\langle i, m_{z} r_{x}, r_{x}^{2}, r_{z}^{2} t_{x}, r_{z}^{2} t_{y}^{-1}\right\rangle$ & 20 & 29 & {$[[1,0,1],[0,1,1],[0,0,2]]$} \\
\hline$H_{749}=\left\langle i, i t_{x}, i t_{y}^{-1}, i t_{z}^{-1}, r_{x}^{2}, r_{z}^{2}\right\rangle$ & 21 & 21 & {$[[1,0,0],[0,1,0],[0,0,1]]$} \\
\hline$H_{750}=\left\langle i, i t_{x}, i t_{y}^{-1}, r_{x}^{2}, r_{y}^{2} r_{z} t_{z}^{-1}, r_{z}^{2}\right\rangle$ & 21 & 29 & {$[[1,0,0],[0,1,0],[0,0,2]]$} \\
\hline$H_{751}=\left\langle i, i t_{x}, r_{x}^{2}, r_{z}^{2}, r_{z}^{2} r_{x} t_{y}^{-1}\right\rangle$ & 21 & 29 & {$[[1,0,0],[0,1,1],[0,0,2]]$} \\
\hline$H_{752}=\left\langle i, r_{x}^{2}, r_{z}^{2}, r_{z}^{2} r_{x} t_{x}, r_{z}^{2} r_{x} t_{y}^{-1}\right\rangle$ & 21 & 29 & {$[[1,0,1],[0,1,1],[0,0,2]]$} \\
\hline$H_{753}=\left\langle i, i t_{x}, i t_{y}^{-1}, m_{x} r_{x}, r_{x}^{2}\right\rangle$ & 22 & 22 & {$[[1,0,0],[0,1,0],[0,0,1]]$} \\
\hline$H_{754}=\left\langle i, i t_{y}^{-1}, m_{x} r_{x}, r_{x}^{2}, r_{z}^{2} t_{x}\right\rangle$ & 22 & 29 & {$[[2,0,0],[0,1,0],[0,0,1]]$} \\
\hline$H_{755}=\left\langle i, i t_{x}, m_{x} r_{x}, r_{x}^{2}, r_{z}^{2} t_{y}^{-1}\right\rangle$ & 22 & 29 & {$[[1,0,0],[0,1,1],[0,0,2]]$} \\
\hline$H_{756}=\left\langle i, m_{x} r_{x}, r_{x}^{2}, r_{z}^{2} t_{x}, r_{z}^{2} t_{y}^{-1}\right\rangle$ & 22 & 29 & $[[1,0,1],[0,1,1],[0,0,2]]]$ \\
\hline$H_{757}=\left\langle m_{x} r_{x}, m_{z}, r_{x}^{2}, r_{z}^{2} r_{x} t_{x}, r_{z}^{2} r_{x} t_{y}^{-1}\right\rangle$ & 23 & 23 & {$[[1,0,0],[0,1,0],[0,0,1]]$} \\
\hline$H_{758}=\left\langle i t_{x}, m_{x} r_{x}, m_{z}, r_{x}^{2}, r_{z}^{2} r_{x} t_{y}^{-1}\right\rangle$ & 23 & 29 & {$[[2,0,0],[0,1,0],[0,0,1]]$} \\
\hline$H_{759}=\left\langle i t_{y}^{-1}, m_{x} r_{x}, m_{z}, r_{x}^{2}, r_{z}^{2} r_{x} t_{x}\right\rangle$ & 23 & 29 & {$[[1,0,0],[0,1,1],[0,0,2]]$} \\
\hline$H_{760}=\left\langle i t_{x}, i t_{y}^{-1}, m_{x} r_{x}, m_{z}, r_{x}^{2}\right\rangle$ & 23 & 29 & {$[[1,0,1],[0,1,1],[0,0,2]]$} \\
\hline$H_{761}=\left\langle r_{x}^{2}, r_{z}^{2}, r_{z}^{2} r_{x}, r_{z}^{2} t_{x}, r_{z}^{2} t_{y}^{-1}\right\rangle$ & 24 & 24 & {$[[1,0,0],[0,1,0],[0,0,1]]$} \\
\hline$H_{762}=\left\langle i t_{x}, r_{x}^{2}, r_{z}^{2}, r_{z}^{2} r_{x}, r_{z}^{2} t_{y}^{-1}\right\rangle$ & 24 & 29 & {$[[2,0,0],[0,1,0],[0,0,1]]$} \\
\hline$H_{763}=\left\langle i t_{y}^{-1}, r_{x}^{2}, r_{z}^{2}, r_{z}^{2} r_{x}, r_{z}^{2} t_{x}\right\rangle$ & 24 & 29 & {$[[1,0,0],[0,1,1],[0,0,2]]$} \\
\hline$H_{764}=\left\langle i t_{x}, i t_{y}^{-1}, r_{x}^{2}, r_{z}^{2}, r_{z}^{2} r_{x}\right\rangle$ & 24 & 29 & {$[[1,0,1],[0,1,1],[0,0,2]]$} \\
\hline$H_{765}=\left\langle m_{z}, m_{z} r_{x}, r_{x}^{2}, r_{x}^{2} t_{x}, r_{x}^{2} t_{y}^{-1}\right\rangle$ & 25 & 25 & {$[[1,0,0],[0,1,0],[0,0,1]]$} \\
\hline$H_{766}=\left\langle i t_{x}, i t_{x}^{-1}, m_{z}, m_{z} r_{x}, r_{x}^{2}, r_{x}^{2} t_{y}^{-1}\right\rangle$ & 25 & 29 & {$[[2,0,0],[0,1,0],[0,0,1]]$} \\
\hline$H_{767}=\left\langle i t_{y}^{-1}, m_{z}, m_{z} r_{x}, r_{x}^{2}, r_{x}^{2} t_{x}\right\rangle$ & 25 & 29 & {$[[1,0,0],[0,1,1],[0,0,2]]$} \\
\hline$H_{768}=\left\langle i t_{x}, i t_{x}^{-1}, i t_{y}^{-1}, m_{z}, m_{z} r_{x}, r_{x}^{2}\right\rangle$ & 25 & 29 & {$[[1,0,1],[0,1,1],[0,0,2]]$} \\
\hline$H_{769}=\left\langle m_{z} r_{x}, r_{x}^{2}, r_{z}^{2}, r_{z}^{2} t_{x}, r_{z}^{2} t_{y}^{-1}\right\rangle$ & 26 & 26 & {$[[1,0,0],[0,1,0],[0,0,1]]$} \\
\hline$H_{770}=\left\langle i t_{x}, m_{z} r_{x}, r_{x}^{2}, r_{z}^{2}, r_{z}^{2} t_{y}^{-1}\right\rangle$ & 26 & 29 & {$[[2,0,0],[0,1,0],[0,0,1]]$} \\
\hline$H_{771}=\left\langle i t_{y}^{-1}, m_{z} r_{x}, r_{x}^{2}, r_{z}^{2}, r_{z}^{2} t_{x}\right\rangle$ & 26 & 29 & {$[[1,0,0],[0,1,1],[0,0,2]]$} \\
\hline$H_{772}=\left\langle i t_{x}, i t_{y}^{-1}, m_{z} r_{x}, r_{x}^{2}, r_{z}^{2}\right\rangle$ & 26 & 29 & {$[[1,0,1],[0,1,1],[0,0,2]]$} \\
\hline$H_{773}=\left\langle r_{x}^{2}, r_{y} r_{x}, r_{z}^{2}, r_{z}^{2} t_{x}\right\rangle$ & 27 & 27 & {$[[1,0,0],[0,1,0],[0,0,1]]$} \\
\hline$H_{774}=\left\langle i t_{x}, r_{x}^{2}, r_{y} r_{x}, r_{z}^{2}\right\rangle$ & 27 & 30 & {$[[1,0,1],[0,1,1],[0,0,2]]$} \\
\hline$H_{775}=\left\langle m_{x} r_{x}^{-1} t_{x}, r_{x}^{2}, r_{y} r_{x}, r_{z}^{2}\right\rangle$ & 27 & 31 & {$[[1,0,1],[0,1,1],[0,0,2]]$} \\
\hline$H_{776}=\left\langle r_{x}^{2}, r_{y} r_{x}, r_{z}^{2}, r_{z}^{2} r_{x} t_{x}\right\rangle$ & 27 & 32 & {$[[1,0,1],[0,1,1],[0,0,2]]$} \\
\hline$H_{777}=\left\langle i, i t_{x}, m_{z} r_{x}, r_{y} r_{x}\right\rangle$ & 28 & 28 & {$[[1,0,0],[0,1,0],[0,0,1]]$} \\
\hline$H_{778}=\left\langle i, m_{z} r_{x}, r_{y} r_{x}, r_{y}^{2} r_{x} t_{x}\right\rangle$ & 28 & 33 & {$[[1,1,1],[0,2,0],[0,0,2]]$} \\
\hline$H_{779}=\left\langle i, i t_{x}, i t_{y}^{-1}, m_{z} r_{x}, r_{x}^{2}, r_{z}^{2}\right\rangle$ & 29 & 29 & {$[[1,0,0],[0,1,0],[0,0,1]]$} \\
\hline$H_{780}=\left\langle i, i t_{x}, r_{x}^{2}, r_{y} r_{x}, r_{z}^{2}\right\rangle$ & 30 & 30 & {$[[1,0,0],[0,1,0],[0,0,1]]$} \\
\hline$H_{781}=\left\langle i, r_{x}^{2}, r_{y} r_{x}, r_{z}^{2}, r_{z}^{2} r_{x} t_{x}\right\rangle$ & 30 & 33 & {$[[1,0,1],[0,1,1],[0,0,2]]$} \\
\hline$H_{782}=\left\langle m_{z} r_{x}, r_{x}^{2}, r_{y} r_{x}, r_{z}^{2}, r_{z}^{2} t_{x}\right\rangle$ & 31 & 31 & {$[[1,0,0],[0,1,0],[0,0,1]]$} \\
\hline$H_{783}=\left\langle i t_{x}, m_{z} r_{x}, r_{x}^{2}, r_{y} r_{x}, r_{z}^{2}\right\rangle$ & 31 & 33 & {$[[1,0,1],[0,1,1],[0,0,2]]$} \\
\hline$H_{784}=\left\langle r_{x}^{2}, r_{y} r_{x}, r_{z}^{2}, r_{z}^{2} r_{x}, r_{z}^{2} t_{x}\right\rangle$ & 32 & 32 & {$[[1,0,0],[0,1,0],[0,0,1]]$} \\
\hline$H_{785}=\left\langle i t_{x}, r_{x}^{2}, r_{y} r_{x}, r_{z}^{2}, r_{z}^{2} r_{x}\right\rangle$ & 32 & 33 & {$[[1,0,1],[0,1,1],[0,0,2]]$} \\
\hline$H_{786}=\left\langle i, i t_{x}, m_{z} r_{x}, r_{x}^{2}, r_{y} r_{x}, r_{z}^{2}\right\rangle$ & 33 & 33 & {$[[1,0,0],[0,1,0],[0,0,1]]$} \\
\hline
\end{tabular}

According to [5], we say that the realization $R=(\Gamma, G, \sigma, \varphi)$ of symmetrical 2extension of $\Lambda^{3}$ satisfies the $\left[p_{x}, p_{y}, p_{z}\right]$-periodicity condition, where $p_{x}, p_{y}, p_{z}$ are positive integers, if there exist $g_{1}, g_{2}, g_{3} \in \mathrm{A} u t_{\sigma}(\Gamma)$ such that $\left[g_{1}, g_{2}\right]=1,\left[g_{2}, g_{3}\right]=1,\left[g_{1}, g_{3}\right]=1$ and $\varphi g_{1}^{\sigma} \varphi^{-1}=t_{x}^{p_{x}}, \varphi g_{2}^{\sigma} \varphi^{-1}=t_{y}^{p_{y}}, \varphi g_{3}^{\sigma} \varphi^{-1}=t_{z}^{p_{z}}$.

\section{Main result}

We have done a computer implementation of the proposed in [7] approach, which can be called a coordinatization of symmetrical extensions of graphs. According to it, the 
realization of symmetrical 2-extension of the grid $\Lambda^{3}$ of class I can be defined by a triple $H, L, X$, where $H$ is a vertex-transitive subgroup of $\operatorname{Aut}\left(\Lambda^{3}\right), L$ is subgroup of index 2 of the stabilizer of the vertex $(0,0,0)$ in $H$, and $X$ is some subset of elements of $H$ mapping the vertex $(0,0,0)$ of $\Lambda^{3}$ to some its adjacent vertices (see [3]for details).

Below we give adaptations of Algorithm 1 and Algorithm 2 from [3] to $\Lambda^{3}$ :

Algorithm 1. Generation of all saturated realizations of symmetrical 2-extensions of $\Lambda^{3}$. Output: A list of realizations $R_{H_{i}, L_{i}, \mathcal{P}_{i}}, i=1, \ldots, n$.

Description. Look over all groups $H$ from $\mathbf{H}_{\mathrm{I}}$. For each such group let $K=H_{(0,0)}$. Choose elements $h_{1}, \ldots, h_{6} \in H$ such that $\left\{h_{1}(0,0,0), \ldots, h_{6}(0,0,0)\right\}=\{(1,0,0)$, $(-1,0,0),(0,1,0),(0,-1,0),(0,0,1),(0,0,-1)\}$. Look over all subgroups $L$ of $K$ of index 2. For each such group $L$, choose $g \in K$, such that $K=L \cup g L$. Look over all subsets $N$ of the set $\left\{h_{1} L, \ldots, h_{6} L, h_{1} g L, \ldots, h_{6} g L\right\}$ such that $N$ is invariant relative left multiplication by elements from $L$ and such that $h L \in N$ imply $h^{-1} L \in N$. For each of such set $N$ let $\mathcal{P}=\left\{\{L, g L\} \cup\left\{\left\{L, L_{1}\right\}: L_{1} \in N\right\}\right\}$ and we obtain the graph $\Gamma_{H, L, \mathcal{P}}$. If between the block $\{L, g L\}$ and the blocks $\left\{h_{j} L, h_{j} g L\right\}, j=1, \ldots, 6$, there are connections of a type non-equal to $2_{\|}$or 4 , then the realization $\left(\Gamma_{H, L, \mathcal{P}}, \lambda_{H / L}(H), \sigma_{H, K, L}, \tilde{\varphi}_{H, K, L}\right)$ is of type I, and we put it into the output list.

Let $R_{H_{i}, L_{i}, \mathcal{P}_{i}}, i=1,2$, be two realizations generated by the Algorithm 1 . Further we describe an algorithm which tests them for equivalency. Let $K_{i}=\left(H_{i}\right)_{(0,0)}$ for $i=$ 1,2. Sets of cosets $H_{1} / K_{1}$ and $H_{2} / K_{2}$ are essentially identified with the grid $\Lambda^{2}$. If the realizations are equivalent, then there exists an isomorphism $\psi$ between them which preserves blocks, and therefore, can be extended to some automorphism $g \in \operatorname{Aut}\left(\Lambda^{2}\right)$. Vertex symmetry of the extension $\Gamma_{H_{2}, L_{2}, \mathcal{P}_{2}}$ implies that $\psi$ can be multiplied by some automorphism of the extension $\Gamma_{H_{2}, L_{2}, \mathcal{P}_{2}}$, which preserves blocks, in such a way that the resulting isomorphism between $\Gamma_{H_{1}, L_{1}, \mathcal{P}_{1}}$ and $\Gamma_{H_{2}, L_{2}, \mathcal{P}_{2}}$, while mapping $H_{1} / K_{1}$ onto $H_{2} / K_{2}$, will give some element $\tilde{g}$ already from $\operatorname{Aut}\left(\Lambda^{2}\right)_{(0,0)}$. An element $\tilde{g}$ takes one of 48 possible values. Algorithm 2, described below, allows to check whether two realizations are equivalent or not, under the assumption that $\tilde{g}=1$. In general case, to test two realizations $R_{H_{i}, L_{i}, \mathcal{P}_{i}}, i=1,2$, for equivalency we must look over all $\tilde{g} \in \operatorname{Aut}\left(\Lambda^{2}\right)_{(0,0)}$, and for each $\tilde{g}$ execute algorithm 2 to check realizations $R_{H_{1}, L_{1}, \mathcal{P}_{1}}$ and $\tilde{g}^{-1}\left(R_{H_{2}, L_{2}, \mathcal{P}_{2}}\right)$ for equivalence.

Algorithm 2. Test two realizations for equivalency, under assumption $\tilde{g}=1$.

Input: Realizations $R_{H_{i}, L_{i}, \mathcal{P}_{i}}=\left(\Gamma_{H_{i}, L_{i}, \mathcal{P}_{i}}, G_{i}, \sigma_{i}, \varphi_{i}\right), i=1,2$.

Output: Test result ('yes' or 'no').

Description. Let $\left(n_{1}, n_{2}, n_{3}\right)$ be the lexicographically minimal triple of positive integers such that both implementations satisfy the condition $\left[n_{1}, n_{2}\right]$ - periodicity.

Let $F_{i}, i=1,2$, be a subgraph of $\Gamma_{H_{i}, L_{i}, \mathcal{P}_{i}}$ generated by the vertex set $\varphi_{i}^{-1}(0,0,0) \cup$ $\ldots \cup \varphi_{i}^{-1}\left(n_{1}-1,0,0\right) \cup \ldots \cup \varphi_{i}^{-1}\left(0, n_{2}-1,0\right) \cup \ldots \cup \varphi_{i}^{-1}\left(n_{1}-1, n_{2}-1,0\right) \cup \ldots \cup$ $\varphi_{i}^{-1}\left(0,0, n_{3}-1\right) \cup \ldots \cup \varphi_{i}^{-1}\left(n_{1}-1,0, n_{3}-1\right) \cup \ldots \cup \varphi_{i}^{-1}\left(0, n_{2}-1, n_{3}-1\right) \cup \ldots \cup \varphi_{i}^{-1}\left(n_{1}-\right.$ $\left.1, n_{2}-1, n_{3}-1\right)$ of $\Lambda^{3}$. Comparing the blocks $\varphi_{1}^{-1}(k, l, m)$ and $\varphi_{2}^{-1}(k, l, m)$ for all $k \in$ $\left\{0, \ldots, n_{1}\right\}, l \in\left\{0, \ldots, n_{2}\right\}, m \in\left\{0, \ldots, n_{3}\right\}$, we can construct $2^{n_{1} n_{2} n_{3}}$ correspondences of the vertices of the subgraphs $F_{1}$ and $F_{2}$. If among them there is a correspondence defining an isomorphism of the subgraphs $F_{1}$ and $F_{2}$ with additional matching on the boundary, so that this correspondence in periodicity can be extended to an isomorphism of the graphs $\Gamma_{H_{i}, L_{i}, \mathcal{P}_{i}}, i=1,2$, then these realizations are equivalent, and if not, then nonequivalent. Looking over $2^{n_{1} n_{2} n_{3}}$ correspondences of the vertices of subgraphs is accelerated by using a backtracking. 
We applied these algorithms and get the following results. A list of saturated realizations generated by Algorithm 1 and thinned out by Algorithm 2, contains 2872 realizations given in Table 4 below. When executing Algorithm 2, the realization with maximal by inclusion group $H_{i}$ was selected in each class of equivalent realizations. Due to this, the realizations in the resulting list are maximal.

We split the set of all realizations of the symmetrical 2-extensions of $\Lambda^{3}$ of the class I into subclasses defined by the types of connections in the neighborhood of vertex. In the first column of Table 3 below, we give all occurring combinations of connection types in the neighborhood of vertex (59 combinations). Combinations are of the form $x_{1} x_{2} y_{1} y_{2} z_{1} z_{2}$, where $x_{1}$ is the type of the first connection by the first direction (the grid $\Lambda^{3}$, and therefore a 2-extension, has three directions along coordinate axes), $x_{2}$ is the type of the second connection by the first direction, $y_{1}$ is the type of the first connection by the second direction, $y_{2}$ is the type of the second connection by the second direction, $z_{1}$ is the type of the first connection by the third direction, $z_{2}$ is the type of the second connection by the third direction. Here, for each extension, the numbering of directions and connections within the direction is performed so that the combination turned out to be lexicographically minimal. In the pictures of combinations in the first column of Table 3 the first direction is shown horizontally (first connection to the left, second to the right), the second direction is shown vertically (bottom connection is first, top connection is second), the third direction is shown diagonally (bottom-left connection is first, top-right connection is second). For each combination of connection types, the remaining columns in Table 3 contain pictures of all found corresponding extensions of vertex neighborhood up to equivalence. In these pictures, the edges in blocks are not shown because we use these types of vertex neighborhood both for saturated and non-saturated realizations.

Table 3

\section{Vertex neighborhood extensions for 2-extensions of $\Lambda^{3}$ of class I}

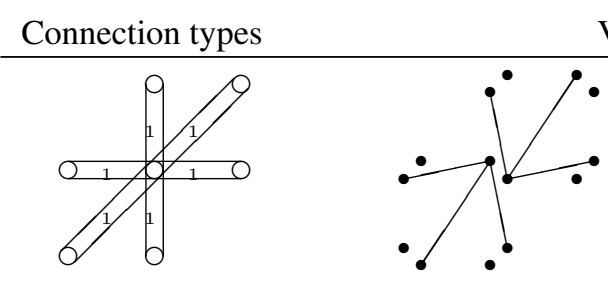

11_11_11

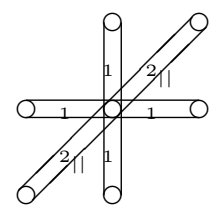

$11 \_11 \_2 \| 2$ ||

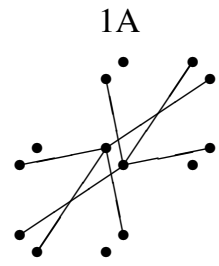

2A

Vertex neighborhood extensions

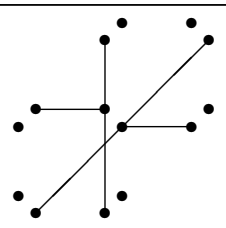

1B

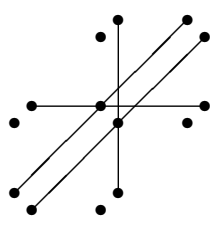

2B 


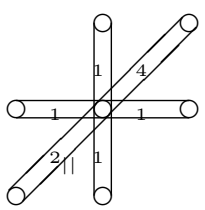

$11 \_11 \_2$ || 4

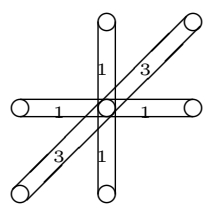

11_11_33

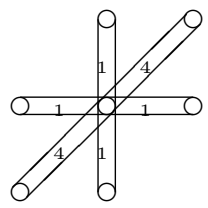

11_11_44

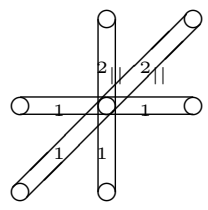

$11 \_12_{||-12||}$

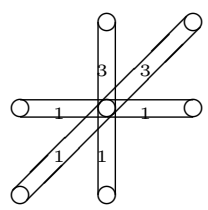

11_13_13

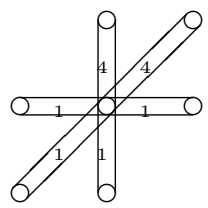

11_14_14

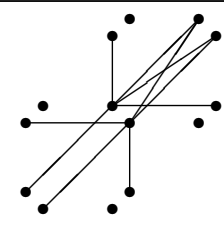

$3 \mathrm{~A}$

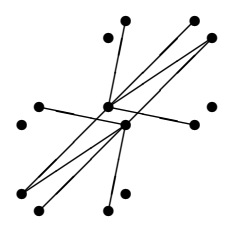

$4 \mathrm{~A}$

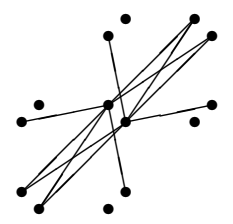

$5 \mathrm{~A}$
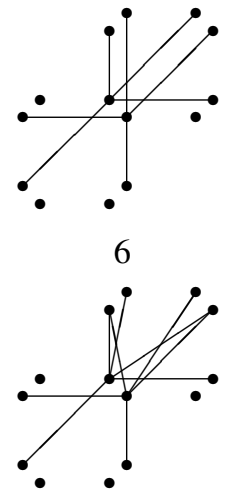

7A

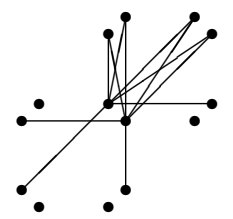

8

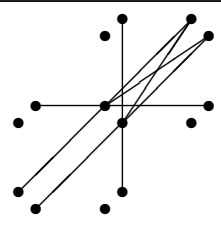

3B

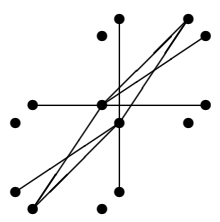

4B

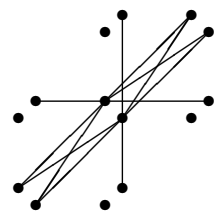

5B

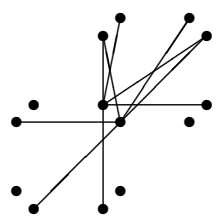

7B 

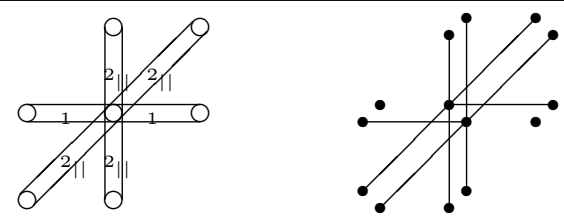

$11_{-} 2_{||} 2_{||-} 2_{||} 2_{\|}$

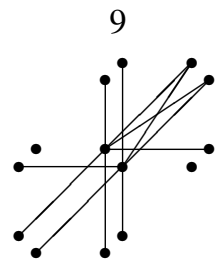

$11_{-} 2_{\|} 2_{\|} \_2{ }_{\|} 4$

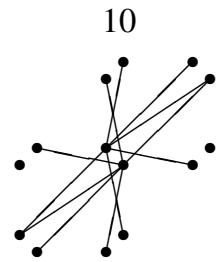

$11 \_22_{||} 2_{||-33}$

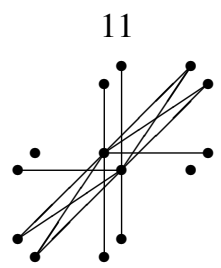

$11 \_22_{||} 2_{||-44}$

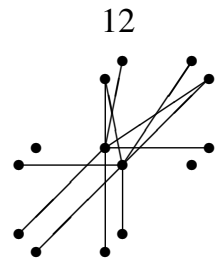

$\left.11 \_2{ }_{||} 3 \_2\right|_{||} 3$

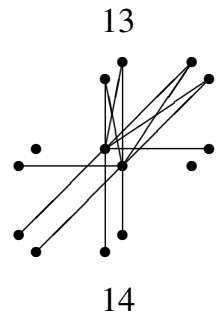

$11 \_2$ || $4 \_2$ || 4 

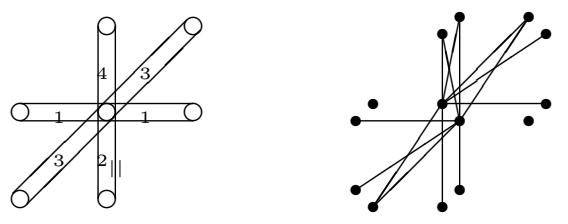

$11 \_2$ || $4 \_33$
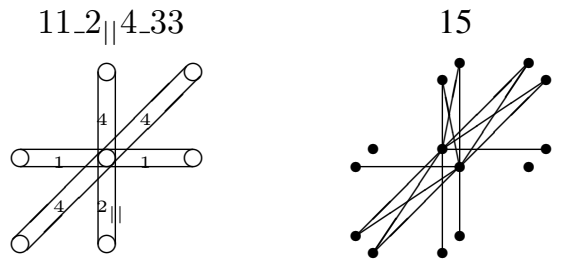

$11 \_2$ || $4 \_44$

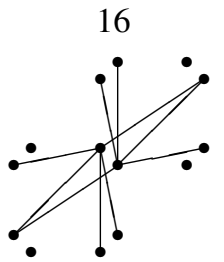

$17 \mathrm{~A}$

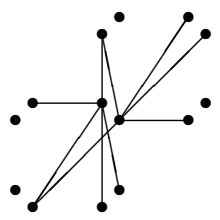

$11 \_2_{V} 2_{V} \_2_{V} 2_{V}$

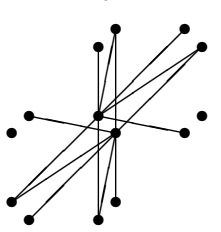

11_33_33

$18 \mathrm{~A}$
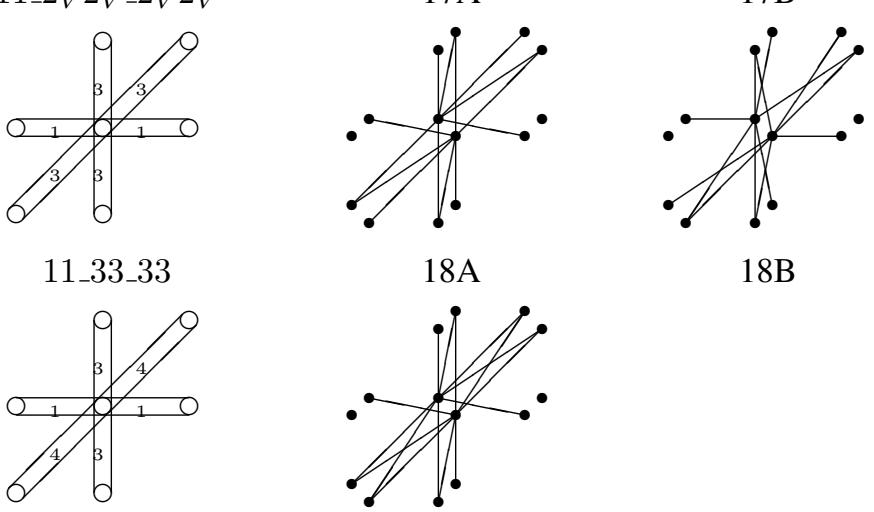

$18 \mathrm{~B}$

11_33_44

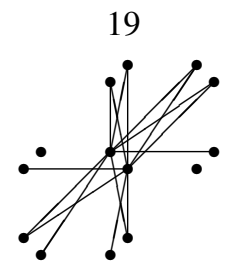

$11 \_34 \_34$

20 


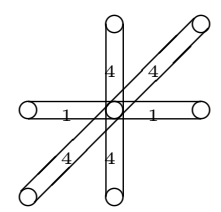

11_44_44
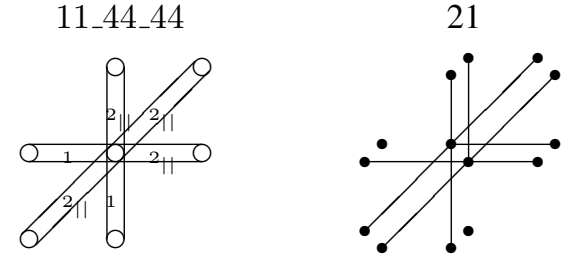

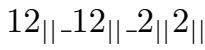

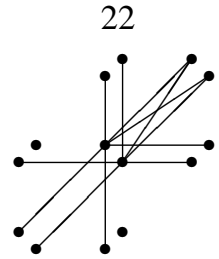

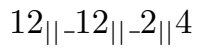

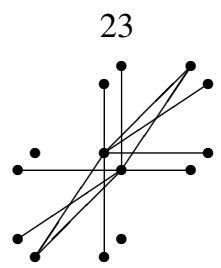

$12_{||-12}||-33$
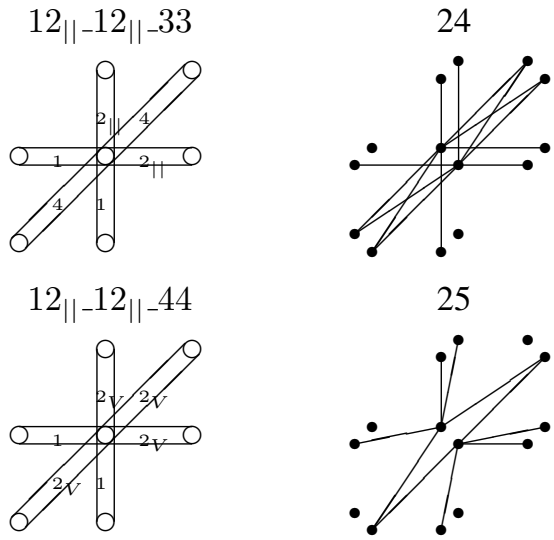

$12_{V-1} 12_{V-} 2_{V} 2_{V}$

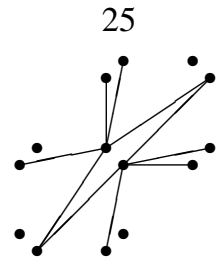

$26 \mathrm{~A}$

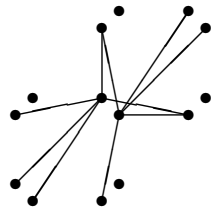

26B

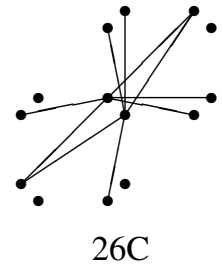




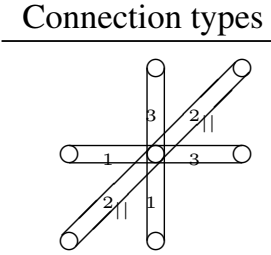

$13 \_13 \_2$ || 2 ||

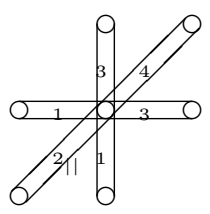

$13 \_13 \_2$ || 4

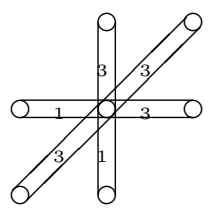

13_13_33

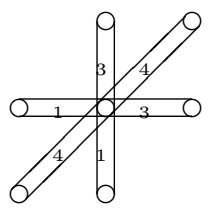

13_13_44

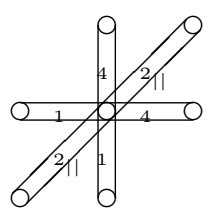

$14 \_14 \_22_{||} 2_{\|}$

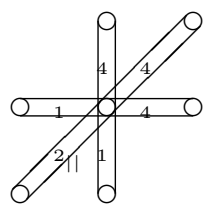

$14 \_14 \_2{ }_{||} 4$

Vertex neighborhood extensions

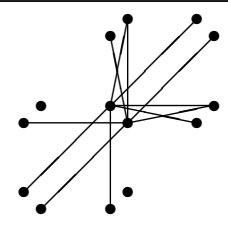

$27 \mathrm{~A}$

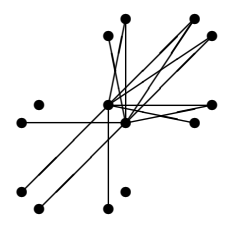

$28 \mathrm{~A}$

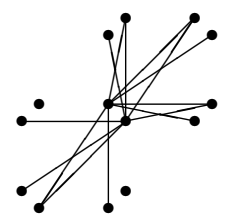

29A

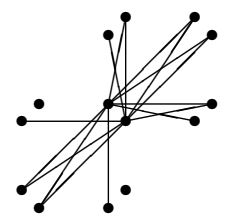

$30 \mathrm{~A}$
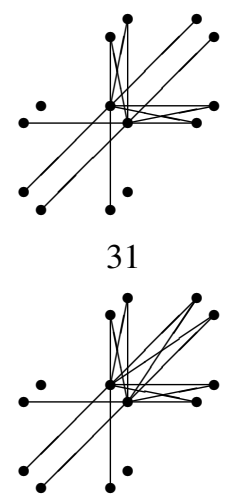

32

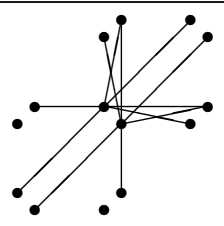

27B

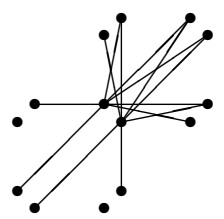

28B

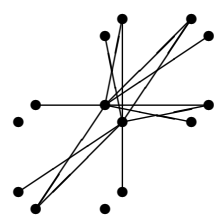

29B

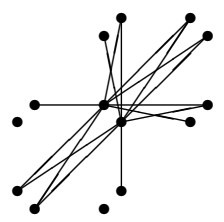

30B 


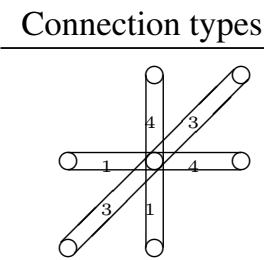

14_14_33

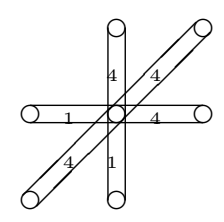

14_14_44
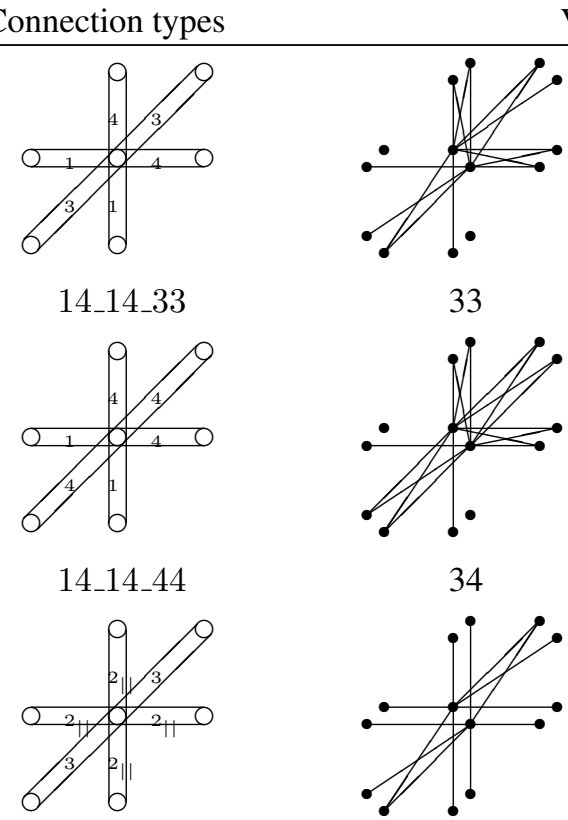
Vertex neighborhood extensions

$2_{||} 2_{||-2} 2_{||} 2_{||-33}$
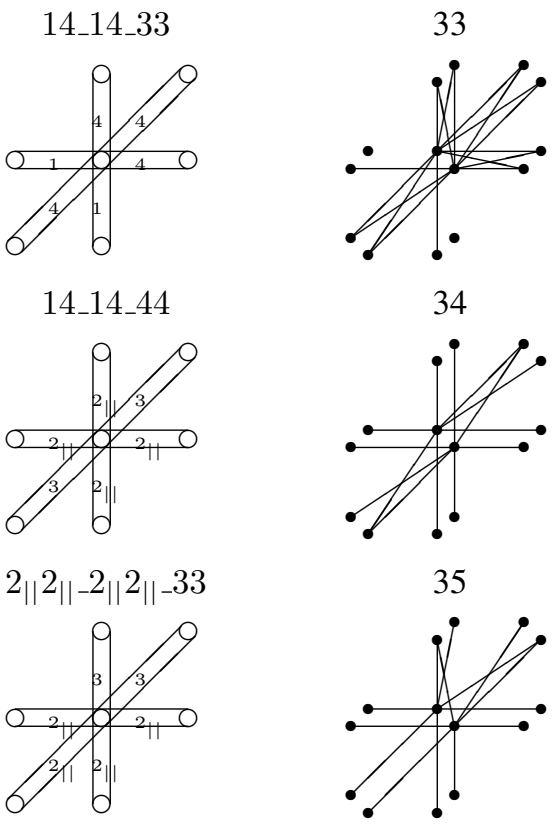

$2_{||} 2_{||-2} 2_{||} 3 \_22_{||} 3$
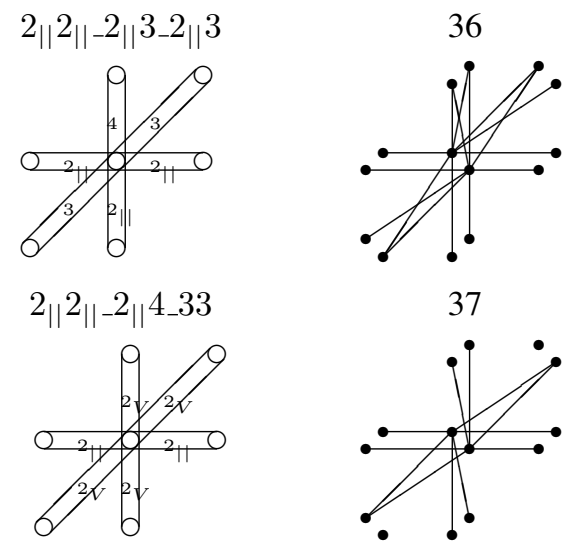

$2_{\|} 2_{\| \mid} 2_{V} 2_{V-} 2_{V} 2_{V}$

$38 \mathrm{~A}$

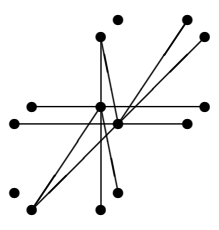

38B 


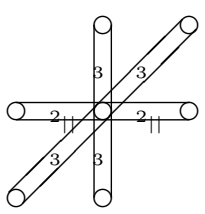

$2_{||} 2_{||-33 \_33}$

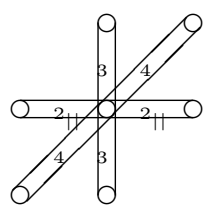

$2_{||} 2_{||-33 \_44}$

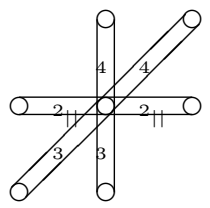

$2_{||} 2_{||-34 \_34}$

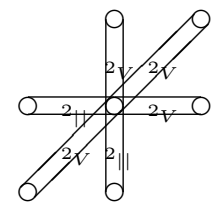

$2_{||} 2_{V \_} 2_{\|} 2_{V \_} 2_{V} 2_{V}$

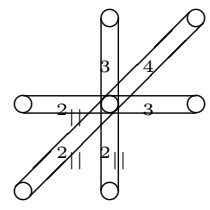

$2_{||} 3 \_2{ }_{\|} 3{ }_{-} 2_{\|} 4$

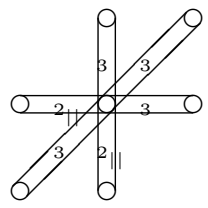

$2_{||} 3 \_2$ || $3 \_33$

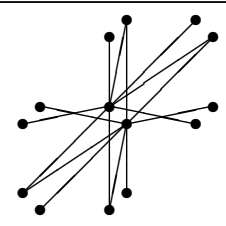

$39 \mathrm{~A}$

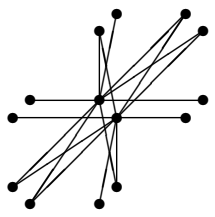

40

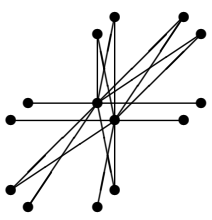

41

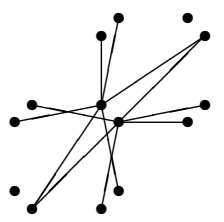

$42 \mathrm{~A}$

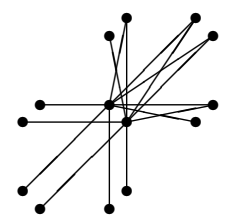

43

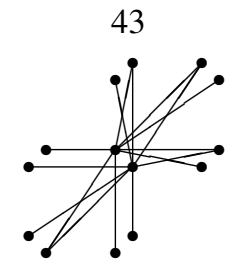

44

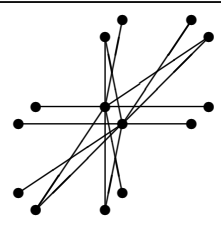

39B

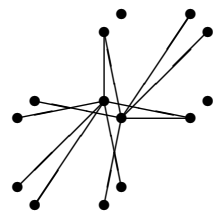

42B 


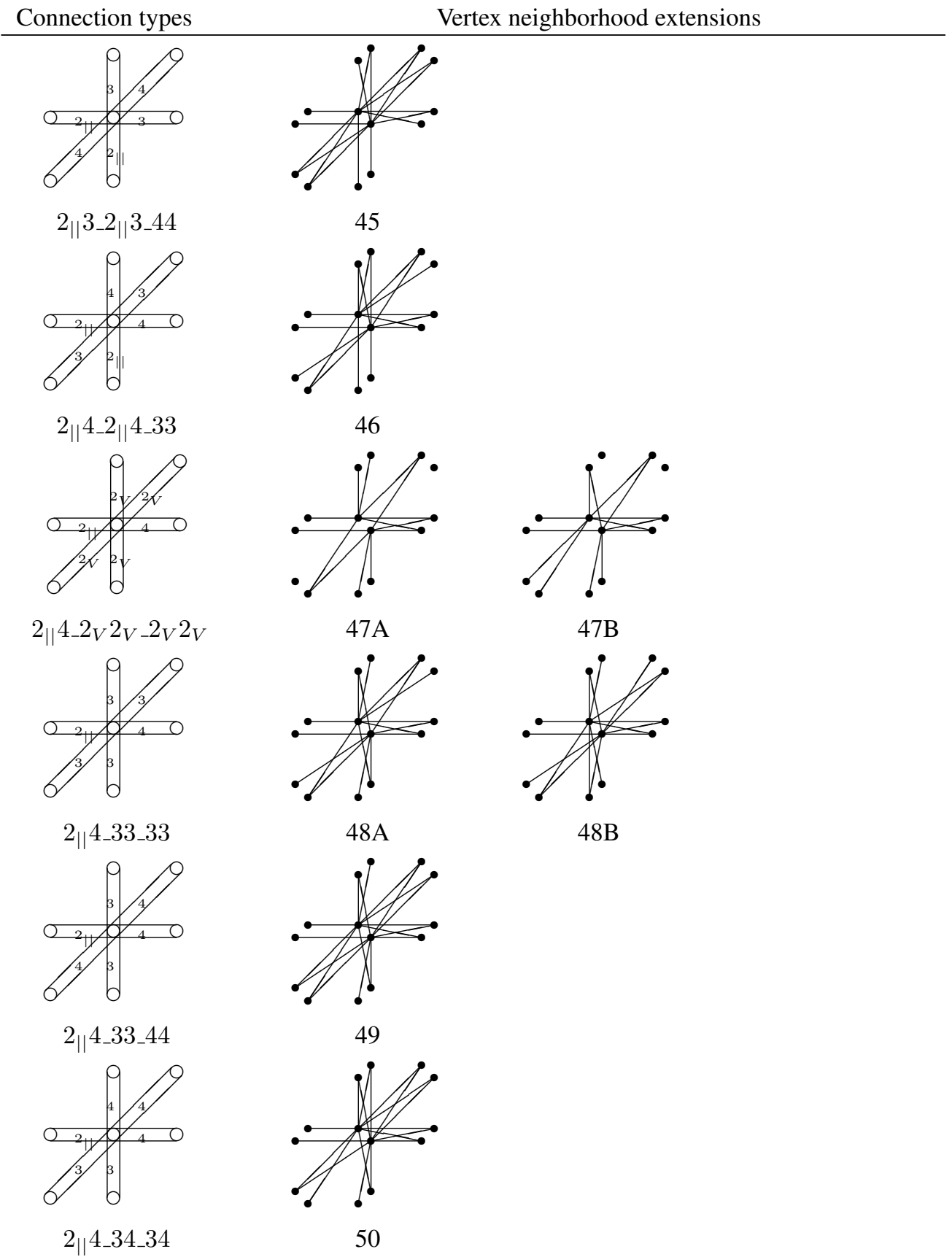




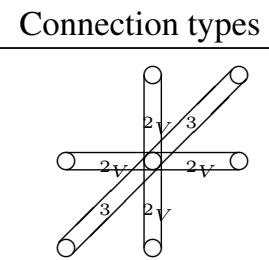

$2_{V} 2_{V} \_2_{V} 2_{V \_} 33$

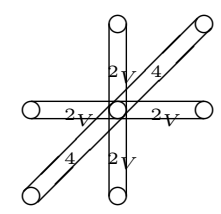

$2_{V} 2_{V \_} 2_{V} 2_{V \_} 44$

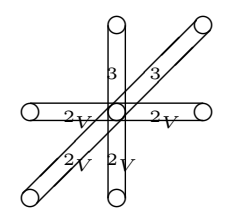

$2_{V} 2_{V \_} 2_{V} 3 \_2 V_{V} 3$

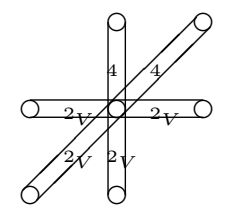

$2_{V} 2_{V} 2_{V} 4 \_2{ }_{V} 4$

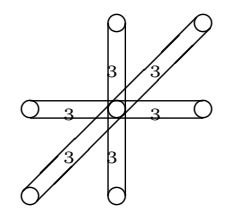

33_33_33

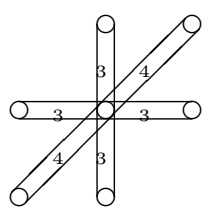

33_33_44

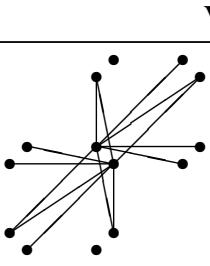

$51 \mathrm{~A}$

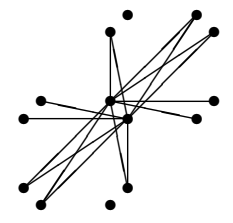

$52 \mathrm{~A}$

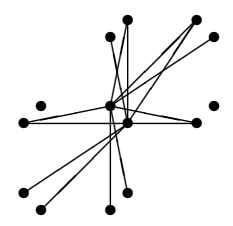

$53 \mathrm{~A}$

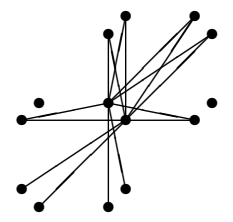

$54 \mathrm{~A}$

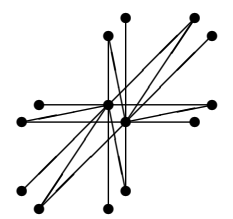

$55 \mathrm{~A}$

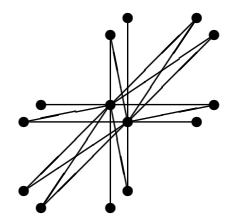

$56 \mathrm{~A}$

Vertex neighborhood extensions

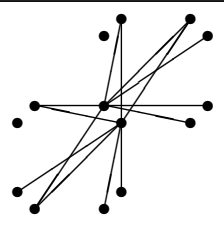

51B

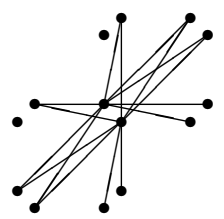

52B

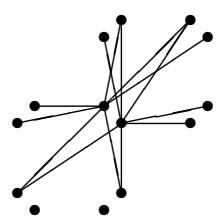

53B

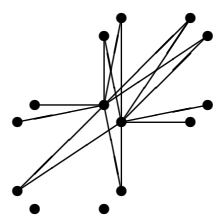

54B

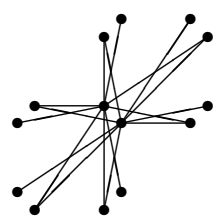

$55 \mathrm{~B}$

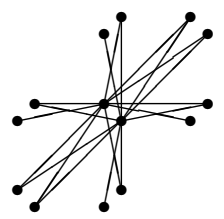

56B

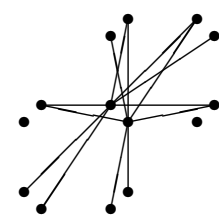

$53 \mathrm{C}$ 


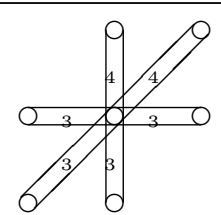

33_34_34

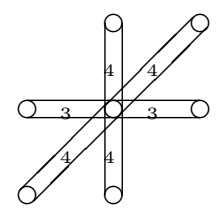

33_44_44

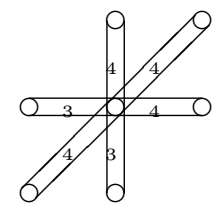

34_34_44

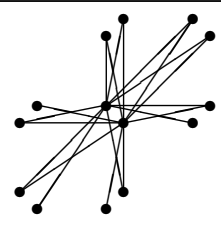

57

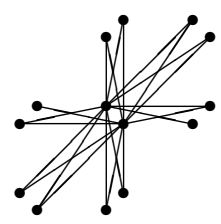

58

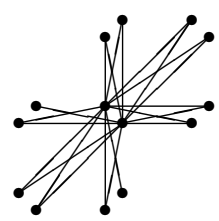

59

A list of 2872 saturated realizations generated using Algorithm 1 and thinned out using Algorithm 2 is given below in Table 5 at the end of the parer. Saturated realizations are defined by triples $H, L, X$ (see above): group $H \in \mathbf{H}$ is given in the fourth column, subgroup $L$ of index 2 in $H_{(0,0,0)}$ is presented in the fifth column, a subset of $X$ of elements of the group $H$ is in the seventh column. In addition, the sixth column contains an element $m$ such that $L \cup m L=H_{(0,0,0)}$. The realization № is given in the third column. Saturated realizations are sorted by vertex neighborhood extensions given in Table 3. (№ of vertex neighborhood extension is given in the first column of Table 5). The set of realizations with given vertex neighborhood extension, in turn, are divided into classes defined by sizes of spheres of radii $1,2, \ldots, 10$ of graph of a realization. We call such a set of sizes of spheres of radii 1, 2,..., 10 growth and give it in the second column of Table 5. In this subdivision, classes are lexicographically sorted by growth increasing. In Table 5, along with saturated realizations, we give non-saturated realizations by № of saturated realizations with an asterisk in the third column (see details before Corollary 1 below).

Theorem 3.1. The saturated realizations of symmetrical 2-extensions of the grid $\Lambda^{3}$ of class I up to equivalence are exhausted by 2872 pairwise nonequivalent saturated realizations given in Table 5.

Note that listing all, up to equivalence, realizations of the symmetrical 2-extensions of the grid $\Lambda^{3}$ is reduced to listing all, up to equivalence, saturated realizations of symmetrical 2-extensions of $\Lambda^{3}$. Indeed, it is obvious that every non-saturated realization of a symmetrical 2-extension of $\Lambda^{3}$ is obtained from a uniquely defined saturated realization by removing an edge in each block. All maximal non-saturated realizations obtained in this way are given in Table 5 by № of saturated maximal realizations taken with an asterisk. 
Corollary 3.2. The non-saturated realizations of symmetrical 2-extensions of the grid $\Lambda^{3}$ of class I up to equivalence are exhausted by 2701 pairwise nonequivalent non-saturated realizations given in Table 5.

Proof. Using a computer, it is easily verified that when removing edges inside blocks of 2872 realizations given in Table 5, 171 their graphs become disconnected and the remaining 2701 realizations (see № with an asterisk in Table 5) give all, up to equivalence, nonsaturated realizations of symmetrical 2 -extensions of the grid $\Lambda^{3}$ of class I.

According to Theorem 1 and Corollary 1, all realizations given in Table 5 are pairwise nonequivalent. However, among the graphs of these realizations, isomorphic ones are found. With GAP, we built partition of the set of graphs of realizations from Table 5 into classes of isomorphic graphs (for details, see the proof of Corollary 3.3 below). In the following Table 4, we give all the non-singleton classes of this partition.

Table 4

\section{Non-singleton classes of isomorphic graphs of realizations of symmetrical 2-extensions of $\Lambda^{3}$ of class I}

\begin{tabular}{|c|c|c|c|}
\hline 1) $1,45^{*}$ & 51) $1282,1412 *$ & 102) 2492,2495 & 153) $1759^{*}, 1760^{*}$ \\
\hline 2) $7,8,76^{*}$ & 52) $1283,1414 *$ & 103) 2552,2553 & 154) $1762^{*}, 1763^{*}$ \\
\hline 3) 9,10 & 53) $1288,664^{*}, 1423^{*}$ & 104) 2651,2659 & 155) $1857^{*}, 1858^{*}$ \\
\hline 4) 16,17 & 54) $1289,1420^{*}$ & 105) 2652,2660 & 156) $1919^{*}, 1920^{*}$ \\
\hline 5) 18,19 & 55) $1290,1424 *$ & 106) 2653,2658 & 157) $1928^{*}, 1929^{*}$ \\
\hline 6) $26,64 *$ & 56) $1294,672^{*}$ & 107) 2654,2655 & 158) $1941^{*}, 1943^{*}$ \\
\hline 7) $30,97 *$ & 57) $1295,673^{*}$ & 108) 2656,2657 & 159) $1944^{*}, 1945^{*}$ \\
\hline 8) $36,198^{*}, 199^{*}, 337^{*}$ & 58) $1307,1437^{*}$ & 109) 2702,2703 & 160) $1962^{*}, 1963^{*}$ \\
\hline 9) $37,38,202^{*}, 203^{*}, 204^{*}, 205^{*}, 206^{*}$, & 59) $1313,712^{*}$ & 110) 2707,2708 & 161) $1990^{*}, 1991^{*}$ \\
\hline $207^{*}, 1119^{*}, 1120^{*}, 1484^{*}$ & 60) $1314,713^{*}$ & 111) 2713,2714 & 162) $1998^{*}, 1999^{*}$ \\
\hline 10) $39,1121^{*}$ & 61) 1716,1717 & 112) 2718,2720 & 163) $2002^{*}, 2006^{*}$ \\
\hline 11) 41,279 & 62) 1720,1721 & 113) 2724,2726 & 164) $2004^{*}, 2005^{*}$ \\
\hline 12) 42,280 & 63) 1722,1724 & 114) $63^{*}, 64^{*}, 65^{*}$ & 165) $2043^{*}, 2047^{*}$ \\
\hline 13) $43,44,214^{*}, 215^{*}, 343^{*}$ & 64) 1725,1726 & 115) $89^{*}, 314^{*}$ & 166) $2050^{*}, 2051^{*}$ \\
\hline 14) $46,123^{*}$ & 65) 1727,1728 & 116) $102^{*}, 105^{*}$ & 167) $2053^{*}, 2054^{*}$ \\
\hline 15) $47,122 *$ & 66) 1756,1757 & 117) $116^{*}, 1121^{*}$ & 168) $2055^{*}, 2056^{*}$ \\
\hline 16) 54,293 & 67) 1759,1760 & 118) $117^{*}, 339^{*}$ & 169) $2058^{*}, 2059^{*}$ \\
\hline 17) 55,294 & 68) 1762,1763 & 119) $121^{*}, 219^{*}$ & 170) $2071^{*}, 2085^{*}$ \\
\hline 18) $57,348^{*}, 352^{*}$ & 69) 1857,1858 & 120) $130^{*}, 1127^{*}$ & 171) $2072^{*}, 2086^{*}$ \\
\hline 19) $59,144^{*}$ & 70) 1919,1920 & 121) $141^{*}, 1125^{*}$ & 172) $2075^{*}, 2084^{*}$ \\
\hline 20) 64,65 & 71) 1928,1929 & 122) $146^{*}, 353^{*}$ & 173) $2080^{*}, 2082^{*}$ \\
\hline 21) $110,250^{*}$ & 72) 1941,1943 & 123) $167^{*}, 245^{*}$ & 174) $2089^{*}, 2090^{*}$ \\
\hline 22) 122,123 & 73) 1944,1945 & 124) $175^{*}, 1139^{*}$ & 175) $2091^{*}, 2092^{*}$ \\
\hline 23) 198,199 & 74) 1962,1963 & 125) $209^{*}, 210^{*}$ & 176) $2093^{*}, 2094^{*}$ \\
\hline 24) 202,203 & 75) 1990,1991 & 126) $211^{*}, 212^{*}$ & 177) $2109^{*}, 2110^{*}$ \\
\hline 25) 206,207 & 76) 1998,1999 & 127) $228^{*}, 350^{*}$ & 178) $2346^{*}, 2349^{*}$ \\
\hline 26) 216,343 & 77) 2002, 2006 & 128) $234^{*}, 235^{*}$ & 179) $2347^{*}, 2348^{*}$ \\
\hline 27) 229,348 & 78) 2004,2005 & 129) $328^{*}, 329^{*}$ & 180) $2353^{*}, 2355^{*}$ \\
\hline 28) 233,352 & 79) 2043,2047 & 130) $360 *, 361^{*}$ & 181) $2357^{*}, 2358^{*}$ \\
\hline 29) $286,216^{*}$ & 80) 2050,2051 & 131) $651^{*}, 652^{*}, 653^{*}, 654^{*}, 2114^{*}$ & 182) $2362^{*}, 2365^{*}$ \\
\hline 30) $296,1123^{*}, 1124^{*}$ & 81) 2053,2054 & 132) $660^{*}, 2115^{*}$ & 183) $2363^{*}, 2364^{*}$ \\
\hline 31) $299,229^{*}$ & 82) 2055,2056 & 133) $662^{*}, 2116^{*}$ & 184) $2368^{*}, 2369^{*}$ \\
\hline 32) $301,233^{*}$ & 83) 2058,2059 & 134) $817^{*}, 818^{*}$ & 185) $2371^{*}, 2372^{*}$ \\
\hline 33) $324,363^{*}$ & 84) 2071,2085 & 135) $819^{*}, 820^{*}$ & 186) $2374^{*}, 2375^{*}$ \\
\hline 34) $332,366^{*}$ & 85) 2072,2086 & 136) $821 *, 2117 *$ & 187) $2376^{*}, 2378^{*}$ \\
\hline 35) $427,2114^{*}$ & 86) 2075,2084 & 137) $825^{*}, 2118^{*}$ & 188) $2492^{*}, 2495^{*}$ \\
\hline 36) $428,503^{*}, 658^{*}$ & 87) 2080,2082 & 138) $1091^{*}, 1094^{*}$ & 189) $2552^{*}, 2553^{*}$ \\
\hline 37) $431,667^{*}, 1489^{*}$ & 88) 2089,2090 & 139) $1092^{*}, 1093^{*}$ & 190) $2651^{*}, 2659^{*}$ \\
\hline 38) $439,1422^{*}$ & 89) 2091,2092 & 140) $1133^{*}, 1134^{*}$ & 191) $2652^{*}, 2660^{*}$ \\
\hline 39) $440,1421^{*}$ & 90) 2093, 2094 & 141) $1136^{*}, 1137^{*}$ & 192) $2653^{*}, 2658^{*}$ \\
\hline 40) $455,1440 *$ & 91) 2109,2110 & 142) $1143^{*}, 1144^{*}$ & 193) $2654^{*}, 2655^{*}$ \\
\hline 41) $456,1439^{*}$ & 92) 2346,2349 & 143) $1147^{*}, 1148^{*}$ & 194) $2656^{*}, 2657^{*}$ \\
\hline 42) 651,652 & 93) 2347,2348 & 144) $1149^{*}, 1150^{*}$ & 195) $2702^{*}, 2703^{*}$ \\
\hline 43) 653,654 & 94) 2353,2355 & 145) $1152^{*}, 2521^{*}$ & 196) $2707^{*}, 2708^{*}$ \\
\hline 44) 664,1489 & 95) 2357,2358 & 146) $1163^{*}, 1622^{*}$ & 197) $2713^{*}, 2714^{*}$ \\
\hline 45) 672,1421 & 96) 2362,2365 & 147) $1716^{*}, 1717^{*}$ & 198) $2718^{*}, 2720^{*}$ \\
\hline 46) 673,1422 & 97) 2363,2364 & 148) $1720^{*}, 1721^{*}$ & 199) $2724^{*}, 2726^{*}$ \\
\hline 47) 712,1439 & 98) 2368,2369 & 149) $1722^{*}, 1724^{*}$ & \\
\hline 48) 713,1440 & 99) 2371,2372 & 150) $1725^{*}, 1726^{*}$ & \\
\hline 49) 1091,1094 & 100) 2374,2375 & 151) $1727^{*}, 1728^{*}$ & \\
\hline 50) 1092,1093 & 101) 2376,2378 & 152) $1756^{*}, 1757^{*}$ & \\
\hline
\end{tabular}

Corollary 3.3. (i) Up to isomorphism, there are 2792 graphs of saturated realizations of symmetrical 2-extensions of the grid $\Lambda^{3}$ of class $I$. 
(ii) Up to isomorphism, there are 2594 graphs of non-saturated realizations of symmetrical 2-extensions of the grid $\Lambda^{3}$ of class $I$.

Corollary 3.4. Up to isomorphism, there are 5350 graphs of realizations of symmetrical 2-extensions of the grid $\Lambda^{3}$ of class I.

Proof. (for Corollaries 3.3 and 3.4) Using GAP for each of the graphs of 5573 (2872 saturated and 2701 non-saturated) realizations of symmetrical 2-extensions of $\Lambda^{3}$ of class I, a subgraph $B$ was generated by a set of vertices that are at a distance of $\leq 4$ from some arbitrary vertex (i.e., $B$ is a ball of radius 4 ). In the obtained set of 5573 finite graphs, balls are isomorphic if and only if they correspond to realizations which are in the same line of Table 4. After that, we continued each isomorphism $\varphi_{b}$ between the balls $B_{1}$ and $B_{2}$ to the isomorphism $\varphi$ of whole graphs of the corresponding realizations $R_{1}=\left(\Gamma_{1}, G_{1}, \sigma_{1}, \varphi_{1}\right)$ and $R_{2}=\left(\Gamma_{2}, G_{2}, \sigma_{2}, \varphi_{2}\right)$ as follows.

Let a realization $R_{1}$ satisfies the condition of $\left[p_{x 1}, p_{y 1}, p_{z 1}\right]$ - periodicity and $R_{2}$ satisfies the condition of $\left[p_{x 2}, p_{y 2}, p_{z 2}\right]$ - periodicity. Recall that, according to [5], a realization $R=(\Gamma, G, \sigma, \varphi)$ of a symmetrical 2-extension of $\Lambda^{3}$ satisfies the condition of $\left[p_{x}, p_{y}, p_{z}\right]$ periodicity, where $p_{x}, p_{y}, p_{z}$ are positive integers, if there exist $g_{1}, g_{2}, g_{3} \in G$ such that $\left[g_{i}, g_{j}\right]=1$ for $i \neq j$ and $\varphi g_{1}^{\sigma} \varphi^{-1}=t_{x}^{p_{x}}, \varphi g_{2}^{\sigma} \varphi^{-1}=t_{y}^{p_{y}}, \varphi g_{3}^{\sigma} \varphi^{-1}=t_{z}^{p_{y}}$. We identify the set of vertices of $\Gamma_{1}$ with the set $\{(x, y, z, w): x, y, z \in \mathbb{Z}, w \in\{0,1\}\}$, so that $\sigma_{1}=\{\{(x, y, z, 0),(x, y, z, 1)\}: x, y, z \in \mathbb{Z}\}$ and $\left\{\left(x_{1}, y_{1}, z_{1}, w_{1}\right),\left(x_{2}, y_{2}, z_{2}, w_{2}\right)\right\} \in$ $\mathrm{E}\left(\Gamma_{1}\right) \Leftrightarrow\left\{\left(x_{1}+p_{x 1}, y_{1}, z_{1}, w_{1}\right),\left(x_{2}+p_{x 1}, y_{2}, z_{2}, w_{2}\right)\right\} \in \mathrm{E}\left(\Gamma_{1}\right) \Leftrightarrow\left\{\left(x_{1}, y_{1}+p_{y 1}, z_{1}, w_{1}\right)\right.$, $\left.\left(x_{2}, y_{2}+p_{y 1}, z_{2}, w_{2}\right)\right\} \in \mathrm{E}\left(\Gamma_{1}\right) \Leftrightarrow\left\{\left(x_{1}, y_{1}, z_{1}+p_{z 1}, w_{1}\right),\left(x_{2}, y_{2}, z_{2}+p_{z 1}, w_{2}\right)\right\} \in$ $\mathrm{E}\left(\Gamma_{1}\right)$. Similarly, we identify the set of vertices of $\Gamma_{2}$ with the set $\{(x, y, z, w): x, y, z \in$ $\mathbb{Z}, w \in\{0,1\}\}$.

We select positive integers $p_{x}, p_{y}, p_{z}$ so that the realization $R_{1}$ satisfies the conditions of $\left[p_{x}, p_{y}, p_{z}\right]$-periodicity, $p_{x}\left|p_{x 1}, p_{y}\right| p_{y 1}, p_{z} \mid p_{z 1}$ and $\left(p_{x}, 0,0\right) M,\left(0, p_{y}, 0\right) M,\left(0,0, p_{z}\right) M \in$ $\left\langle\left(p_{x 2}, 0,0\right),\left(0, p_{y 2}, 0\right),\left(0,0, p_{z 2}\right)\right\rangle$, where angle brackets mean generation in the additive group of row-vectors and the $3 \times 3$-matrix $M$ is obtained from the $3 \times 4$ matrix

$$
\left(\begin{array}{c}
\left(p_{x}, 0,0,0\right) \varphi_{b} / p_{x} \\
\left(0, p_{y}, 0,0\right) \varphi_{b} / p_{y} \\
\left(0,0, p_{z}, 0\right) \varphi_{b} / p_{z}
\end{array}\right)
$$

by deleting the last column. We need to ensure that the extension of the fragment $\left[0 \ldots p_{x}-\right.$ $1] \times\left[0 \ldots p_{y}-1\right] \times\left[0 \ldots p_{z}-1\right]$ is inside of the ball $B_{1}$. To choose $p_{x}, p_{y}, p_{z}$ in such a way for some pairs of realizations $R_{1}$ and $R_{2}$ we had to take the radii of balls $B_{1}$ and $B_{2}$ greater than 4.

The image of an arbitrary vertex $u$ of the extension $\Gamma_{1}$ is now defined by $u \varphi=$ $=u t^{-1} \varphi_{b} t^{M}$, where the shift $t^{-1}=t_{x}^{p_{x} n_{1}} t_{y}^{p_{y} n_{2}} t_{z}^{p_{z} n_{3}}$ maps $u$ into the extension of the fragment $\left[0 \ldots p_{x}-1\right] \times\left[0 \ldots p_{y}-1\right] \times\left[0 \ldots p_{z}-1\right]$, where $n_{1}, n_{2}, n_{3}$ are suitable positive integers. 


\section{of symmetrical 2 -extensions of the grid $\Lambda^{3}$ of class I}

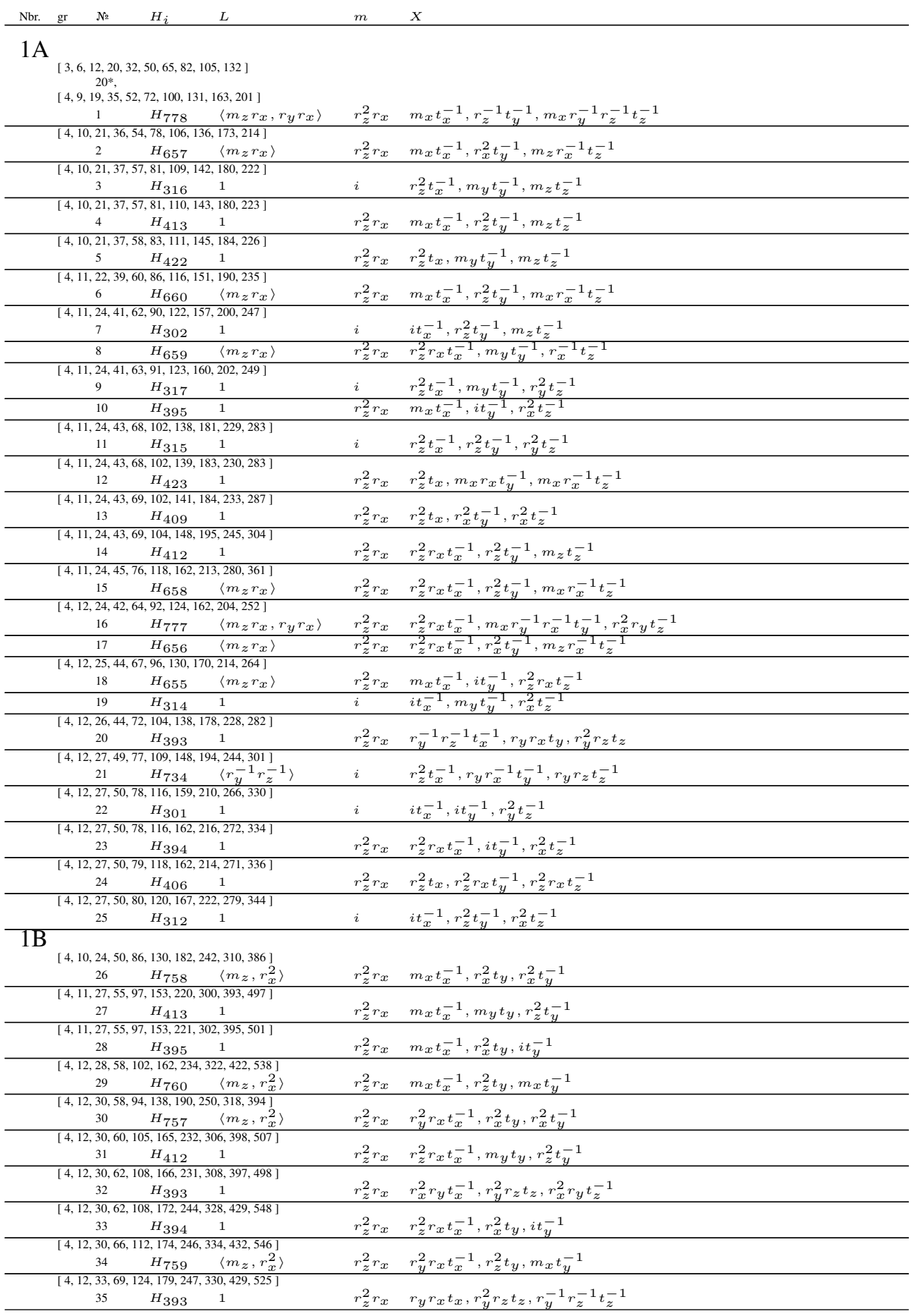




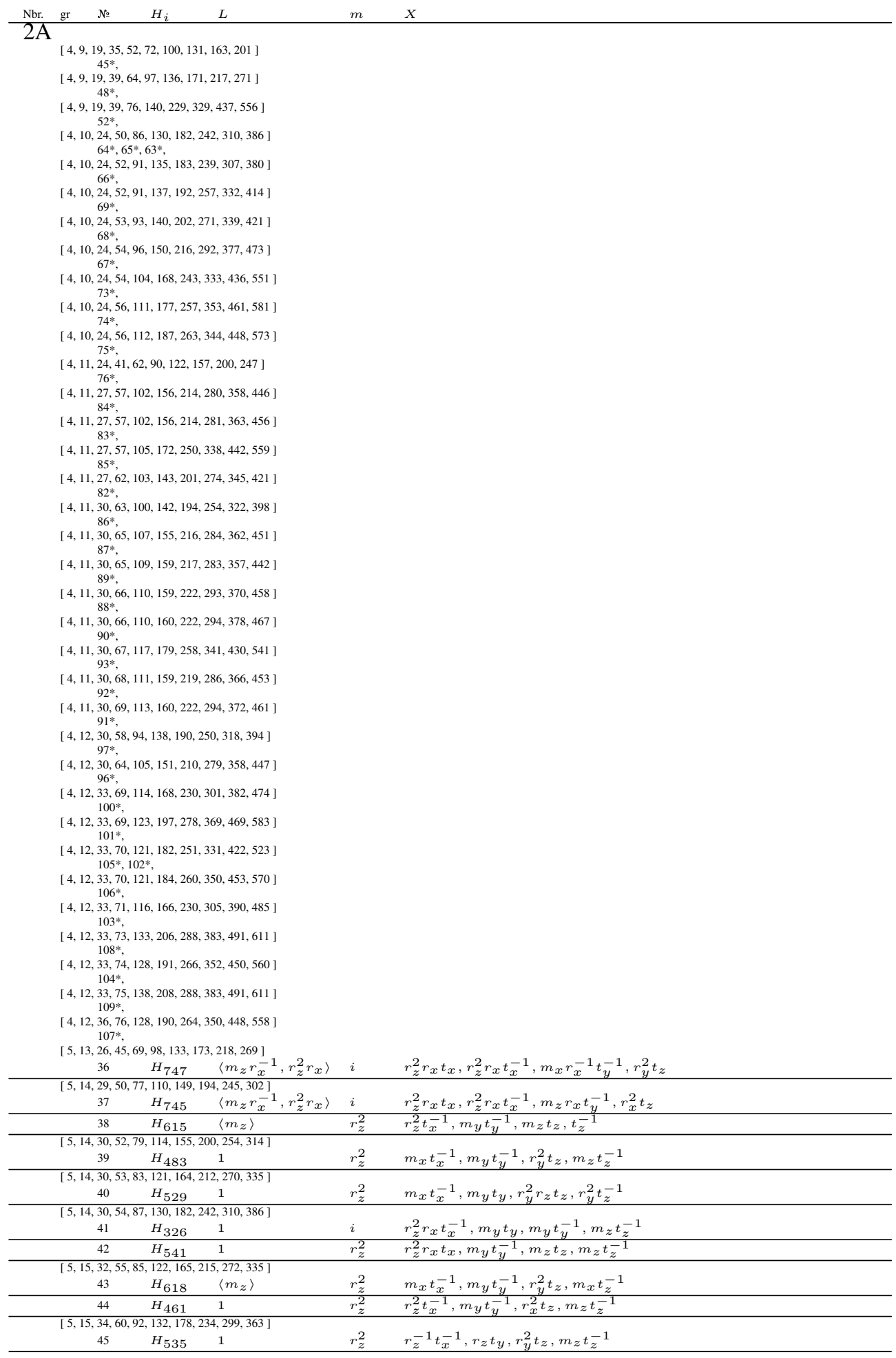




\begin{tabular}{|c|c|c|c|c|c|}
\hline Nbr. & gr $\quad$ № & $H_{i}$ & $L$ & $m$ & $X$ \\
\hline & $\begin{array}{r}{[5,15,34,} \\
46\end{array}$ & 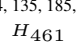 & $\begin{array}{l}42,306,378] \\
1\end{array}$ & $r_{z}^{2}$ & $r_{z}^{2} t_{x}^{-1}, m_{y} t_{y}^{-1}, r_{y}^{2} t_{z}, m_{z} t_{z}^{-1}$ \\
\hline & $\begin{array}{r}{[5,15,34,} \\
47\end{array}$ & $\begin{array}{c}138,189, \\
H_{675}\end{array}$ & $\begin{array}{c}47,314,389] \\
\left\langle r_{y}^{2} r_{x}\right\rangle\end{array}$ & $r_{z}^{2} r_{x}$ & $r_{y}^{2} t_{x}, r_{z}^{2} t_{x}^{-1}, m_{y} t_{y}^{-1}, m_{x} r_{x}^{-1} t_{z}^{-1}$ \\
\hline & $\begin{array}{r}{[5,15,34,} \\
48\end{array}$ & $\begin{array}{c}3,142,191, \\
H_{512}\end{array}$ & $\begin{array}{l}49,316,385] \\
1\end{array}$ & $r_{z}^{2}$ & $r_{z}^{-1} t_{x}^{-1}, r_{z} t_{y}, r_{y}^{2} r_{z} t_{z}, r_{y}^{2} t_{z}^{-1}$ \\
\hline & $\begin{array}{r}{[5,15,34,} \\
49\end{array}$ & $\begin{array}{c}33,155,216 \\
H_{460}\end{array}$ & $\begin{array}{l}285,363,450] \\
1\end{array}$ & $r_{z}^{2}$ & $r_{z}^{2} t_{x}^{-1}, r_{z}^{2} t_{y}^{-1}, r_{y}^{2} t_{z}, m_{z} t_{z}^{-1}$ \\
\hline & $\begin{array}{r}{[5,15,34,} \\
50\end{array}$ & $\begin{array}{c}5,159,22 \\
H_{503}\end{array}$ & $\begin{array}{l}293,373,463] \\
1\end{array}$ & $r_{z}^{2}$ & $r_{z}^{2} t_{x}^{-1}, r_{z}^{2} t_{y}^{-1}, r_{x}^{2} r_{z} t_{z}, r_{y}^{2} t_{z}^{-1}$ \\
\hline & $\begin{array}{r}{[5,15,35,} \\
51\end{array}$ & $\begin{array}{c}5,137,191, \\
H_{640}\end{array}$ & $\begin{array}{l}49,313,389] \\
\left\langle m_{z} r_{x}^{-1}\right\rangle\end{array}$ & $r_{x}^{2}$ & $r_{z}^{2} r_{x} t_{x}, r_{y}^{2} r_{x} t_{x}^{-1}, r_{x}^{-1} t_{y}^{-1}, m_{z} t_{z}$ \\
\hline & $\begin{array}{r}{[5,15,38,} \\
52\end{array}$ & $\begin{array}{c}26,201,285 \\
H_{541}\end{array}$ & $\begin{array}{l}386,497,630] \\
1\end{array}$ & $r_{z}^{2}$ & $r_{z}^{2} r_{x} t_{x}, m_{y} t_{y}^{-1}, m_{z} t_{z}, i t_{z}^{-1}$ \\
\hline & $\begin{array}{r}{[5,16,33,} \\
53\end{array}$ & $\begin{array}{c}128,173, \\
H_{616}\end{array}$ & $\begin{array}{c}26,285,352] \\
\left\langle m_{z}\right\rangle\end{array}$ & $r_{z}^{2}$ & $r_{z}^{2} t_{x}^{-1}, m_{y} t_{y}^{-1}, r_{x}^{2} t_{z}, m_{y} t_{z}^{-1}$ \\
\hline & $\begin{array}{r}{[5,16,34,} \\
54\end{array}$ & $\begin{array}{c}, 138,190, \\
H_{308}\end{array}$ & 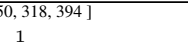 & $i$ & $r_{z}^{2} t_{x}^{-1}, r_{z}^{2} t_{y}, r_{z}^{2} t_{y}^{-1}, r_{y}^{2} r_{z} t_{z}^{-1}$ \\
\hline & 55 & $H_{511}$ & 1 & $r_{z}^{2}$ & $r_{z}^{2} r_{x} t_{x}, r_{x}^{2} t_{y}^{-1}, r_{x}^{2} t_{z}, r_{x}^{2} t_{z}^{-1}$ \\
\hline & $\begin{array}{r}{[5,16,36,} \\
56\end{array}$ & $\begin{array}{c}145,197, \\
H_{629}\end{array}$ & $\begin{array}{l}\overline{88,330,407]} \\
\left\langle m_{z} r_{x}^{-1}\right\rangle\end{array}$ & $r_{x}^{2}$ & $r_{z}^{2} r_{x} t_{x}, r_{y}^{2} r_{x} t_{x}^{-1}, m_{z} r_{x} t_{y}^{-1}, r_{x}^{2} t_{z}$ \\
\hline & $\begin{array}{r}{[5,16,37,} \\
57\end{array}$ & $\begin{array}{c}3,150,205 \\
H_{616}\end{array}$ & $\begin{array}{c}268,341,422] \\
\left\langle m_{z}\right\rangle\end{array}$ & $r_{z}^{2}$ & $r_{z}^{2} t_{x}^{-1}, m_{y} t_{y}^{-1}, r_{y}^{2} t_{z}, m_{x} t_{z}^{-1}$ \\
\hline & $\begin{array}{r}{[5,16,37,} \\
58 \\
\end{array}$ & $\begin{array}{c}3,150,205 \\
H_{483}\end{array}$ & $\begin{array}{l}269,343,424] \\
1\end{array}$ & $r_{z}^{2}$ & $m_{x} t_{x}^{-1}, m_{y} t_{y}^{-1}, r_{y}^{2} t_{z}, i t_{z}^{-1}$ \\
\hline & $\begin{array}{r}{[5,16,37,} \\
59 \\
\end{array}$ & $\begin{array}{c}4,151,206 \\
H_{469}\end{array}$ & $\begin{array}{l}271,344,426] \\
1\end{array}$ & $r_{z}^{2}$ & $r_{z}^{2} t_{x}^{-1}, m_{y} t_{y}^{-1}, r_{x}^{2} t_{z}, r_{y}^{2} t_{z}^{-1}$ \\
\hline & $\begin{array}{r}{[5,16,37,} \\
60\end{array}$ & $\begin{array}{c}14,152,208 \\
H_{529}\end{array}$ & $\begin{array}{l}274,349,431] \\
1\end{array}$ & $r_{z}^{2}$ & $m_{x} t_{x}^{-1}, m_{y} t_{y}^{-1}, r_{y}^{2} r_{z} t_{z}, r_{y}^{2} t_{z}^{-1}$ \\
\hline & $\begin{array}{r}{[5,16,37,} \\
61 \\
\end{array}$ & $\begin{array}{c}5,151,206 \\
H_{468} \\
\end{array}$ & $\begin{array}{l}271,345,427] \\
1\end{array}$ & $r_{z}^{2}$ & $r_{z}^{2} t_{x}^{-1}, m_{y} t_{y}^{-1}, m_{z} t_{z}, i t_{z}^{-1}$ \\
\hline & $\begin{array}{r}{[5,16,37,} \\
62 \\
\end{array}$ & $\begin{array}{c}2,168,230 \\
H_{614} \\
\end{array}$ & $\begin{array}{c}304,386,480] \\
\left\langle m_{z}\right\rangle\end{array}$ & $r_{z}^{2}$ & $r_{z}^{2} t_{x}^{-1}, r_{z}^{2} t_{y}^{-1}, r_{y}^{2} t_{z}, m_{x} t_{z}^{-1}$ \\
\hline & $\begin{array}{r}{[5,16,38,} \\
63 \\
\end{array}$ & $\begin{array}{c}13,168,232 \\
H_{531}\end{array}$ & $\begin{array}{l}305,388,480] \\
1\end{array}$ & $r_{z}^{2}$ & $m_{x} r_{z} t_{x}^{-1}, m_{x} r_{z} t_{y}^{-1}, r_{y}^{2} t_{z}, m_{z} t_{z}^{-1}$ \\
\hline & $\begin{array}{r}{[5,16,38,} \\
64\end{array}$ & $\begin{array}{c}4,170,237 \\
H_{305}\end{array}$ & $\begin{array}{l}314,401,498] \\
1\end{array}$ & $i$ & $i t_{x}^{-1}, t_{y}, t_{y}^{-1}, r_{y}^{2} r_{z} t_{z}^{-1}$ \\
\hline & 65 & $H_{504}$ & 1 & $r_{z}^{2}$ & $r_{z}^{2} t_{x}^{-1}, r_{x}^{2} r_{y} t_{y}^{-1}, t_{z}, t_{z}^{-1}$ \\
\hline & $\begin{array}{r}{[5,16,38,} \\
66\end{array}$ & $\begin{array}{c}14,162,220 \\
H_{479}\end{array}$ & $\begin{array}{l}288,364,449] \\
1\end{array}$ & $r_{z}^{2}$ & $r_{y}^{2} t_{x}^{-1}, m_{y} t_{y}^{-1}, r_{x}^{2} t_{z}, r_{y}^{2} t_{z}^{-1}$ \\
\hline & $\begin{array}{r}{[5,16,38,} \\
67\end{array}$ & $\begin{array}{c}19,183,25 \\
H_{533} \\
\end{array}$ & $\begin{array}{l}336,426,530] \\
1\end{array}$ & $r_{z}^{2}$ & $m_{x} r_{z}^{-1} t_{x}^{-1}, m_{x} r_{z}^{-1} t_{y}, r_{y}^{2} r_{z} t_{z}, r_{y}^{2} t_{z}^{-1}$ \\
\hline & $\begin{array}{r}{[5,16,38,} \\
68\end{array}$ & $\begin{array}{c}15,167,233 \\
H_{571} \\
\end{array}$ & $\begin{array}{l}302,379,470] \\
1\end{array}$ & $m_{z} r_{x}$ & $m_{x} t_{x}, r_{y}^{2} r_{x} t_{x}^{-1}, r_{z}^{2} t_{y}^{-1}, m_{z} t_{z}$ \\
\hline & $\begin{array}{r}{[5,16,38,} \\
69 \\
\end{array}$ & $\begin{array}{c}16,167,228 \\
H_{486} \\
\end{array}$ & $\begin{array}{l}297,377,466] \\
1\end{array}$ & $r_{z}^{2}$ & $r_{y}^{2} t_{x}^{-1}, m_{y} t_{y}^{-1}, m_{z} t_{z}, i t_{z}^{-1}$ \\
\hline & $\begin{array}{r}{[5,16,38,} \\
70\end{array}$ & $\begin{array}{c}2,191,271 \\
H_{540} \\
\end{array}$ & $\begin{array}{l}367,473,595] \\
1 \\
\end{array}$ & $r_{z}^{2}$ & $i t_{x}^{-1}, r_{x}^{2} r_{y} t_{y}^{-1}, m_{y} t_{z}, m_{y} t_{z}^{-1}$ \\
\hline & $\begin{array}{r}{[5,16,38,} \\
71\end{array}$ & $\begin{array}{c}27,197,281 \\
H_{323}\end{array}$ & $\begin{array}{l}380,492,620] \\
1\end{array}$ & $i$ & $r_{z}^{2} t_{x}, r_{z}^{2} t_{x}^{-1}, r_{x}^{2} r_{y} t_{y}^{-1}, r_{x}^{2} t_{z}^{-1}$ \\
\hline & $\begin{array}{r}{[5,16,39,} \\
72\end{array}$ & $\begin{array}{c}9,178,244 \\
H_{669} \\
\end{array}$ & $\begin{array}{c}322,410,508] \\
\left\langle r_{y}^{2} r_{x}\right\rangle\end{array}$ & $r_{z}^{2} r_{x}$ & $r_{y}^{2} t_{x}, r_{z}^{2} t_{x}^{-1}, r_{x}^{2} t_{y}^{-1}, r_{x}^{2} t_{z}^{-1}$ \\
\hline & $\begin{array}{r}{[5,16,41,} \\
73\end{array}$ & $\begin{array}{c}31,200,28 \\
H_{308}\end{array}$ & $\begin{array}{l}380,487,608] \\
1\end{array}$ & $i$ & $r_{z}^{2} t_{x}^{-1}, m_{z} t_{y}, m_{z} t_{y}^{-1}, r_{y}^{2} r_{z} t_{z}^{-1}$ \\
\hline & $\begin{array}{r}{[5,16,41,} \\
74\end{array}$ & $\begin{array}{c}4,205,292 \\
H_{511}\end{array}$ & $\begin{array}{l}388,500,620] \\
1\end{array}$ & $r_{z}^{2}$ & $r_{z}^{2} r_{x} t_{x}, r_{x}^{2} t_{y}^{-1}, r_{y}^{2} t_{z}, r_{y}^{2} t_{z}^{-1}$ \\
\hline & $\begin{array}{r}{[5,16,41,} \\
75\end{array}$ & $\begin{array}{c}15,216,290 \\
H_{511}\end{array}$ & $\begin{array}{l}387,501,629] \\
1\end{array}$ & $r_{z}^{2}$ & $r_{z}^{2} r_{x} t_{x}, r_{x}^{2} t_{y}^{-1}, r_{x}^{2} t_{z}, r_{y}^{2} t_{z}^{-1}$ \\
\hline & $\begin{array}{r}{[5,17,38,} \\
76 \\
\end{array}$ & $\begin{array}{c}3,148,203 \\
H_{626} \\
\end{array}$ & $\begin{array}{l}263,332,411] \\
\left\langle m_{z}\right\rangle\end{array}$ & $r_{z}^{2}$ & $r_{z}^{-1} t_{x}^{-1}, r_{z} t_{y}, r_{y}^{2} t_{z}, m_{x} t_{z}^{-1}$ \\
\hline & $\begin{array}{r}{[5,17,38,} \\
77\end{array}$ & $\begin{array}{c}9,159,217 \\
H_{461} \\
\end{array}$ & $\begin{array}{l}285,362,450] \\
1\end{array}$ & $r_{z}^{2}$ & $r_{z}^{2} t_{x}^{-1}, m_{y} t_{y}^{-1}, r_{x}^{2} t_{z}, i t_{z}^{-1}$ \\
\hline & $\begin{array}{r}{[5,17,39,} \\
78 \\
\end{array}$ & $\begin{array}{c}18,179,249 \\
H_{460} \\
\end{array}$ & $\begin{array}{l}335,427,532] \\
1 \\
\end{array}$ & $r_{z}^{2}$ & $r_{z}^{2} t_{x}^{-1}, r_{z}^{2} t_{y}^{-1}, r_{y}^{2} t_{z}, i t_{z}^{-1}$ \\
\hline & $\begin{array}{r}{[5,17,39,} \\
79\end{array}$ & $\begin{array}{c}19,179,253 \\
H_{503} \\
\end{array}$ & $\begin{array}{l}338,434,539] \\
1\end{array}$ & $r_{z}^{2}$ & $r_{z}^{2} t_{x}^{-1}, r_{z}^{2} t_{y}^{-1}, r_{y}^{2} r_{z} t_{z}, r_{y}^{2} t_{z}^{-1}$ \\
\hline & $\begin{array}{r}{[5,17,40,} \\
80\end{array}$ & $\begin{array}{c}1,164,225 \\
H_{747} \\
\end{array}$ & $\begin{array}{l}293,376,467] \\
\left\langle m_{z} r_{x}^{-1}, r_{z}^{2} r_{x}\right\rangle\end{array}$ & $i$ & $r_{y}^{2} r_{x} t_{x}, m_{z} r_{x} t_{x}^{-1}, m_{x} r_{x}^{-1} t_{y}^{-1}, r_{y}^{2} t_{z}$ \\
\hline & $\begin{array}{r}{[5,17,40,} \\
81\end{array}$ & $\begin{array}{c}14,166,228 \\
H_{461}\end{array}$ & $\begin{array}{l}300,381,473] \\
1\end{array}$ & $r_{z}^{2}$ & $r_{z}^{2} t_{x}^{-1}, m_{y} t_{y}^{-1}, r_{y}^{2} t_{z}, i t_{z}^{-1}$ \\
\hline & $\begin{array}{r}{[5,17,40,} \\
82\end{array}$ & $\begin{array}{c}16,166,236 \\
H_{639} \\
\end{array}$ & $\begin{array}{l}302,370,468] \\
\left\langle m_{z} r_{x}^{-1}\right\rangle\end{array}$ & $r_{x}^{2}$ & $r_{z}^{2} r_{x} t_{x}, r_{y}^{2} r_{x} t_{x}^{-1}, m_{x} r_{x}^{-1} t_{y}^{-1}, r_{y}^{2} t_{z}$ \\
\hline & $\begin{array}{r}{[5,17,41,} \\
83\end{array}$ & $\begin{array}{l}25,182,248 \\
H_{475}\end{array}$ & $\begin{array}{l}325,413,510] \\
1\end{array}$ & $r_{z}^{2}$ & $i t_{x}^{-1}, m_{y} t_{y}^{-1}, m_{z} t_{z}, i t_{z}^{-1}$ \\
\hline
\end{tabular}




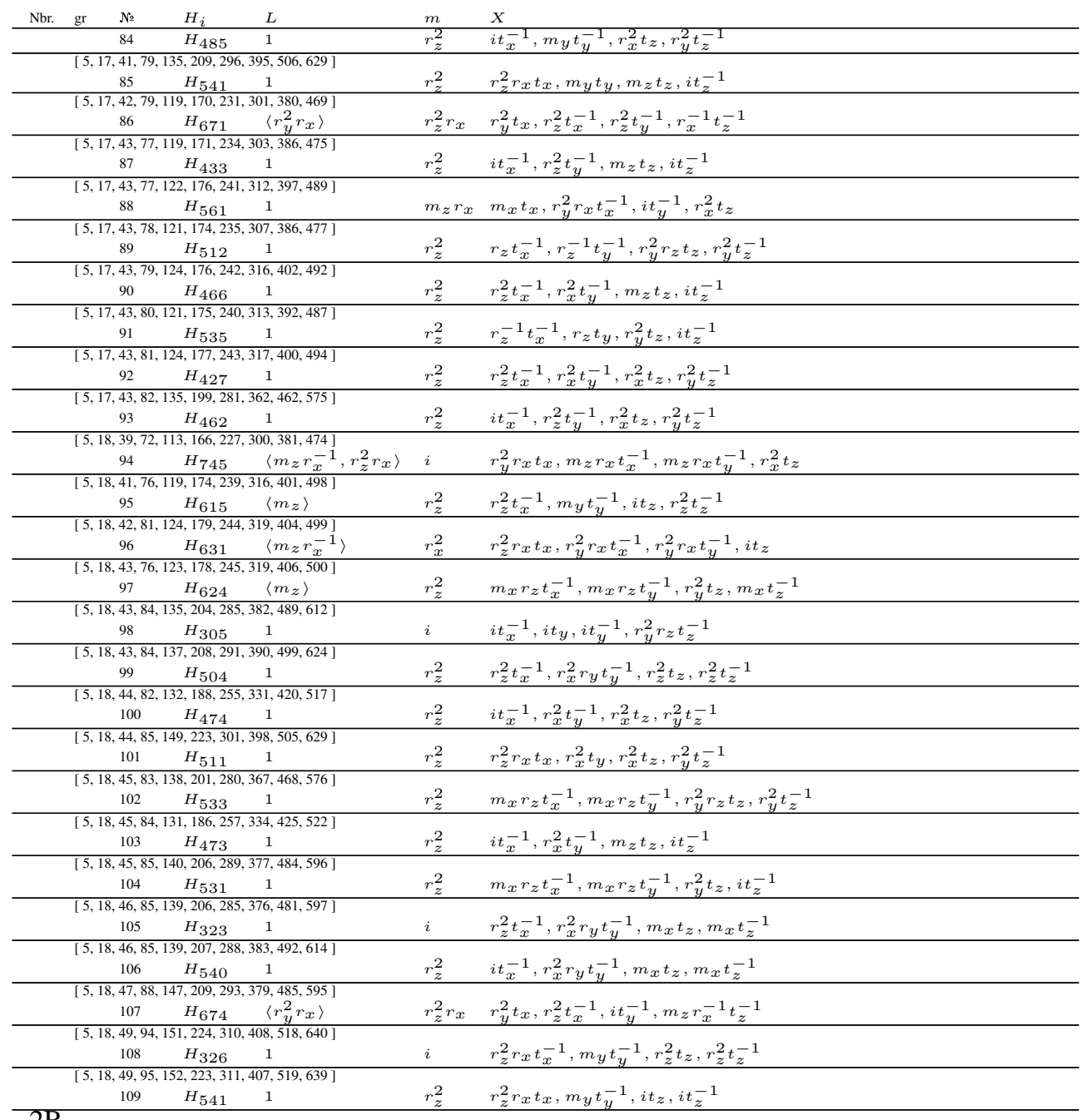

2B

$[4,11,30,73,147,243,346,462,602,756]$ $112^{*}$,

$[4,11,30,76,156,253,350,466,608,762]$ $113^{*}$,

$[4,12,33,85,153,240,344,464,600,752]$ $115^{*}$,

$[5,14,32,64,112,176,256,352,464,592]$ $110 \quad H_{779} \quad\left\langle i, r_{x}^{2}, r_{z}^{2}\right\rangle \quad r_{z}^{2} r_{x} \quad m_{x} t_{x}, t_{x}^{-1}, m_{y} t_{y}, t_{y}^{-1}$

$[5,16,42,88,152,232,328,440,568,712]$ $111 \quad H_{766}\left\langle m_{y}, r_{x}^{2}\right\rangle$

$5,17,46,101,177,262,364,490,628,774]$ $112 \quad H_{571} \quad 1$ $113 \quad H_{561} \quad 1$

$5,18,48,96,160,240,336,448,576,720]$ $114 \quad H_{779} \quad\left\langle i, r_{x}^{2}, r_{z}^{2}\right\rangle \quad r_{z}^{2} r_{x} \quad m_{z} r_{x}^{-1} t_{x}, r_{z}^{2} r_{x} t_{x}^{-1}, m_{y} t_{y}, t_{y}^{-1}$

$[5,18,48,106,174,266,366,490,622,778]$ ${ }_{115} H_{768}\left\langle m_{y}, r_{x}^{2}\right\rangle \quad m_{z} r_{x}^{-1} r_{z}^{2} r_{x} t_{x}, m_{x} t_{x}^{-1}, r_{z}^{2} t_{y}, m_{x} t_{y}^{-1}$

$3 \mathrm{~A}$

$[5,14,30,52,79,114,155,200,254,314]$ $116^{*}$,

$[5,14,31,57,90,131,181,238,303,377]$ $117^{*}$,

$[5,14,31,58,95,141,195,260,335,416]$ $119^{*}$

$[5,14,31,59,96,141,200,268,338,421]$ 
$\begin{array}{llllllll}\text { Nbr. } & \text { gr } & \text { № } & H_{i} & L & m & X\end{array}$

$[5,14,31,61,100,143,201,274,345,421]$ $120^{*}$

$[5,15,34,61,94,135,185,242,306,378]$ $123^{*}$,

$[5,15,34,61,95,138,189,247,314,389]$ $122^{*}$

$[5,15,34,61,96,141,193,253,324,401]$ $121^{*}$

$[5,15,34,62,97,139,190,249,314,387]$ $125^{*}$,

$[5,15,34,62,98,142,194,254,322,398]$ $128^{*}$

$[5,15,34,62,99,145,199,262,334,415]$ $124 *$

$[5,15,34,63,102,151,210,279,358,447]$ $131^{*}$,

$[5,15,34,63,103,154,214,284,366,458]$ $129 *$

$[5,15,35,64,100,146,201,264,337,418]$

$[5,15,35,65,103,150,206,271,345,427]$ $127 *$

$[5,15,35,67,114,178,255,344,446,560]$ $132^{*}, 133^{*}$,

$[5,15,37,68,108,158,218,287,365,453]$ $134^{*}$

$[5,15,37,68,108,159,222,293,370,458]$ $135^{*}$

$[5,15,37,69,111,163,224,297,381,469]$ $136^{*}$,

$[5,15,37,70,111,166,228,301,392,487]$

$137^{*}$,
$[5,15,37,70,113,166,230,305,390,485]$ $138^{*}$

$[5,15,37,74,122,178,244,322,410,508]$

$130^{*}$,
$[5,15,37,84,147,214,293,387,495,615]$ $139^{*}$

$[5,16,36,62,96,140,189,246,314,386]$ $140 *$

$[5,16,36,63,97,139,189,247,312,384$ $141^{*}$,

$[5,16,36,64,100,144,196,256,324,400]$ $147^{*}, 142^{*}, 143^{*}$,

$[5,16,37,66,104,151,206,271,344,426]$ $144^{*}$

$[5,16,37,66,105,157,217,283,357,442]$ $146^{*}$

$[5,16,37,67,106,155,213,280,357,443]$ $145^{*}$,

$[5,16,37,67,107,159,222,294,372,461]$ $148^{*}$

$[5,16,37,67,108,159,218,289,369,457]$ 154

$[5,16,39,72,113,164,223,292,373,461]$ $152^{*}$,

$152^{*}$,
$[5,16,39,73,115,165,224,294,375,467]$ $151^{*}$,

$[5,16,39,73,115,166,228,300,381,473]$ $153^{*}$ [ $5,16,39,75,124,186,259,342,436,541]$

$[5,16,39,76,125,189,265,349,444,550]$ $150^{*}$,

[ $5,16,40,75,115,165,228,298,378,471]$ $155^{*}$

$[5,16,42,77,118,172,234,305,389,480]$ $158^{*}$

$[5,16,42,82,132,192,263,346,440,545]$ $156^{*}$,

$[5,16,42,82,132,199,278,360,454,571]$ $159^{*}$

$[5,16,42,83,133,194,266,349,444,550]$ $157^{*}$

$[5,16,43,89,144,210,292,387,495,615]$ $160^{*}$,

$[5,17,39,71,112,162,221,289,369,455]$ $161^{*}$,

[ 5, 17, 39, 72, 115, 168, 231, 303, 384, 476 ] $166^{*}$

$[5,17,40,76,129,197,277,369,470,585]$ $170^{*}$,

[ $5,17,40,76,129,198,279,368,469,587]$ $171^{*}$,

[ $5,17,41,73,114,168,229,298,381,471]$ $164^{*}$

$[5,17,41,74,114,165,228,298,378,471]$ $162^{*}, 163^{*}$,

$[5,17,41,74,116,170,232,303,387,478]$ $169^{*}$,

$[5,17,41,75,118,171,234,307,390,483]$ $165^{*}$ 
\begin{tabular}{clccc} 
Nbr. & gr & № & $H_{i}$ & $L$ \\
\hline & {$[5,17,41,76,124,184,253,333,424,525]$}
\end{tabular} m $\quad X$

$$
167^{*},
$$

$[5,17,41,77,127,191,266,352,450,560]$

$[5,17,42,78,124,179,244,320,406,503]$ $172^{*}$,

$[5,17,42,79,125,179,244,321,408,505]$ $173^{*}$

$[5,17,43,86,142,210,292,387,495,615]$ $180^{*}$,

$[5,17,44,82,131,194,268,352,448,556]$ $174^{*}$,

$[5,17,44,83,131,190,259,339,430,531]$ $175^{*}$,

$[5,17,44,84,134,196,270,356,454,564]$ $177^{*}, 178^{*}$

$[5,17,44,84,135,198,273,360,459,570]$ 44,84,
$179^{*}$,

$[5,17,44,84,139,206,282,370,470,582]$ $176^{*}$,

$[5,18,41,73,114,165,228,298,378,471]$ $181^{*}$

$[5,18,43,76,118,171,234,307,390,483]$ $182^{*}, 183^{*}$,

$[5,18,44,79,123,178,244,321,408,505]$ $184^{*}, 185^{*}$,

$[5,18,46,87,141,210,292,387,495,615]$ $186^{*}$

$[5,19,43,75,118,171,234,307,390,483]$ $187^{*}$,

$[5,19,44,78,123,178,244,321,408,505]$ $188^{*}$

$[5,19,45,80,127,185,256,340,433,536]$ $189^{*}$

$[6,18,37,63,99,142,189,249,317,384]$

$116 \quad H_{645}\left\langle m_{z} r_{x}^{-1}\right\rangle \quad r_{x}^{2} \quad r_{z}^{2} r_{x} t_{x}, r_{z}^{2} t_{x}^{-1}, m_{x} r_{x} t_{x}^{-1}, r_{x}^{-1} t_{y}^{-1}, m_{z} t_{z}$

$[6,18,38,66,102,146,198,258,326,402]$ $117 \quad H_{640}\left\langle m_{z} r_{x}^{-1}\right\rangle \quad r_{x}^{2} \quad r_{z}^{2} r_{x} t_{x}, r_{y}^{2} r_{x} t_{x}^{-1}, r_{z}^{2} r_{x} t_{x}^{-1}, r_{x}^{-1} t_{y}^{-1}, m_{z} t_{z}$

$[6,18,40,74,116,168,233,302,379,470]$ $118 \quad H_{571} \quad m_{z} r_{x} \quad m_{x} t_{x}, r_{y}^{2} r_{x} t_{x}^{-1}, m_{x} t_{x}^{-1}, r_{z}^{2} t_{y}^{-1}, m_{z} t_{z}$

$[6,18,40,74,117,168,229,299,378,467]$ $119 \quad H_{486} \quad 1$

$[6,18,42,78,116,166,236,302,370,468$ $120 \quad H_{639} \quad\left\langle m_{z} r_{x}^{-1}\right\rangle \quad r_{x}^{2} \quad r_{z}^{2} r_{x} t_{x}, r_{y}^{2} r_{x} t_{x}^{-1}, r_{z}^{2} r_{x} t_{x}^{-1}, m_{x} r_{x}^{-1} t_{y}^{-1}, r_{y}^{2} t_{z}$

$[6,19,40,69,108,154,208,273,344,423]$ $121 \quad H_{629}\left\langle m_{z} r_{x}^{-1}\right\rangle$

$6,19,41,71,110,158,214,279,353,435$

\begin{tabular}{|c|c|c|c|c|}
\hline 122 & $H_{461}$ & 1 & $r_{z}^{2}$ & $r_{z}^{2} t_{x}^{-1}, m_{y} t_{y}^{-1}, r_{y}^{2} t_{z}, r_{x}^{2} t_{z}, m_{z} t_{z}^{-1}$ \\
\hline 123 & $H_{482}$ & 1 & $r_{z}^{2}$ & $m_{x} t_{x}^{-1}, m_{y} t_{y}^{-1}, r_{y}^{2} t_{z}, r_{y}^{2} t_{z}^{-1}, r_{x}^{2} t_{z}^{-1}$ \\
\hline \multicolumn{5}{|c|}{$[6,19,41,72,112,160,217,283,358,442]$} \\
\hline 124 & $H_{468}$ & 1 & $r_{z}^{2}$ & $r_{z}^{2} t_{x}^{-1}, m_{y} t_{y}^{-1}, m_{z} t_{z}, i t_{z}^{-1}, m_{z} t_{z}^{-1}$ \\
\hline \multicolumn{5}{|c|}{$[6,19,41,72,112,161,219,285,360,444]$} \\
\hline 125 & $H_{530}$ & 1 & $r_{z}^{2}$ & $m_{x} t_{x}^{-1}, m_{y} t_{y}^{-1}, m_{z} r_{z}^{-1} t_{z}, m_{z} r_{z} t_{z}, r_{y}^{2} t_{z}^{-1}$ \\
\hline \multicolumn{5}{|c|}{$[6,19,42,72,111,161,217,283,359,441]$} \\
\hline 126 & $H_{483}$ & 1 & $r_{z}^{2}$ & $m_{x} t_{x}^{-1}, m_{y} t_{y}^{-1}, r_{y}^{2} t_{z}, i t_{z}^{-1}, m_{z} t_{z}^{-1}$ \\
\hline \multicolumn{5}{|c|}{$[6,19,42,73,113,164,222,289,366,450]$} \\
\hline 127 & $H_{529}$ & 1 & $r_{z}^{2}$ & $m_{x} t_{x}^{-1}, m_{y} t_{y}^{-1}, r_{y}^{2} r_{z} t_{z}, r_{x}^{2} r_{z} t_{z}, r_{y}^{2} t_{z}^{-1}$ \\
\hline \multicolumn{5}{|c|}{$[6,19,43,77,119,170,231,301,380,469]$} \\
\hline 128 & $H_{479}$ & 1 & $r_{z}^{2}$ & $r_{y}^{2} t_{x}^{-1}, m_{y} t_{y}^{-1}, r_{x}^{2} t_{z}, r_{y}^{2} t_{z}^{-1}, r_{x}^{2} t_{z}^{-1}$ \\
\hline \multicolumn{5}{|c|}{$[6,19,43,79,126,182,249,327,414,511]$} \\
\hline 129 & $H_{475}$ & 1 & $r_{z}^{2}$ & $i t_{x}^{-1}, m_{y} t_{y}^{-1}, m_{z} t_{z}, i t_{z}^{-1}, m_{z} t_{z}^{-1}$ \\
\hline \multicolumn{5}{|c|}{$[6,19,43,80,131,192,262,342,432,534]$} \\
\hline 130 & $H_{638}$ & $\left\langle m_{z} r_{x}^{-1}\right\rangle$ & $r_{x}^{2}$ & $r_{z}^{2} r_{x} t_{x}, r_{z}^{2} t_{x}^{-1}, m_{x} r_{x} t_{x}^{-1}, m_{z} r_{x} t_{y}^{-1}, r_{x}^{2} t_{z}$ \\
\hline \multicolumn{5}{|c|}{$[6,19,44,79,124,179,244,319,404,499]$} \\
\hline 131 & $H_{631}$ & $\left\langle m_{z} r_{x}^{-1}\right\rangle$ & $r_{x}^{2}$ & $r_{z}^{2} r_{x} t_{x}, r_{y}^{2} r_{x} t_{x}^{-1}, r_{z}^{2} r_{x} t_{x}^{-1}, r_{y}^{2} r_{x} t_{y}^{-1}, i t_{z}$ \\
\hline \multicolumn{5}{|c|}{$[6,19,44,84,141,212,299,398,507,629]$} \\
\hline 132 & $H_{541}$ & 1 & $r_{z}^{2}$ & $r_{z}^{2} r_{x} t_{x}, m_{y} t_{y}^{-1}, m_{z} t_{z}, i t_{z}^{-1}, m_{z} t_{z}^{-1}$ \\
\hline \multirow{2}{*}{\multicolumn{5}{|c|}{$\begin{array}{ccc}133 & H_{541} & 1 \\
{[6,19,45,77,121,172,235,305,387,476]}\end{array}$}} \\
\hline & & & & \\
\hline 134 & $H_{433}$ & 1 & $r_{z}^{2}$ & $i t_{x}^{-1}, r_{z}^{2} t_{y}^{-1}, m_{z} t_{z}, i t_{z}^{-1}, m_{z} t_{z}^{-1}$ \\
\hline \multicolumn{5}{|c|}{$[6,19,45,77,123,176,241,312,397,489]$} \\
\hline 135 & $H_{561}$ & 1 & $m_{z} r_{x}$ & $m_{x} t_{x}, r_{y}^{2} r_{x} t_{x}^{-1}, m_{x} t_{x}^{-1}, i t_{y}^{-1}, r_{x}^{2} t_{z}$ \\
\hline \multicolumn{5}{|c|}{$[6,19,45,79,126,177,243,318,403,493]$} \\
\hline 136 & $H_{466}$ & 1 & $r_{z}^{2}$ & $r_{z}^{2} t_{x}^{-1}, r_{x}^{2} t_{y}^{-1}, m_{z} t_{z}, i t_{z}^{-1}, m_{z} t_{z}^{-1}$ \\
\hline \multicolumn{5}{|c|}{$[6,19,47,82,130,189,263,343,445,545]$} \\
\hline 137 & $H_{539}$ & 1 & $r_{z}^{2}$ & $i t_{x}^{-1}, r_{x}^{2} t_{y}, m_{z} r_{z}^{-1} t_{z}, m_{z} r_{z} t_{z}, r_{y}^{2} r_{z} t_{z}^{-1}$ \\
\hline \multicolumn{5}{|c|}{$[6,19,47,82,131,186,257,334,425,522]$} \\
\hline 138 & $H_{473}$ & 1 & $r_{z}^{2}$ & $i t_{x}^{-1}, r_{x}^{2} t_{y}^{-1}, m_{z} t_{z}, i t_{z}^{-1}, m_{z} t_{z}^{-1}$ \\
\hline \multicolumn{5}{|c|}{$[6,19,50,100,159,226,312,410,520,642]$} \\
\hline 139 & $H_{511}$ & 1 & $r_{z}^{2}$ & $r_{z}^{2} r_{x} t_{x}, r_{x}^{2} t_{y}^{-1}, r_{y}^{2} t_{z}, r_{x}^{2} t_{z}, r_{y}^{2} t_{z}^{-1}$ \\
\hline
\end{tabular}




\begin{tabular}{|c|c|c|c|c|c|c|}
\hline Nbr. & $\mathrm{gr}$ & № & $H_{i}$ & $L$ & $m$ & $X$ \\
\hline & & 140 & $H_{479}$ & 1 & $r_{z}^{2}$ & $r_{y}^{2} t_{x}^{-1}, m_{y} t_{y}^{-1}, r_{y}^{2} t_{z}, r_{y}^{2} t_{z}^{-1}, r_{x}^{2} t_{z}^{-1}$ \\
\hline & {$[6$,} & $0,43,7$ & $7,167,22$ & $296,374,461]$ & & \\
\hline & & 141 & $H_{537}$ & 1 & $r_{z}^{2}$ & $r_{z}^{-1} t_{x}^{-1}, r_{z} t_{y}, m_{z} r_{z}^{-1} t_{z}, m_{z} r_{z} t_{z}, r_{y}^{2} t_{z}^{-1}$ \\
\hline & {$[6$,} & $\overline{0,43,7}$ & $9,170,23$ & $01,380,469]$ & & \\
\hline & & 142 & $H_{524}$ & 1 & $r_{z}^{2}$ & $m_{z} r_{z}^{-1} t_{x}^{-1}, m_{z} r_{z} t_{y}, r_{y}^{2} t_{z}, r_{y}^{2} t_{z}^{-1}, r_{x}^{2} t_{z}^{-1}$ \\
\hline & & 143 & $H_{509}$ & 1 & $r_{z}^{2}$ & $r_{z}^{-1} t_{x}^{-1}, r_{z} t_{y}, r_{y}^{2} t_{z}, r_{y}^{2} t_{z}^{-1}, r_{x}^{2} t_{z}^{-1}$ \\
\hline & {$[6$,} & $0,44,7$ & $8,168,22$ & $298,377,465]$ & & \\
\hline & & 144 & $H_{469}$ & 1 & $r_{z}^{2}$ & $r_{z}^{2} t_{x}^{-1}, m_{y} t_{y}^{-1}, r_{x}^{2} t_{z}, r_{y}^{2} t_{z}^{-1}, r_{x}^{2} t_{z}^{-1}$ \\
\hline & {$[6$,} & $0,44,7$ & $8,170,23$ & $300,380,468]$ & & \\
\hline & & 145 & $H_{461}$ & 1 & $r_{z}^{2}$ & $r_{z}^{2} t_{x}^{-1}, m_{y} t_{y}^{-1}, r_{x}^{2} t_{z}, i t_{z}^{-1}, m_{z} t_{z}^{-1}$ \\
\hline & {$[6$,} & $\begin{array}{c}0,44,7 \\
146\end{array}$ & $\begin{array}{l}0,174,23 \\
H_{512}\end{array}$ & $\begin{array}{l}307,386,477] \\
1\end{array}$ & $r_{z}^{2}$ & $r_{z}^{-1} t_{x}^{-1}, r_{z} t_{y}, r_{y}^{2} r_{z} t_{z}, r_{x}^{2} r_{z} t_{z}, r_{y}^{2} t_{z}^{-1}$ \\
\hline & {$[6$,} & $0,44,7$ & $9,170,23$ & $301,380,469]$ & & \\
\hline & & 147 & $H_{429}$ & 1 & $r_{z}^{2}$ & $r_{y}^{2} t_{x}^{-1}, r_{x}^{2} t_{y}^{-1}, r_{y}^{2} t_{z}, r_{y}^{2} t_{z}^{-1}, r_{x}^{2} t_{z}^{-1}$ \\
\hline & {$[6$,} & $0,44,7$ & $1,175,24$ & $313,392,487]$ & & \\
\hline & & 148 & $H_{535}$ & 1 & $r_{z}^{2}$ & $r_{z}^{-1} t_{x}^{-1}, r_{z} t_{y}, r_{y}^{2} t_{z}, i t_{z}^{-1}, m_{z} t_{z}^{-1}$ \\
\hline & {$[6$,} & $0,45,8$ & $4,197,27$ & {$[59,456,563]$} & & \\
\hline & & 149 & $H_{460}$ & 1 & $r_{z}^{2}$ & $r_{z}^{2} t_{x}^{-1}, r_{z}^{2} t_{y}^{-1}, r_{y}^{2} t_{z}, i t_{z}^{-1}, m_{z} t_{z}^{-1}$ \\
\hline & & $0,45,8$ & $4,201,27$ & $666,463,572]$ & & \\
\hline & & 150 & $H_{503}$ & 1 & $r_{z}^{2}$ & $r_{z}^{2} t_{x}^{-1}, r_{z}^{2} t_{y}^{-1}, r_{y}^{2} r_{z} t_{z}, r_{x}^{2} r_{z} t_{z}, r_{y}^{2} t_{z}^{-1}$ \\
\hline & {$[6$,} & $\overline{0,46,8}$ & $4,176,23$ & $13,395,488]$ & & \\
\hline & & $\frac{151}{0.46,8}$ & $H_{645}$ & $\left\langle m_{z} r_{x}^{-1}\right\rangle$ & $r_{x}^{2}$ & $r_{y}^{2} r_{x} t_{x}, r_{z}^{2} t_{x}^{-1}, m_{x} r_{x} t_{x}^{-1}, r_{x}^{-1} t_{y}^{-1}, m_{z} t_{z}$ \\
\hline & & 152 & $H_{469}$ & 1 & $r_{z}^{2}$ & $r_{z}^{2} t_{x}^{-1}, m_{y} t_{y}^{-1}, r_{y}^{2} t_{z}, r_{y}^{2} t_{z}^{-1}, r_{x}^{2} t_{z}^{-1}$ \\
\hline & {$[6$,} & $0,46,8$ & $5,179,24$ & $18,402,497]$ & & \\
\hline & & 153 & $H_{461}$ & 1 & $r_{z}^{2}$ & $r_{z}^{2} t_{x}^{-1}, m_{y} t_{y}^{-1}, r_{y}^{2} t_{z}, i t_{z}^{-1}, m_{z} t_{z}^{-1}$ \\
\hline & & $0,46,8$ & $0,186,25$ & $31,419,517]$ & & \\
\hline & & 154 & $H_{485}$ & 1 & $r_{z}^{2}$ & $i t_{x}^{-1}, m_{y} t_{y}^{-1}, r_{x}^{2} t_{z}, r_{y}^{2} t_{z}^{-1}, r_{x}^{2} t_{z}^{-1}$ \\
\hline & & $0,47,8$ & $3,178,24$ & $13,398,493]$ & & \\
\hline & & 155 & $H_{640}$ & $\left\langle m_{z} r_{x}^{-1}\right\rangle$ & $r_{x}^{2}$ & $r_{y}^{2} r_{x} t_{x}, r_{y}^{2} r_{x} t_{x}^{-1}, r_{z}^{2} r_{x} t_{x}^{-1}, r_{x}^{-1} t_{y}^{-1}, m_{z} t_{z}$ \\
\hline & & $\overline{0,48,8}$ & $3,204,27$ & $\begin{array}{l}363,460,567] \\
1\end{array}$ & $r^{2}$ & $r_{z}^{2} t_{x}^{-1}, r_{z}^{2} t_{u}^{-1}, r_{u}^{2} t_{z}, r_{u}^{2} t_{z}^{-1}, r_{x}^{2} t_{z}^{-1}$ \\
\hline & & $\overline{0,48,8}$ & $3,207,28$ & $67,463,572]$ & & \\
\hline & & 157 & $H_{519}$ & 1 & $r_{z}^{2}$ & $r_{z}^{2} t_{x}^{-1}, r_{z}^{2} t_{y}^{-1}, m_{z} r_{z}^{-1} t_{z}, m_{z} r_{z} t_{z}, r_{y}^{2} t_{z}^{-1}$ \\
\hline & & $\overline{0,50,8}$ & $1,188,25$ & $32,421,518]$ & & \\
\hline & & 158 & $H_{427}$ & 1 & $r_{z}^{2}$ & $r_{z}^{2} t_{x}^{-1}, r_{x}^{2} t_{y}^{-1}, r_{y}^{2} t_{z}, r_{y}^{2} t_{z}^{-1}, r_{x}^{2} t_{z}^{-1}$ \\
\hline & & $0,50,9$ & $7,216,29$ & $879,483,602]$ & & \\
\hline & & 159 & $H_{462}$ & 1 & $r_{z}^{2}$ & $i t_{x}^{-1}, r_{z}^{2} t_{y}^{-1}, r_{x}^{2} t_{z}, r_{y}^{2} t_{z}^{-1}, r_{x}^{2} t_{z}^{-1}$ \\
\hline & & $\overline{0,54,1}$ & $55,226,3$ & $410,520,642]$ & & \\
\hline & & 160 & $H_{541}$ & 1 & $r_{z}^{2}$ & $r_{z}^{2} r_{x} t_{x}, m_{y} t_{y}^{-1}, i t_{z}, m_{z} t_{z}, i t_{z}^{-1}$ \\
\hline & {$[6$,} & $1,45,8$ & $6,178,24$ & $19,401,495]$ & $n^{2}$ & $r^{2}-1 r^{2}+1-1 r^{2}+r^{2}-1 r^{2}-1$ \\
\hline & & $\frac{161}{1,47,8}$ & $\frac{H_{427}}{3,178,24}$ & 1 & $r_{z}^{2}$ & $r_{z}^{t} t_{x}, r_{x}^{t} y, r_{x}^{t} t_{z}, r_{y}^{t} z^{t}, r_{x}^{t} z$ \\
\hline & & 162 & $H_{486}$ & 1 & $r_{z}^{2}$ & $r_{y}^{2} t_{x}^{-1}, m_{y} t_{y}^{-1}, i t_{z}, i t_{z}^{-1}, m_{z} t_{z}^{-1}$ \\
\hline & & 163 & $H_{571}$ & 1 & $m_{z} r_{x}$ & $r_{y}^{2} r_{x} t_{x}, r_{y}^{2} r_{x} t_{x}^{-1}, m_{x} t_{x}^{-1}, r_{z}^{2} t_{y}^{-1}, m_{z} t_{z}$ \\
\hline & & $1,47,8$ & $8,184,24$ & $325,412,507]$ & & \\
\hline & & & $H_{485}$ & 1 & $r_{z}^{2}$ & $i t_{x}^{-1}, m_{y} t_{y}^{-1}, r_{y}^{2} t_{z}, r_{y}^{2} t_{z}^{-1}, r_{x}^{2} t_{z}^{-1}$ \\
\hline & & $1,47,8$ & $7,182,24$ & $22,407,502]$ & 2 & $r^{2} r r^{2} r t^{-1} r^{2} r t^{-1} m r^{-1} r^{2} t$ \\
\hline & & $\begin{array}{l}1,47,8 \\
166\end{array}$ & $\begin{array}{c}1,187,25 \\
H_{474}\end{array}$ & $\begin{array}{c}331,420,517] \\
1\end{array}$ & $r_{z}^{2}$ & $i t_{x}^{-1}, r_{x}^{2} t_{y}^{-1}, r_{x}^{2} t_{z}, r_{y}^{2} t_{z}^{-1}, r_{x}^{2} t_{z}^{-1}$ \\
\hline & & $1,47,8$ & $8,201,28$ & $367,468,576]$ & & \\
\hline & & 167 & $H_{533}$ & 1 & $r_{z}^{2}$ & $m_{x} r_{z} t_{x}^{-1}, m_{x} r_{z} t_{y}^{-1}, r_{y}^{2} r_{z} t_{z}, r_{x}^{2} r_{z} t_{z}, r_{y}^{2} t_{z}^{-1}$ \\
\hline & {$[6$,} & $1,47,8$ & $0,206,28$ & $877,484,596]$ & & \\
\hline & & 168 & $H_{531}$ & 1 & $r_{z}^{2}$ & $m_{x} r_{z} t_{x}^{-1}, m_{x} r_{z} t_{y}^{-1}, r_{y}^{2} t_{z}, i t_{z}^{-1}, m_{z} t_{z}^{-1}$ \\
\hline & & $1,48,8$ & $1,188,25$ & $32,421,518]$ & & $i t^{-1}, r^{2} t^{-1}, r^{2} t z, r^{2} t^{-1}, r^{2} t^{-1}$ \\
\hline & & 169 & $H_{474}$ & 1 & $r_{z}^{2}$ & \\
\hline & {$[6$,} & $1,48,8$ & $0,222,30$ & $97,506,628]$ & $r^{2}$ & $r_{z}^{2} r_{x} t_{x}, r_{x}^{2} t_{y}, r_{x}^{2} t_{z}, r_{z}^{2} t_{\gamma}^{-1}, r_{x}^{2} t_{\alpha}^{-1}$ \\
\hline & & $1,48,9$ & $\frac{511}{9,220,30}$ & $997,508,628]$ & & \\
\hline & & 171 & $H_{511}$ & 1 & $r_{z}^{2}$ & $r_{z}^{2} r_{x} t_{x}, r_{x}^{2} t_{y}^{-1}, r_{x}^{2} t_{z}, r_{y}^{2} t_{z}^{-1}, r_{x}^{2} t_{z}^{-1}$ \\
\hline & {$[6$,} & $1,49,8$ & $4,190,25$ & $36,426,525]$ & & \\
\hline & & 172 & $H_{461}$ & 1 & $r_{z}^{2}$ & $r_{z}^{2} t_{x}^{-1}, m_{y} t_{y}^{-1}, r_{y}^{2} t_{z}, r_{x}^{2} t_{z}, i t_{z}^{-1}$ \\
\hline & & $1,49,8$ & $4,190,25$ & $38,427,526]$ & & \\
\hline & & 173 & $H_{468}$ & 1 & $r_{z}^{2}$ & $r_{z}^{2} t_{x}^{-1}, m_{y} t_{y}^{-1}, i t_{z}, i t_{z}^{-1}, m_{z} t_{z}^{-1}$ \\
\hline & {$[6$,} & $1,49,8$ & $3,205,28$ & $668,468,576]$ & & \\
\hline & & 174 & $H_{462}$ & 1 & $r_{z}^{2}$ & $i t_{x}^{-1}, r_{z}^{2} t_{y}^{-1}, r_{y}^{2} t_{z}, r_{y}^{2} t_{z}^{-1}, r_{x}^{2} t_{z}^{-1}$ \\
\hline & & $1,50,8$ & $6,208,28$ & $770,469,577]$ & & \\
\hline & & 175 & $H_{526}$ & 1 & $r_{z}^{2}$ & $m_{x} r_{z} t_{x}^{-1}, m_{x} r_{z} t_{y}^{-1}, m_{z} r_{z}^{-1} t_{z}, m_{z} r_{z} t_{z}, r_{y}^{2} t_{z}^{-1}$ \\
\hline & {$[6$,} & $\begin{array}{l}1,50,8 \\
176\end{array}$ & $\begin{array}{c}7,212,29 \\
H_{638}\end{array}$ & $\begin{array}{l}384,488,600] \\
\left\langle m_{z} r_{x}^{-1}\right\rangle\end{array}$ & $r_{x}^{2}$ & $r_{y}^{2} r_{x} t_{x}, r_{z}^{2} t_{x}^{-1}, m_{x} r_{x} t_{x}^{-1}, m_{z} r_{x} t_{y}^{-1}, r_{x}^{2} t_{z}$ \\
\hline & {$[6$,} & $1,50,9$ & $8,212,29$ & $\frac{x}{80,484,596]}$ & & \\
\hline & & 177 & $H_{507}$ & 1 & $r_{z}^{2}$ & $r_{y}^{2} r_{z} t_{x}^{-1}, r_{y}^{2} r_{z} t_{y}^{-1}, r_{y}^{2} t_{z}, r_{y}^{2} t_{z}^{-1}, r_{x}^{2} t_{z}^{-1}$ \\
\hline & & 178 & $H_{522}$ & 1 & $r_{z}^{2}$ & $m_{x} r_{z} t_{x}^{-1}, m_{x} r_{z} t_{y}^{-1}, r_{y}^{2} t_{z}, r_{y}^{2} t_{z}^{-1}, r_{x}^{2} t_{z}^{-1}$ \\
\hline
\end{tabular}




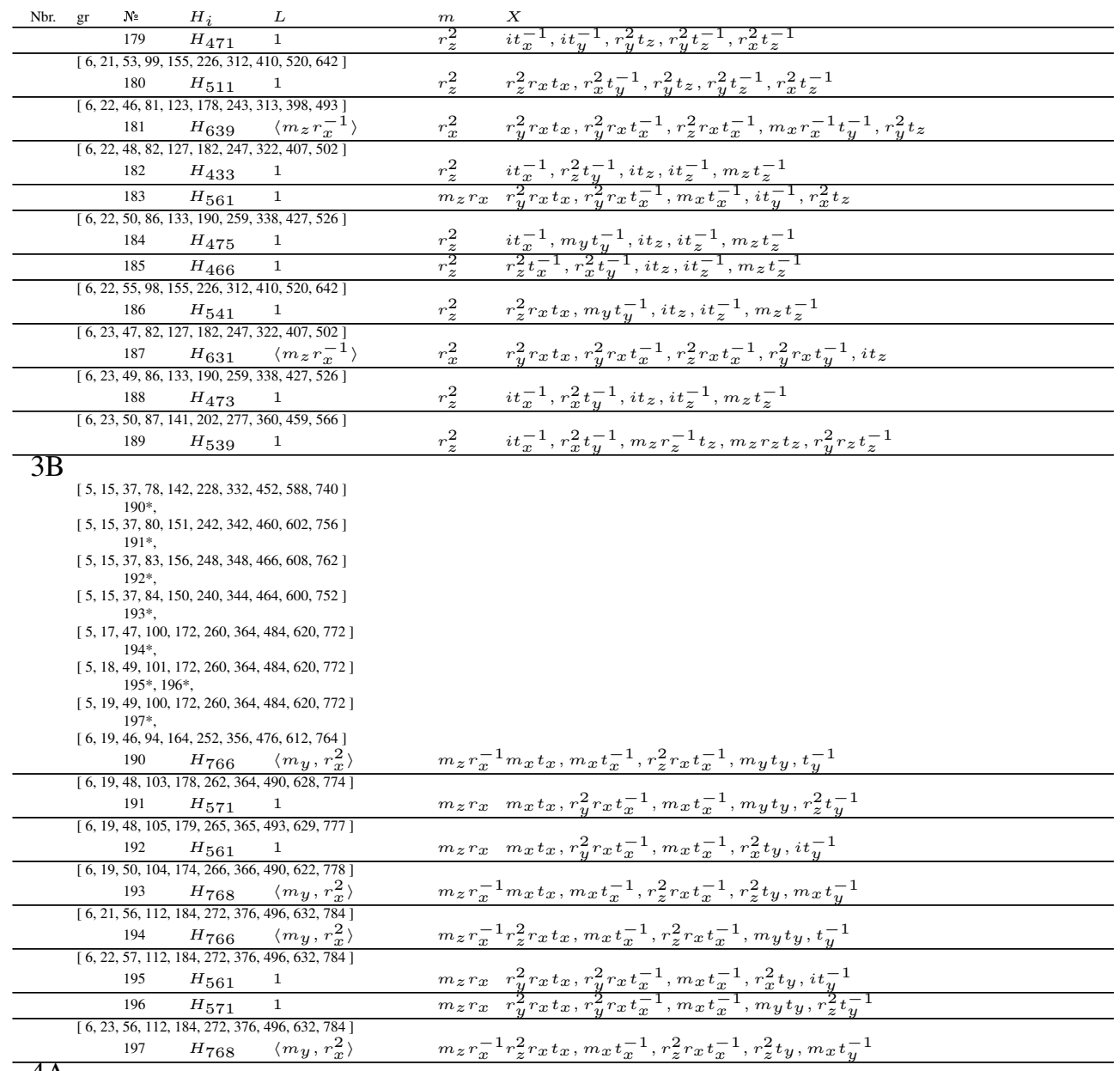

$4 \mathrm{~A}$

$[5,13,26,45,69,98,133,173,218,269]$ $198^{*}, 199^{*}$,

$[5,13,26,46,75,112,157,213,275,343]$ $200^{*}$,

$[5,13,26,47,81,126,176,236,309,396]$ $201 *$

$[5,14,29,50,77,110,149,194,245,302]$ $202^{*}, 206^{*}, 203^{*}, 207^{*}, 204^{*}, 205^{*}$,

$[5,14,31,57,88,125,173,228,287,355]$ $208^{*}$,

$[5,14,32,59,94,139,193,256,327,406]$ $209^{*}, 210^{*}$,

$[5,14,32,59,94,139,195,262,335,414]$ $212^{*}, 211^{*}$

$[5,14,32,68,117,178,256,346,449,564]$ $213^{*}$,

$[5,15,32,55,85,122,165,215,272,335]$ $214^{*}, 215^{*}$

$[5,15,33,58,89,127,173,226,285,351]$ $216^{*}$

$[5,15,33,59,94,138,190,250,318,394]$ $217^{*}$,

[ $5,15,34,61,96,141,193,253,324,401]$ $219^{*}$,

$[5,15,35,64,99,141,192,251,318,393]$ $218^{*}$,

$[5,15,35,65,103,152,212,279,357,447]$ $220^{*}$,

$[5,15,37,70,112,166,228,299,383,474]$ $221^{*}$

$[5,15,37,71,113,165,227,299,381,473]$ $222 *$

$[5,15,37,76,130,193,264,353,458,566]$ 


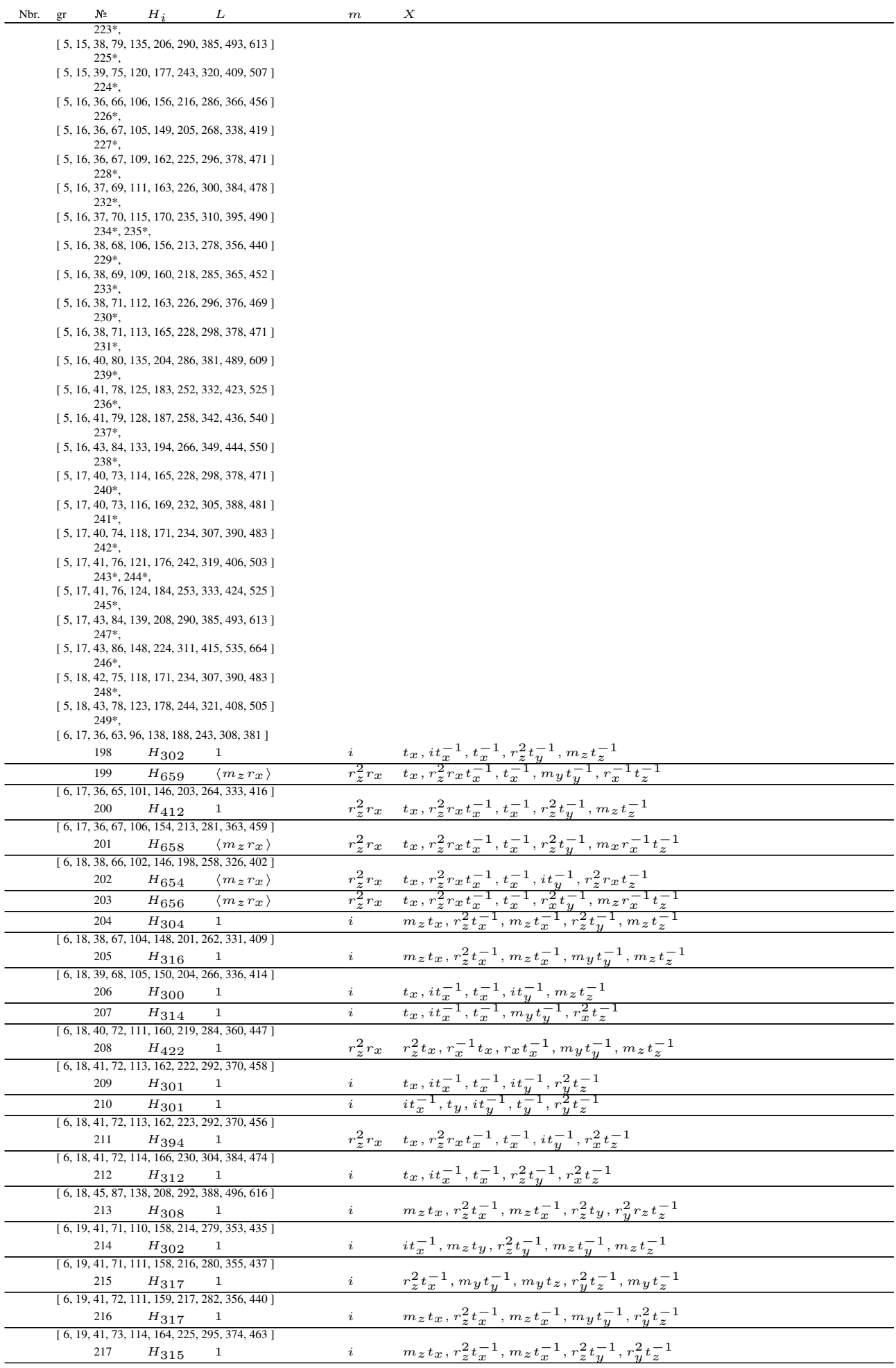




\begin{tabular}{|c|c|c|c|c|c|}
\hline Nbr. & $\begin{array}{ll}\text { gr } \quad \text { № } \\
6,19,42,7\end{array}$ & $\frac{H_{i}}{16,166,22}$ & $\frac{L}{93,372,458]}$ & $m$ & $x$ \\
\hline & 218 & $H_{423}$ & 1 & $r_{z}^{2} r_{x}$ & $r_{z}^{2} t_{x}, r_{x}^{-1} t_{x}, r_{x} t_{x}^{-1}, m_{x} r_{x} t_{y}^{-1}, m_{x} r_{x}^{-1} t_{z}^{-1}$ \\
\hline & {$[6,19,43,7$} & $8,170,23$ & $02,383,472]$ & & $r^{2} t^{-1}, m_{u} t^{-1}, m_{r} t_{z}, m_{r} t^{-1}, r^{2} t_{\gamma}^{-1}$ \\
\hline & $\frac{219}{[6,19,43,7}$ & $\frac{H_{318}}{17,171,23}$ & 1 & $i$ & \\
\hline & $\begin{array}{r}{[6,19,43,7} \\
220\end{array}$ & $\begin{array}{c}17,171,23 \\
H_{661}\end{array}$ & $03,387,480]$ & $r_{\gamma}^{2} r_{x}$ & $m_{z} r_{x}^{-1} t_{x}, m_{x} t_{x}^{-1}, m_{z} r_{x}^{-1} t_{x}^{-1}, m_{y} t_{u}^{-1}, r_{x}^{-1} t_{z}^{-1}$ \\
\hline & {$[6,19,45,7$} & & $\frac{\left\langle m_{z} x^{x}\right\rangle}{27,416.513]}$ & & \\
\hline & 221 & $H_{315}$ & 1 & $i$ & $r_{z}^{2} t_{x}^{-1}, m_{z} t_{y}, r_{z}^{2} t_{y}^{-1}, m_{z} t_{y}^{-1}, r_{y}^{2} t_{z}^{-1}$ \\
\hline & {$[6,19,45,8$} & $34,193,26$ & $45,438,541]$ & & \\
\hline & 222 & $H_{315}$ & 1 & $i$ & $r_{z}^{2} t_{x}^{-1}, r_{z}^{2} t_{y}^{-1}, m_{y} t_{z}, r_{y}^{2} t_{z}^{-1}, m_{y} t_{z}^{-1}$ \\
\hline & {$[6,19,45,8$} & $+3,206,28$ & $75,480,590]$ & & \\
\hline & 223 & $H_{311}$ & 1 & $i$ & $i t_{x}^{-1}, r_{z}^{2} t_{y}^{-1}, m_{y} t_{z}, r_{y}^{2} t_{z}^{-1}, m_{y} t_{z}^{-1}$ \\
\hline & {$[6,19,47,8$} & 10,201,27 & $60,458,564]$ & & \\
\hline & 224 & $H_{409}$ & 1 & $r_{z}^{2} r_{x}$ & $r_{z}^{2} t_{x}, r_{x}^{-1} t_{x}, r_{x} t_{x}^{-1}, r_{x}^{2} t_{y}^{-1}, r_{x}^{2} t_{z}^{-1}$ \\
\hline & {$[6,19,50,9$} & $2,225,31$ & $09,519,641]$ & & $n^{2} r^{\prime}-1-1-1 r^{2} t-m+r^{2}+1$ \\
\hline & $\frac{225}{[6,20,43,7}$ & $\frac{H_{326}}{20,174,23}$ & $\frac{1}{11,394,488]}$ & ¿ & $r_{z}^{2} r_{x} t_{x}^{-1}, m_{y} t_{y}^{-1}, r_{z}^{2} t_{z}, m_{z} t_{z}, r_{z}^{2} t_{z}^{-1}$ \\
\hline & 226 & $H_{657}$ & $\left\langle m_{z} r_{x}\right\rangle$ & $r_{z}^{2} r_{x}$ & $m_{z} r_{x}^{-1} t_{x}, m_{x} t_{x}^{-1}, m_{z} r_{x}^{-1} t_{x}^{-1}, r_{x}^{2} t_{y}^{-1}, m_{z} r_{x}^{-1} t_{z}^{-1}$ \\
\hline & {$[6,20,44,7$} & $23,174,23$ & $11,392,485]$ & & \\
\hline & 227 & $H_{319}$ & 1 & $i$ & $r_{z}^{2} t_{x}^{-1}, m_{x} t_{y}, m_{x} t_{y}^{-1}, r_{x}^{2} t_{y}^{-1}, r_{y}^{2} t_{z}^{-1}$ \\
\hline & {$[6,20,44,8$} & $26,180,24$ & $26,414,512]$ & & \\
\hline & 228 & $H_{312}$ & 1 & $i$ & $i t_{x}^{-1}, m_{z} t_{y}, r_{z}^{2} t_{y}^{-1}, m_{z} t_{y}^{-1}, r_{x}^{2} t_{z}^{-1}$ \\
\hline & {$[6,20,45,7$} & $20,173,23$ & $05,387,476]$ & & \\
\hline & 229 & $H_{303}$ & 1 & $i$ & $i t_{x}^{-1}, m_{x} t_{y}, m_{x} t_{y}^{-1}, r_{x}^{2} t_{y}^{-1}, r_{x}^{2} t_{z}^{-1}$ \\
\hline & {$[6,20,45,8$} & $22,177,24$ & $12,397,492]$ & & \\
\hline & 230 & $H_{316}$ & 1 & $i$ & $r_{z}^{2} t_{x}^{-1}, r_{y}^{2} t_{y}, r_{y}^{2} t_{y}^{-1}, m_{y} t_{y}^{-1}, m_{z} t_{z}^{-1}$ \\
\hline & {$[6,20,45,8$} & $23,178,24$ & $13,398,493]$ & & \\
\hline & 231 & $H_{413}$ & 1 & $r_{z}^{2} r_{x}$ & $m_{z} r_{x}^{-1} t_{x}, m_{x} t_{x}^{-1}, m_{z} r_{x}^{-1} t_{x}^{-1}, r_{z}^{2} t_{y}^{-1}, m_{z} t_{z}^{-1}$ \\
\hline & {$[6,20,45,8$} & $26,182,24$ & $27,414,512]$ & & \\
\hline & 232 & $H_{316}$ & 1 & $i$ & $r_{z}^{2} t_{x}^{-1}, m_{y} t_{y}^{-1}, r_{z}^{2} t_{z}, r_{z}^{2} t_{z}^{-1}, m_{z} t_{z}^{-1}$ \\
\hline & {$[6,20,46,8$} & $25,179,24$ & $17,402,495]$ & & \\
\hline & 233 & $H_{314}$ & 1 & $i$ & $i t_{x}^{-1}, m_{y} t_{y}^{-1}, m_{x} t_{z}, m_{x} t_{z}^{-1}, r_{x}^{2} t_{z}^{-1}$ \\
\hline & {$[6,20,46,8$} & $34,197,27$ & $58,456,565]$ & & \\
\hline & 234 & $H_{305}$ & 1 & $i$ & $t_{x}, i t_{x}^{-1}, t_{x}^{-1}, i t_{y}^{-1}, r_{y}^{2} r_{z} t_{z}^{-1}$ \\
\hline & {$[6,20,47,8$} & $37,201,27$ & $66,466,577]$ & & \\
\hline & 235 & $H_{305}$ & 1 & $i$ & $t_{x}, i t_{x}^{-1}, t_{x}^{-1}, i t_{y}, r_{y}^{2} r_{z} t_{z}^{-1}$ \\
\hline & {$[6,20,48,8$} & $38,197,27$ & $51,446,549]$ & & \\
\hline & 236 & $H_{301}$ & 1 & $i$ & $i t_{x}^{-1}, i t_{y}^{-1}, m_{y} t_{z}, r_{y}^{2} t_{z}^{-1}, m_{y} t_{z}^{-1}$ \\
\hline & $\begin{array}{r}{[6,20,48,8} \\
237\end{array}$ & $\begin{array}{c}13,203,28 \\
H_{312}\end{array}$ & $\begin{array}{l}65,463,569] \\
1\end{array}$ & $i$ & $i t_{x}^{-1}, r_{z}^{2} t_{y}^{-1}, m_{x} t_{z}, m_{x} t_{z}^{-1}, r_{x}^{2} t_{z}^{-1}$ \\
\hline & {$[6,20,49,8$} & $13,207,28$ & $67,463,572]$ & & \\
\hline & 238 & $H_{406}$ & 1 & $r_{z}^{2} r_{x}$ & $r_{z}^{2} t_{x}, r_{x}^{-1} t_{x}, r_{x} t_{x}^{-1}, r_{z}^{2} r_{x} t_{y}^{-1}, r_{z}^{2} r_{x} t_{z}^{-1}$ \\
\hline & {$[6,20,50,9$} & $0,221,30$ & $05,515,637]$ & & \\
\hline & 239 & $H_{308}$ & 1 & $i$ & $m_{z} t_{x}, r_{z}^{2} t_{x}^{-1}, m_{z} t_{x}^{-1}, r_{z}^{2} t_{y}^{-1}, r_{y}^{2} r_{z} t_{z}^{-1}$ \\
\hline & {$[6,21,46,8$} & $23,178,24$ & $13,398,493]$ & & \\
\hline & 240 & $H_{660}$ & $\left\langle m_{z} r_{x}\right\rangle$ & $r_{z}^{2} r_{x}$ & $m_{z} r_{x}^{-1} t_{x}, m_{x} t_{x}^{-1}, m_{z} r_{x}^{-1} t_{x}^{-1}, r_{z}^{2} t_{y}^{-1}, m_{x} r_{x}^{-1} t_{z}^{-1}$ \\
\hline & {$[6,21,46,8$} & $26,181,24$ & $21,406,501]$ & & \\
\hline & 241 & $H_{302}$ & 1 & $i$ & $i t_{x}^{-1}, r_{z}^{2} t_{y}^{-1}, r_{z}^{2} t_{z}, r_{z}^{2} t_{z}^{-1}, m_{z} t_{z}^{-1}$ \\
\hline & $\begin{array}{r}{[6,21,46,8} \\
242\end{array}$ & $\begin{array}{c}27,182,24 \\
H_{395}\end{array}$ & $\begin{array}{l}22,407,502] \\
1\end{array}$ & $r_{z}^{2} r_{x}$ & $m_{z} r_{r}^{-1} t_{x}, m_{x} t_{x}^{-1}, m_{z} r_{x}^{-1} t_{x}^{-1}, i t_{u}^{-1}, r_{x}^{2} t_{z}^{-1}$ \\
\hline & $\frac{242}{[6,21,48,8}$ & $\frac{11395}{32,189,25}$ & $\frac{1}{37,426,525]}$ & & 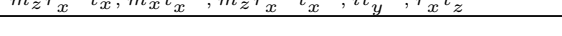 \\
\hline & 243 & $H_{313}$ & 1 & $i$ & $i t_{x}^{-1}, m_{y} t_{y}^{-1}, r_{z}^{2} t_{z}, r_{z}^{2} t_{z}^{-1}, m_{z} t_{z}^{-1}$ \\
\hline & 244 & $H_{317}$ & 1 & $i$ & $r_{z}^{2} t_{x}^{-1}, r_{y}^{2} t_{y}, r_{y}^{2} t_{y}^{-1}, m_{y} t_{y}^{-1}, r_{y}^{2} t_{z}^{-1}$ \\
\hline & {$[6,21,50,9$} & $46,213,29$ & $84,488,603]$ & & \\
\hline & 245 & $H_{323}$ & 1 & $i$ & $r_{z}^{2} t_{x}^{-1}, r_{x}^{2} r_{y} t_{y}^{-1}, m_{x} t_{z}, r_{x}^{2} t_{z}, m_{x} t_{z}^{-1}$ \\
\hline & {$[6,21,51,9$} & $7,232,32$ & $28,549,680]$ & & \\
\hline & 246 & $H_{323}$ & 1 & $i$ & $r_{z}^{2} t_{x}^{-1}, r_{x}^{2} r_{y} t_{y}^{-1}, m_{x} t_{z}, m_{x} t_{z}^{-1}, r_{x}^{2} t_{z}^{-1}$ \\
\hline & $\begin{array}{r}{[6,21,53,9} \\
247\end{array}$ & $\begin{array}{r}4,225,31 \\
H_{326}\end{array}$ & $\begin{array}{l}09,519,641] \\
1\end{array}$ & $i$ & $r_{z}^{2} r_{x} t_{x}^{-1}, m_{y} t_{u}^{-1}, r_{z}^{2} t_{z}, r_{z}^{2} t_{z}^{-1}, m_{z} t_{z}^{-1}$ \\
\hline & {$[6,22,47,8$} & $\frac{326}{27,182,24}$ & $22,407,502]$ & & \\
\hline & 248 & $H_{655}$ & $\left\langle m_{z} r_{x}\right\rangle$ & $r_{z}^{2} r_{x}$ & $m_{z} r_{x}^{-1} t_{x}, m_{x} t_{x}^{-1}, m_{z} r_{x}^{-1} t_{x}^{-1}, i t_{y}^{-1}, r_{z}^{2} r_{x} t_{z}^{-1}$ \\
\hline & {$[6,22,49,8$} & $3,190,25$ & $38,427,526]$ & & \\
\hline & 249 & $H_{314}$ & 1 & $i$ & $i t_{x}^{-1}, r_{y}^{2} t_{y}, r_{y}^{2} t_{y}^{-1}, m_{y} t_{y}^{-1}, r_{x}^{2} t_{z}^{-1}$ \\
\hline
\end{tabular}

$[5,14,32,64,112,176,256,352,464,592]$ $250^{*}$,

$[5,14,32,65,120,201,300,409,537,692]$ $251^{*}$,

[ $5,14,32,68,124,208,312,424,555,711]$ $252^{*}$,

$[5,14,32,68,130,210,310,430,566,718]$

$253^{*}$,
$[5,16,42,88,152,232,328,440,568,712]$ 254*,

$[5,17,46,98,171,260,364,484,620,772]$

$$
255^{*},
$$

$[5,17,46,98,172,261,364,484,620,772]$ $256^{*}$, 


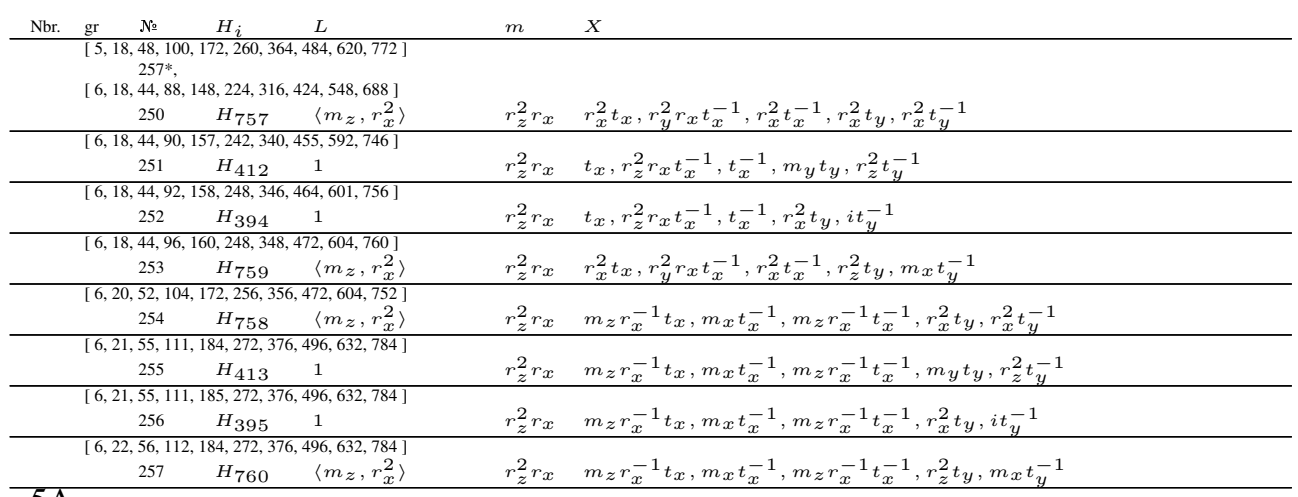

$5 \mathrm{~A}$

$[6,18,38,66,102,146,198,258,326,402]$ $258^{*}$

$[6,18,39,71,114,165,224,294,375,467]$ $259^{*}$,

$[6,18,40,73,114,165,228,298,378,471]$ $260^{*}$

$[6,19,42,75,118,171,234,307,390,483]$ $261^{*}$,

$[6,19,43,76,118,172,234,305,389,480]$ $262^{*}$,

$[6,19,43,77,122,178,244,320,406,503]$ $263^{*}$

$[6,19,43,78,123,178,244,321,408,505]$ $264^{*}$,

$[6,19,44,80,127,185,256,340,433,536]$ $265^{*}$,

$[6,19,45,84,139,206,282,370,470,582]$ $266^{*}$

$[6,19,45,86,141,210,292,387,495,615]$ $268^{*}, 269^{*}, 270^{*}, 271^{*}$,

$[6,19,48,90,142,206,282,370,470,582]$ $267^{*}$,

[6, 21, 51, 95, 153, 224, 308, 406, 516, 638 ] $273^{*}$,

$[6,21,51,95,154,227,313,413,526,652]$ $272^{*}$,

$[6,21,51,95,155,230,318,420,535,663]$ $274^{*}$,

$[6,21,51,95,156,233,323,427,545,677]$ $275^{*}$,

$[7,21,44,77,119,170,231,301,380,469]$ $258 \quad H_{748} \quad\left\langle m_{z} r_{x}^{-1}, r_{z}^{2} r_{x}\right\rangle \quad i \quad r_{z}^{2} t_{x}, m_{x} r_{x} t_{x}, m_{y} t_{x}^{-1}, r_{x} t_{x}^{-1}, m_{x} r_{x}^{-1} t_{y}^{-1}, r_{y}^{2} t_{z}$

$[7,21,45,80,124,176,239,313,395,488]$ $259 \quad H_{645}\left\langle m_{z} r_{x}^{-1}\right\rangle \quad r_{x}^{2} \quad r_{y}^{2} r_{x} t_{x}, r_{z}^{2} r_{x} t_{x}, r_{z}^{2} t_{x}^{-1}, m_{x} r_{x} t_{x}^{-1}, r_{x}^{-1} t_{y}^{-1}, m_{z} t_{z}$

$[7,21,46,81,123,178,243,313,398,493]$ $260 \quad H_{747}\left\langle m_{z} r_{x}^{-1}, r_{z}^{2} r_{x}\right\rangle \quad i \quad r_{y}^{2} r_{x} t_{x}, r_{z}^{2} r_{x} t_{x}, m_{z} r_{x} t_{x}^{-1}, r_{z}^{2} r_{x} t_{x}^{-1}, m_{x} r_{x}^{-1} t_{y}^{-1}, r_{y}^{2} t_{z}$

$\left[\begin{array}{c}{[7,22,47,82,127,182,247,322,407,502]} \\ 261\end{array} H_{745}\left\langle m_{z} r_{x}^{-1}, r_{z}^{2} r_{x}\right\rangle \quad i \quad r_{y}^{2} r_{x} t_{x}, r_{z}^{2} r_{x} t_{x}, m_{z} r_{x} t_{x}^{-1}, r_{z}^{2} r_{x} t_{x}^{-1}, m_{z} r_{x} t_{y}^{-1}, r_{x}^{2} t_{z}\right.$

$7,22,49,84,131,188,255,332,421,518]$ $262 \quad H_{616}\left\langle m_{z}\right\rangle \quad r_{z}^{2} \quad r_{z}^{2} t_{x}^{-1}, m_{y} t_{y}^{-1}, r_{y}^{2} t_{z}, r_{x}^{2} t_{z}, m_{x} t_{z}^{-1}, m_{y} t_{z}^{-1}$

$[7,22,49,85,133,190,259,336,426,525]$ $263 \quad H_{461} \quad 1 \quad r_{z}^{2} \quad r_{z}^{2} t_{x}^{-1}, m_{y} t_{y}^{-1}, r_{y}^{2} t_{z}, r_{x}^{2} t_{z}, i t_{z}^{-1}, m_{z} t_{z}^{-1}$

$[7,22,49,86,133,190,259,338,427,526]$ $264 \quad H_{615} \quad\left\langle m_{z}\right\rangle$

$[7,22,50,87,141,202,277,360,459,566]$ $265 \quad H_{539} \quad 1 \quad r_{z}^{2} \quad i t_{x}^{-1}, r_{x}^{2} t_{y}^{-1}, m_{z} r_{z}^{-1} t_{z}, m_{z} r_{z} t_{z}, r_{y}^{2} r_{z} t_{z}^{-1}, r_{x}^{2} r_{z} t_{z}^{-1}$

$\left[\begin{array}{r}\text { 7, 22, 50, 89, 147, 212, 296, 384, 488,600] } \\ 266 \quad H_{638}\left\langle m_{z} r_{x}^{-1}\right\rangle\end{array} \quad r_{x}^{2} \quad r_{y}^{2} r_{x} t_{x}, r_{z}^{2} r_{x} t_{x}, r_{z}^{2} t_{x}^{-1}, m_{x} r_{x} t_{x}^{-1}, m_{z} r_{x} t_{y}^{-1}, r_{x}^{2} t_{z}\right.$

$\begin{array}{r}{[7,22,53,93,152,216,296,384,488,600]} \\ 267\end{array} H_{746}\left\langle m_{z} r_{x}^{-1}, r_{z}^{2} r_{x}\right\rangle \quad i \quad r_{z}^{2} t_{x}, m_{x} r_{x} t_{x}, m_{y} t_{x}^{-1}, r_{x} t_{x}^{-1}, m_{z} r_{x} t_{y}^{-1}, r_{x}^{2} t_{z}$

7, 22, 54, 98, 155, 226, 312, 410, 520, 642]

\begin{tabular}{cccll}
268 & $H_{308}$ & 1 & $i$ & $r_{z}^{2} t_{x}^{-1}, r_{z}^{2} t_{y}, m_{z} t_{y}, r_{z}^{2} t_{y}^{-1}, m_{z} t_{y}^{-1}, r_{y}^{2} r_{z} t_{z}^{-1}$ \\
\hline 269 & $H_{326}$ & 1 & $i$ & $r_{z}^{2} r_{x} t_{x}^{-1}, m_{y} t_{y}^{-1}, r_{z}^{2} t_{z}, m_{z} t_{z}, r_{z}^{2} t_{z}^{-1}, m_{z} t_{z}^{-1}$ \\
\hline 270 & $H_{511}$ & 1 & $r_{z}^{2}$ & $r_{z}^{2} r_{x} t_{x}, r_{x}^{2} t_{y}^{-1}, r_{y}^{2} t_{z}, r_{x}^{2} t_{z}, r_{y}^{2} t_{z}^{-1}, r_{x}^{2} t_{z}^{-1}$ \\
\hline 271 & $H_{541}$ & 1 & $r_{z}^{2}$ & $r_{z}^{2} r_{x} t_{x}, m_{y} t_{y}^{-1}, i t_{z}, m_{z} t_{z}, i t_{z}^{-1}, m_{z} t_{z}^{-1}$ \\
\hline
\end{tabular}

$[7,24,57,102,163,236,325,426,541,668]$

\begin{tabular}{lllll}
272 & $H_{305}$ & 1 & $i$ & $i t_{x}^{-1}, i t_{y}, t_{y}, i t_{y}^{-1}, t_{y}^{-1}, r_{y}^{2} r_{z} t_{z}^{-1}$ \\
\hline 273 & $H_{540}$ & 1 & $r_{z}^{2}$ & $i t_{x}^{-1}, r_{x}^{2} r_{y} t_{y}^{-1}, m_{x} t_{z}, m_{y} t_{z}, m_{x} t_{z}^{-1}, m_{y} t_{z}^{-1}$ \\
\hline $\begin{array}{c}{[7,24,57,102,165,240,331,434,551,680]} \\
274\end{array} H_{504} \quad 1$ & $r_{z}^{2}$ & $r_{z}^{2} t_{x}^{-1}, r_{x}^{2} r_{y} t_{y}^{-1}, r_{z}^{2} t_{z}, t_{z}, r_{z}^{2} t_{z}^{-1}, t_{z}^{-1}$
\end{tabular}

$[7,24,57,102,165,240,331,434,554,686]$ 


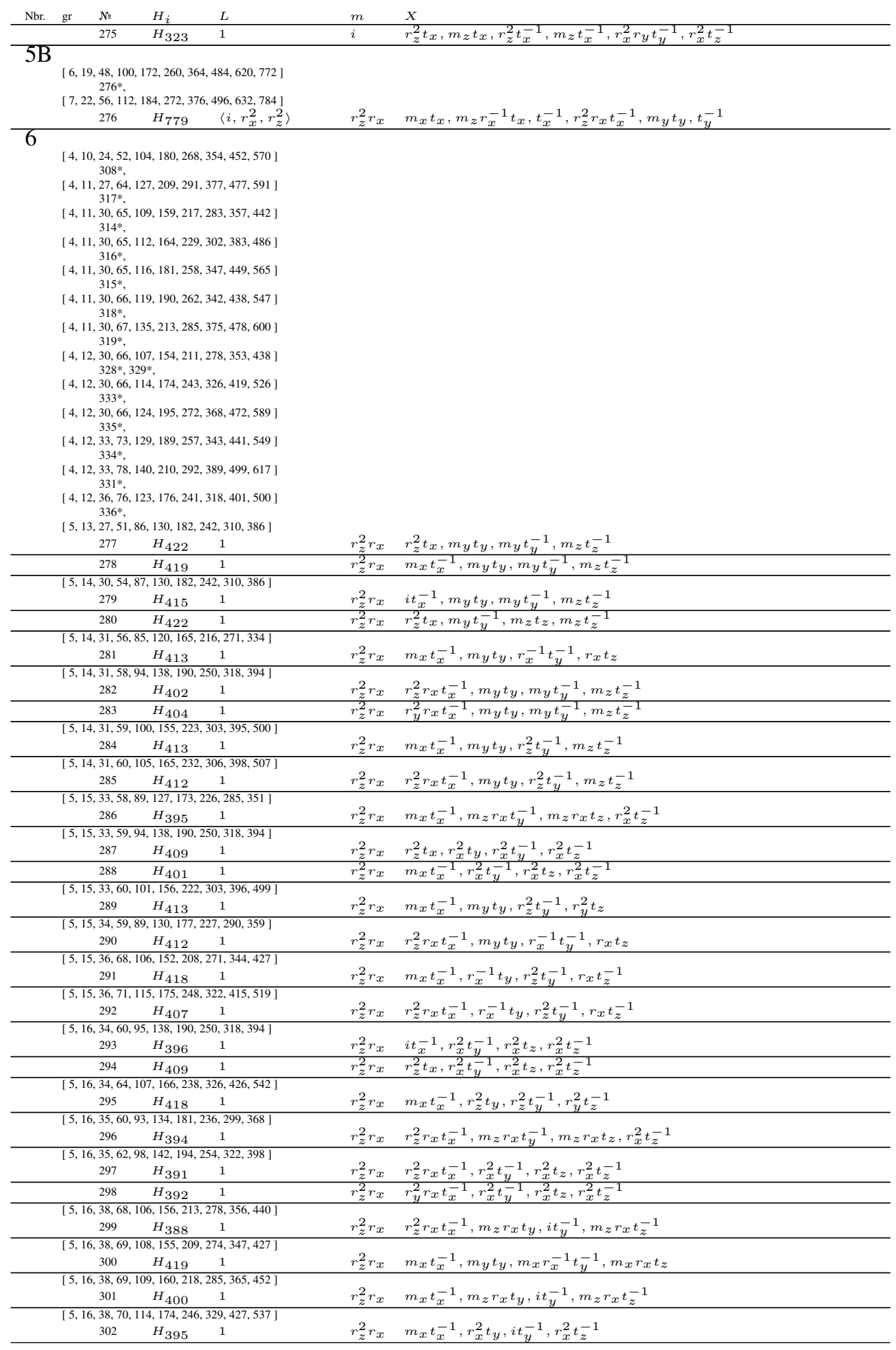




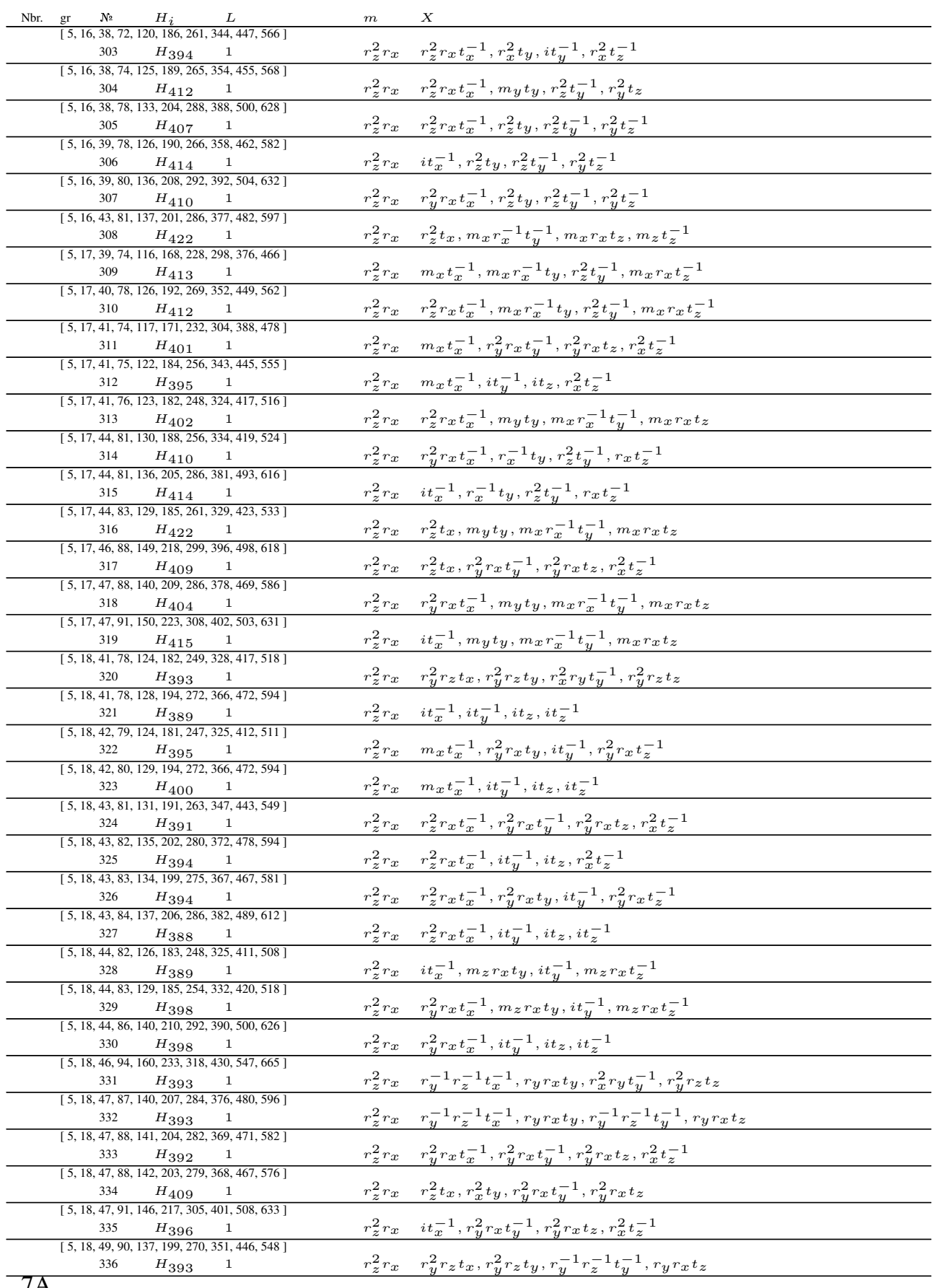

$7 \mathrm{~A}$

[ $5,13,26,45,69,98,133,173,218,269]$ $337^{*}$,

$[5,14,31,57,90,131,181,238,303,377]$ $339^{*}$,

$[5,14,31,57,91,134,185,244,313,390]$ $338^{*}$,

$[5,14,31,57,91,136,192,253,320,399$ ] $340^{*}$, 


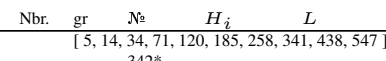
$342^{*}$,

[ $5,14,34,72,127,193,269,359,461,573]$ $341^{*}$,

$[5,15,32,55,85,122,165,215,272,335]$ $343^{*}$,

$[5,15,33,61,99,146,202,267,341,425]$ $344^{*}$,

$[5,15,34,67,111,162,229,302,383,486]$ $345^{*}$,

$[5,15,38,74,127,194,270,356,457,571]$ $346^{*}$,

$[5,16,36,67,109,162,225,296,378,471]$ $350^{*}$,

$[5,16,37,66,103,150,205,268,341,422]$ $348^{*}, 352^{*}$,

$[5,16,37,66,105,157,217,283,357,442]$ $353^{*}$

$[5,16,37,67,107,158,217,286,367,455]$ $349^{*}$,

$[5,16,37,71,117,170,236,314,399,498]$ $351^{*}$

$[5,16,37,72,117,176,245,328,421,528]$ $355^{*}$,

$[5,16,37,72,122,188,271,355,445,559]$ $347^{*}$,

[ $5,16,39,78,134,202,280,374,485,604]$ $354^{*}$,

$[5,16,39,79,135,204,281,373,481,602]$ $356^{*}$,

$[5,17,40,73,116,170,235,310,395,491]$ $357^{*}$,

[ $5,17,41,77,127,187,258,345,443,551]$ $358^{*}$

$[5,17,41,81,137,204,283,373,478,597]$ $359 *$

$[5,18,39,70,109,158,215,282,357,442]$ $360^{*}, 361^{*}$

$[5,18,43,76,121,176,241,318,401,500]$ $362^{*}$,

$[5,18,43,81,131,191,263,347,443,549]$ $363^{*}$

$[5,18,43,83,136,203,282,377,481,599]$ $365^{*}$,

$[5,18,46,88,140,204,284,376,478,594]$ $364^{*}$,

$[5,18,47,87,140,207,284,376,480,596]$ $366^{*}$

$[6,17,36,64,98,140,191,248,313,387]$

$$
337 \quad H_{413} \quad 1
$$
$r_{z}^{2} r_{x} \quad m_{x} t_{x}^{-1}, r_{z}^{2} t_{y}^{-1}, r_{x}^{-1} t_{y}^{-1}, r_{x} t_{z}, m_{z} t_{z}^{-1}$

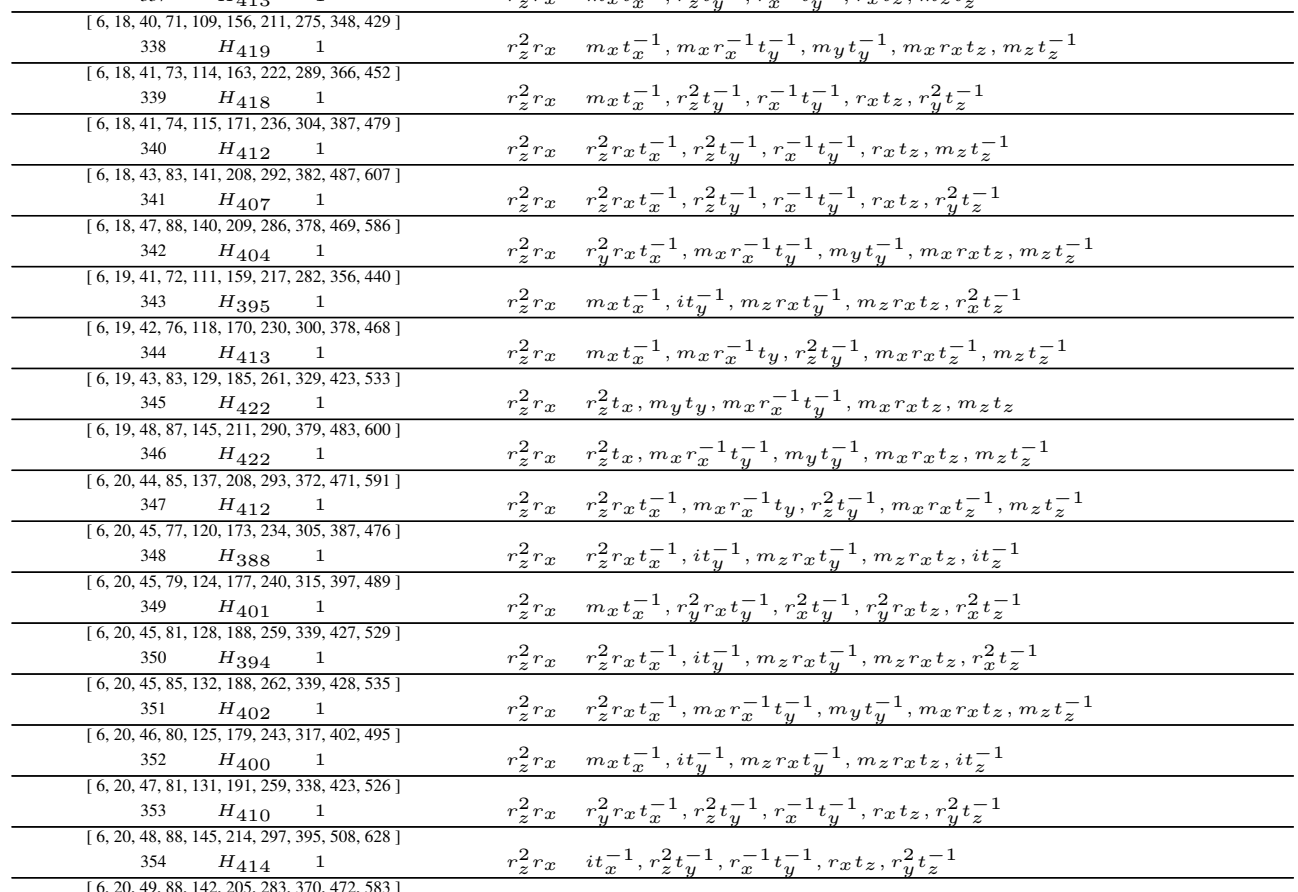




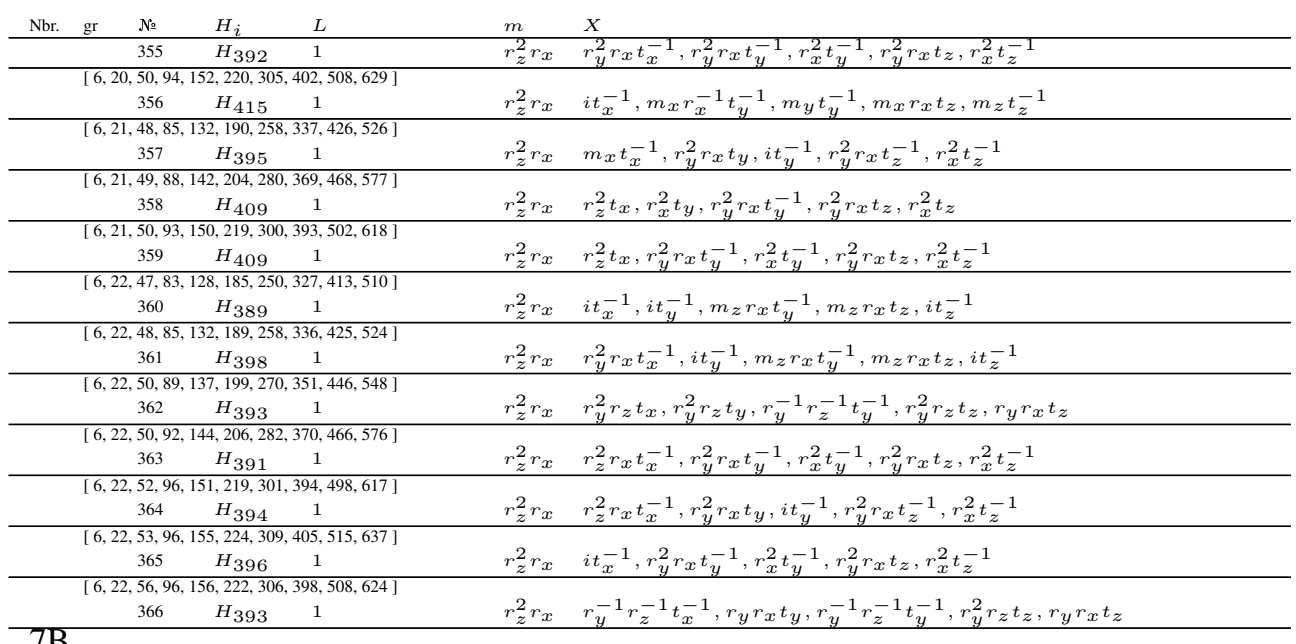

$[5,14,34,68,112,170,244,326,420,532]$ $367^{*}$,

$[5,15,37,77,129,189,267,358,454,569]$ $369^{*}$,

$[5,15,38,77,126,183,253,336,429,535]$ $368^{*}$

$[5,15,39,77,128,193,266,350,453,570]$ $370^{* *}$

$[5,15,39,81,135,202,284,380,490,614]$ $371^{*}$,

$[5,15,41,84,134,200,278,367,471,591]$ $372^{*}$

$[5,15,41,86,147,226,320,418,530,661]$ $374 *$,

$[5,15,41,91,152,230,320,421,537,670]$ $375^{*}$,

[ $5,15,42,90,151,228,315,412,525,654]$ $373^{*}$

[ $5,16,41,84,142,211,294,390,499,624]$ $376^{*}$

$[5,16,41,84,142,212,289,382,493,613]$ $377^{*}$,

[ $5,16,42,89,149,214,289,380,485,599]$ $378^{*}$

[ $5,16,42,89,152,227,313,413,530,661]$ $379^{*}$

$[5,16,42,95,157,229,317,421,534,663]$ $382^{*}$,

$[5,16,45,95,155,225,308,403,513,637]$ $380^{*}$,

[ 5, 16, 45, 96, 154, 217, 297, 392, 499, 618 ] 381 *,

$[5,16,45,98,161,234,319,418,530,655]$ $383^{*}$,

$[5,17,45,87,141,207,285,378,482,597]$ $385^{*}$,

[ $5,17,45,88,153,236,323,420,531,661]$ $384^{*}$

[ $5,17,45,96,165,242,330,430,544,674]$ $386^{*}$

$[5,17,47,93,155,232,317,419,541,672]$ $389^{*}$,

$[5,17,47,94,155,229,318,419,536,665]$ $387^{*}$,

$[5,17,49,96,155,227,316,415,528,655]$ $388^{*}$

$[5,18,48,102,161,233,320,423,538,669]$ $392^{*}$,

$[5,18,50,100,155,223,305,399,509,629]$ $390^{*}$,

[ $5,18,50,101,160,225,309,407,509,632]$ $395^{*}$

$[5,18,50,102,161,233,321,423,539,669]$ $396^{*}$,

$[5,18,50,104,162,231,318,419,532,661]$ $393^{*}$,

[ $5,18,51,103,166,239,327,429,542,672]$ $391^{*}$

$[5,18,52,102,156,222,303,396,503,622]$ $394 *$

$[6,18,45,87,138,205,287,374,477,599]$

$$
367 \quad H_{413} \quad 1
$$

$r_{z}^{2} r_{x} \quad m_{x} t_{x}^{-1}, m_{y} t_{y}, r_{z}^{2} t_{y}^{-1}, r_{x}^{-1} t_{y}^{-1}, r_{x} t_{z}$

$[6,19,48,92,143,204,281,367,464,577]$ 


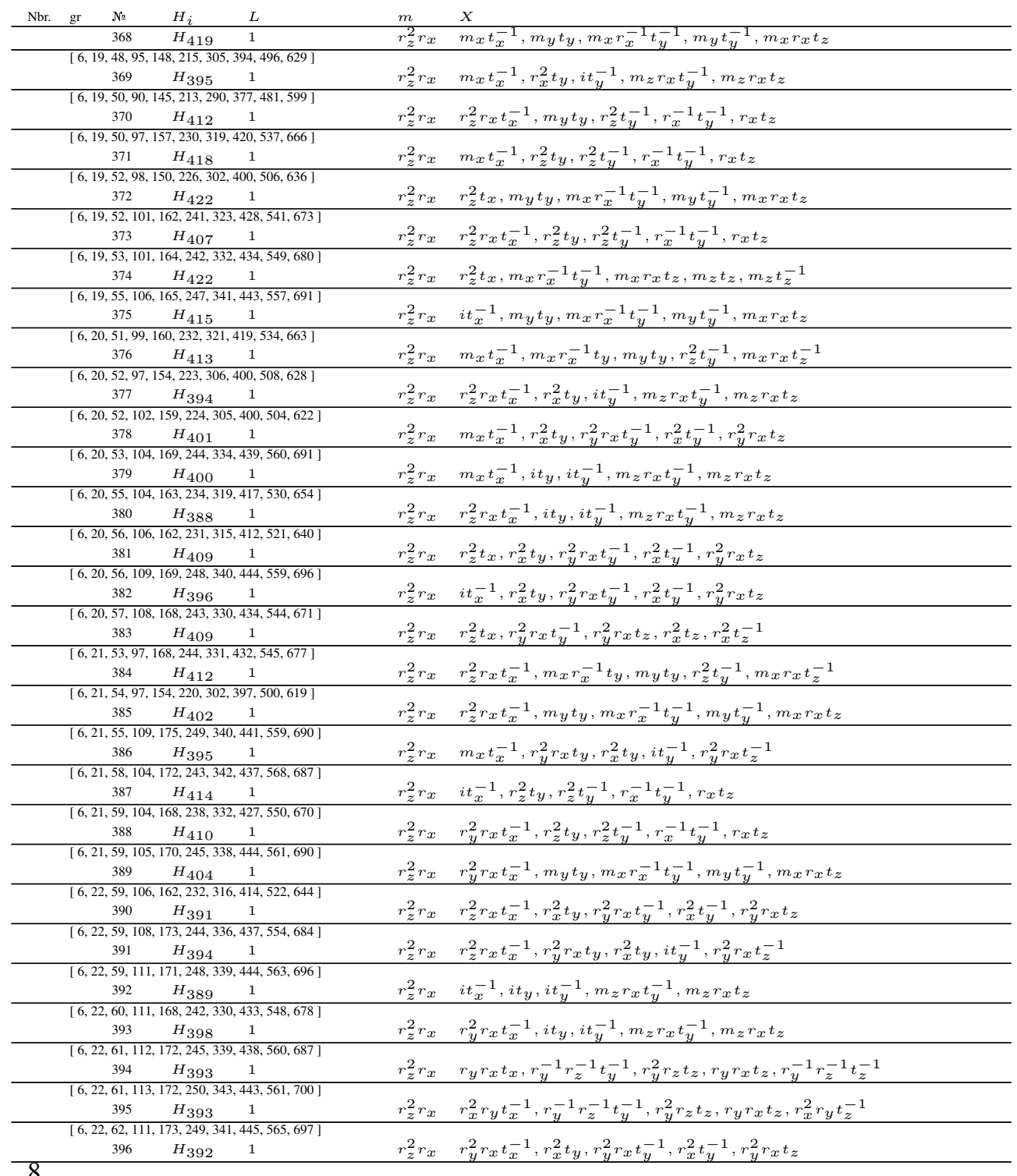

$[6,17,37,70,115,173,246,329,423,534]$ $397 *$,

$[6,17,38,75,125,183,253,336,429,535]$ $398^{*}$,

$[6,17,40,82,134,200,278,367,471,591]$ $399^{*}$,

[ $6,18,42,82,136,204,286,382,492,616]$ $400^{*}$,

$[6,18,42,83,140,210,294,390,499,624]$ $401^{*}$,

$[6,18,44,85,140,208,285,375,481,600]$ $403^{*}$,

$[6,18,44,86,141,207,285,378,482,597]$ $404^{*}$,

$[6,18,44,87,153,236,323,420,531,661]$ $402^{*}$

$[6,18,45,90,153,229,317,416,532,663]$ $405^{*}$,

$[6,18,45,92,154,232,317,419,540,672]$ 407 *

$[6,18,46,92,155,232,317,419,541,672]$ 


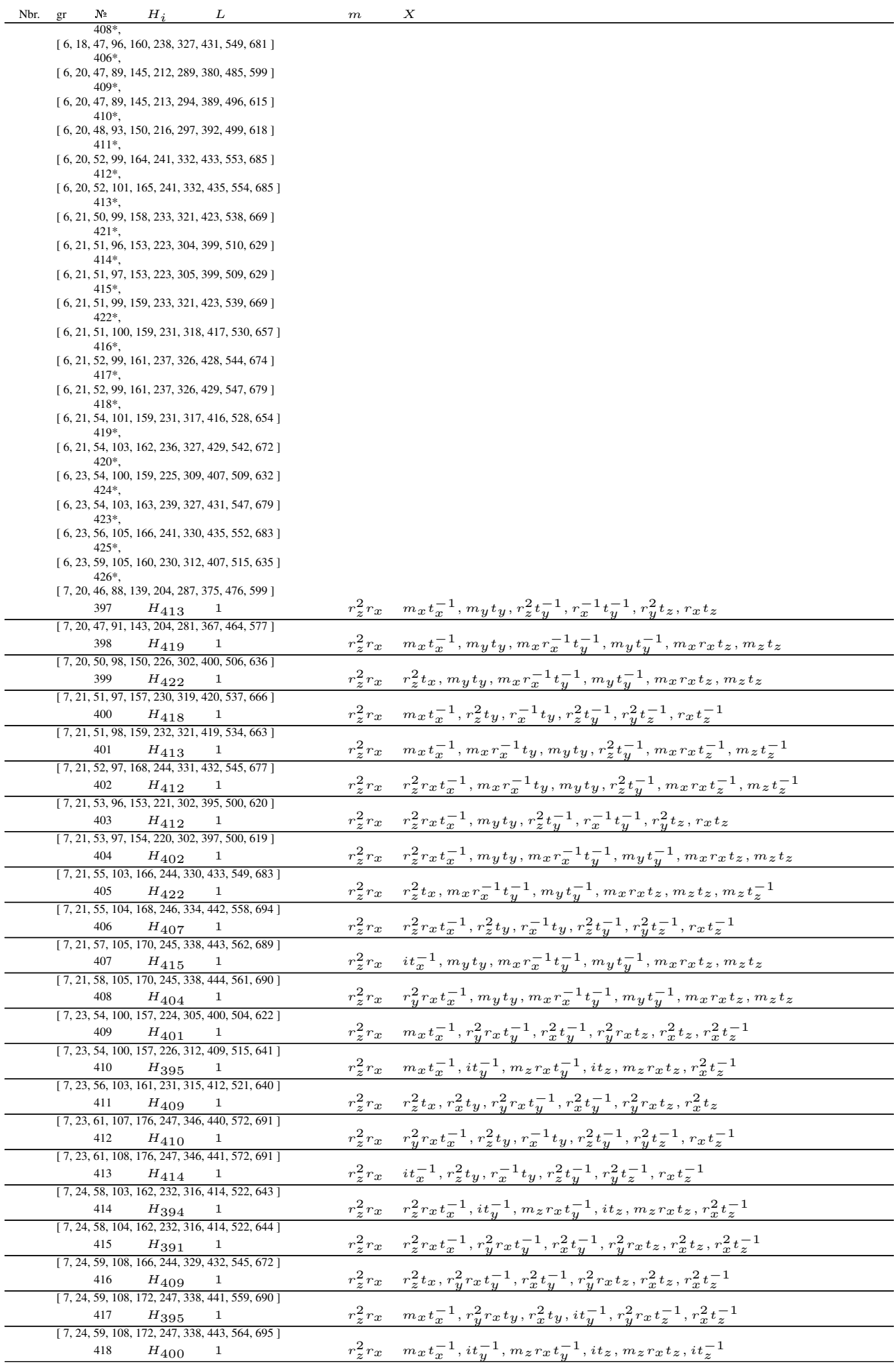




\begin{tabular}{|c|c|c|c|c|c|}
\hline \multirow[t]{2}{*}{ Nbr. } & gr № & $H_{i}$ & $L$ & $m$ & $X$ \\
\hline & $\begin{array}{r}{[7,24,60,1]} \\
419\end{array}$ & & {$[7,24,60,105,166,238,326,425,540,666]$} & $r_{z}^{2} r_{x}$ & $r_{z}^{2} r_{x} t_{x}^{-1}, i t_{y}^{-1}, m_{z} r_{x} t_{y}^{-1}, i t_{z}, m_{z} r_{x} t_{z}, i t_{z}^{-1}$ \\
\hline & \multicolumn{3}{|c|}{$[7,24,60,107,170,244,336,437,554,684]$} & & \\
\hline & 420 & $H_{394}$ & 1 & $r_{z}^{2} r_{x}$ & $r_{z}^{2} r_{x} t_{x}^{-1}, r_{y}^{2} r_{x} t_{y}, r_{x}^{2} t_{y}, i t_{y}^{-1}, r_{y}^{2} r_{x} t_{z}^{-1}, r_{x}^{2} t_{z}^{-1}$ \\
\hline & \multicolumn{3}{|c|}{$[7,24,60,109,172,250,340,445,564,698]$} & $r_{z}^{2} r_{x}$ & $i t_{x}^{-1}, r_{u}^{2} r_{x} t_{u}^{-1}, r_{x}^{2} t_{u}^{-1}, r_{u}^{2} r_{x} t_{z}, r_{x}^{2} t_{z}, r_{x}^{2} t_{z}^{-1}$ \\
\hline & \multicolumn{3}{|c|}{$[7,24,61,109,173,249,341,445,565,697]$} & & \\
\hline & 422 & $H_{392}$ & 1 & $r_{z}^{2} r_{x}$ & $r_{y}^{2} r_{x} t_{x}^{-1}, r_{y}^{2} r_{x} t_{y}^{-1}, r_{x}^{2} t_{y}^{-1}, r_{y}^{2} r_{x} t_{z}, r_{x}^{2} t_{z}, r_{x}^{2} t_{z}^{-1}$ \\
\hline & \multicolumn{3}{|c|}{$[7,26,61,110,173,250,341,446,565,698]$} & & \\
\hline & 423 & $H_{389}$ & 1 & $r_{z}^{2} r_{x}$ & $i t_{x}^{-1}, i t_{y}^{-1}, m_{z} r_{x} t_{y}^{-1}, i t_{z}, m_{z} r_{x} t_{z}, i t_{z}^{-1}$ \\
\hline & {$\left[\begin{array}{l}1,20,01 \\
424\end{array}\right.$} & $\begin{array}{ll}7,200,3 \\
H_{393}\end{array}$ & 1 & $r_{z}^{2} r_{x}$ & $r_{y}^{2} r_{z} t_{x}, r_{y}^{2} r_{z} t_{y}, r_{x}^{2} r_{y} t_{u}^{-1}, r_{y}^{-1} r_{z}^{-1} t_{y}^{-1}, r_{y}^{2} r_{z} t_{z}, r_{y} r_{x} t_{z}$ \\
\hline & \multicolumn{3}{|c|}{$[7,26,63,111,174,249,342,446,566,697]$} & & \\
\hline & 425 & $H_{398}$ & 1 & $r_{z}^{2} r_{x}$ & $r_{y}^{2} r_{x} t_{x}^{-1}, i t_{y}^{-1}, m_{z} r_{x} t_{y}^{-1}, i t_{z}, m_{z} r_{x} t_{z}, i t_{z}^{-1}$ \\
\hline & \multicolumn{3}{|c|}{$[7,26,66,110,176,249,344,444,566,694]$} & & \\
\hline & 426 & $H_{393}$ & 1 & $r_{z}^{2} r_{x}$ & $r_{y}^{-1} r_{z}^{-1} t_{x}^{-1}, r_{y} r_{x} t_{y}, r_{x}^{2} r_{y} t_{y}^{-1}, r_{y}^{-1} r_{z}^{-1} t_{y}^{-1}, r_{y}^{2} r_{z} t_{z}, r_{y} r_{x} t_{z}$ \\
\hline
\end{tabular}

$[5,14,32,66,117,179,251,335,431,539]$ $435^{*}$,

$[5,14,33,68,119,182,254,336,431,539]$ $437^{*}$,

$[5,14,33,68,119,183,259,347,448,563]$

$438^{*}$,
$[5,15,38,77,126,186,260,342,434,541$

$[5,15,39,85,152,236,336,452,584,732]$ $445^{*}$

$[5,15,39,87,158,247,353,474,610,762]$ $446^{*}$

$[5,15,40,89,154,226,310,408,520,646]$ $448^{*}$

$[5,15,40,89,158,231,313,413,526,653]$ $449^{*}$

$[5,15,40,89,158,236,322,421,536,667]$ $450^{*}$

$[5,15,40,91,155,228,316,415,530,660]$ $451^{*}$

$[5,15,41,85,137,198,271,356,455,566]$ $447^{*}$,

$447^{*}$,
$[5,16,42,86,141,204,279,367,467,579]$ $457^{*}$,

$[5,16,42,86,143,211,292,387,495,615$

$461^{*}$,
$[5,16,42,86,145,216,297,391,499,619$ $458^{*}$

$[5,16,42,96,160,229,317,419,535,665]$ $464^{*}$,

$[5,16,42,96,165,235,319,425,547,674]$ $465^{*}$

$[5,16,43,90,150,220,302,396,505,629]$ $459 *$

$[5,16,45,97,168,256,360,480,616,768]$ $462^{*}$,

$[5,16,45,98,163,237,327,431,549,681]$ $466^{*}$

$[5,16,45,99,170,256,360,480,616,768]$ $463^{*}$

$[5,16,45,102,168,236,323,427,543,671]$

$467^{*}$,
$[5,16,45,102,171,243,331,435,553,685]$ $468^{*}$,

[ $5,16,46,90,144,216,299,389,491,607]$

$460^{*}$,
$[5,17,44,83,132,196,268,350,450,556]$ 469 *

$[5,17,44,85,138,203,276,362,464,575]$ $470^{*}$,

$[5,17,45,93,158,235,322,422,538,666]$ $472^{*}$,

$[5,17,47,96,155,225,309,405,516,641]$ $471^{*}$

$[5,17,47,99,170,259,364,484,620,772]$ $473^{*}$,

$[5,17,47,101,174,264,371,492,628,780]$ $474^{*}$

$[5,17,48,101,167,240,327,430,545,675]$ $475^{*}$

$[5,17,50,103,162,232,318,416,528,654]$ $476^{*}$,

$[5,17,50,112,188,276,383,505,640,790]$ $477^{*}$,

$[5,17,51,112,188,278,383,503,639,790]$ $478^{*}$,

$[5,18,47,90,142,205,281,369,469,581$ $479^{*}$

$[5,18,48,89,142,213,294,386,492,609]$ $481^{*}$,

$[5,18,48,90,143,210,289,379,480,597]$ $482^{*}$, 


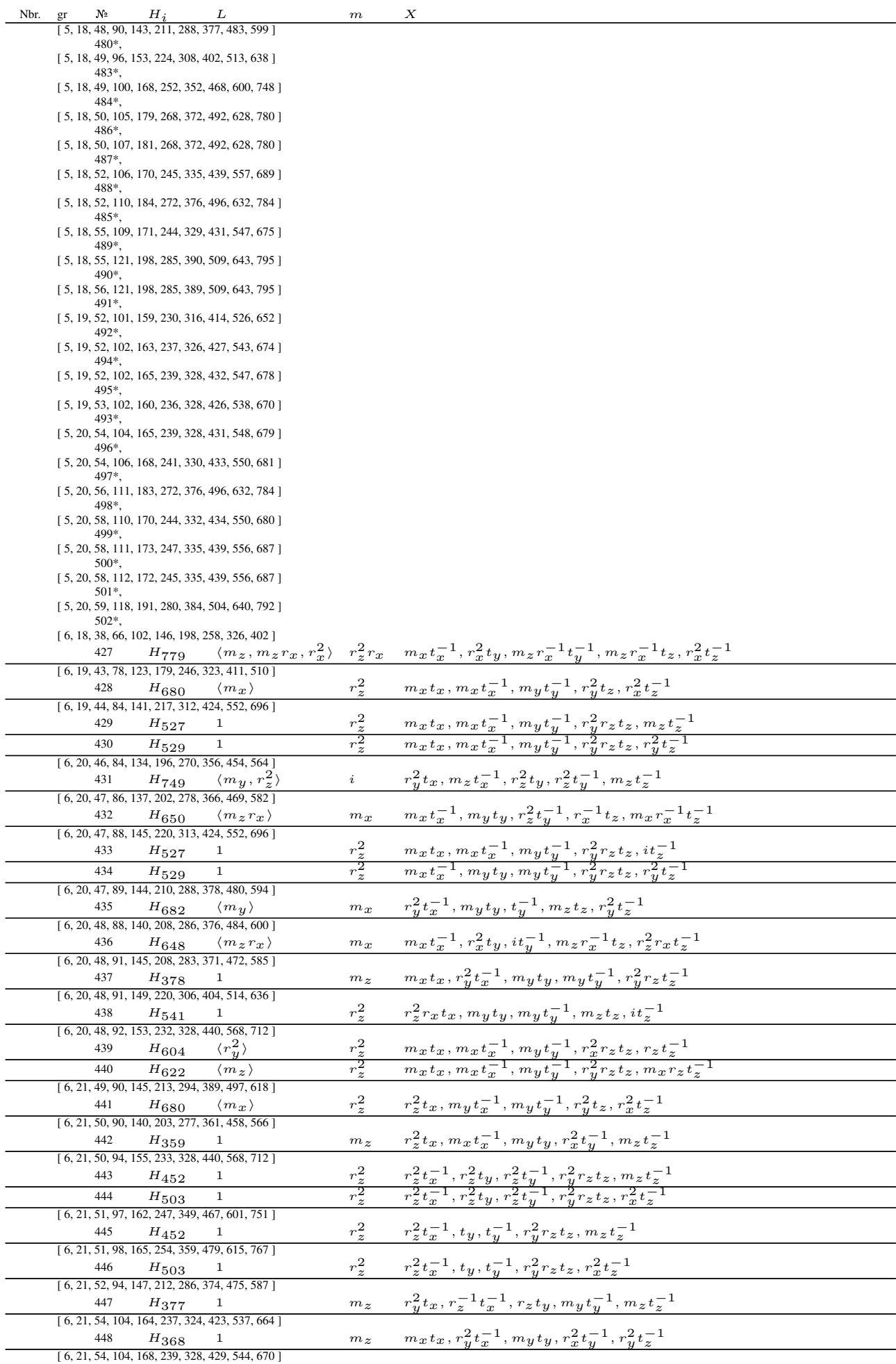




\begin{tabular}{|c|c|c|c|c|c|}
\hline \multirow[t]{2}{*}{ Nbr. } & № & $H_{i}$ & $L$ & & $X$ \\
\hline & 449 & $H_{409}$ & 1 & $r_{z}^{2} r_{x}$ & $r_{z}^{2} t_{x}, r_{x}^{2} t_{y}, r_{y}^{2} r_{x} t_{y}^{-1}, r_{y}^{2} r_{x} t_{z}, r_{x}^{2} t_{z}^{-1}$ \\
\hline \multicolumn{6}{|c|}{$[6,21,54,104,169,245,333,434,553,685]$} \\
\hline \multicolumn{6}{|c|}{$[6,21,54,105,167,243,332,434,553,683]$} \\
\hline & 451 & $H_{361}$ & 1 & $m_{z}$ & $r_{z}^{2} t_{x}, i t_{x}^{-1}, r_{z}^{2} t_{y}, i t_{y}^{-1}, r_{y}^{2} t_{z}^{-1}$ \\
\hline \multicolumn{6}{|c|}{$[6,22,50,94,150,222,306,406,518,646]$} \\
\hline & 452 & $H_{779}$ & $\left\langle m_{z}, m_{z} r_{x}, r_{x}^{2}\right\rangle$ & $r_{z}^{2} r_{x}$ & $m_{x} t_{x}^{-1}, r_{z}^{2} t_{y}, m_{x} t_{y}^{-1}, m_{x} r_{x}^{-1} t_{z}, r_{y}^{2} r_{x} t_{z}^{-1}$ \\
\hline \multicolumn{6}{|c|}{$[6,22,51,96,157,234,329,440,568,712]$} \\
\hline & 453 & $H_{452}$ & 1 & $r_{z}^{2}$ & $r_{z}^{2} t_{x}^{-1}, r_{z}^{2} t_{y}, r_{z}^{2} t_{y}^{-1}, r_{y}^{2} r_{z} t_{z}, i t_{z}^{-1}$ \\
\hline & 454 & $H_{503}$ & 1 & $r_{z}^{2}$ & $r_{z}^{2} t_{x}^{-1}, r_{z}^{2} t_{y}, r_{z}^{2} t_{y}^{-1}, r_{y}^{2} r_{z} t_{z}, r_{y}^{2} t_{z}^{-1}$ \\
\hline \multicolumn{6}{|c|}{$[6,22,52,98,161,240,336,448,576,720]$} \\
\hline & 456 & $H_{611}$ & $\frac{\langle y}{\left\langle m_{z}\right\rangle}$ & $\frac{r_{z}}{r_{z}^{2}}$ & 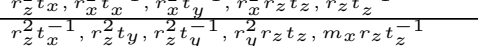 \\
\hline \multicolumn{6}{|c|}{$[6,22,53,98,153,218,296,386,488,602]$} \\
\hline & 457 & $H_{370}$ & 1 & $m_{z}$ & $r_{z}^{2} t_{x}, m_{x} t_{x}^{-1}, m_{y} t_{y}, r_{x}^{2} t_{y}^{-1}, r_{x}^{2} t_{z}^{-1}$ \\
\hline \multicolumn{6}{|c|}{$[6,22,54,100,158,228,312,407,516,639]$} \\
\hline \multirow{2}{*}{\multicolumn{6}{|c|}{$[6,22,55,101,158,230,315,411,522,647]$}} \\
\hline & & & & & \\
\hline & 459 & $H_{359}$ & 1 & $m_{z}$ & ${ }^{i t_{x}}, m_{x} t_{x}^{-1}, m_{y} t_{y}, r_{x}^{2} t_{y}^{-1}, m_{z} t_{z}^{-1}$ \\
\hline \multicolumn{6}{|c|}{$[6,22,56,100,157,226,308,401,505,624]$} \\
\hline & 460 & $H_{382}$ & 1 & $m_{z}$ & $r_{y}^{2} t_{x}, r_{z}^{-1} t_{x}^{-1}, r_{z} t_{y}, m_{y} t_{y}^{-1}, r_{y}^{2} r_{z} t_{z}^{-1}$ \\
\hline \multicolumn{6}{|c|}{$[6,22,56,102,160,230,316,414,524,646]$} \\
\hline & 461 & $H_{750}$ & $\left\langle m_{y}, r_{z}^{2}\right\rangle$ & $i$ & $r_{y}^{2} t_{x}, m_{z} t_{x}^{-1}, r_{z}^{2} t_{y}, r_{z}^{2} t_{y}^{-1}, r_{x}^{2} r_{z} t_{z}^{-1}$ \\
\hline & {$[6,22,56,1$} & $74,259,36$ & $482,618,770]$ & & \\
\hline & 462 & $H_{503}$ & 1 & $r_{z}^{2}$ & $r_{z}^{2} t_{x}^{-1}, t_{y}, t_{y}^{-1}, r_{y}^{2} r_{z} t_{z}, r_{y}^{2} t_{z}^{-1}$ \\
\hline & {$[6,22,56,1$} & $74,259,36$ & $482,618,770]$ & & \\
\hline & 463 & $H_{452}$ & 1 & $r_{z}^{2}$ & $r_{z}^{2} t_{x}^{-1}, t_{y}, t_{y}^{-1}, r_{y}^{2} r_{z} t_{z}, i t_{z}^{-1}$ \\
\hline & $\begin{array}{r}{[6,22,56,1} \\
464\end{array}$ & $\begin{array}{l}71,247,33 \\
H_{649}\end{array}$ & $\begin{array}{c}443,563,695] \\
\left\langle m_{z} r_{x}\right\rangle\end{array}$ & $m_{x}$ & $r_{z}^{2} r_{x} t_{x}^{-1}, r_{x}^{2} t_{y}, i t_{u}^{-1}, m_{z} r_{x}^{-1} t_{z}, r_{\gamma}^{2} r_{x} t^{-1}$ \\
\hline & {$[6,22,56,1$} & $\frac{11649}{72,245,34}$ & $\frac{1}{448,563,690]}$ & & \\
\hline & 465 & $H_{652}$ & $\left\langle m_{z} r_{x}\right\rangle$ & $m_{x}$ & $r_{z}^{2} r_{x} t_{x}^{-1}, m_{y} t_{y}, r_{z}^{2} t_{y}^{-1}, r_{x}^{-1} t_{z}, m_{x} r_{x}^{-1} t_{z}^{-1}$ \\
\hline & {$[6,22,59,1$} & $72,248,34$ & $444,564,696]$ & & \\
\hline & 466 & $H_{682}$ & $\left\langle m_{y}\right\rangle$ & $m_{x}$ & $r_{y}^{2} t_{x}^{-1}, r_{z}^{2} t_{y}, m_{x} t_{y}^{-1}, m_{z} t_{z}, r_{y}^{2} t_{z}^{-1}$ \\
\hline & {$[6,22,59,1$} & $71,245,33$ & $441,557,686]$ & & \\
\hline & 467 & $H_{541}$ & 1 & $r_{z}^{2}$ & $r_{z}^{2} r_{x} t_{x}, m_{x} t_{y}, m_{x} t_{y}^{-1}, m_{z} t_{z}, i t_{z}^{-1}$ \\
\hline & {$[6,22,59,1$} & $75,250,34$ & $446,566,698]$ & & \\
\hline & 468 & $H_{378}$ & 1 & $m_{z}$ & $r_{y}^{2} t_{x}, r_{y}^{2} t_{x}^{-1}, m_{y} t_{y}, r_{x}^{2} t_{y}^{-1}, r_{y}^{2} r_{z} t_{z}^{-1}$ \\
\hline & {$[6,23,53,9$} & $5,209,282$ & $71,469,576]$ & & \\
\hline & 469 & $H_{682}$ & $\left\langle m_{x}\right\rangle$ & $r_{z}^{2}$ & $r_{y}^{2} t_{x}, r_{y}^{2} t_{x}^{-1}, m_{y} t_{y}^{-1}, r_{y}^{2} t_{z}, r_{x}^{2} t_{z}^{-1}$ \\
\hline & {$[6,23,53,9$} & $0,215,290$ & $81,482,595]$ & & \\
\hline & 470 & $H_{343}$ & 1 & $m_{z}$ & $r_{z}^{2} t_{x}, m_{x} r_{z} t_{x}^{-1}, i t_{y}, m_{x} r_{z} t_{y}^{-1}, m_{z} t_{z}^{-1}$ \\
\hline & {$[6,23,57,1$} & $65,239,32$ & $424,539,665]$ & & \\
\hline & 471 & $H_{370}$ & 1 & $m_{z}$ & ${ }^{i t_{x}}, m_{x} t_{x}^{-1}, m_{y} t_{y}, r_{x}^{2} t_{y}^{-1}, r_{x}^{2} t_{z}^{-1}$ \\
\hline & {$[6,23,57,1$} & $68,243,33$ & $435,552,681]$ & & \\
\hline & 472 & $H_{359}$ & 1 & $m_{z}$ & $i t_{x}, r_{y}^{2} t_{x}^{-1}, m_{y} t_{y}, r_{x}^{2} t_{y}^{-1}, m_{z} t_{z}^{-1}$ \\
\hline & {$[6,23,58,1$} & $80,268,37$ & $492,628,780]$ & & \\
\hline & 473 & $H_{527}$ & 1 & $r_{z}^{2}$ & $m_{x} t_{x}^{-1}, m_{x} t_{y}, m_{x} t_{y}^{-1}, r_{y}^{2} r_{z} t_{z}, m_{z} t_{z}^{-1}$ \\
\hline & {$[6,23,58,1$} & $82,271,37$ & $496,632,784]$ & & $\begin{array}{lllll}-1 & -1 & 2 & 2 & -1\end{array}$ \\
\hline & 474 & $H_{529}$ & $\frac{1}{441558689]}$ & $r_{z}^{2}$ & $m_{y} t_{x}, m_{y} t_{x}^{-1}, m_{y} t_{y}^{-1}, r_{y}^{2} r_{z} t_{z}, r_{y}^{2} t_{z}^{-1}$ \\
\hline & $\begin{array}{r}{[6,23,59,1} \\
475\end{array}$ & $\begin{array}{c}72,246,33 \\
H_{377}\end{array}$ & $\begin{array}{l}441,558,689] \\
1\end{array}$ & $m_{z}$ & $r_{u}^{2} t_{x}, m_{z} r_{z}^{-1} t_{x}^{-1}, m_{z} r_{z} t_{y}, m_{y} t_{u}^{-1}, m_{z} t_{z}^{-1}$ \\
\hline & {$[6,23,60,1$} & $67,241,32$ & $427,541,668]$ & & \\
\hline & 476 & $H_{370}$ & 1 & $m_{z}$ & $r_{z}^{2} t_{x}, r_{y}^{2} t_{x}^{-1}, m_{y} t_{y}, r_{x}^{2} t_{y}^{-1}, r_{x}^{2} t_{z}^{-1}$ \\
\hline & {$[6,23,61,1$} & $90,279,38$ & $506,639,792]$ & & \\
\hline & 477 & $H_{541}$ & 1 & $r_{z}^{2}$ & $r_{x} t_{x}, r_{x}^{-1} t_{x}^{-1}, m_{y} t_{y}^{-1}, m_{z} t_{z}, i t_{z}^{-1}$ \\
\hline & {$[6,23,61,1$} & $91,280,38$ & $505,639,792]$ & & \\
\hline & 478 & $H_{541}$ & 1 & $r_{z}^{2}$ & $r_{z}^{2} r_{x} t_{x}, r_{y}^{2} r_{x} t_{x}^{-1}, m_{y} t_{y}^{-1}, m_{z} t_{z}, i t_{z}^{-1}$ \\
\hline & {$[6,24,55,9$} & $2,218,296$ & $86,488,602]$ & & \\
\hline & 479 & $H_{683}$ & $\frac{\left\langle m_{x}\right\rangle}{400,506,622]}$ & $r_{z}^{2}$ & $r_{y}^{2} t_{x}, r_{y}^{2} t_{x}^{-1}, r_{x}^{2} t_{y}^{-1}, r_{y}^{2} t_{z}, r_{x}^{2} t_{z}^{-1}$ \\
\hline & $\begin{array}{r}{[6,24,56,1} \\
480\end{array}$ & $\begin{array}{c}58,225,30 \\
H_{344}\end{array}$ & $\begin{array}{l}400,506,622] \\
1\end{array}$ & $m_{z}$ & $r_{z}^{2} t_{x}, i t_{x}^{-1}, r_{z}^{2} t_{y}, r_{z}^{2} t_{y}^{-1}, r_{y}^{2} r_{z} t_{z}^{-1}$ \\
\hline & {$[6,24,56,1$} & $59,231,31$ & $412,519,644]$ & & \\
\hline & 481 & $H_{347}$ & 1 & $m_{z}$ & $r_{z}^{2} t_{x}, m_{x} r_{z} t_{x}^{-1}, i t_{y}, m_{x} r_{z} t_{y}^{-1}, r_{x}^{2} r_{z} t_{z}^{-1}$ \\
\hline & {$[6,24,56,1$} & $60,230,31$ & $407,516,638]$ & & \\
\hline & 482 & $H_{511}$ & 1 & $r_{z}^{2}$ & $r_{z}^{2} r_{x} t_{x}, r_{x}^{2} t_{y}, r_{x}^{2} t_{y}^{-1}, r_{x}^{2} t_{z}, r_{y}^{2} t_{z}^{-1}$ \\
\hline & {$[6,24,58,1$} & $61,234,31$ & $416,529,653]$ & & \\
\hline & 483 & $H_{350}$ & 1 & $m_{z}$ & $r_{z}^{2} t_{x}, i t_{x}^{-1}, m_{y} t_{y}, r_{x}^{2} t_{y}^{-1}, m_{z} t_{z}^{-1}$ \\
\hline & $\begin{array}{r}{[6,24,58,1} \\
484\end{array}$ & $\begin{array}{c}77,263,36 \\
H_{611}\end{array}$ & $\begin{array}{c}483,617,767] \\
\left\langle m_{z}\right\rangle\end{array}$ & $r_{z}^{2}$ & $r_{z}^{2} t_{x}^{-1}, t_{y}, t_{y}^{-1}, r_{y}^{2} r_{z} t_{z}, m_{x} r_{z} t_{z}^{-1}$ \\
\hline & {$[6,24,60,1$} & $86,274,37$ & $498,634,786]$ & & \\
\hline & 485 & $H_{598}$ & $\left\langle r_{y}^{2}\right\rangle$ & $r_{z}^{2}$ & $r_{y}^{2} t_{x}, r_{y}^{2} t_{x}^{-1}, r_{x}^{2} t_{y}^{-1}, r_{x}^{2} r_{z} t_{z}, r_{z} t_{z}^{-1}$ \\
\hline & {$[6,24,61,1$} & $86,273,37$ & $496,632,784]$ & & \\
\hline & 486 & $H_{529}$ & 1 & $r_{z}^{2}$ & $m_{x} t_{x}^{-1}, m_{x} t_{y}, m_{x} t_{y}^{-1}, r_{y}^{2} r_{z} t_{z}, r_{y}^{2} t_{z}^{-1}$ \\
\hline & {$[6,24,61,1$} & $86,273,37$ & $496,632,784]$ & & \\
\hline & 487 & $H_{527}$ & 1 & $r_{z}^{2}$ & $m_{x} t_{x}^{-1}, m_{x} t_{y}, m_{x} t_{y}^{-1}, r_{y}^{2} r_{z} t_{z}, i t_{z}^{-1}$ \\
\hline
\end{tabular}




\begin{tabular}{|c|c|c|c|c|c|}
\hline Nbr. & gr № & $H_{i}$ & $L$ & $m$ & $x$ \\
\hline & $\begin{array}{r}{[6,24,62,} \\
488\end{array}$ & $\begin{array}{c}76,252,34 \\
H_{370}\end{array}$ & $\begin{array}{l}448,568,700] \\
1\end{array}$ & $m_{z}$ & $i t_{x}, r_{y}^{2} t_{x}^{-1}, m_{y} t_{y}, r_{x}^{2} t_{y}^{-1}, r_{x}^{2} t_{z}^{-1}$ \\
\hline & {$[6,24,64$,} & $77,250,33$ & $443,560,689]$ & & \\
\hline & 489 & $H_{382}$ & 1 & $m_{z}$ & $r_{y}^{2} t_{x}, m_{z} r_{z}^{-1} t_{x}^{-1}, m_{z} r_{z} t_{y}, m_{y} t_{y}^{-1}, r_{y}^{2} r_{z} t_{z}^{-1}$ \\
\hline & {$[6,24,65$, } & $96,285,38$ & $508,643,796]$ & & \\
\hline & 490 & $H_{511}$ & 1 & $r_{z}^{2}$ & $r_{x} t_{x}, r_{x}^{-1} t_{x}^{-1}, r_{x}^{2} t_{y}^{-1}, r_{x}^{2} t_{z}, r_{y}^{2} t_{z}^{-1}$ \\
\hline & {$[6,24,65$,} & $97,284,38$ & $508,643,796]$ & & \\
\hline & 491 & $H_{511}$ & 1 & $r_{z}^{2}$ & $r_{z}^{2} r_{x} t_{x}, r_{y}^{2} r_{x} t_{x}^{-1}, r_{x}^{2} t_{y}^{-1}, r_{x}^{2} t_{z}, r_{y}^{2} t_{z}^{-1}$ \\
\hline & {$[6,25,59$,} & $67,241,32$ & $427,541,668]$ & & \\
\hline & 492 & $H_{365}$ & 1 & $m_{z}$ & $r_{z}^{2} t_{x}, i t_{x}^{-1}, m_{y} t_{y}, r_{x}^{2} t_{y}^{-1}, r_{x}^{2} t_{z}^{-1}$ \\
\hline & {$[6,25,60$,} & $70,248,33$ & $434,554,686]$ & & \\
\hline & 493 & $H_{372}$ & 1 & $m_{z}$ & $r_{z}^{2} t_{x}, i t_{x}^{-1}, m_{y} t_{y}, r_{x}^{2} t_{y}^{-1}, r_{y}^{2} t_{z}^{-1}$ \\
\hline & {$[6,25,61$,} & $70,246,33$ & $438,557,687]$ & & \\
\hline & 494 & $H_{682}$ & $\left\langle m_{x}\right\rangle$ & $r_{z}^{2}$ & $i t_{x}, r_{x}^{2} t_{x}^{-1}, m_{y} t_{y}^{-1}, r_{y}^{2} t_{z}, r_{x}^{2} t_{z}^{-1}$ \\
\hline & {$[6,25,61$,} & $72,247,33$ & $442,560,692]$ & & \\
\hline & 495 & $H_{343}$ & 1 & $m_{z}$ & $r_{z}^{2} t_{x}, r_{y}^{2} r_{z} t_{x}^{-1}, i t_{y}, r_{y}^{2} r_{z} t_{y}^{-1}, m_{z} t_{z}^{-1}$ \\
\hline & $\begin{array}{r}{[6,26,61,} \\
496\end{array}$ & $\begin{array}{c}74,250,34 \\
H_{683}\end{array}$ & $\begin{array}{l}46,566,698] \\
\left\langle m_{x}\right\rangle\end{array}$ & $r_{z}^{2}$ & $i t_{x}, r_{x}^{2} t_{x}^{-1}, r_{x}^{2} t_{y}^{-1}, r_{y}^{2} t_{z}, r_{x}^{2} t_{z}^{-1}$ \\
\hline & {$[6,26,62$,} & $76,252,34$ & $448,568,700]$ & & \\
\hline & 497 & $H_{364}$ & 1 & $m_{z}$ & $r_{z}^{2} t_{x}, i t_{x}^{-1}, m_{y} t_{y}, r_{x}^{2} t_{y}^{-1}, i t_{z}^{-1}$ \\
\hline & {$[6,26,64$,} & $90,278,38$ & $\overline{002,638,790]}$ & & \\
\hline & 498 & $H_{622}$ & $\left\langle m_{z}\right\rangle$ & $r_{z}^{2}$ & $m_{x} t_{x}^{-1}, m_{x} t_{y}, m_{x} t_{y}^{-1}, r_{y}^{2} r_{z} t_{z}, m_{x} r_{z} t_{z}^{-1}$ \\
\hline & {$[6,26,65$,} & $76,251,34$ & $444,563,693]$ & & \\
\hline & 499 & $H_{347}$ & 1 & $m_{z}$ & $r_{z}^{2} t_{x}, r_{y}^{2} r_{z} t_{x}^{-1}, i t_{y}, r_{y}^{2} r_{z} t_{y}^{-1}, r_{x}^{2} r_{z} t_{z}^{-1}$ \\
\hline & {$[6,26,65$,} & $77,251,34$ & $447,566,697]$ & & \\
\hline & 500 & $H_{511}$ & 1 & $r_{z}^{2}$ & $r_{z}^{2} r_{x} t_{x}, r_{y}^{2} t_{y}, r_{y}^{2} t_{y}^{-1}, r_{x}^{2} t_{z}, r_{y}^{2} t_{z}^{-1}$ \\
\hline & $\begin{array}{r}{[6,26,65,} \\
501\end{array}$ & $\begin{array}{c}76,252,34 \\
H_{344}\end{array}$ & $\begin{array}{l}448,568,700] \\
1\end{array}$ & $m z$ & $r_{z}^{2} t_{x}, i t_{x}^{-1}, i t_{y}, i t_{u}^{-1}, r_{u}^{2} r_{z} t_{z}^{-1}$ \\
\hline & {$[6,26,66$,} & $94,282,38$ & $506,642,794]$ & & \\
\hline & 502 & $H_{604}$ & $\left\langle r_{y}^{2}\right\rangle$ & $r_{z}^{2}$ & $i t_{x}, m_{y} t_{x}^{-1}, m_{y} t_{y}^{-1}, r_{x}^{2} r_{z} t_{z}, r_{z} t_{z}^{-1}$ \\
\hline
\end{tabular}

10

$6,19,43,78,123,179,246,323,411,510]$ $503^{*}$

$[6,19,43,79,127,187,259,343,439,547]$ $504^{*}$,

$[6,19,43,79,128,190,262,344,439,547]$ $505^{*}$

$[6,19,43,79,128,191,267,355,456,571]$ $506^{*}$

$[6,20,47,85,132,192,265,347,440,546]$ $507 *$

$[6,20,47,87,139,203,279,367,467,579]$ $510^{*}$

$[6,20,48,89,139,200,274,360,459,569]$ $508^{*}$,

$[6,20,48,90,143,207,283,371,471,583]$ $509^{*}$,

$[6,20,48,90,144,211,290,381,486,602]$ $511^{*}$,

$[6,20,48,91,147,216,297,388,491,607]$ $512^{*}$

$[6,20,49,94,151,220,303,399,508,631]$ $513^{*}$

$[6,20,49,96,161,245,348,468,604,756]$ $514^{*}, 515^{*}$,

$[6,20,50,101,172,260,364,484,620,772]$ $516^{*}$

$[6,20,51,100,160,232,318,416,528,654]$ $517^{*}$

$[6,21,49,86,135,199,270,353,453,558]$ $518^{*}$,

$[6,21,49,89,141,205,281,369,469,581]$ $520^{*}$,

$[6,21,49,89,143,210,289,379,480,597]$ $523^{*}$

[ $6,21,49,89,143,211,288,377,483,599]$ $522^{*}$,

$[6,21,50,90,141,205,279,366,468,578]$ $519^{*}$,

$[6,21,50,91,143,207,283,371,471,583]$ $521^{*}$,

$[6,21,50,91,145,213,292,385,492,609]$ $526^{*}$

[ $6,21,51,93,144,207,283,371,471,583]$ $524^{*}$,

$[6,21,51,94,149,217,295,386,492,607]$ $525^{*}$,

$[6,21,51,95,151,219,300,395,503,623]$ $536^{*}, 537^{*}$,

$[6,21,51,96,155,227,312,411,523,648]$ $530^{*}$,

$[6,21,51,97,157,228,311,408,519,643]$ $531^{*}$

$[6,21,52,97,152,218,295,386,492,607]$ $527^{*}$

$[6,21,52,97,153,221,302,397,504,624]$ 
\begin{tabular}{llllllll} 
Nbr. & gr & № & $H_{i}$ & $L$ & $m$ & $X$ \\
\hline & & $529 *$ & & &
\end{tabular}

$[6,21,52,97,153,223,306,401,511,634]$ $528^{*}$,

$[6,21,52,97,153,224,309,405,516,641]$ $532 *$,

$[6,21,52,98,157,230,315,413,526,651]$ $533^{*}$,

$[6,21,52,101,164,236,320,419,531,656]$ $534^{*}$,

$[6,21,52,104,170,242,327,433,555,682]$ $541^{*}$

$[6,21,53,100,161,237,324,425,542,670]$ $538^{*}$,

$[6,21,53,104,169,242,327,433,555,682]$ $543^{*}$,

$[6,21,53,104,173,260,364,484,620,772]$ $535^{*}$,

$535^{*}$,
$[6,21,54,108,180,268,372,492,628,780]$ $539^{*}, 540^{*}$

$[6,21,55,103,160,232,318,416,528,654]$ $542 *$,

$[6,21,56,107,169,245,335,439,557,689]$ $544^{*}$,

$[6,21,57,110,174,250,339,443,561,693]$ $545^{*}$

$[6,22,52,92,143,207,283,371,471,583]$ $546^{*}, 547^{*}$,

$[6,22,53,96,152,221,302,397,504,624]$ $548^{*}$,

$[6,22,54,97,150,217,295,386,492,607]$ $549^{*}$

$[6,22,54,97,152,224,305,398,510,629]$ $550^{*}$,

$[6,22,54,98,154,226,310,405,516,640]$ $551^{*}, 552^{*}$

$[6,22,54,100,158,230,316,414,526,652]$ $556^{*}$,

$556^{*}$,
$[6,22,54,102,165,241,331,435,553,685]$ $562^{*}$,

$[6,22,54,102,169,256,360,480,616,768]$ $553^{*}, 554^{*}$

$[6,22,55,101,158,228,307,401,513,631]$ $555^{*}$,

$[6,22,55,101,159,236,328,426,538,670]$ $557^{*}$

$[6,22,55,102,160,232,318,416,528,654]$ $558^{*}$

$[6,22,55,102,160,234,323,423,538,668]$ $559 *$

$[6,22,55,103,162,233,318,416,528,654]$ $560^{*}$,

$[6,22,55,103,164,238,325,427,543,671]$ $561^{*}$,

$[6,22,55,106,175,261,364,484,620,772]$ $564^{*}, 565^{*}$,

$[6,22,56,104,161,232,318,416,528,654]$ $563^{*}$,

$[6,22,56,109,180,268,372,492,628,780]$ $566^{*}$,

$[6,22,57,106,168,245,335,439,557,689]$ $568^{*}$

$[6,22,57,109,173,248,335,436,552,682]$ $567^{*}$,

$[6,22,58,110,173,249,339,443,561,693]$ $569^{*}$,

$[6,22,59,110,170,243,329,431,547,675]$ $570^{*}$,

$[6,22,59,112,174,249,339,443,561,693]$ $571^{*}$

$[6,23,52,91,143,207,283,371,471,583]$ $572^{*}$,

$[6,23,53,95,151,219,300,395,503,623]$ $574^{*}, 575^{*}$,

$[6,23,55,102,162,233,318,417,529,654]$ $576^{*}$

$[6,23,55,103,164,237,325,427,543,673]$

$586^{*}$,
$[6,23,56,99,155,227,307,401,513,631]$ $573^{*}$,

$[6,23,56,103,163,237,325,427,543,673]$ $590^{*}$, $[6,23,56,103,163,239,330,429,541,672]$
$578^{*}$,

$[6,23,56,103,164,239,328,431,548,679]$ $585^{*}$,

$[6,23,56,104,164,235,320,419,531,656]$ $579^{*}$,

$[6,23,56,104,164,237,325,426,541,670]$ $580^{*}$

$[6,23,56,105,167,241,330,433,550,681]$ $591^{*}, 592^{*}$,

$[6,23,57,101,156,227,307,401,513,631]$ $577^{*}$, 


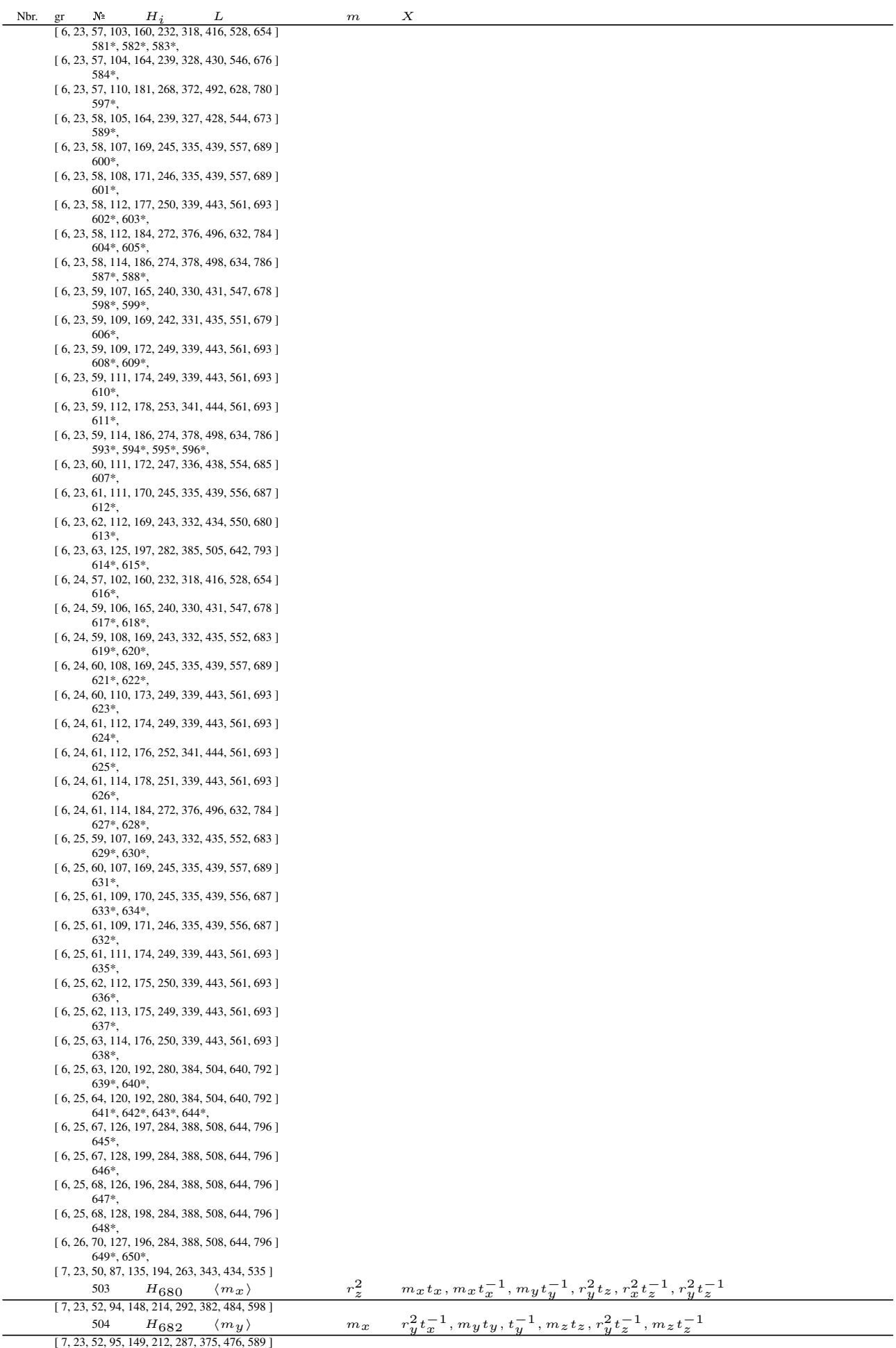




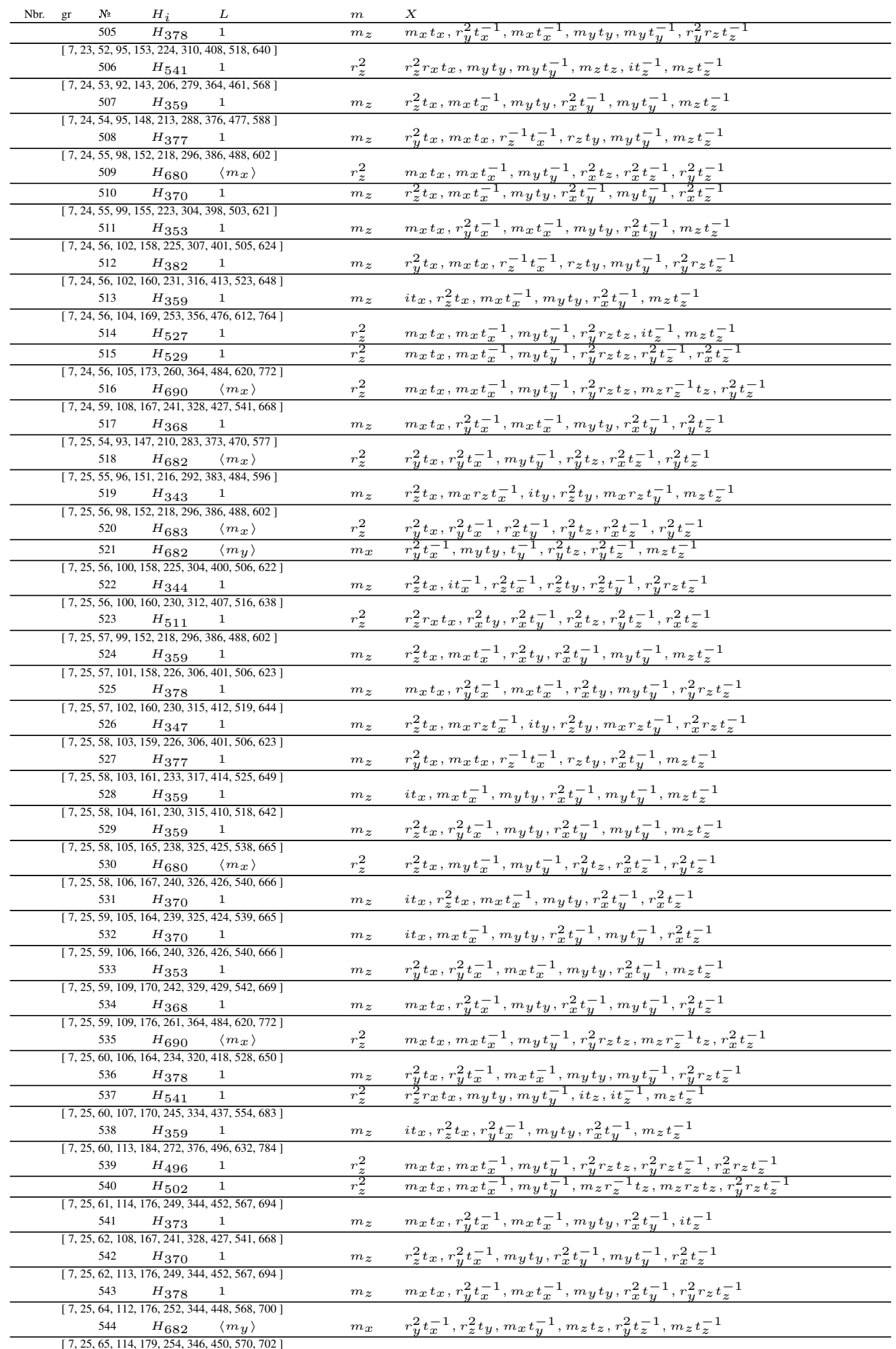




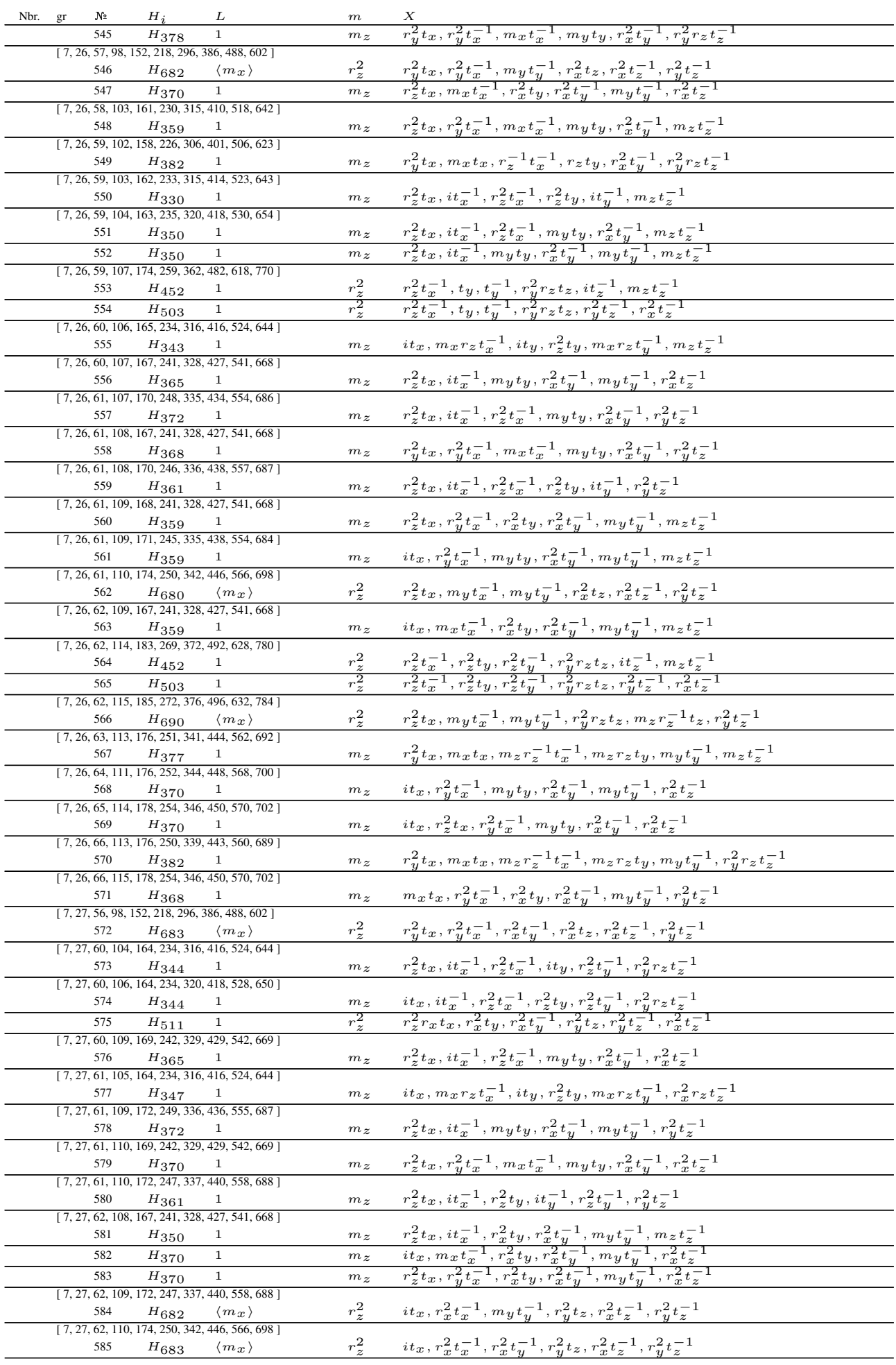




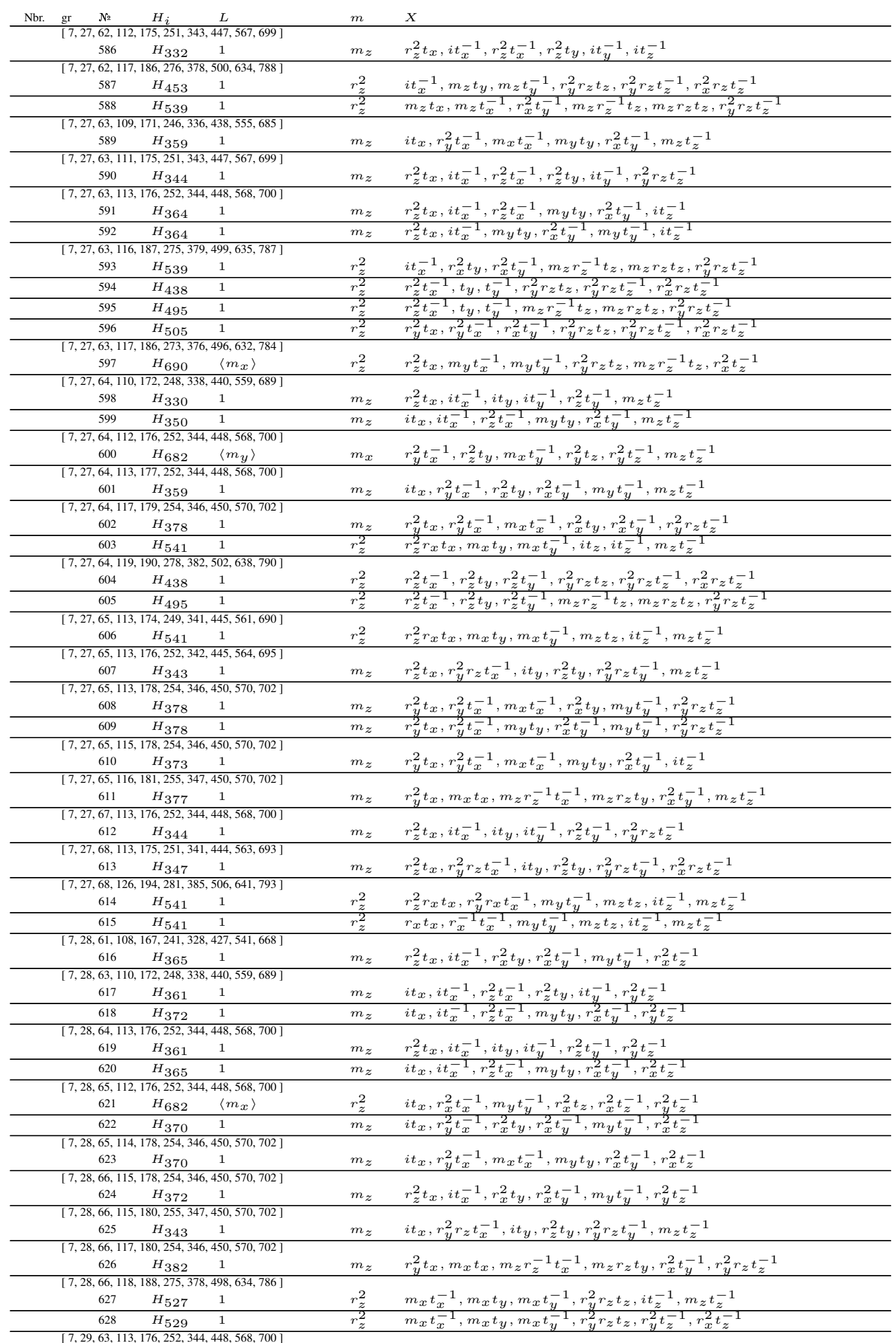




\begin{tabular}{|c|c|c|c|c|c|}
\hline \multirow[t]{2}{*}{ Nbr. } & № & $H_{i}$ & $L$ & $m$ & $x$ \\
\hline & 629 & $H_{332}$ & 1 & $m_{z}$ & $r_{z}^{2} t_{x}, i t_{x}^{-1}, i t_{y}, i t_{y}^{-1}, r_{z}^{2} t_{y}^{-1}, i t_{z}^{-1}$ \\
\hline & 630 & $H_{364}$ & 1 & $m_{z}$ & $i t_{x}, i t_{x}^{-1}, r_{z}^{2} t_{x}^{-1}, m_{y} t_{y}, r_{x}^{2} t_{y}^{-1}, i t_{z}^{-1}$ \\
\hline \multicolumn{6}{|c|}{$[7,29,64,112,176,252,344,448,568,700]$} \\
\hline & 631 & $H_{683}$ & $\left\langle m_{x}\right\rangle$ & $r_{z}^{2}$ & $i t_{x}, r_{x}^{2} t_{x}^{-1}, r_{x}^{2} t_{y}^{-1}, r_{x}^{2} t_{z}, r_{x}^{2} t_{z}^{-1}, r_{y}^{2} t_{z}^{-1}$ \\
\hline \multicolumn{6}{|c|}{$[7,29,65,113,176,251,343,447,566,697]$} \\
\hline & 632 & $H_{511}$ & 1 & $r_{z}^{2}$ & $r_{z}^{2} r_{x} t_{x}, r_{y}^{2} t_{y}, r_{y}^{2} t_{y}^{-1}, r_{x}^{2} t_{z}, r_{y}^{2} t_{z}^{-1}, r_{x}^{2} t_{z}^{-1}$ \\
\hline \multicolumn{6}{|c|}{$[7,29,65,113,176,252,344,448,568,700]$} \\
\hline & 633 & $H_{344}$ & 1 & $m_{z}$ & $r_{z}^{2} t_{x}, i t_{x}^{-1}, r_{z}^{2} t_{x}^{-1}, i t_{y}, i t_{y}^{-1}, r_{y}^{2} r_{z} t_{z}^{-1}$ \\
\hline & 634 & $H_{344}$ & 1 & $m_{z}$ & $r_{z}^{2} t_{x}, i t_{x}^{-1}, i t_{y}, r_{z}^{2} t_{y}, i t_{y}^{-1}, r_{y}^{2} r_{z} t_{z}^{-1}$ \\
\hline \multicolumn{6}{|c|}{$[7,29,65,115,178,254,346,450,570,702]$} \\
\hline & 635 & $H_{364}$ & 1 & $m_{z}$ & $r_{z}^{2} t_{x}, i t_{x}^{-1}, r_{x}^{2} t_{y}, r_{x}^{2} t_{y}^{-1}, m_{y} t_{y}^{-1}, i t_{z}^{-1}$ \\
\hline \multicolumn{6}{|c|}{$[7,29,66,115,179,254,346,450,570,702]$} \\
\hline & 636 & $H_{511}$ & 1 & $r_{z}^{2}$ & $r_{z}^{2} r_{x} t_{x}, r_{y}^{2} t_{y}, r_{y}^{2} t_{y}^{-1}, r_{y}^{2} t_{z}, r_{y}^{2} t_{z}^{-1}, r_{x}^{2} t_{z}^{-1}$ \\
\hline \multicolumn{6}{|c|}{$[7,29,66,116,178,254,346,450,570,702]$} \\
\hline & 637 & $H_{344}$ & 1 & $m_{z}$ & $i t_{x}, i t_{x}^{-1}, r_{z}^{2} t_{x}^{-1}, i t_{y}, i t_{y}^{-1}, r_{y}^{2} r_{z} t_{z}^{-1}$ \\
\hline \multirow{2}{*}{\multicolumn{6}{|c|}{$\begin{array}{c}{[7,29,67,116,179,254,346,450,570,702]} \\
638\end{array}$}} \\
\hline & 638 & $H_{347}$ & 1 & $m_{z}$ & $i t_{x}, r_{y}^{2} r_{z} t_{x}^{-1}, i t_{y}, r_{z}^{2} t_{y}, r_{y}^{2} r_{z} t_{y}^{-1}, r_{x}^{2} r_{z} t_{z}^{-1}$ \\
\hline \multicolumn{6}{|c|}{$[7,29,67,124,193,283,385,507,641,795]$} \\
\hline & 639 & $H_{539}$ & 1 & $r_{z}^{2}$ & $i t_{x}^{-1}, r_{y}^{2} t_{y}, r_{y}^{2} t_{y}^{-1}, m_{z} r_{z}^{-1} t_{z}, m_{z} r_{z} t_{z}, r_{y}^{2} r_{z} t_{z}^{-1}$ \\
\hline & 640 & $H_{505}$ & 1 & $r_{z}^{2}$ & $r_{y}^{2} t_{x}^{-1}, r_{y}^{2} t_{y}, r_{y}^{2} t_{y}^{-1}, r_{y}^{2} r_{z} t_{z}, r_{y}^{2} r_{z} t_{z}^{-1}, r_{x}^{2} r_{z} t_{z}^{-1}$ \\
\hline \multicolumn{6}{|c|}{$[7,29,68,123,194,282,386,506,642,794]$} \\
\hline & 641 & $H_{453}$ & 1 & $r_{z}^{2}$ & $i t_{x}^{-1}, i t_{y}, i t_{y}^{-1}, r_{y}^{2} r_{z} t_{z}, r_{y}^{2} r_{z} t_{z}^{-1}, r_{x}^{2} r_{z} t_{z}^{-1}$ \\
\hline & 642 & $H_{539}$ & 1 & $r_{z}^{2}$ & $i t_{x}, i t_{x}^{-1}, r_{x}^{2} t_{y}^{-1}, m_{z} r_{z}^{-1} t_{z}, m_{z} r_{z} t_{z}, r_{y}^{2} r_{z} t_{z}^{-1}$ \\
\hline & 643 & $H_{496}$ & 1 & $r_{z}^{2}$ & $m_{x} t_{x}^{-1}, m_{x} t_{y}, m_{x} t_{y}^{-1}, r_{y}^{2} r_{z} t_{z}, r_{y}^{2} r_{z} t_{z}^{-1}, r_{x}^{2} r_{z} t_{z}^{-1}$ \\
\hline & 644 & $H_{502}$ & 1 & $r_{z}^{2}$ & $m_{x} t_{x}^{-1}, m_{x} t_{y}, m_{x} t_{y}^{-1}, m_{z} r_{z}^{-1} t_{z}, m_{z} r_{z} t_{z}, r_{y}^{2} r_{z} t_{z}^{-1}$ \\
\hline \multicolumn{6}{|c|}{$[7,29,71,126,196,284,388,508,644,796]$} \\
\hline & 645 & $H_{511}$ & 1 & $r_{z}^{2}$ & $r_{x} t_{x}, r_{x}^{-1} t_{x}^{-1}, r_{x}^{2} t_{y}^{-1}, r_{y}^{2} t_{z}, r_{y}^{2} t_{z}^{-1}, r_{x}^{2} t_{z}^{-1}$ \\
\hline \multicolumn{6}{|c|}{$[7,29,71,128,196,284,388,508,644,796]$} \\
\hline \multicolumn{6}{|c|}{$[7,29,72,125,196,284,388,508,644,796]$} \\
\hline & 647 & $H_{511}$ & 1 & $r_{z}^{2}$ & $r_{z}^{2} r_{x} t_{x}, r_{y}^{2} r_{x} t_{x}^{-1}, r_{x}^{2} t_{y}^{-1}, r_{y}^{2} t_{z}, r_{y}^{2} t_{z}^{-1}, r_{x}^{2} t_{z}^{-1}$ \\
\hline \multicolumn{6}{|c|}{$[7,29,72,127,196,284,388,508,644,796]$} \\
\hline & 648 & $H_{511}$ & 1 & $r_{z}^{2}$ & $r_{z}^{2} r_{x} t_{x}, r_{y}^{2} r_{x} t_{x}^{-1}, r_{x}^{2} t_{y}^{-1}, r_{x}^{2} t_{z}, r_{y}^{2} t_{z}^{-1}, r_{x}^{2} t_{z}^{-1}$ \\
\hline \multicolumn{6}{|c|}{$[7,30,73,125,196,284,388,508,644,796]$} \\
\hline & 650 & $H_{541}$ & 1 & $r_{z}^{2}$ & $r_{x} t_{x}, r_{x}^{-1} t_{x}^{-1}, m_{y} t_{y}^{-1}, i t_{z}, i t_{z}^{-1}, m_{z} t_{z}^{-1}$ \\
\hline
\end{tabular}

$[6,18,38,66,102,146,198,258,326,402]$ $651^{*}, 652^{*}, 653^{*}, 654^{*}$

$[6,19,43,78,122,175,239,314,398,491]$ $655^{*}$,

$[6,19,43,78,123,179,246,323,411,510]$ $658^{*}$

$[6,19,43,79,127,187,259,343,439,547]$ $656^{*}$,

$[6,19,43,79,128,191,267,355,455,567]$ $657^{*}$

$[6,19,46,90,148,220,306,404,516,642]$ $659^{*}$

$[6,20,46,82,128,186,254,332,422,522]$ $660^{*}$,

$[6,20,46,84,134,194,263,343,434,535]$ $662^{*}$

$[6,20,46,84,134,196,270,356,454,564]$ $667^{*}$

$[6,20,46,84,134,196,272,362,465,582]$ $663^{*}$,

$[6,20,46,84,135,199,275,363,463,575]$ $661^{*}$,

$[6,20,47,87,139,203,279,367,467,579]$ $664^{*}, 665^{*}$,

$[6,20,47,87,140,207,287,380,487,607]$ $668^{*}$

$[6,20,48,89,140,204,281,368,468,581]$ 666

$[6,20,48,90,143,207,283,370,469,581]$ $670^{*}$

$[6,20,48,90,144,211,290,380,484,602]$ $669^{*}$

[ 6, 20, 48, 91, 147, 215, 296, 391, 499, 619] $674^{*}, 675^{*}$,

$[6,20,48,94,160,244,344,460,592,740]$ $671^{*}$

$[6,20,49,96,160,240,336,448,576,720]$ $672^{*}, 673^{*}$,

$[6,20,51,98,156,228,314,412,524,650]$ $676^{*}$,

$[6,20,51,99,161,237,327,431,549,681]$ $677^{*}$

$[6,21,49,89,141,205,281,369,469,581]$ $678^{*}$,

$[6,21,49,91,147,217,301,399,511,637]$ $681^{*}$ 


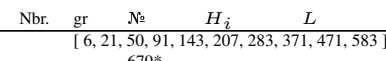
$679^{*}$,

$[6,21,50,94,150,217,298,392,499,619]$ $687^{*}$,

$[6,21,50,95,153,221,303,399,507,627]$ $688^{*}$,

$[6,21,51,93,145,211,289,376,477,591]$ 683*,

$[6,21,51,94,148,216,298,391,497,617]$

$684^{*}$,
$[6,21,51,95,151,221,306,404,516,642]$ $680^{*}$,

$[6,21,51,95,151,222,306,401,512,637]$ 686*,

$[6,21,51,96,155,227,312,410,521,645]$ $692^{*}$,

$[6,21,51,97,156,228,314,412,524,650]$ $689^{*}$,

$[6,21,52,95,148,217,298,389,496,614]$ $682^{*}$,

$[6,21,52,97,155,230,319,418,532,661]$ $685^{*}$

$[6,21,52,98,153,218,296,386,488,602]$ $694^{*}$,

$[6,21,52,98,156,228,314,412,524,650]$ $690^{*}, 691^{*}$

$[6,21,52,100,162,239,329,428,543,679]$ $695^{*}$

$[6,21,53,100,161,237,327,431,549,681]$ $693^{*}$,

$[6,21,53,104,174,263,368,488,624,776]$ $696^{*}$,

$[6,21,53,106,179,268,373,493,629,781]$ $697^{*}$

$[6,21,56,110,181,269,373,493,629,781]$ $698^{*}$,

$[6,21,56,115,188,275,383,506,640,789]$ $699^{*}$,

$[6,21,57,110,171,243,331,435,551,679$ ] $700^{*}$,

$[6,22,51,91,143,207,283,371,471,583$ $701 *$,

$[6,22,52,95,151,219,300,395,503,623]$ $705^{*}, 706^{*}$,

$[6,22,52,96,151,216,294,384,486,600]$

$[6,22,52,97,154,222,304,399,507,628]$ 703*,

$[6,22,53,97,154,224,307,403,512,634]$ $702^{*}$,

$[6,22,53,98,157,230,317,418,533,662]$ $708^{*}$,

$[6,22,53,100,159,229,314,413,525,650]$ $709^{*}$

$[6,22,53,100,163,240,331,435,553,685]$ $711^{*}$,

$[6,22,53,100,164,244,340,452,580,724]$ $712^{*}, 713^{*}$,

$[6,22,53,101,163,237,326,429,546,677]$ $710^{*}$

$[6,22,54,100,158,228,312,410,520,642]$ $717^{*}$,

$[6,22,54,100,158,230,316,414,526,652]$ $707^{*}$,

$[6,22,54,100,159,231,317,417,530,656]$ $716^{*}$,

$[6,22,54,100,161,237,327,431,549,681]$ $718^{*}$

$[6,22,54,102,165,241,331,435,553,685]$ $719^{*}$,

$[6,22,54,104,172,256,356,472,604,752]$ $720^{*}$,

$[6,22,55,102,160,232,318,416,528,654]$ $714^{*}, 715^{*}$

$[6,22,55,103,165,241,331,435,553,685]$ $725^{*}$

$[6,22,56,104,163,238,328,429,545,676]$ $723^{*}, 724^{*}$,

$[6,22,56,109,179,265,367,485,619,769]$

$722^{*}$,
$[6,22,56,109,180,268,372,492,628,780]$ $721^{*}$,

$[6,22,57,109,172,247,337,441,559,691]$ $733^{*}, 734^{*}$,

$[6,22,57,111,183,272,376,496,632,784]$ 735,

$[6,22,57,111,183,273,378,498,634,786]$ $731^{*}$

$[6,22,57,113,186,274,378,498,634,786]$ $726^{*}$,

$[6,22,58,110,173,247,335,439,556,687]$ $737^{*}$,

$[6,22,58,110,177,264,368,488,624,776]$ 


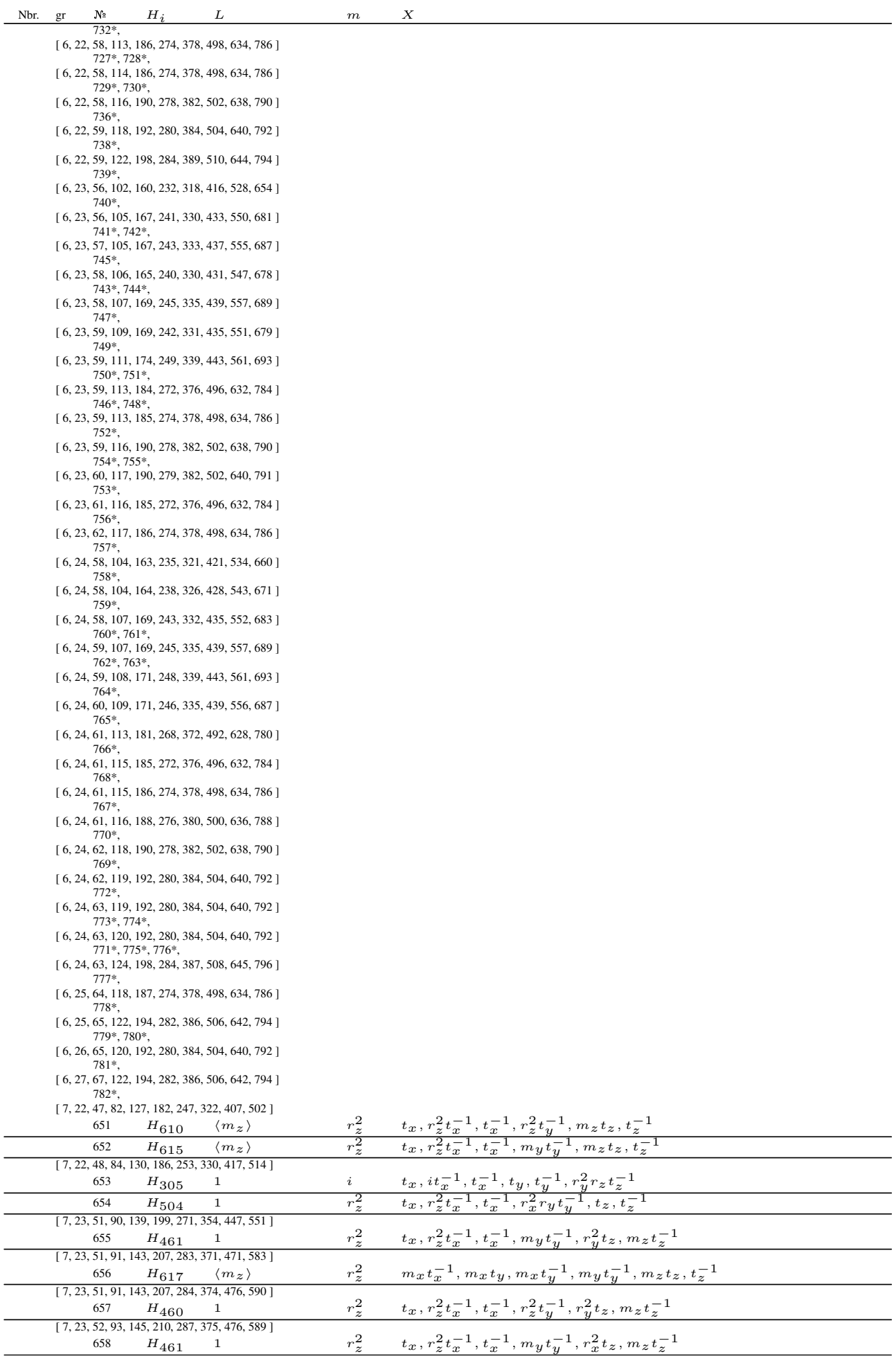




\begin{tabular}{|c|c|c|c|c|c|}
\hline Nbr. & gr № & $H_{i}$ & $L$ & $m$ & $X$ \\
\hline & $\begin{array}{r}{[7,23,54,9} \\
659 \\
\end{array}$ & $\begin{array}{c}77,231,318 \\
H_{460} \\
\end{array}$ & $\begin{array}{l}117,531,658] \\
1 \\
\end{array}$ & $r_{z}^{2}$ & $r_{z}^{2} t_{x}^{-1}, t_{y}, r_{z}^{2} t_{y}^{-1}, t_{y}^{-1}, r_{y}^{2} t_{z}, m_{z} t_{z}^{-1}$ \\
\hline & $\begin{array}{r}{[7,24,53,9} \\
660 \\
\end{array}$ & $\begin{array}{c}43,206,275 \\
H_{616}\end{array}$ & $\begin{array}{c}364,461,568] \\
\left\langle m_{z}\right\rangle\end{array}$ & $r_{z}^{2}$ & $t_{x}, r_{z}^{2} t_{x}^{-1}, t_{x}^{-1}, m_{y} t_{y}^{-1}, r_{y}^{2} t_{z}, m_{x} t_{z}^{-1}$ \\
\hline & $\begin{array}{r}{[7,24,53,9} \\
661\end{array}$ & $\begin{array}{c}47,212,290 \\
H_{614}\end{array}$ & $\begin{array}{c}\langle 80,482,596] \\
\left\langle m_{z}\right\rangle\end{array}$ & $r_{z}^{2}$ & $t_{x}, r_{z}^{2} t_{x}^{-1}, t_{x}^{-1}, r_{z}^{2} t_{y}^{-1}, r_{y}^{2} t_{z}, m_{x} t_{z}^{-1}$ \\
\hline & $\begin{array}{r}{[7,24,54,9} \\
662\end{array}$ & $\begin{array}{c}49,216,295 \\
H_{323}\end{array}$ & $\begin{array}{l}86,491,607] \\
1\end{array}$ & $i$ & $m_{z} t_{x}, r_{z}^{2} t_{x}^{-1}, m_{z} t_{x}^{-1}, r_{x}^{2} r_{y} t_{y}^{-1}, m_{x} t_{z}, m_{x} t_{z}^{-1}$ \\
\hline & $\begin{array}{r}{[7,24,54,9} \\
663 \\
\end{array}$ & $\begin{array}{c}49,217,298 \\
H_{540}\end{array}$ & $\begin{array}{l}93,502,624] \\
1\end{array}$ & $r_{z}^{2}$ & $m_{z} t_{x}, i t_{x}^{-1}, m_{z} t_{x}^{-1}, r_{x}^{2} r_{y} t_{y}^{-1}, m_{x} t_{z}, m_{x} t_{z}^{-1}$ \\
\hline & $\begin{array}{r}{[7,24,54,9} \\
664\end{array}$ & $\begin{array}{c}50,216,294 \\
H_{615}\end{array}$ & $\begin{array}{c}84,486,600] \\
\left\langle m_{z}\right\rangle\end{array}$ & $r_{z}^{2}$ & $r_{z}^{2} t_{x}^{-1}, m_{x} t_{y}, m_{x} t_{y}^{-1}, m_{y} t_{y}^{-1}, m_{z} t_{z}, t_{z}^{-1}$ \\
\hline & 665 & $H_{483}$ & 1 & $r_{z}^{2}$ & $m_{y} t_{x}, m_{x} t_{x}^{-1}, m_{y} t_{x}^{-1}, m_{y} t_{y}^{-1}, r_{y}^{2} t_{z}, m_{z} t_{z}^{-1}$ \\
\hline & $\begin{array}{r}{[7,24,55,9]} \\
666\end{array}$ & $\begin{array}{c}0,217,295 \\
H_{431}\end{array}$ & $\begin{array}{l}84,487,601] \\
1\end{array}$ & $r_{z}^{2}$ & $t_{x}, r_{z}^{2} t_{x}^{-1}, t_{x}^{-1}, r_{z}^{2} t_{y}^{-1}, m_{z} t_{z}, i t_{z}^{-1}$ \\
\hline & $\begin{array}{r}{[7,24,55,9} \\
667\end{array}$ & $\begin{array}{c}5,224,307 \\
H_{616}\end{array}$ & $\begin{array}{c}02,511,632] \\
\left\langle m_{z}\right\rangle\end{array}$ & $r_{z}^{2}$ & $t_{x}, r_{z}^{2} t_{x}^{-1}, t_{x}^{-1}, m_{y} t_{y}^{-1}, r_{x}^{2} t_{z}, m_{y} t_{z}^{-1}$ \\
\hline & $\begin{array}{r}{[7,24,55,9} \\
668\end{array}$ & $\begin{array}{l}36,227,311 \\
H_{483}\end{array}$ & $\begin{array}{l}08,519,643] \\
1\end{array}$ & $r_{z}^{2}$ & $m_{x} t_{x}^{-1}, m_{x} t_{y}, m_{x} t_{y}^{-1}, m_{y} t_{y}^{-1}, r_{y}^{2} t_{z}, m_{z} t_{z}^{-1}$ \\
\hline & $\begin{array}{r}{[7,24,56,1} \\
669 \\
\end{array}$ & $\begin{array}{c}157,226,30 \\
H_{468} \\
\end{array}$ & $\begin{array}{l}400,508,628] \\
1\end{array}$ & $r_{z}^{2}$ & $t_{x}, r_{z}^{2} t_{x}^{-1}, t_{x}^{-1}, m_{y} t_{y}^{-1}, m_{z} t_{z}, i t_{z}^{-1}$ \\
\hline & $\begin{array}{r}{[7,24,56,1} \\
670\end{array}$ & $\begin{array}{c}157,226,30 \\
H_{469}\end{array}$ & $\begin{array}{l}401,508,628] \\
1\end{array}$ & $r_{z}^{2}$ & $t_{x}, r_{z}^{2} t_{x}^{-1}, t_{x}^{-1}, m_{y} t_{y}^{-1}, r_{x}^{2} t_{z}, r_{y}^{2} t_{z}^{-1}$ \\
\hline & $\begin{array}{r}{[7,24,56,1} \\
671\end{array}$ & $\begin{array}{c}170,255,35 \\
H_{452}\end{array}$ & $\begin{array}{l}475,609,759] \\
1\end{array}$ & $r_{z}^{2}$ & $t_{x}, r_{z}^{2} t_{x}^{-1}, t_{x}^{-1}, r_{z}^{2} t_{y}^{-1}, r_{x}^{2} r_{z} t_{z}, m_{z} t_{z}^{-1}$ \\
\hline & $\begin{array}{r}7,24,56,1 \\
672\end{array}$ & $\begin{array}{c}172,256,35 \\
H_{326}\end{array}$ & $\begin{array}{l}472,604,752] \\
1\end{array}$ & $i$ & $m_{z} r_{x} t_{x}, r_{z}^{2} r_{x} t_{x}^{-1}, m_{z} r_{x} t_{x}^{-1}, m_{y} t_{y}, m_{y} t_{y}^{-1}, m_{z} t_{z}^{-1}$ \\
\hline & 673 & $H_{541}$ & 1 & $r_{z}^{2}$ & $r_{z}^{2} r_{x} t_{x}, r_{x} t_{x}, r_{x}^{-1} t_{x}^{-1}, m_{y} t_{y}^{-1}, m_{z} t_{z}, m_{z} t_{z}^{-1}$ \\
\hline & $\begin{array}{r}{[7,24,58,1} \\
674\end{array}$ & $\begin{array}{c}162,232,31 \\
H_{326}\end{array}$ & $\begin{array}{l}416,526,648] \\
1\end{array}$ & $i$ & $r_{z}^{2} r_{x} t_{x}^{-1}, m_{y} t_{y}, m_{y} t_{y}^{-1}, r_{z}^{2} t_{z}, r_{z}^{2} t_{z}^{-1}, m_{z} t_{z}^{-1}$ \\
\hline & 675 & $H_{541}$ & 1 & $r_{z}^{2}$ & $r_{z}^{2} r_{x} t_{x}, m_{x} t_{y}, m_{x} t_{y}^{-1}, m_{y} t_{y}^{-1}, m_{z} t_{z}, m_{z} t_{z}^{-1}$ \\
\hline & $\begin{array}{c}{[7,24,58,1} \\
676\end{array}$ & $\begin{array}{c}163,237,32 \\
H_{425}\end{array}$ & $\begin{array}{l}423,537,664] \\
1\end{array}$ & $r_{z}^{2}$ & $t_{x}, r_{z}^{2} t_{x}^{-1}, t_{x}^{-1}, r_{z}^{2} t_{y}^{-1}, r_{x}^{2} t_{z}, r_{y}^{2} t_{z}^{-1}$ \\
\hline & $\begin{array}{r}{[7,24,59,1} \\
677\end{array}$ & $\begin{array}{c}170,246,33 \\
H_{614}\end{array}$ & $\begin{array}{l}442,562,694] \\
\left\langle m_{z}\right\rangle\end{array}$ & $r_{z}^{2}$ & $r_{z}^{2} t_{x}^{-1}, t_{y}, r_{z}^{2} t_{y}^{-1}, t_{y}^{-1}, r_{y}^{2} t_{z}, m_{x} t_{z}^{-1}$ \\
\hline & $\begin{array}{r}{[7,25,55,9} \\
678\end{array}$ & $\begin{array}{c}51,217,295 \\
H_{618}\end{array}$ & $\begin{array}{l}\langle 85,487,601] \\
\left\langle m_{z}\right\rangle\end{array}$ & $r_{z}^{2}$ & $m_{x} t_{x}^{-1}, m_{x} t_{y}, m_{x} t_{y}^{-1}, m_{y} t_{y}^{-1}, r_{x}^{2} t_{z}, m_{y} t_{z}^{-1}$ \\
\hline & $\begin{array}{r}{[7,25,56,9} \\
679 \\
\end{array}$ & $\begin{array}{c}2,218,296 \\
H_{461}\end{array}$ & $\begin{array}{l}886,488,602] \\
1\end{array}$ & $r_{z}^{2}$ & $r_{z}^{2} t_{x}^{-1}, m_{x} t_{y}, m_{x} t_{y}^{-1}, m_{y} t_{y}^{-1}, r_{x}^{2} t_{z}, m_{z} t_{z}^{-1}$ \\
\hline & $\begin{array}{r}{[7,25,57,1} \\
680 \\
\end{array}$ & $\begin{array}{c}159,231,31 \\
H_{460} \\
\end{array}$ & $\begin{array}{l}415,529,656] \\
1\end{array}$ & $r_{z}^{2}$ & $t_{x}, r_{z}^{2} t_{x}^{-1}, t_{x}^{-1}, r_{z}^{2} t_{y}^{-1}, r_{y}^{2} t_{z}, i t_{z}^{-1}$ \\
\hline & $\begin{array}{r}{[7,25,57,1} \\
681\end{array}$ & $\begin{array}{c}163,237,32 \\
H_{618}\end{array}$ & $\begin{array}{l}427,543,673] \\
\left\langle m_{z}\right\rangle\end{array}$ & $r_{z}^{2}$ & $m_{x} t_{x}^{-1}, m_{x} t_{y}, m_{x} t_{y}^{-1}, m_{y} t_{y}^{-1}, r_{y}^{2} t_{z}, m_{x} t_{z}^{-1}$ \\
\hline & $\begin{array}{r}{[7,25,58,1} \\
682\end{array}$ & $\begin{array}{c}158,228,30 \\
H_{433}\end{array}$ & $\begin{array}{l}404,512,630] \\
1\end{array}$ & $r_{z}^{2}$ & $m_{z} t_{x}, i t_{x}^{-1}, m_{z} t_{x}^{-1}, r_{z}^{2} t_{y}^{-1}, m_{z} t_{z}, i t_{z}^{-1}$ \\
\hline & $\begin{array}{r}{[7,25,58,1} \\
683\end{array}$ & $\begin{array}{c}160,231,31 \\
H_{485}\end{array}$ & $\begin{array}{l}409,519,640] \\
1\end{array}$ & $r_{z}^{2}$ & $m_{z} t_{x}, i t_{x}^{-1}, m_{z} t_{x}^{-1}, m_{y} t_{y}^{-1}, r_{x}^{2} t_{z}, r_{y}^{2} t_{z}^{-1}$ \\
\hline & $\begin{array}{r}{[7,25,58,1} \\
684 \\
\end{array}$ & $\begin{array}{c}159,230,31 \\
H_{461}\end{array}$ & $\begin{array}{l}408,517,639] \\
1\end{array}$ & $r_{z}^{2}$ & $t_{x}, r_{z}^{2} t_{x}^{-1}, t_{x}^{-1}, m_{y} t_{y}^{-1}, r_{y}^{2} t_{z}, i t_{z}^{-1}$ \\
\hline & $\begin{array}{r}{[7,25,58,1} \\
685\end{array}$ & $\begin{array}{c}163,238,32 \\
H_{462}\end{array}$ & $\begin{array}{l}428,545,673] \\
1\end{array}$ & $r_{z}^{2}$ & $m_{z} t_{x}, i t_{x}^{-1}, m_{z} t_{x}^{-1}, r_{z}^{2} t_{y}^{-1}, r_{x}^{2} t_{z}, r_{y}^{2} t_{z}^{-1}$ \\
\hline & $\begin{array}{r}{[7,25,58,1} \\
686\end{array}$ & $\begin{array}{c}164,237,32 \\
H_{475}\end{array}$ & $\begin{array}{l}423,537,662] \\
1\end{array}$ & $r_{z}^{2}$ & $m_{z} t_{x}, i t_{x}^{-1}, m_{z} t_{x}^{-1}, m_{y} t_{y}^{-1}, m_{z} t_{z}, i t_{z}^{-1}$ \\
\hline & $\begin{array}{r}{[7,25,58,1} \\
687\end{array}$ & $\begin{array}{c}163,234,31 \\
H_{433}\end{array}$ & $\begin{array}{l}416,527,650] \\
1\end{array}$ & $r_{z}^{2}$ & $i t_{x}^{-1}, t_{y}, r_{z}^{2} t_{y}^{-1}, t_{y}^{-1}, m_{z} t_{z}, i t_{z}^{-1}$ \\
\hline & $\begin{array}{r}{[7,25,58,1} \\
688\end{array}$ & $\begin{array}{c}165,235,31 \\
H_{466}\end{array}$ & $\begin{array}{l}417,529,651] \\
1\end{array}$ & $r_{z}^{2}$ & $t_{x}, r_{z}^{2} t_{x}^{-1}, t_{x}^{-1}, r_{x}^{2} t_{y}^{-1}, m_{z} t_{z}, i t_{z}^{-1}$ \\
\hline & $\begin{array}{r}{[7,25,58,1} \\
689\end{array}$ & $\begin{array}{c}165,239,32 \\
H_{482}\end{array}$ & $\begin{array}{l}425,539,666] \\
1\end{array}$ & $r_{z}^{2}$ & $m_{x} t_{x}^{-1}, m_{x} t_{y}, m_{x} t_{y}^{-1}, m_{y} t_{y}^{-1}, r_{x}^{2} t_{z}, r_{y}^{2} t_{z}^{-1}$ \\
\hline & $\begin{array}{r}{[7,25,59,1} \\
690\end{array}$ & $\begin{array}{c}165,239,32 \\
H_{461}\end{array}$ & $\begin{array}{l}425,539,666] \\
1\end{array}$ & $r_{z}^{2}$ & $r_{z}^{2} t_{x}^{-1}, m_{x} t_{y}, m_{x} t_{y}^{-1}, m_{y} t_{y}^{-1}, r_{y}^{2} t_{z}, m_{z} t_{z}^{-1}$ \\
\hline & 691 & $H_{481}$ & 1 & $r_{z}^{2}$ & $m_{x} t_{x}^{-1}, m_{x} t_{y}, m_{x} t_{y}^{-1}, m_{y} t_{y}^{-1}, m_{z} t_{z}, i t_{z}^{-1}$ \\
\hline & $\begin{array}{r}{[7,25,59,1} \\
692\end{array}$ & $\begin{array}{c}167,240,32 \\
H_{461}\end{array}$ & $\begin{array}{l}426,540,666] \\
1\end{array}$ & $r_{z}^{2}$ & $t_{x}, r_{z}^{2} t_{x}^{-1}, t_{x}^{-1}, m_{y} t_{y}^{-1}, r_{x}^{2} t_{z}, i t_{z}^{-1}$ \\
\hline & $\begin{array}{r}{[7,25,60,1} \\
693\end{array}$ & $\begin{array}{c}169,244,33 \\
H_{460}\end{array}$ & $\begin{array}{l}440,560,692] \\
1\end{array}$ & $r_{z}^{2}$ & $r_{z}^{2} t_{x}^{-1}, t_{y}, r_{z}^{2} t_{y}^{-1}, t_{y}^{-1}, r_{y}^{2} t_{z}, i t_{z}^{-1}$ \\
\hline & $\begin{array}{r}{[7,25,60,1} \\
694\end{array}$ & $\begin{array}{c}165,236,32 \\
H_{427}\end{array}$ & $\begin{array}{l}418,529,652] \\
1\end{array}$ & $r_{z}^{2}$ & $t_{x}, r_{z}^{2} t_{x}^{-1}, t_{x}^{-1}, r_{x}^{2} t_{y}^{-1}, r_{x}^{2} t_{z}, r_{y}^{2} t_{z}^{-1}$ \\
\hline & $\begin{array}{r}{[7,25,60,1} \\
695 \\
\end{array}$ & $\begin{array}{c}171,249,34 \\
H_{462} \\
\end{array}$ & $\begin{array}{l}441,563,699] \\
1\end{array}$ & $r_{z}^{2}$ & $i t_{x}^{-1}, t_{y}, r_{z}^{2} t_{y}^{-1}, t_{y}^{-1}, r_{x}^{2} t_{z}, r_{y}^{2} t_{z}^{-1}$ \\
\hline & $\begin{array}{r}{[7,25,60,1} \\
696 \\
\end{array}$ & $\begin{array}{c}82,270,37 \\
H_{527} \\
\end{array}$ & $\begin{array}{l}494,630,782] \\
1\end{array}$ & $r_{z}^{2}$ & $m_{x} t_{x}^{-1}, m_{x} t_{y}, m_{y} t_{y}, m_{x} t_{y}^{-1}, r_{y}^{2} r_{z} t_{z}, m_{z} t_{z}^{-1}$ \\
\hline & $\begin{array}{r}{[7,25,60,1} \\
697\end{array}$ & $\begin{array}{c}182,270,37 \\
H_{503}\end{array}$ & $\begin{array}{l}494,631,782] \\
1\end{array}$ & $r_{z}^{2}$ & $t_{x}, r_{z}^{2} t_{x}^{-1}, t_{x}^{-1}, r_{z}^{2} t_{y}^{-1}, r_{x}^{2} r_{z} t_{z}, r_{y}^{2} t_{z}^{-1}$ \\
\hline
\end{tabular}




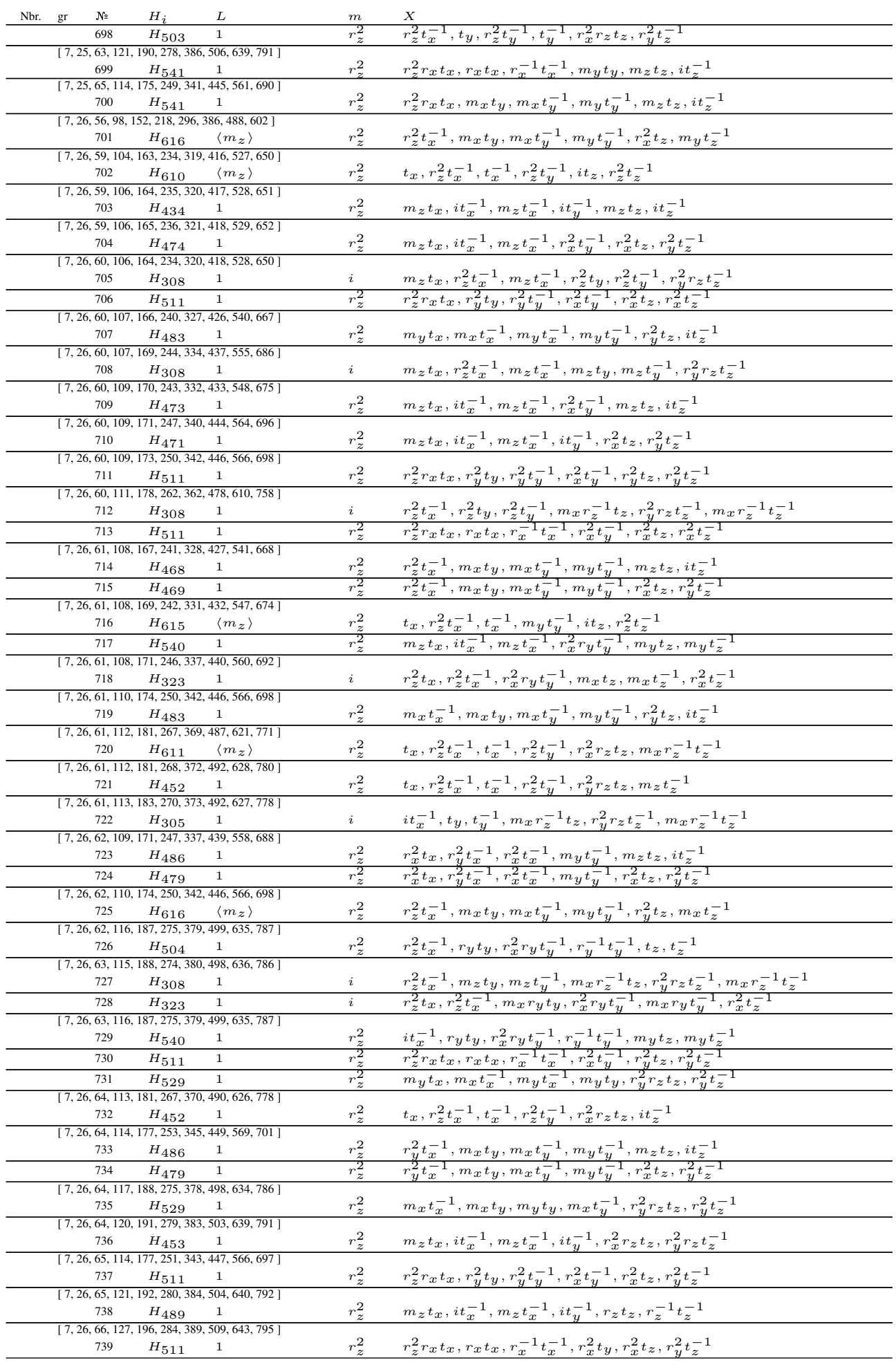




\begin{tabular}{|c|c|c|c|c|c|}
\hline \multirow[t]{2}{*}{ Nbr. } & gr № & $H_{i}$ & $L$ & $m$ & \multirow[t]{2}{*}{$x$} \\
\hline & \multicolumn{3}{|c|}{$[7,27,61,108,167,241,328,427,541,668]$} & $r_{z}^{2}$ & \\
\hline & {$[7,27,62,1$} & $75,251,34$ & $447,567,699]$ & & \\
\hline & 741 & $H_{477}$ & 1 & $r_{z}^{2}$ & $r_{y}^{2} t_{x}^{-1}, r_{y}^{2} t_{y}, r_{y}^{2} t_{y}^{-1}, r_{x}^{2} t_{y}^{-1}, m_{z} t_{z}, i t_{z}^{-1}$ \\
\hline & 742 & $H_{429}$ & 1 & $r_{z}^{2}$ & $r_{y}^{2} t_{x}^{-1}, r_{y}^{2} t_{y}, r_{y}^{2} t_{y}^{-1}, r_{x}^{2} t_{y}^{-1}, r_{x}^{2} t_{z}, r_{y}^{2} t_{z}^{-1}$ \\
\hline & {$[7,27,63,1$} & $72,248,33$ & $440,559,689]$ & & \\
\hline & 743 & $H_{466}$ & 1 & $r_{z}^{2}$ & $r_{z}^{2} t_{x}^{-1}, r_{y}^{2} t_{y}, r_{y}^{2} t_{y}^{-1}, r_{x}^{2} t_{y}^{-1}, m_{z} t_{z}, i t_{z}^{-1}$ \\
\hline & 744 & $H_{427}$ & 1 & $r_{z}^{2}$ & $r_{z}^{2} t_{x}^{-1}, r_{y}^{2} t_{y}, r_{y}^{2} t_{y}^{-1}, r_{x}^{2} t_{y}^{-1}, r_{x}^{2} t_{z}, r_{y}^{2} t_{z}^{-1}$ \\
\hline & {$[7,27,63,1$} & $75,251,34$ & $447,567,699]$ & & \\
\hline & 745 & $H_{617}$ & $\left\langle m_{z}\right\rangle$ & $r_{z}^{2}$ & $m_{x} t_{x}^{-1}, m_{x} t_{y}, m_{x} t_{y}^{-1}, m_{y} t_{y}^{-1}, i t_{z}, r_{z}^{2} t_{z}^{-1}$ \\
\hline & {$[7,27,63,1$} & $85,272,37$ & $496,632,784]$ & & \\
\hline & 746 & $H_{503}$ & 1 & $r_{z}^{2}$ & $t_{x}, r_{z}^{2} t_{x}^{-1}, t_{x}^{-1}, r_{z}^{2} t_{y}^{-1}, r_{y}^{2} r_{z} t_{z}, r_{y}^{2} t_{z}^{-1}$ \\
\hline & {$[7,27,64,1$} & $76,252,34$ & $448,568,700]$ & & \\
\hline & 747 & $H_{461}$ & 1 & $r_{z}^{2}$ & $r_{z}^{2} t_{x}^{-1}, m_{x} t_{y}, m_{x} t_{y}^{-1}, m_{y} t_{y}^{-1}, r_{y}^{2} t_{z}, i t_{z}^{-1}$ \\
\hline & {$[7,27,64,1$} & $87,274,37$ & $498,634,786]$ & & \\
\hline & 748 & $H_{527}$ & 1 & $r_{z}^{2}$ & $m_{x} t_{x}^{-1}, m_{x} t_{y}, m_{x} t_{y}^{-1}, m_{y} t_{y}^{-1}, r_{y}^{2} r_{z} t_{z}, m_{z} t_{z}^{-1}$ \\
\hline & {$[7,27,65,1$} & $74,249,34$ & $445,561,690]$ & & \\
\hline & 749 & $H_{541}$ & 1 & $r_{z}^{2}$ & $r_{z}^{2} r_{x} t_{x}, m_{x} t_{y}, m_{y} t_{y}, m_{x} t_{y}^{-1}, m_{z} t_{z}, i t_{z}^{-1}$ \\
\hline & {$[7,27,65,1$} & $78,254,34$ & $450,570,702]$ & & \\
\hline & 750 & $H_{475}$ & 1 & $r_{z}^{2}$ & $i t_{x}^{-1}, m_{x} t_{y}, m_{x} t_{y}^{-1}, m_{y} t_{y}^{-1}, m_{z} t_{z}, i t_{z}^{-1}$ \\
\hline & 751 & $H_{485}$ & 1 & $r_{z}^{2}$ & $i t_{x}^{-1}, m_{x} t_{y}, m_{x} t_{y}^{-1}, m_{y} t_{y}^{-1}, r_{x}^{2} t_{z}, r_{y}^{2} t_{z}^{-1}$ \\
\hline & {$[7,27,65,1$} & $91,279,38$ & $503,639,791]$ & & \\
\hline & 752 & $H_{622}$ & $\left\langle m_{z}\right\rangle$ & $r_{z}^{2}$ & $m_{x} t_{x}^{-1}, m_{x} t_{y}, m_{y} t_{y}, m_{x} t_{y}^{-1}, r_{y}^{2} r_{z} t_{z}, m_{x} r_{z} t_{z}^{-1}$ \\
\hline & {$[7,27,65,1$} & $92,280,38$ & $505,640,792]$ & & \\
\hline & 753 & $H_{541}$ & 1 & $r_{z}^{2}$ & $r_{z}^{2} r_{x} t_{x}, r_{x} t_{x}, r_{x}^{-1} t_{x}^{-1}, m_{y} t_{y}^{-1}, m_{z} t_{z}, i t_{z}^{-1}$ \\
\hline & {$[7,27,65,1$} & $93,281,38$ & $505,641,793]$ & & \\
\hline & 754 & $H_{505}$ & 1 & $r_{z}^{2}$ & $r_{y}^{2} t_{x}^{-1}, r_{y}^{2} t_{y}, r_{y}^{2} t_{y}^{-1}, r_{x}^{2} t_{y}^{-1}, r_{x}^{2} r_{z} t_{z}, r_{y}^{2} r_{z} t_{z}^{-1}$ \\
\hline & 755 & $H_{506}$ & 1 & $r_{z}^{2}$ & $r_{y}^{2} t_{x}^{-1}, r_{y}^{2} t_{y}, r_{y}^{2} t_{y}^{-1}, r_{x}^{2} t_{y}^{-1}, r_{z} t_{z}, r_{z}^{-1} t_{z}^{-1}$ \\
\hline & {$[7,27,66,1$} & $86,273,37$ & $496,632,784]$ & & \\
\hline & 756 & $H_{503}$ & 1 & $r_{z}^{2}$ & $r_{z}^{2} t_{x}^{-1}, t_{y}, r_{z}^{2} t_{y}^{-1}, t_{y}^{-1}, r_{y}^{2} r_{z} t_{z}, r_{y}^{2} t_{z}^{-1}$ \\
\hline & {$[7,27,68,1$} & $89,276,37$ & $499,635,787]$ & & \\
\hline & 757 & $H_{527}$ & 1 & $r_{z}^{2}$ & $m_{x} t_{x}^{-1}, m_{x} t_{y}, m_{y} t_{y}, m_{x} t_{y}^{-1}, r_{y}^{2} r_{z} t_{z}, i t_{z}^{-1}$ \\
\hline & {$[7,28,63,1$} & $71,244,33$ & $434,549,676]$ & & \\
\hline & 758 & $H_{305}$ & 1 & $i$ & $t_{x}, i t_{x}^{-1}, t_{x}^{-1}, i t_{y}, i t_{y}^{-1}, r_{y}^{2} r_{z} t_{z}^{-1}$ \\
\hline & {$[7,28,63,1$} & $73,248,33$ & $442,559,688]$ & & \\
\hline & 759 & $H_{504}$ & 1 & $r_{z}^{2}$ & $t_{x}, r_{z}^{2} t_{x}^{-1}, t_{x}^{-1}, r_{x}^{2} r_{y} t_{y}^{-1}, r_{z}^{2} t_{z}, r_{z}^{2} t_{z}^{-1}$ \\
\hline & {$[7,28,63,1$} & $76,252,34$ & $448,568,700]$ & & \\
\hline & 760 & $H_{473}$ & 1 & $r_{z}^{2}$ & $i t_{x}^{-1}, r_{y}^{2} t_{y}, r_{y}^{2} t_{y}^{-1}, r_{x}^{2} t_{y}^{-1}, m_{z} t_{z}, i t_{z}^{-1}$ \\
\hline & 761 & $H_{474}$ & 1 & $r_{z}^{2}$ & $i t_{x}^{-1}, r_{y}^{2} t_{y}, r_{y}^{2} t_{y}^{-1}, r_{x}^{2} t_{y}^{-1}, r_{x}^{2} t_{z}, r_{y}^{2} t_{z}^{-1}$ \\
\hline & {$[7,28,64,1$} & $76,252,34$ & $448,568,700]$ & & \\
\hline & 762 & $H_{615}$ & $\left\langle m_{z}\right\rangle$ & $r_{z}^{2}$ & $r_{z}^{2} t_{x}^{-1}, m_{x} t_{y}, m_{x} t_{y}^{-1}, m_{y} t_{y}^{-1}, i t_{z}, r_{z}^{2} t_{z}^{-1}$ \\
\hline & 763 & $H_{326}$ & 1 & $\underline{i}$ & $r_{z}^{2} r_{x} t_{x}^{-1}, r_{y}^{2} t_{y}, r_{y}^{2} t_{y}^{-1}, m_{y} t_{y}^{-1}, r_{z}^{2} t_{z}, r_{z}^{2} t_{z}^{-1}$ \\
\hline & {$[7,28,64,1$} & $77,254,34$ & $450,570,702]$ & & \\
\hline & 764 & $H_{541}$ & 1 & $r_{z}^{2}$ & $r_{z}^{2} r_{x} t_{x}, m_{x} t_{y}, m_{x} t_{y}^{-1}, m_{y} t_{y}^{-1}, i t_{z}, i t_{z}^{-1}$ \\
\hline & $\begin{array}{r}{[7,28,65,1} \\
765\end{array}$ & $\begin{array}{c}76,251,34 \\
H_{511}\end{array}$ & $\begin{array}{c}447,566,697] \\
1\end{array}$ & $r^{2}$ & $r^{2} r_{x} t_{x}, r^{2} t_{\psi}, r^{2} t_{y}, r^{2} t^{-1}, r^{2} t_{z}, r^{2} t^{-1}$ \\
\hline & {$[7,28,65,1$} & $\frac{11511}{83,269,37}$ & $\frac{1}{492,628,780]}$ & & \\
\hline & 766 & $H_{452}$ & 1 & $r_{z}^{2}$ & $t_{x}, r_{z}^{2} t_{x}^{-1}, t_{x}^{-1}, r_{z}^{2} t_{y}^{-1}, r_{y}^{2} r_{z} t_{z}, i t_{z}^{-1}$ \\
\hline & {$[7,28,65,1$} & $88,275,37$ & $499,635,787]$ & & \\
\hline & 767 & $H_{529}$ & 1 & $r_{z}^{2}$ & $m_{y} t_{x}, m_{x} t_{x}^{-1}, m_{y} t_{x}^{-1}, m_{y} t_{y}^{-1}, r_{y}^{2} r_{z} t_{z}, r_{y}^{2} t_{z}^{-1}$ \\
\hline & {$[7,28,66,1$} & $88,275,37$ & $498,634,786]$ & & \\
\hline & 768 & $H_{529}$ & 1 & $r_{z}^{2}$ & $m_{x} t_{x}^{-1}, m_{x} t_{y}, m_{x} t_{y}^{-1}, m_{y} t_{y}^{-1}, r_{y}^{2} r_{z} t_{z}, r_{y}^{2} t_{z}^{-1}$ \\
\hline & {$[7,28,66,1$} & $91,279,38$ & $503,639,791]$ & & $r^{2} t^{-1} t^{-1} r^{2} t^{-1} r^{2} r r t r^{2} r-t^{-1}$ \\
\hline & 769 & $H_{438}$ & 1 & $r_{z}^{2}$ & \\
\hline & $\begin{array}{r}{[7,28,66,1} \\
770\end{array}$ & $\begin{array}{c}92,280,38 \\
H_{323}\end{array}$ & $\begin{array}{l}504,640,792] \\
1\end{array}$ & $i$ & $r_{z}^{2} t_{x}^{-1}, m_{x} r_{y} t_{y}, r_{x}^{2} r_{y} t_{u}^{-1}, m_{x} r_{y} t_{u}^{-1}, m_{x} t_{z}, m_{x} t_{z}^{-1}$ \\
\hline & {$[7,28,67,1$} & $\frac{323}{92,280,38}$ & $504,640,792]$ & & \\
\hline & 771 & $H_{446}$ & 1 & $r_{z}^{2}$ & $t_{x}, r_{z}^{2} t_{x}^{-1}, t_{x}^{-1}, r_{z}^{2} t_{y}^{-1}, r_{z} t_{z}, r_{z}^{-1} t_{z}^{-1}$ \\
\hline & {$[7,28,67,1$} & $94,282,38$ & $506,642,794]$ & & \\
\hline & 772 & $H_{540}$ & 1 & $r_{z}^{2}$ & $i t_{x}^{-1}, r_{y} t_{y}, r_{x}^{2} r_{y} t_{y}^{-1}, r_{y}^{-1} t_{y}^{-1}, m_{x} t_{z}, m_{x} t_{z}^{-1}$ \\
\hline & {$[7,28,68,1$} & $95,281,38$ & $505,643,793]$ & & \\
\hline & 773 & $H_{305}$ & 1 & $i$ & $i t_{x}^{-1}, i t_{y}, i t_{y}^{-1}, m_{x} r_{z}^{-1} t_{z}, r_{y}^{2} r_{z} t_{z}^{-1}, m_{x} r_{z}^{-1} t_{z}^{-1}$ \\
\hline & 774 & $H_{326}$ & 1 & $i$ & $m_{z} r_{x} t_{x}, r_{z}^{2} r_{x} t_{x}^{-1}, m_{z} r_{x} t_{x}^{-1}, m_{y} t_{y}^{-1}, r_{z}^{2} t_{z}, r_{z}^{2} t_{z}^{-1}$ \\
\hline & {$[7,28,68,1$} & $94,282,38$ & $506,642,794]$ & & \\
\hline & 775 & $H_{504}$ & 1 & $r_{z}^{2}$ & $r_{z}^{2} t_{x}^{-1}, r_{y} t_{y}, r_{x}^{2} r_{y} t_{y}^{-1}, r_{y}^{-1} t_{y}^{-1}, r_{z}^{2} t_{z}, r_{z}^{2} t_{z}^{-1}$ \\
\hline & 776 & $H_{541}$ & 1 & $r_{z}^{2}$ & $r_{z}^{2} r_{x} t_{x}, r_{x} t_{x}, r_{x}^{-1} t_{x}^{-1}, m_{y} t_{y}^{-1}, i t_{z}, i t_{z}^{-1}$ \\
\hline & $\begin{array}{r}{[7,28,68,1} \\
777\end{array}$ & $\begin{array}{c}96,284,38 \\
H_{511}\end{array}$ & $\begin{array}{l}509,644,796] \\
1\end{array}$ & $r_{z}^{2}$ & $r_{z}^{2} r_{x} t_{x}, r_{x} t_{x}, r_{x}^{-1} t_{x}^{-1}, r_{x}^{2} t_{u}^{-1}, r_{x}^{2} t_{z}, r_{u}^{2} t_{z}^{-1}$ \\
\hline & {$[7,29,68,1$} & $89,276,37$ & $499,635,787]$ & & \\
\hline & 778 & $H_{527}$ & 1 & $r_{z}^{2}$ & $m_{x} t_{x}^{-1}, m_{x} t_{y}, m_{x} t_{y}^{-1}, m_{y} t_{y}^{-1}, r_{y}^{2} r_{z} t_{z}, i t_{z}^{-1}$ \\
\hline & {$[7,29,69,1$} & $95,283,38$ & $507,643,795]$ & & \\
\hline & 779 & $H_{496}$ & 1 & $r_{z}^{2}$ & $m_{x} t_{x}^{-1}, m_{x} t_{y}, m_{x} t_{y}^{-1}, m_{y} t_{y}^{-1}, r_{x}^{2} r_{z} t_{z}, r_{y}^{2} r_{z} t_{z}^{-1}$ \\
\hline & 780 & $H_{514}$ & 1 & $r_{z}^{2}$ & $m_{x} t_{x}^{-1}, m_{x} t_{y}, m_{x} t_{y}^{-1}, m_{y} t_{y}^{-1}, r_{z} t_{z}, r_{z}^{-1} t_{z}^{-1}$ \\
\hline
\end{tabular}




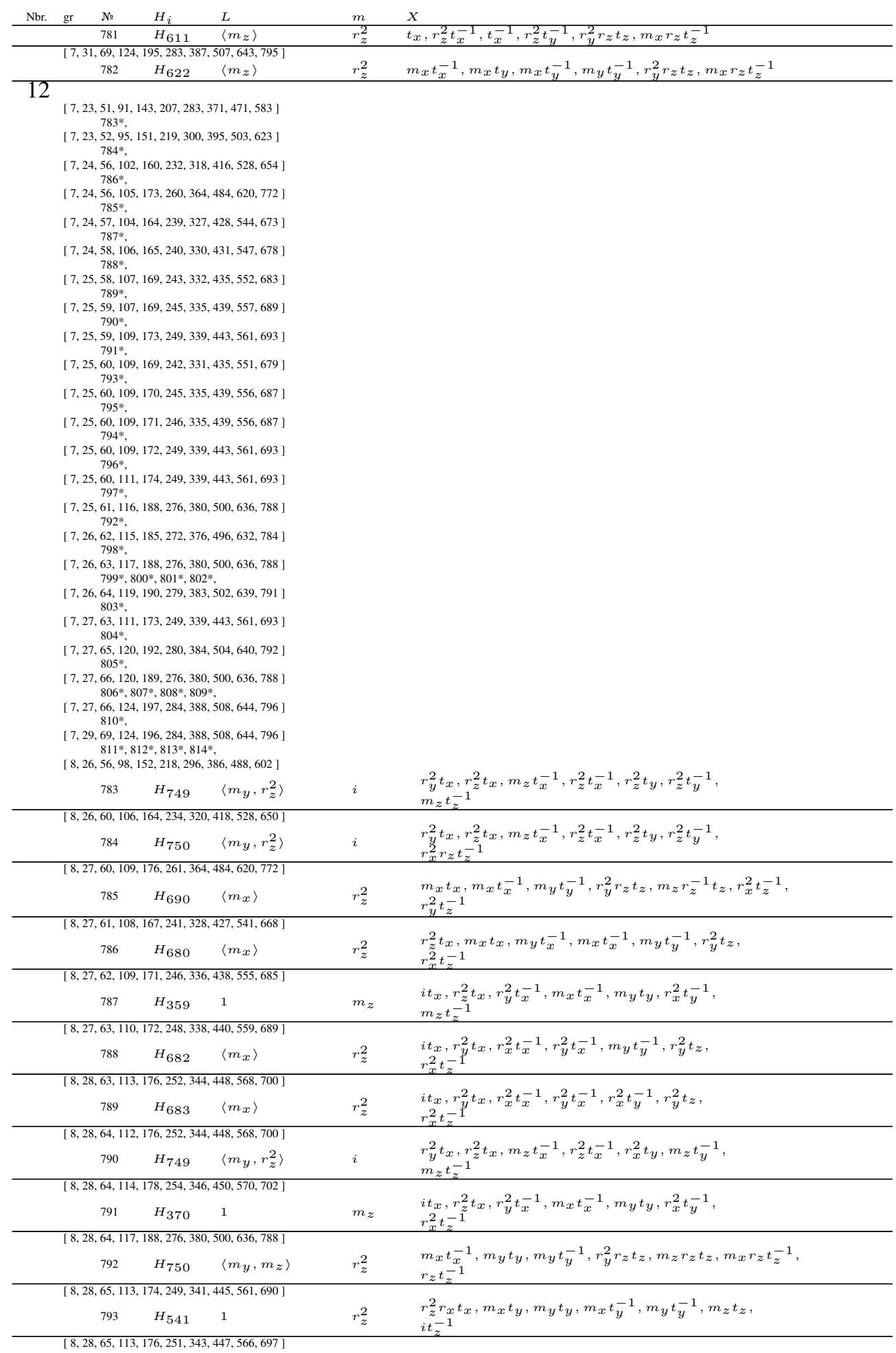




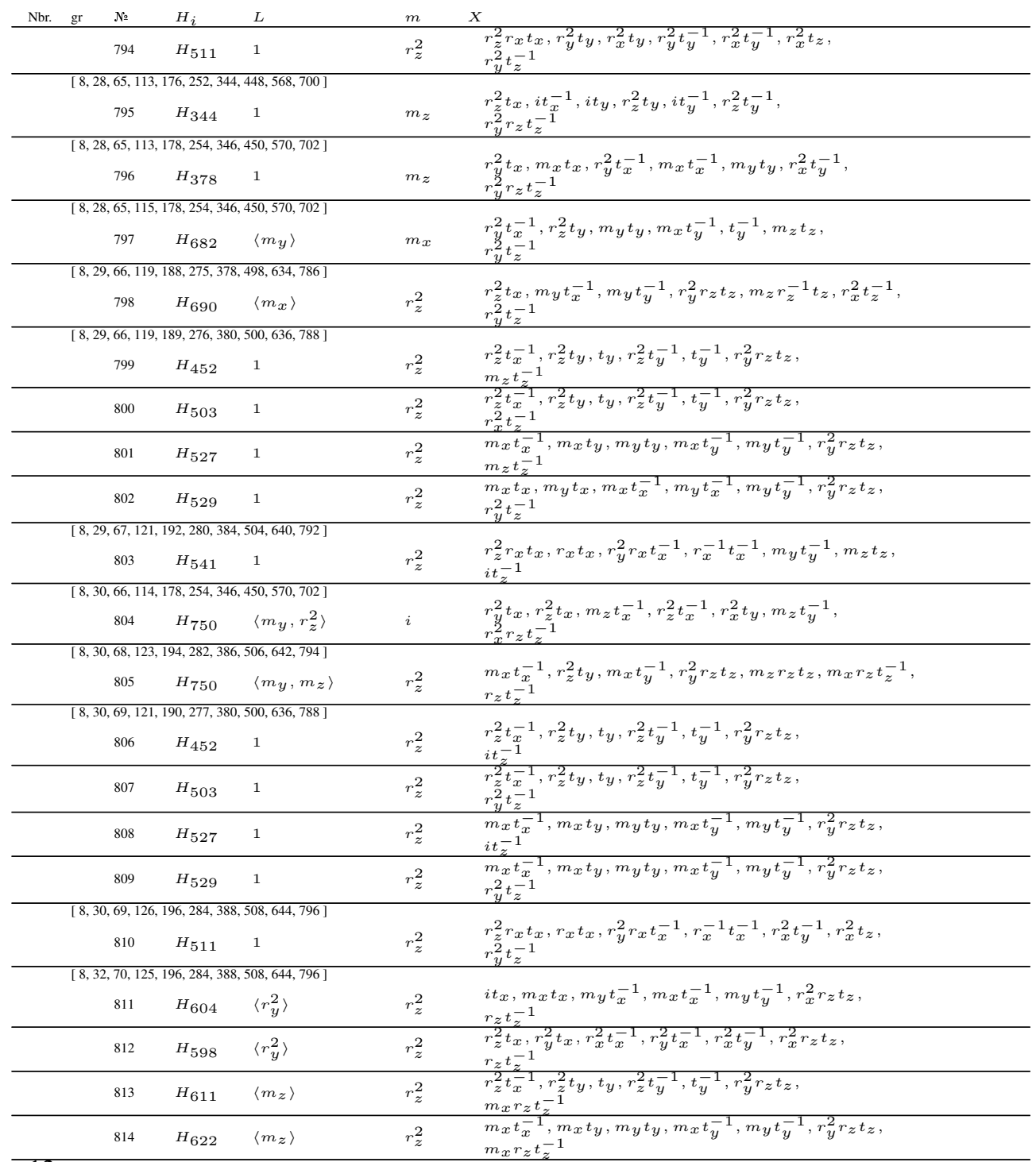

$[6,19,44,83,134,196,272,361,460,573]$ $816^{*}$,

$[6,19,44,84,138,201,272,357,460,577]$ $815^{*}$,

$[6,20,45,78,121,176,238,310,395,486]$ $817^{*}, 818^{*}$,

$[6,20,45,80,125,180,245,320,405,500]$ $819^{*}, 820^{*}$

$[6,20,47,85,132,191,261,340,431,533]$ $821^{*}$,

$[6,20,47,87,137,195,263,343,434,535]$ $825^{*}$,

$[6,20,47,87,139,203,280,370,471,585]$ $823^{*}$,

$[6,20,47,87,141,211,292,382,486,602]$ $822^{*}$

$[6,20,47,87,141,211,297,397,509,635]$ $826^{*}$,

$[6,20,47,89,147,216,292,384,496,615]$ $824^{*}$,

$[6,20,49,95,154,221,296,386,492,607]$

$[6,20,52,105,170,243,329,429,544,675]$ 


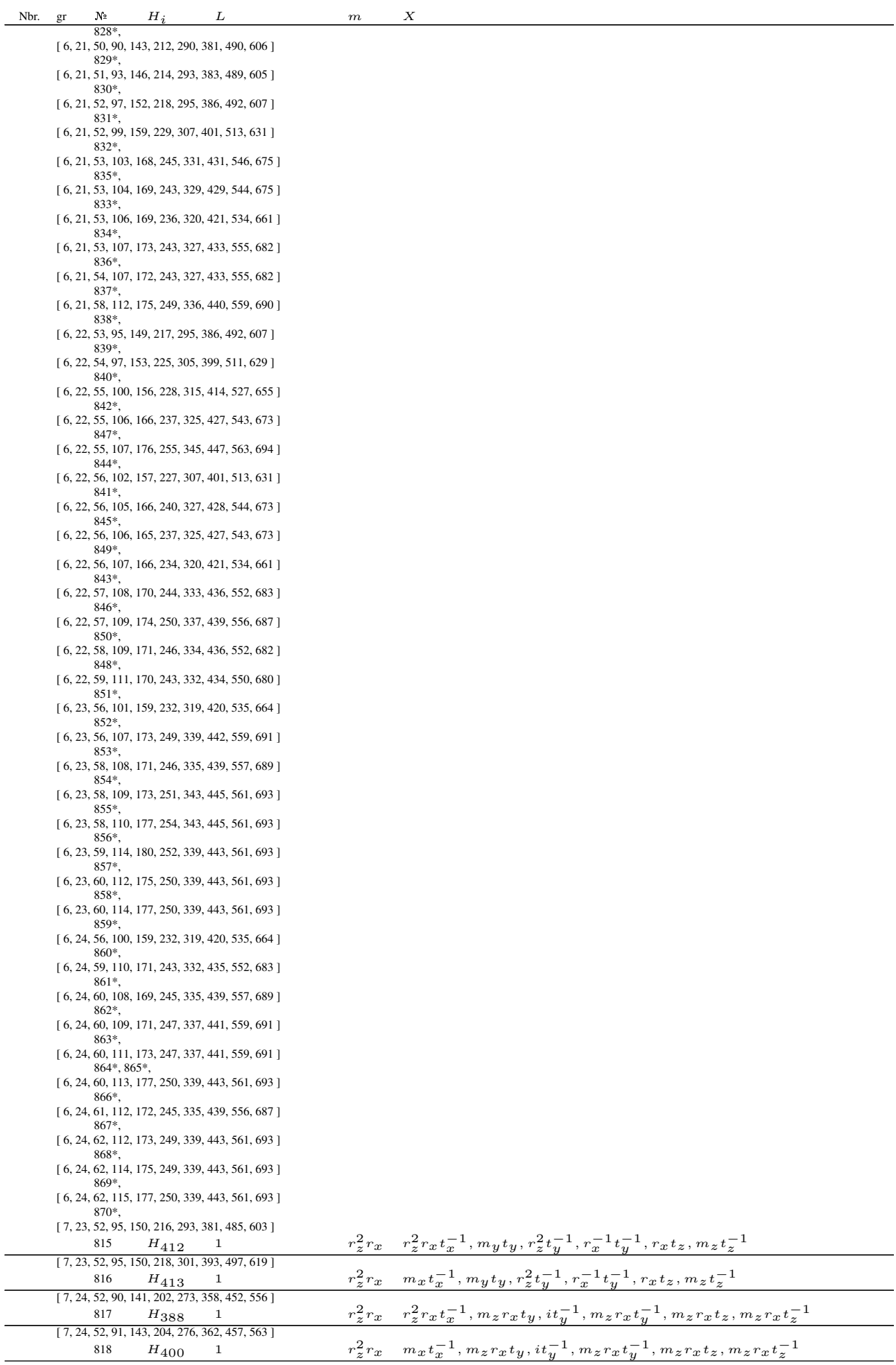




\begin{tabular}{|c|c|c|c|c|c|}
\hline \multirow[t]{2}{*}{ Nbr. } & \multicolumn{3}{|c|}{$\begin{array}{l}\text { gr } \quad \text { № } \quad H_{i} \\
{[7,24,53,93,145,208,283,369,467,576]}\end{array}$} & $m$ & $X$ \\
\hline & $\begin{array}{r}{[7,24,53,9} \\
819\end{array}$ & $\begin{array}{c}5,208,283 \\
H_{389}\end{array}$ & $\begin{array}{l}69,467,576] \\
1\end{array}$ & $r_{z}^{2} r_{x}$ & $i t_{x}^{-1}, m_{z} r_{x} t_{y}, i t_{y}^{-1}, m_{z} r_{x} t_{y}^{-1}, m_{z} r_{x} t_{z}, m_{z} r_{x} t_{z}^{-1}$ \\
\hline & \multicolumn{3}{|c|}{$[7,24,53,94,147,210,287,375,474,584]$} & & \\
\hline & 820 & $H_{398}$ & 1 & $r_{z}^{2} r_{x}$ & $r_{y}^{2} r_{x} t_{x}^{-1}, m_{z} r_{x} t_{y}, i t_{y}^{-1}, m_{z} r_{x} t_{y}^{-1}, m_{z} r_{x} t_{z}, m_{z} r_{x} t_{z}^{-1}$ \\
\hline & \multicolumn{3}{|c|}{$[7,24,54,95,148,213,288,376,477,588]$} & & \\
\hline & 821 & $H_{418}$ & 1 & $r_{z}^{2} r_{x}$ & $m_{x} t_{x}^{-1}, r_{x}^{-1} t_{y}, r_{z}^{2} t_{y}^{-1}, r_{x}^{-1} t_{y}^{-1}, r_{x} t_{z}, r_{x} t_{z}^{-1}$ \\
\hline & \multicolumn{3}{|c|}{$[7,24,54,96,152,221,301,395,501,618]$} & & \\
\hline & 822 & $H_{407}$ & 1 & $r_{z}^{2} r_{x}$ & $r_{z}^{2} r_{x} t_{x}^{-1}, r_{x}^{-1} t_{y}, r_{z}^{2} t_{y}^{-1}, r_{x}^{-1} t_{y}^{-1}, r_{x} t_{z}, r_{x} t_{z}^{-1}$ \\
\hline & \multicolumn{3}{|c|}{$[7,24,54,98,154,222,307,400,504,630]$} & & \\
\hline & 823 & $H_{395}$ & 1 & $r_{z}^{2} r_{x}$ & $m_{x} t_{x}^{-1}, r_{x}^{2} t_{y}, i t_{y}^{-1}, m_{z} r_{x} t_{y}^{-1}, m_{z} r_{x} t_{z}, r_{x}^{2} t_{z}^{-1}$ \\
\hline & \multicolumn{3}{|c|}{$[7,24,54,98,156,225,306,402,510,629]$} & & \\
\hline & 824 & $H_{394}$ & 1 & $r_{z}^{2} r_{x}$ & $r_{z}^{2} r_{x} t_{x}^{-1}, r_{x}^{2} t_{y}, i t_{y}^{-1}, m_{z} r_{x} t_{y}^{-1}, m_{z} r_{x} t_{z}, r_{x}^{2} t_{z}^{-1}$ \\
\hline & \multicolumn{3}{|c|}{$[7,24,55,99,154,219,298,389,493,610]$} & & \\
\hline & 825 & $H_{410}$ & 1 & $r_{z}^{2} r_{x}$ & $r_{y}^{2} r_{x} t_{x}^{-1}, r_{x}^{-1} t_{y}, r_{z}^{2} t_{y}^{-1}, r_{x}^{-1} t_{y}^{-1}, r_{x} t_{z}, r_{x} t_{z}^{-1}$ \\
\hline & \multicolumn{3}{|c|}{$[7,24,55,99,157,231,320,423,543,677]$} & $r_{\gamma}^{2} r_{x}$ & $i t_{x}^{-1}, r_{x}^{-1} t_{y}, r_{z}^{2} t^{-1}, r_{x}^{-1} t^{-1}, r_{x} t_{z}, r_{x} t_{\alpha}^{-1}$ \\
\hline & \multicolumn{3}{|c|}{$[7,24,56,103,161,227,306,401,506,623]$} & & \\
\hline & 827 & $H_{419}$ & 1 & $r_{z}^{2} r_{x}$ & $m_{x} t_{x}^{-1}, m_{y} t_{y}, m_{x} r_{x}^{-1} t_{y}^{-1}, m_{y} t_{y}^{-1}, m_{x} r_{x} t_{z}, m_{z} t_{z}^{-1}$ \\
\hline & {$[7,24,60,1$} & $75,249,33$ & $438,557,689]$ & & \\
\hline & 828 & $H_{422}$ & 1 & $r_{z}^{2} r_{x}$ & $r_{z}^{2} t_{x}, m_{y} t_{y}, m_{x} r_{x}^{-1} t_{y}^{-1}, m_{y} t_{y}^{-1}, m_{x} r_{x} t_{z}, m_{z} t_{z}^{-1}$ \\
\hline & {$[7,25,56,9$} & $7,226,307$ & $05,512,631]$ & & \\
\hline & 829 & $H_{395}$ & 1 & $r_{z}^{2} r_{x}$ & $m_{x} t_{x}^{-1}, r_{y}^{2} r_{x} t_{y}, m_{z} r_{x} t_{y}^{-1}, m_{z} r_{x} t_{z}, r_{y}^{2} r_{x} t_{z}^{-1}, r_{x}^{2} t_{z}^{-1}$ \\
\hline & {$[7,25,57,1$} & $56,225,30$ & $399,505,622]$ & & \\
\hline & 830 & $H_{413}$ & 1 & $r_{z}^{2} r_{x}$ & $m_{x} t_{x}^{-1}, m_{x} r_{x}^{-1} t_{y}, m_{y} t_{y}, r_{x}^{-1} t_{y}^{-1}, r_{x} t_{z}, m_{x} r_{x} t_{z}^{-1}$ \\
\hline & {$[7,25,58,1$} & $59,226,30$ & $401,506,623]$ & & \\
\hline & 831 & $H_{402}$ & 1 & $r_{z}^{2} r_{x}$ & $r_{z}^{2} r_{x} t_{x}^{-1}, m_{y} t_{y}, m_{x} r_{x}^{-1} t_{y}^{-1}, m_{y} t_{y}^{-1}, m_{x} r_{x} t_{z}, m_{z} t_{z}^{-1}$ \\
\hline & {$[7,25,58,1$} & $66,234,31$ & $416,524,644]$ & & \\
\hline & 832 & $H_{401}$ & 1 & $r_{z}^{2} r_{x}$ & $m_{x} t_{x}^{-1}, r_{x}^{2} t_{y}, r_{y}^{2} r_{x} t_{y}^{-1}, r_{x}^{2} t_{y}^{-1}, r_{y}^{2} r_{x} t_{z}, r_{x}^{2} t_{z}^{-1}$ \\
\hline & {$[7,25,60,1$} & $75,249,33$ & $438,557,689]$ & & \\
\hline & 833 & $H_{422}$ & 1 & $r_{z}^{2} r_{x}$ & $r_{z}^{2} t_{x}, m_{y} t_{y}, m_{x} r_{x}^{-1} t_{y}^{-1}, m_{x} r_{x} t_{z}, m_{z} t_{z}, m_{z} t_{z}^{-1}$ \\
\hline & {$[7,25,60,1$} & $72,243,33$ & $433,548,674]$ & & \\
\hline & 834 & $H_{409}$ & 1 & $r_{z}^{2} r_{x}$ & $r_{z}^{2} t_{x}, r_{x}^{2} t_{y}, r_{y}^{2} r_{x} t_{y}^{-1}, r_{x}^{2} t_{y}^{-1}, r_{y}^{2} r_{x} t_{z}, r_{x}^{2} t_{z}^{-1}$ \\
\hline & {$[7,25,61,1$} & $77,253,34$ & $447,566,698]$ & & \\
\hline & 835 & $H_{418}$ & 1 & $r_{z}^{2} r_{x}$ & $m_{x} t_{x}^{-1}, r_{z}^{2} t_{y}, r_{z}^{2} t_{y}^{-1}, r_{x}^{-1} t_{y}^{-1}, r_{x} t_{z}, r_{y}^{2} t_{z}^{-1}$ \\
\hline & {$[7,25,62,1$} & $77,249,34$ & $452,567,694]$ & & \\
\hline & 836 & $H_{415}$ & 1 & $r_{z}^{2} r_{x}$ & $i t_{x}^{-1}, m_{y} t_{y}, m_{x} r_{x}^{-1} t_{y}^{-1}, m_{y} t_{y}^{-1}, m_{x} r_{x} t_{z}, m_{z} t_{z}^{-1}$ \\
\hline & {$[7,25,63,1$} & $77,249,34$ & $452,567,694]$ & & \\
\hline & 837 & $H_{404}$ & 1 & $r_{z}^{2} r_{x}$ & $r_{y}^{2} r_{x} t_{x}^{-1}, m_{y} t_{y}, m_{x} r_{x}^{-1} t_{y}^{-1}, m_{y} t_{y}^{-1}, m_{x} r_{x} t_{z}, m_{z} t_{z}^{-1}$ \\
\hline & {$[7,25,65,1$} & $78,251,34$ & $448,567,699]$ & $r^{2} r_{x}$ & $r^{2} r_{x} t^{-1}, r^{2} t_{y}, r^{2} t^{-1}, r^{-1} t^{-1}, r_{x} t z, r^{2} t_{-1}^{-1}$ \\
\hline & $\frac{838}{[7,26,58,1}$ & $\frac{H_{407}}{58,226,30}$ & $\frac{1}{401,506,623]}$ & & \\
\hline & 839 & $H_{412}$ & 1 & $r_{z}^{2} r_{x}$ & $r_{z}^{2} r_{x} t_{x}^{-1}, m_{x} r_{x}^{-1} t_{y}, m_{y} t_{y}, r_{x}^{-1} t_{y}^{-1}, r_{x} t_{z}, m_{x} r_{x} t_{z}^{-1}$ \\
\hline & {$[7,26,59,1$} & $63,233,31$ & $415,523,643]$ & & \\
\hline & 840 & $H_{394}$ & 1 & $r_{z}^{2} r_{x}$ & $r_{z}^{2} r_{x} t_{x}^{-1}, r_{y}^{2} r_{x} t_{y}, m_{z} r_{x} t_{y}^{-1}, m_{z} r_{x} t_{z}, r_{y}^{2} r_{x} t_{z}^{-1}, r_{x}^{2} t_{z}^{-1}$ \\
\hline & {$[7,26,61,1$} & $64,234,31$ & $416,524,644]$ & & \\
\hline & 841 & $H_{391}$ & 1 & $r_{z}^{2} r_{x}$ & $r_{z}^{2} r_{x} t_{x}^{-1}, r_{x}^{2} t_{y}, r_{y}^{2} r_{x} t_{y}^{-1}, r_{x}^{2} t_{y}^{-1}, r_{y}^{2} r_{x} t_{z}, r_{x}^{2} t_{z}^{-1}$ \\
\hline & {$[7,26,62,1$} & $68,243,33$ & $433,551,680]$ & & \\
\hline & 842 & $H_{395}$ & 1 & $r_{z}^{2} r_{x}$ & $m_{x} t_{x}^{-1}, r_{y}^{2} r_{x} t_{y}, i t_{y}^{-1}, m_{z} r_{x} t_{y}^{-1}, m_{z} r_{x} t_{z}, r_{y}^{2} r_{x} t_{z}^{-1}$ \\
\hline & {$[7,26,62,1$} & $70,243,33$ & $433,548,674]$ & & \\
\hline & 843 & $H_{409}$ & 1 & $r_{z}^{2} r_{x}$ & $r_{z}^{2} t_{x}, r_{x}^{2} t_{y}, r_{y}^{2} r_{x} t_{y}^{-1}, r_{y}^{2} r_{x} t_{z}, r_{x}^{2} t_{z}, r_{x}^{2} t_{z}^{-1}$ \\
\hline & {$[7,26,62,1$} & $81,257,34$ & $451,571,702]$ & & \\
\hline & 844 & $H_{413}$ & 1 & $r_{z}^{2} r_{x}$ & $m_{x} t_{x}^{-1}, m_{x} r_{x}^{-1} t_{y}, m_{y} t_{y}, r_{z}^{2} t_{y}^{-1}, r_{y}^{2} t_{z}, m_{x} r_{x} t_{z}^{-1}$ \\
\hline & {$\left[\begin{array}{l}{[7,26,63,1} \\
845\end{array}\right.$} & $\begin{array}{c}74,249,33 \\
H_{413}\end{array}$ & $\begin{array}{l}441,559,688] \\
1\end{array}$ & $r^{2} r_{x}$ & 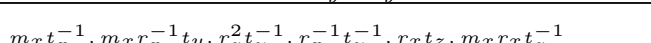 \\
\hline & {$[7,26,63,1$} & $\frac{113}{75,250,34}$ & $\frac{1}{444,563,694]}$ & $r_{z}^{2} r_{x}$ & $y^{1}, r_{x}{ }^{t} t_{y}, r_{x} t_{z}, m_{x} r_{x} t_{z}$ \\
\hline & 846 & $H_{394}$ & 1 & $r_{z}^{2} r_{x}$ & $r_{z}^{2} r_{x} t_{x}^{-1}, r_{y}^{2} r_{x} t_{y}, i t_{y}^{-1}, m_{z} r_{x} t_{y}^{-1}, m_{z} r_{x} t_{z}, r_{y}^{2} r_{x} t_{z}^{-1}$ \\
\hline & {$[7,26,63,1$} & $75,251,34$ & $447,567,699]$ & & \\
\hline & 847 & $H_{396}$ & 1 & $r_{z}^{2} r_{x}$ & $i t_{x}^{-1}, r_{x}^{2} t_{y}, r_{y}^{2} r_{x} t_{y}^{-1}, r_{x}^{2} t_{y}^{-1}, r_{y}^{2} r_{x} t_{z}, r_{x}^{2} t_{z}^{-1}$ \\
\hline & {$[7,26,64,1$} & $75,250,34$ & $444,562,692]$ & & \\
\hline & 848 & $H_{412}$ & 1 & $r_{z}^{2} r_{x}$ & $r_{z}^{2} r_{x} t_{x}^{-1}, m_{x} r_{x}^{-1} t_{y}, r_{z}^{2} t_{y}^{-1}, r_{x}^{-1} t_{y}^{-1}, r_{x} t_{z}, m_{x} r_{x} t_{z}^{-1}$ \\
\hline & $\begin{array}{c}{[7,26,64,1} \\
849\end{array}$ & $\begin{array}{c}75,251,34 \\
H, 302\end{array}$ & $447,567,699]$ & $r_{z}^{2} r_{x}$ & $r_{u}^{2} r_{x} t_{x}^{-1}, r_{x}^{2} t_{y}, r_{u}^{2} r_{x} t_{u}^{-1}, r_{x}^{2} t_{u}^{-1}, r_{u}^{2} r_{x} t_{z}, r_{x}^{2} t_{z}^{-1}$ \\
\hline & {$[7,26,64,1$} & $79,254,34$ & $448,568,700]$ & & \\
\hline & 850 & $H_{400}$ & 1 & $r_{z}^{2} r_{x}$ & $m_{x} t_{x}^{-1}, m_{z} r_{x} t_{y}, i t_{y}^{-1}, i t_{z}, i t_{z}^{-1}, m_{z} r_{x} t_{z}^{-1}$ \\
\hline & {$[7,26,65,1$} & $74,249,33$ & $442,560,690]$ & & \\
\hline & 851 & $H_{388}$ & 1 & $r_{z}^{2} r_{x}$ & $r_{z}^{2} r_{x} t_{x}^{-1}, m_{z} r_{x} t_{y}, i t_{y}^{-1}, i t_{z}, i t_{z}^{-1}, m_{z} r_{x} t_{z}^{-1}$ \\
\hline & {$[7,27,62,1$} & $70,245,33$ & $438,556,687]$ & & \\
\hline & 852 & $H_{401}$ & 1 & $r_{z}^{2} r_{x}$ & $m_{x} t_{x}^{-1}, r_{y}^{2} r_{x} t_{y}, r_{y}^{2} r_{x} t_{y}^{-1}, r_{x}^{2} t_{y}^{-1}, r_{y}^{2} r_{x} t_{z}, r_{y}^{2} r_{x} t_{z}^{-1}$ \\
\hline & {$[7,27,62,1$} & $78,254,34$ & $449,569,701]$ & & \\
\hline & 853 & $H_{409}$ & 1 & $r_{z}^{2} r_{x}$ & $r_{z}^{2} t_{x}, r_{y}^{2} r_{x} t_{y}, r_{y}^{2} r_{x} t_{y}^{-1}, r_{y}^{2} r_{x} t_{z}, r_{y}^{2} r_{x} t_{z}^{-1}, r_{x}^{2} t_{z}^{-1}$ \\
\hline & {$[7,27,64,1$} & $77,252,34$ & $448,568,700]$ & & \\
\hline & 854 & $H_{419}$ & 1 & $r_{z}^{2} r_{x}$ & $m_{x} t_{x}^{-1}, m_{x} r_{x}^{-1} t_{y}, m_{x} r_{x}^{-1} t_{y}^{-1}, m_{y} t_{y}^{-1}, m_{x} r_{x} t_{z}, m_{x} r_{x} t_{z}^{-1}$ \\
\hline & $\begin{array}{r}{[7,27,64,1]} \\
855\end{array}$ & $\begin{array}{c}78,256,34 \\
H_{422}\end{array}$ & $\begin{array}{l}450,570,702] \\
1\end{array}$ & $r_{\sim}^{2} r_{x}$ & $x^{2}$ \\
\hline & $\frac{833}{[7,27,64,1}$ & $\frac{11422}{81,256,34}$ & $450,570,702]$ & & $r_{z} \iota_{x}, m_{x} r_{x}{ }^{\imath} y, m_{x} r_{x}{ }_{y} y \quad, m_{x} r_{x} \iota_{z}, m_{x} r_{x} t_{z} \quad, m_{z} \iota_{z}$ \\
\hline
\end{tabular}




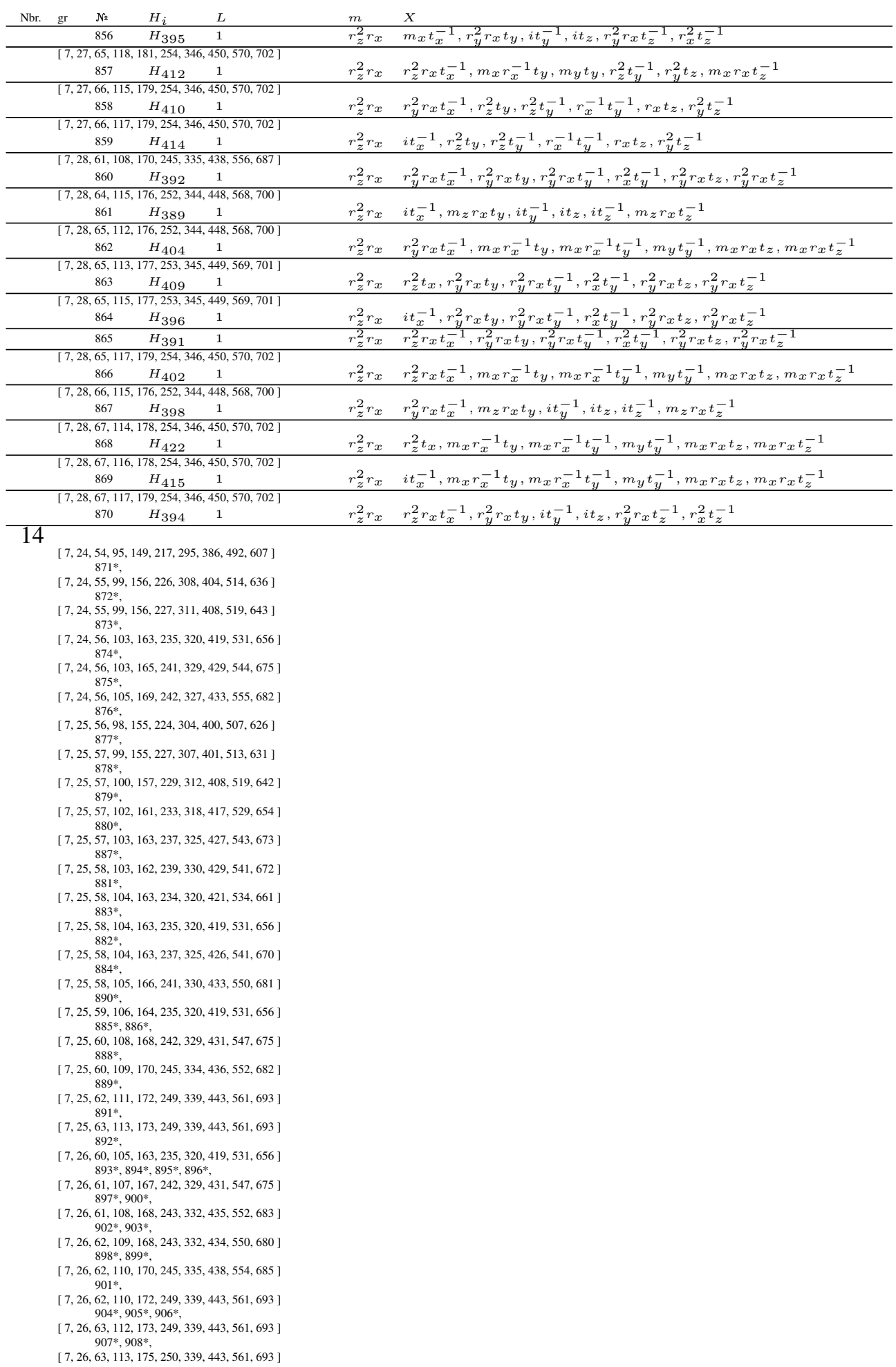




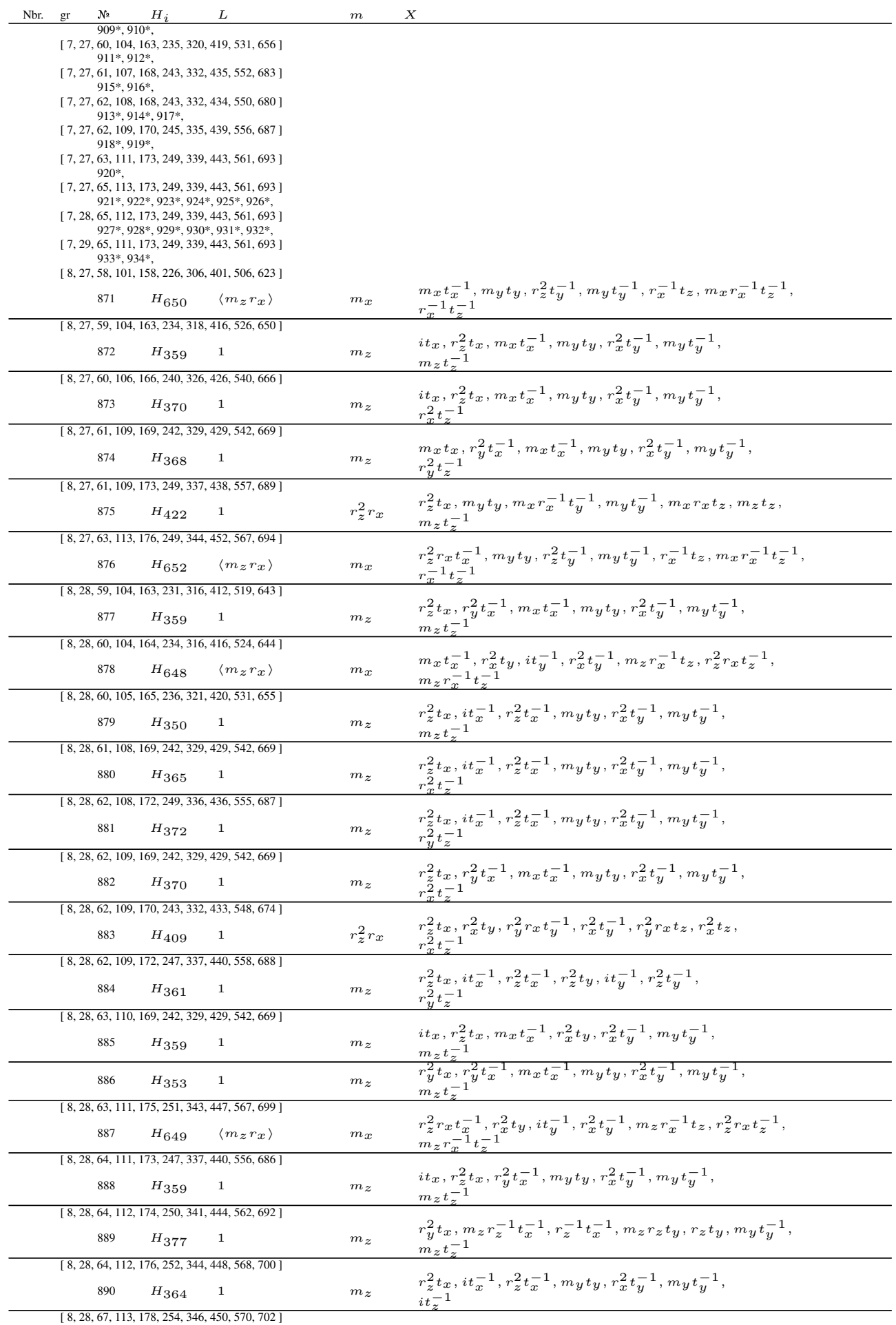




\begin{tabular}{|c|c|c|c|c|c|}
\hline Nbr. & № & $H_{i}$ & $L$ & $m$ & $x$ \\
\hline & 891 & $H_{370}$ & 1 & $m_{z}$ & $\begin{array}{l}i t_{x}, r_{z}^{2} t_{x}, r_{y}^{2} t_{x}^{-1}, m_{y} t_{y}, r_{x}^{2} t_{y}^{-1}, m_{y} t_{y}^{-1} \\
r_{x}^{2} t_{z}^{-1}\end{array}$ \\
\hline & \multicolumn{5}{|c|}{$[8,28,68,114,178,254,346,450,570,702]$} \\
\hline & 892 & $H_{368}$ & 1 & $m_{z}$ & $\begin{array}{l}m_{x} t_{x}, r_{y}^{2} t_{x}^{-1}, m_{x} t_{x}^{-1}, r_{x}^{2} t_{y}, r_{x}^{2} t_{y}^{-1}, m_{y} t_{y}^{-1} \\
r_{y}^{2} t_{z}^{-1}\end{array}$ \\
\hline & \multicolumn{4}{|c|}{$[8,29,63,109,169,242,329,429,542,669]$} & \\
\hline & 893 & $H_{350}$ & 1 & $m_{z}$ & $\begin{array}{l}r_{z}^{2} t_{x}, i t_{x}^{-1}, r_{z}^{2} t_{x}^{-1}, r_{x}^{2} t_{y}, r_{x}^{2} t_{y}^{-1}, m_{y} t_{y}^{-1} \\
m_{z} t_{z}^{-1}\end{array}$ \\
\hline & 894 & $H_{370}$ & 1 & $m_{z}$ & $\begin{array}{l}i t_{x}, r_{z}^{2} t_{x}, m_{x} t_{x}^{-1}, r_{x}^{2} t_{y}, r_{x}^{2} t_{y}^{-1}, m_{y} t_{y}^{-1} \\
r_{x}^{2} t_{z}^{-1}\end{array}$ \\
\hline & 895 & $H_{359}$ & 1 & $m_{z}$ & $\begin{array}{l}r_{z}^{2} t_{x}, r_{y}^{2} t_{x}^{-1}, m_{x} t_{x}^{-1}, r_{x}^{2} t_{y}, r_{x}^{2} t_{y}^{-1}, m_{y} t_{y}^{-1} \\
m_{z} t_{z}^{-1}\end{array}$ \\
\hline & 896 & $H_{368}$ & 1 & $m_{z}$ & $\begin{array}{l}r_{y}^{2} t_{x}, r_{y}^{2} t_{x}^{-1}, m_{x} t_{x}^{-1}, m_{y} t_{y}, r_{x}^{2} t_{y}^{-1}, m_{y} t_{y}^{-1}, \\
r_{y}^{2} t_{z}^{-1}\end{array}$ \\
\hline & \multicolumn{5}{|c|}{$[8,29,64,110,173,247,337,440,556,686]$} \\
\hline & 897 & $H_{359}$ & 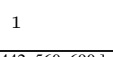 & $m_{z}$ & $\begin{array}{l}i t_{x}, r_{y}^{2} t_{x}^{-1}, m_{x} t_{x}^{-1}, m_{y} t_{y}, r_{x}^{2} t_{y}^{-1}, m_{y} t_{y}^{-1} \\
m_{z} t_{z}^{-1}\end{array}$ \\
\hline & \multicolumn{4}{|c|}{$[8,29,65,111,174,249,339,442,560,690]$} & \\
\hline & 898 & $H_{330}$ & 1 & $m_{z}$ & $\begin{array}{l}r_{z}^{2} t_{x}, \imath t_{x}, r_{z}^{t} t_{x}, \imath t_{y}, \imath t_{y}, r_{z}^{t} y \\
m_{z} t_{z}^{-1}\end{array}$ \\
\hline & 899 & $H_{350}$ & 1 & $m_{z}$ & $\begin{array}{l}i t_{x}, i t_{x}^{-1}, r_{z}^{2} t_{x}^{-1}, m_{y} t_{y}, r_{x}^{2} t_{y}^{-1}, m_{y} t_{y}^{-1} \\
m_{z} t_{z}^{-1}\end{array}$ \\
\hline & \multicolumn{4}{|c|}{$[8,29,65,111,175,250,339,443,560,689]$} & \\
\hline & 900 & $H_{382}$ & 1 & $m_{z}$ & $\begin{array}{l}r_{y}^{t_{x}, m_{z} r_{z}{ }^{t} x}, r_{z}{ }^{t_{x}}, m_{z^{2} z^{t} y}, r_{z} z_{y}, m_{y^{t} y} \\
r_{y}^{2} r_{z}{ }^{-1}\end{array}$ \\
\hline & \multicolumn{4}{|c|}{$[8,29,65,112,175,251,342,445,564,695]$} & \\
\hline & 901 & $H_{343}$ & $140.569 .700]$ & $m_{z}$ & $\begin{array}{l}r_{z}^{2} t_{x}, r_{y}^{2} r_{z}{ }^{-1}, m_{x} r_{z}{ }^{-1}, i t_{y}, r_{y}^{2} r_{z} t_{y}^{-1}, m_{x} r_{z} t_{y}^{-1} \\
m_{z} t_{z}^{-1}\end{array}$ \\
\hline & \multicolumn{4}{|c|}{$[8,29,65,112,176,252,344,448,568,700]$} & $r^{2} t r i t-1, r^{2} t-1, i t, i t-1, r^{2} t-1$ \\
\hline & 902 & $H_{361}$ & 1 & $m_{z}$ & $\begin{array}{l}r^{\imath} z_{x}, i \iota x \\
r_{y}^{2} t-z^{-1}\end{array}$ \\
\hline & 903 & $H_{365}$ & 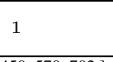 & $m_{z}$ & $\begin{array}{l}i_{x}, i t_{x}^{-1}, r_{z}^{2} t_{x}^{-1}, m_{y} t_{y}, r_{x}^{2} t_{y}^{-1}, m_{y} t_{y}^{-1}, \\
r_{x}^{2} t_{z}^{-1}\end{array}$ \\
\hline & \multicolumn{5}{|c|}{$[8,29,66,113,178,254,346,450,570,702]$} \\
\hline & 904 & $H_{370}$ & 1 & $m_{z}$ & $\begin{array}{l}i t_{x}, r_{y}^{2} t_{x}^{-1}, m_{x} t_{x}^{-1}, m_{y} t_{y}, r_{x}^{2} t_{y}^{-1}, m_{y} t_{y}^{-1} \\
r_{x}^{2} t_{z}^{-1}\end{array}$ \\
\hline & 905 & $H_{378}$ & 1 & $m_{z}$ & $\begin{array}{l}r_{y}^{2} t_{x}, r_{y}^{2} t_{x}^{-1}, m_{x} t_{x}^{-1}, m_{y} t_{y}, r_{x}^{2} t_{y}^{-1}, m_{y} t_{y}^{-1} \\
r_{y}^{2} r_{z} t_{z}^{-1}\end{array}$ \\
\hline & 906 & $H_{378}$ & 1 & $m_{z}$ & $\begin{array}{l}r_{y}^{2} t_{x}, r_{y}^{2} t_{x}^{-1}, m_{x} t_{x}^{-1}, r_{x}^{2} t_{y}, m_{y} t_{y}, m_{y} t_{y}^{-1} \\
r_{y}^{2} r_{z} t_{z}^{-1}\end{array}$ \\
\hline & \multicolumn{4}{|c|}{$[8,29,67,114,178,254,346,450,570,702]$} & \\
\hline & 907 & $H_{372}$ & 1 & $m_{z}$ & $\begin{array}{l}r_{z}^{2} t_{x}, i t_{x}^{-1}, r_{z}^{2} t_{x}^{-1}, r_{x}^{2} t_{y}, r_{x}^{2} t_{y}^{-1}, m_{y} t_{y}^{-1} \\
r_{y}^{2} t_{z}^{-1}\end{array}$ \\
\hline & 908 & $H_{373}$ & 1 & $m_{z}$ & $\begin{array}{l}r_{y}^{2} t_{x}, r_{y}^{2} t_{x}^{-1}, m_{x} t_{x}^{-1}, m_{y} t_{y}, r_{x}^{2} t_{y}^{-1}, m_{y} t_{y}^{-1}, \\
i t_{z}^{-1}\end{array}$ \\
\hline & \multicolumn{5}{|c|}{$[8,29,67,115,179,254,346,450,570,702]$} \\
\hline & 909 & $H_{359}$ & 1 & $m_{z}$ & $\begin{array}{l}i t_{x}, r_{z}^{2} t_{x}, r_{y}^{2} t_{x}^{-1}, r_{x}^{2} t_{y}, r_{x}^{2} t_{y}^{-1}, m_{y} t_{y}^{-1} \\
m_{z} t_{z}^{-1}\end{array}$ \\
\hline & 910 & $H_{650}$ & $\left\langle m_{z} r_{x}\right\rangle$ & $m_{x}$ & $\begin{array}{l}m_{x} t_{x}^{-1}, r_{z}^{2} t_{y}, r_{z}^{2} t_{y}^{-1}, m_{y} t_{y}^{-1}, m_{x} r_{x}^{-1} t_{z}, m_{x} r_{x}^{-1} t_{z}^{-1}, \\
r_{x}^{-1} t_{z}^{-1}\end{array}$ \\
\hline & \multicolumn{4}{|c|}{$[8,30,62,109,169,242,329,429,542,669]$} & \\
\hline & 911 & $H_{365}$ & 1 & $m_{z}$ & $\begin{array}{l}r_{z}^{2} t_{x}, i t_{x}^{-1}, r_{z}^{2} t_{x}^{-1}, r_{x}^{2} t_{y}, r_{x}^{2} t_{y}^{-1}, m_{y} t_{y}^{-1} \\
r_{x}^{2} t_{z}^{-1}\end{array}$ \\
\hline & 912 & $H_{370}$ & 1 & $m_{z}$ & $\begin{array}{l}r_{z}^{2} t_{x}, r_{y}^{2} t_{x}^{-1}, m_{x} t_{x}^{-1}, r_{x}^{2} t_{y}, r_{x}^{2} t_{y}^{-1}, m_{y} t_{y}^{-1}, \\
r_{x}^{2} t_{z}^{-1}\end{array}$ \\
\hline & \multicolumn{5}{|c|}{$[8,30,64,111,174,249,339,442,560,690]$} \\
\hline & 913 & $H_{361}$ & 1 & $m_{z}$ & $\begin{array}{l}i t_{x}, i t_{x}^{-1}, r_{z}^{2} t_{x}^{-1}, r_{z}^{2} t_{y}, i t_{y}^{-1}, r_{z}^{2} t_{y}^{-1} \\
r_{y}^{2} t_{z}^{-1}\end{array}$ \\
\hline & 914 & $H_{372}$ & 1 & $m_{z}$ & $\begin{array}{l}i_{x}, i t_{x}^{-1}, r_{z}^{2} t_{x}^{-1}, m_{y} t_{y}, r_{x}^{2} t_{y}^{-1}, m_{y} t_{y}^{-1}, \\
r_{y}^{2} t_{z}^{-1}\end{array}$ \\
\hline & \multicolumn{4}{|c|}{$[8,30,64,112,176,252,344,448,568,700]$} & \\
\hline & 915 & $H_{332}$ & 1 & $m_{z}$ & $\begin{array}{l}r_{z}^{2} t_{x}, i t_{x}^{-1}, r_{z}^{2} t_{x}^{-1}, i t_{y}, i t_{y}^{-1}, r_{z}^{2} t_{y}^{-1} \\
i t_{z}^{-1}\end{array}$ \\
\hline & 916 & $H_{364}$ & 1 & $m_{z}$ & $\begin{array}{l}i t_{x}, i t_{x}^{-1}, r_{z}^{2} t_{x}^{-1}, m_{y} t_{y}, r_{x}^{2} t_{y}^{-1}, m_{y} t_{y}^{-1}, \\
i t_{z}^{-1}\end{array}$ \\
\hline & \multicolumn{4}{|c|}{$[8,30,65,112,175,251,341,444,563,693]$} & \\
\hline & 917 & $H_{347}$ & 1 & $m_{z}$ & $\begin{array}{l}r_{z}^{2} t_{x}, r_{y}^{2} r_{z} t_{x}^{-1}, m_{x} r_{z} t_{x}^{-1}, i t_{y}, r_{y}^{2} r_{z} t_{y}^{-1}, m_{x} r_{z} t_{y}^{-1}, \\
r_{x}^{2} r_{z} t_{z}^{-1}\end{array}$ \\
\hline & \multicolumn{4}{|c|}{$[8,30,65,113,176,252,344,448,568,700]$} & \\
\hline & 918 & $H_{344}$ & 1 & $m_{z}$ & $\begin{array}{l}z^{2} x, t t_{x} \\
r_{y}^{2} r_{z} t_{z}^{-1}\end{array}$ \\
\hline
\end{tabular}




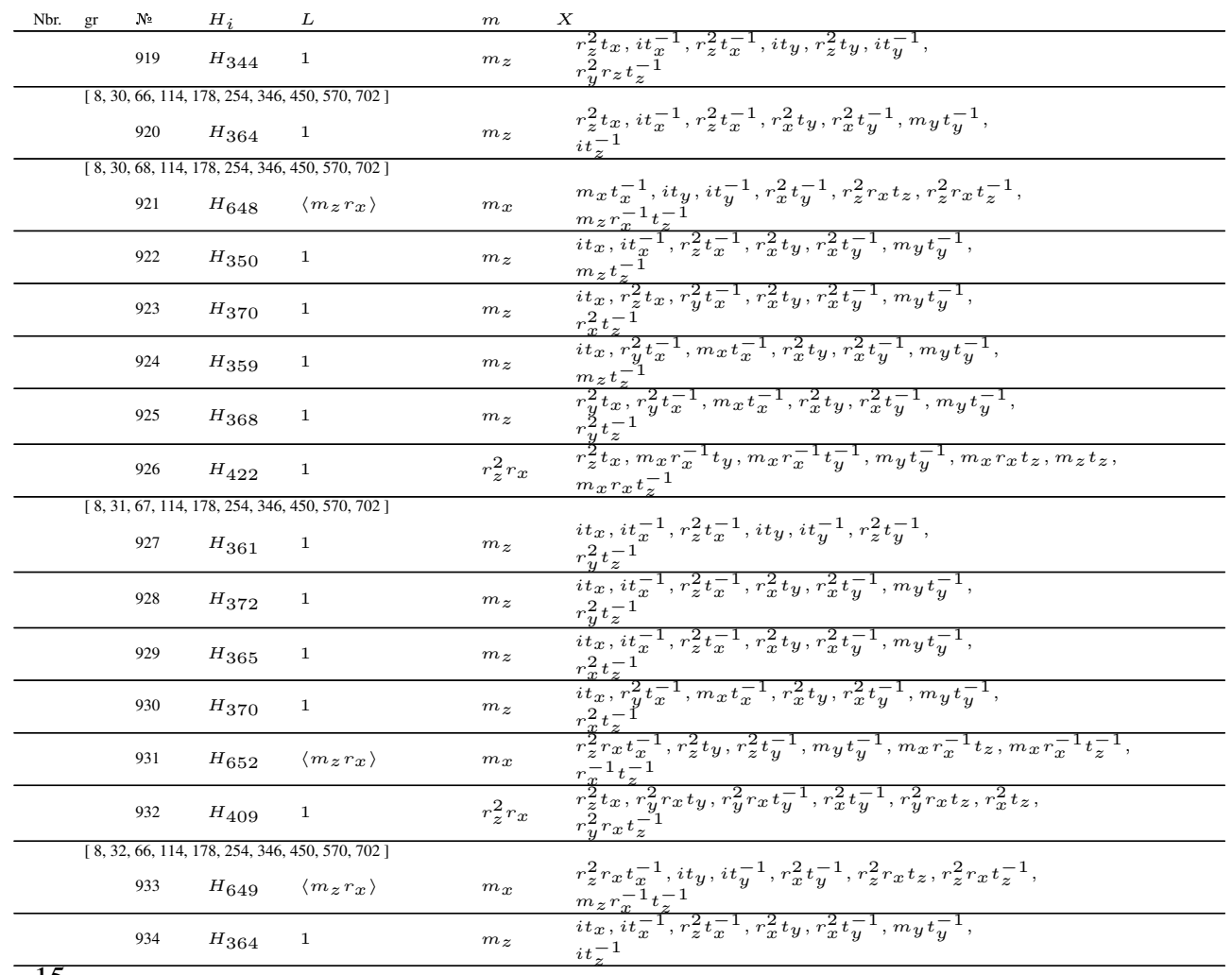

15

$[7,23,50,87,135,194,263,343,434,535]$ $936^{*}$

[ $7,23,51,91,143,207,283,371,471,583$ ] $935^{*}$,

$[7,23,51,92,147,214,292,383,487,604]$ $937^{*}$

$[7,23,52,94,149,217,298,392,499,619]$ $939^{*}$,

[ $7,23,52,95,152,221,303,399,507,627]$ $940^{*}$,

[ 7, 23, 52, 96, 155, 227, 312, 411, 523, 648 ] $938^{*}$,

$[7,24,53,93,146,210,285,373,472,583]$

$$
941^{*}
$$

[ $7,24,54,95,148,214,291,379,480,593]$ $944^{*}$,

$[7,24,54,96,150,216,294,384,486,600]$ $950^{*}$,

$[7,24,54,96,151,219,300,394,500,619]$ $945^{*}$

$[7,24,54,97,153,222,304,399,507,628]$ 949*,

$[7,24,54,97,154,224,308,407,519,644]$ $942^{*}$,

$[7,24,54,97,154,225,308,404,515,639]$ $948^{*}$,

$[7,24,55,97,151,220,300,392,499,616]$ $943^{*}$,

$[7,24,55,98,152,218,296,386,488,602]$ $953^{*}, 954^{*}$,

$[7,24,55,99,158,233,321,421,535,663]$ $946^{*}$

$[7,24,55,100,158,229,314,413,525,650]$ 956*,

$[7,24,55,100,159,231,316,415,527,652]$ $947^{*}, 951^{*}, 952^{*}$,

$[7,24,55,100,161,239,329,428,543,679]$ $955^{*}$

$[7,24,55,101,162,237,326,429,546,677]$ $957^{*}$,

[ $7,24,56,101,159,231,316,414,525,649$ ] $960^{*}$,

$[7,24,57,109,180,268,372,492,628,780]$ $958^{*}, 959^{*}$, 


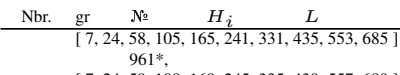

$[7,24,59,108,169,245,335,439,557,689]$ $962^{*}$

$[7,24,59,109,171,247,337,441,559,691]$ $963^{*}$

[ $7,25,56,98,152,218,296,386,488,602]$ $964^{*}$

[ $7,25,57,99,152,218,296,386,488,602]$ $968^{*}$,

$[7,25,57,102,161,233,318,417,529,654]$ $965^{*}, 966^{*}$,

[ 7, 25, 58, 102, 158, 228, 311, 407, 516, 638 ] $967^{*}, 969^{*}$,

[ $7,25,58,102,159,231,316,414,525,649$ ] $972 *$,

$[7,25,58,104,163,235,320,419,531,656]$ $970^{*}, 971^{*}$,

$[7,25,58,105,166,241,330,433,550,681]$ $977^{*}, 978^{*}$,

$[7,25,59,105,163,235,321,421,534,660]$ $982^{*}, 983^{*}$,

$[7,25,59,105,165,241,331,435,553,685$ ] $973^{*}$,

$[7,25,59,106,166,241,330,432,548,678]$ $974^{*}, 975^{*}$,

$[7,25,59,107,169,245,335,439,557,689]$ $976^{*}, 985^{*}, 986^{*}$,

$[7,25,59,109,177,264,368,488,624,776]$ $979^{*}$,

$[7,25,60,108,169,245,335,439,557,689]$ $984^{*}$,

$[7,25,60,109,169,242,331,435,551,679$ ] $990^{*}, 991^{*}$,

$[7,25,60,109,171,247,337,441,559,691]$ $992^{*}$,

$[7,25,60,112,182,269,373,493,629,781]$ $987^{*}$,

$[7,25,60,113,184,272,376,496,632,784$ ] $980^{*}, 981^{*}, 988^{*}, 989^{*}$,

$[7,25,61,111,173,249,339,443,561,693]$ $993^{*}$

$[7,25,62,116,185,272,376,496,632,784]$ $994^{*}, 995^{*}$,

[ $7,26,57,98,152,218,296,386,488,602]$ $998^{*}$

[ $7,26,58,101,158,228,311,407,516,638$ ] $996^{*}, 997^{*}$,

$[7,26,59,104,163,235,320,419,531,656]$ 999*, 1000*,

$[7,26,59,104,163,235,321,421,534,660]$ $1001^{*}, 1002^{*}$,

$[7,26,60,107,168,243,332,435,552,683]$ $1006^{*}, 1007^{*}$,

$[7,26,60,107,169,245,335,439,557,689]$ 1003*,

$[7,26,61,108,168,243,332,434,550,680]$ $1004^{*}, 1005^{*}$

$[7,26,61,109,171,246,335,439,556,687]$ $1008^{*}, 1009^{*}$,

$[7,26,61,109,171,247,337,441,559,691]$ $1010^{*}$,

$[7,26,61,115,186,274,378,498,634,786]$ $1015^{*}$,

$[7,26,62,110,171,247,337,441,559,691]$ $1017^{*}, 1018^{*}, 1019^{*}, 1020^{*}$

$[7,26,62,111,173,249,339,443,561,693]$ $1021^{*}$,

$[7,26,62,112,175,250,339,443,561,693]$ 1022*, 1023*,

$[7,26,62,113,181,268,372,492,628,780]$ $1011^{*}, 1012^{*}$,

$[7,26,62,115,185,272,376,496,632,784$ ] $1024^{*}, 1025^{*}$,

$[7,26,62,115,186,274,378,498,634,786]$ $1013^{*}, 1014^{*}$,

$[7,26,62,118,190,278,382,502,638,790]$ $1016^{*}$,

$[7,26,63,112,173,249,339,443,561,693$ ] $1028^{*}, 1029^{*}$,

$[7,26,63,116,186,274,378,498,634,786]$ $1030 *, 1031 *$

$[7,26,63,118,190,278,382,502,638,790]$ 1026*, 1027*,

$[7,26,65,123,195,282,385,505,642,793]$ $1032^{*}, 1033^{*}$

$[7,26,67,126,197,284,388,508,644,796$ ] $1034^{*}$,

$[7,27,62,109,171,247,337,441,559,691]$ $1036^{*}, 1037 *, 1038^{*}$

$[7,27,62,112,181,268,372,492,628,780]$ 1035*,

$[7,27,63,111,173,249,339,443,561,693]$ 


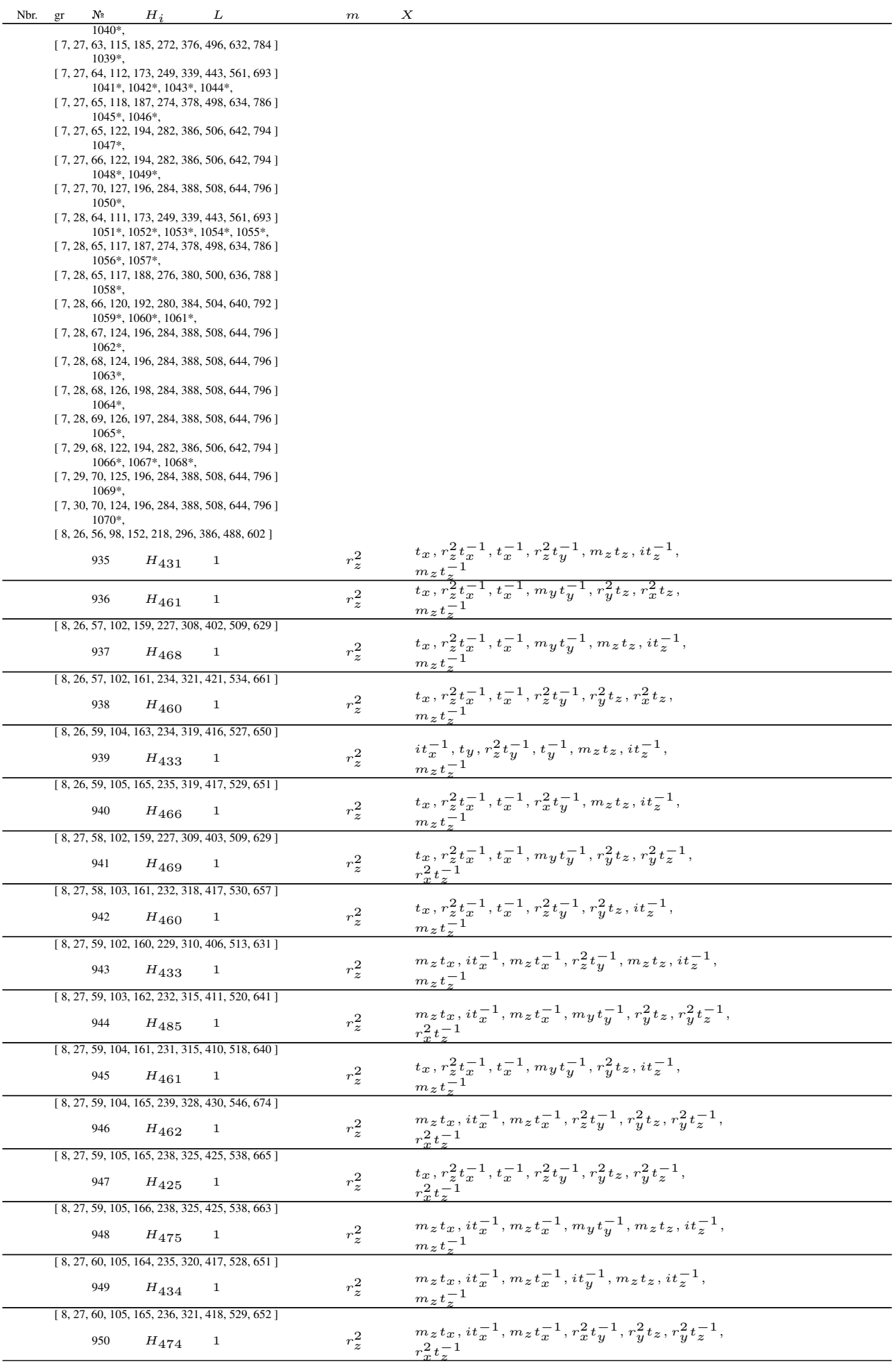




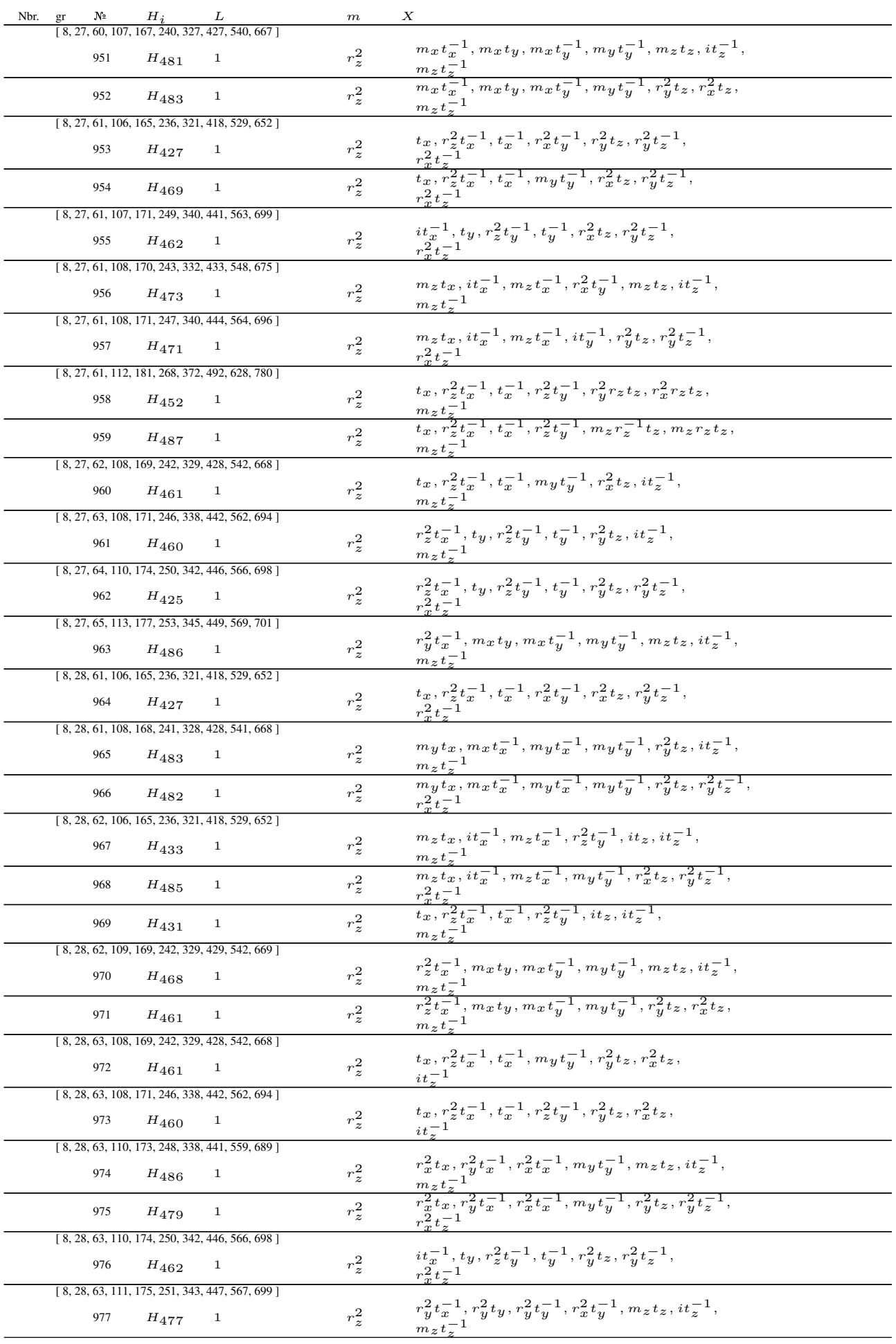




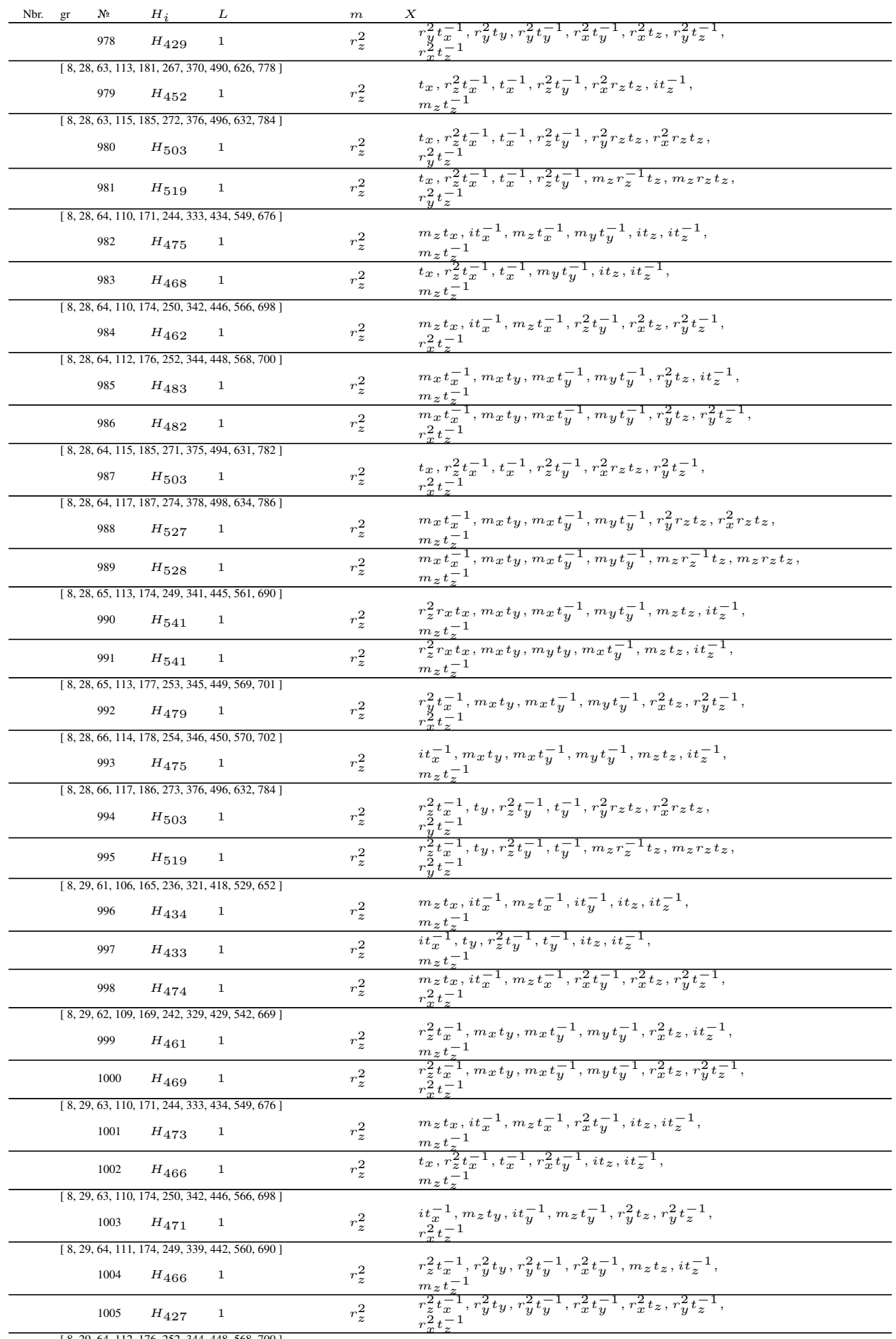

$[8,29,64,112,176,252,344,448,568,700]$ 


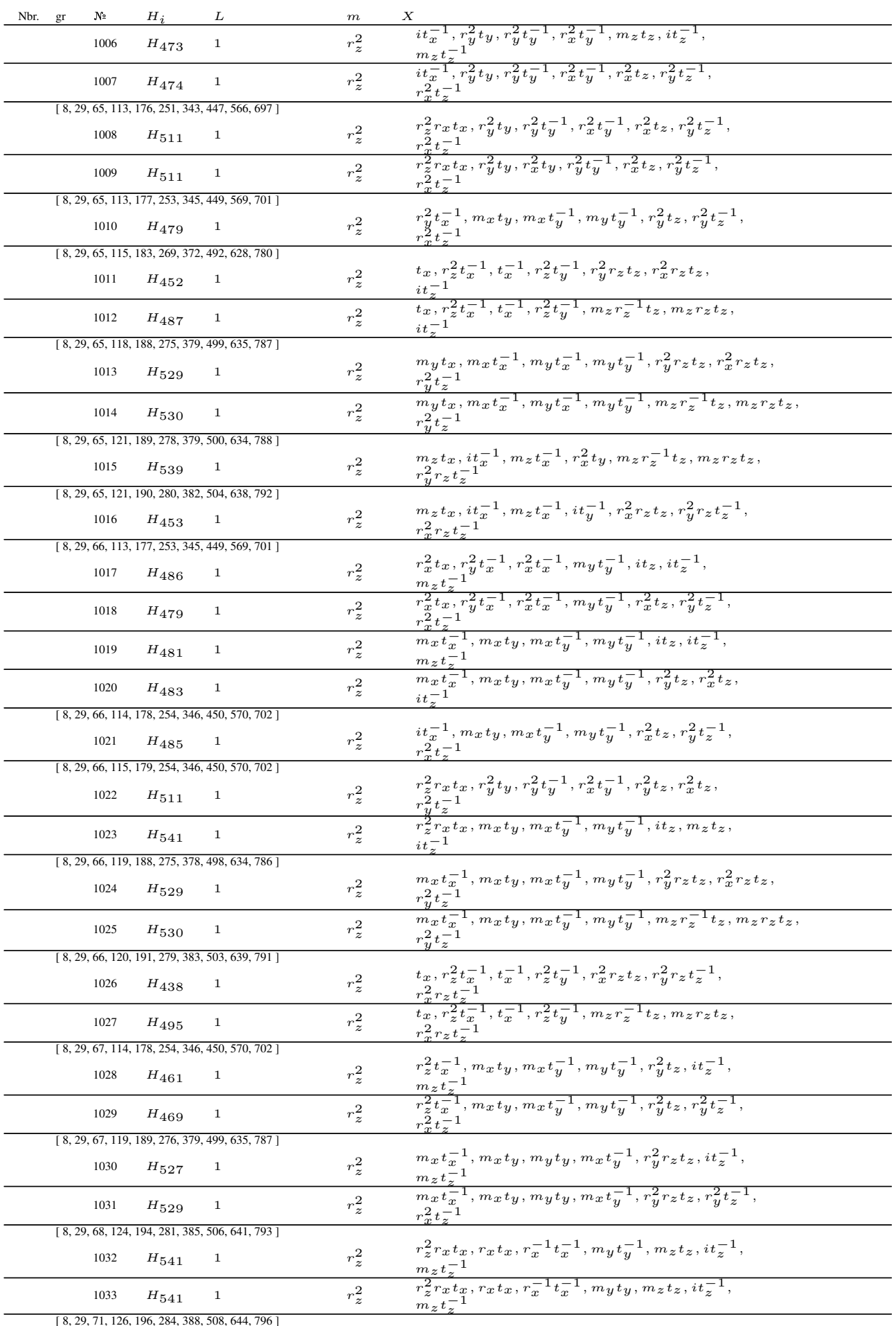




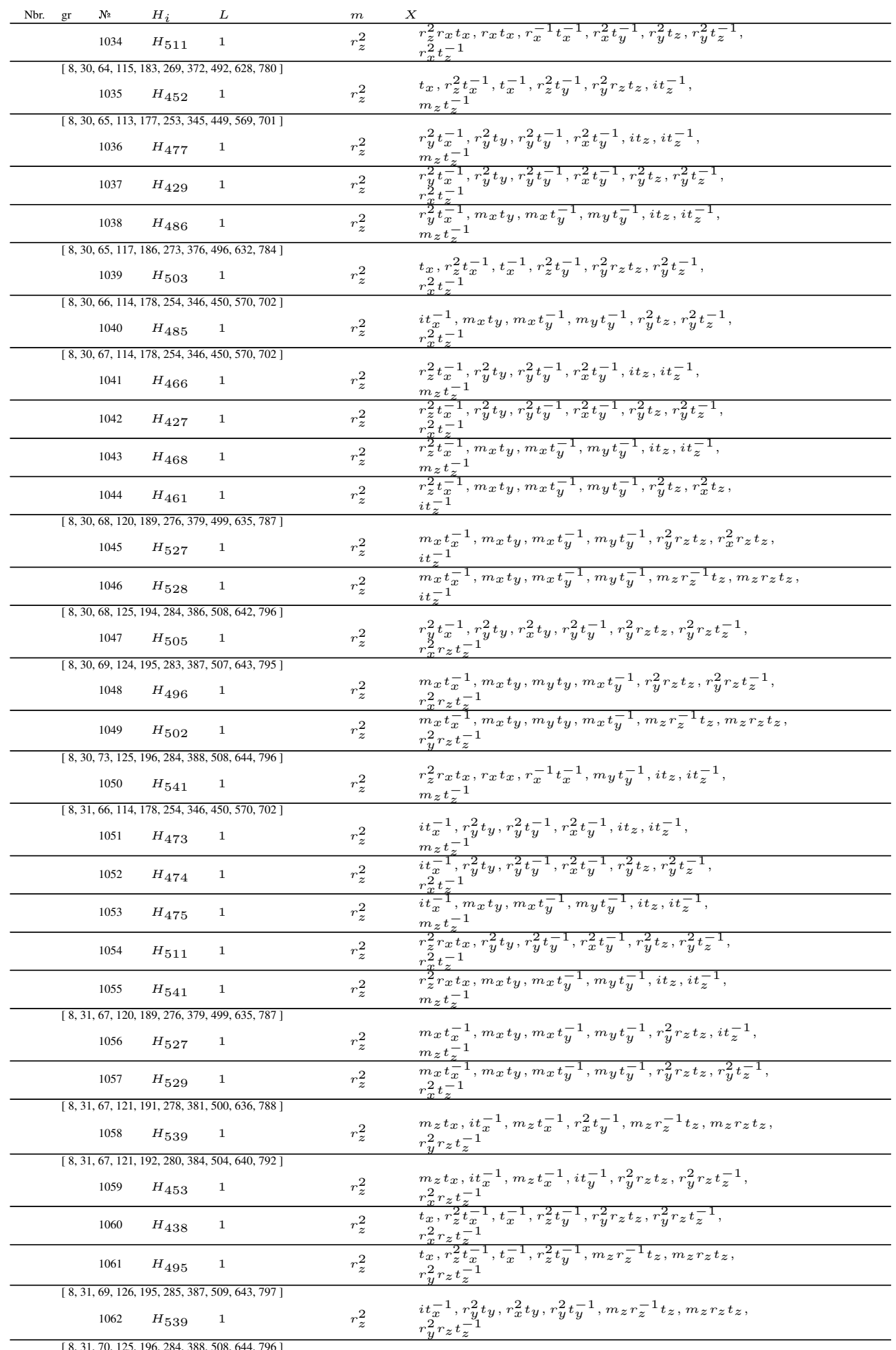




\begin{tabular}{|c|c|c|c|c|c|c|}
\hline Nbr. & gr & № & $H_{i}$ & $L$ & $m$ & $X$ \\
\hline & & 1063 & $H_{511}$ & 1 & $r_{z}^{2}$ & $\begin{array}{l}r_{z}^{2} r_{x} t_{x}, r_{x} t_{x}, r_{x}^{-1} t_{x}^{-1}, r_{x}^{2} t_{y}^{-1}, r_{y}^{2} t_{z}, r_{x}^{2} t_{z} \\
r_{y}^{2} t_{z}^{-1}\end{array}$ \\
\hline \multicolumn{7}{|c|}{$[8,31,70,127,196,284,388,508,644,796]$} \\
\hline & & 1064 & $H_{511}$ & 1 & $r_{z}^{2}$ & $\begin{array}{l}r_{z}^{2} r_{x} t_{x}, r_{x} t_{x}, r_{x}^{-1} t_{x}^{-1}, r_{x}^{2} t_{y}, r_{x}^{2} t_{z}, r_{y}^{2} t_{z}^{-1} \\
r_{x}^{2} t_{z}^{-1}\end{array}$ \\
\hline \multicolumn{7}{|c|}{$[8,31,71,126,196,284,388,508,644,796]$} \\
\hline & & 1065 & $H_{511}$ & 1 & $r_{z}^{2}$ & $\begin{array}{l}r_{z}^{2} r_{x} t_{x}, r_{x} t_{x}, r_{x}^{-1} t_{x}^{-1}, r_{x}^{2} t_{y}^{-1}, r_{x}^{2} t_{z}, r_{y}^{2} t_{z}^{-1} \\
r_{x}^{2} t_{z}^{-1}\end{array}$ \\
\hline \multicolumn{7}{|c|}{$[8,32,69,124,195,283,387,507,643,795]$} \\
\hline & & 1066 & $H_{505}$ & 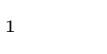 & $r_{z}^{2}$ & $\begin{array}{l}r_{y}^{2} t_{x}^{-1}, r_{y}^{2} t_{y}, r_{y}^{2} t_{y}^{-1}, r_{x}^{2} t_{y}^{-1}, r_{y}^{2} r_{z} t_{z}, r_{y}^{2} r_{z} t_{z}^{-1}, \\
r_{x}^{2} r_{z} t-1\end{array}$ \\
\hline & & 1067 & $H_{496}$ & 1 & $r_{z}^{2}$ & $\begin{array}{l}m_{x} t_{x}^{-1}, m_{x} t_{y}, m_{x} t_{y}^{-1}, m_{y} t_{y}^{-1}, r_{y}^{2} r_{z} t_{z}, r_{y}^{2} r_{z} t_{z}^{-1}, \\
r_{x}^{2} r_{z} t_{z}^{-1}\end{array}$ \\
\hline & & 1068 & $H_{502}$ & 1 & $r_{z}^{2}$ & $\begin{array}{l}m_{x} t_{x}^{-1}, m_{x} t_{y}, m_{x} t_{y}^{-1}, m_{y} t_{y}^{-1}, m_{z} r_{z}^{-1} t_{z}, m_{z} r_{z} t_{z}, \\
r_{y}^{2} r_{z}{ }_{z}^{-1}\end{array}$ \\
\hline \multicolumn{7}{|c|}{$[8,32,71,125,196,284,388,508,644,796]$} \\
\hline & & 1069 & $H_{541}$ & 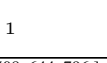 & $r_{z}^{2}$ & $\begin{array}{l}r_{z}^{2} r_{x} t_{x}, r_{x} t_{x}, r_{x}^{-1} t_{x}^{-1}, m_{y} t_{y}^{-1}, i t_{z}, m_{z} t_{z} \\
i t_{z}^{-1}\end{array}$ \\
\hline \multicolumn{7}{|c|}{$[8,33,70,125,196,284,388,508,644,796]$} \\
\hline & & 1070 & $H_{539}$ & 1 & $r_{z}^{2}$ & $\begin{array}{l}i t_{x}^{-1}, r_{y}^{2} t_{y}, r_{y}^{2} t_{y}^{-1}, r_{x}^{2} t_{y}^{-1}, m_{z} r_{z}^{-1} t_{z}, m_{z} r_{z} t_{z} \\
r_{y}^{2} r_{z} t_{z}^{-1}\end{array}$ \\
\hline
\end{tabular}

$[8,27,59,104,163,235,320,419,531,656]$ $1071^{*}$

$[8,27,60,106,167,242,329,431,547,675]$ $1072 *, 1075 *$

$[8,27,60,107,168,243,332,435,552,683]$ 1077*,

$[8,27,60,107,169,245,334,436,552,682]$ $1073^{*}$

$[8,27,61,108,168,243,332,434,550,680]$ $1074^{*}, 1078^{*}$

$[8,27,61,109,169,242,331,435,551,679]$ $1079 *$

$[8,27,61,109,170,245,335,438,554,685]$ $1076^{*}$,

$[8,27,61,109,170,245,335,439,556,687]$ $1081^{*}$,

$[8,27,61,109,171,246,335,439,556,687]$ $1080^{*}$

$[8,27,61,109,172,249,339,443,561,693$ ] $1082^{*}, 1083^{*}$

$[8,27,62,111,173,249,339,443,561,693]$ $1084^{*}$,

$[8,28,64,112,173,249,339,443,561,693]$ $1086^{*}, 1087^{*}, 1088^{*}, 1089^{*}, 1090^{*}$

$[8,28,64,117,188,276,380,500,636,788]$ $1085^{*}$,

$[8,29,64,111,173,249,339,443,561,693]$ $1091^{*}, 1092^{*}, 1093^{*}, 1094^{*}, 1095^{*}, 1096^{*}, 1097^{*}, 1098^{*}, 1099^{*}, 1100^{*}, 1101^{*}$,

$[8,29,67,120,189,276,380,500,636,788$ ] $1103^{*}$,

[ $8,29,67,121,193,282,385,505,642,793$ ] $1102 *$

$[8,29,68,124,196,284,388,508,644,796]$ $1104^{*}$,

$[8,30,67,119,189,276,380,500,636,788]$ $1105^{*}, 1106^{*}, 1107^{*}, 1108^{*}$,

$[8,30,70,125,196,284,388,508,644,796]$ $1109^{*}$,

$[8,31,70,124,196,284,388,508,644,796]$

$1110^{*}, 1111^{*}, 1112^{*}, 1113^{*}, 1114^{*}, 1115^{*}, 1116^{*}, 1117^{*}, 1118^{*}$

$[9,29,62,109,169,242,329,429,542,669]$

$1071 \quad H_{680} \quad\left\langle m_{x}\right\rangle \quad r_{z}^{2} \quad r_{z}^{2} t_{x}, m_{x} t_{x}, m_{y} t_{x}^{-1}, m_{x} t_{x}^{-1}, m_{y}{ }^{-1}, r_{y}^{2} t_{z}$,

$[9,29,63,110,173,247,337,440,556,686]$ $r_{x}^{2} t_{z}^{-1}, r_{y}^{2} t_{z}^{-1}$

$1072 \quad H_{359} \quad 1$ $i t_{x}, r_{z}^{2} t_{x}, r_{y}^{2} t_{x}^{-1}, m_{x} t_{x}^{-1}, m_{y} t_{y}, r_{x}^{2} t_{y}^{-1}$,

$9,29,63,111,174,250,341,444,562,692]$

$1073 \quad H_{377} \quad 1$

$r_{y}^{2} t_{x}, m_{x} t_{x}, m_{z} r_{z}^{-1} t_{x}^{-1}, r_{z}^{-1} t_{x}^{-1}, m_{z} r_{z} t_{y}, r_{z} t_{y}$,

$29,64,111,174,249,339,442,560,690]$

$1074 \quad H_{682} \quad\left\langle m_{x}\right\rangle$ $m_{y} t_{y}^{-1}, m_{z} t_{z}^{-1}$

$[9,29,64,111,175,250,339,443,560,689]$

$i t_{x}, r_{y}^{2} t_{x}, r_{x}^{2} t_{x}^{-1}, r_{y}^{2} t_{x}^{-1}, m_{y} t_{y}^{-1}, r_{y}^{2} t_{z}$,

$1075 \quad H_{382} \quad 1$

$r_{y}^{2} t_{x}, m_{x} t_{x}, m_{z} r_{z}^{-1} t_{x}^{-1}, r_{z}^{-1} t_{x}^{-1}, m_{z} r_{z} t_{y}, r_{z} t_{y}$,

$[9,29,64,112,175,251,342,445,564,695]$

$1076 \quad H_{343} \quad 1$ $m_{y} t_{y}^{-1}, r_{y}^{2} r_{z} t_{z}^{-1}$

$[9,29,64,112,175,251,342,445,564,695]$
$1076 \quad H_{343} \quad 1$

$r_{z}^{2} t_{x}, r_{y}^{2} r_{z} t_{x}^{-1}, m_{x} r_{z} t_{x}^{-1}, i t_{y}, r_{z}^{2} t_{y}, r_{y}^{2} r_{z} t_{y}^{-1}$ $m_{x} r_{z} t_{y}^{-1}, m_{z} t_{z}^{-1}$ 


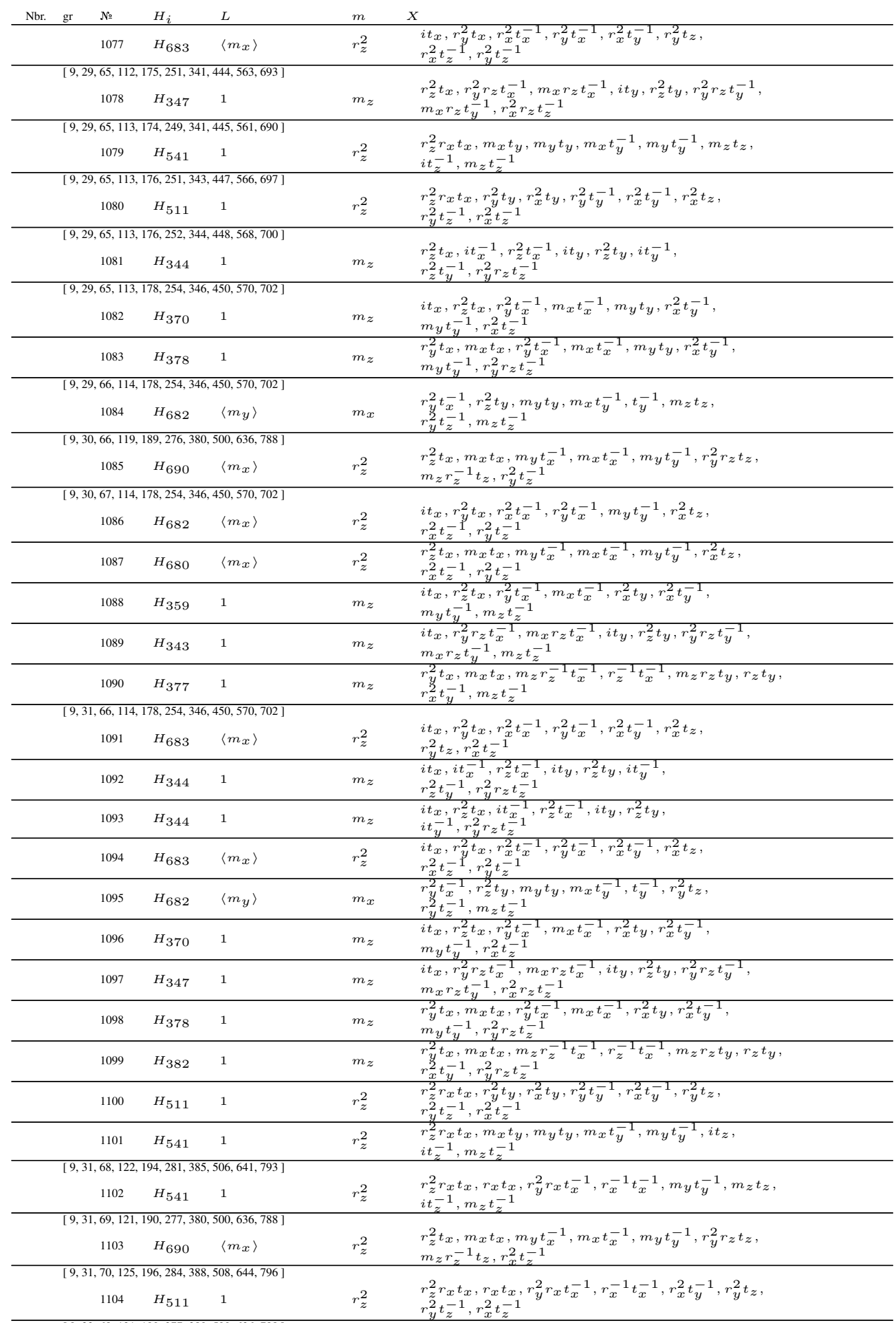




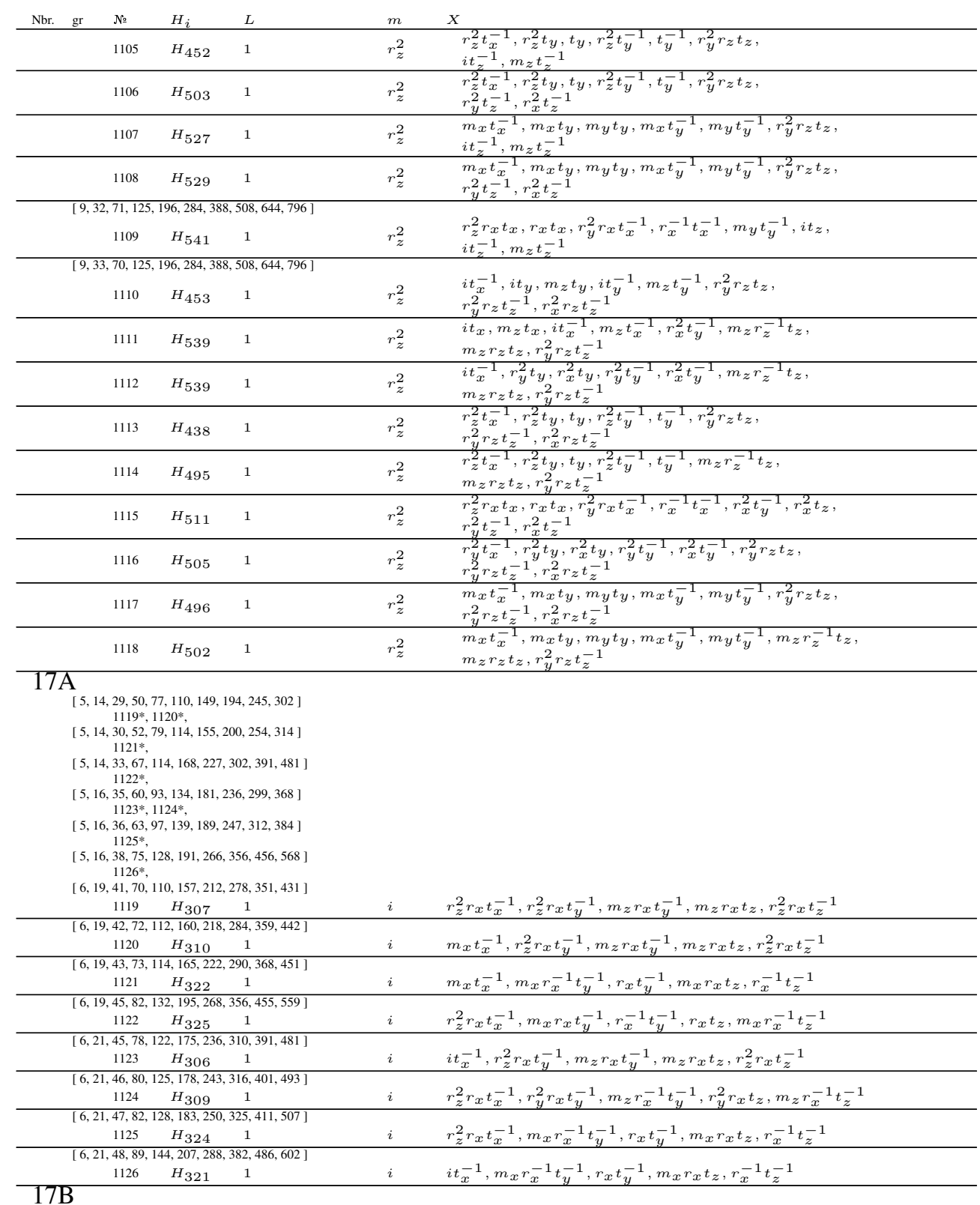

[ $5,15,37,74,122,178,244,322,410,508]$ $1127^{*}$,

$[5,15,38,78,132,200,282,378,488,612]$ $1128^{*}$,

$[5,15,40,82,134,200,278,368,472,590]$ $1129 *$

[ $5,15,40,83,142,216,300,393,500,622]$ 1130 *

$[5,15,40,89,152,230,319,422,541,670]$ $1131 *$

$[5,15,41,87,146,221,306,403,513,637]$ 1132 *,

$[5,16,39,74,119,174,239,314,399,494]$ $1133^{*}, 1134^{*}$,

$[5,16,39,76,127,192,271,364,471,592]$ 1135*,

$[5,16,42,80,131,196,274,364,467,584]$ 


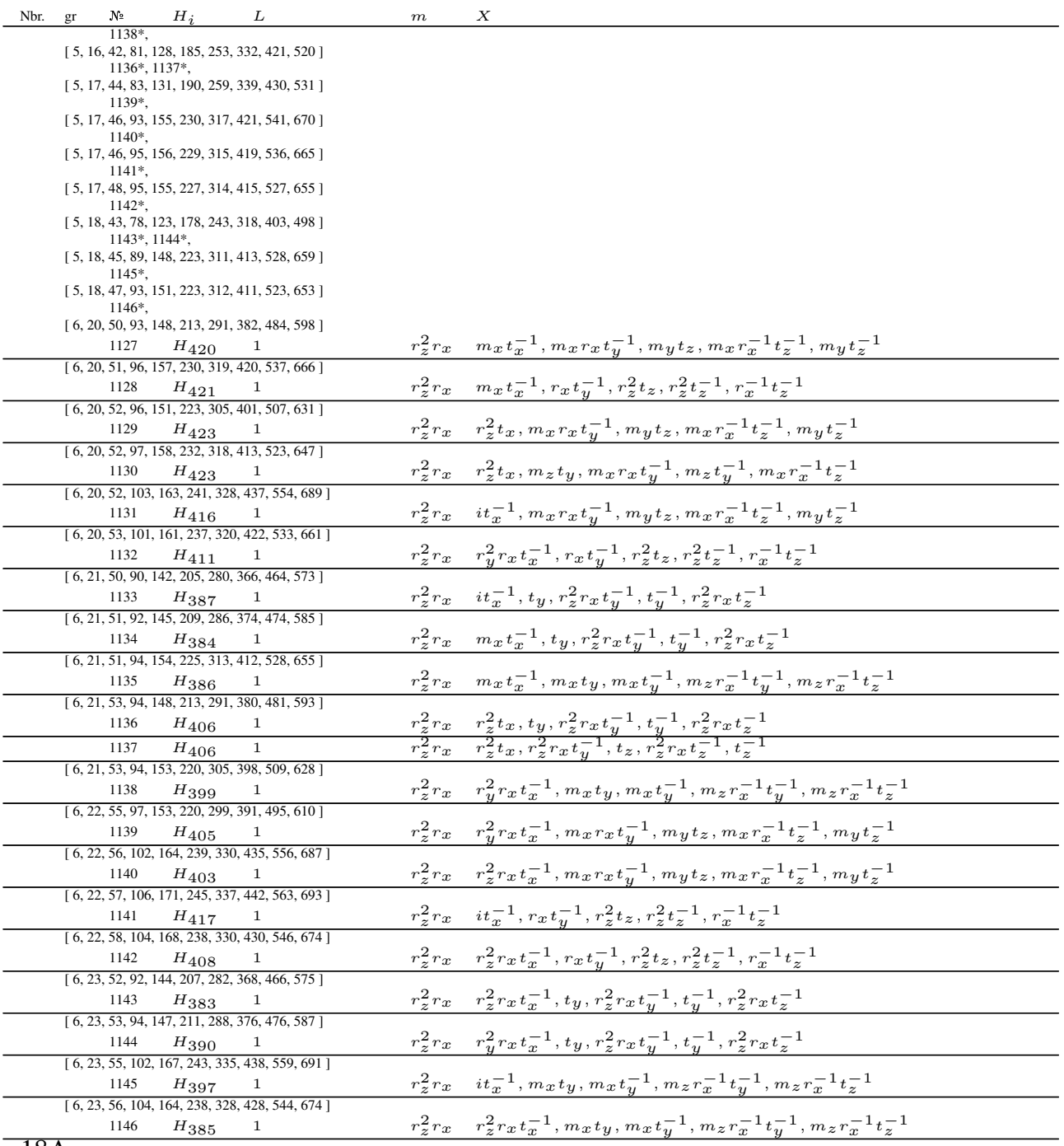

$18 \mathrm{~A}$

$[7,22,47,82,127,182,247,322,407,502]$ $1147^{*}, 1148^{*}$,

$[7,22,48,85,132,189,257,336,425,524]$ $1149^{*}, 1150^{*}$

$[7,23,50,87,135,194,263,343,434,535$ $1152 *$

$[7,23,51,90,140,202,275,359,455,562]$ $1151^{*}$,

$[7,23,51,91,143,206,280,366,464,574]$ $1153^{*}$,

$[7,23,51,91,143,207,283,371,471,583]$ $1154 *$

$[7,23,52,94,148,214,292,382,484,598]$ $1156^{*}$,

$[7,23,52,94,150,220,302,396,504,626]$ $1157^{*}$,

$[7,23,52,95,152,222,305,401,510,632]$ $1155^{*}$,

$[7,23,52,96,155,226,311,411,523,647]$ $1158^{*}$

$[7,23,52,97,159,234,321,425,545,674]$ $1159^{*}$,

$[7,23,54,99,156,228,315,414,527,655]$ $1160^{*}$,

$[7,23,55,103,165,241,331,435,553,685]$ $1161 *$ 


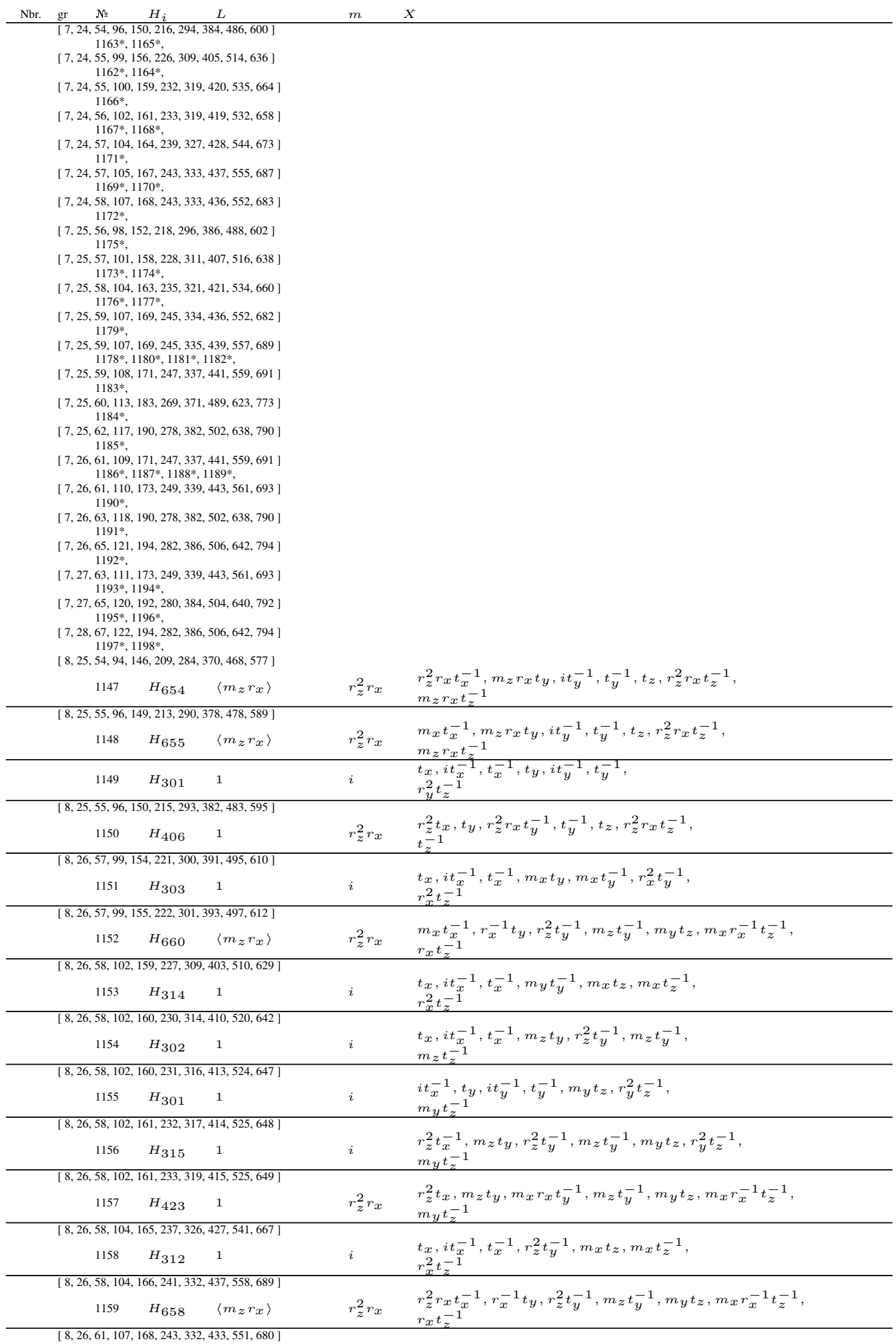




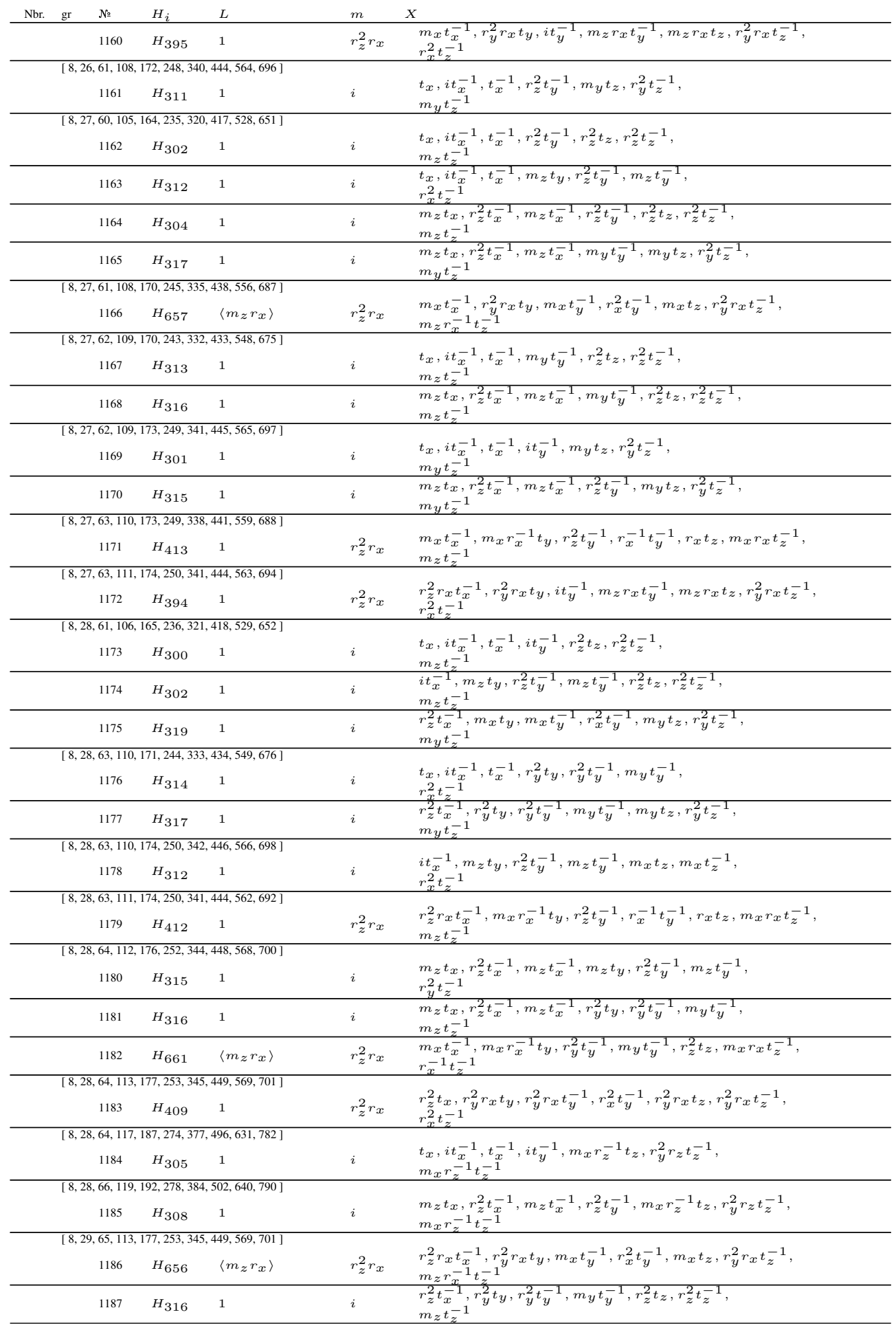




\begin{tabular}{|c|c|c|c|c|c|c|}
\hline Nbr. & $\mathrm{gr}$ & № & $H_{i}$ & $L$ & $m$ & $X$ \\
\hline & & 1188 & $H_{317}$ & 1 & $i$ & $\begin{array}{l}m_{z} t_{x}, r_{z}^{2} t_{x}^{-1}, m_{z} t_{x}^{-1}, r_{y}^{2} t_{y}, r_{y}^{2} t_{y}^{-1}, m_{y} t_{y}^{-1} \\
r_{y}^{2} t_{z}^{-1}\end{array}$ \\
\hline & & 1189 & $H_{318}$ & 1 & $i$ & $\begin{array}{l}r_{z}^{2} t_{x}^{-1}, r_{y}^{2} t_{y}, r_{y}^{2} t_{y}^{-1}, m_{y} t_{y}^{-1}, m_{x} t_{z}, m_{x} t_{z}^{-1}, \\
r_{x}^{2} t_{z}^{-1}\end{array}$ \\
\hline \multicolumn{7}{|c|}{$[8,29,65,114,178,254,346,450,570,702]$} \\
\hline & & 1190 & $H_{422}$ & 1 & $r_{z}^{2} r_{x}$ & $\begin{array}{l}r_{z}^{2} t_{x}, m_{x} r_{x}^{-1} t_{y}, m_{x} r_{x}^{-1} t_{y}^{-1}, m_{y} t_{y}^{-1}, m_{x} r_{x} t_{z}, m_{x} r_{x} t_{z}^{-1}, \\
m_{z} t_{z}^{-1}\end{array}$ \\
\hline \multicolumn{7}{|c|}{$[8,29,67,122,193,281,385,505,641,793]$} \\
\hline & & 1191 & $H_{323}$ & 1 & $i$ & $\begin{array}{l}r_{z}^{2} t_{x}^{-1}, m_{x} r_{y} t_{y}, r_{x}^{2} r_{y} t_{y}^{-1}, m_{x} r_{y} t_{y}^{-1}, m_{x} t_{z}, m_{x} t_{z}^{-1} \\
r_{x}^{2} t_{z}^{-1}\end{array}$ \\
\hline \multicolumn{7}{|c|}{$[8,29,69,123,196,282,388,506,644,794]$} \\
\hline & & 1192 & $H_{326}$ & 1 & $i$ & $\begin{array}{l}m_{z} r_{x} t_{x}, r_{z}^{2} r_{x} t_{x}^{-1}, m_{z} r_{x} t_{x}^{-1}, m_{y} t_{y}^{-1}, r_{z}^{2} t_{z}, r_{z}^{2} t_{z}^{-1} \\
m_{z} t_{z}^{-1}\end{array}$ \\
\hline \multicolumn{7}{|c|}{$[8,30,66,114,178,254,346,450,570,702]$} \\
\hline & & 1193 & $H_{659}$ & $\left\langle m_{z} r_{x}\right\rangle$ & $r_{z}^{2} r_{x}$ & $\begin{array}{l}r_{z}^{2} r_{x} t_{x}^{-1}, m_{x} r_{x}^{-1} t_{y}, r_{y}^{2} t_{y}^{-1}, m_{y} t_{y}^{-1}, r_{z}^{2} t_{z}, m_{x} r_{x} t_{z}^{-1}, \\
r_{x}^{-1} t_{z}^{-1}\end{array}$ \\
\hline & & 1194 & $H_{314}$ & 1 & $i$ & $\begin{array}{l}i t_{x}^{-1}, r_{y}^{2} t_{y}, r_{y}^{2} t_{y}^{-1}, m_{y} t_{y}^{-1}, m_{x} t_{z}, m_{x} t_{z}^{-1} \\
r_{x}^{2} t_{z}^{-1}\end{array}$ \\
\hline \multicolumn{7}{|c|}{$[8,30,67,121,192,280,384,504,640,792]$} \\
\hline & & 1195 & $H_{305}$ & 1 & $i$ & $\begin{array}{l}t_{x}, i t_{x}^{-1}, t_{x}^{-1}, i t_{y}, m_{x} r_{z}^{-1} t_{z}, r_{y}^{2} r_{z} t_{z}^{-1} \\
m_{x} r_{z}^{-1} t_{z}^{-1}\end{array}$ \\
\hline & & 1196 & $H_{308}$ & 1 & $i$ & $\begin{array}{l}m_{z} t_{x}, r_{z}^{2} t_{x}^{-1}, m_{z} t_{x}^{-1}, r_{z}^{2} t_{y}, m_{x} r_{z}^{-1} t_{z}, r_{y}^{2} r_{z} t_{z}^{-1} \\
m_{x} r_{z}^{-1} t_{z}^{-1}\end{array}$ \\
\hline \multicolumn{7}{|c|}{$[8,31,69,124,195,283,387,507,643,795]$} \\
\hline & & 1197 & $H_{323}$ & 1 & $i$ & $\begin{array}{l}r_{z}^{2} t_{x}^{-1}, m_{x} r_{y} t_{y}, r_{x}^{2} r_{y} t_{y}^{-1}, m_{x} r_{y} t_{y}^{-1}, m_{x} t_{z}, r_{x}^{2} t_{z} \\
m_{x} \bar{z}^{-1}\end{array}$ \\
\hline & & 1198 & $H_{326}$ & 1 & $i$ & $\begin{array}{l}m_{z} r_{x} t_{x}, r_{z}^{2} r_{x} t_{x}^{-1}, m_{z} r_{x} t_{x}^{-1}, m_{y} t_{y}^{-1}, r_{z}^{2} t_{z}, m_{z} t_{z}, \\
r_{z}^{2} t_{z}^{-1}\end{array}$ \\
\hline
\end{tabular}

18B

[ $7,25,57,103,163,237,325,427,543,673$ ] $1200^{*}$,

$[7,25,58,103,161,235,322,421,535,663]$ 1199*,

[ 7, 26, 61, 107, 167, 242, 329, 431, 547, 675 ] $1201 *$,

$[7,26,62,109,168,243,332,434,550,680]$ $1202^{*}$

[ 7, 27, 61, 107, 169, 245, 334, 436, 552, 682 ] 1203*,

$[7,27,62,109,170,245,335,438,554,685]$ 1204*,

$[7,27,65,113,173,249,339,443,561,693]$ 1205*,

$[7,29,65,111,173,249,339,443,561,693$ ] $1206^{*}$,

$[8,28,62,108,170,244,332,434,550,678]$

$1199 \quad H_{759} \quad\left\langle m_{z}, r_{x}^{2}\right\rangle$

$r_{z}^{2} r_{x} \quad r_{y}^{2} r_{x} t_{x}^{-1}, r_{z}^{2} t_{y}, r_{x}^{-1} t_{y}, m_{x} t_{y}^{-1}, m_{z} r_{x}^{-1} t_{y}^{-1}, m_{z} r_{x}^{-1} t_{z}$,

$[8,28,62,110,174,250,342,446,566,698]$ $1200 \quad H_{760} \quad\left\langle m_{z}, r_{x}^{2}\right\rangle$ $r_{z}^{2} r_{x} \quad m_{x} t_{x}^{-1}, r_{z}^{2} t_{y}, r_{x}^{-1} t_{y}, m_{x} t_{y}^{-1}, m_{z} r_{x}^{-1} t_{y}^{-1}, m_{z} r_{x}^{-1} t_{z}$

$[8,29,65,111,175,250,339,443,560,689]$ $1201 \quad H_{413} \quad 1$

$[8,29,66,112,175,251,341,444,563,693]$ $1202 \quad H_{395} \quad 1$

$[8,30,63,111,174,250,341,444,562,692]$ $1203 \quad H_{412} \quad 1$

$[8,30,64,112,175,251,342,445,564,695]$ $1204 \quad H_{394} \quad 1$

$8,30,68,114,178,254,346,450,570,702]$ $1205 \quad H_{758} \quad\left\langle m_{z}, r_{x}^{2}\right\rangle$

$[8,32,66,114,178,254,346,450,570,702]$ $1206 \quad H_{757} \quad\left\langle m_{z}, r_{x}^{2}\right\rangle$ $r_{z}^{2} r_{x} \quad m_{x} t_{x}^{-1}, m_{x} r_{x}^{-1} t_{y}, m_{y} t_{y}, r_{z}^{2} t_{y}^{-1}, r_{x}^{-1} t_{y}^{-1}, r_{x} t_{z}$, $m_{x} r_{x} t_{z}^{-1}$ $r_{z}^{2} r_{x} \quad m_{x} t_{x}^{-1}, r_{y}^{2} r_{x} t_{y}, r_{x}^{2} t_{y}, i t_{y}^{-1}, m_{z} r_{x} t_{y}^{-1}, m_{z} r_{x} t_{z}$,

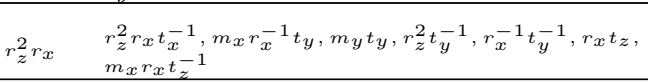

$r_{z}^{2} r_{x} \quad r_{z}^{2} r_{x} t_{x}^{-1}, r_{y}^{2} r_{x} t_{y}, r_{x}^{2} t_{y}, i t_{y}^{-1}, m_{z} r_{x} t_{y}^{-1}, m_{z} r_{x} t_{z}$

\begin{tabular}{|c|c|c|c|c|}
\hline \multicolumn{5}{|c|}{$[8,30,68,114,178,254,346,450,570,702]$} \\
\hline 1205 & $H_{758}$ & $\left\langle m_{z}, r_{x}^{2}\right\rangle$ & $r_{z}^{2} r_{x}$ & $\begin{array}{l}m_{x} t_{x}^{-1}, m_{x} r_{x} t_{y}, r_{x}^{2} t_{y}, r_{y}^{2} r_{x} t_{y}^{-1}, r_{x}^{2} t_{y}^{-1}, m_{x} r_{x}^{-1} t_{z} \\
r_{y}^{2} r_{x} t_{z}^{-1}\end{array}$ \\
\hline \multicolumn{5}{|c|}{$[8,32,66,114,178,254,346,450,570,702]$} \\
\hline 1206 & $H_{757}$ & $\left\langle m_{z}, r_{x}^{2}\right\rangle$ & $r_{z}^{2} r_{x}$ & $\begin{array}{l}r_{y}^{2} r_{x} t_{x}^{-1}, m_{x} r_{x} t_{y}, r_{x}^{2} t_{y}, r_{y}^{2} r_{x} t_{y}^{-1}, r_{x}^{2} t_{y}^{-1}, m_{x} r_{x}^{-1} t_{z}, \\
r_{y}^{2} r_{x} t_{z}\end{array}$ \\
\hline
\end{tabular}

19

$[8,26,56,98,152,218,296,386,488,602]$ $1208 *$

$[8,26,57,101,158,228,311,407,516,638]$ $1207^{*}$,

$[8,26,57,101,159,231,316,414,525,649]$ $1209^{*}$,

$[8,26,58,104,162,232,316,414,524,646]$

1213*, 


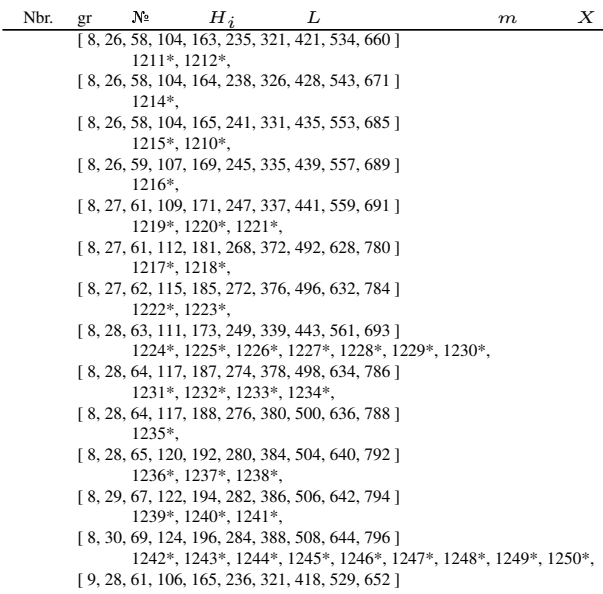

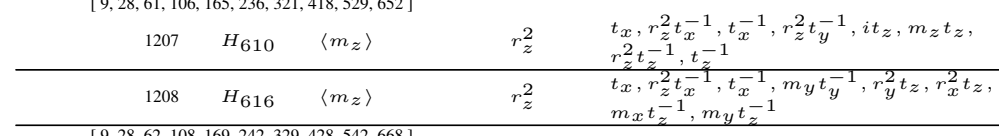

$[9,28,62,108,169,242,329,428,542,668]$

$1209 \quad H_{461} \quad 1 \quad r_{z}^{2} \quad \begin{aligned} & t_{x}, r_{z}^{2} t_{x}^{-1}, t_{x}^{-1}, m_{y} t_{y}^{-1}, r_{y}^{2} t_{z}, r_{x}^{2} t_{z}, \\ & i t_{z}^{-1}, m_{z} t^{-1}\end{aligned}$

$[9,28,62,108,171,246,338,442,562,694]$ $i t_{z}^{-1}, m_{z} t_{z}^{-1}$

\begin{tabular}{|c|c|c|c|c|}
\hline \multicolumn{5}{|c|}{$[9,28,62,108,171,246,338,442,562,694]$} \\
\hline 1210 & $H_{460}$ & 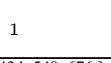 & $r_{z}^{2}$ & $\begin{array}{l}t_{x}, r_{z}^{2} t_{x}^{-1}, t_{x}^{-1}, r_{z}^{2} t_{y}^{-1}, r_{y}^{2} t_{z}, r_{x}^{2} t_{z} \\
i t_{z}^{-1}, m_{z} t_{z}^{-1}\end{array}$ \\
\hline \multicolumn{5}{|c|}{$[9,28,63,110,171,244,333,434,549,676]$} \\
\hline 1211 & $H_{615}$ & $\left\langle m_{z}\right\rangle$ & $r_{z}^{2}$ & $\begin{array}{l}t_{x}, r_{z}^{2} t_{x}^{-1}, t_{x}^{-1}, m_{y} t_{y}^{-1}, i t_{z}, m_{z} t_{z} \\
r_{z}^{2} t_{z}^{-1}, t_{z}^{-1}\end{array}$ \\
\hline 1212 & $H_{305}$ & 1 & $i$ & $\begin{array}{l}t_{x}, i t_{x}^{-1}, t_{x}^{-1}, i t_{y}, t_{y}, i t_{y}^{-1} \\
t_{y}^{-1}, r_{y}^{2} r_{z} t_{z}^{-1}\end{array}$ \\
\hline 1213 & $H_{540}$ & 1 & $r_{z}^{2}$ & $\begin{array}{l}m_{z} t_{x}, i t_{x}^{-1}, m_{z} t_{x}^{-1}, r_{x}^{2} r_{y} t_{y}^{-1}, m_{x} t_{z}, m_{y} t_{z} \\
m_{x} t_{z}^{-1}, m_{y} t_{z}^{-1}\end{array}$ \\
\hline \multicolumn{5}{|c|}{$[9,28,63,110,173,248,339,442,559,688]$} \\
\hline 1214 & $H_{504}$ & 1 & $r_{z}^{2}$ & $\begin{array}{l}t_{x}, r_{z}^{2} t_{x}^{-1}, t_{x}^{-1}, r_{x}^{2} r_{y} t_{y}^{-1}, r_{z}^{2} t_{z}, t_{z} \\
r_{z}^{2} t_{z}^{-1}, t_{z}^{-1}\end{array}$ \\
\hline \multicolumn{5}{|c|}{$[9,28,63,110,173,248,339,442,562,694]$} \\
\hline 1215 & $H_{323}$ & 1 & $i$ & $\begin{array}{l}r_{z}^{2} t_{x}, m_{z} t_{x}, r_{z}^{2} t_{x}^{-1}, m_{z} t_{x}^{-1}, r_{x}^{2} r_{y} t_{y}^{-1}, m_{x} t_{z} \\
m_{x} t_{z}^{-1}, r_{x}^{2} t_{z}^{-1}\end{array}$ \\
\hline \multicolumn{5}{|c|}{$[9,28,63,110,174,250,342,446,566,698]$} \\
\hline 1216 & $H_{614}$ & $\left\langle m_{z}\right\rangle$ & $r_{z}^{2}$ & $\begin{array}{l}t_{x}, r_{z}^{2} t_{x}^{-1}, t_{x}^{-1}, r_{z}^{2} t_{y}^{-1}, r_{y}^{2} t_{z}, r_{x}^{2} t_{z} \\
m_{x} t_{z}^{-1}, m_{y} t_{z}^{-1}\end{array}$ \\
\hline \multicolumn{5}{|c|}{$[9,29,64,115,183,269,372,492,628,780]$} \\
\hline 1217 & $H_{452}$ & 1 & $r_{z}^{2}$ & $\begin{array}{l}t_{x}, r_{z}^{2} t_{x}^{-1}, t_{x}^{-1}, r_{z}^{2} t_{y}^{-1}, r_{y}^{2} r_{z} t_{z}, r_{x}^{2} r_{z} t_{z} \\
i t_{z}^{-1}, m_{z} t_{z}^{-1}\end{array}$ \\
\hline 1218 & $H_{487}$ & 1 & $r_{z}^{2}$ & $\begin{array}{l}t_{x}, r_{z}^{2} t_{x}^{-1}, t_{x}^{-1}, r_{z}^{2} t_{y}^{-1}, m_{z} r_{z}^{-1} t_{z}, m_{z} r_{z} t_{z} \\
i t_{z}^{-1}, m_{z} t_{z}^{-1}\end{array}$ \\
\hline \multicolumn{5}{|c|}{$[9,29,65,113,177,253,345,449,569,701]$} \\
\hline 1219 & $H_{617}$ & $\left\langle m_{z}\right\rangle$ & $r_{z}^{2}$ & $\begin{array}{l}m_{x} t_{x}^{-1}, m_{x} t_{y}, m_{x} t_{y}^{-1}, m_{y} t_{y}^{-1}, i t_{z}, m_{z} t_{z} \\
r_{z}^{2} t_{z}^{-1}, t_{z}^{-1}\end{array}$ \\
\hline 1220 & $H_{618}$ & $\left\langle m_{z}\right\rangle$ & $r_{z}^{2}$ & $\begin{array}{l}m_{x} t_{x}^{-1}, m_{x} t_{y}, m_{x} t_{y}^{-1}, m_{y} t_{y}^{-1}, r_{y}^{2} t_{z}, r_{x}^{2} t_{z}, \\
m_{x} t_{z}^{-1}, m_{y} t_{z}^{-1}\end{array}$ \\
\hline 1221 & $H_{483}$ & 1 & $r_{z}^{2}$ & $\begin{array}{l}m_{x} t_{x}^{-1}, m_{x} t_{y}, m_{x} t_{y}^{-1}, m_{y} t_{y}^{-1}, r_{y}^{2} t_{z}, r_{x}^{2} t_{z} \\
i t_{z}^{-1}, m_{z} t_{z}^{-1}\end{array}$ \\
\hline \multicolumn{5}{|c|}{$[9,29,65,117,186,273,376,496,632,784]$} \\
\hline 1222 & $H_{503}$ & 1 & $r_{z}^{2}$ & $\begin{array}{l}{ }^{t} x, r_{z}^{2} t_{x}, t_{x}^{1}, r_{z}^{2} t_{y}, r_{y}^{2} r^{t} z, r_{x}^{2} r_{z} t_{z} \\
r_{y}^{2} t_{z}^{-1}, r_{x}^{2} t_{z}^{-1}\end{array}$ \\
\hline 1223 & $H_{519}$ & 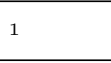 & $r_{z}^{2}$ & $\begin{array}{l}t_{x}, r_{z}^{2} t_{x}^{-1}, t_{x}^{-1}, r_{z}^{2} t_{y}^{-1}, m_{z} r_{z}^{-1} t_{z}, m_{z} r_{z} t_{z} \\
r_{y}^{2} t_{z}^{-1}, r_{x}^{2} t_{z}^{-1}\end{array}$ \\
\hline \multicolumn{5}{|c|}{$[9,30,66,114,178,254,346,450,570,702]$} \\
\hline 1224 & $H_{615}$ & $\left\langle m_{z}\right\rangle$ & $r_{z}^{2}$ & $\begin{array}{l}r_{z}^{2} t_{x}^{-1}, m_{x} t_{y}, m_{x} t_{y}^{-1}, m_{y} t_{y}^{-1}, i t_{z}, m_{z} t_{z} \\
r_{z}^{2} t_{z}^{-1}, t_{z}^{-1}\end{array}$ \\
\hline 1225 & $H_{616}$ & $\left\langle m_{z}\right\rangle$ & $r_{z}^{2}$ & $\begin{array}{l}r_{z}^{2} t_{x}^{-1}, m_{x} t_{y}, m_{x} t_{y}^{-1}, m_{y} t_{y}^{-1}, r_{y}^{2} t_{z}, r_{x}^{2} t_{z} \\
m_{x} t_{z}^{-1}, m_{y} t_{z}^{-1}\end{array}$ \\
\hline 1226 & $H_{308}$ & 1 & $i$ & $\begin{array}{l}m_{z} t_{x}, r_{z}^{2} t_{x}^{-1}, m_{z} t_{x}^{-1}, r_{z}^{2} t_{y}, m_{z} t_{y}, r_{z}^{2} t_{y}^{-1} \\
m_{z} t_{y}^{-1}, r_{y}^{2} r_{z} t_{z}^{-1}\end{array}$ \\
\hline
\end{tabular}




\begin{tabular}{|c|c|c|c|c|c|c|}
\hline Nbr. & gr & № & $H_{i}$ & $L$ & $m$ & $x$ \\
\hline & & 1227 & $H_{326}$ & 1 & $i$ & $\begin{array}{l}r_{z}^{2} r_{x} t_{x}^{-1}, r_{y}^{2} t_{y}, m_{y} t_{y}, r_{y}^{2} t_{y}^{-1}, m_{y} t_{y}^{-1}, r_{z}^{2} t_{z} \\
r_{z}^{2} t_{z}^{-1}, m_{z} t_{z}^{-1}\end{array}$ \\
\hline & & 1228 & $H_{461}$ & 1 & $r_{z}^{2}$ & $\begin{array}{l}r_{z}^{2} t_{x}^{-1}, m_{x} t_{y}, m_{x} t_{y}^{-1}, m_{y} t_{y}^{-1}, r_{y}^{2} t_{z}, r_{x}^{2} t_{z} \\
i t_{z}^{-1}, m_{z} t_{z}^{-1}\end{array}$ \\
\hline & & 1229 & $H_{511}$ & 1 & $r_{z}^{2}$ & $\begin{array}{l}r_{z}^{2} r_{x} t_{x}, r_{y}^{2} t_{y}, r_{y}^{2} t_{y}^{-1}, r_{x}^{2} t_{y}^{-1}, r_{y}^{2} t_{z}, r_{x}^{2} t_{z} \\
r_{y}^{2} t_{z}^{-1}, r_{x}^{2} t_{z}^{-1}\end{array}$ \\
\hline & & 1230 & $H_{541}$ & 1 & $r_{z}^{2}$ & $\begin{array}{l}r_{z}^{2} r_{x} t_{x}, m_{x} t_{y}, m_{x} t_{y}^{-1}, m_{y} t_{y}^{-1}, i t_{z}, m_{z} t_{z} \\
i t_{z}^{-1}, m_{z} t_{z}^{-1}\end{array}$ \\
\hline \multicolumn{7}{|c|}{$[9,30,67,120,189,276,379,499,635,787]$} \\
\hline & & 1231 & $H_{527}$ & 1 & $r_{z}^{2}$ & $\begin{array}{l}m_{x} t_{x}^{-1}, m_{x} t_{y}, m_{x} t_{y}^{-1}, m_{y} t_{y}^{-1}, r_{y}^{2} r_{z} t_{z}, r_{x}^{2} r_{z} t_{z} \\
i t_{z}^{-1}, m_{z} t_{z}^{-1}\end{array}$ \\
\hline & & 1232 & $H_{528}$ & 1 & $r_{z}^{2}$ & $\begin{array}{l}m_{x} t_{x}^{-1}, m_{x} t_{y}, m_{x} t_{y}^{-1}, m_{y} t_{y}^{-1}, m_{z} r_{z}^{-1} t_{z}, m_{z} r_{z} t_{z}, \\
i t_{z}^{-1}, m_{z} t_{z}^{-1}\end{array}$ \\
\hline & & 1233 & $H_{529}$ & 1 & $r_{z}^{2}$ & $\begin{array}{l}m_{x} t_{x}^{-1}, m_{x} t_{y}, m_{x} t_{y}^{-1}, m_{y} t_{y}^{-1}, r_{y}^{2} r_{z} t_{z}, r_{x}^{2} r_{z} t_{z} \\
r_{y}^{2} t_{z}^{-1}, r_{x}^{2} t_{z}^{-1}\end{array}$ \\
\hline & & 1234 & $H_{530}$ & 1 & $r_{z}^{2}$ & $\begin{array}{l}m_{x} t_{x}^{-1}, m_{x} t_{y}, m_{x} t_{y}^{-1}, m_{y} t_{y}^{-1}, m_{z} r_{z}^{-1} t_{z}, m_{z} r_{z} t_{z} \\
r_{y}^{2} t_{z}^{-1}, r_{x}^{2} t_{z}^{-1}\end{array}$ \\
\hline \multicolumn{7}{|c|}{$[9,30,67,121,191,278,381,500,636,788]$} \\
\hline & & 1235 & $H_{539}$ & 1 & $r_{z}^{2}$ & $\begin{array}{l}m_{z} t_{x}, i t_{x}^{-1}, m_{z} t_{x}^{-1}, r_{x}^{2} t_{y}^{-1}, m_{z} r_{z}^{-1} t_{z}, m_{z} r_{z} t_{z}, \\
r_{y}^{2} r_{z} t_{z}^{-1}, r_{x}^{2} r_{z} t_{z}^{-1}\end{array}$ \\
\hline \multicolumn{7}{|c|}{$[9,30,67,121,192,280,384,504,640,792]$} \\
\hline & & 1236 & $H_{611}$ & $\left\langle m_{z}\right\rangle$ & $r_{z}^{2}$ & $\begin{array}{l}t_{x}, r_{z}^{2} t_{x}^{-1}, t_{x}^{-1}, r_{z}^{2} t_{y}^{-1}, r_{y}^{2} r_{z} t_{z}, r_{x}^{2} r_{z} t_{z} \\
m_{x} r_{z} t_{z}^{-1}, m_{x} r_{z}^{-1} t_{z}^{-1}\end{array}$ \\
\hline & & 1237 & $H_{619}$ & $\left\langle m_{z}\right\rangle$ & $r_{z}^{2}$ & $\begin{array}{l}t_{x}, r_{z}^{2} t_{x}^{-1}, t_{x}^{-1}, r_{z}^{2} t_{y}^{-1}, m_{z} r_{z}^{-1} t_{z}, m_{z} r_{z} t_{z} \\
r_{z}^{-1} t_{z}^{-1}, r_{z} t_{z}^{-1}\end{array}$ \\
\hline & & 1238 & $H_{495}$ & 1 & $r_{z}^{2}$ & $\begin{array}{l}t_{x}, r_{z}^{2} t_{x}^{-1}, t_{x}^{-1}, r_{z}^{2} t_{y}^{-1}, m_{z} r_{z}^{-1} t_{z}, m_{z} r_{z} t_{z} \\
r_{y}^{2} r_{z} t_{z}^{-1}, r_{x}^{2} r_{z} t_{z}^{-1}\end{array}$ \\
\hline \multicolumn{7}{|c|}{$[9,31,69,124,195,283,387,507,643,795]$} \\
\hline & & 1239 & $H_{622}$ & $\left\langle m_{z}\right\rangle$ & $r_{z}^{2}$ & $\begin{array}{l}m_{x} t_{x}^{-1}, m_{x} t_{y}, m_{x} t_{y}^{-1}, m_{y} t_{y}^{-1}, r_{y}^{2} r_{z} t_{z}, r_{x}^{2} r_{z} t_{z} \\
m_{x} r_{z} t_{z}^{-1}, m_{x} r_{z}^{-1} t_{z}^{-1}\end{array}$ \\
\hline & & 1240 & $H_{623}$ & $\left\langle m_{z}\right\rangle$ & $r_{z}^{2}$ & $\begin{array}{l}m_{x} t_{x}^{-1}, m_{x} t_{y}, m_{x} t_{y}^{-1}, m_{y} t_{y}^{-1}, m_{z} r_{z}^{-1} t_{z}, m_{z} r_{z} t_{z}, \\
r_{z}^{-1} t_{z}^{-1}, r_{z} t_{z}^{-1}\end{array}$ \\
\hline & & 1241 & $H_{502}$ & 1 & $r_{z}^{2}$ & $\begin{array}{l}m_{x} t_{x}^{-1}, m_{x} t_{y}, m_{x} t_{y}^{-1}, m_{y} t_{y}^{-1}, m_{z} r_{z}^{-1} t_{z}, m_{z} r_{z} t_{z}, \\
r_{y}^{2} r_{z} t_{z}^{-1}, r_{x}^{2} r_{z} t_{z}^{-1}\end{array}$ \\
\hline \multicolumn{7}{|c|}{$[9,32,70,125,196,284,388,508,644,796]$} \\
\hline & & 1242 & $H_{305}$ & 1 & $i$ & $\begin{array}{l}i t_{x}^{-1}, i t_{y}, t_{y}, i t_{y}^{-1}, t_{y}^{-1}, m_{x} r_{z}^{-1} t_{z} \\
r_{y}^{2} r_{z} t_{z}^{-1}, m_{x} r_{z}^{-1} t_{z}^{-1}\end{array}$ \\
\hline & & 1243 & $H_{308}$ & 1 & $i$ & $\begin{array}{l}r_{z}^{2} t_{x}^{-1}, r_{z}^{2} t_{y}, m_{z} t_{y}, r_{z}^{2} t_{y}^{-1}, m_{z} t_{y}^{-1}, m_{x} r_{z}^{-1} t_{z} \\
r_{y}^{2} r_{z} t_{z}^{-1}, m_{x} r_{z}^{-1} t_{z}^{-1}\end{array}$ \\
\hline & & 1244 & $H_{323}$ & 1 & $i$ & $\begin{array}{l}r_{z}^{2} t_{x}, m_{z} t_{x}, r_{z}^{2} t_{x}^{-1}, m_{z} t_{x}^{-1}, m_{x} r_{y} t_{y}, r_{x}^{2} r_{y} t_{y}^{-1} \\
m_{x} r_{y} t_{y}^{-1}, r_{x}^{2} t_{z}^{-1}\end{array}$ \\
\hline & & 1245 & $H_{326}$ & 1 & $i$ & $\begin{array}{l}m_{z} r_{x} t_{x}, r_{z}^{2} r_{x} t_{x}^{-1}, m_{z} r_{x} t_{x}^{-1}, m_{y} t_{y}^{-1}, r_{z}^{2} t_{z}, m_{z} t_{z}, \\
r_{z}^{2} t_{z}^{-1}, m_{z} t_{z}^{-1}\end{array}$ \\
\hline & & 1246 & $H_{539}$ & 1 & $r_{z}^{2}$ & $\begin{array}{l}i t_{x}^{-1}, r_{y}^{2} t_{y}, r_{y}^{2} t_{y}^{-1}, r_{x}^{2} t_{y}^{-1}, m_{z} r_{z}^{-1} t_{z}, m_{z} r_{z} t_{z} \\
r_{y}^{2} r_{z} t_{z}^{-1}, r_{x}^{2} r_{z} t_{z}^{-1}\end{array}$ \\
\hline & & 1247 & $H_{540}$ & 1 & $r_{z}^{2}$ & $\begin{array}{l}i t_{x}^{-1}, r_{y} t_{y}, r_{x}^{2} r_{y} t_{y}^{-1}, r_{y}^{-1} t_{y}^{-1}, m_{x} t_{z}, m_{y} t_{z} \\
m_{x} t_{z}^{-1}, m_{y} t_{z}^{-1}\end{array}$ \\
\hline & & 1248 & $H_{504}$ & 1 & $r_{z}^{2}$ & $\begin{array}{l}r_{z}^{2} t_{x}^{-1}, r_{y} t_{y}, r_{x}^{2} r_{y} t_{y}^{-1}, r_{y}^{-1} t_{y}^{-1}, r_{z}^{2} t_{z}, t_{z} \\
r_{z}^{2} t_{z}^{-1}, t_{z}^{-1}\end{array}$ \\
\hline & & 1249 & $H_{511}$ & 1 & $r_{z}^{2}$ & $\begin{array}{l}r_{z}^{2} r_{x} t_{x}, r_{x} t_{x}, r_{x}^{-1} t_{x}^{-1}, r_{x}^{2} t_{y}^{-1}, r_{y}^{2} t_{z}, r_{x}^{2} t_{z} \\
r_{y}^{2} t_{z}^{-1}, r_{x}^{2} t_{z}^{-1}\end{array}$ \\
\hline & & 1250 & $H_{541}$ & 1 & $r_{z}^{2}$ & $\begin{array}{l}r_{z}^{2} r_{x} t_{x}, r_{x} t_{x}, r_{x}^{-1} t_{x}^{-1}, m_{y} t_{y}^{-1}, i t_{z}, m_{z} t_{z} \\
i t_{z}^{-1}, m_{z} t_{z}^{-1}\end{array}$ \\
\hline
\end{tabular}

$[8,27,59,105,165,239,327,429,545,675]$ $1252^{*}$,

$[8,27,60,106,167,242,329,431,547,675]$ $1254^{*}$

$[8,27,60,107,168,243,332,435,552,683]$ $1257^{*}$,

$[8,27,60,107,169,245,334,436,552,682]$ $1251^{*}$,

$[8,27,61,108,168,243,332,434,550,680]$ $1253^{*}, 1258^{*}$,

$[8,27,61,108,170,247,336,440,559,690]$ $1255^{*}$,

$[8,27,61,109,170,245,335,438,554,685]$ $1256^{*}$,

$[8,27,61,109,170,245,335,439,556,687]$ $1259^{*}, 1260^{*}$,

$[8,27,61,109,172,249,339,443,561,693]$ $1261^{*}$,

$[8,27,62,111,173,249,339,443,561,693]$ 1262*, 


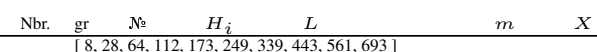

$1263^{*}, 1264^{*}, 1265^{*}, 1266^{*}, 1267^{*}, 1268^{*}$

$[8,29,64,111,173,249,339,443,561,693]$

$1269^{*}, 1270^{*}, 1271^{*}, 1272^{*}, 1273^{*}, 1274^{*}, 1275^{*}, 1276^{*}, 1277^{*}, 1278^{*}$

$[9,29,63,111,174,250,341,444,562,692]$

$1251 \quad H_{412} \quad 1 \quad r_{z}^{2} r_{x} \quad r_{z}^{2} r_{x} t_{x}^{-1}, m_{x} r_{x}^{-1} t_{y}, m_{y} t_{y}, r_{z}^{2} t_{y}^{-1}, r_{x}^{-1} t_{y}^{-1}, r_{x} t_{z}$

$[9,29,63,111,174,250,342,446,566,698]$

$[9,29,63,111,174,250,342,446,566,698]$
$1252 \quad H_{418} \quad 1$ $m_{x} r_{x} t_{z}^{-1}, m_{z} t_{z}^{-1}$

$[9,29,64,111,174,249,339,442,560,690]$

$\begin{array}{lll}1253 & H_{388} & 1\end{array}$

$[9,29,64,111,175,250,339,443,560,689]$

$1254 \quad H_{413} \quad 1$

$[9,29,64,111,176,251,343,448,567,699]$

$1255 \quad H_{407} \quad 1$

$[9,29,64,112,175,251,342,445,564,695]$

$\begin{array}{lll}1256 & H_{394} \quad 1\end{array}$

$[9,29,64,112,176,252,344,448,568,700]$

$1257 \quad H_{389} \quad 1$

$[9,29,65,112,175,251,341,444,563,693]$

$1258 \quad H_{395} \quad 1$

$[9,29,65,113,176,252,344,448,568,700]$

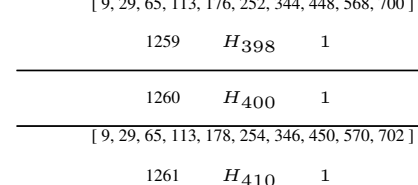

$[9,29,66,114,178,254,346,450,570,702]$

$1262 \quad H_{414} \quad 1$

$[9,30,67,114,178,254,346,450,570,702]$

\begin{tabular}{|c|c|c|c|c|}
\hline \multicolumn{3}{|c|}{$[9,30,67,114,178,254,346,450,570,702]$} & \multirow[b]{2}{*}{$r_{z}^{2} r_{x}$} & \multirow{2}{*}{$\begin{array}{l}r_{z}^{2} t_{x}, m_{x} r_{x}^{-1} t_{y}, m_{y} t_{y}, m_{x} r_{x}^{-1} t_{y}^{-1}, m_{y} t_{y}^{-1}, m_{x} r_{x} t_{z} \\
m_{x} r_{x} t_{z}^{-1}, m_{z} t_{z}^{-1}\end{array}$} \\
\hline 1263 & $H_{422}$ & 等, & & \\
\hline 1264 & $H_{409}$ & 1 & $r_{z}^{2} r_{x}$ & $\begin{array}{l}r_{z}^{2} t_{x}, r_{y}^{2} r_{x} t_{y}, r_{x}^{2} t_{y}, r_{y}^{2} r_{x} t_{y}^{-1}, r_{x}^{2} t_{y}^{-1}, r_{y}^{2} r_{x} t_{z} \\
r_{y}^{2} r_{x} t_{z}^{-1}, r_{x}^{2} t_{z}^{-1}\end{array}$ \\
\hline 1265 & $H_{395}$ & 1 & $r_{z}^{2} r_{x}$ & $\begin{array}{l}m_{x} t_{x}^{-1}, r_{y}^{2} r_{x} t_{y}, i t_{y}^{-1}, m_{z} r_{x} t_{y}^{-1}, i t_{z}, m_{z} r_{x} t_{z} \\
r_{y}^{2} r_{x} t_{z}^{-1}, r_{x}^{2} t_{z}^{-1}\end{array}$ \\
\hline 1266 & $H_{413}$ & 1 & $r_{z}^{2} r_{x}$ & $\begin{array}{l}m_{x} t_{x}^{-1}, m_{x} r_{x}^{-1} t_{y}, m_{y} t_{y}, r_{z}^{2} t_{y}^{-1}, r_{x}^{-1} t_{y}^{-1}, r_{y}^{2} t_{z} \\
r_{x} t_{z}, m_{x} r_{x} t_{z}^{-1}\end{array}$ \\
\hline 1267 & $H_{419}$ & 1 & $r_{z}^{2} r_{x}$ & $\begin{array}{l}m_{x} t_{x}^{-1}, m_{x} r_{x}^{-1} t_{y}, m_{y} t_{y}, m_{x} r_{x}^{-1} t_{y}^{-1}, m_{y} t_{y}^{-1}, m_{x} r_{x} t_{z} \\
m_{x} r_{x} t_{z}^{-1}, m_{z} t_{z}^{-1}\end{array}$ \\
\hline 1268 & $H_{401}$ & 1 & $r_{z}^{2} r_{x}$ & $\begin{array}{l}m_{x} t_{x}^{-1}, r_{y}^{2} r_{x} t_{y}, r_{y}^{2} r_{x} t_{y}^{-1}, r_{x}^{2} t_{y}^{-1}, r_{y}^{2} r_{x} t_{z}, r_{x}^{2} t_{z} \\
r_{y}^{2} r_{x} t_{z}^{-1}, r_{x}^{2} t_{z}^{-1}\end{array}$ \\
\hline \multicolumn{3}{|c|}{$[9,31,66,114,178,254,346,450,570,702]$} & & \\
\hline 1269 & $H_{415}$ & 1 & $r_{z}^{2} r_{x}$ & $\begin{array}{l}i t_{x}^{-1}, m_{x} r_{x}^{-1} t_{y}, m_{y} t_{y}, m_{x} r_{x}^{-1} t_{y}^{-1}, m_{y}{ }_{y}^{-1}, m_{x} r_{x} t_{z} \\
m_{x} r_{x} t_{z}^{-1}, m_{z} t_{z}^{-1}\end{array}$ \\
\hline 1270 & $H_{396}$ & 1 & $r_{z}^{2} r_{x}$ & $\begin{array}{l}i t_{x}^{-1}, r_{y}^{2} r_{x} t_{y}, r_{y}^{2} r_{x} t_{y}^{-1}, r_{x}^{2} t_{y}^{-1}, r_{y}^{2} r_{x} t_{z}, r_{x}^{2} t_{z} \\
r_{y}^{2} r_{x} t_{z}^{-1}, r_{x}^{2} t_{z}^{-1}\end{array}$ \\
\hline 1271 & $H_{422}$ & 1 & $r_{z}^{2} r_{x}$ & $\begin{array}{l}r_{z}^{2} t_{x}, m_{x} r_{x}^{-1} t_{y}, m_{x} r_{x}^{-1} t_{y}^{-1}, m_{y} t_{y}^{-1}, m_{x} r_{x} t_{z}, m_{z} t_{z}, \\
m_{x} r_{x} t_{z}^{-1}, m_{z} t_{z}^{-1}\end{array}$ \\
\hline 1272 & $H_{409}$ & 1 & $r_{z}^{2} r_{x}$ & $\begin{array}{l}r_{z}^{2} t_{x}, r_{y}^{2} r_{x} t_{y}, r_{y}^{2} r_{x} t_{y}^{-1}, r_{x}^{2} t_{y}^{-1}, r_{y}^{2} r_{x} t_{z}, r_{x}^{2} t_{z} \\
r_{y}^{2} r_{x} t_{z}^{-1}, r_{x}^{2} t_{z}^{-1}\end{array}$ \\
\hline 1273 & $H_{394}$ & 1 & $r_{z}^{2} r_{x}$ & $\begin{array}{l}r_{z}^{2} r_{x} t_{x}^{-1}, r_{y}^{2} r_{x} t_{y}, i t_{y}^{-1}, m_{z} r_{x} t_{y}^{-1}, i t_{z}, m_{z} r_{x} t_{z} \\
r_{y}^{2} r_{x} t_{z}^{-1}, r_{x}^{2} t_{z}^{-1}\end{array}$ \\
\hline 1274 & $H_{412}$ & 1 & $r_{z}^{2} r_{x}$ & $\begin{array}{l}r_{z}^{2} r_{x} t_{x}^{-1}, m_{x} r_{x}^{-1} t_{y}, m_{y} t_{y}, r_{z}^{2} t_{y}^{-1}, r_{x}^{-1} t_{y}^{-1}, r_{y}^{2} t_{z} \\
r_{x} t_{z}, m_{x} r_{x} t_{z}^{-1}\end{array}$ \\
\hline 1275 & $H_{402}$ & 1 & $r_{z}^{2} r_{x}$ & $\begin{array}{l}r_{z}^{2} r_{x} t_{x}^{-1}, m_{x} r_{x}^{-1} t_{y}, m_{y} t_{y}, m_{x} r_{x}^{-1} t_{y}^{-1}, m_{y} t_{y}^{-1}, m_{x} r_{x} t_{z} \\
m_{x} r_{x} t_{z}^{-1}, m_{z} t_{z}^{-1}\end{array}$ \\
\hline 1276 & $H_{391}$ & 1 & $r_{z}^{2} r_{x}$ & $\begin{array}{l}r_{z}^{2} r_{x} t_{x}^{-1}, r_{y}^{2} r_{x} t_{y}, r_{y}^{2} r_{x} t_{y}^{-1}, r_{x}^{2} t_{y}^{-1}, r_{y}^{2} r_{x} t_{z}, r_{x}^{2} t_{z} \\
r_{y}^{2} r_{x} t_{z}^{-1}, r_{x}^{2} t_{z}^{-1}\end{array}$ \\
\hline 1277 & $H_{404}$ & 1 & $r_{z}^{2} r_{x}$ & $\begin{array}{l}r_{y}^{2} r_{x} t_{x}^{-1}, m_{x} r_{x}^{-1} t_{y}, m_{y} t_{y}, m_{x} r_{x}^{-1} t_{y}^{-1}, m_{y}{ }^{-1}, m_{x} r_{x} t_{z}, \\
m_{x} r_{x} t_{z}^{-1}, m_{z} t_{z}^{-1}\end{array}$ \\
\hline
\end{tabular}




\begin{tabular}{|c|c|c|c|c|c|c|}
\hline Nbr. & gr & № & $H_{i}$ & $L$ & $m$ & $X$ \\
\hline & & 1278 & $H_{392}$ & 1 & $r_{z}^{2} r_{x}$ & $\begin{array}{l}r_{y}^{2} r_{x} t_{x}^{-1}, r_{y}^{2} r_{x} t_{y}, r_{y}^{2} r_{x} t_{y}^{-1}, r_{x}^{2} t_{y}^{-1}, r_{y}^{2} r_{x} t_{z}, r_{x}^{2} t_{z} \\
r_{y}^{2} r_{x} t_{z}^{-1}, r_{x}^{2} t_{z}^{-1}\end{array}$ \\
\hline \multirow{11}{*}{21} & \multicolumn{6}{|c|}{$[9,29,63,111,173,249,339,443,561,693]$} \\
\hline & \multirow{2}{*}{\multicolumn{6}{|c|}{ 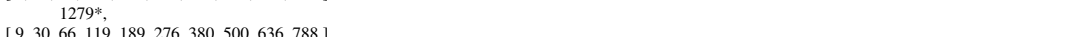 }} \\
\hline & & \\
\hline & \multirow{2}{*}{\multicolumn{6}{|c|}{$\begin{array}{l}1280^{*}, \\
{[9,31,69,124,196,284,388,508,644,796]}\end{array}$}} \\
\hline & \multirow{2}{*}{\multicolumn{6}{|c|}{$1281^{*}}}$, \\
\hline & & & & & & \\
\hline & & 1279 & $H_{779}$ & $\left\langle m_{z}, m_{z} r_{x}, r_{x}^{2}\right\rangle$ & $r_{z}^{2} r_{x}$ & $\begin{array}{l}m_{x} t_{x}^{-1}, r_{z}^{2} t_{y}, r_{x}^{2} t_{y}, m_{x} t_{y}^{-1}, m_{z} r_{x}^{-1} t_{y}^{-1}, m_{x} r_{x}^{-1} t_{z}, \\
m_{z} r^{-1} t_{z}, r_{y}^{2} r_{x} t_{z}^{-1}, r_{x}^{2} t_{z}^{-1}\end{array}$ \\
\hline & \multicolumn{6}{|c|}{$[10,31,68,121,190,277,380,500,636,788]$} \\
\hline & & 1280 & $H_{690}$ & $\left\langle m_{x}\right\rangle$ & $r_{z}^{2}$ & $\begin{array}{l}r_{z}^{2} t_{x}, m_{x} t_{x}, m_{y} t_{x}^{-1}, m_{x} t_{x}^{-1}, m_{y} t_{y}^{-1}, r_{y}^{2} r_{z} t_{z} \\
m_{z} r_{z}^{-1} t_{z}, r_{x}^{2} t_{z}^{-1}, r_{y}^{2} t_{z}^{-1}\end{array}$ \\
\hline & \multicolumn{6}{|c|}{$[10,32,70,125,196,284,388,508,644,796]$} \\
\hline & & 1281 & $H_{750}$ & $\left\langle m_{y}, m_{z}\right\rangle$ & $r_{z}^{2}$ & $\begin{array}{l}m_{x} t_{x}^{-1}, r_{z}^{2} t_{y}, m_{y} t_{y}, m_{x} t_{y}^{-1}, m_{y} t_{y}^{-1}, r_{y}^{2} r_{z} t_{z} \\
m_{z} r_{z} t_{z}, m_{x} r_{z} t_{z}^{-1}, r_{z} t_{z}^{-1}\end{array}$ \\
\hline
\end{tabular}

$[5,14,34,78,153,246,345,461,602,756]$ $1298^{*}$

$[5,15,42,101,174,248,340,443,558,697]$ $1306^{*}$

[ $5,16,43,89,147,216,296,388,499,621]$ $1317 *$

$[5,16,45,98,167,247,340,447,569,706]$ $1320^{*}$

$[5,16,45,102,182,272,371,491,635,789]$ $1321^{*}$,

$[5,16,46,101,170,248,340,448,570,705]$ $1318^{*}$

$[5,16,48,106,172,247,339,445,567,703]$ $1319 *$

$[5,17,44,88,146,216,298,394,504,626]$ $1323^{*}$

$[5,17,44,91,163,258,365,481,615,771]$ $1322 *$,

$[5,17,47,101,172,252,344,452,575,713$ ] $1324^{*}$

$[5,17,48,103,169,244,333,439,557,687]$ $1325^{*}$,

$[5,17,50,108,173,247,336,441,563,694]$ $1326^{*}$,

$[5,17,51,109,177,257,356,468,591,732]$ $1327^{*}$,

$[5,17,53,111,178,259,357,470,597,739]$ $1328^{*}$,

$[5,18,47,98,171,258,362,482,618,770]$ $1329^{*}$,

$[5,18,48,103,171,246,339,446,566,702]$ $1331^{*}$,

[ $5,18,49,102,169,244,336,443,560,695$ ] $1330^{*}$

$[5,18,51,110,180,260,359,470,596,738]$ $1333^{*}$,

$[5,18,52,109,174,255,361,482,618,770]$ $1332 *$

$[5,18,53,114,183,259,355,468,595,738]$ $1334 *$

$[5,19,51,100,161,233,321,424,539,669]$ $1335^{*}$,

$[5,19,52,108,172,243,337,444,559,697]$ $1336^{*}$,

$[5,19,53,110,178,254,349,460,581,719]$ $1337^{*}$,

$[5,19,53,110,183,272,376,495,632,785]$ $1338^{*}$

$[5,19,57,114,178,256,351,460,586,726]$ $1339^{*}$,

$[5,19,57,116,184,264,361,474,601,742]$ $1340 *$,

$[5,20,50,98,156,230,316,418,532,662]$ $1341 *$

$[5,20,56,115,180,258,353,464,589,730]$ $1342 *$

$[5,20,56,115,185,270,373,496,632,784]$ $1343^{*}$

$[5,20,56,117,186,266,363,476,604,748]$ $1346^{*}$,

$[5,20,57,113,177,254,349,460,583,720]$ $1344^{*}$

$[5,20,57,113,182,272,376,496,632,784]$ $1345^{*}$,

$[5,20,63,117,177,260,356,466,592,732]$ $1347^{*}$,

$[6,18,39,72,120,184,264,360,472,600]$ $1282 \quad H_{650} \quad\left\langle m_{x}\right\rangle$ 


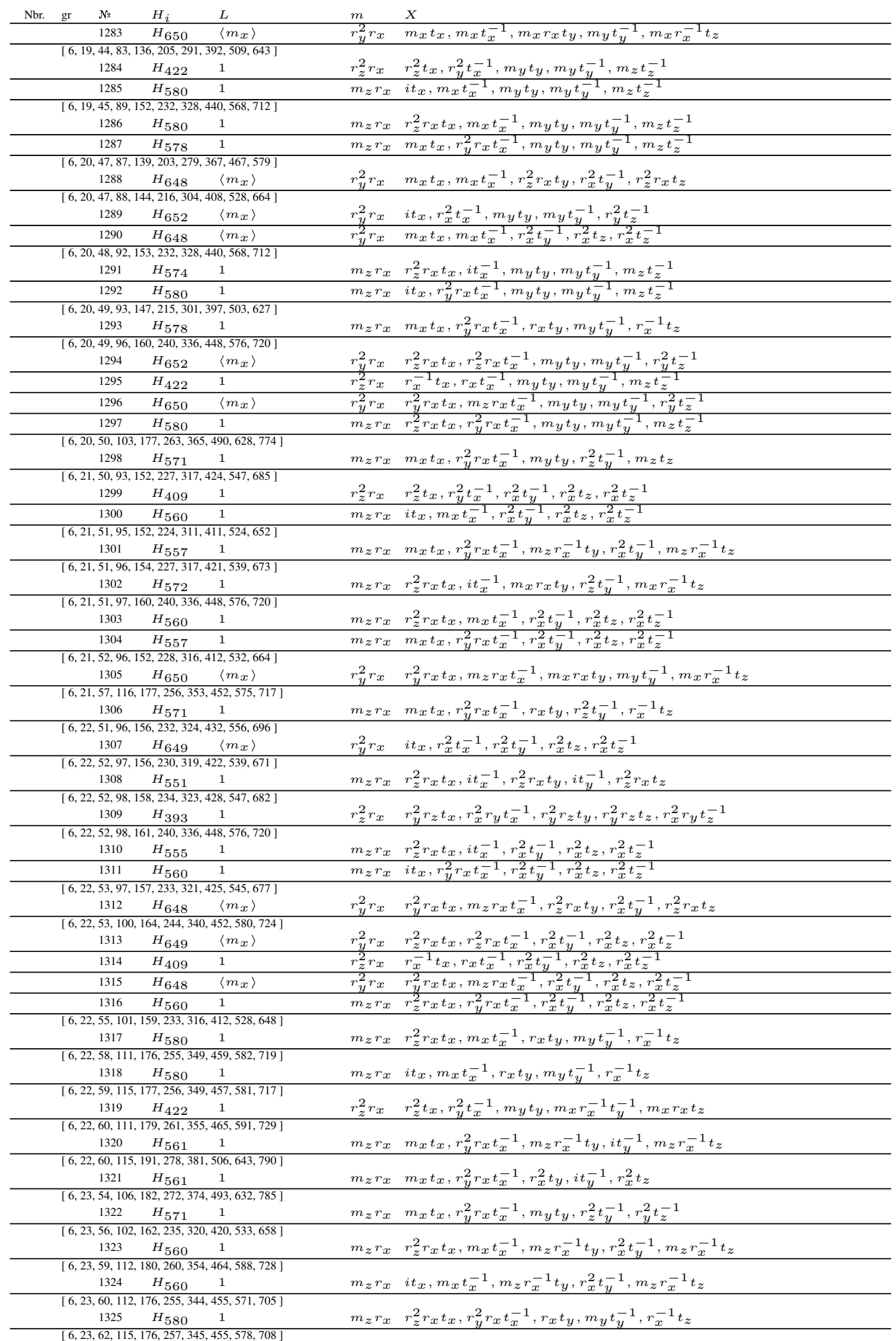




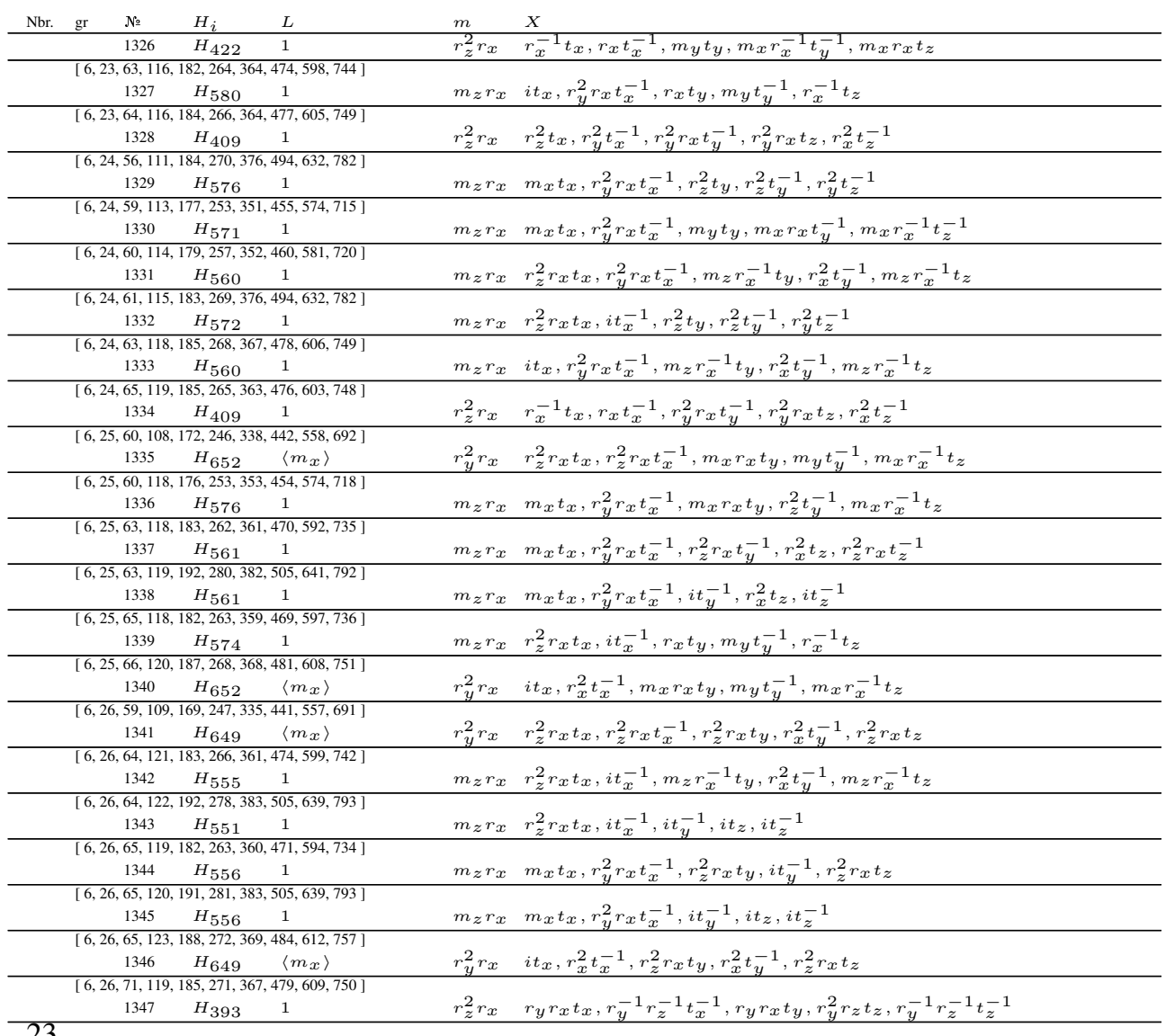

$[6,19,44,85,146,229,332,452,588,740]$ $1348^{*}$,

$[6,19,44,87,154,241,341,460,602,756]$ 1349*,

$[6,20,49,95,154,224,308,406,516,640]$ 1351 *,

$[6,20,49,95,156,234,333,452,588,740]$ 1350 *,

$[6,20,49,95,157,237,336,453,588,740]$ $1352 *$

$[6,20,50,101,172,260,364,484,620,772]$ $1353^{*}, 1354^{*}$,

$[6,20,52,108,172,244,339,443,558,697]$ $1355^{*}$,

$[6,21,49,94,165,259,365,481,615,771]$ $1356^{*}$,

$[6,21,49,97,170,258,362,482,618,770]$ $1357 *$

$[6,21,52,100,162,237,326,429,546,677]$ $1359^{*}$,

$[6,21,52,100,164,244,341,456,589,740]$ $1358 *$

$[6,21,52,101,168,253,356,476,612,764]$ $1361^{*}$,

$[6,21,53,103,168,247,340,448,570,705]$ $1360^{*}$,

$[6,21,53,104,173,260,364,484,620,772]$ $1362^{*}, 1363^{*}, 1364^{*}$,

$[6,21,54,105,167,244,339,442,559,697]$ $1365^{*}$

$[6,21,56,107,169,247,340,447,569,706]$ $1366^{*}$,

$[6,21,56,110,180,267,369,491,635,789]$ $1367^{*}$,

$[6,22,54,103,172,260,364,484,620,772]$ $1368^{*}$,

$[6,22,54,104,170,246,337,443,560,695]$ $1369^{*}$

$[6,22,54,107,171,243,337,444,559,697]$ 
\begin{tabular}{lllllll} 
Nbr. & gr & № & $H_{i}$ & $L$ & $m$ & $X$ \\
\hline & & $1370^{*}$ & & & &
\end{tabular}

[ $6,22,55,105,170,249,343,452,575,713]$ $1371^{*}$,

$[6,22,55,105,172,256,357,476,612,764]$ $1372 *$,

$[6,22,55,105,173,257,358,477,612,764]$ $1373^{*}$,

[ $6,22,56,107,172,253,348,456,580,718]$ $1375^{*}$,

$[6,22,56,108,175,256,354,467,591,732]$ $1374 *$

$[6,22,56,109,180,268,372,492,628,780]$ $1376^{*}, 1377^{*}$

$[6,22,58,111,171,245,339,442,559,697]$ $1378^{*}$,

$[6,23,54,102,172,260,364,484,620,772]$ $1379^{*}$,

$[6,23,56,108,176,260,361,478,613,764]$ $1381 *$

$[6,23,57,106,170,255,361,482,618,770]$ $1380^{*}$,

$[6,23,57,109,177,259,357,469,596,738]$ $1384^{*}$,

$[6,23,57,110,181,268,372,492,628,780]$ $1386^{*}, 1387^{*}$

$[6,23,58,106,168,245,333,439,557,687]$ $1382 *$,

$[6,23,58,111,178,255,349,460,581,719$ ] $1385^{*}$,

$[6,23,58,111,183,273,376,495,632,785]$ $1388^{*}$,

$[6,23,59,110,170,245,339,442,559,697]$ $1389^{*}$,

$[6,23,59,110,175,260,364,484,620,772]$ $1383^{*}$

$[6,23,59,112,176,254,349,460,583,720]$ $1390^{*}$,

$[6,23,59,112,181,272,376,496,632,784]$ $1391 *$,

$[6,23,60,112,176,256,350,458,582,720]$ $1392 *$,

$[6,23,60,114,184,272,376,496,632,784]$ $1394 *$,

$[6,23,61,114,178,257,354,467,591,732]$ 1393*,

$[6,24,59,108,172,249,341,448,568,704]$ $1397^{*}$,

$[6,24,59,109,170,245,339,442,559,697]$ $1396 *$

$[6,24,59,109,173,252,347,457,584,726]$ $1395^{*}$,

$[6,24,60,111,176,256,351,460,586,726]$ $1398^{*}$,

$[6,24,62,113,176,256,350,458,582,720]$ $1400 *$,

$[6,24,62,115,184,272,376,496,632,784]$ $1403^{*}$,

$[6,24,62,116,182,262,359,471,598,740]$ $1401 *$,

$[6,24,62,117,184,264,361,474,601,742]$ $1399^{*}$,

$[6,24,63,117,183,264,361,474,601,742]$ $1402^{*}$,

$[6,25,59,110,173,252,344,452,573,710]$ $1404^{*}$,

$[6,25,60,112,177,258,353,464,589,730]$ $1405^{*}$,

$[6,25,61,114,185,272,376,496,632,784]$ $1406 *$,

$[6,25,62,112,176,256,350,458,582,720]$ $1407 *$,

$[6,25,62,114,184,272,376,496,632,784]$ $1408^{*}$,

$[6,25,62,116,184,270,373,496,632,784]$ $1409^{*}$,

$[6,25,62,118,185,266,363,476,604,748]$ $1410^{*}$,

$[6,25,63,118,184,266,363,476,604,748]$ $1411^{*}$,

$[7,23,52,98,165,252,356,476,612,764]$

${ }_{1348} H_{578} \quad m_{z} r_{x} \quad m_{x} t_{x}, r_{y}^{2} r_{x} t_{x}^{-1}, m_{x} t_{x}^{-1}, m_{y} t_{y}, m_{y} t_{y}^{-1}, m_{z} t_{z}^{-1}$

\begin{tabular}{|c|c|c|c|c|}
\hline 1348 & $H_{578}$ & 1 & $m_{z} r_{x}$ & $m_{x} t_{x}, r_{y}^{2} r_{x} t_{x}^{-1}, m_{x} t_{x}^{-1}, m_{y} t_{y}, m_{y} t_{y}^{-1}, m_{z} t_{z}^{-1}$ \\
\hline \multicolumn{5}{|c|}{$[7,23,54,106,177,261,364,490,628,774]$} \\
\hline 1349 & $H_{571}$ & 1 & $m_{z} r_{x}$ & $m_{x} t_{x}, r_{y}^{2} r_{x} t_{x}^{-1}, m_{x} t_{x}^{-1}, m_{y} t_{y}, r_{z}^{2} t_{y}^{-1}, m_{z} t_{z}$ \\
\hline \multicolumn{5}{|c|}{$[7,24,56,104,169,253,356,476,612,764]$} \\
\hline 1350 & $H_{580}$ & 1 & $m_{z} r_{x}$ & $i t_{x}, r_{z}^{2} r_{x} t_{x}, m_{x} t_{x}^{-1}, m_{y} t_{y}, m_{y} t_{y}^{-1}, m_{z} t_{z}^{-1}$ \\
\hline \multicolumn{5}{|c|}{$[7,24,57,106,166,238,326,426,538,666]$} \\
\hline 1351 & $H_{578}$ & 1 & $m_{z} r_{x}$ & $m_{x} t_{x}, r_{y}^{2} r_{x} t_{x}^{-1}, m_{x} t_{x}^{-1}, r_{x} t_{y}, m_{y} t_{y}^{-1}, r_{x}^{-1} t_{z}$ \\
\hline \multicolumn{5}{|c|}{$[7,24,57,106,171,255,357,476,612,764]$} \\
\hline 1352 & $H_{580}$ & 1 & $m_{z} r_{x}$ & $i t_{x}, r_{y}^{2} r_{x} t_{x}^{-1}, m_{x} t_{x}^{-1}, m_{y} t_{y}, m_{y} t_{y}^{-1}, m_{z} t_{z}^{-1}$ \\
\hline
\end{tabular}




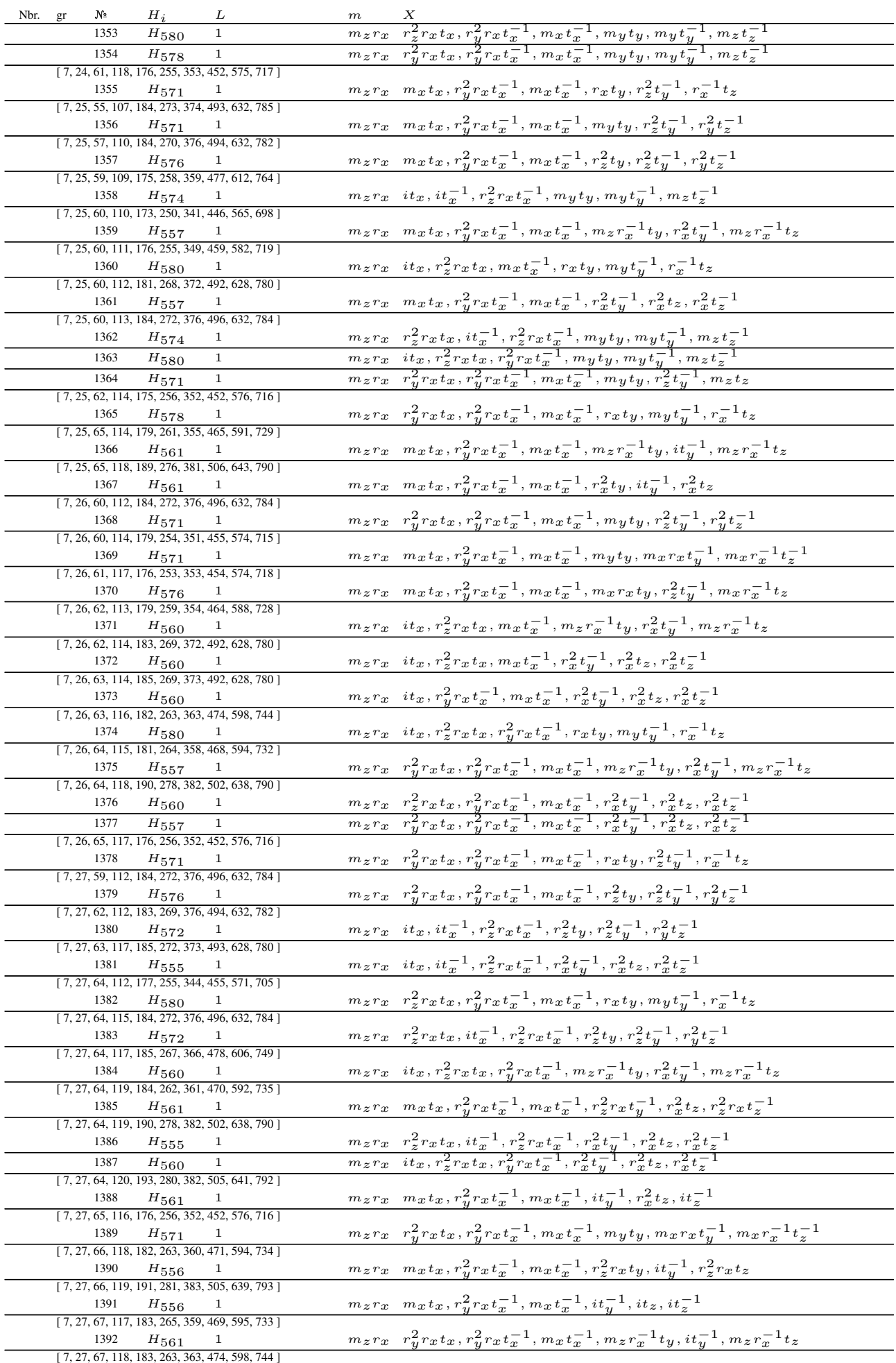




\begin{tabular}{|c|c|c|c|c|}
\hline Nbr. & № & $H_{i}$ & $L$ & $m \quad X$ \\
\hline & 1393 & $H_{580}$ & 1 & $m_{z} r_{x} i t_{x}, r_{y}^{2} r_{x} t_{x}^{-1}, m_{x} t_{x}^{-1}, r_{x} t_{y}, m_{y} t_{y}^{-1}, r_{x}^{-1} t_{z}$ \\
\hline & \multicolumn{4}{|c|}{$[7,27,67,120,192,280,384,504,640,792]$} \\
\hline & \multicolumn{4}{|c|}{$[7,28,64,115,180,260,356,466,594,735]$} \\
\hline & 1395 & $H_{572}$ & 1 & $m_{z} r_{x} \quad r_{z}^{2} r_{x} t_{x}, i t_{x}^{-1}, r_{z}^{2} r_{x} t_{x}^{-1}, m_{x} r_{x} t_{y}, r_{z}^{2} t_{y}^{-1}, m_{x} r_{x}^{-1} t_{z}$ \\
\hline & \multicolumn{4}{|c|}{$[7,28,64,116,176,256,352,452,576,716]$} \\
\hline & 1396 & $H_{576}$ & 1 & $m_{z} r_{x} \quad r_{y}^{2} r_{x} t_{x}, r_{y}^{2} r_{x} t_{x}^{-1}, m_{x} t_{x}^{-1}, m_{x} r_{x} t_{y}, r_{z}^{2} t_{y}^{-1}, m_{x} r_{x}^{-1} t_{z}$ \\
\hline & \multicolumn{4}{|c|}{$[7,28,65,114,181,258,353,461,582,721]$} \\
\hline & 1397 & $H_{560}$ & 1 & $m_{z} r_{x} r_{z}^{2} r_{x} t_{x}, r_{y}^{2} r_{x} t_{x}^{-1}, m_{x} t_{x}^{-1}, m_{z} r_{x}^{-1} t_{y}, r_{x}^{2} t_{y}^{-1}, m_{z} r_{x}^{-1} t_{z}$ \\
\hline & \multicolumn{4}{|c|}{$[7,28,65,116,182,263,359,469,597,736]$} \\
\hline & 1398 & $H_{574}$ & 1 & $m_{z} r_{x} \quad r_{z}^{2} r_{x} t_{x}, i t_{x}^{-1}, r_{z}^{2} r_{x} t_{x}^{-1}, r_{x} t_{y}, m_{y} t_{y}^{-1}, r_{x}^{-1} t_{z}$ \\
\hline & \multicolumn{4}{|c|}{$[7,28,67,121,187,268,368,481,608,751]$} \\
\hline & 1399 & $H_{572}$ & 1 & $m_{z} r_{x} i t_{x}, i t_{x}^{-1}, r_{z}^{2} r_{x} t_{x}^{-1}, m_{x} r_{x} t_{y}, r_{z}^{2} t_{y}^{-1}, m_{x} r_{x}^{-1} t_{z}$ \\
\hline & \multicolumn{4}{|c|}{$[7,28,68,117,183,265,359,469,595,733]$} \\
\hline & 1400 & $H_{561}$ & 1 & $m_{z} r_{x} \quad r_{y}^{2} r_{x} t_{x}, r_{y}^{2} r_{x} t_{x}^{-1}, m_{x} t_{x}^{-1}, r_{z}^{2} r_{x} t_{y}^{-1}, r_{x}^{2} t_{z}, r_{z}^{2} r_{x} t_{z}^{-1}$ \\
\hline & \multicolumn{4}{|c|}{$[7,28,68,120,187,268,367,479,607,750]$} \\
\hline & 1401 & $H_{560}$ & 1 & $m_{z} r_{x} \quad i t_{x}, r_{y}^{2} r_{x} t_{x}^{-1}, m_{x} t_{x}^{-1}, m_{z} r_{x}^{-1} t_{y}, r_{x}^{2} t_{y}^{-1}, m_{z} r_{x}^{-1} t_{z}$ \\
\hline & \multicolumn{4}{|c|}{$[7,28,68,120,187,268,368,481,608,751]$} \\
\hline & 1402 & $H_{574}$ & 1 & $m_{z} r_{x} \quad i t_{x}, i t_{x}^{-1}, r_{z}^{2} r_{x} t_{x}^{-1}, r_{x} t_{y}, m_{y} t_{y}^{-1}, r_{x}^{-1} t_{z}$ \\
\hline & \multicolumn{4}{|c|}{$[7,28,68,120,192,280,384,504,640,792]$} \\
\hline & 1403 & $H_{561}$ & 1 & $m_{z} r_{x} \quad r_{y}^{2} r_{x} t_{x}, r_{y}^{2} r_{x} t_{x}^{-1}, m_{x} t_{x}^{-1}, i t_{y}^{-1}, r_{x}^{2} t_{z}, i t_{z}^{-1}$ \\
\hline & \\
\hline & \multicolumn{4}{|c|}{$\begin{array}{c}{[7,29,64,117,180,262,354,465,586,726]} \\
1404 \quad H_{551} \quad 1 \\
\end{array}$} \\
\hline & \multicolumn{4}{|c|}{$[7,29,65,118,183,266,361,474,599,742]$} \\
\hline & \multicolumn{4}{|c|}{$\frac{14555,1}{[7,29,66,121,192,280,384,504,640,792]}$} \\
\hline & 1406 & $H_{551}$ & 1 & $m_{z} r_{x} \quad r_{z}^{2} r_{x} t_{x}, i t_{x}^{-1}, r_{z}^{2} r_{x} t_{x}^{-1}, i t_{y}^{-1}, i t_{z}, i t_{z}^{-1}$ \\
\hline & \multicolumn{4}{|c|}{$[7,29,67,117,183,265,359,469,595,733]$} \\
\hline & 1407 & $H_{556}$ & 1 & $m_{z} r_{x} \quad r_{y}^{2} r_{x} t_{x}, r_{y}^{2} r_{x} t_{x}^{-1}, m_{x} t_{x}^{-1}, r_{z}^{2} r_{x} t_{y}, i t_{y}^{-1}, r_{z}^{2} r_{x} t_{z}$ \\
\hline & \multicolumn{4}{|c|}{$[7,29,67,120,192,280,384,504,640,792]$} \\
\hline & 1408 & $H_{556}$ & 1 & $m_{z} r_{x} \quad r_{y}^{2} r_{x} t_{x}, r_{y}^{2} r_{x} t_{x}^{-1}, m_{x} t_{x}^{-1}, i t_{y}^{-1}, i t_{z}, i t_{z}^{-1}$ \\
\hline & \multicolumn{4}{|c|}{$[7,29,67,121,192,278,383,505,639,793]$} \\
\hline & 1409 & $H_{551}$ & 1 & $m_{z} r_{x} \quad i t_{x}, i t_{x}^{-1}, r_{z}^{2} r_{x} t_{x}^{-1}, i t_{y}^{-1}, i t_{z}, i t_{z}^{-1}$ \\
\hline & \multicolumn{4}{|c|}{$[7,29,67,123,188,272,369,484,612,757]$} \\
\hline & 1410 & $H_{551}$ & 1 & $m_{z} r_{x} i t_{x}, i t_{x}^{-1}, r_{z}^{2} r_{x} t_{x}^{-1}, r_{z}^{2} r_{x} t_{y}, i t_{y}^{-1}, r_{z}^{2} r_{x} t_{z}$ \\
\hline & \multicolumn{4}{|c|}{$[7,29,68,122,188,272,369,484,612,757]$} \\
\hline & 1411 & $H_{555}$ & 1 & $m_{z} r_{x} \quad i t_{x}, i t_{x}^{-1}, r_{z}^{2} r_{x} t_{x}^{-1}, m_{z} r_{x}^{-1} t_{y}, r_{x}^{2} t_{y}^{-1}, m_{z} r_{x}^{-1} t_{z}$ \\
\hline
\end{tabular}

$[6,18,39,72,120,184,264,360,472,600]$ $1412 *$

$[6,18,39,73,128,209,308,417,545,700]$ $1413^{*}$,

$[6,19,44,82,130,188,258,338,428,530]$ $1414^{*}$,

$[6,19,45,89,152,232,328,440,568,712]$ $1415^{*}, 1416^{*}$

$[6,19,47,97,161,235,328,432,548,686]$ $1417 *$,

$[6,20,46,88,152,238,344,465,598,749]$ $1418 *$,

$[6,20,46,90,161,249,353,473,609,761]$ $1419 *$

$[6,20,47,87,139,203,279,367,467,579]$ 1423 *

$[6,20,47,88,144,216,304,408,528,664]$ $1424^{*}, 1420^{*}$

$[6,20,48,92,153,232,328,440,568,712]$ $1421^{*}, 1422^{*}$

$[6,20,49,93,148,219,307,404,517,649$ $1425^{*}$

$[6,20,50,101,172,260,364,484,620,772]$ $1426 *$

$[6,20,51,97,154,225,309,406,517,641]$ $1427^{*}$

$[6,20,51,100,166,250,349,465,602,760]$ $1428 *$

$[6,21,51,95,153,227,315,417,535,667]$ $1432^{*}$,

$[6,21,51,97,160,240,336,448,576,720]$ $1433^{*}, 1434^{*}$

$[6,21,51,98,160,235,327,432,549,685]$ $1431^{*}$

$[6,21,51,100,163,238,332,437,553,691]$ $1430^{*}$,

$[6,21,51,100,171,260,364,484,620,772]$ $1429^{*}$,

$[6,21,55,108,170,245,339,442,559,697]$ $1435^{*}$

$[6,21,56,106,167,246,336,441,563,694]$ $1436 *$

$[6,22,51,96,156,232,324,432,556,696]$ $1437^{*}$,

$[6,22,52,98,161,240,336,448,576,720]$ $1439^{*}, 1440^{*}$ 


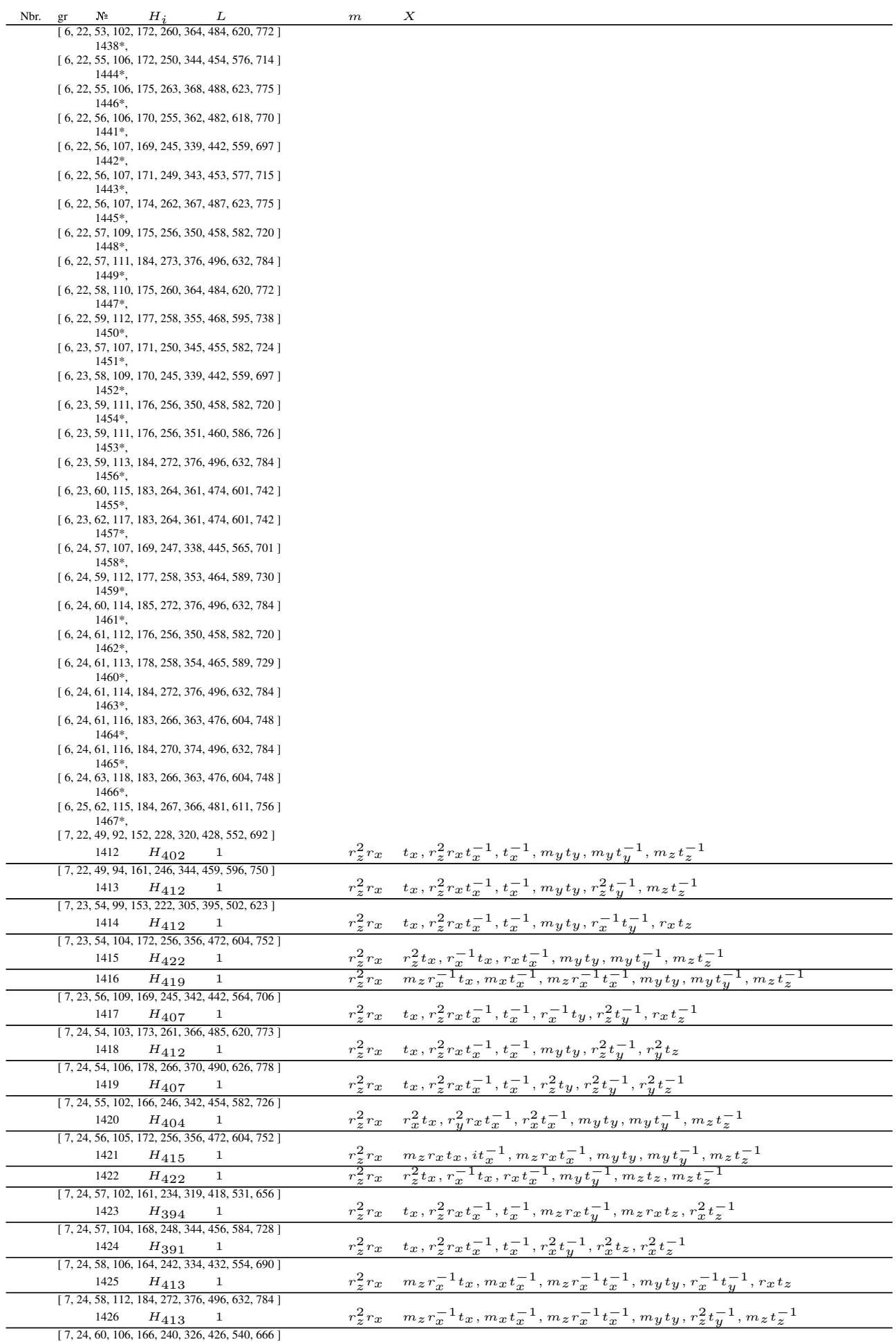




\begin{tabular}{|c|c|c|c|c|c|}
\hline \multirow[t]{2}{*}{ Nbr. } & № & $H_{i}$ & $L$ & $m$ & $x$ \\
\hline & 1427 & $H_{388}$ & 1 & $r_{z}^{2} r_{x}$ & $t_{x}, r_{z}^{2} r_{x} t_{x}^{-1}, t_{x}^{-1}, m_{z} r_{x} t_{y}, i t_{y}^{-1}, m_{z} r_{x} t_{z}^{-1}$ \\
\hline \multicolumn{5}{|c|}{$[7,24,60,110,180,268,369,488,626,780]$} & $t_{x}, r_{z}^{2} r_{x} t_{x}^{-1}, t_{x}^{-1}, r_{x}^{2} t_{y}, i t_{y}^{-1}, r_{x}^{2} t_{z}^{-1}$ \\
\hline \multicolumn{6}{|c|}{$[7,25,58,111,184,272,376,496,632,784]$} \\
\hline & 1429 & $H_{413}$ & 1 & $r_{z}^{2} r_{x}$ & $m_{z} r_{x}^{-1} t_{x}, m_{x} t_{x}^{-1}, m_{z} r_{x}^{-1} t_{x}^{-1}, m_{y} t_{y}, r_{z}^{2} t_{y}^{-1}, r_{y}^{2} t_{z}$ \\
\hline \multicolumn{6}{|c|}{$[7,25,58,112,173,252,349,450,571,714]$} \\
\hline & 1430 & $H_{412}$ & 1 & $r_{z}^{2} r_{x}$ & $t_{x}, r_{z}^{2} r_{x} t_{x}^{-1}, t_{x}^{-1}, m_{x} r_{x}^{-1} t_{y}, r_{z}^{2} t_{y}^{-1}, m_{x} r_{x} t_{z}^{-1}$ \\
\hline \multicolumn{6}{|c|}{$[7,25,59,110,172,250,346,448,570,710]$} \\
\hline \multicolumn{6}{|c|}{$[7,25,60,107,170,249,340,447,570,705]$} \\
\hline & 1432 & $H_{395}$ & 1 & $r_{z}^{2} r_{x}$ & $m_{z} r_{x}^{-1} t_{x}, m_{x} t_{x}^{-1}, m_{z} r_{x}^{-1} t_{x}^{-1}, m_{z} r_{x} t_{y}^{-1}, m_{z} r_{x} t_{z}, r_{x}^{2} t_{z}^{-1}$ \\
\hline \multicolumn{6}{|c|}{$[7,25,60,110,178,262,362,478,610,758]$} \\
\hline & 1433 & $H_{409}$ & 1 & $r_{z}^{2} r_{x}$ & $r_{z}^{2} t_{x}, r_{x}^{-1} t_{x}, r_{x} t_{x}^{-1}, r_{x}^{2} t_{y}, r_{x}^{2} t_{y}^{-1}, r_{x}^{2} t_{z}^{-1}$ \\
\hline & 1434 & $H_{401}$ & 1 & $r_{z}^{2} r_{x}$ & $m_{z} r_{x}^{-1} t_{x}, m_{x} t_{x}^{-1}, m_{z} r_{x}^{-1} t_{x}^{-1}, r_{x}^{2} t_{y}^{-1}, r_{x}^{2} t_{z}, r_{x}^{2} t_{z}^{-1}$ \\
\hline \multicolumn{6}{|c|}{$[7,25,63,116,176,256,352,452,576,716]$} \\
\hline & 1435 & $H_{418}$ & 1 & $r_{z}^{2} r_{x}$ & $m_{z} r_{x}^{-1} t_{x}, m_{x} t_{x}^{-1}, m_{z} r_{x}^{-1} t_{x}^{-1}, r_{x}^{-1} t_{y}, r_{z}^{2} t_{y}^{-1}, r_{x} t_{z}^{-1}$ \\
\hline \multicolumn{6}{|c|}{$[7,25,64,112,175,257,345,455,578,708]$} \\
\hline & 1436 & $H_{422}$ & 1 & $r_{z}^{2} r_{x}$ & $r_{z}^{2} t_{x}, r_{x}^{-1} t_{x}, r_{x} t_{x}^{-1}, m_{y} t_{y}, m_{x} r_{x}^{-1} t_{y}^{-1}, m_{x} r_{x} t_{z}$ \\
\hline \multicolumn{6}{|c|}{$[7,26,59,110,174,258,354,470,598,746]$} \\
\hline \multicolumn{6}{|c|}{$[7,26,59,112,184,272,376,496,632,784]$} \\
\hline & 1438 & $H_{418}$ & 1 & $r_{z}^{2} r_{x}$ & $m_{z} r_{x}^{-1} t_{x}, m_{x} t_{x}^{-1}, m_{z} r_{x}^{-1} t_{x}^{-1}, r_{z}^{2} t_{y}, r_{z}^{2} t_{y}^{-1}, r_{y}^{2} t_{z}^{-1}$ \\
\hline \multicolumn{6}{|c|}{$[7,26,60,111,178,262,362,478,610,758]$} \\
\hline & 1439 & $H_{396}$ & 1 & $r_{z}^{2} r_{x}$ & $m_{z} r_{x} t_{x}, i t_{x}^{-1}, m_{z} r_{x} t_{x}^{-1}, r_{x}^{2} t_{y}^{-1}, r_{x}^{2} t_{z}, r_{x}^{2} t_{z}^{-1}$ \\
\hline & 1440 & $H_{409}$ & 1 & $r_{z}^{2} r_{x}$ & $r_{z}^{2} t_{x}, r_{x}^{-1} t_{x}, r_{x} t_{x}^{-1}, r_{x}^{2} t_{y}^{-1}, r_{x}^{2} t_{z}, r_{x}^{2} t_{z}^{-1}$ \\
\hline \multicolumn{6}{|c|}{$[7,26,62,113,182,270,376,494,632,782]$} \\
\hline & 1441 & $H_{410}$ & 1 & $r_{z}^{2} r_{x}$ & $r_{x}^{2} t_{x}, r_{y}^{2} r_{x} t_{x}^{-1}, r_{x}^{2} t_{x}^{-1}, r_{z}^{2} t_{y}, r_{z}^{2} t_{y}^{-1}, r_{y}^{2} t_{z}^{-1}$ \\
\hline & $\begin{array}{r}{[7,26,63,11} \\
1442\end{array}$ & $\begin{array}{l}76,256,3 \\
H_{419}\end{array}$ & $\begin{array}{l}452,576,716] \\
1\end{array}$ & $r_{\gamma}^{2} r_{x}$ & $m_{z} r_{x}^{-1} t_{x}, m_{x} t_{x}^{-1}, m_{z} r_{x}^{-1} t_{x}^{-1}, m_{y} t_{y}, m_{x} r_{x}^{-1} t^{-1}, m_{x} r_{x} t_{z}$ \\
\hline & {$[7,26,63,11$} & $80,261,3$ & $467,591,731]$ & & \\
\hline & 1443 & $H_{394}$ & 1 & $r_{z}^{2} r_{x}$ & $t_{x}, r_{z}^{2} r_{x} t_{x}^{-1}, t_{x}^{-1}, r_{y}^{2} r_{x} t_{y}, i t_{y}^{-1}, r_{y}^{2} r_{x} t_{z}^{-1}$ \\
\hline & {$[7,26,63,11$} & $81,261,3$ & $467,591,731]$ & & \\
\hline & 1444 & $H_{391}$ & 1 & $r_{z}^{2} r_{x}$ & $t_{x}, r_{z}^{2} r_{x} t_{x}^{-1}, t_{x}^{-1}, r_{y}^{2} r_{x} t_{y}^{-1}, r_{y}^{2} r_{x} t_{z}, r_{x}^{2} t_{z}^{-1}$ \\
\hline & {$[7,26,63,11$} & $86,276,3$ & $500,634,788]$ & & \\
\hline & 1445 & $H_{388}$ & 1 & $r_{z}^{2} r_{x}$ & $t_{x}, r_{z}^{2} r_{x} t_{x}^{-1}, t_{x}^{-1}, i t_{y}^{-1}, i t_{z}, i t_{z}^{-1}$ \\
\hline & {$[7,26,63,11$} & $87,276,3$ & $500,635,788]$ & & \\
\hline & 1446 & $H_{394}$ & 1 & $r_{z}^{2} r_{x}$ & $t_{x}, r_{z}^{2} r_{x} t_{x}^{-1}, t_{x}^{-1}, i t_{y}^{-1}, i t_{z}, r_{x}^{2} t_{z}^{-1}$ \\
\hline & {$[7,26,64,11$} & $84,272,3$ & $496,632,784]$ & & \\
\hline & 1447 & $H_{414}$ & 1 & $r_{z}^{2} r_{x}$ & $m_{z} r_{x} t_{x}, i t_{x}^{-1}, m_{z} r_{x} t_{x}^{-1}, r_{z}^{2} t_{y}, r_{z}^{2} t_{y}^{-1}, r_{y}^{2} t_{z}^{-1}$ \\
\hline & {$[7,26,65,11$} & $83,265,3$ & $469,595,733]$ & & \\
\hline & 1448 & $H_{400}$ & 1 & $r_{z}^{2} r_{x}$ & $m_{z} r_{x}^{-1} t_{x}, m_{x} t_{x}^{-1}, m_{z} r_{x}^{-1} t_{x}^{-1}, m_{z} r_{x} t_{y}, i t_{y}^{-1}, m_{z} r_{x} t_{z}^{-1}$ \\
\hline & $\begin{array}{c}{[7,26,65,11} \\
1449\end{array}$ & $\begin{array}{ll}93,280,3 \\
H_{395}\end{array}$ & $\begin{array}{l}504,640,792] \\
1\end{array}$ & $r_{\gamma}^{2} r_{r}$ & $m_{z} r^{-1} t_{r}, m_{r} t^{-1}, m_{z} r^{-1} t^{-1}, r^{2} t_{\psi}, i t^{-1}, r^{2} t^{-1}$ \\
\hline & $\frac{147,}{[7,26,67,11}$ & $\frac{11395}{84,265,3}$ & $\frac{1}{476,603,748]}$ & & 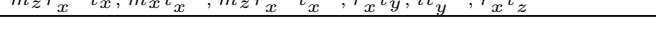 \\
\hline & 1450 & $H_{409}$ & 1 & $r_{z}^{2} r_{x}$ & $r_{z}^{2} t_{x}, r_{x}^{-1} t_{x}, r_{x} t_{x}^{-1}, r_{x}^{2} t_{y}, r_{y}^{2} r_{x} t_{y}^{-1}, r_{y}^{2} r_{x} t_{z}$ \\
\hline & {$[7,27,63,11$} & $79,259,3$ & $465,593,734]$ & & \\
\hline & 1451 & $H_{414}$ & 1 & $r_{z}^{2} r_{x}$ & $m_{z} r_{x} t_{x}, i t_{x}^{-1}, m_{z} r_{x} t_{x}^{-1}, r_{x}^{-1} t_{y}, r_{z}^{2} t_{y}^{-1}, r_{x} t_{z}^{-1}$ \\
\hline & {$[7,27,64,11$} & $76,256,3$ & $452,576,716]$ & & \\
\hline & 1452 & $H_{413}$ & 1 & $r_{z}^{2} r_{x}$ & $m_{z} r_{x}^{-1} t_{x}, m_{x} t_{x}^{-1}, m_{z} r_{x}^{-1} t_{x}^{-1}, m_{x} r_{x}^{-1} t_{y}, r_{z}^{2} t_{y}^{-1}, m_{x} r_{x} t_{z}^{-1}$ \\
\hline & $\begin{array}{r}{[7,27,65,11} \\
1453\end{array}$ & $\begin{array}{l}82,263,3 \\
H_{415}\end{array}$ & $\begin{array}{l}469,597,736] \\
1\end{array}$ & $r_{z}^{2} r_{x}$ & $m_{z} r_{x} t_{x}, i t_{x}^{-1}, m_{z} r_{x} t_{x}^{-1}, m_{y} t_{y}, m_{x} r_{x}^{-1} t_{y}^{-1}, m_{x} r_{x} t_{z}$ \\
\hline & {$[7,27,66,11$} & $83,265,3$ & $469,595,733]$ & & \\
\hline & 1454 & $H_{401}$ & 1 & $r_{z}^{2} r_{x}$ & $m_{z} r_{x}^{-1} t_{x}, m_{x} t_{x}^{-1}, m_{z} r_{x}^{-1} t_{x}^{-1}, r_{y}^{2} r_{x} t_{y}^{-1}, r_{y}^{2} r_{x} t_{z}, r_{x}^{2} t_{z}^{-1}$ \\
\hline & {$[7,27,66,12$} & $87,268,3$ & $481,608,751]$ & & \\
\hline & 1455 & $H_{410}$ & 1 & $r_{z}^{2} r_{x}$ & $r_{x}^{2} t_{x}, r_{y}^{2} r_{x} t_{x}^{-1}, r_{x}^{2} t_{x}^{-1}, r_{x}^{-1} t_{y}, r_{z}^{2} t_{y}^{-1}, r_{x} t_{z}^{-1}$ \\
\hline & {$[7,27,66,12$} & $92,280,3$ & $504,640,792]$ & $r^{2} r_{x}$ & $r-1+-1$ it -1 it $r^{2} t-1$ \\
\hline & $\frac{1456}{[7,27,68,12}$ & $\frac{H_{395}}{87,268,3}$ & $\frac{1}{481,608,751]}$ & $r_{z}^{2} r_{x}$ & 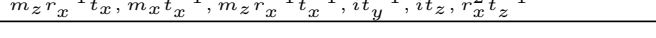 \\
\hline & 1457 & $\mathrm{H}_{404}$ & 1 & $r_{z}^{2} r_{x}$ & $r_{x}^{2} t_{x}, r_{y}^{2} r_{x} t_{x}^{-1}, r_{x}^{2} t_{x}^{-1}, m_{y} t_{y}, m_{x} r_{x}^{-1} t_{y}^{-1}, m_{x} r_{x} t_{z}$ \\
\hline & {$[7,28,63,11$} & $78,259,3$ & $461,582,721]$ & & \\
\hline & 1458 & $H_{389}$ & 1 & $r_{z}^{2} r_{x}$ & $m_{z} r_{x} t_{x}, i t_{x}^{-1}, m_{z} r_{x} t_{x}^{-1}, m_{z} r_{x} t_{y}, i t_{y}^{-1}, m_{z} r_{x} t_{z}^{-1}$ \\
\hline & {$[7,28,65,11$} & $83,266,3$ & $474,599,742]$ & & \\
\hline & 1459 & $H_{396}$ & 1 & $r_{z}^{2} r_{x}$ & $m_{z} r_{x} t_{x}, i t_{x}^{-1}, m_{z} r_{x} t_{x}^{-1}, r_{y}^{2} r_{x} t_{y}^{-1}, r_{y}^{2} r_{x} t_{z}, r_{x}^{2} t_{z}^{-1}$ \\
\hline & $\begin{array}{c}{[7,28,66,11} \\
1460\end{array}$ & $\begin{array}{ll}83,265,3 \\
H_{4} 22\end{array}$ & $\begin{array}{l}473,598,741] \\
1\end{array}$ & $r_{r}^{2} r_{x}$ & $r_{z}^{2} t_{x}, r^{-1} t_{x}, r_{x} t^{-1}, m_{x} r^{-1} t^{-1}, m_{x} r_{x} t_{z}, m_{z} t^{-1}$ \\
\hline & $\frac{1400}{[7,28,66,12}$ & $\frac{H_{422}}{92,280,3}$ & $\frac{1}{504,640,792]}$ & & $r_{z}^{\tau_{x}, r_{x}} \tau_{x}, r_{x} \tau_{x}, m_{x} r_{x} \tau_{y}, m_{x} r_{x} \tau_{z}, m_{z} \tau_{z}$ \\
\hline & 1461 & $H_{389}$ & 1 & $r_{z}^{2} r_{x}$ & $m_{z} r_{x} t_{x}, i t_{x}^{-1}, m_{z} r_{x} t_{x}^{-1}, i t_{y}^{-1}, i t_{z}, i t_{z}^{-1}$ \\
\hline & {$[7,28,67,11$} & $83,265,3$ & $469,595,733]$ & & \\
\hline & 1462 & $H_{395}$ & 1 & $r_{z}^{2} r_{x}$ & $m_{z} r_{x}^{-1} t_{x}, m_{x} t_{x}^{-1}, m_{z} r_{x}^{-1} t_{x}^{-1}, r_{y}^{2} r_{x} t_{y}, i t_{y}^{-1}, r_{y}^{2} r_{x} t_{z}^{-1}$ \\
\hline & {$[7,28,67,12$} & $92,280,3$ & $504,640,792]$ & & 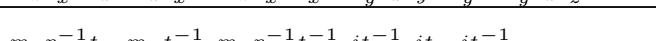 \\
\hline & 1463 & $H_{400}$ & 1 & $r_{x}$ & $m_{z} r_{x}^{-1} t_{x}, m_{x} t_{x}^{-1}, m_{z} r_{x}^{-1} t_{x}^{-1}, i t_{y}^{-1}, i t_{z}, i t_{z}^{-1}$ \\
\hline & {$[7,28,67,12$} & $\begin{array}{c}88,272,3 \\
H_{398}\end{array}$ & $\begin{array}{l}484,612,757] \\
1\end{array}$ & $r_{z}^{2} r_{x}$ & $r_{x}^{2} t_{x}, r_{y}^{2} r_{x} t_{x}^{-1}, r_{x}^{2} t_{x}^{-1}, m_{z} r_{x} t_{y}, i t_{y}^{-1}, m_{z} r_{x} t_{z}^{-1}$ \\
\hline & {$[7,28,67,12$} & $91,279,3$ & $505,639,793]$ & & \\
\hline & 1465 & $H_{398}$ & 1 & $r_{z}^{2} r_{x}$ & $r_{x}^{2} t_{x}, r_{y}^{2} r_{x} t_{x}^{-1}, r_{x}^{2} t_{x}^{-1}, i t_{y}^{-1}, i t_{z}, i t_{z}^{-1}$ \\
\hline
\end{tabular}




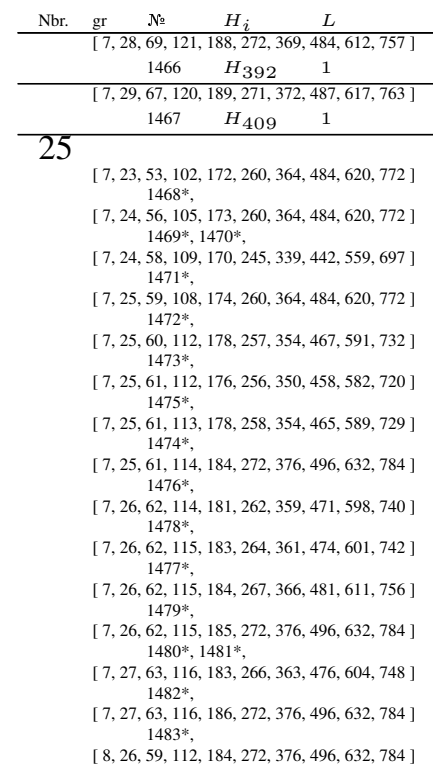

$[8,26,59,112,184,272,376,496,632,784]$

$1468 \quad H_{650}\left\langle m_{x}\right\rangle \quad \begin{array}{ll}r_{y}^{2} r_{x} & r_{y}^{2} r_{x} t_{x}, m_{x} t_{x}, m_{z} r_{x} t_{x}^{-1}, m_{x} t_{x}^{-1}, m_{y} t_{y}, m_{y} t_{y}^{-1},\end{array}$

$[8,27,61,113,184,272,376,496,632,784]$

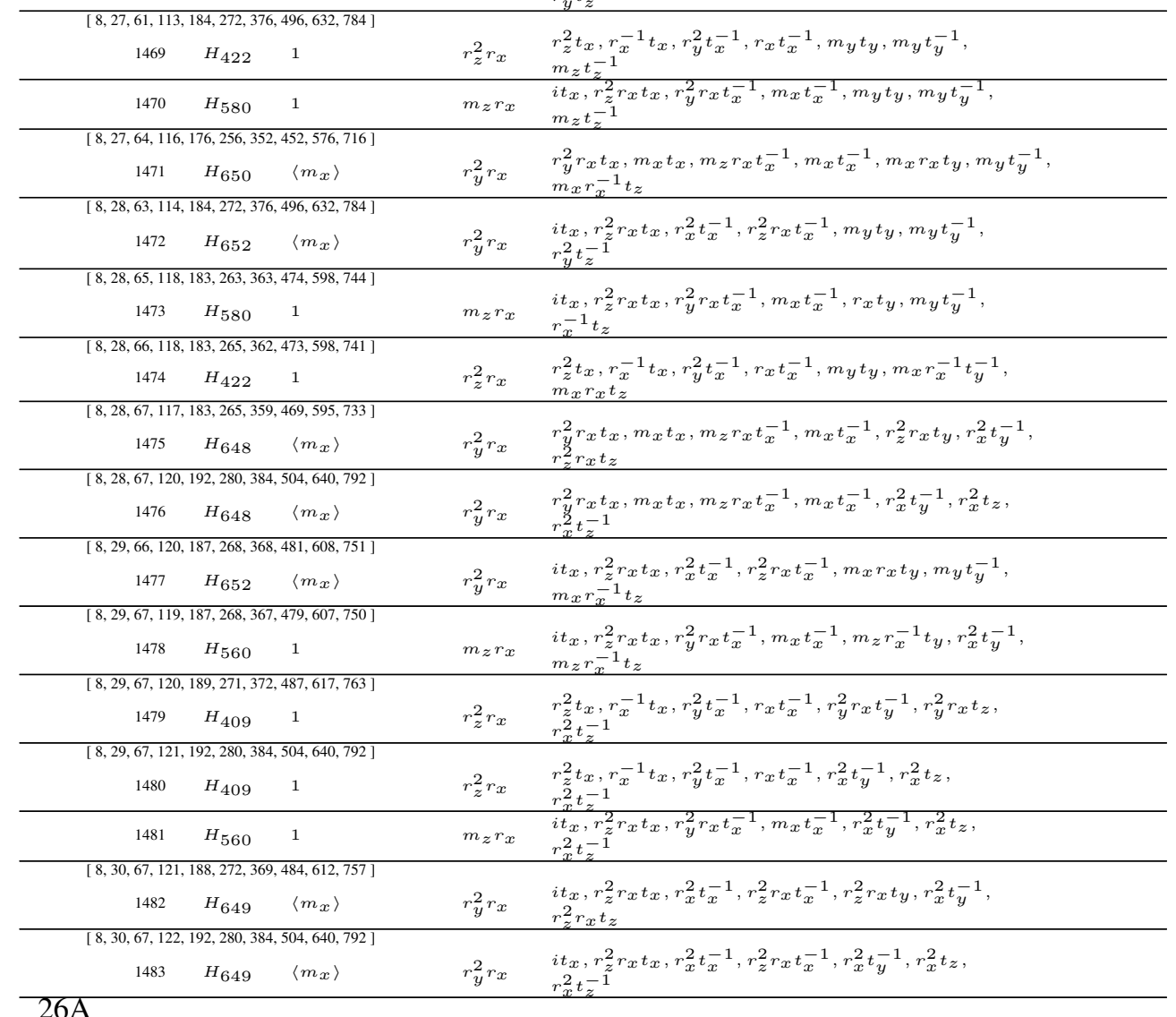




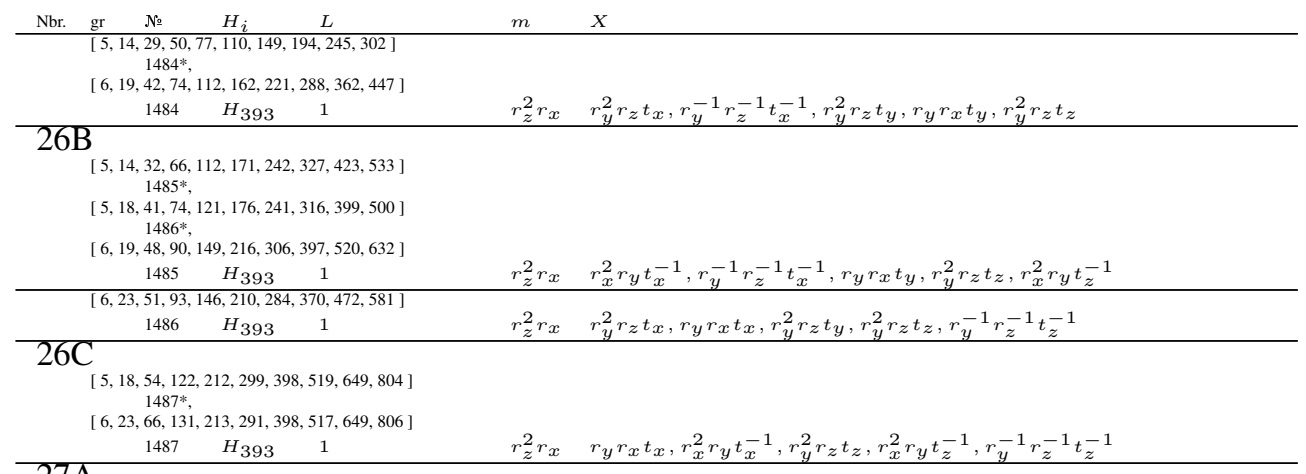

$27 \mathrm{~A}$

$[6,18,39,71,114,167,231,306,391,487]$ $1488^{*}$

$[6,20,46,84,134,196,270,356,454,564]$ $1489^{*}$,

$[6,20,48,90,145,215,296,388,499,621]$ $1490 *$,

$[6,20,49,94,151,221,307,406,516,640]$ $1491 *$,

$[6,21,52,102,167,243,336,443,560,695]$ $1492 *$,

[ $6,21,52,107,172,244,339,443,558,697]$ $1493^{*}$

$[6,22,52,94,150,220,302,398,508,630]$

$1494^{*}$,
$[6,22,53,99,160,233,321,424,539,669]$ $1495^{*}$,
,

$[6,22,53,107,172,243,337,444,559,697]$ $1496^{*}$,

$[6,22,55,103,164,240,330,433,550,681]$ $1498^{*}$,

$[6,22,55,104,168,247,340,448,570,705]$ $1497^{*}$,

$[6,22,55,105,168,245,339,442,559,697]$ $1500^{*}$,

$[6,22,56,106,169,247,339,445,567,703]$ $1499^{*}$,

$[6,22,56,109,174,252,347,457,584,726]$ $1501 *$,

$[6,22,57,106,167,246,336,441,563,694]$ $1502 *$,

$[6,22,58,107,168,245,333,439,557,687]$ 1503*,

$[6,23,57,108,172,249,342,449,571,708]$ $1504^{*}$,

$[6,23,58,111,178,256,351,462,583,721]$ $1505^{*}$,

$[6,24,54,100,158,232,318,420,534,664]$ $1506 *$

$[6,24,59,114,177,254,346,454,575,712]$ $1509^{*}$,

$[6,24,60,110,174,253,347,456,579,717]$ $1507^{*}$,

$[6,24,60,111,176,256,351,460,586,726]$ $1508^{*}$,

$[6,24,60,112,178,258,352,460,584,722]$ $1512^{*}$,

$[6,24,60,114,179,256,351,462,585,722]$ $1513^{*}$,

$[6,24,61,111,174,251,343,450,570,706]$ $1514^{*}$,

$[6,24,61,113,178,257,354,467,591,732]$ $1511^{*}$,

$[6,24,61,113,179,261,359,472,599,741]$ $1510^{*}$,

$[6,24,62,114,179,260,357,470,597,740]$ $1515^{*}$,

$[6,26,62,114,179,260,355,466,591,732]$ $1516^{*}$,

$[6,26,63,115,183,264,361,474,601,742]$ $1517 *$

$[6,26,65,117,183,264,361,473,600,742]$ $1518^{*}$,

$[6,28,66,118,185,268,365,478,606,750]$ $1519^{*}$,

$[7,22,48,86,134,192,262,342,432,534]$

$1488 H_{650}\left\langle m_{x}\right\rangle$

$[7,24,54,96,150,216,294,384,486,600]$ $r_{y}^{2} r_{x} \quad m_{x} t_{x}, m_{x} t_{x}^{-1}, m_{y} t_{y}^{-1}, m_{x} r_{x} t_{y}^{-1}, r_{y}^{2} t_{z}, m_{x} r_{x}^{-1} t_{z}^{-1}$ $1489 \quad H_{648}\left\langle m_{x}\right\rangle$ 


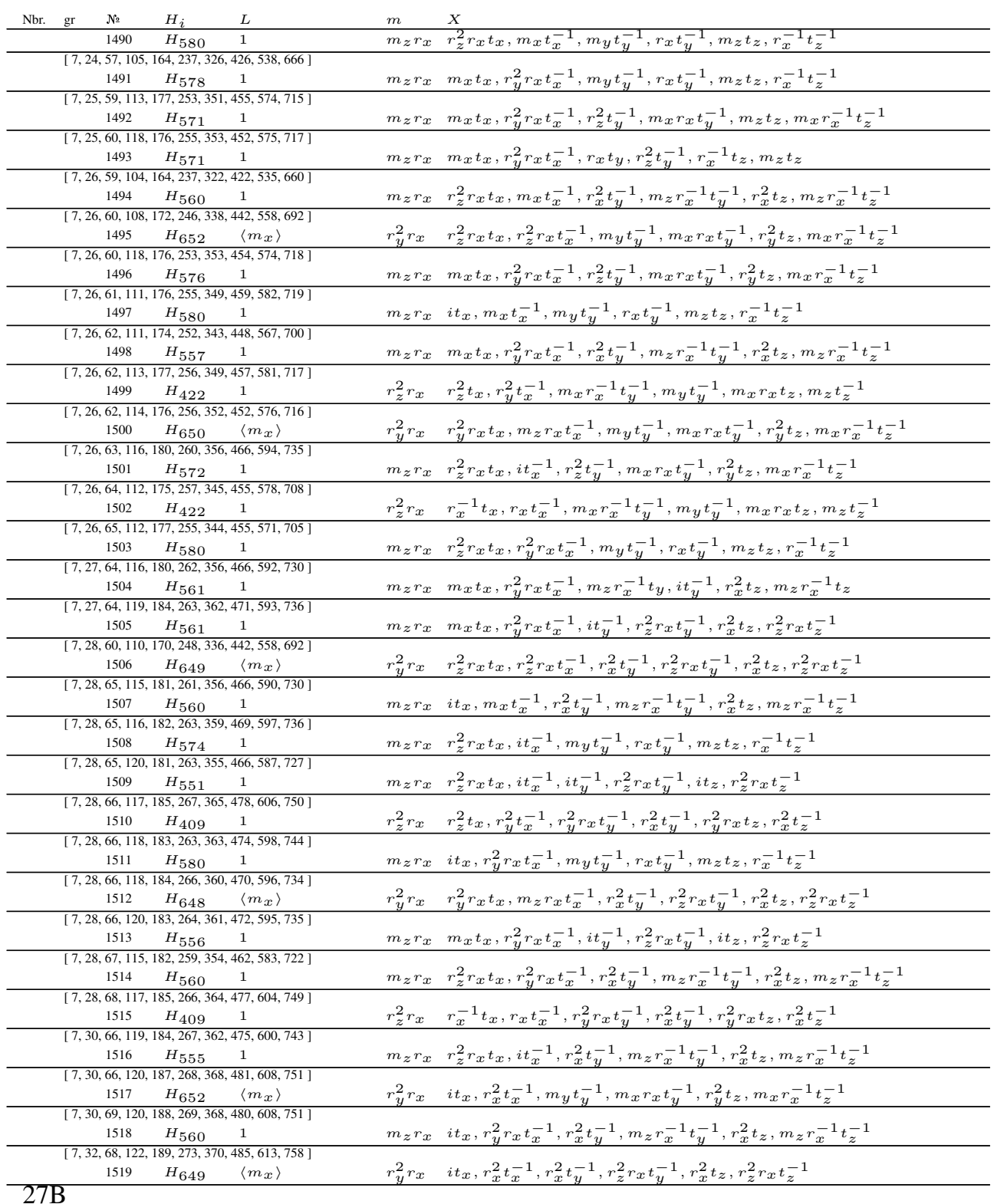

$[6,19,47,95,160,240,336,448,576,720]$ $1520^{*}$

$[6,20,51,107,184,274,378,498,634,786]$ $1521^{*}$

$[6,21,58,119,193,281,387,508,644,796]$ $1522^{*}$,

$[6,21,58,120,196,284,388,508,644,796]$ $1523^{*}$,

$[6,21,58,124,201,288,392,512,648,800]$ $1524 *$

$[6,22,60,120,195,285,391,512,648,800]$ $1525^{*}$,

$[6,22,60,126,208,296,397,516,652,804]$ $1526^{*}$,

[ $6,22,62,128,198,283,393,517,647,795$ ] $1527 *$

$[6,22,62,129,207,293,396,516,652,804]$ 1528*,

$[6,22,64,130,205,292,396,516,652,804]$ 


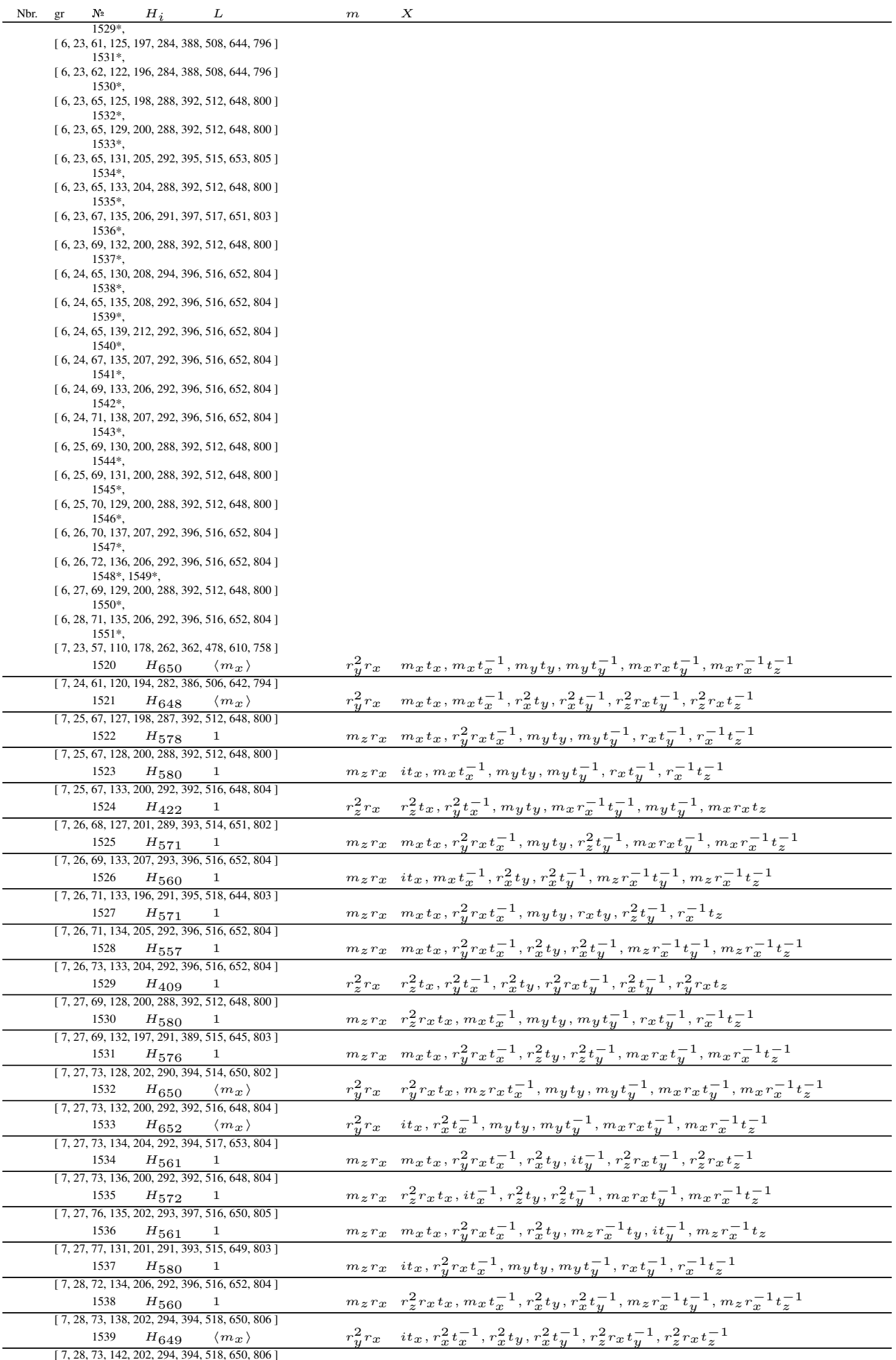




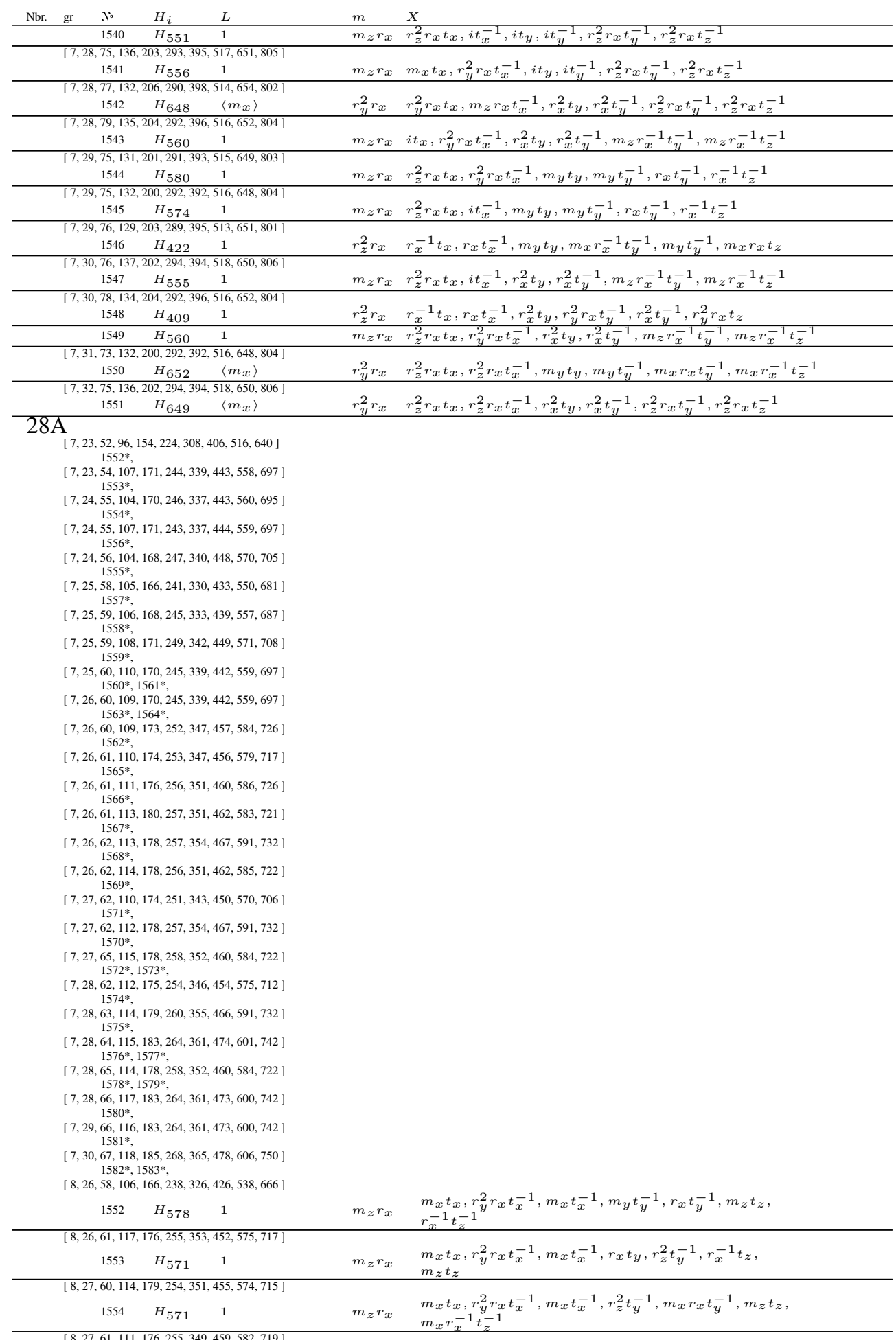




\begin{tabular}{lllll} 
Nbr. & gr & № & $H_{i}$ & $L$ \\
\hline & & 1555 & $H_{580}$ & 1
\end{tabular}

$[8,27,61,117,176,253,353,454,574,718]$

$1556 \quad H_{576} \quad 1$

$[8,28,63,112,175,252,343,448,567,700]$

$1557 \quad H_{557} \quad 1$

$[8,28,64,112,177,255,344,455,571,705]$

$1558 \quad H_{580} \quad 1$

$[8,28,65,115,180,262,356,466,592,730]$

$1559 \quad H_{561} \quad 1$

$[8,28,65,116,176,256,352,452,576,716]$

$\begin{array}{lll}1560 & H_{571} & 1 \\ 1561 & H_{578} & 1\end{array}$

$[8,29,64,115,180,260,356,466,594,735]$

$\begin{array}{lll}1562 & H_{572} & 1\end{array}$

$[8,29,64,116,176,256,352,452,576,716]$

$\begin{array}{lll}1563 & H_{576} & 1 \\ 1564 & H_{571} & 1\end{array}$

$[8,29,65,115,181,261,356,466,590,730]$

$$
1565 \quad H_{560} \quad 1
$$

$[8,29,65,116,182,263,359,469,597,736]$

$1566 \quad H_{574} \quad 1$

$[8,29,65,120,185,263,362,471,593,736]$

$1567 \quad H_{561} \quad 1$

$[8,29,66,118,183,263,363,474,598,744]$

$$
1568 \quad H_{580} \quad 1
$$

$[8,29,67,119,183,264,361,472,595,735]$

$$
1569 \quad H_{556} \quad 1
$$

$[8,30,65,118,183,263,363,474,598,744]$

$$
1570 \quad H_{580} \quad 1
$$

$[8,30,66,115,182,259,354,462,583,722]$

$$
1571 \quad H_{560} \quad 1
$$

$[8,30,69,118,184,266,360,470,596,734]$

$\begin{array}{lll}1572 & H_{561} & 1 \\ 1573 & H_{557} & 1\end{array}$

$[8,31,65,118,181,263,355,466,587,727]$

$1574 \quad H_{551} \quad 1$

$[8,31,66,119,184,267,362,475,600,743]$

$1575 \quad H_{555} \quad 1$

$[8,31,66,120,187,268,368,481,608,751]$

$\begin{array}{lll}1576 & H_{572} & 1 \\ 1577 & H_{574} & 1\end{array}$

$[8,31,68,118,184,266,360,470,596,734]$

$\begin{array}{lll}1578 & H_{556} & 1 \\ 1579 & H_{561} & 1\end{array}$

$[8,31,69,120,188,269,368,480,608,751]$

$$
1580 \quad H_{560} \quad 1
$$

$[8,32,68,120,188,269,368,480,608,751]$

$$
1581 \quad H_{560} \quad 1
$$

$\begin{array}{ll}m & X \\ m_{z} r_{x} & \begin{array}{l}i t_{x}, r_{z}^{2} r_{x} t_{x}, m_{x} t_{x}^{-1}, m_{y} t_{y}^{-1}, r_{x} t_{y}^{-1}, m_{z} t_{z}, \\ r_{x}^{-1} t_{z}-1\end{array}\end{array}$

$m_{z} r_{x} \quad m_{x} t_{x}, r_{y}^{2} r_{x} t_{x}^{-1}, m_{x} t_{x}^{-1}, r_{z}^{2} t_{y}^{-1}, m_{x} r_{x} t_{y}^{-1}, r_{y}^{2} t_{z}$, $m_{x} r_{x}^{-1} t_{z}^{-1}$

$m_{z} r_{x} \quad m_{x} t_{x}, r_{y}^{2} r_{x} t_{x}^{-1}, m_{x} t_{x}^{-1}, r_{x}^{2} t_{y}^{-1}, m_{z} r_{x}^{-1} t_{y}^{-1}, r_{x}^{2} t_{z}$, $m_{z} r_{x}^{-1} t_{z}^{-1}$

$r_{z}^{2} r_{x} t_{x}, r_{y}^{2} r_{x} t_{x}^{-1}, m_{x} t_{x}^{-1}, m_{y} t_{y}^{-1}, r_{x} t_{y}^{-1}, m_{z} t_{z}$, $m_{z} r_{x} \quad r_{z}^{2} r_{x} t_{x}$

$m_{z} r_{x} \quad m_{x} t_{x}, r_{y}^{2} r_{x} t_{x}^{-1}, m_{x} t_{x}^{-1}, m_{z} r_{x}^{-1} t_{y}, i t_{y}^{-1}, r_{x}^{2} t_{z}$, $m_{z} r_{x}^{-1} t_{z}$

$m_{z} r_{x} \quad r_{y}^{2} r_{x} t_{x}, r_{y}^{2} r_{x} t_{x}^{-1}, m_{x} t_{x}^{-1}, r_{z}^{2} t_{y}^{-1}, m_{x} r_{x} t_{y}^{-1}, m_{z} t_{z}$, $\begin{array}{ll}m_{z} r_{x} & r_{x}^{2} r_{x} t_{x}, r_{y}^{2} r_{x} t_{x}^{-1}, m_{x} t_{x}^{-1}, m_{y} t_{y}^{-1}, r_{x} t_{y}^{-1}, m_{z} t_{z}, \\ r_{x}^{-1} t_{z}^{-1}\end{array}$

$m_{z} r_{x} \quad r_{z}^{2} r_{x} t_{x}, i t_{x}^{-1}, r_{z}^{2} r_{x} t_{x}^{-1}, r_{z}^{2} t_{y}^{-1}, m_{x} r_{x} t_{y}^{-1}, r_{y}^{2} t_{z}$, $m_{x} r_{x}^{-1} t_{z}^{-1}$

$r_{y}^{2} r_{x} t_{x}, r_{y}^{2} r_{x} t_{x}^{-1}, m_{x} t_{x}^{-1}, r_{z}^{2} t_{y}^{-1}, m_{x} r_{x} t_{y}^{-1}, r_{y}^{2} t_{z}$,

$m_{z} r_{x} \quad m_{x} r_{x}^{-1} r_{z}^{-1}$

$m_{z} r_{x} \quad r_{y}^{2} r_{x} t_{x}, r_{y}^{2} r_{x} t_{x}^{-1}, m_{x} t_{x}^{-1}, r_{x} t_{y}, r_{z}^{2} t_{y}^{-1}, r_{x}^{-1} t_{z}$, $m_{z} t_{z}$

$m_{z} r_{x} \quad i t_{x}, r_{z}^{2} r_{x} t_{x}, m_{x} t_{x}^{-1}, r_{x}^{2} t_{y}^{-1}, m_{z} r_{x}^{-1} t_{y}^{-1}, r_{x}^{2} t_{z}$ $m_{z} r_{x}^{-1} t_{z}^{-1}$

$m_{z} r_{x} \quad r_{z}^{2} r_{x} t_{x}, i t_{x}^{-1}, r_{z}^{2} r_{x} t_{x}^{-1}, m_{y} t_{y}^{-1}, r_{x} t_{y}^{-1}, m_{z} t_{z}$, $r_{x}^{-1} t_{z}^{-1}$

$m_{z} r_{x} \quad m_{x} t_{x}, r_{y}^{2} r_{x} t_{x}^{-1}, m_{x} t_{x}^{-1}, i t_{y}^{-1}, r_{z}^{2} r_{x} t_{y}^{-1}, r_{x}^{2} t_{z}$,

$r_{z}^{2} r_{x} t_{z}^{-1}$

$m_{z} r_{x} \quad{ }^{i t_{x}}, r_{z}^{2} r_{x} t_{x}, r_{y}^{2} r_{x} t_{x}^{-1}, m_{y} t_{y}^{-1}, r_{x} t_{y}^{-1}, m_{z} t_{z}$, $r_{x}^{-1} t_{z}^{-1}$

$m_{z} r_{x} \quad m_{x} t_{x}, r_{y}^{2} r_{x} t_{x}^{-1}, m_{x} t_{x}^{-1}, i t_{y}^{-1}, r_{z}^{2} r_{x} t_{y}^{-1}, i t_{z}$, $r_{z}^{2} r_{x} t_{z}^{-1}$

$m_{z} r_{x} \quad i t_{x}, r_{y}^{2} r_{x} t_{x}^{-1}, m_{x} t_{x}^{-1}, m_{y} t_{y}^{-1}, r_{x} t_{y}^{-1}, m_{z} t_{z}$, $r_{x}^{-1} t_{z}^{-1}$

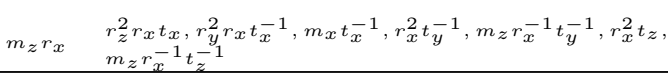

$m_{z} r_{x} \quad r_{y}^{2} r_{x} t_{x}, r_{y}^{2} r_{x} t_{x}^{-1}, m_{x} t_{x}^{-1}, i t_{y}^{-1}, r_{z}^{2} r_{x} t_{y}^{-1}, r_{x}^{2} t_{z}$, $\begin{array}{ll} & r_{z}^{2} r_{x} t_{z}^{-1} \\ m_{z} r_{x} & r_{y}^{2} r_{x} t_{x}, r_{y}^{2} r_{x} t_{x}^{-1}, m_{x} t_{x}^{-1}, r_{x}^{2} t_{y}^{-1}, m_{z} r_{x}^{-1} t_{y}^{-1}, r_{x}^{2} t_{z}, \\ m_{z} r_{x}^{-1} t_{z}^{-1}\end{array}$

$m_{z} r_{x} \quad r_{z}^{2} r_{x} t_{x}, i t_{x}^{-1}, r_{z}^{2} r_{x} t_{x}^{-1}, i t_{y}^{-1}, r_{z}^{2} r_{x} t_{y}^{-1}, i t_{z}$, $r_{z}^{2} r_{x} t_{z}^{-1}$

$m_{z} r_{x} \quad r_{z}^{2} r_{x} t_{x}, i t_{x}^{-1}, r_{z}^{2} r_{x} t_{x}^{-1}, r_{x}^{2} t_{y}^{-1}, m_{z} r_{x}^{-1} t_{y}^{-1}, r_{x}^{2} t_{z}$, $m_{z} r_{x}^{-1} t_{z}^{-1}$

$m_{z} r_{x} \quad i t_{x}, i t_{x}^{-1}, r_{z}^{2} r_{x} t_{x}^{-1}, r_{z}^{2} t_{y}^{-1}, m_{x} r_{x} t_{y}^{-1}, r_{y}^{2} t_{z}$,

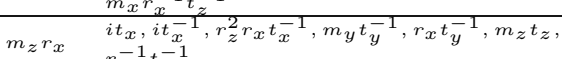
$r_{x}^{-1} t_{z}^{-1}$ $m_{z} r_{x} \quad r_{y}^{2} r_{x} t_{x}, r_{y}^{2} r_{x} t_{x}^{-1}, m_{x} t_{x}^{-1}, i t_{y}^{-1}, r_{z}^{2} r_{x} t_{y}^{-1}, i t_{z}$, $m_{z} r_{x} \quad r_{y}^{2} r_{x} t_{x}, r_{y}^{2} r_{x} t_{x}^{-1}, m_{x} t_{x}^{-1}, m_{z} r_{x}^{-1} t_{y}, i t_{y}^{-1}, r_{x}^{2} t_{z}$, $m_{z} r_{x}^{-1} t_{z}$

$m_{z} r_{x} \quad i t_{x}, r_{z}^{2} r_{x} t_{x}, r_{y}^{2} r_{x} t_{x}^{-1}, r_{x}^{2} t_{y}^{-1}, m_{z} r_{x}^{-1} t_{y}^{-1}, r_{x}^{2} t_{z}$, $m_{z} r_{x}^{-1} t_{z}^{-1}$

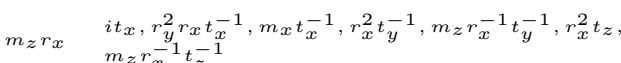




\begin{tabular}{|c|c|c|c|c|c|}
\hline Nbr. & $\begin{array}{ll}\mathrm{gr} & \text { № } \\
{[8.33 .68} & 12\end{array}$ & $\frac{H_{i}}{89.273,3}$ & $\frac{L}{485.613 .758}$ & $m$ & $x$ \\
\hline & 1582 & $H_{551}$ & $x^{2}+x_{0}$ & $m_{z} r_{x}$ & $\begin{array}{l}i t_{x}, i t_{x}^{-1}, r_{z}^{2} r_{x} t_{x}^{-1}, i t_{y}^{-1}, r_{z}^{2} r_{x} t_{y}^{-1}, i t_{z}, \\
r_{z}^{2} r_{x} t_{z}^{-1}\end{array}$ \\
\hline & 1583 & $H_{555}$ & 1 & $m_{z} r_{x}$ & $\begin{array}{l}i t_{x}, i t_{x}^{-1}, r_{z}^{2} r_{x} t_{x}^{-1}, r_{x}^{2} t_{y}^{-1}, m_{z} r_{x}^{-1} t_{y}^{-1}, r_{x}^{2} t_{z}, \\
m_{z} r_{x}^{-1} t_{z}\end{array}$ \\
\hline
\end{tabular}

28B

$[7,24,61,121,196,284,388,508,644,796]$ $1584^{*}$,

$[7,24,64,128,197,283,393,517,647,795]$ $1585^{*}$,

$[7,25,63,122,196,284,388,508,644,796]$ $1586^{*}$,

$[7,25,63,122,198,288,392,512,648,800]$ $1587 *$

$[7,25,63,125,196,284,388,508,644,796]$ $1588^{*}$,

$[7,25,65,131,209,294,396,516,652,804]$ $1589 *$

$[7,25,69,135,205,291,397,517,651,803]$ $1590^{*}$,

$[7,26,66,130,208,294,396,516,652,804]$ $1591^{*}$,

$[7,26,68,133,207,293,395,515,653,805]$ $1592^{*}$,

$[7,26,69,135,206,292,396,516,652,804]$ $1596^{*}$,

$[7,26,70,130,200,288,392,512,648,800]$ $1593^{*}, 1594^{*}$,

[ $7,26,70,131,200,288,392,512,648,800$ ] $1595^{*}$,

$[7,27,69,131,201,288,392,512,648,800]$ $1597^{*}$,

$[7,27,70,129,200,288,392,512,648,800]$ $1598^{*}, 1599^{*}$,

$[7,27,70,130,200,288,392,512,648,800]$ $1600^{*}$,

$[7,27,70,131,200,288,392,512,648,800]$ $1601^{*}$

[ 7, 27, 72, 137, 207, 292, 396, 516, 652, 804$]$ $1602 *$,

$[7,27,74,136,206,292,396,516,652,804]$ $1603^{*}, 1604 *$

$[7,28,70,129,200,288,392,512,648,800]$ $1605^{*}$,

[ 7, 28, 70, 137, 208, 292, 396, 516, 652, 804 ] $1606^{*}$,

$[7,28,71,137,207,292,396,516,652,804]$ $1607 *$

$[7,28,73,136,206,292,396,516,652,804]$

$1608^{*}$,
$[7,28,74,135,206,292,396,516,652,804]$ $1609^{*}, 1610^{*}$

$[7,29,70,129,200,288,392,512,648,800]$ $1611^{*}, 1612^{*}$,

[ $7,29,73,135,206,292,396,516,652,804$ ] $1613^{*}$,

$[7,30,72,135,206,292,396,516,652,804]$ $1614^{*}, 1615^{*}$,

$[8,27,68,128,200,288,392,512,648,800]$

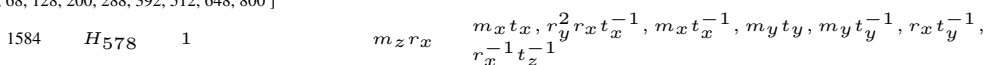

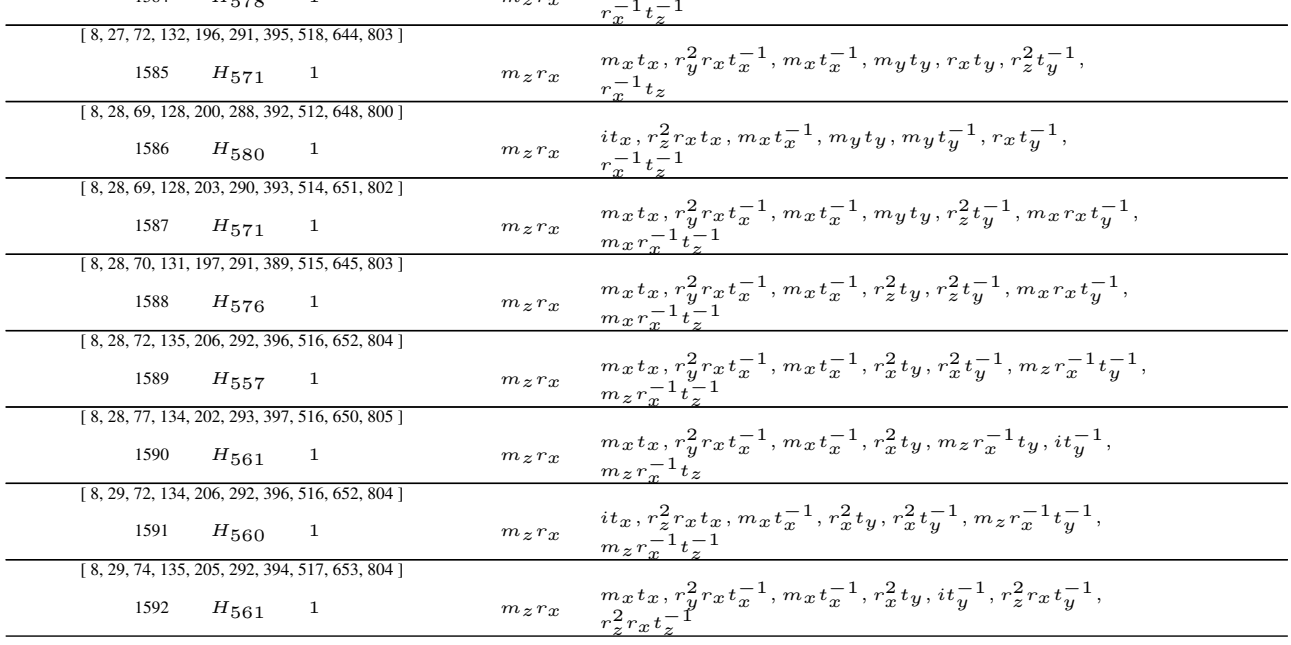




\begin{tabular}{|c|c|c|c|c|c|}
\hline Nbr. & gr № & $H_{i}$ & $L$ & $m$ & $x$ \\
\hline & {$[8,29,76,13$} & $H_{571}$ & $514,650,802]$ & $m_{z} r_{x}$ & $\begin{array}{l}r_{y}^{2} r_{x} t_{x}, r_{y}^{2} r_{x} t_{x}^{-1}, m_{x} t_{x}^{-1}, m_{y} t_{y}, r_{z}^{2} t_{y}^{-1}, m_{x} r_{x} t_{y}^{-1} \\
m_{x} r_{x}^{-1} t_{z}^{-1}\end{array}$ \\
\hline & 1594 & $H_{578}$ & 1 & $m_{z} r_{x}$ & $\begin{array}{l}r_{y}^{2} r_{x} t_{x}, r_{y}^{2} r_{x} t_{x}^{-1}, m_{x} t_{x}^{-1}, m_{y} t_{y}, m_{y} t_{y}^{-1}, r_{x} t_{y}^{-1} \\
r_{x}^{-1} t_{z}^{-1}\end{array}$ \\
\hline & {$[8,29,76,13$} & $H_{580}$ & $515,649,803]$ & $m_{z} r_{x}$ & $\begin{array}{l}i t_{x}, r_{y}^{2} r_{x} t_{x}^{-1}, m_{x} t_{x}^{-1}, m_{y} t_{y}, m_{y} t_{y}^{-1}, r_{x} t_{y}^{-1} \\
r_{x}^{-1} t_{z}^{-1}\end{array}$ \\
\hline & {$[8,29,76,13$} & $\begin{array}{l}03,293,3 \\
H_{556}\end{array}$ & $\begin{array}{l}517,651,805] \\
1\end{array}$ & $m_{z} r_{x}$ & $\begin{array}{l}m_{x} t_{x}, r_{y}^{2} r_{x} t_{x}^{-1}, m_{x} t_{x}^{-1}, i t_{y}, i t_{y}^{-1}, r_{z}^{2} r_{x} t_{y}^{-1} \\
r_{z}^{2} r_{x} t_{z}^{-1}\end{array}$ \\
\hline & {$[8,30,74,13$} & $H_{572}$ & $516,648,804]$ & $m_{z} r_{x}$ & $\begin{array}{l}i t_{x}, i t_{x}^{-1}, r_{z}^{2} r_{x} t_{x}^{-1}, r_{z}^{2} t_{y}, r_{z}^{2} t_{y}^{-1}, m_{x} r_{x} t_{y}^{-1} \\
m_{x} r_{x}^{-1} t_{z}^{-1}\end{array}$ \\
\hline & {$[8,30,75,13$} & $\begin{array}{l}02,290,3 \\
H_{576}\end{array}$ & $514,650,802]$ & $m_{z} r_{x}$ & $\begin{array}{l}r_{y}^{2} r_{x} t_{x}, r_{y}^{2} r_{x} t_{x}^{-1}, m_{x} t_{x}^{-1}, r_{z}^{2} t_{y}, r_{z}^{2} t_{y}^{-1}, m_{x} r_{x} t_{y}^{-1} \\
m_{x} r_{x}^{-1} t_{z}^{-1}\end{array}$ \\
\hline & 1599 & $H_{571}$ & 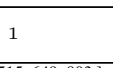 & $m_{z} r_{x}$ & $\begin{array}{l}m_{x} r_{x} L_{z}^{2} \\
r_{y}^{2} r_{x} t_{x}, r_{y}^{2} r_{x} t_{x}^{-1}, m_{x} t_{x}^{-1}, m_{y} t_{y}, r_{x} t_{y}, r_{z}^{2} t_{y}^{-1} \\
r_{x}^{-1} t_{z}\end{array}$ \\
\hline & {$[8,30,75,13$} & $H_{580}$ & $515,649,803]$ & $m_{z} r_{x}$ & $\begin{array}{l}i t_{x}, r_{z}^{2} r_{x} t_{x}, r_{y}^{2} r_{x} t_{x}^{-1}, m_{y} t_{y}, m_{y} t_{y}^{-1}, r_{x} t_{y}^{-1} \\
r_{x}^{-1} t_{z}^{-1}\end{array}$ \\
\hline & {$[8,30,75,13$} & $H_{574}$ & $\begin{array}{l}516,648,804] \\
1\end{array}$ & $m_{z} r_{x}$ & $\begin{array}{l}i t_{x}, i t_{x}^{-1}, r_{z}^{2} r_{x} t_{x}^{-1}, m_{y} t_{y}, m_{y} t_{y}^{-1}, r_{x} t_{y}^{-1} \\
r_{x}^{-1} t_{z}^{-1}\end{array}$ \\
\hline & $\begin{array}{r}{[8,30,78,13} \\
1602\end{array}$ & $H_{560}$ & $\begin{array}{l}516,652,804] \\
1\end{array}$ & $m_{z} r_{x}$ & $\begin{array}{l}i t_{x}, r_{y}^{2} r_{x} t_{x}^{-1}, m_{x} t_{x}^{-1}, r_{x}^{2} t_{y}, r_{x}^{2} t_{y}^{-1}, m_{z} r_{x}^{-1} t_{y}^{-1} \\
m_{z} r_{x}^{-1} t_{z}^{-1}\end{array}$ \\
\hline & {$[8,30,80,13$} & $H_{561}$ & $514,654,802]$ & $m_{z} r_{x}$ & $\begin{array}{l}r_{y}^{2} r_{x} t_{x}, r_{y}^{2} r_{x} t_{x}^{-1}, m_{x} t_{x}^{-1}, r_{x}^{2} t_{y}, i t_{y}^{-1}, r_{z}^{2} r_{x} t_{y}^{-1} \\
r_{z}^{2} r_{x} t_{z}^{-1}\end{array}$ \\
\hline & 1604 & $H_{557}$ & 1 & $m_{z} r_{x}$ & $\begin{array}{l}r_{y}^{2} r_{x} t_{x}, r_{y}^{2} r_{x} t_{x}^{-1}, m_{x} t_{x}^{-1}, r_{x}^{2} t_{y}, r_{x}^{2} t_{y}^{-1}, m_{z} r_{x}^{-1} t_{y}^{-1}, \\
m_{z} r_{x}^{-1} t_{z}^{-1}\end{array}$ \\
\hline & {$[8,31,74,13$} & $\begin{array}{r}01,291,3 \\
H_{580}\end{array}$ & $515,649,803]$ & $m_{z} r_{x}$ & $\begin{array}{l}r_{z}^{2} r_{x} t_{x}, r_{y}^{2} r_{x} t_{x}^{-1}, m_{x} t_{x}^{-1}, m_{y}{ }^{\prime}, m_{y} t_{y}^{-1}, r_{x} t_{y}^{-1} \\
r_{x}^{-1} t_{z}^{-1}\end{array}$ \\
\hline & $\begin{array}{r}{[8,31,75,13} \\
1606\end{array}$ & $\begin{array}{l}02,294,3 \\
H_{551}\end{array}$ & $\begin{array}{l}518,650,806] \\
1\end{array}$ & $m_{z} r_{x}$ & $\begin{array}{l}i t_{x}, i t_{x}^{-1}, r_{z}^{2} r_{x} t_{x}^{-1}, i t_{y}, i t_{y}^{-1}, r_{z}^{2} r_{x} t_{y}^{-1} \\
r_{z}^{2} r_{x} t_{z}^{-1}\end{array}$ \\
\hline & $\begin{array}{r}{[8,31,76,13} \\
1607\end{array}$ & $\begin{array}{l}02,294,3 \\
H_{555}\end{array}$ & $518,650,806]$ & $m_{z} r_{x}$ & $\begin{array}{l}i t_{x}, i t_{x}^{-1}, r_{z}^{2} r_{x} t_{x}^{-1}, r_{x}^{2} t_{y}, r_{x}^{2} t_{y}^{-1}, m_{z} r_{x}^{-1} t_{y}^{-1} \\
m_{z} r_{x}^{-1} t_{z}^{-1}\end{array}$ \\
\hline & {$[8,31,78,13$} & $H_{560}$ & $516,652,804]$ & $m_{z} r_{x}$ & $\begin{array}{l}{ }^{i t_{x}}, r_{z}^{2} r_{x} t_{x}, r_{y}^{2} r_{x} t_{x}^{-1}, r_{x}^{2} t_{y}, r_{x}^{2} t_{y}^{-1}, m_{z} r_{x}^{-1} t_{y}^{-1}, \\
m_{z} r_{x}^{-1} t_{z}^{-1}\end{array}$ \\
\hline & {$[8,31,79,13$} & $H_{556}$ & $514,654,802]$ & $m_{z} r_{x}$ & $\begin{array}{l}r_{y}^{2} r_{x} t_{x}, r_{y}^{2} r_{x} t_{x}^{-1}, m_{x} t_{x}^{-1}, i t_{y}, i t_{y}^{-1}, r_{z}^{2} r_{x} t_{y}^{-1} \\
r_{z}^{2} r_{x} t_{z}^{-1}\end{array}$ \\
\hline & 1610 & $H_{561}$ & 1 & $m_{z} r_{x}$ & $\begin{array}{l}r_{y}^{2} r_{x} t_{x}, r_{y}^{2} r_{x} t_{x}^{-1}, m_{x} t_{x}^{-1}, r_{x}^{2} t_{y}, m_{z} r_{x}^{-1} t_{y}, i t_{y}^{-1}, \\
m_{z} r_{x}^{-1} t_{z}\end{array}$ \\
\hline & {$[8,32,73,13$} & $\begin{array}{r}00,292,3 \\
H_{572}\end{array}$ & $\begin{array}{l}516,648,804] \\
1\end{array}$ & $m_{z} r_{x}$ & $\begin{array}{l}r_{z}^{2} r_{x} t_{x}, i t_{x}^{-1}, r_{z}^{2} r_{x} t_{x}^{-1}, r_{z}^{2} t_{y}, r_{z}^{2} t_{y}^{-1}, m_{x} r_{x} t_{y}^{-1} \\
m_{x} r_{x}^{-1} t_{z}^{-1}\end{array}$ \\
\hline & 1612 & $H_{574}$ & 1 & $m_{z} r_{x}$ & $\begin{array}{l}r_{z}^{2} r_{x} t_{x}, i t_{x}^{-1}, r_{z}^{2} r_{x} t_{x}^{-1}, m_{y} t_{y}, m_{y} t_{y}^{-1}, r_{x} t_{y}^{-1} \\
r_{x}^{-1} t_{z}^{-1}\end{array}$ \\
\hline & $\begin{array}{r}{[8,32,77,13} \\
1613\end{array}$ & $\begin{array}{r}04,292,3 \\
H_{560}\end{array}$ & $\begin{array}{l}516,652,804] \\
1\end{array}$ & $m_{z} r_{x}$ & $\begin{array}{l}r_{z}^{2} r_{x} t_{x}, r_{y}^{2} r_{x} t_{x}^{-1}, m_{x} t_{x}^{-1}, r_{x}^{2} t_{y}, r_{x}^{2} t_{y}^{-1}, m_{z} r_{x}^{-1} t_{y}^{-1}, \\
m_{z} r_{x}^{-1} t_{z}^{-1}\end{array}$ \\
\hline & $\begin{array}{r}{[8,33,75,13} \\
1614\end{array}$ & $\begin{array}{r}02,294,3 \\
H_{551}\end{array}$ & $\begin{array}{l}518,650,806] \\
1\end{array}$ & $m_{z} r_{x}$ & $\begin{array}{l}r_{z}^{2} r_{x} t_{x}, i t_{x}^{-1}, r_{z}^{2} r_{x} t_{x}^{-1}, i t_{y}, i t_{y}^{-1}, r_{z}^{2} r_{x} t_{y}^{-1} \\
r_{z}^{2} r_{x} t_{z}^{-1}\end{array}$ \\
\hline & 1615 & $H_{555}$ & 1 & $m_{z} r_{x}$ & $\begin{array}{l}r_{z}^{2} r_{x} t_{x}, i t_{x}^{-1}, r_{z}^{2} r_{x} t_{x}^{-1}, r_{x}^{2} t_{y}, r_{x}^{2} t_{y}^{-1}, m_{z} r_{x}^{-1} t_{y}^{-1}, \\
m_{z} r_{x}^{-1} t_{z}^{-1}\end{array}$ \\
\hline
\end{tabular}

[ 7, 22, 48, 86, 134, 192, 262, 342, 432, 534 ] $1616^{*}$

$[7,22,50,98,161,235,328,432,548,686]$ $1617^{*}$,

$[7,23,52,98,160,235,327,432,549,685]$ $1619 *$

$[7,23,52,100,163,238,332,437,553,691]$ $1618^{*}$

$[7,23,57,106,167,246,336,441,563,694]$ $1620^{*}$,

$[7,24,54,96,150,216,294,384,486,600]$ 1622 *

$[7,24,55,99,156,227,311,408,519,643]$ 


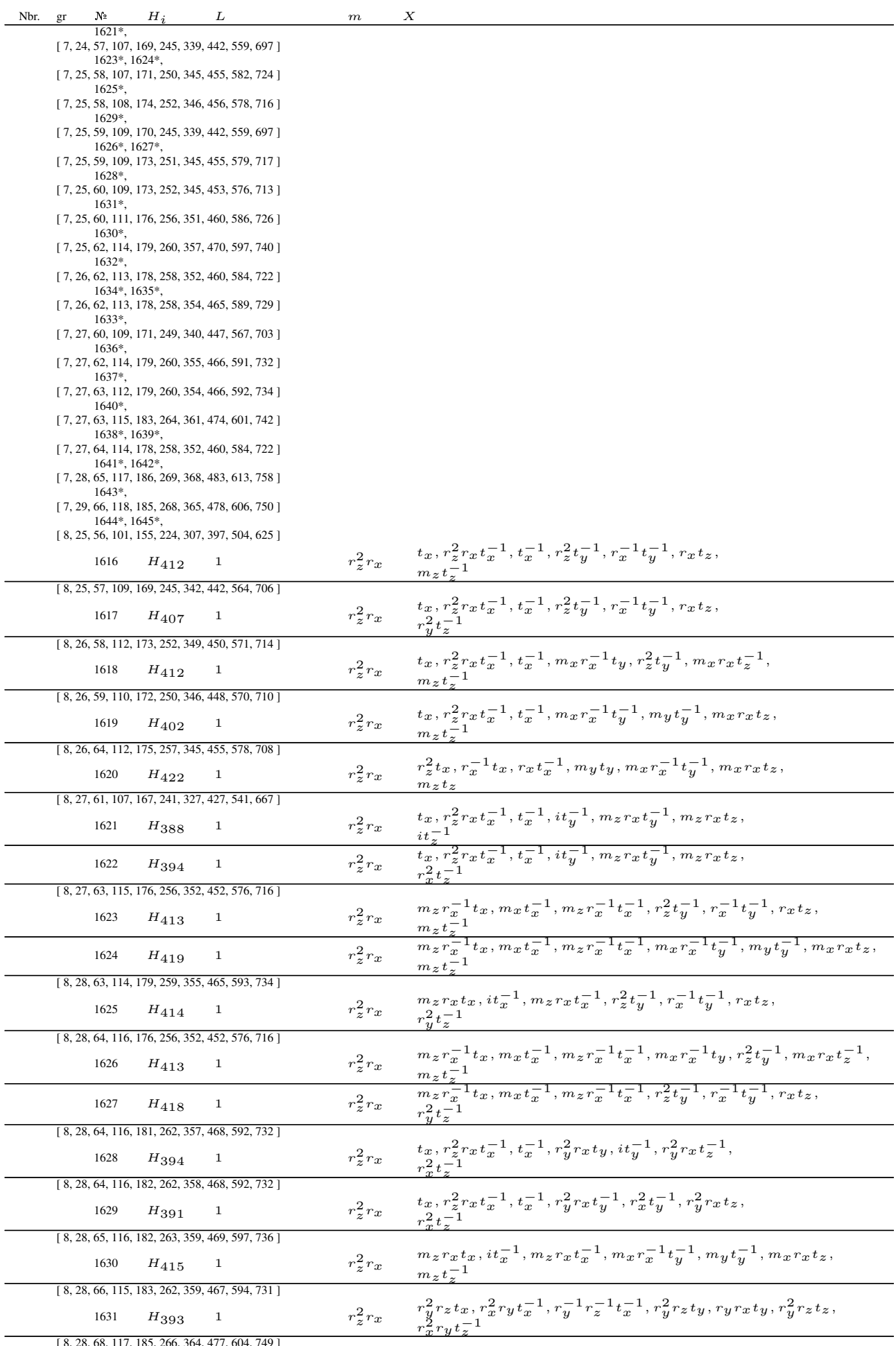




\begin{tabular}{|c|c|c|c|c|c|}
\hline Nbr. & № & $H_{i}$ & $L$ & $m$ & $x$ \\
\hline & 1632 & $H_{409}$ & 1 & $r_{z}^{2} r_{x}$ & $\begin{array}{l}r_{z}^{2} t_{x}, r_{x}^{-1} t_{x}, r_{x} t_{x}^{-1}, r_{x}^{2} t_{y}, r_{y}^{2} r_{x} t_{y}^{-1}, r_{y}^{2} r_{x} t_{z} \\
r_{x}^{2} t_{z}\end{array}$ \\
\hline & \multicolumn{4}{|c|}{$[8,29,66,118,183,265,362,473,598,741]$} & \\
\hline & 1633 & $H_{422}$ & 1 & $r_{z}^{2} r_{x}$ & $\begin{array}{l}r_{z}^{2} t_{x}, r_{x}^{-1} t_{x}, r_{x} t_{x}^{-1}, m_{x} r_{x}^{-1} t_{y}^{-1}, m_{y} t_{y}^{-1}, m_{x} r_{x} t_{z} \\
m_{z} t_{z}^{-1}\end{array}$ \\
\hline & \multicolumn{5}{|c|}{$[8,29,67,118,184,266,360,470,596,734]$} \\
\hline & 1634 & $H_{395}$ & 1 & $r_{z}^{2} r_{x}$ & $\begin{array}{l}m_{z} r_{x}^{-1} t_{x}, m_{x} t_{x}^{-1}, m_{z} r_{x}^{-1} t_{x}^{-1}, i t_{y}^{-1}, m_{z} r_{x} t_{y}^{-1}, m_{z} r_{x} t_{z}, \\
r_{x}^{2} t_{z}^{-1}\end{array}$ \\
\hline & 1635 & $H_{401}$ & 1 & $r_{z}^{2} r_{x}$ & $\begin{array}{l}m_{z} r_{x}^{-1} t_{x}, m_{x} t_{x}^{-1}, m_{z} r_{x}^{-1} t_{x}^{-1}, r_{y}^{2} r_{x} t_{y}^{-1}, r_{x}^{2} t_{y}^{-1}, r_{y}^{2} r_{x} t_{z}, \\
r_{x}^{2} t_{z}^{-1}\end{array}$ \\
\hline & \multicolumn{5}{|c|}{$[8,30,64,116,179,260,352,462,583,722]$} \\
\hline & 1636 & $H_{389}$ & 1 & $r_{z}^{2} r_{x}$ & $\begin{array}{l}m_{z} r_{x} t_{x}, i t_{x}^{-1}, m_{z} r_{x} t_{x}^{-1}, i t_{y}^{-1}, m_{z} r_{x} t_{y}^{-1}, m_{z} r_{x} t_{z} \\
i t_{z}^{-1}\end{array}$ \\
\hline & \multicolumn{5}{|c|}{$[8,30,66,119,184,267,362,475,600,743]$} \\
\hline & 1637 & $H_{396}$ & 1 & $r_{z}^{2} r_{x}$ & $\begin{array}{l}m_{z} r_{x} t_{x}, i t_{x}^{-1}, m_{z} r_{x} t_{x}^{-1}, r_{y}^{2} r_{x} t_{y}^{-1}, r_{x}^{2} t_{y}^{-1}, r_{y}^{2} r_{x} t_{z} \\
r_{x}^{2} t_{z}^{-1}\end{array}$ \\
\hline & \multicolumn{5}{|c|}{$[8,30,66,120,187,268,368,481,608,751]$} \\
\hline & 1638 & $H_{410}$ & 1 & $r_{z}^{2} r_{x}$ & 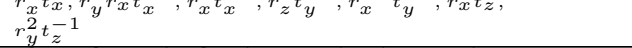 \\
\hline & 1639 & $H_{404}$ & 1 & $r_{z}^{2} r_{x}$ & $\begin{array}{l}r_{x}^{2} t_{x}, r_{y}^{2} r_{x} t_{x}^{-1}, r_{x}^{2} t_{x}^{-1}, m_{x} r_{x}^{-1} t_{y}^{-1}, m_{y} t_{y}^{-1}, m_{x} r_{x} t_{z}, \\
m_{z} t_{z}^{-1}\end{array}$ \\
\hline & \multicolumn{5}{|c|}{$[8,30,67,119,187,269,367,479,609,752]$} \\
\hline & 1640 & $H_{393}$ & 1 & $r_{z}^{2} r_{x}$ & $\begin{array}{l}r_{y}^{2} r_{z} t_{x}, r_{y} r_{x} t_{x}, r_{y}^{-1} r_{z}^{-1} t_{x}^{-1}, r_{y}^{2} r_{z} t_{y}, r_{y} r_{x} t_{y}, r_{y}^{2} r_{z} t_{z} \\
r_{y}^{-1} r_{z}^{-1} t_{z}^{-1}\end{array}$ \\
\hline & \multicolumn{5}{|c|}{$[8,30,68,118,184,266,360,470,596,734]$} \\
\hline & 1641 & $H_{400}$ & 1 & $r_{z}^{2} r_{x}$ & $\begin{array}{l}m_{z} r_{x}^{-1} t_{x}, m_{x} t_{x}^{-1}, m_{z} r_{x}^{-1} t_{x}^{-1}, i t_{y}^{-1}, m_{z} r_{x} t_{y}^{-1}, m_{z} r_{x} t_{z}, \\
i t_{z}^{-1}\end{array}$ \\
\hline & 1642 & $H_{395}$ & 1 & $r_{z}^{2} r_{x}$ & $\begin{array}{l}m_{z} r_{x}^{-1} t_{x}, m_{x} t_{x}^{-1}, m_{z} r_{x}^{-1} t_{x}^{-1}, r_{y}^{2} r_{x} t_{y}, i t_{y}^{-1}, r_{y}^{2} r_{x} t_{z}^{-1} \\
r_{x}^{2} t_{z}^{-1}\end{array}$ \\
\hline & \multicolumn{5}{|c|}{$[8,31,68,121,190,272,373,488,618,764]$} \\
\hline & 1643 & $H_{409}$ & 1 & $r_{z}^{2} r_{x}$ & $\begin{array}{l}r_{z}^{2} t_{x}, r_{x}^{-1} t_{x}, r_{x} t_{x}^{-1}, r_{y}^{2} r_{x} t_{y}^{-1}, r_{x}^{2} t_{y}^{-1}, r_{y}^{2} r_{x} t_{z} \\
r_{x}^{2} t_{z}^{-1}\end{array}$ \\
\hline & \multicolumn{5}{|c|}{$[8,32,68,122,189,273,370,485,613,758]$} \\
\hline & 1644 & $H_{398}$ & 1 & $r_{z}^{2} r_{x}$ & $\begin{array}{l}r_{x}^{2} t_{x}, r_{y}^{2} r_{x} t_{x}^{-1}, r_{x}^{2} t_{x}^{-1}, i t_{y}^{-1}, m_{z} r_{x} t_{y}^{-1}, m_{z} r_{x} t_{z} \\
i t_{z}^{-1}\end{array}$ \\
\hline & 1645 & $H_{392}$ & 1 & $r_{z}^{2} r_{x}$ & $\begin{array}{l}r_{x}^{z} t_{x}, r_{y}^{2} r_{x} t_{x}^{-1}, r_{x}^{2} t_{x}^{-1}, r_{y}^{2} r_{x} t_{y}^{-1}, r_{x}^{2} t_{y}^{-1}, r_{y}^{2} r_{x} t_{z} \\
r_{x}^{2} t_{z}^{-1}\end{array}$ \\
\hline
\end{tabular}

$[7,23,57,110,182,273,376,493,630,785]$ 1646*,

$[7,23,60,119,192,282,385,505,641,793]$ $1647^{*}$,

$[7,24,60,114,184,272,376,496,632,784]$ $1649^{*}$,

$[7,24,60,116,190,281,384,503,639,792$ ] $1648^{*}$,

$[7,24,61,121,196,284,387,507,644,796]$ $1650^{*}$,

$[7,24,65,126,197,283,387,507,643,795$ ] $1651^{*}$

$[7,25,65,127,198,284,388,508,644,796]$ $1653^{*}$,

$[7,25,66,127,197,283,388,508,643,795]$ $1652 *$

$[7,25,67,127,199,288,392,512,648,800]$ $1654^{*}, 1655^{*}$,

$[7,26,67,129,200,288,392,512,648,800]$ $1656^{*}$,

$[7,26,69,129,200,288,392,512,648,800]$ $1657^{*}, 1658^{*}$

[ $7,26,69,131,200,288,392,512,648,800$ ] $1659^{*}$,

$[7,26,70,129,200,288,392,512,648,800]$ $1660^{*}$,

$[7,26,71,134,206,292,396,516,652,804]$ $1661^{*}, 1662^{*}$,

$[7,27,69,135,206,292,396,516,652,804]$ $1664^{*}$,

$[7,27,70,128,200,288,392,512,648,800]$ $1663^{*}$,

$[7,27,71,137,206,292,396,516,652,804]$ $1665^{*}$,

$[7,27,72,136,206,292,396,516,652,804]$ $1666^{*}$

$[7,27,73,135,206,292,396,516,652,804]$ $1667^{*}, 1668^{*}$,

$[7,28,69,129,200,288,392,512,648,800]$ $1669^{*}, 1670^{*}$,

$[7,28,72,135,206,292,396,516,652,804]$ $1671^{*}$

$[7,29,71,135,206,292,396,516,652,804]$ $1672^{*}, 1673^{*}$

$[7,29,71,135,210,291,395,518,651,803]$ 
\begin{tabular}{lllllll} 
Nbr. & gr & № & $H_{i}$ & $L$ & $m$ & $X$ \\
\hline & $1674^{*}$, & & &
\end{tabular}

[ $7,29,75,135,206,291,395,518,651,803$ ] $1675^{*}$,

$[8,26,66,120,193,282,385,504,641,794]$

$1646 \quad H_{412} \quad 1$

$[8,26,68,126,197,288,388,512,644,800]$

$1647 \quad H_{407} \quad 1$

$[8,27,67,124,198,287,388,510,645,799]$

$1648 \quad H_{412} \quad 1$

$[8,27,68,122,194,282,386,506,642,794]$ $1649 \quad H_{402} \quad 1$

$[8,27,70,127,200,287,392,511,648,799]$ $\begin{array}{lll}1650 & H_{394} \quad 1\end{array}$

$[8,27,73,128,200,286,392,510,648,798]$ $1651 \quad H_{388} \quad 1$

$[8,28,73,129,200,287,392,511,648,799]$ $1652 \quad H_{394} \quad 1$

$[8,28,73,130,200,288,392,512,648,800]$ $1653 \quad H_{391} \quad 1$

$[8,28,74,129,202,290,394,514,650,802]$

$\begin{array}{lll}1654 & H_{413} & 1 \\ 1655 & H_{419} & 1\end{array}$

$[8,29,73,132,200,292,392,516,648,804]$ $1656 \quad H_{410} \quad 1$

$[8,29,75,130,202,290,394,514,650,802]$

$\begin{array}{lll}1657 & H_{413} & 1 \\ 1658 & H_{418} & 1\end{array}$

$[8,29,75,132,200,292,392,516,648,804]$ $1659 \quad H_{404} \quad 1$

$[8,29,76,129,203,289,395,513,651,801]$ $1660 \quad H_{422} \quad 1$

$[8,29,78,132,206,290,398,514,654,802]$

$\begin{array}{lll}1661 & H_{395} & 1 \\ 1662 & H_{401} & 1\end{array}$

$[8,30,75,129,203,289,395,513,651,801]$ $1663 \quad H_{422} \quad 1$

$[8,30,75,136,202,294,394,518,650,806]$ $1664 \quad H_{398} \quad 1$

$[8,30,77,136,202,294,394,518,650,806]$ $1665 \quad H_{392} \quad 1$

$[8,30,78,134,204,292,396,516,652,804]$

$$
1666 \quad H_{409} \quad 1
$$

$[8,30,79,132,206,290,398,514,654,802]$

$\begin{array}{lll}1667 & H_{400} & 1 \\ 1668 & H_{395} & 1\end{array}$

$[8,31,73,132,200,292,392,516,648,804]$

\begin{tabular}{ccc}
1669 & $H_{414}$ & 1 \\
\hline 1670 & $H_{415}$ & 1
\end{tabular}

$[8,31,77,134,204,292,396,516,652,804]$

$$
1671 \quad H_{409} \quad 1
$$

$[8,32,75,136,202,294,394,518,650,806]$

$r_{z}^{2} r_{x} \quad t_{x}, r_{z}^{2} r_{x} t_{x}^{-1}, t_{x}^{-1}, m_{y} t_{y}, r_{z}^{2} t_{y}^{-1}, r_{x}^{-1} t_{y}^{-1}$

$r_{z}^{2} r_{x} \quad t_{x}, r_{z}^{2} r_{x} t_{x}^{-1}, t_{x}^{-1}, r_{z}^{2} t_{y}, r_{z}^{2} t_{y}^{-1}, r_{x}^{-1} t_{y}^{-1}$,

$r_{z}^{2} r_{x} \quad t_{x}, r_{z}^{2} r_{x} t_{x}^{-1}, t_{x}^{-1}, m_{x} r_{x}^{-1} t_{y}, m_{y} t_{y}, r_{z}^{2} t_{y}^{-1}$,

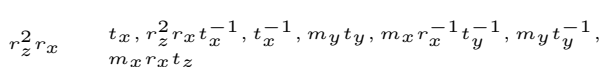

$r_{z}^{2} r_{x} \quad t_{x}, r_{z}^{2} r_{x} t_{x}^{-1}, t_{x}^{-1}, r_{x}^{2} t_{y}, i t_{y}^{-1}, m_{z} r_{x} t_{y}^{-1}$,

$r_{z}^{2} r_{x} \quad t_{x}, r_{z}^{2} r_{x} t_{x}^{-1}, t_{x}^{-1}, i t_{y}, i t_{y}^{-1}, m_{z} r_{x} t_{y}^{-1}$,

$r_{z}^{2} r_{x} \quad t_{x}, r_{z}^{2} r_{x} t_{x}^{-1}, t_{x}^{-1}, r_{y}^{2} r_{x} t_{y}, r_{x}^{2} t_{y}, i t_{y}^{-1}$, $r_{y}^{2} r_{x} t_{z}^{-1}$

$\begin{array}{ll}r_{z}^{2} r_{x} & \begin{array}{l}t_{x}, r_{z}^{2} r_{x} t_{x}^{-1}, t_{x}^{-1}, r_{x}^{2} t_{y}, r_{y}^{2} r_{x} t_{y}^{-1}, r_{x}^{2} t_{y}^{-1}, \\ r_{y}^{2} r_{x} t_{z}\end{array}\end{array}$

$r_{z}^{2} r_{x} \quad m_{z} r_{x}^{-1} t_{x}, m_{x} t_{x}^{-1}, m_{z} r_{x}^{-1} t_{x}^{-1}, m_{y} t_{y}, r_{z}^{2} t_{y}^{-1}, r_{x}^{-1} t_{y}^{-1}$,

$r_{z}^{2} r_{x} \quad m_{z} r_{x}^{-1} t_{x}, m_{x} t_{x}^{-1}, m_{z} r_{x}^{-1} t_{x}^{-1}, m_{y} t_{y}, m_{x} r_{x}^{-1} t_{y}^{-1}, m_{y} t_{y}^{-1}$, $m_{x} r_{x} t_{z}$

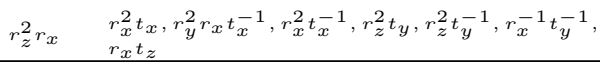

$r_{z}^{2} r_{x} \quad m_{z} r_{x}^{-1} t_{x}, m_{x} t_{x}^{-1}, m_{z} r_{x}^{-1} t_{x}^{-1}, m_{x} r_{x}^{-1} t_{y}, m_{y} t_{y}, r_{z}^{2} t_{y}^{-1}$, $r_{z}^{2} r_{x} r_{x} t_{z}^{-1}$

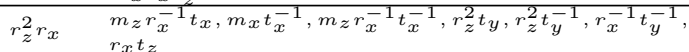

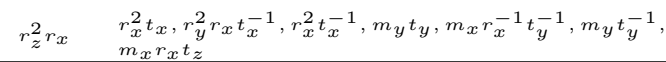
$r_{z}^{2} r_{x} \quad \begin{aligned} & r_{z}^{2} t_{x}, r_{x}^{-1} t_{x}, r_{x} t_{x}^{-1}, m_{y} t_{y}, m_{x} r_{x}^{-1} t_{y}^{-1}, m_{y} t_{y}^{-1}, \\ & m_{x} r_{x} t^{-1}\end{aligned}$

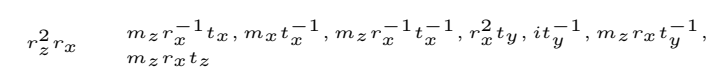
$\begin{array}{ll}r_{z}^{2} r_{x} & m_{z} r_{x} r_{x}^{-1} t_{x}, m_{x} t_{x}^{-1}, m_{z} r_{x}^{-1} t_{x}^{-1}, r_{x}^{2} t_{y}, r_{y}^{2} r_{x} t_{y}^{-1}, r_{x}^{2} t_{y}^{-1}, \\ & r_{y}^{2} r_{x} t_{z}\end{array}$

$r_{z}^{2} r_{x} \quad r_{z}^{2} t_{x}, r_{x}^{-1} t_{x}, r_{x} t_{x}^{-1}, m_{x} r_{x}^{-1} t_{y}^{-1}, m_{x} r_{x} t_{z}, m_{z} t_{z}$, $r_{z}^{2} r_{x} \quad \begin{aligned} & r_{x}^{2} t_{x}, r_{y}^{2} r_{x} t_{x}^{-1}, r_{x}^{2} t_{x}^{-1}, i t_{y}, i t_{y}^{-1}, m_{z} r_{x} t_{y}^{-1}, \\ & m_{z} r_{x} t_{z}\end{aligned}$ $\begin{array}{ll}r_{z}^{2} r_{x} & r_{x}^{2} t_{x}, r_{y}^{2} r_{x} t_{x}^{-1}, r_{x}^{2} t_{x}^{-1}, r_{x}^{2} t_{y}, r_{y}^{2} r_{x} t_{y}^{-1}, r_{x}^{2} t_{y}^{-1}, \\ r_{y}^{2} r_{x} t_{z}\end{array}$ $r_{z}^{2} r_{x} \quad r_{z}^{2} t_{x}, r_{x}^{-1} t_{x}, r_{x} t_{x}^{-1}, r_{x}^{2} t_{y}, r_{y}^{2} r_{x} t_{y}^{-1}, r_{x}^{2} t_{y}^{-1}$,

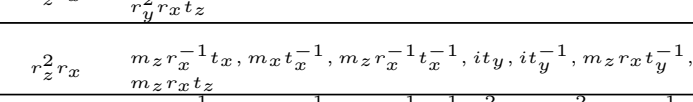
$r_{z}^{2} r_{x} \quad m_{z} r_{x}^{-1} t_{x}, m_{x} t_{x}^{-1}, m_{z} r_{x}^{-1} t_{x}^{-1}, r_{y}^{2} r_{x} t_{y}, r_{x}^{2} t_{y}, i t_{y}^{-1}$,

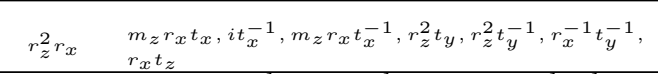

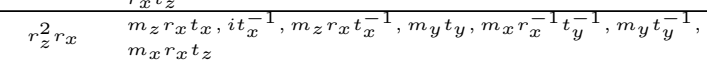
$r_{z}^{2} r_{x} \quad r_{z}^{2} t_{x}, r_{x}^{-1} t_{x}, r_{x} t_{x}^{-1}, r_{y}^{2} r_{x} t_{y}^{-1}, r_{y}^{2} r_{x} t_{z}, r_{x}^{2} t_{z}$, 


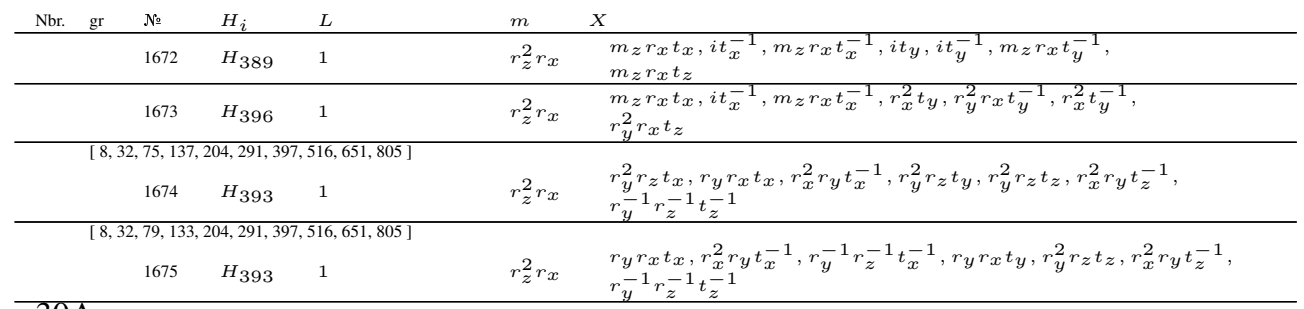

$30 \mathrm{~A}$

$[8,26,59,109,170,245,339,442,559,697]$ $1676^{*}$,

$[8,27,61,112,178,257,354,467,591,732]$ $1677^{*}$

$[8,27,62,113,178,258,354,465,589,729]$ $1678^{*}$,

$[8,28,63,115,183,264,361,474,601,742]$ $1679 *$

$[8,28,64,114,178,258,352,460,584,722]$ $1680 *$

$[8,29,65,116,183,264,361,473,600,742]$ $1681^{*}$,

$[8,29,65,117,186,269,368,483,613,758]$ $1682 *$,

$[8,30,66,118,185,268,365,478,606,750]$ $1683^{*}$,

$[9,28,64,116,176,256,352,452,576,716]$
$1676 H_{650}\left\langle m_{x}\right\rangle$

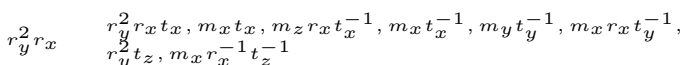

$[9,29,65,118,183,263,363,474,598,744]$

$1677 \quad H_{580} \quad 1$

$m_{z} r_{x} \quad i t_{x}, r_{z}^{2} r_{x} t_{x}, r_{y}^{2} r_{x} t_{x}^{-1}, m_{x} t_{x}^{-1}, m_{y} t_{y}^{-1}, r_{x} t_{y}^{-1}$

$[9,29,66,118,183,265,362,473,598,741]$

$1678 \quad H_{422} \quad 1$

$0,66,120,187,268,368,481,608,751]$

$1679 \quad H_{652} \quad\left\langle m_{x}\right\rangle$

$r_{z}^{2} t_{x}, r_{x}^{-1} t_{x}, r_{y}^{2} t_{x}^{-1}, r_{x} t_{x}^{-1}, m_{x} r_{x}^{-1} t_{y}^{-1}, m_{y} t_{y}^{-1}$, $m_{x} r_{x} t_{z}, m_{z} t_{z}^{-1}$

$30,68,118,184,266,360,470,596,734]$

$1680 \quad H_{648} \quad\left\langle m_{x}\right\rangle$

$i t_{x}, r_{z}^{2} r_{x} t_{x}, r_{x}^{2} t_{x}^{-1}, r_{z}^{2} r_{x} t_{x}^{-1}, m_{y} t_{y}^{-1}, m_{x} r_{x} t_{y}^{-1}$ $r_{y}^{2} t_{z}, m_{x} r_{x}^{-1} t_{z}^{-1}$

$68,120,188,269,368,480,608,751$

$1681 \quad H_{560} \quad 1$

$r_{y}^{2} r_{x} t_{x}, m_{x} t_{x}, m_{z} r_{x} t_{x}^{-1}, m_{x} t_{x}^{-1}, r_{x}^{2} t_{y}^{-1}, r_{z}^{2} r_{x} t_{y}^{-1}$,

\begin{tabular}{c}
\hline $9,31,68,120,188,269,368,480,608,751]$ \\
$1681 \quad H_{5} 601$ \\
{$[9,31,68,121,190,272,373,488,618,764]$} \\
$1682 \quad H_{409} 1$ \\
\hline$[9,32,68,122,189,273,370,485,613,758]$ \\
$1683 \quad H_{649}\left\langle m_{x}\right\rangle$ \\
30B $\quad[8,27,69,129,200,288,392,512,648,800]$ \\
$\quad 1684^{*}$, \\
{$[8,28,69,129,200,288,392,512,648,800]$} \\
$1685^{*}$, \\
{$[8,28,70,128,200,288,392,512,648,800]$} \\
$1686^{*}$, \\
{$[8,28,73,135,206,292,396,516,652,804]$} \\
$1687^{*}$, \\
{$[8,29,69,129,200,288,392,512,648,800]$} \\
$1688^{*}$, \\
{$[8,29,72,135,206,292,396,516,652,804]$} \\
$1689^{*}, 1690^{*}$, \\
{$[8,30,71,135,206,292,396,516,652,804]$} \\
$1691^{*}$, \\
{$[9,29,75,130,202,290,394,514,650,802]$}
\end{tabular}

$[9,29,75,130,202,290,394,514,650,802]$

$$
1684 \quad H_{650} \quad\left\langle m_{x}\right\rangle
$$

$[9,30,74,131,201,291,393,515,649,803]$

$$
1685 \quad H_{580} \quad 1
$$

$r_{y}^{2} r_{x} \quad r_{y}^{2} r_{x} t_{x}, m_{x} t_{x}, m_{z} r_{x} t_{x}^{-1}, m_{x} t_{x}^{-1}, m_{y} t_{y}, m_{y} t_{y}^{-1}$,

$0,75,129,203,289,395,513,651,801]$

$$
1686 \quad H_{422} \quad 1
$$

$m_{z r_{x}} \quad i t_{x}, r_{z}^{2} r_{x} t_{x}, r_{y}^{2} r_{x} t_{x}^{-1}, m_{x} t_{x}^{-1}, m_{y} t_{y}, m_{y} t_{y}^{-1}$, $r_{x} t_{y}^{-1}, r_{x}^{-1} t_{z}^{-1}$

, 79, 132, 206, 290, 398, 514, 654, 802 ]

$1687 \quad H_{648} \quad\left\langle m_{x}\right\rangle$

$r_{z}^{2} t_{x}, r_{x}^{-1} t_{x}, r_{y}^{2} t_{x}^{-1}, r_{x} t_{x}^{-1}, m_{y} t_{y}, m_{x} r_{x}^{-1} t_{y}^{-1}$, $m_{y} t_{y}^{-1}, m_{x} r_{x} t_{z}$

$r_{y}^{2} r_{x} \quad r_{y}^{2} r_{x} t_{x}, m_{x} t_{x}, m_{z} r_{x} t_{x}^{-1}, m_{x} t_{x}^{-1}, r_{x}^{2} t_{y}, r_{x}^{2} t_{y}^{-1}$ 


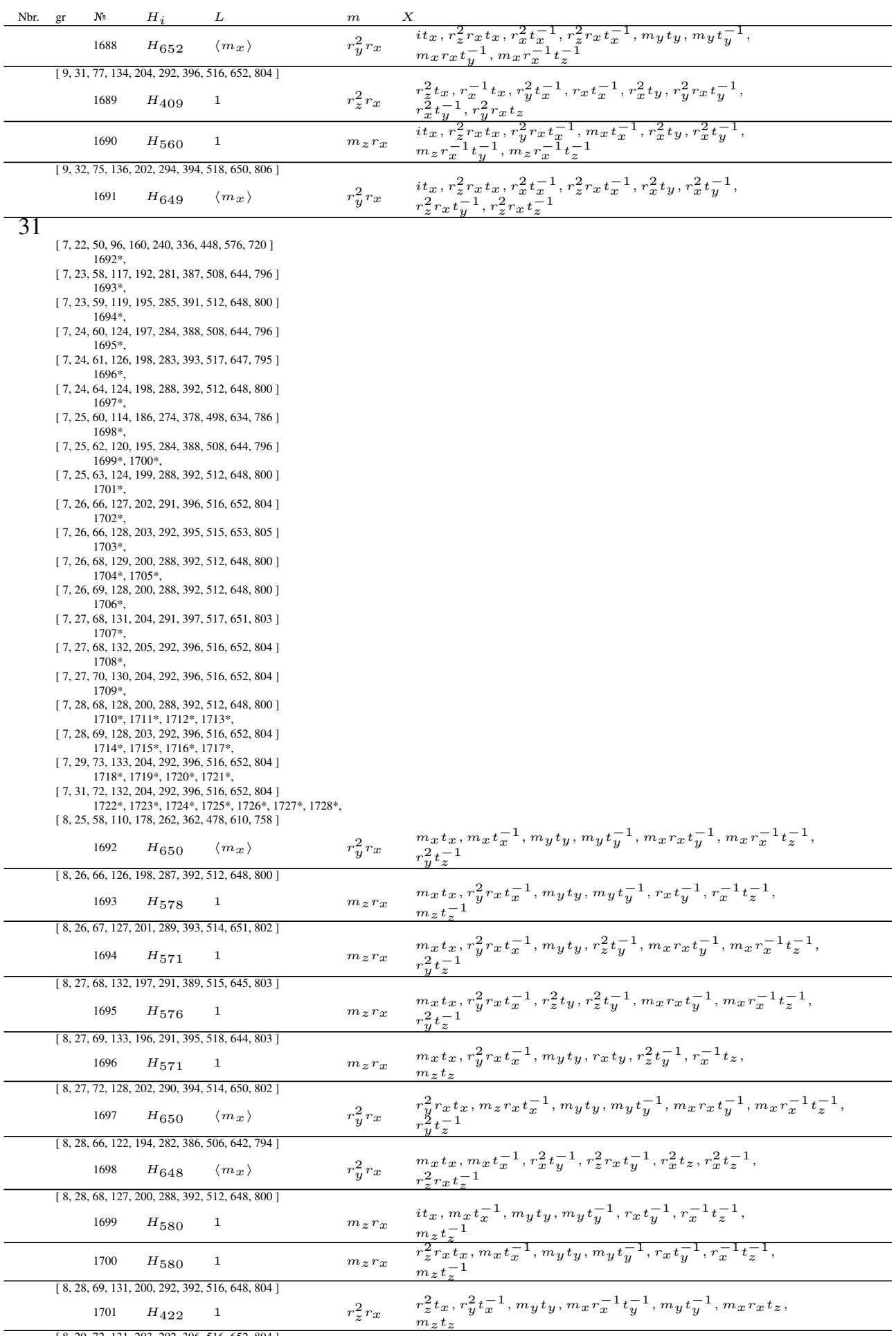

$[8,29,72,131,203,292,396,516,652,804]$ 


\begin{tabular}{|c|c|c|c|c|c|}
\hline Nbr. & № & $H_{i}$ & $L$ & $m$ & $x$ \\
\hline & 1702 & $H_{557}$ & 1 & $m_{z} r_{x}$ & $\begin{array}{l}m_{x} t_{x}, r_{y}^{2} r_{x} t_{x}^{-1}, r_{x}^{2} t_{y}^{-1}, m_{z} r_{x}^{-1} t_{y}^{-1}, r_{x}^{2} t_{z}, r_{x}^{2} t_{z}^{-1} \\
m_{z} r_{x}^{-1} t_{z}^{-1}\end{array}$ \\
\hline \multicolumn{6}{|c|}{$[8,29,72,132,204,292,394,517,653,804]$} \\
\hline & 1703 & $H_{561}$ & 1 & $m_{z} r_{x}$ & $\begin{array}{l}m_{x} t_{x}, r_{y}^{2} r_{x} t_{x}^{-1}, i t_{y}^{-1}, r_{z}^{2} r_{x} t_{y}^{-1}, r_{x}^{2} t_{z}, i t_{z}^{-1} \\
r_{z}^{2} r_{x} t_{z}^{-1}\end{array}$ \\
\hline \multicolumn{6}{|c|}{$[8,29,74,131,201,291,393,515,649,803]$} \\
\hline & 1704 & $H_{580}$ & 1 & $m_{z} r_{x}$ & $\begin{array}{l}i t_{x}, r_{y}^{2} r_{x} t_{x}^{-1}, m_{y}{ }^{t}, m_{y} t_{y}^{-1}, r_{x} t_{y}^{-1}, r_{x}^{-1} t_{z}^{-1} \\
m_{z} t_{z}^{-1}\end{array}$ \\
\hline & 1705 & $H_{580}$ & 1 & $m_{z} r_{x}$ & $\begin{array}{l}r_{z}^{2} r_{x} t_{x}, r_{y}^{2} r_{x} t_{x}^{-1}, m_{y} t_{y}, m_{y} t_{y}^{-1}, r_{x} t_{y}^{-1}, r_{x}^{-1} t_{z}^{-1}, \\
m_{z} t_{z}^{-1}\end{array}$ \\
\hline \multicolumn{6}{|c|}{$[8,29,75,129,203,289,395,513,651,801]$} \\
\hline & 1706 & $H_{422}$ & 1 & $r_{z}^{2} r_{x}$ & $\begin{array}{l}r_{x}^{-1} t_{x}, r_{x} t_{x}^{-1}, m_{y} t_{y}, m_{x} r_{x}^{-1} t_{y}^{-1}, m_{y} t_{y}^{-1}, m_{x} r_{x} t_{z} \\
m_{z} t_{z}\end{array}$ \\
\hline \multicolumn{6}{|c|}{$[8,30,74,133,202,293,397,516,650,805]$} \\
\hline & 1707 & $H_{561}$ & 1 & $m_{z} r_{x}$ & $\begin{array}{l}m_{x} t_{x}, r_{y}^{2} r_{x} t_{x}^{-1}, r_{x}^{2} t_{y}, m_{z} r_{x}^{-1} t_{y}, i t_{y}^{-1}, r_{x}^{2} t_{z} \\
m_{z} r_{x}^{-1} t_{z}\end{array}$ \\
\hline \multicolumn{6}{|c|}{$[8,30,74,134,203,293,395,517,651,805]$} \\
\hline & 1708 & $H_{556}$ & 1 & $m_{z} r_{x}$ & $\begin{array}{l}m_{x} t_{x}, r_{y}^{2} r_{x} t_{x}^{-1}, i t_{y}^{-1}, r_{z}^{2} r_{x} t_{y}^{-1}, i t_{z}, i t_{z}^{-1} \\
r_{z}^{2} r_{x} t_{z}^{-1}\end{array}$ \\
\hline \multicolumn{6}{|c|}{$[8,30,76,130,206,290,398,514,654,802]$} \\
\hline & 1709 & $H_{648}$ & $\left\langle m_{x}\right\rangle$ & $r_{y}^{2} r_{x}$ & $\begin{array}{l}r_{y}^{2} r_{x} t_{x}, m_{z} r_{x} t_{x}^{-1}, r_{x}^{2} t_{y}^{-1}, r_{z}^{2} r_{x} t_{y}^{-1}, r_{x}^{2} t_{z}, r_{x}^{2} t_{z}^{-1} \\
r_{z}^{2} r_{x} t_{z}^{-1}\end{array}$ \\
\hline \multicolumn{6}{|c|}{$[8,31,72,132,200,292,392,516,648,804]$} \\
\hline & 1710 & $H_{652}$ & $\left\langle m_{x}\right\rangle$ & $r_{y}^{2} r_{x}$ & $\begin{array}{l}i t_{x}, r_{x}^{2} t_{x}^{-1}, m_{y} t_{y}, m_{y} t_{y}^{-1}, m_{x} r_{x} t_{y}^{-1}, m_{x} r_{x}^{-1} t_{z}^{-1} \\
r_{y}^{2} t_{z}^{-1}\end{array}$ \\
\hline & 1711 & $H_{652}$ & $\left\langle m_{x}\right\rangle$ & $r_{y}^{2} r_{x}$ & $\begin{array}{l}r_{z}^{2} r_{x} t_{x}, r_{z}^{2} r_{x} t_{x}^{-1}, m_{y} t_{y}, m_{y} t_{y}^{-1}, m_{x} r_{x} t_{y}^{-1}, m_{x} r_{x}^{-1} t_{z}^{-1}, \\
r_{y}^{2} t_{z}^{-1}\end{array}$ \\
\hline & 1712 & $H_{572}$ & 1 & $m_{z} r_{x}$ & $\begin{array}{l}r_{z}^{2} r_{x} t_{x}, i t_{x}^{-1}, r_{z}^{2} t_{y}, r_{z}^{2} t_{y}^{-1}, m_{x} r_{x} t_{y}^{-1}, m_{x} r_{x}^{-1} t_{z}^{-1} \\
r_{y}^{2} t_{z}^{-1}\end{array}$ \\
\hline & 1713 & $H_{574}$ & 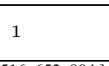 & $m_{z} r_{x}$ & $\begin{array}{l}r_{z}^{2} r_{x} t_{x}, i t_{x}^{-1}, m_{y} t_{y}, m_{y} t_{y}^{-1}, r_{x} t_{y}^{-1}, r_{x}^{-1} t_{z}^{-1} \\
m_{z} t_{z}^{-1}\end{array}$ \\
\hline \multicolumn{6}{|c|}{$[8,31,73,131,204,292,396,516,652,804]$} \\
\hline & 1714 & $H_{409}$ & 1 & $r_{z}^{2} r_{x}$ & $\begin{array}{l}r_{z}^{2} t_{x}, r_{y}^{2} t_{x}^{-1}, r_{y}^{2} r_{x} t_{y}^{-1}, r_{x}^{2} t_{y}^{-1}, r_{y}^{2} r_{x} t_{z}, r_{x}^{2} t_{z} \\
r_{x}^{2} t_{z}^{-1}\end{array}$ \\
\hline & 1715 & $H_{560}$ & 1 & $m_{z} r_{x}$ & $\begin{array}{l}\text { it } x, m_{x} t_{x}^{-1}, r_{x}^{2} t_{y}^{-1}, m_{z} r_{x}^{-1} t_{y}^{-1}, r_{x}^{2} t_{z}, r_{x}^{2} t_{z}^{-1} \\
m_{z} r_{x}^{-1} t_{z}^{-1}\end{array}$ \\
\hline & 1716 & $H_{560}$ & 1 & $m_{z} r_{x}$ & $\begin{array}{l}r_{z}^{2} r_{x} t_{x}, m_{x} t_{x}^{-1}, r_{x}^{2} t_{y}^{-1}, m_{z} r_{x}^{-1} t_{y}^{-1}, r_{x}^{2} t_{z}, r_{x}^{2} t_{z}^{-1} \\
m_{z} r_{x}^{-1} t_{z}^{-1}\end{array}$ \\
\hline & 1717 & $H_{560}$ & 1 & $m_{z} r_{x}$ & $\begin{array}{l}r_{z}^{2} r_{x} t_{x}, m_{x} t_{x}^{-1}, r_{x}^{2} t_{y}, m_{z} r_{x}^{-1} t_{y}, r_{x}^{2} t_{y}^{-1}, r_{x}^{2} t_{z} \\
m_{z} r_{x}^{-1} t_{z}\end{array}$ \\
\hline \multicolumn{6}{|c|}{$[8,32,77,132,204,292,396,516,652,804]$} \\
\hline & 1718 & $H_{409}$ & 1 & $r_{z}^{2} r_{x}$ & $\begin{array}{l}r_{x} t_{x}, r_{x} t_{x}, r_{y}^{2} r_{x} t_{y}, r_{x}^{2} y, r_{y}^{2} r_{x} t_{z}, r_{x}^{2} t_{z} \\
r_{x}^{2} t_{z}^{-1}\end{array}$ \\
\hline & 1719 & $H_{560}$ & 1 & $m_{z} r_{x}$ & $\begin{array}{l}i t_{x}, r_{y}^{2} r_{x} t_{x}^{-1}, r_{x}^{2} t_{y}^{-1}, m_{z} r_{x}^{-1} t_{y}^{-1}, r_{x}^{2} t_{z}, r_{x}^{2} t_{z}^{-1} \\
m_{z} r_{x}^{-1} t_{z}^{-1}\end{array}$ \\
\hline & 1720 & $H_{560}$ & 1 & $m_{z} r_{x}$ & $\begin{array}{l}r_{z}^{2} r_{x} t_{x}, r_{y}^{2} r_{x} t_{x}^{-1}, r_{x}^{2} t_{y}^{-1}, m_{z} r_{x}^{-1} t_{y}^{-1}, r_{x}^{2} t_{z}, r_{x}^{2} t_{z}^{-1}, \\
m_{z} r_{x}^{-1} t_{z}^{-1}\end{array}$ \\
\hline & 1721 & $H_{560}$ & 1 & $m_{z} r_{x}$ & $\begin{array}{l}r_{z}^{2} r_{x} t_{x}, r_{y}^{2} r_{x} t_{x}^{-1}, r_{x}^{2} t_{y}, m_{z} r_{x}^{-1} t_{y}, r_{x}^{2} t_{y}^{-1}, r_{x}^{2} t_{z} \\
m_{z} r_{x}^{-1} t_{z}\end{array}$ \\
\hline \multicolumn{6}{|c|}{$[8,34,74,134,202,294,394,518,650,806]$} \\
\hline & 1722 & $H_{649}$ & $\left\langle m_{x}\right\rangle$ & $r_{y}^{2} r_{x}$ & $\begin{array}{l}t_{x}, r_{x}^{2} t_{x}, r_{x}^{2} t_{y}, r_{z}^{z} r_{x} t_{y}, r_{x}^{t} t^{2}, r_{x}^{2} t_{z} \\
r_{z}^{2} r_{x} t_{z}\end{array}$ \\
\hline & 1723 & $H_{649}$ & $\left\langle m_{x}\right\rangle$ & $r_{y}^{2} r_{x}$ & $\begin{array}{l}r_{z}^{2} r_{x} t_{x}, r_{z}^{2} r_{x} t_{x}^{-1}, r_{x}^{2} t_{y}^{-1}, r_{z}^{2} r_{x} t_{y}^{-1}, r_{x}^{2} t_{z}, r_{x}^{2} t_{z}^{-1} \\
r_{z}^{2} r_{x} t_{z}^{-1}\end{array}$ \\
\hline & 1724 & $H_{649}$ & $\left\langle m_{x}\right\rangle$ & $r_{y}^{2} r_{x}$ & $\begin{array}{l}i t_{x}, r_{x}^{2} t_{x}^{-1}, r_{x}^{2} t_{y}^{-1}, r_{z}^{2} r_{x} t_{y}^{-1}, r_{x}^{2} t_{z}, r_{x}^{2} t_{z}^{-1} \\
r_{z}^{2} r_{x} t_{z}^{-1}\end{array}$ \\
\hline & 1725 & $H_{551}$ & 1 & $m_{z} r_{x}$ & $\begin{array}{l}r_{z}^{2} r_{x} t_{x}, i t_{x}^{-1}, i t_{y}^{-1}, r_{z}^{2} r_{x} t_{y}^{-1}, i t_{z}, i t_{z}^{-1} \\
r_{z}^{2} r_{x} t_{z}^{-1}\end{array}$ \\
\hline & 1726 & $H_{551}$ & 1 & $m_{z} r_{x}$ & $\begin{array}{l}r_{z}^{2} r_{x} t_{x}, i t_{x}^{-1}, i t_{y}, r_{z}^{2} r_{x} t_{y}, i t_{y}^{-1}, i t_{z} \\
r_{z}^{2} r_{x} t_{z}\end{array}$ \\
\hline & 1727 & $H_{555}$ & 1 & $m_{z} r_{x}$ & $\begin{array}{l}r_{z}^{2} r_{x} t_{x}, i t_{x}^{-1}, r_{x}^{2} t_{y}^{-1}, m_{z} r_{x}^{-1} t_{y}^{-1}, r_{x}^{2} t_{z}, r_{x}^{2} t_{z}^{-1} \\
m_{z} r_{x}^{-1} t_{z}^{-1}\end{array}$ \\
\hline & 1728 & $H_{555}$ & 1 & $m_{z} r_{x}$ & $\begin{array}{l}i t_{x}, r_{z}^{2} r_{x} t_{x}^{-1}, r_{x}^{2} t_{y}, m_{z} r_{x}^{-1} t_{y}, r_{x}^{2} t_{y}^{-1}, r_{x}^{2} t_{z} \\
m_{z} r_{x}^{-1} t_{z}\end{array}$ \\
\hline
\end{tabular}

$[8,26,62,121,198,288,392,512,648,800$ ] $1730^{*}$,

$[8,26,62,124,196,284,388,508,644,796]$ $1731^{*}$

$[8,26,63,126,197,283,393,517,647,795]$ $1732 *$

$[8,27,63,120,195,284,388,508,644,796]$ 


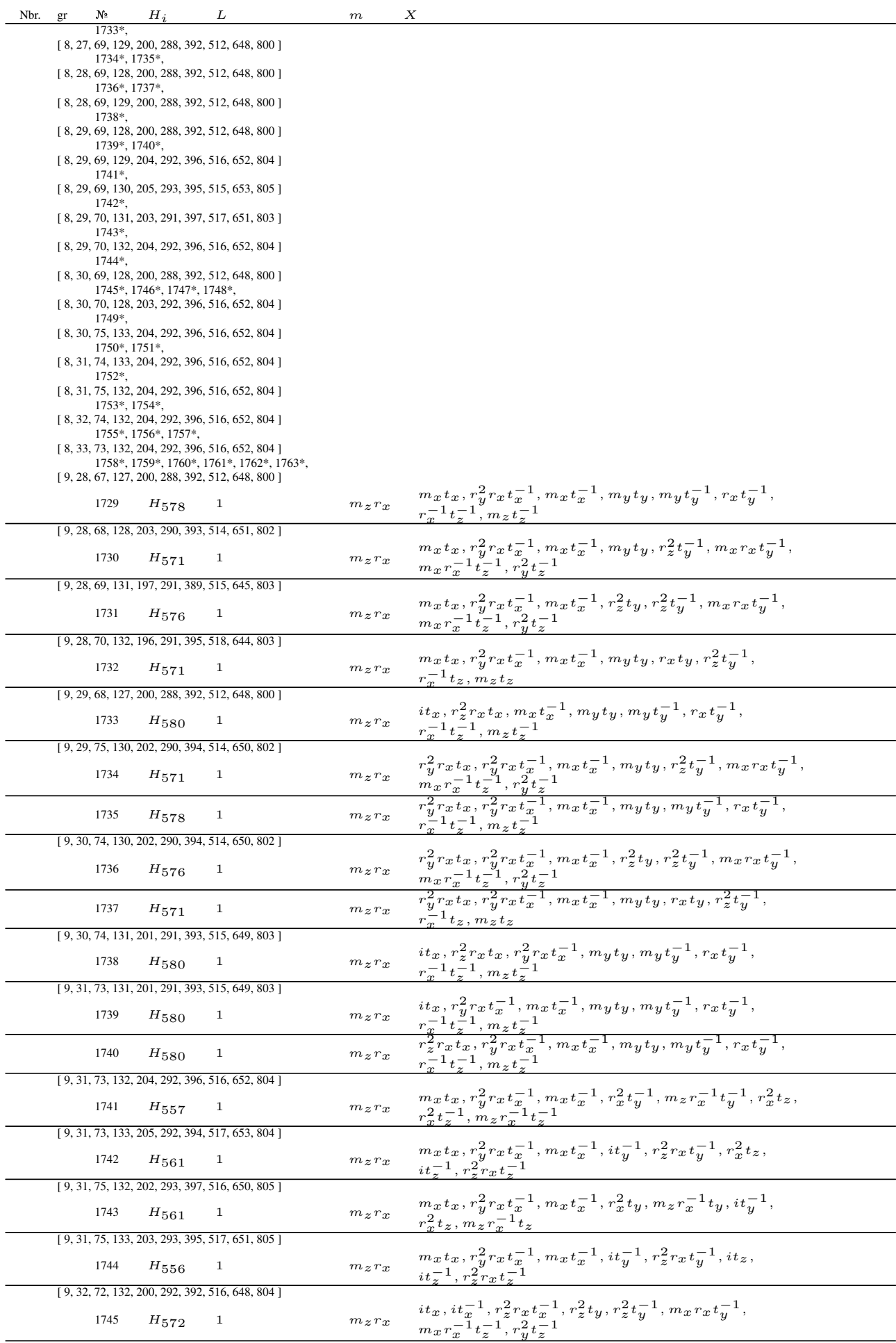




\begin{tabular}{|c|c|c|c|c|c|c|}
\hline Nbr. & gr & № & $H_{i}$ & $L$ & $m$ & $\mathrm{X}$ \\
\hline & & 1746 & $H_{572}$ & 1 & $m_{z} r_{x}$ & $\begin{array}{l}r_{z}^{2} r_{x} t_{x}, i t_{x}^{-1}, r_{z}^{2} r_{x} t_{x}^{-1}, r_{z}^{2} t_{y}, r_{z}^{2} t_{y}^{-1}, m_{x} r_{x} t_{y}^{-1} \\
m_{x} r_{x}^{-1} t_{z}^{-1}, r_{y}^{2} t_{z}^{-1}\end{array}$ \\
\hline & & 1747 & $H_{574}$ & 1 & $m_{z} r_{x}$ & $\begin{array}{l}i t_{x}, i t_{x}^{-1}, r_{z}^{2} r_{x} t_{x}^{-1}, m_{y} t_{y}, m_{y} t_{y}^{-1}, r_{x} t_{y}^{-1} \\
r_{x}^{-1} t_{z}^{-1}, m_{z} t_{z}^{-1}\end{array}$ \\
\hline & & 1748 & $H_{574}$ & 1 & $m_{z} r_{x}$ & $\begin{array}{l}r_{z}^{2} r_{x} t_{x}, i t_{x}^{-1}, r_{z}^{2} r_{x} t_{x}^{-1}, m_{y} t_{y}, m_{y} t_{y}^{-1}, r_{x} t_{y}^{-1} \\
r_{x}^{-1} t_{z}^{-1}, m_{z} t_{z}^{-1}\end{array}$ \\
\hline & \multicolumn{6}{|c|}{$[9,32,73,131,204,292,396,516,652,804]$} \\
\hline & & 1749 & $H_{560}$ & 1 & $m_{z} r_{x}$ & $\begin{array}{l}i t_{x}, r_{z}^{2} r_{x} t_{x}, m_{x} t_{x}^{-1}, r_{x}^{2} t_{y}^{-1}, m_{z} r_{x}^{-1} t_{y}^{-1}, r_{x}^{2} t_{z} \\
r_{x}^{2} t_{z}^{-1}, m_{z} r_{x}^{-1} t_{z}^{-1}\end{array}$ \\
\hline & \multicolumn{6}{|c|}{$[9,32,79,130,206,290,398,514,654,802]$} \\
\hline & & 1750 & $H_{561}$ & 1 & $m_{z} r_{x}$ & $\begin{array}{l}r_{y}^{2} r_{x} t_{x}, r_{y}^{2} r_{x} t_{x}^{-1}, m_{x} t_{x}^{-1}, i t_{y}^{-1}, r_{z}^{2} r_{x} t_{y}^{-1}, r_{x}^{2} t_{z} \\
i t_{z}^{-1}, r_{z}^{2} r_{x} t_{z}^{-1}\end{array}$ \\
\hline & & 1751 & $H_{557}$ & 5565090 & $m_{z} r_{x}$ & $\begin{array}{l}r_{y}^{2} r_{x} t_{x}, r_{y}^{2} r_{x} t_{x}^{-1}, m_{x} t_{x}^{-1}, r_{x}^{2} t_{y}^{-1}, m_{z} r_{x}^{-1} t_{y}^{-1}, r_{x}^{2} t_{z}, \\
r_{x}^{2} t_{z}^{-1}, m_{z} r_{x}^{-1} t_{z}^{-1}\end{array}$ \\
\hline & \multicolumn{6}{|c|}{$[9,33,77,132,204,292,396,516,652,804]$} \\
\hline & & 1752 & $H_{560}$ & 1 & $m_{z} r_{x}$ & $\begin{array}{l}i t_{x}, r_{z}^{2} r_{x} t_{x}, r_{y}^{2} r_{x} t_{x}^{-1}, r_{x}^{2} t_{y}^{-1}, m_{z} r_{x}^{-1} t_{y}^{-1}, r_{x}^{2} t_{z}, \\
r_{x}^{2} t_{z}^{-1}, m_{z} r_{x}^{-1} t_{z}^{-1}\end{array}$ \\
\hline & \multicolumn{6}{|c|}{$[9,33,78,130,206,290,398,514,654,802]$} \\
\hline & & 1753 & $H_{556}$ & 1 & $m_{z} r_{x}$ & $\begin{array}{l}r_{y}^{2} r_{x} t_{x}, r_{y}^{2} r_{x} t_{x}^{-1}, m_{x} t_{x}^{-1}, i t_{y}^{-1}, r_{z}^{2} r_{x} t_{y}^{-1}, i t_{z} \\
i t_{z}^{-1}, r_{z}^{2} r_{x} t_{z}^{-1}\end{array}$ \\
\hline & & 1754 & $H_{561}$ & $516-652804$ & $m_{z} r_{x}$ & $\begin{array}{l}r_{y}^{2} r_{x} t_{x}, r_{y}^{2} r_{x} t_{x}^{-1}, m_{x} t_{x}^{-1}, r_{x}^{2} t_{y}, m_{z} r_{x}^{-1} t_{y}, i t_{y}^{-1}, \\
r_{x}^{2} t_{z}, m_{z} r_{x}^{-1} t_{z}\end{array}$ \\
\hline & \multicolumn{6}{|c|}{$[9,34,76,132,204,292,396,516,652,804]$} \\
\hline & & 1755 & $H_{560}$ & 1 & $m_{z} r_{x}$ & $\begin{array}{l}i t_{x}, r_{y}^{2} r_{x} t_{x}^{-1}, m_{x} t_{x}^{-1}, r_{x}^{2} t_{y}^{-1}, m_{z} r_{x}^{-1} t_{y}^{-1}, r_{x}^{2} t_{z} \\
r_{x}^{2} t_{z}^{-1}, m_{z} r_{x}^{-1} t_{z}^{-1}\end{array}$ \\
\hline & & 1756 & $H_{560}$ & 1 & $m_{z} r_{x}$ & $\begin{array}{l}r_{z}^{2} r_{x} t_{x}, r_{y}^{2} r_{x} t_{x}^{-1}, m_{x} t_{x}^{-1}, r_{x}^{2} t_{y}^{-1}, m_{z} r_{x}^{-1} t_{y}^{-1}, r_{x}^{2} t_{z}, \\
r_{x}^{2} t_{z}^{-1}, m_{z} r_{x}^{-1} t_{z}^{-1}\end{array}$ \\
\hline & & 1757 & $H_{560}$ & 55065806 & $m_{z} r_{x}$ & $\begin{array}{l}r_{z}^{2} r_{x} t_{x}, r_{y}^{2} r_{x} t_{x}^{-1}, m_{x} t_{x}^{-1}, r_{x}^{2} t_{y}, m_{z} r_{x}^{-1} t_{y}, r_{x}^{2} t_{y}^{-1} \\
r_{x}^{2} t_{z}, m_{z} r_{x}^{-1} t_{z}\end{array}$ \\
\hline & \multicolumn{6}{|c|}{$[9,35,74,134,202,294,394,518,650,806]$} \\
\hline & & 1758 & $H_{551}$ & 1 & $m_{z} r_{x}$ & $\begin{array}{l}i t_{x}, i t_{x}^{-1}, r_{z}^{2} r_{x} t_{x}^{-1}, i t_{y}^{-1}, r_{z}^{2} r_{x} t_{y}^{-1}, i t_{z} \\
i t_{z}^{-1}, r_{z}^{2} r_{x} t_{z}^{-1}\end{array}$ \\
\hline & & 1759 & $H_{551}$ & 1 & $m_{z} r_{x}$ & $\begin{array}{l}r_{z}^{2} r_{x} t_{x}, i t_{x}^{-1}, r_{z}^{2} r_{x} t_{x}^{-1}, i t_{y}^{-1}, r_{z}^{2} r_{x} t_{y}^{-1}, i t_{z} \\
i t_{z}^{-1}, r_{z}^{2} r_{x} t_{z}^{-1}\end{array}$ \\
\hline & & 1760 & $H_{551}$ & 1 & $m_{z} r_{x}$ & $\begin{array}{l}i t_{x}, r_{z}^{2} r_{x} t_{x}, r_{z}^{2} r_{x} t_{x}^{-1}, i t_{y}, r_{z}^{2} r_{x} t_{y}, i t_{y}^{-1} \\
i t_{z}, r_{z}^{2} r_{x} t_{z}\end{array}$ \\
\hline & & 1761 & $H_{555}$ & 1 & $m_{z} r_{x}$ & $\begin{array}{l}i t_{x}, i t_{x}^{-1}, r_{z}^{2} r_{x} t_{x}^{-1}, r_{x}^{2} t_{y}^{-1}, m_{z} r_{x}^{-1} t_{y}^{-1}, r_{x}^{2} t_{z} \\
r_{x}^{2} t_{z}^{-1}, m_{z} r_{x}^{-1} t_{z}^{-1}\end{array}$ \\
\hline & & 1762 & $H_{555}$ & 1 & $m_{z} r_{x}$ & $\begin{array}{l}r_{z}^{2} r_{x} t_{x}, i t_{x}^{-1}, r_{z}^{2} r_{x} t_{x}^{-1}, r_{x}^{2} t_{y}^{-1}, m_{z} r_{x}^{-1} t_{y}^{-1}, r_{x}^{2} t_{z}, \\
r_{x}^{2} t_{z}^{-1}, m_{z} r_{x}^{-1} t_{z}^{-1}\end{array}$ \\
\hline & & 1763 & $H_{555}$ & 1 & $m_{z} r_{x}$ & $\begin{array}{l}r_{z}^{2} r_{x} t_{x}, i t_{x}^{-1}, r_{z}^{2} r_{x} t_{x}^{-1}, r_{x}^{2} t_{y}, m_{z} r_{x}^{-1} t_{y}, r_{x}^{2} t_{y}^{-1} \\
r_{x}^{2} t_{z}, m_{z} r_{x}^{-1} t_{z}\end{array}$ \\
\hline
\end{tabular}

$[8,25,58,110,182,273,376,493,630,785]$ $1764^{*}$

$[8,25,59,113,184,272,376,496,632,784]$ $1766^{*}$,

$[8,25,59,115,190,281,384,503,639,792]$ $1765^{*}$,

$[8,25,60,118,192,282,385,505,641,793]$ $1767^{*}$

$[8,26,66,126,199,288,392,512,648,800]$ $1768^{*}, 1769^{*}$,

$[8,27,68,128,200,288,392,512,648,800]$ $1770^{*}, 1771^{*}$

$[8,27,69,128,200,288,392,512,648,800]$ 1772 *

$[8,28,66,123,196,284,387,507,644,796]$ $1774^{*}$,

$[8,28,66,124,196,284,388,508,644,796]$ $1776^{*}$,

$[8,28,67,123,195,283,387,507,643,795]$ $1773^{*}$,

$[8,28,67,124,195,283,388,508,643,795]$ $1775^{*}$,

$[8,28,69,127,200,288,392,512,648,800]$ $1777^{*}$,

$[8,29,68,128,200,288,392,512,648,800]$ $1778^{*}, 1779^{*}, 1780^{*}, 1781^{*}$,

$[8,29,72,131,204,292,396,516,652,804]$ $1782^{*}, 1783^{*}$,

$[8,30,73,133,204,292,396,516,652,804]$ $1784^{*}$,

$[8,30,74,132,204,292,396,516,652,804]$ $1785^{*}, 1786^{*}$

$[8,31,73,132,204,292,396,516,652,804]$ $1787^{*}$

$[8,32,72,132,204,292,396,516,652,804]$ $1788 *, 1789 *, 1790 *, 1791 *$

$[9,27,66,120,193,282,385,504,641,794]$ 


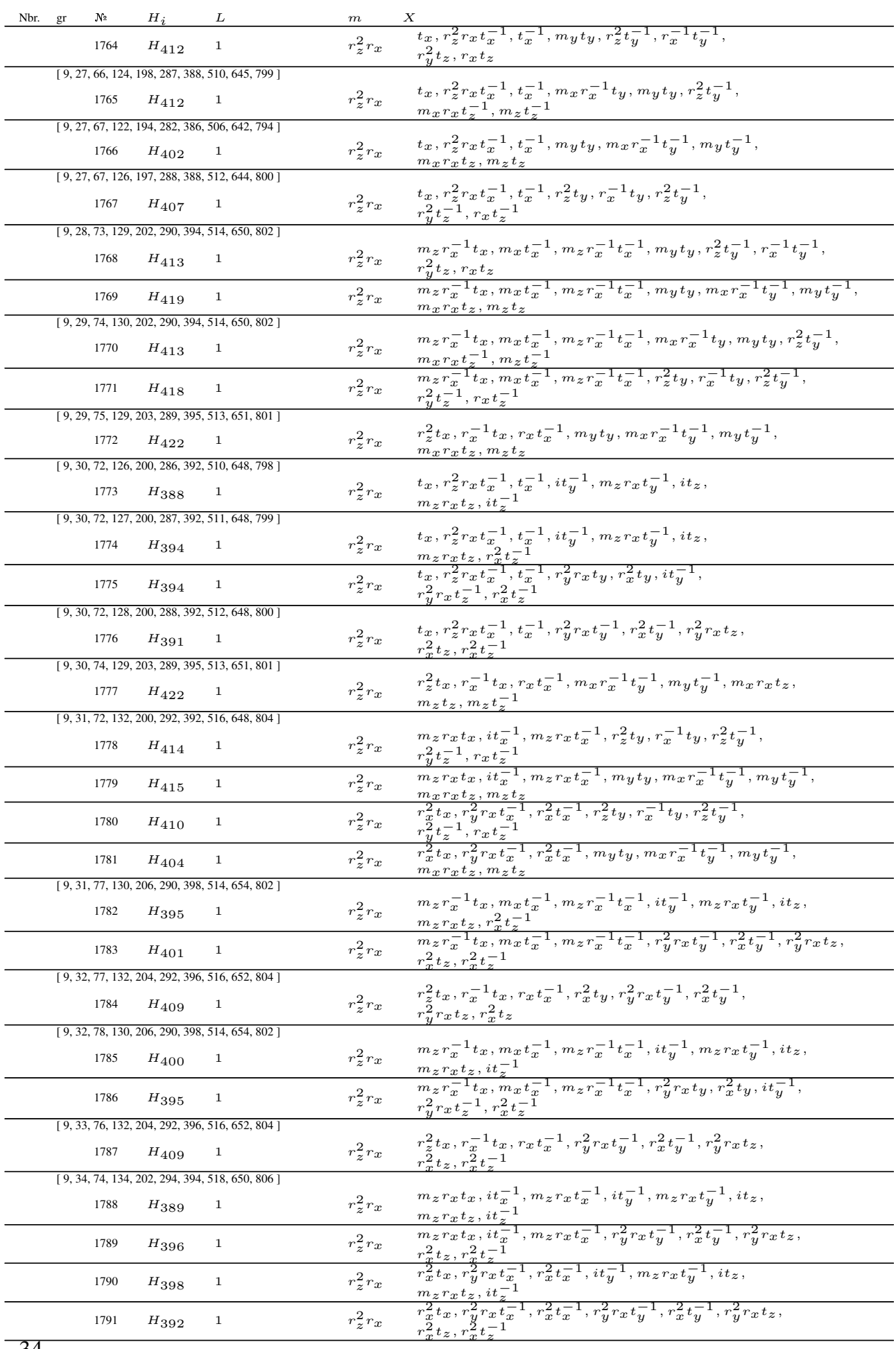




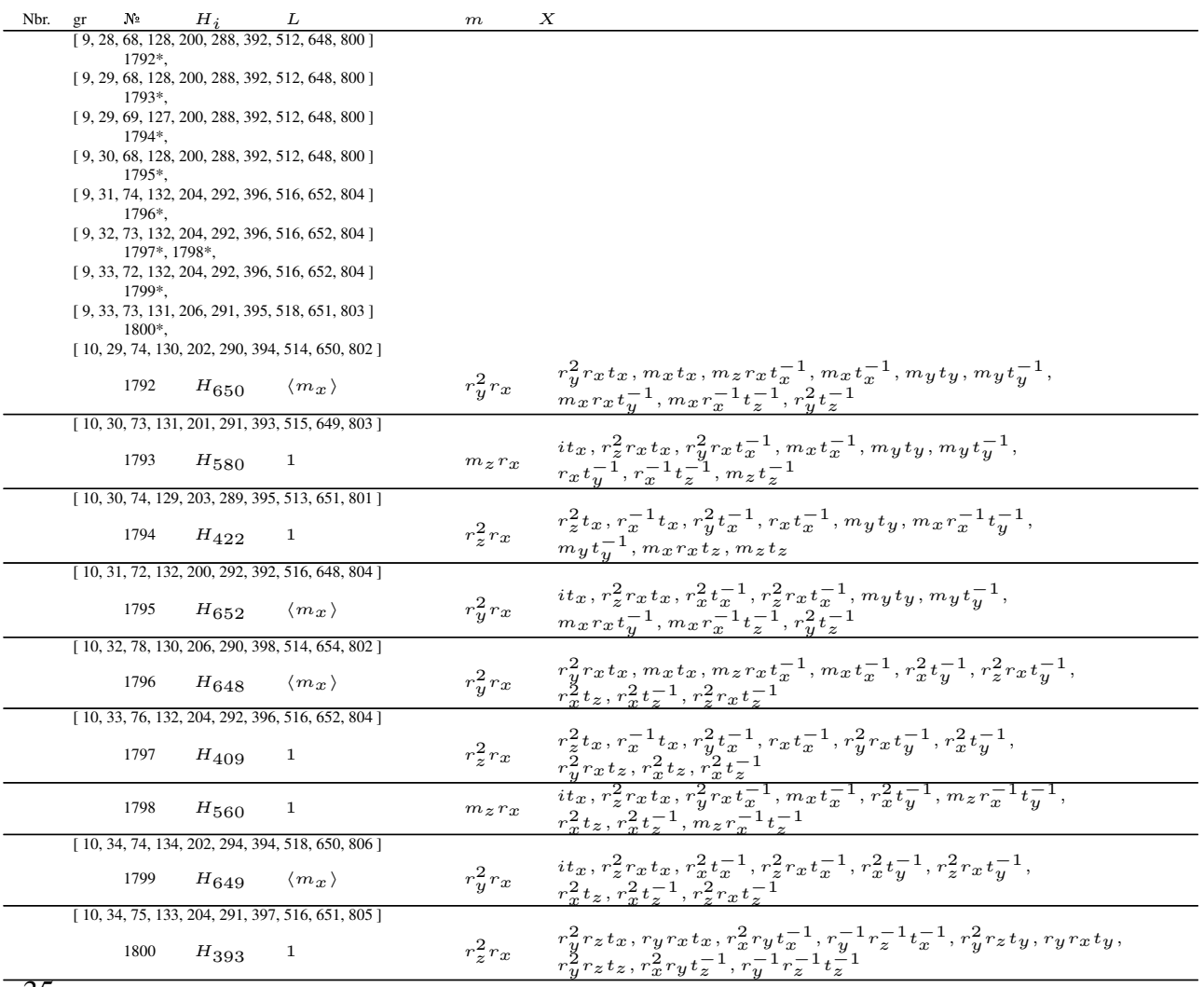

$[7,24,56,104,168,248,344,456,584,728]$ $1801 *$

$[7,25,60,112,180,264,364,480,612,760]$ $1802^{*}$,

$[7,25,60,113,184,273,379,500,636,788$ ] $1803^{*}$,

$[7,25,61,116,188,276,380,500,636,788]$ $1804^{*}$,

$[7,26,62,114,182,266,366,482,614,762]$ $1805^{*}$,

$[7,26,64,120,192,280,384,504,640,792]$ $1806^{*}, 1807^{*}$,

$[7,26,64,122,196,284,388,508,644,796]$ $1808^{*}$,

$[7,26,65,123,196,284,388,508,644,796$ ] $1809^{*}, 1810^{*}$

$[7,26,65,123,197,286,390,510,646,798]$ $1811^{*}$

[ $7,26,65,124,198,286,390,510,646,798$ ] $1812^{*}$,

[ 7, 26, 66, 124, 196, 284, 388, 508, 644, 796 ] $1813^{*}$,

$[7,26,66,126,198,284,388,508,644,796]$ $1814^{*}$,

$[7,27,67,124,196,284,388,508,644,796]$ $1815^{*}, 1816^{*}, 1817^{*}, 1818^{*}, 1819^{*}, 1820^{*}$,

$[7,27,67,125,199,289,395,516,652,804]$ $1821^{*}$

$[7,27,68,125,196,284,388,508,644,796]$ $1822^{*}, 1823^{*}$,

$[7,27,68,127,200,288,392,512,648,800]$ $1824^{*}$,

$[7,27,70,130,203,292,396,516,652,804]$ $1825^{*}$,

[ $7,28,68,124,196,284,388,508,644,796$ ] $1826^{*}, 1827^{*}, 1828^{*}, 1829^{*}, 1830^{*}, 1831^{*}, 1832^{*}, 1833^{*}$,

$[7,28,70,128,200,288,392,512,648,800]$ $1834^{*}, 1835^{*}, 1836^{*}$

[ $7,28,70,129,203,292,396,516,652,804$ ] $1837^{*}$,

$[7,28,70,130,204,292,396,516,652,804]$ $1838 *, 1839^{*}$ 


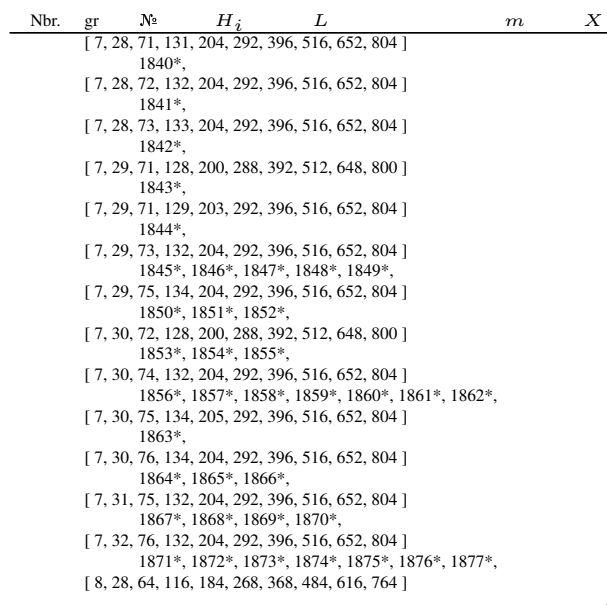

$[8,28,64,116,184,268,368,484,616,764]$
$1801 \quad H_{779} \quad\left\langle m_{z}, m_{z} r_{x}, r\right.$
$m_{z} r_{x}^{-1} t_{x}, m_{x} t_{x}^{-1}, m_{z} r_{x}^{-1} t_{x}^{-1}, r_{x}^{2} t_{y}, m_{z} r_{x}^{-1} t_{y}^{-1}, m_{z} r_{x}^{-1} t_{z}$,

$[8,29,67,121,191,277,379,497,631,781]$

\begin{tabular}{|c|c|c|c|c|}
\hline 1802 & $H_{452}$ & 1 & $r_{z}^{2}$ & $\begin{array}{l}t_{x}, r_{z}^{2} t_{x}^{-1}, t_{x}^{-1}, t_{y}, t_{y}^{-1}, r_{y}^{2} r_{z} t_{z}, \\
m_{z} t_{z}^{-1}\end{array}$ \\
\hline
\end{tabular}

$[8,29,67,122,194,283,388,508,644,796]$

$1803 \quad H_{503} \quad 1 \quad r_{z}^{2} \quad t_{x}, r_{z}^{2} t_{x}^{-1}, t_{x}^{-1}, t_{y}, t_{y}^{-1}, r_{y}^{2} r_{z} t_{z}$,

$[8,29,68,124,196,284,388,508,644,796]$

$1804 \quad H_{680} \quad\left\langle m_{x}\right\rangle \quad r_{z}^{2} \quad m_{x} t_{x}, m_{x} t_{x}^{-1}, m_{x} t_{y}, m_{y} t_{y}^{-1}, m_{x} t_{y}^{-1}, r_{y}^{2} t_{z}$,

$[8,30,68,122,192,278,380,498,632,782]$

$1805 \quad H_{611}\left\langle m_{z}\right\rangle \quad r_{z}^{2} \quad \begin{aligned} & t_{x}, r_{z}^{2} t_{x}^{-1}, t_{x}^{-1}, t_{y}, t_{y}^{-1}, r_{y}^{2} r_{z} t_{z}, \\ & m_{x} r_{z} t_{z}^{-1}\end{aligned}$

$[8,30,70,126,198,286,390,510,646,798]$

$r_{y}^{2} t_{x}, m_{z} t_{x}^{-1}, r_{z}^{2} t_{y}, r_{z}^{2} t_{y}^{-1}, r_{z}^{2} t_{z}, m_{z} t_{z}^{-1}$

$\begin{array}{lllll}1806 & H_{749} & \left\langle m_{y}, r_{z}^{2}\right\rangle & i & \begin{array}{l}r_{y}^{2} t_{x}, m_{z} t_{x}^{-1}, r_{z}^{2} t_{y}, r_{z}^{2} t_{y}^{-1}, r_{z}^{2} t_{z}, m_{z} t_{z}^{-1}, \\ r_{z}^{2} t_{z}^{-1}\end{array} \\ 1807 & H_{682}\left\langle m_{y}\right\rangle & m_{x} & \begin{array}{l}m_{z} t_{x}, r_{y}^{2} t_{x}^{-1}, m_{z} t_{x}^{-1}, m_{y} t_{y}, t_{y}^{-1}, m_{z} t_{z}, \\ r_{t}^{2}-1\end{array}\end{array}$

$[8,30,70,128,200,288,392,512,648,800]$ $1808 \quad H_{598} \quad\left\langle r_{y}^{2}\right\rangle \quad r_{z}^{2} \quad r_{y}^{2} t_{x}, r_{y}^{2} t_{x}^{-1}, r_{y}^{2} t_{y}, r_{x}^{2} t_{y}^{-1}, r_{y}^{2} t_{y}^{-1}, r_{x}^{2} r_{z} t_{z}$,

$[8,30,71,128,200,288,392,512,648,800]$

\begin{tabular}{|c|c|c|c|c|}
\hline 1809 & $H_{650}$ & $\left\langle m_{z} r_{x}\right\rangle$ & $m_{x}$ & $t_{x}, m_{x} t_{x}^{-1}, t_{x}^{-1}, m_{y} t_{y}, r_{z}^{2} t_{y}^{-1}, r_{x}^{-1} t_{z}$ \\
\hline 1810 & $H_{378}$ & 1 & $m z$ & $m_{x} t_{x}, r_{y}^{2} t_{x}^{-1}, m_{y} t_{y}, m_{y} t_{y}^{-1}, m_{x} r_{z} t_{z}, r_{y}^{2} r_{z} t_{z}^{-1}$, \\
\hline
\end{tabular}

$[8,30,71,128,201,289,393,513,649,801]$

$1811 \quad H_{503} \quad 1$

$r_{z}^{2} \quad{ }_{x}{ }_{r^{2}}, r_{z}^{2} t_{x}^{-1}, t_{x}^{-1}, t_{y}, t_{y}^{-1}, r_{y}^{2} r_{z} t_{z}$

$[8,30,71,129,201,289,393,513,649,801]$ $1812 \quad H_{452} \quad 1 \quad r_{z}^{2} \quad \begin{aligned} & t_{x}, r_{z}^{2} t_{x}^{-1}, t_{x}^{-1}, t_{y}, t_{y}^{-1}, r_{y}^{2} r_{z} t_{z}, \\ & i t^{-1}\end{aligned}$

$[8,30,72,128,200,288,392,512,648,800]$

$$
1813 \quad H_{648} \quad\left\langle m_{z} r_{x}\right\rangle
$$

$[8,30,72,130,200,288,392,512,648,800]$

$$
1814 \quad H_{541} \quad 1
$$

\begin{tabular}{|c|c|c|c|c|}
\hline 1815 & $H_{361}$ & 1 & $m_{z}$ & $\begin{array}{l}r_{z}^{2} t_{x}, i t_{x}^{-1}, r_{z}^{2} t_{y}, i t_{y}^{-1}, m_{x} t_{z}, r_{y}^{2} t_{z}^{-1} \\
m_{x} t_{z}^{-1}\end{array}$ \\
\hline 1816 & $H_{680}$ & $\left\langle m_{x}\right\rangle$ & $r_{z}^{2}$ & $\begin{array}{l}r_{z}^{2} t_{x}, m_{y} t_{x}^{-1}, m_{x} t_{y}, m_{y} t_{y}^{-1}, m_{x} t_{y}^{-1}, r_{y}^{2} t_{z}, \\
r_{x}^{2} t_{z}^{-1}\end{array}$ \\
\hline 1817 & $H_{368}$ & 1 & $m_{z}$ & $\begin{array}{l}m_{x} t_{x}, r_{y}^{2} t_{x}^{-1}, m_{y} t_{y}, r_{x}^{2} t_{y}^{-1}, m_{x} t_{z}, r_{y}^{2} t_{z}^{-1}, \\
m_{x} t_{z}^{-1}\end{array}$ \\
\hline 1818 & $H_{527}$ & 1 & $r_{z}^{2}$ & $\begin{array}{l}m_{x} t_{x}, m_{x} t_{x}^{-1}, m_{x} t_{y}, m_{x} t_{y}^{-1}, m_{y} t_{y}^{-1}, r_{y}^{2} r_{z} t_{z}, \\
m_{z} t_{z}^{-1}\end{array}$ \\
\hline 1819 & $H_{527}$ & 1 & $r_{z}^{2}$ & $\begin{array}{l}m_{y} t_{x}, m_{x} t_{x}^{-1}, m_{y} t_{x}^{-1}, m_{x} t_{y}, m_{x} t_{y}^{-1}, r_{y}^{2} r_{z} t_{z} \\
m_{z} t_{z}^{-1}\end{array}$ \\
\hline 1820 & $H_{529}$ & 1 & $r_{z}^{2}$ & $\begin{array}{l}m_{x} t_{x}, m_{x} t_{x}^{-1}, m_{x} t_{y}, m_{x} t_{y}^{-1}, m_{y} t_{y}^{-1}, r_{y}^{2} r_{z} t_{z}, \\
r_{y}^{2} t_{z}^{-1}\end{array}$ \\
\hline
\end{tabular}

$[8,31,72,128,200,288,392,512,648,800]$ 
$1821 \quad H_{529} \quad 1$

$[8,31,73,128,200,288,392,512,648,800]$

$\begin{array}{lll}1822 & H_{422} & 1 \\ 1823 & H_{409} & 1\end{array}$

$[8,31,73,130,202,290,394,514,650,802]$

$1824 \quad H_{359} \quad 1$

$[8,31,75,131,204,292,396,516,652,804]$

$\begin{array}{lll}1825 & H_{377} & 1\end{array}$

$[8,32,72,128,200,288,392,512,648,800]$

$\begin{array}{lll}1826 & H_{779} & m_{z} \\ & & \left\langle m_{z}, m_{z} r_{x}, r_{x}^{2}\right\rangle\end{array}$

$1828 \quad H_{622}\left\langle m_{z}\right\rangle \quad r_{z}^{2}\left\langle m_{y} t_{x}, m_{x} t_{x}^{-1}, m_{y} t_{x}^{-1}, m_{x} t_{y}, m_{x} t_{y}^{-1}, r_{y}^{2} r_{z} t_{z}\right.$,

$\begin{array}{lllll}1829 & H_{649} & \left\langle m_{z} r_{x}\right\rangle & m_{x} & m_{x} r_{z} t_{z}^{-1} \\ & & & m_{z}^{2} r_{x} t_{z}^{-1}\end{array}$

$1830 \quad H_{682} \quad\left\langle m_{y}\right\rangle \quad m_{x} \quad m_{z} t_{x}, r_{y}^{2} t_{x}^{-1}, m_{z} t_{x}^{-1}, r_{z}^{2} t_{y}, m_{x} t_{y}^{-1}, m_{z} t_{z}$

$r_{y}^{2} t_{z}$

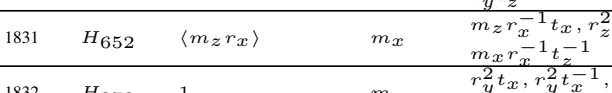

$\begin{array}{lllll}1832 & H_{378} & 1 & m_{z} & \begin{array}{l}r_{y}^{2} t_{x}, r_{y}^{2} t_{x}^{-1}, m_{y} t_{y}, r_{x}^{2} t_{y}^{-1}, m_{x} r_{z} t_{z}, r_{y}^{2} r_{z} t_{z}^{-1}, \\ 1833\end{array} H_{541} \quad 1\end{array}$

$[8,32,74,130,202,290,394,514,650,802]$

\begin{tabular}{|c|c|c|c|c|}
\hline 1834 & $H_{370}$ & 1 & $m_{z}$ & $\begin{array}{l}r_{z}^{2} t_{x}, m_{x} t_{x}^{-1}, m_{y} t_{y}, r_{x}^{2} t_{y}^{-1}, m_{y} t_{z}, r_{x}^{2} t_{z}^{-1} \\
m_{y} t_{z}^{-1}\end{array}$ \\
\hline 1835 & $H_{527}$ & 1 & $r_{z}^{2}$ & $\begin{array}{l}m_{x} t_{x}, m_{x} t_{x}^{-1}, m_{x} t_{y}, m_{x} t_{y}^{-1}, m_{y} t_{y}^{-1}, r_{y}^{2} r_{z} t_{z}, \\
i t_{z}^{-1}\end{array}$ \\
\hline 1836 & $H_{529}$ & 1 & $r_{z}^{2}$ & $\begin{array}{l}m_{y} t_{x}, m_{x} t_{x}^{-1}, m_{y} t_{x}^{-1}, m_{y} t_{y}, m_{y} t_{y}^{-1}, r_{y}^{2} r_{z} t_{z}, \\
r_{y}^{2} t_{z}^{-1}\end{array}$ \\
\hline
\end{tabular}

$[8,32,74,131,204,292,396,516,652,804]$

$1837 \quad H_{529} \quad 1$

$[8,32,74,132,204,292,396,516,652,804]$

$\begin{array}{lll}1838 & H_{604} & \left\langle r_{y}^{2}\right\rangle \\ 1839 & H_{527} & 1\end{array}$

$[8,32,75,132,204,292,396,516,652,804]$

$1840 \quad H_{359} \quad 1$

$[8,32,76,132,204,292,396,516,652,804]$

$$
1841 \quad H_{359} \quad 1
$$

$[8,32,77,132,204,292,396,516,652,804]$

$$
1842 \quad H_{382} \quad 1
$$

$[8,33,74,130,202,290,394,514,650,802]$

$$
1843 \quad H_{682} \quad\left\langle m_{x}\right\rangle
$$

$[8,33,74,131,204,292,396,516,652,804]$

\begin{tabular}{|c|c|c|c|c|}
\hline 1845 & $H_{370}$ & 1 & $m_{z}$ & $\begin{array}{l}i t_{x}, m_{x} t_{x}^{-1}, m_{y} t_{y}, r_{x}^{2} t_{y}^{-1}, m_{y} t_{z}, r_{x}^{2} t_{z}^{-1}, \\
m_{y} t_{z}^{-1}\end{array}$ \\
\hline 1846 & $H_{370}$ & 1 & $m_{z}$ & $\begin{array}{l}r_{z}^{2} t_{x}, r_{y}^{2} t_{x}^{-1}, m_{y} t_{y}, r_{x}^{2} t_{y}^{-1}, m_{y} t_{z}, r_{x}^{2} t_{z}^{-1}, \\
m_{y} t_{z}^{-1}\end{array}$ \\
\hline 1847 & $H_{359}$ & 1 & $m_{z}$ & $\begin{array}{l}i t_{x}, r_{y}^{2} t_{x}^{-1}, m_{y} t_{y}, r_{x}^{2} t_{y}^{-1}, t_{z}, m_{z} t_{z}^{-1} \\
t_{z}^{-1}\end{array}$ \\
\hline 1848 & $H_{452}$ & 1 & $r_{z}^{2}$ & $\begin{array}{l}t_{x}, r_{z}^{2} t_{x}^{-1}, t_{x}^{-1}, r_{z}^{2} t_{y}, r_{z}^{2} t_{y}^{-1}, r_{y}^{2} r_{z} t_{z}, \\
m_{z} t_{z}^{-1}\end{array}$ \\
\hline 1849 & $H_{503}$ & 1 & $r_{z}^{2}$ & $\begin{array}{l}t_{x}, r_{z}^{2} t_{x}^{-1}, t_{x}^{-1}, r_{z}^{2} t_{y}, r_{z}^{2} t_{y}^{-1}, r_{y}^{2} r_{z} t_{z}, \\
r_{x}^{2} t_{z}^{-1}\end{array}$ \\
\hline
\end{tabular}

$$
1844 \quad H_{343} \quad 1
$$

$[8,33,76,132,204,292,396,516,652,804]$ 


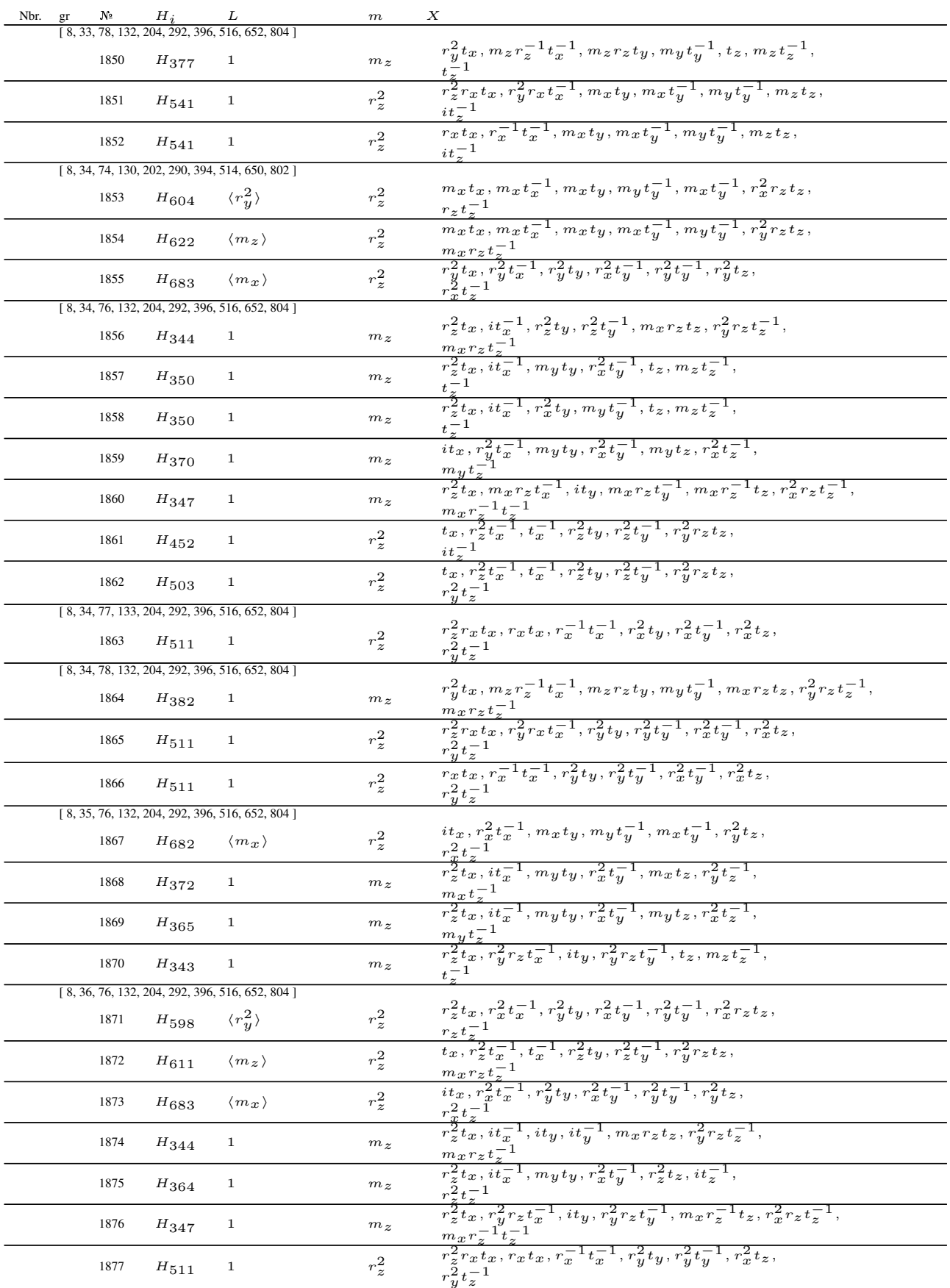

$[7,25,62,121,199,291,396,516,652,804]$ $1878^{*}$,

$[7,26,63,116,184,268,368,484,616,764]$ $1879^{*}$,

$[7,26,65,123,196,284,388,508,644,796]$ $1880^{*}$

$[7,26,65,125,202,292,396,516,652,804]$ $1881^{*}$,

$[7,26,67,131,209,296,397,516,652,804]$ 


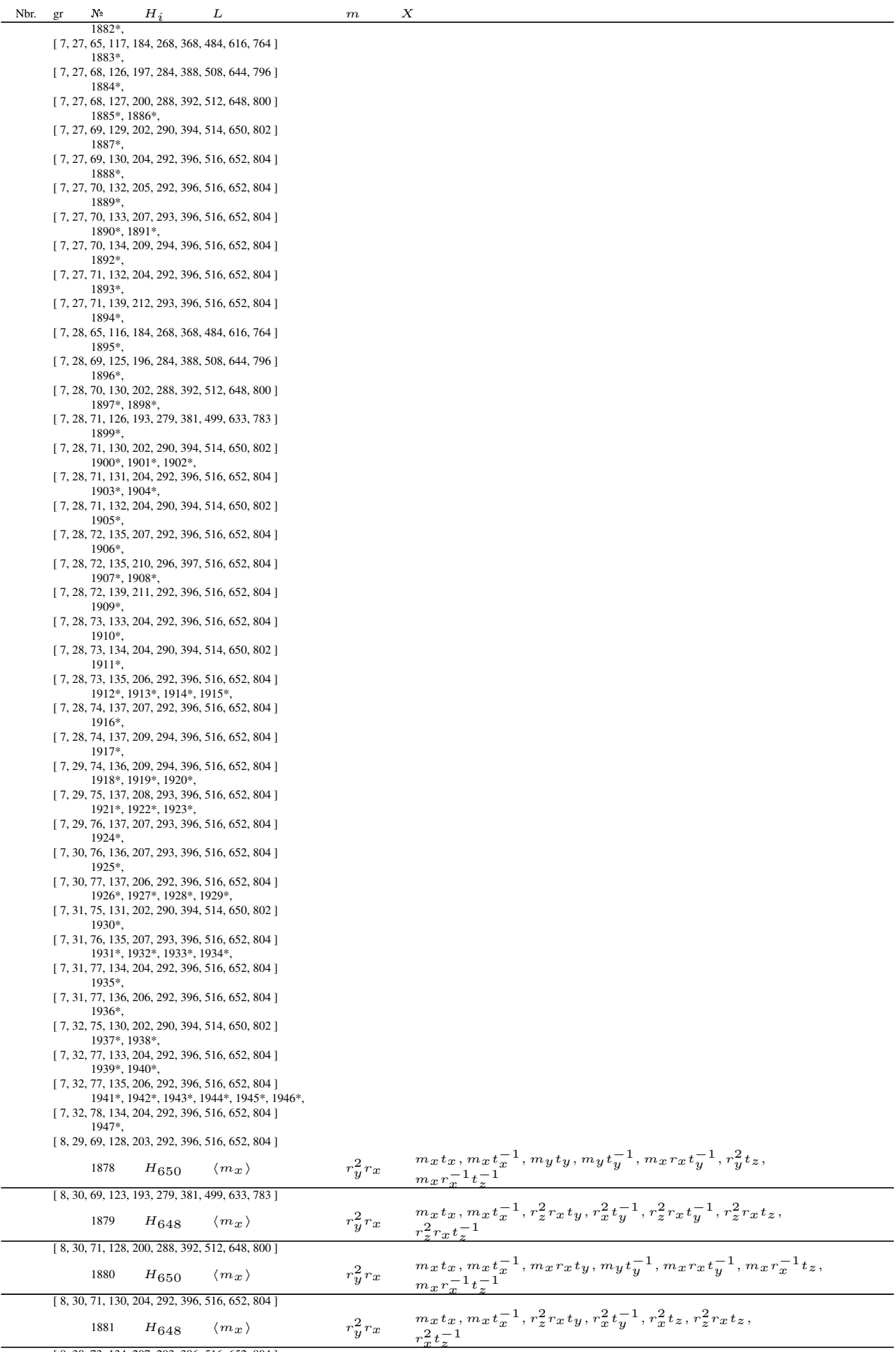




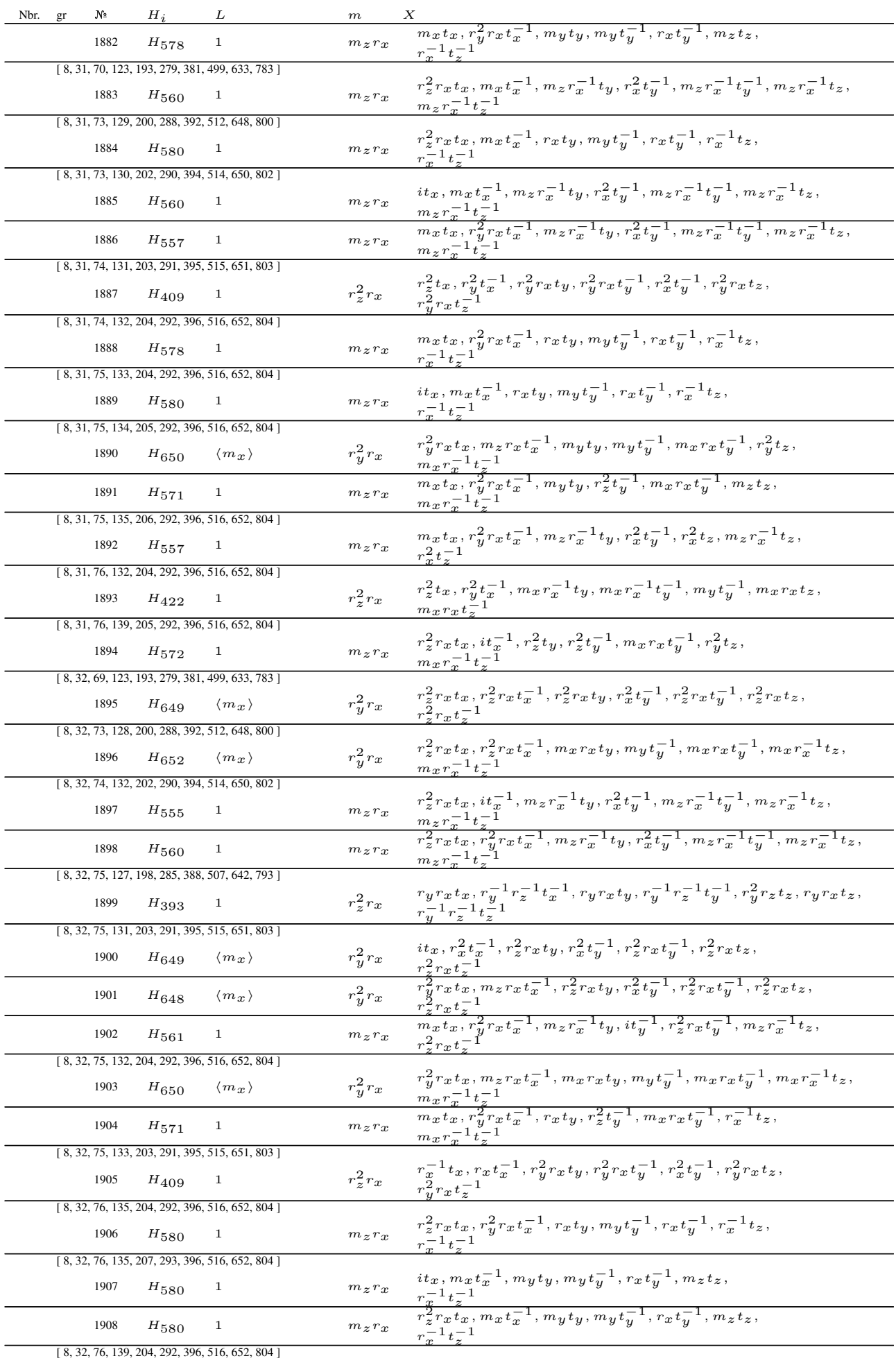




\begin{tabular}{lllll} 
Nbr. & gr & № & $H_{i}$ & $L$ \\
\hline & & \multirow{2}{*}{1909} & $H_{551}$ & 1
\end{tabular}

$[8,32,77,132,204,292,396,516,652,804]$

$1910 \quad H_{652} \quad\left\langle m_{x}\right\rangle$

$[8,32,77,133,203,291,395,515,651,803]$

$1911 \quad H_{560} \quad 1$

$[8,32,77,134,204,292,396,516,652,804]$

$\begin{array}{lll}1912 & H_{422} & 1 \\ 1913 & H_{648} & \left\langle m_{x}\right\rangle \\ 1914 & H_{574} & 1 \\ 1915 & H_{561} & 1\end{array}$

$[8,32,78,135,204,292,396,516,652,804]$

$1916 \quad H_{580} \quad 1$

$[8,32,78,135,206,292,396,516,652,804]$ $1917 \quad H_{422} \quad 1$

$[8,33,77,135,206,292,396,516,652,804]$

$\begin{array}{lll}1918 & H_{560} & 1 \\ 1919 & H_{560} & 1 \\ 1920 & H_{560} & 1\end{array}$

$[8,33,78,135,205,292,396,516,652,804]$

\begin{tabular}{ccc}
1921 & $H_{409}$ & 1 \\
\hline 1922 & $H_{580}$ & 1 \\
\hline 1923 & $H_{580}$ & 1
\end{tabular}

$[8,33,79,134,205,292,396,516,652,804]$ $1924 \quad H_{422} \quad 1$

$[8,34,78,134,205,292,396,516,652,804]$ $1925 \quad H_{571} \quad 1$

$[8,34,79,134,204,292,396,516,652,804]$

\begin{tabular}{ccc}
1926 & $H_{409}$ & 1 \\
\hline 1927 & $H_{560}$ & 1 \\
\hline 1928 & $H_{560}$ & 1 \\
\hline 1929 & $H_{560}$ & 1
\end{tabular}

[ $8,35,76,131,203,291,395,515,651,803]$
$1930 \quad H_{561} \quad 1$

$[8,35,77,134,205,292,396,516,652,804]$

\begin{tabular}{lll}
1931 & $H_{652}$ & $\left\langle m_{x}\right\rangle$ \\
\hline 1932 & $H_{652}$ & $\left\langle m_{x}\right\rangle$ \\
\hline 1933 & $H_{574}$ & 1 \\
\hline 1934 & $H_{576}$ & 1
\end{tabular}

$[8,35,78,132,204,292,396,516,652,804]$

$$
1935 \quad H_{571} \quad 1
$$

[ $8,35,78,134,204,292,396,516,652,804]$

$$
1936 \quad H_{561} \quad 1
$$

[ $8,36,75,131,203,291,395,515,651,803]$

$$
1937 \quad H_{551} \quad 1
$$

$m \quad X$
$m_{z} r_{x}$
$r_{z}^{2} r_{x} t_{x}, i t_{x}^{-1}, r_{z}^{2} r_{x} t_{y}, i t_{y}^{-1}, i t_{z}, r_{z}^{2} r_{x} t_{z}$

$r_{y}^{2} r_{x} \quad i t_{x}, r_{x}^{2} t_{x}^{-1}, m_{x} r_{x} t_{y}, m_{y} t_{y}^{-1}, m_{x} r_{x} t_{y}^{-1}, m_{x} r_{x}^{-1} t_{z}$, $m_{x} r_{x}^{-1} t_{z}^{-1}$

$m_{z} r_{x} \quad i t_{x}, r_{y}^{2} r_{x} t_{x}^{-1}, m_{z} r_{x}^{-1} t_{y}, r_{x}^{2} t_{y}^{-1}, m_{z} r_{x}^{-1} t_{y}^{-1}, m_{z} r_{x}^{-1} t_{z}$, $m_{z} r_{x}^{-1} t_{z}^{-1}$

$r_{z}^{2} r_{x} \quad r_{x}^{-1} t_{x}, r_{x} t_{x}^{-1}, m_{x} r_{x}^{-1} t_{y}, m_{x} r_{x}^{-1} t_{y}^{-1}, m_{y} t_{y}^{-1}, m_{x} r_{x} t_{z}$, $\begin{array}{ll}r_{y}^{2} r_{x} & r_{y}^{2} r_{x} t_{x}, m_{z} r_{x} t_{x}^{-1}, r_{z}^{2} r_{x} t_{y}, r_{x}^{2} t_{y}^{-1}, r_{x}^{2} t_{z}, r_{z}^{2} r_{x} t_{z}, \\ & r_{x}^{2} t_{z}^{-1}\end{array}$ $r_{z}^{2} r_{x} t_{x}, i t_{x}^{-1}, r_{x} t_{y}, m_{y} t_{y}^{-1}, r_{x} t_{y}^{-1}, r_{x}^{-1} t_{z}$

$\begin{array}{ll}m_{z} r_{x} & r_{z}^{2} r_{x} t_{x}, i_{x}, r_{x} t_{y}, m_{y} t_{y}, r_{x} t_{y}, r_{x} t_{z}, \\ & r_{x}^{-1} t_{z}^{-1} \\ m_{z} r_{x} & m_{x} t_{x}, r_{y}^{2} r_{x} t_{x}^{-1}, r_{x}^{2} t_{y}, i t_{y}^{-1}, r_{z}^{2} r_{x} t_{y}^{-1}, r_{x}^{2} t_{z},\end{array}$ $\begin{array}{ll}m_{z} r_{x} & m_{z} r_{x}, r_{z}^{2} r^{-1}\end{array}$

$m_{z} r_{x} \quad i t_{x}, r_{y}^{2} r_{x} t_{x}^{-1}, r_{x} t_{y}, m_{y} t_{y}^{-1}, r_{x} t_{y}^{-1}, r_{x}^{-1} t_{z}$,

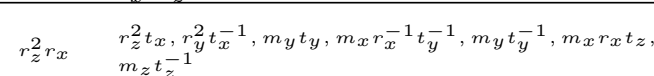

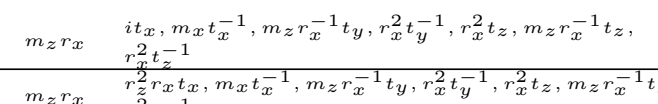
$\begin{array}{ll}m_{z} r_{x} & i t_{x}, m_{x} t_{x}^{-1}, m_{z} r_{x}^{-1} t_{y}, r_{x}^{2} t_{y}^{-1}, r_{x}^{2} t_{z}, m_{z} r_{x}^{-1} t_{z} \\ & r_{x}^{2} t_{z}^{-1} \\ m_{z} r_{x} & r_{z}^{2} r_{x} t_{x}, m_{x} t_{x}^{-1}, m_{z} r_{x}^{-1} t_{y}, r_{x}^{2} t_{y}^{-1}, r_{x}^{2} t_{z}, m_{z} r_{x}^{-1} t\end{array}$ $\begin{array}{ll}m_{z} r_{x} & r_{x}^{2} t_{z}^{-1} \\ r^{2} r_{x} t_{x},{ }^{2}\end{array}$ $m_{z} r_{x} \quad r_{z}^{2} r_{x} t_{x}, m_{x} t_{x}^{-1}, r_{x}^{2} t_{y}, r_{x}^{2} t_{y}^{-1}, m_{z} r_{x}^{-1} t_{y}^{-1}, r_{x}^{2} t_{z}$ $m_{z} r_{x}^{-1} t_{z}^{-1}$

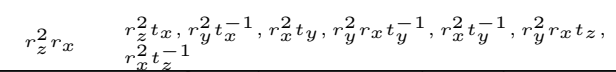

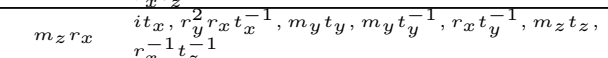
$\begin{array}{ll}m_{z} r_{x} & r_{z}^{2} r_{x} t_{x}, r_{y}^{2} r_{x} t_{x}^{-1}, m_{y} t_{y}, m_{y} t_{y}^{-1}, r_{x} t_{y}^{-1}, m_{z} t_{z}, \\ r_{x}^{-1} t_{z}^{-1}\end{array}$ $r_{z}^{2} r_{x} \quad r_{x}^{-1} t_{x}, r_{x} t_{x}^{-1}, m_{y} t_{y}, m_{x} r_{x}^{-1} t_{y}^{-1}, m_{y} t_{y}^{-1}, m_{x} r_{x} t_{z}$, $m_{z} t_{z}^{-1}$ $\begin{array}{ll}m_{z} r_{x} & \begin{array}{l}m_{x} t_{x}, r_{y}^{2} r_{x} t_{x}^{-1}, m_{y} t_{y}, r_{x} t_{y}, r_{z}^{2} t_{y}^{-1}, r_{x}^{-1} t_{z} \\ r_{y}^{2} t_{z}^{-1}\end{array}\end{array}$

$r_{z}^{2} r_{x} \quad r_{x}^{-1} t_{x}, r_{x} t_{x}^{-1}, r_{x}^{2} t_{y}, r_{y}^{2} r_{x} t_{y}^{-1}, r_{x}^{2} t_{y}^{-1}, r_{y}^{2} r_{x} t_{z}$, $\begin{array}{ll}m_{z} r_{x} & r_{x}^{2} t_{z}^{-1} \\ & r_{x}^{2} t_{z}^{-1}\end{array}$

\begin{aligned}$m_{z} r_{x} & r_{z}^{2} r_{x} t_{x}, r_{y}^{2} r_{x} t_{x}^{-1}, m_{z} r_{x}^{-1} t_{y}, r_{x}^{2} t_{y}^{-1}, r_{x}^{2} t_{z}, m_{z} r_{x}^{-1} t_{z} \\ & , r_{x}^{2} t_{z}^{-1} \\ \multirow{2}{*}{m_{z} r_{x}} & r_{z}^{2} r_{x} t_{x}, r_{y}^{2} r_{x} t_{x}^{-1}, r_{x}^{2} t_{y}, r_{x}^{2} t_{y}^{-1}, m_{z} r_{x}^{-1} t_{y}^{-1}, r_{x}^{2} t_{z} \\ & , m_{z} r_{x}^{-1} t_{z}^{-1}\end{aligned}$

$m_{z} r_{x} \quad m_{x} t_{x}, r_{y}^{2} r_{x} t_{x}^{-1}, m_{z} r_{x}^{-1} t_{y}, r_{z}^{2} r_{x} t_{y}^{-1}, r_{x}^{2} t_{z}, m_{z} r_{x}^{-1} t_{z}$, $\begin{array}{ll}r_{y}^{2} r_{x} & \begin{array}{l}i t_{x}, r_{x}^{2} t_{z}^{-1}, m_{y} t_{y}, m_{y} t_{y}^{-1}, m_{x} r_{x} t_{y}^{-1}, r_{y}^{2} t_{z}, \\ m_{x} r_{x}^{-1} t_{z}^{-1}\end{array}\end{array}$ $\begin{array}{ll}r_{y}^{2} r_{x} & \begin{array}{l}i t_{x}, r_{x}^{2} t_{x}^{-1}, m_{y}{ }_{y}, m_{y} t_{y}^{-1}, m_{x} r_{x} t_{y}^{-1}, r_{y}^{2} t_{z} \\ m_{x} r_{x}^{-1} t_{z}^{-1}\end{array} \\ r_{y}^{2} r_{x} & \begin{array}{l}r_{z}^{2} r_{x} t_{x}, r_{z}^{2} r_{x} t_{x}^{-1}, m_{y} t_{y}, m_{y} t_{y}^{-1}, m_{x} r_{x} t_{y}^{-1}, r_{y}^{2} t_{z} \\ m_{x} r_{x}^{-1} t_{z}^{-1}\end{array} \\ m_{z} r_{x} & \begin{array}{l}r_{z}^{2} r_{x} t_{x}, i t_{x}^{-1}, m_{y} t_{y}, m_{y} t_{y}^{-1}, r_{x} t_{y}^{-1}, m_{z} t_{z} \\ r_{x}^{-1} t_{z}^{-1}\end{array} \\ m_{z} r_{x} & \begin{array}{l}m_{x} t_{x}, r_{y}^{2} r_{x} t_{x}^{-1}, r_{z}^{2} t_{y}, r_{z}^{2} t_{y}^{-1}, m_{x} r_{x} t_{y}^{-1}, r_{y}^{2} t_{z}, \\ m_{x} r_{x}^{-1} t_{z}^{-1}\end{array} \\ m_{z} r_{x} \quad \begin{array}{l}m_{x} t_{x}, r_{y}^{2} r_{x} t_{x}^{-1}, m_{y} t_{y}, r_{x} t_{y}, m_{x} r_{x} t_{y}^{-1}, r_{x}^{-1} t_{z}, \\ m_{x} r_{x}^{-1} t_{z}^{-1}\end{array} \\ m_{z} r_{x} & \begin{array}{l}m_{x} t_{x}, r_{y}^{2} r_{x} t_{x}^{-1}, m_{z} r_{x}^{-1} t_{y}, i t_{y}^{-1}, r_{x}^{2} t_{z}, m_{z} r_{x}^{-1} t_{z}, \\ i t_{z}^{-1}\end{array}\end{array}$ $m_{z} r_{x} \quad r_{z}^{2} r_{x} t_{x}, i t_{x}^{-1}, r_{z}^{2} r_{x} t_{y}, i t_{y}^{-1}, r_{z}^{2} r_{x} t_{y}^{-1}, r_{z}^{2} r_{x} t_{z}$, 


\begin{tabular}{|c|c|c|c|c|c|c|}
\hline Nbr. & $\mathrm{gr}$ & № & $H_{i}$ & $L$ & $m$ & $X$ \\
\hline & & 1938 & $H_{556}$ & 1 & $m_{z} r_{x}$ & $\begin{array}{l}m_{x} t_{x}, r_{y}^{2} r_{x} t_{x}^{-1}, r_{z}^{2} r_{x} t_{y}, i t_{y}^{-1}, r_{z}^{2} r_{x} t_{y}^{-1}, r_{z}^{2} r_{x} t_{z} \\
r_{z}^{2} r_{x} t \frac{1}{z}\end{array}$ \\
\hline \multicolumn{7}{|c|}{$[8,36,77,132,204,292,396,516,652,804]$} \\
\hline & & 1939 & $H_{572}$ & -5 & $m_{z} r_{x}$ & $\begin{array}{l}r_{z}^{2} r_{x} t_{x}, i t_{x}^{-1}, m_{x} r_{x} t_{y}, r_{z}^{2} t_{y}^{-1}, m_{x} r_{x} t_{y}^{-1}, m_{x} r_{x}^{-1} t_{z} \\
m_{x} r_{x}^{-1} t_{z}^{-1}\end{array}$ \\
\hline & & 1940 & $H_{576}$ & 1 & $m_{z} r_{x}$ & $\begin{array}{l}m_{x} t_{x}, r_{y}^{2} r_{x} t_{x}^{-1}, m_{x} r_{x} t_{y}, r_{z}^{2} t_{y}^{-1}, m_{x} r_{x} t_{y}^{-1}, m_{x} r_{x}^{-1} t_{z} \\
m_{x} r_{x}^{-1} t_{z}^{-1}\end{array}$ \\
\hline \multicolumn{7}{|c|}{$[8,36,77,134,204,292,396,516,652,804]$} \\
\hline & & 1941 & $H_{649}$ & $\left\langle m_{x}\right\rangle$ & $r_{y}^{2} r_{x}$ & $\begin{array}{l}i t_{x}, r_{x}^{2} t_{x}^{-1}, r_{x}^{2} t_{y}, r_{x}^{2} t_{y}^{-1}, r_{z}^{2} r_{x} t_{y}^{-1}, r_{x}^{2} t_{z} \\
r_{z}^{2} r_{x} t_{z}^{1}\end{array}$ \\
\hline & & 1942 & $H_{649}$ & $\left\langle m_{x}\right\rangle$ & $r_{y}^{2} r_{x}$ & $\begin{array}{l}r_{z}^{2} r_{x} t_{x}, r_{z}^{2} r_{x} t_{x}^{-1}, r_{z}^{2} r_{x} t_{y}, r_{x}^{2} t_{y}^{-1}, r_{x}^{2} t_{z}, r_{z}^{2} r_{x} t_{z} \\
r_{x}^{2} t_{z}^{-1}\end{array}$ \\
\hline & & 1943 & $H_{649}$ & $\left\langle m_{x}\right\rangle$ & $r_{y}^{2} r_{x}$ & $\begin{array}{l}i t_{x}, r_{x}^{2} t_{x}^{-1}, r_{z}^{2} r_{x} t_{y}, r_{x}^{2} t_{y}^{-1}, r_{x}^{2} t_{z}, r_{z}^{2} r_{x} t_{z} \\
r_{x}^{2} t_{z}^{-1}\end{array}$ \\
\hline & & 1944 & $H_{555}$ & 1 & $m_{z} r_{x}$ & $\begin{array}{l}r_{z}^{2} r_{x} t_{x}, i t_{x}^{-1}, m_{z} r_{x}^{-1} t_{y}, r_{x}^{2} t_{y}^{-1}, r_{x}^{2} t_{z}, m_{z} r_{x}^{-1} t_{z} \\
r_{x}^{2} t_{z}^{-1}\end{array}$ \\
\hline & & 1945 & $H_{555}$ & 1 & $m_{z} r_{x}$ & $\begin{array}{l}i t_{x}, r_{z}^{2} r_{x} t_{x}^{-1}, r_{x}^{2} t_{y}, r_{x}^{2} t_{y}^{-1}, m_{z} r_{x}^{-1} t_{y}^{-1}, r_{x}^{2} t_{z} \\
m_{z} r_{x}^{-1} t_{z}^{-1}\end{array}$ \\
\hline & & 1946 & $H_{556}$ & 1 & $m_{z} r_{x}$ & $\begin{array}{l}m_{x} t_{x}, r_{y}^{2} r_{x} t_{x}^{-1}, r_{z}^{2} r_{x} t_{y}, i t_{y}^{-1}, i t_{z}, r_{z}^{2} r_{x} t_{z} \\
i t_{z}^{-1}\end{array}$ \\
\hline \multicolumn{7}{|c|}{$[8,36,78,132,204,292,396,516,652,804]$} \\
\hline & & 1947 & $H_{393}$ & 1 & $r_{z}^{2} r_{x}$ & $\begin{array}{l}r_{y}^{2} r_{z} t_{x}, r_{x}^{2} r_{y} t_{x}^{-1}, r_{y}^{2} r_{z} t_{y}, r_{y}^{-1} r_{z}^{-1} t_{y}^{-1}, r_{y}^{2} r_{z} t_{z}, r_{y} r_{x} t_{z}, \\
r_{x}^{2} r_{y} t_{z}^{-1}\end{array}$ \\
\hline
\end{tabular}

$[8,29,68,124,196,284,388,508,644,796]$ $1948^{*}, 1949^{*}$

$[8,30,70,126,198,286,390,510,646,798]$ $1950^{*}, 1951^{*}, 1952^{*}, 1953^{*}$,

$[8,30,71,128,200,288,392,512,648,800]$ $1954^{*}, 1955^{*}, 1956^{*}, 1957^{*}, 1958^{*}$

$[8,30,72,131,204,292,396,516,652,804]$ $1959^{*}, 1960^{*}, 1961^{*}, 1962^{*}, 1963^{*}, 1964^{*}$,

[ $8,30,73,134,206,292,396,516,652,804$ ] $1965^{*}$,

$[8,31,72,128,200,288,392,512,648,800]$ $1966^{*}$

$[8,31,73,129,200,288,392,512,648,800]$ $1967^{*}, 1968^{*}, 1969^{*}$,

$[8,31,73,131,204,292,396,516,652,804]$ $1970^{*}$

[ $8,31,74,132,204,292,396,516,652,804$ ]

$1971^{*}, 1972^{*}, 1973^{*}, 1974^{*}, 1975^{*}, 1976^{*}, 1977^{*}, 1978^{*}, 1979^{*}$

$[8,31,75,133,204,292,396,516,652,804$ ] $1980 *$

$[8,31,75,134,205,292,396,516,652,804]$ $1981^{*}, 1982^{*}, 1983^{*}$

$[8,32,73,128,200,288,392,512,648,800]$ 1984*, 1985*, 1986*, 1987*, 1988*, 1989*, 1990*, 1991*, 1992*, 1993*, 1994*, 1995*,

$[8,32,75,132,204,292,396,516,652,804]$

1996*, 1997*, 1998*, 1999*, 2000*, 2001*, 2002*, 2003*, 2004*, 2005*, 2006*, 2007*, 2008* $2009^{*}, 2010^{*}, 2011^{*}, 2012^{*}, 2013^{*}, 2014^{*}, 2015^{*}, 2016^{*}, 2017^{*}, 2018^{*}, 2019^{*}, 2020^{*}, 2021^{*}$ $2022^{*}, 2023^{*}, 2024^{*}, 2025^{*}$,

$[8,32,76,133,204,292,396,516,652,804]$ $2026^{*}, 2027^{*}, 2028^{*}, 2029^{*}, 2030^{*}, 2031^{*}, 2032^{*}$

$[8,32,76,134,205,292,396,516,652,804]$ $2033^{*}, 2034^{*}, 2035^{*}, 2036^{*}$

$[8,32,77,134,204,292,396,516,652,804]$ $2037^{*}, 2038^{*}, 2039^{*}, 2040^{*}$

$[8,32,77,135,205,292,396,516,652,804]$ $2041^{*}$

$[8,33,76,132,204,292,396,516,652,804]$ $2042^{*}, 2043^{*}, 2044^{*}, 2045^{*}, 2046^{*}, 2047^{*}, 2048^{*}, 2049^{*}, 2050^{*}, 2051^{*}, 2052^{*}, 2053^{*}, 2054^{*}$ $2055^{*}, 2056^{*}, 2057^{*}, 2058^{*}, 2059^{*}, 2060^{*}, 2061^{*}, 2062^{*}, 2063^{*}$

$[8,33,77,133,204,292,396,516,652,804]$ $2064^{*}, 2065^{*}, 2066^{*}$,

$[8,33,77,134,205,292,396,516,652,804]$ $2067 *$

$[8,33,78,135,205,292,396,516,652,804]$ $2068^{*}, 2069^{*}, 2070^{*}$

$[8,34,77,132,204,292,396,516,652,804]$

2071*, 2072*, 2073*, 2074*, 2075*, 2076*, 2077*, 2078*, 2079*, 2080*, 2081*, 2082*, 2083*, 2084*, 2085*, 2086*, 2087*, 2088*, 2089*, 2090*, 2091*, 2092*, 2093*, 2094*, 2095*, 2096*, 2097*, 2098*, 2099*, 2100*, 2101*, 2102*, 2103*, 2104*, 2105*, 2106*, 2107*, 2108*, 2109*, $2110^{*}$,

$[8,34,78,134,205,292,396,516,652,804]$ $2111^{*}, 2112^{*}, 2113^{*}$

$[9,32,72,128,200,288,392,512,648,800]$

\begin{tabular}{|c|c|c|c|c|}
\hline 1948 & $H_{680}$ & $\left\langle m_{x}\right\rangle$ & $r_{z}^{2}$ & $\begin{array}{l}m_{x} t_{x}, m_{x} t_{x}^{-1}, m_{x} t_{y}, m_{y} t_{y}^{-1}, m_{x} t_{y}^{-1}, r_{y}^{2} t_{z} \\
r_{x}^{2} t_{z}^{-1}, r_{y}^{2} t_{z}^{-1}\end{array}$ \\
\hline 1949 & $H_{690}$ & $\left\langle m_{x}\right\rangle$ & $r_{z}^{2}$ & $\begin{array}{l}m_{x} t_{x}, m_{x} t_{x}^{-1}, m_{x} t_{y}, m_{y} t_{y}^{-1}, m_{x} t_{y}^{-1}, r_{y}^{2} r_{z} t_{z} \\
m_{z} r_{z}^{-1} t_{z}, r_{y}^{2} t_{z}^{-1}\end{array}$ \\
\hline
\end{tabular}

$[9,33,73,129,201,289,393,513,649,801]$ 


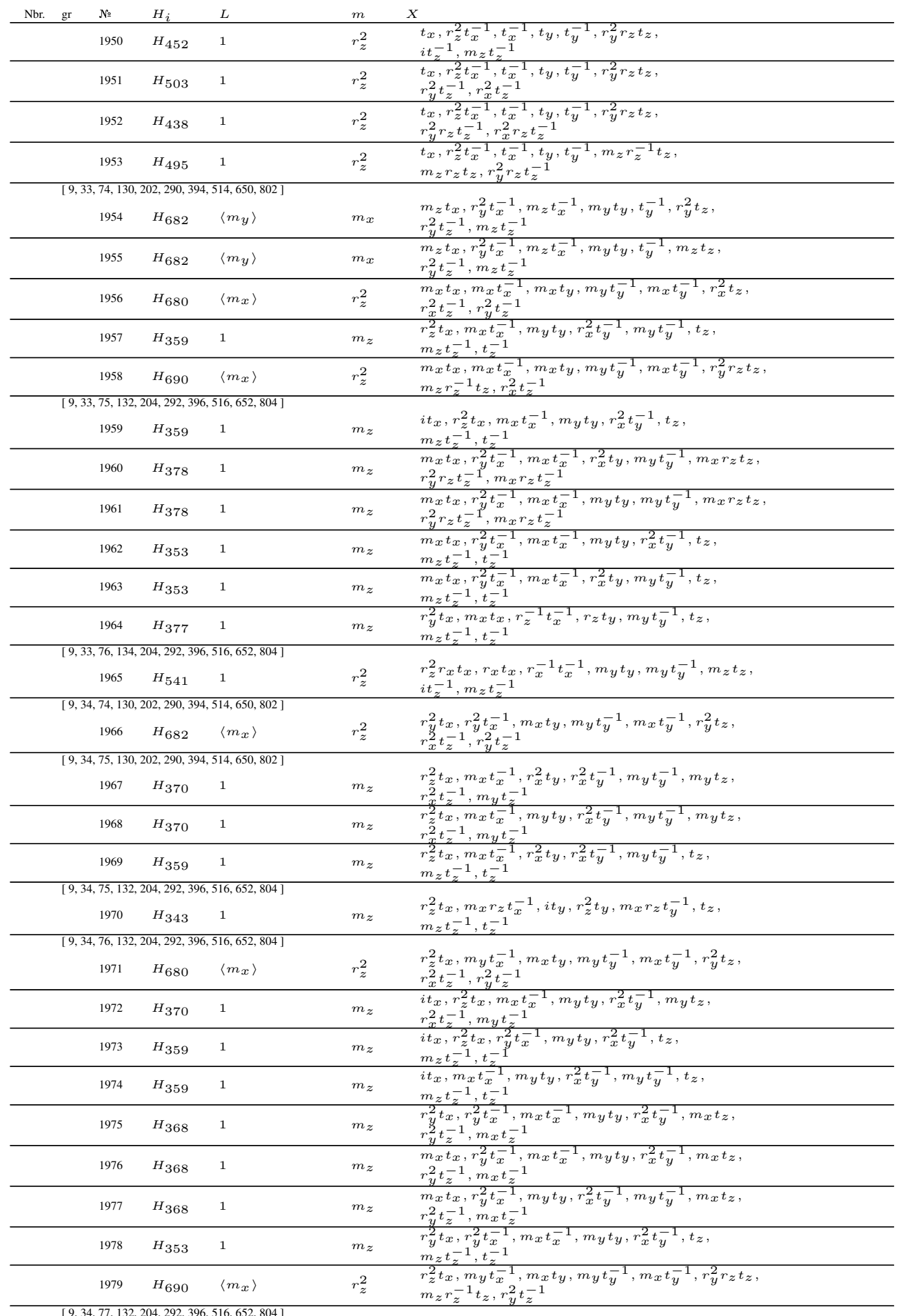




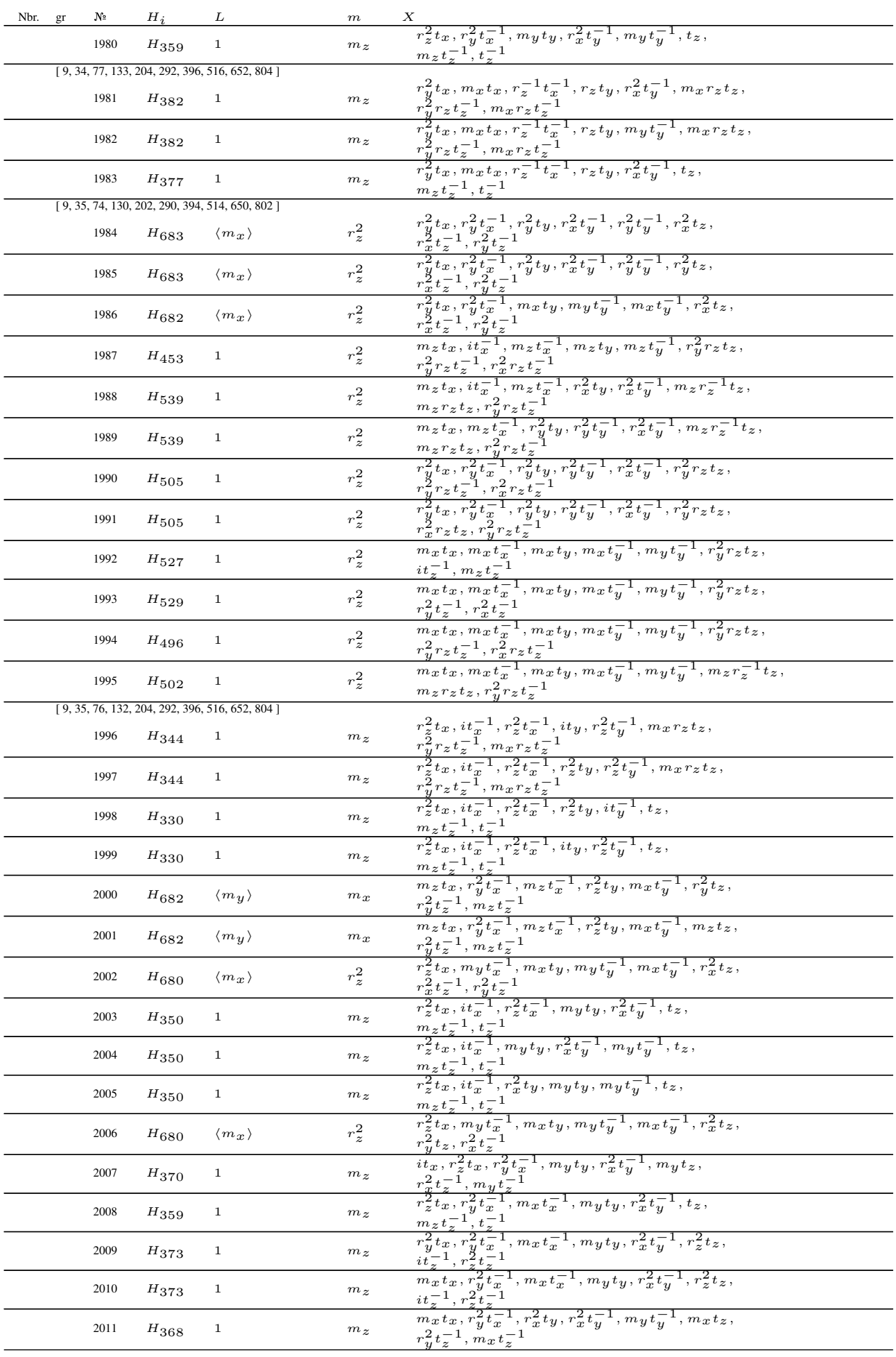




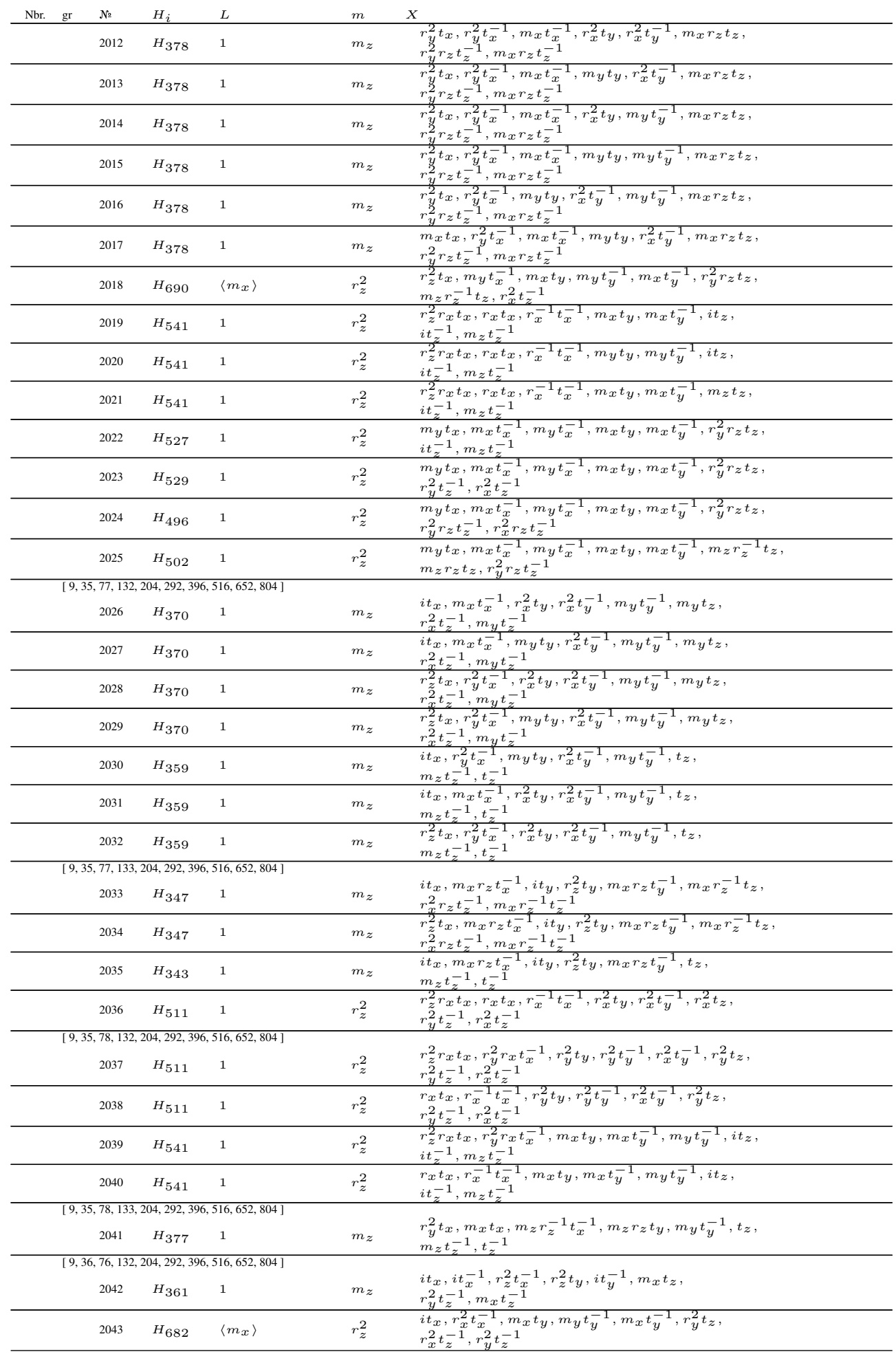




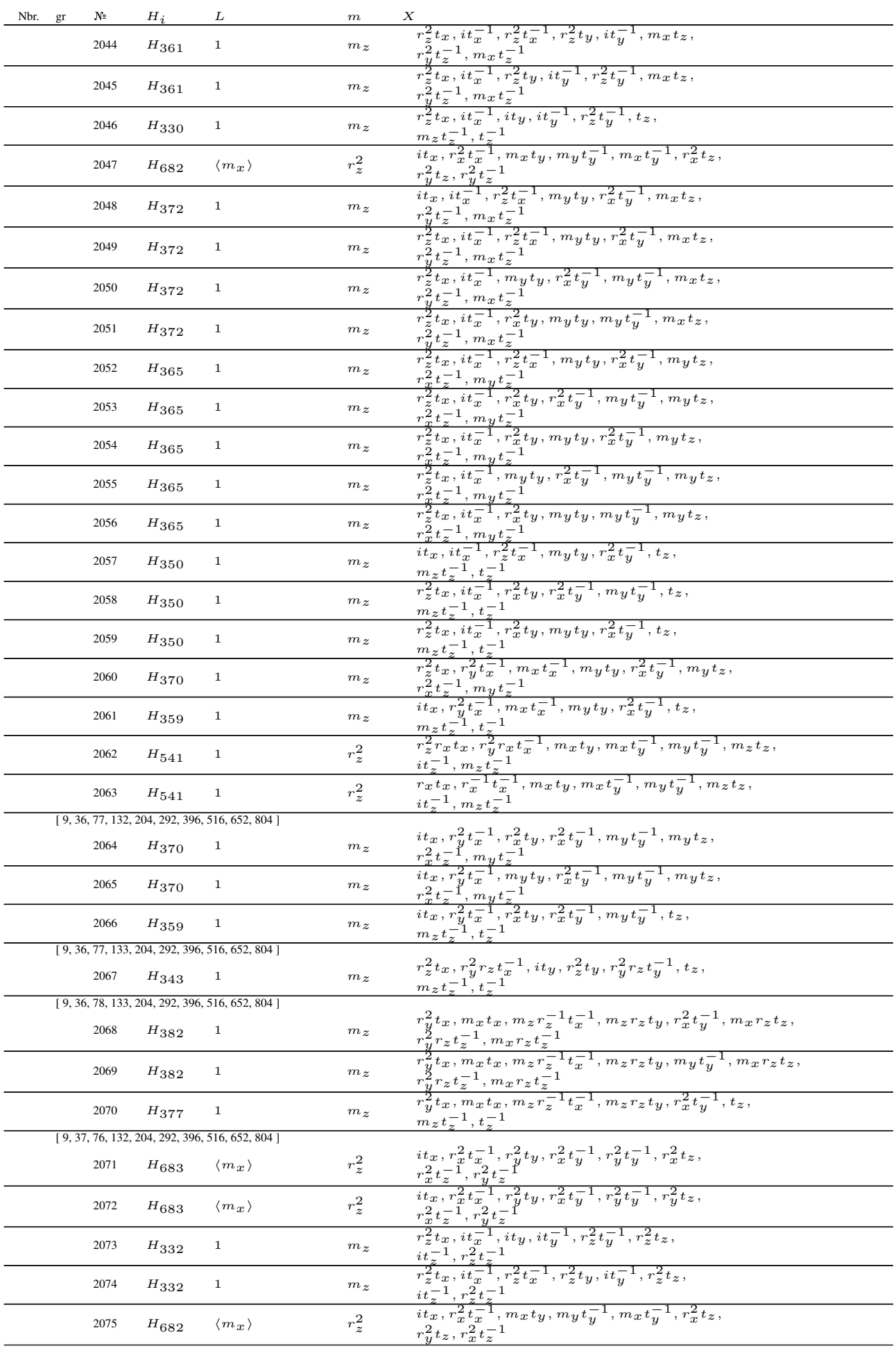




\begin{tabular}{|c|c|c|c|c|c|}
\hline Nbr. & № & $H_{i}$ & $L$ & $m$ & $x$ \\
\hline & 2076 & $H_{361}$ & 1 & $m_{z}$ & $\begin{array}{l}r_{z}^{2} t_{x}, i t_{x}^{-1}, i t_{y}, i t_{y}^{-1}, r_{z}^{2} t_{y}^{-1}, m_{x} t_{z} \\
r_{y}^{2} t_{z}^{-1}, m_{x} t_{z}^{-1}\end{array}$ \\
\hline & 2077 & $H_{344}$ & 1 & $m_{z}$ & $\begin{array}{l}i t_{x}, i t_{x}^{-1}, r_{z}^{2} t_{x}^{-1}, i t_{y}, i t_{y}^{-1}, m_{x} r_{z} t_{z} \\
r_{y}^{2} r_{z} t_{z}^{-1}, m_{x} r_{z} t_{z}^{-1}\end{array}$ \\
\hline & 2078 & $H_{344}$ & 1 & $m_{z}$ & $\begin{array}{l}r_{z}^{2} t_{x}, i t_{x}^{-1}, r_{z}^{2} t_{x}^{-1}, i t_{y}, i t_{y}^{-1}, m_{x} r_{z} t_{z} \\
r_{y}^{2} r_{z} t_{z}^{-1}, m_{x} r_{z}{ }_{z}^{-1}\end{array}$ \\
\hline & 2079 & $H_{344}$ & 1 & $m_{z}$ & $\begin{array}{l}r_{z}^{2} t_{x}, i t_{x}^{-1}, i t_{y}, i t_{y}^{-1}, r_{z}^{2} t_{y}^{-1}, m_{x} r_{z} t_{z} \\
r_{y}^{2} r_{z} t_{z}^{-1}, m_{x} r_{z} t_{z}^{-1}\end{array}$ \\
\hline & 2080 & $H_{344}$ & 1 & $m_{z}$ & $\begin{array}{l}r_{z}^{2} t_{x}, i t_{x}^{-1}, i t_{y}, r_{z}^{2} t_{y}, i t_{y}^{-1}, m_{x} r_{z} t_{z} \\
r_{y}^{2} r_{z} t_{z}^{-1}, m_{x} r_{z} t_{z}^{-1}\end{array}$ \\
\hline & 2081 & $H_{344}$ & 1 & $m_{z}$ & $\begin{array}{l}r_{z}^{2} t_{x}, i t_{x}^{-1}, r_{z}^{2} t_{x}^{-1}, r_{z}^{2} t_{y}, i t_{y}^{-1}, m_{x} r_{z} t_{z}, \\
r_{y}^{2} r_{z} t_{z}^{-1}, m_{x} r_{z} t_{z}^{-1}\end{array}$ \\
\hline & 2082 & $H_{344}$ & 1 & $m_{z}$ & $\begin{array}{l}i t_{x}, i t_{x}^{-1}, r_{z}^{2} t_{x}^{-1}, i t_{y}, r_{z}^{2} t_{y}^{-1}, m_{x} r_{z} t_{z} \\
r_{y}^{2} r_{z} t_{z}^{-1}, m_{x} r_{z} t_{z}^{-1}\end{array}$ \\
\hline & 2083 & $H_{344}$ & 1 & $m_{z}$ & $\begin{array}{l}i t_{x}, i t_{x}^{-1}, r_{z}^{2} t_{x}^{-1}, r_{z}^{2} t_{y}, r_{z}^{2} t_{y}^{-1}, m_{x} r_{z} t_{z} \\
r_{y}^{2} r_{z} t_{z}^{-1}, m_{x} r_{z} t_{z}^{-1}\end{array}$ \\
\hline & 2084 & $H_{682}$ & $\left\langle m_{x}\right\rangle$ & $r_{z}^{2}$ & $\begin{array}{l}i t_{x}, r_{x}^{2} t_{x}^{-1}, m_{x} t_{y}, m_{y} t_{y}^{-1}, m_{x} t_{y}^{-1}, r_{x}^{2} t_{z}, \\
r_{x}^{2} t_{z}^{-1}, r_{y}^{2} t_{z}^{-1}\end{array}$ \\
\hline & 2085 & $H_{683}$ & $\left\langle m_{x}\right\rangle$ & $r_{z}^{2}$ & $\begin{array}{l}i t_{x}, r_{x}^{2} t_{x}^{-1}, r_{y}^{2} t_{y}, r_{x}^{2} t_{y}^{-1}, r_{y}^{2} t_{y}^{-1}, r_{x}^{2} t_{z} \\
r_{y}^{2} t_{z}, r_{x}^{2} t_{z}^{-1}\end{array}$ \\
\hline & 2086 & $H_{683}$ & $\left\langle m_{x}\right\rangle$ & $r_{z}^{2}$ & $\begin{array}{l}i t_{x}, r_{x}^{2} t_{x}^{-1}, r_{y}^{2} t_{y}, r_{x}^{2} t_{y}^{-1}, r_{y}^{2} t_{y}^{-1}, r_{x}^{2} t_{z} \\
r_{y}^{2} t_{z}, r_{y}^{2} t_{z}^{-1}\end{array}$ \\
\hline & 2087 & $H_{364}$ & 1 & $m_{z}$ & $\begin{array}{l}i t_{x}, i t_{x}^{-1}, r_{z}^{2} t_{x}^{-1}, m_{y} t_{y}, r_{x}^{2} t_{y}^{-1}, r_{z}^{2} t_{z} \\
i t_{z}^{-1}, r_{z}^{2} t_{z}^{-1}\end{array}$ \\
\hline & 2088 & $H_{364}$ & 1 & $m_{z}$ & $\begin{array}{l}r_{z}^{2} t_{x}, i t_{x}^{-1}, r_{z}^{2} t_{x}^{-1}, m_{y} t_{y}, r_{x}^{2} t_{y}^{-1}, r_{z}^{2} t_{z} \\
i t_{z}^{-1}, r_{z}^{2} t_{z}^{-1}\end{array}$ \\
\hline & 2089 & $H_{364}$ & 1 & $m_{z}$ & $\begin{array}{l}r_{z}^{2} t_{x}, i t_{x}^{-1}, r_{x}^{2} t_{y}, r_{x}^{2} t_{y}^{-1}, m_{y} t_{y}^{-1}, r_{z}^{2} t_{z} \\
i t_{z}^{-1}, r_{z}^{2} t_{z}^{-1}\end{array}$ \\
\hline & 2090 & $H_{364}$ & 1 & $m_{z}$ & $\begin{array}{l}r_{z}^{2} t_{x}, i t_{x}^{-1}, r_{x}^{2} t_{y}, m_{y} t_{y}, r_{x}^{2} t_{y}^{-1}, r_{z}^{2} t_{z} \\
i t_{z}^{-1}, r_{z}^{2} t_{z}^{-1}\end{array}$ \\
\hline & 2091 & $H_{364}$ & 1 & $m_{z}$ & $\begin{array}{l}r_{z}^{2} t_{x}, i t_{x}^{-1}, m_{y} t_{y}, r_{x}^{2} t_{y}^{-1}, m_{y} t_{y}^{-1}, r_{z}^{2} t_{z}, \\
i t_{z}^{-1}, r_{z}^{2} t_{z}^{-1}\end{array}$ \\
\hline & 2092 & $H_{364}$ & 1 & $m_{z}$ & $\begin{array}{l}r_{z}^{2} t_{x}, i t_{x}^{-1}, r_{x}^{2} t_{y}, m_{y} t_{y}, m_{y} t_{y}^{-1}, r_{z}^{2} t_{z} \\
i t_{z}^{-1}, r_{z}^{2} t_{z}^{-1}\end{array}$ \\
\hline & 2093 & $H_{372}$ & 1 & $m_{z}$ & $\begin{array}{l}r_{z}^{2} t_{x}, i t_{x}^{-1}, r_{x}^{2} t_{y}, r_{x}^{2} t_{y}^{-1}, m_{y} t_{y}^{-1}, m_{x} t_{z}, \\
r_{y}^{2} t_{z}^{-1}, m_{x} t_{z}^{-1}\end{array}$ \\
\hline & 2094 & $H_{372}$ & 1 & $m_{z}$ & $\begin{array}{l}r_{z}^{2} t_{x}, i t_{x}^{-1}, r_{x}^{2} t_{y}, m_{y} t_{y}, r_{x}^{2} t_{y}^{-1}, m_{x} t_{z} \\
r_{y}^{2} t_{z}^{-1}, m_{x} t_{z}^{-1}\end{array}$ \\
\hline & 2095 & $H_{365}$ & 1 & $m_{z}$ & $\begin{array}{l}i t_{x}, i t_{x}^{-1}, r_{z}^{2} t_{x}^{-1}, m_{y} t_{y}, r_{x}^{2} t_{y}^{-1}, m_{y} t_{z} \\
r_{x}^{2} t_{z}^{-1}, m_{y} t_{z}^{-1}\end{array}$ \\
\hline & 2096 & $H_{370}$ & 1 & $m_{z}$ & $\begin{array}{l}i t_{x}, r_{y}^{2} t_{x}^{-1}, m_{x} t_{x}^{-1}, m_{y} t_{y}, r_{x}^{2} t_{y}^{-1}, m_{y} t_{z}, \\
r_{x}^{2} t_{z}^{-1}, m_{y} t_{z}^{-1}\end{array}$ \\
\hline & 2097 & $H_{453}$ & 1 & $r_{z}^{2}$ & $\begin{array}{l}m_{z} t_{x}, i t_{x}^{-1}, m_{z} t_{x}^{-1}, i t_{y}, i t_{y}^{-1}, r_{y}^{2} r_{z} t_{z} \\
r_{y}^{2} r_{z} t_{z}^{-1}, r_{x}^{2} r_{z} t_{z}^{-1}\end{array}$ \\
\hline & 2098 & $H_{539}$ & 1 & $r_{z}^{2}$ & $\begin{array}{l}i t_{x}, i t_{x}^{-1}, r_{y}^{2} t_{y}, r_{y}^{2} t_{y}^{-1}, r_{x}^{2} t_{y}^{-1}, m_{z} r_{z}^{-1} t_{z} \\
m_{z} r_{z} t_{z}, r_{y}^{2} r_{z} t_{z}^{-1}\end{array}$ \\
\hline & 2099 & $H_{539}$ & 1 & $r_{z}^{2}$ & $\begin{array}{l}m_{z} t_{x}, i t_{x}^{-1}, m_{z} t_{x}^{-1}, r_{y}^{2} t_{y}, r_{y}^{2} t_{y}^{-1}, m_{z} r_{z}^{-1} t_{z} \\
m_{z} r_{z} t_{z}, r_{y}^{2} r_{z} t_{z}^{-1}\end{array}$ \\
\hline & 2100 & $H_{452}$ & 1 & $r_{z}^{2}$ & $\begin{array}{l}t_{x}, r_{z}^{2} t_{x}^{-1}, t_{x}^{-1}, r_{z}^{2} t_{y}, r_{z}^{2} t_{y}^{-1}, r_{y}^{2} r_{z} t_{z} \\
i t_{z}^{-1}, m_{z} t_{z}^{-1}\end{array}$ \\
\hline & 2101 & $H_{503}$ & 1 & $r_{z}^{2}$ & $\begin{array}{l}t_{x}, r_{z}^{2} t_{x}^{-1}, t_{x}^{-1}, r_{z}^{2} t_{y}, r_{z}^{2} t_{y}^{-1}, r_{y}^{2} r_{z} t_{z} \\
r_{y}^{2} t_{z}^{-1}, r_{x}^{2} t_{z}^{-1}\end{array}$ \\
\hline & 2102 & $H_{438}$ & 1 & $r_{z}^{2}$ & $\begin{array}{l}t_{x}, r_{z}^{2} t_{x}^{-1}, t_{x}^{-1}, r_{z}^{2} t_{y}, r_{z}^{2} t_{y}^{-1}, r_{y}^{2} r_{z} t_{z} \\
r_{y}^{2} r_{z} t_{z}^{-1}, r_{x}^{2} r_{z} t_{z}^{-1}\end{array}$ \\
\hline & 2103 & $H_{495}$ & 1 & $r_{z}^{2}$ & $\begin{array}{l}t_{x}, r_{z}^{2} t_{x}^{-1}, t_{x}^{-1}, r_{z}^{2} t_{y}, r_{z}^{2} t_{y}^{-1}, m_{z} r_{z}^{-1} t_{z} \\
m_{z} r_{z} t_{z}, r_{y}^{2} r_{z} t_{z}^{-1}\end{array}$ \\
\hline & 2104 & $H_{511}$ & 1 & $r_{z}^{2}$ & $\begin{array}{l}r_{z}^{2} r_{x} t_{x}, r_{y}^{2} r_{x} t_{x}^{-1}, r_{y}^{2} t_{y}, r_{y}^{2} t_{y}^{-1}, r_{x}^{2} t_{y}^{-1}, r_{x}^{2} t_{z} \\
r_{y}^{2} t_{z}^{-1}, r_{x}^{2} t_{z}^{-1}\end{array}$ \\
\hline & 2105 & $H_{511}$ & 1 & $r_{z}^{2}$ & $\begin{array}{l}r_{z}^{2} r_{x} t_{x}, r_{x} t_{x}, r_{x}^{-1} t_{x}^{-1}, r_{y}^{2} t_{y}, r_{y}^{2} t_{y}^{-1}, r_{y}^{2} t_{z} \\
r_{y}^{2} t_{z}^{-1}, r_{x}^{2} t_{z}^{-1}\end{array}$ \\
\hline & 2106 & $H_{511}$ & 1 & $r_{z}^{2}$ & $\begin{array}{l}r_{z}^{2} r_{x} t_{x}, r_{x} t_{x}, r_{x}^{-1} t_{x}^{-1}, r_{y}^{2} t_{y}, r_{y}^{2} t_{y}^{-1}, r_{x}^{2} t_{z} \\
r_{y}^{2} t_{z}^{-1}, r_{x}^{2} t_{z}^{-1}\end{array}$ \\
\hline & 2107 & $H_{511}$ & 1 & $r_{z}^{2}$ & $\begin{array}{l}r_{z}^{2} r_{x} t_{x}, r_{x} t_{x}, r_{x}^{-1} t_{x}^{-1}, r_{x}^{2} t_{y}, r_{x}^{2} t_{y}^{-1}, r_{y}^{2} t_{z} \\
r_{y}^{2} t_{z}^{-1}, r_{x}^{2} t_{z}^{-1}\end{array}$ \\
\hline & 2108 & $H_{511}$ & 1 & $r_{z}^{2}$ & $\begin{array}{l}r_{x} t_{x}, r_{x}^{-1} t_{x}^{-1}, r_{y}^{2} t_{y}, r_{y}^{2} t_{y}^{-1}, r_{x}^{2} t_{y}^{-1}, r_{x}^{2} t_{z} \\
r_{y}^{2} t_{z}^{-1}, r_{x}^{2} t_{z}^{-1}\end{array}$ \\
\hline
\end{tabular}




\begin{tabular}{|c|c|c|c|c|c|c|}
\hline Nbr. & $\mathrm{gr}$ & № & $H_{i}$ & $L$ & $m$ & $X$ \\
\hline & & 2109 & $H_{505}$ & 1 & $r_{z}^{2}$ & $\begin{array}{l}r_{x}^{2} t_{x}, r_{y}^{2} t_{x}^{-1}, r_{x}^{2} t_{x}^{-1}, r_{y}^{2} t_{y}, r_{y}^{2} t_{y}^{-1}, r_{y}^{2} r_{z} t_{z} \\
r_{y}^{2} r_{z} t_{z}^{-1}, r_{x}^{2} r_{z} t_{z}^{-1}\end{array}$ \\
\hline & & 2110 & $H_{505}$ & 1 & $r_{z}^{2}$ & $\begin{array}{l}r_{x}^{2} t_{x}, r_{y}^{2} t_{x}^{-1}, r_{x}^{2} t_{x}^{-1}, r_{y}^{2} t_{y}, r_{y}^{2} t_{y}^{-1}, r_{y}^{2} r_{z} t_{z} \\
r_{x}^{2} r_{z} t_{z}, r_{y}^{2} r_{z} t_{z}^{-1}\end{array}$ \\
\hline & {$[9$,} & 77,13 & $4,292,39$ & $516,652,804]$ & & \\
\hline & & 2111 & $H_{347}$ & 1 & $m_{z}$ & $\begin{array}{l}i t_{x}, r_{y}^{2} r_{z} t_{x}^{-1}, i t_{y}, r_{z}^{2} t_{y}, r_{y}^{2} r_{z} t_{y}^{-1}, m_{x} r_{z}^{-1} t_{z} \\
r_{x}^{2} r_{z} t_{z}^{-1}, m_{x} r_{z}^{-1} t_{z}^{-1}\end{array}$ \\
\hline & & 2112 & $H_{347}$ & 1 & $m_{z}$ & $\begin{array}{l}r_{z}^{2} t_{x}, r_{y}^{2} r_{z} t_{x}^{-1}, i t_{y}, r_{z}^{2} t_{y}, r_{y}^{2} r_{z} t_{y}^{-1}, m_{x} r_{z}^{-1} t_{z}, \\
r_{x}^{2} r_{z} t_{z}^{-1}, m_{x} r_{z}^{-1} t_{z}^{-1}\end{array}$ \\
\hline & & 2113 & $H_{343}$ & 1 & $m_{z}$ & $\begin{array}{l}i t_{x}, r_{y}^{2} r_{z} t_{x}^{-1}, i t_{y}, r_{z}^{2} t_{y}, r_{y}^{2} r_{z} t_{y}^{-1}, t_{z} \\
m_{z} t_{z}^{-1}, t_{z}^{-1}\end{array}$ \\
\hline
\end{tabular}

$38 \mathrm{~A}$

$[6,18,38,66,102,146,198,258,326,402]$ $2114^{*}$,

$[6,20,46,82,128,186,254,332,422,522]$ $2115^{*}$

$[6,20,46,84,134,194,263,343,434,535]$

$2116^{*}$,
$[6,20,47,85,132,191,261,340,431,533]$ $2117^{*}$

$[6,20,47,87,137,195,263,343,434,535]$ $2118^{*}$,

$[6,22,50,90,142,206,282,370,470,582]$ $2119 *$

$[6,22,52,94,148,214,292,382,484,598]$ $2120^{*}$,

$[6,22,54,98,152,218,296,386,488,602]$ $2123^{*}$,

$[6,22,54,99,156,227,311,408,519,643]$ $2121 *, 2122 *$

$[6,22,56,100,152,218,296,386,488,602$ ] 2124*,

$[6,24,58,105,166,240,328,430,545,675]$ $2125^{*}, 2126^{*}$,

$[6,24,59,106,166,240,328,430,545,675]$ $2127^{*}, 2128^{*}$,

$[6,26,62,109,172,249,338,444,564,695]$ $2129^{*}$

$[7,23,50,87,135,194,263,343,434,535]$ $2114 \quad H_{751}\left\langle m_{y}, r_{z}^{2}\right\rangle \quad i \quad r_{z}^{2} t_{x}, r_{z}^{2} t_{x}^{-1}, r_{y}^{2} r_{x} t_{y}, m_{x} r_{x} t_{y}^{-1}, m_{x} r_{x} t_{z}^{-1}, r_{y}^{2} r_{x} t_{z}^{-1}$

$[7,25,56,98,154,222,301,395,500,616]$ $2115 \quad H_{459} \quad 1$

$7,25,56,100,158,226,306,401,506,623]$ $2116 \quad H_{533} \quad 1$ $2117 \quad H_{536} \quad 1$

$[7,25,57,102,159,226,306,401,506,623]$ $2118 H_{512} \quad r_{z}^{2} \quad r_{z} t_{x}, r_{z}^{-1} t_{x}^{-1}, r_{z}^{-1} t_{y}, r_{z} t_{y}, r_{y}^{2} r_{z} t_{z}, r_{y}^{2} t_{z}^{-1}$ $2119 H_{613}\left\langle m_{z\rangle} \quad r_{z}^{2} \quad m_{x} r_{z} t_{x}^{-1}, m_{x} r_{z}^{-1} t_{x}^{-1}, m_{x} r_{z}^{-1} t_{y}, m_{x} r_{z} t_{y}^{-1}, r_{y}^{2} r_{z} t_{z}, m_{x} r_{z} t_{z}^{-1}\right.$

$[7,27,60,107,167,240,327,427,540,667]$ $2120 \quad H_{627} \quad\left\langle m_{z}\right\rangle \quad r_{z}^{2} \quad r_{z}^{-1} t_{x}^{-1}, r_{z} t_{x}^{-1}, r_{z} t_{y}, r_{z}^{-1} t_{y}^{-1}, r_{y}^{2} r_{z} t_{z}, m_{x} r_{z} t_{z}^{-1}$

$[7,27,62,108,168,242,328,428,542,668]$ $2121 \quad H_{693}\left\langle m_{x}\right\rangle \quad r_{z}^{2} \quad r_{x}^{2} r_{z} t_{x}, m_{z} r_{z} t_{x}^{-1}, m_{z} r_{z} t_{y}^{-1}, r_{x}^{2} r_{z} t_{y}^{-1}, r_{y}^{2} t_{z}, r_{x}^{2} t_{z}^{-1}$

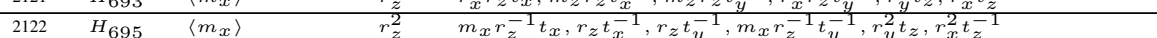

$[7,27,62,109,169,242,329,429,542,669] \quad\left[r_{y}\right]$ $2123 \quad H_{608}\left\langle r_{y}^{2}\right\rangle \quad r_{z}^{2} \quad r_{y}^{2} r_{x} t_{x}, r_{z}^{2} r_{x} t_{x}^{-1}, m_{x} r_{x}^{-1} t_{y}^{-1}, m_{z} r_{x} t_{y}^{-1}, m_{z} r_{x} t_{z}, m_{x} r_{x}^{-1} t_{z}^{-1}$

$\begin{gathered}{[7,27,64,109,169,242,329,429,542,669]} \\ 2124 \quad H_{600}\left\langle r_{y}^{2}\right\rangle\end{gathered} \quad r_{z}^{2} \quad r_{y}^{2} r_{x} t_{x}, r_{z}^{2} r_{x} t_{x}^{-1}, r_{x}^{-1} t_{y}^{-1}, r_{y}^{2} r_{x} t_{y}^{-1}, r_{x} t_{z}, r_{z}^{2} r_{x} t_{z}^{-1}$

\begin{tabular}{lllll}
2125 & $H_{459}$ & 1 & $r_{z}^{2}$ & $m_{x} r_{z} t_{x}^{-1}, m_{x} r_{z}^{-1} t_{x}^{-1}, m_{x} r_{z}^{-1} t_{y}, m_{x} r_{z} t_{y}^{-1}, r_{y}^{2} r_{z} t_{z}, i t$ \\
2126 & $H_{533}$ & 1 & $r_{z}^{2}$ & $m_{x} r_{z} t_{x}^{-1}, m_{x} r_{z}^{-1} t_{x}^{-1}, m_{x} r_{z}^{-1} t_{y}, m_{x} r_{z} t_{y}^{-1}, r_{y}^{2} r_{z} t_{z}, r_{y}^{2} t_{z}^{-1}$ \\
\hline
\end{tabular}

$7,29,65,113,176,251,342,446,563,696]$ $2127 \quad H_{536} \quad 1 \quad r_{z}^{2} \quad r_{z}^{-1} t_{x}^{-1}, r_{z} t_{x}^{-1}, r_{z} t_{y}, r_{z}^{-1} t_{y}^{-1}, r_{y}^{2} r_{z} t_{z}, i t_{z}^{-1}$ $2128 \quad H_{512} \quad 1 \quad r_{z}^{2} \quad r_{z}^{-1} t_{x}^{-1}, r_{z} t_{x}^{-1}, r_{z} t_{y}, r_{z}^{-1} t_{y}^{-1}, r_{y}^{2} r_{z} t_{z}, r_{y}^{2} t_{z}^{-1}$ $2129 \quad H_{751}\left\langle m_{y}, r_{z}^{2}\right\rangle \quad{ }_{i} \quad r_{y}^{2} t_{x}, m_{z} t_{x}^{-1}, r_{y}^{2} r_{x} t_{y}, m_{x} r_{x} t_{y}^{-1}, m_{x} r_{x} t_{z}^{-1}, r_{y}^{2} r_{x} t_{z}^{-1}$

$[6,19,46,92,156,236,332,444,572,716]$ $2130^{*}$,

$[6,20,48,92,152,228,320,428,552,692]$ $2131^{*}$

$[6,21,56,114,188,276,380,500,636,788]$ $2132^{*}$,

$[6,21,57,117,193,283,388,508,644,796]$ 2133*,

$[6,21,57,119,197,287,392,512,648,800]$ $2134 *$

$[6,22,56,108,176,260,360,476,608,756]$ $2135^{*}$,

$[6,22,57,111,182,271,376,496,632,784]$ 


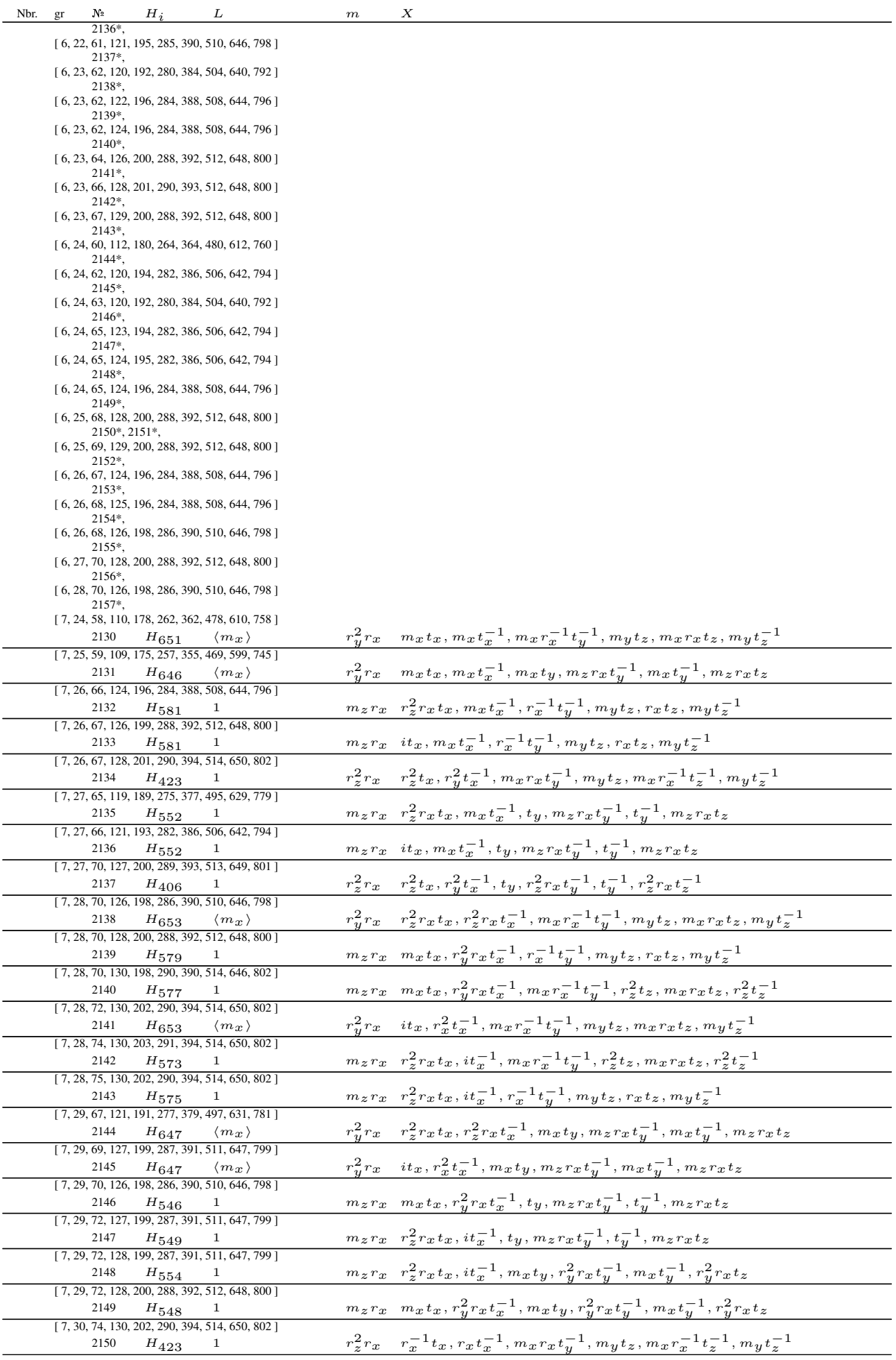




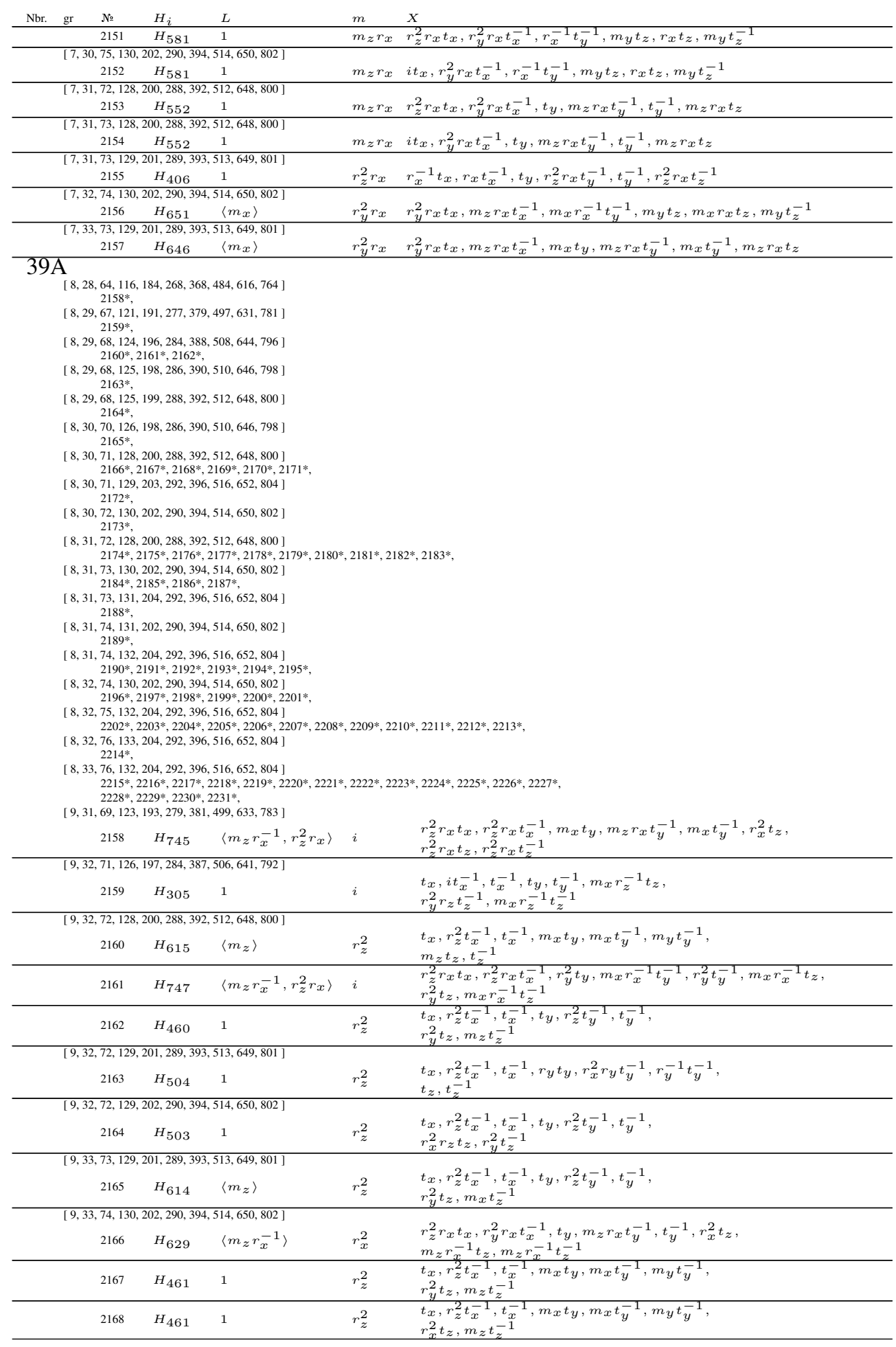




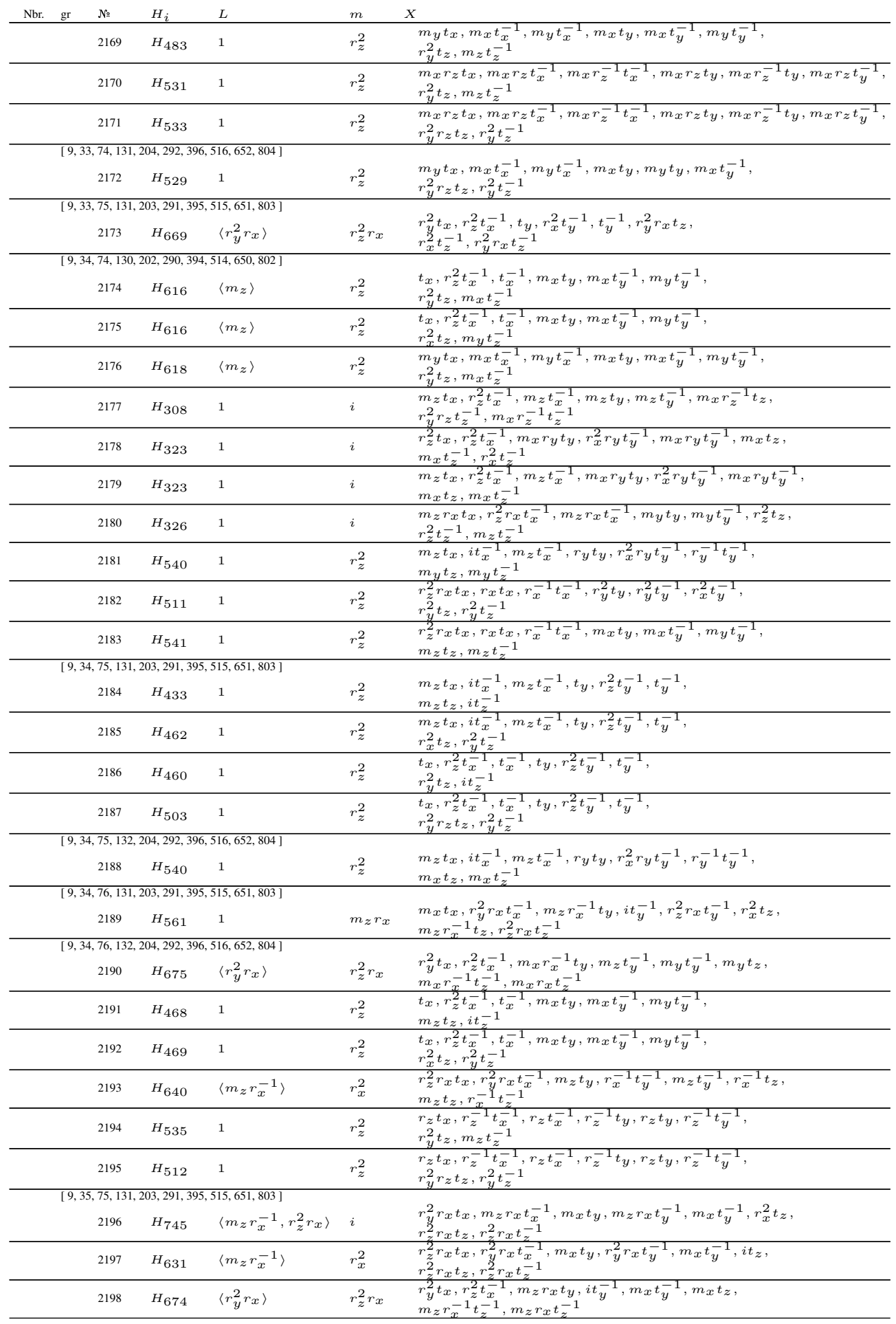




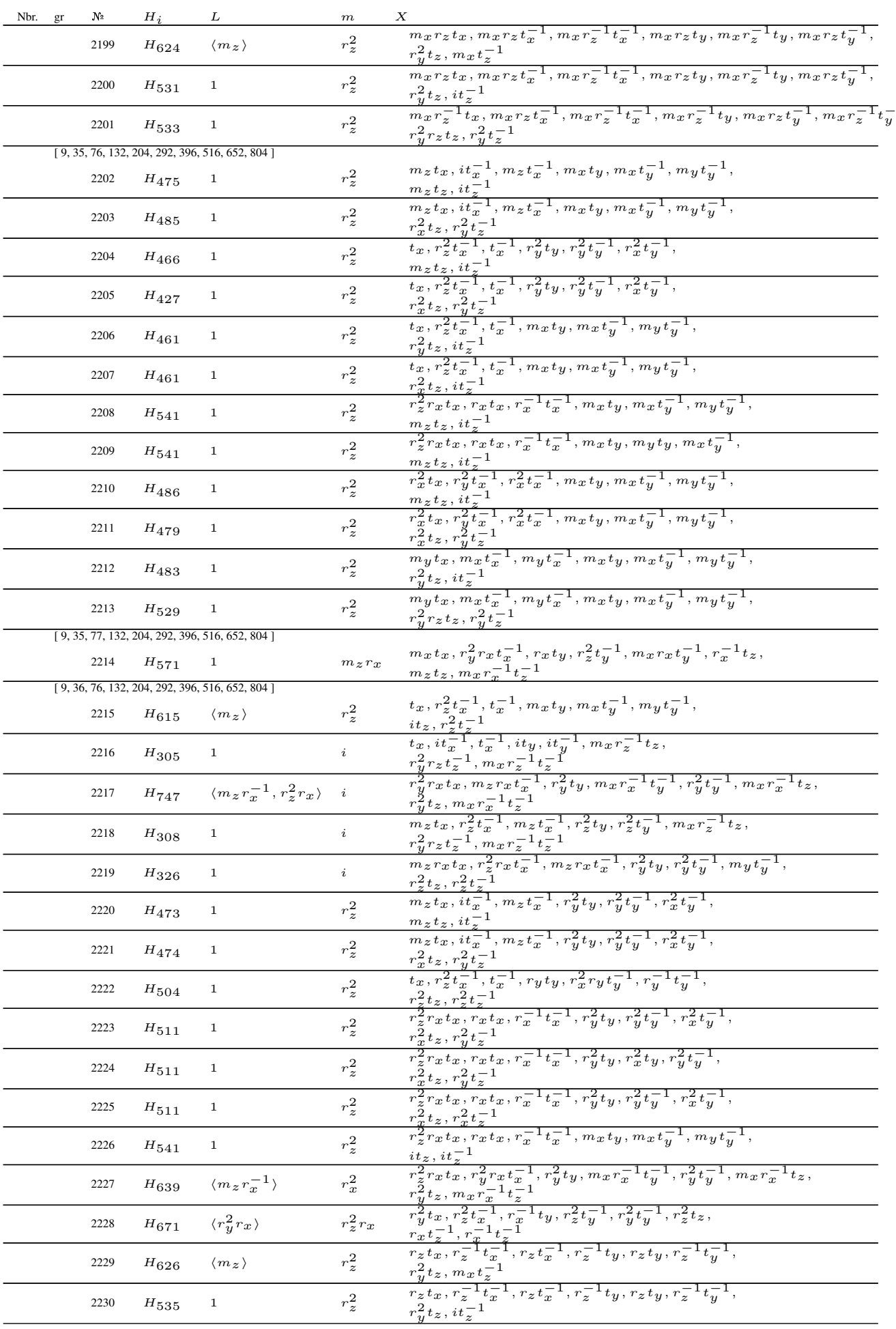




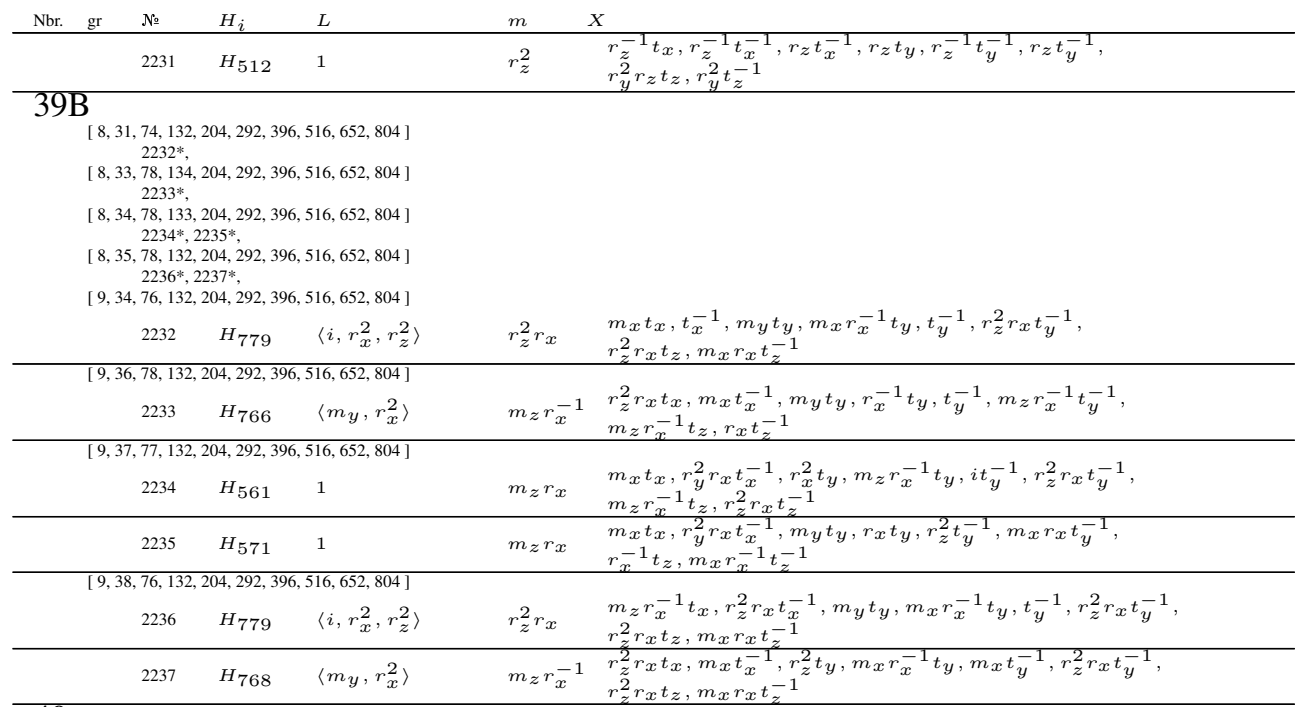

$[9,32,72,128,200,288,392,512,648,800]$ $2238^{*}, 2239^{*}, 2240^{*}$,

$[9,33,75,132,204,292,396,516,652,804]$ $2241^{*}, 2242^{*}, 2243^{*}, 2244^{*}, 2245^{*}, 2246^{*}, 2247^{*}, 2248^{*}$,

$[9,34,76,132,204,292,396,516,652,804]$

$2249^{*}, 2250^{*}, 2251^{*}, 2252^{*}, 2253^{*}, 2254^{*}, 2255^{*}, 2256^{*}, 2257^{*}, 2258^{*}, 2259^{*}, 2260^{*}, 2261^{*}$, $2262^{*}, 2263^{*}, 2264^{*}, 2265^{*}, 2266^{*}, 2267^{*}, 2268^{*}, 2269^{*}$,

$[10,34,74,130,202,290,394,514,650,802]$

\begin{tabular}{lllll}
2238 & $H_{749}$ & $\left\langle m_{y}, r_{z}^{2}\right\rangle$ & $i$ & $\begin{array}{l}r_{y}^{2} t_{x}, r_{z}^{2} t_{x}, m_{z} t_{x}^{-1}, r_{z}^{2} t_{x}^{-1}, r_{z}^{2} t_{y}, r_{z}^{2} t_{y}^{-1}, \\
r_{z}^{2} t_{z}, m_{z} t_{z}^{-1}, r_{z}^{2} t_{z}^{-1}\end{array}$ \\
\hline 2239 & $H_{750}$ & $\left\langle m_{y}, m_{z}\right\rangle$ & $r_{z}^{2}$ & $\begin{array}{l}m_{y} t_{x}, m_{x} t_{x}^{-1}, m_{y} t_{x}^{-1}, m_{y} t_{y}, m_{y} t_{y}^{-1}, r_{y}^{2} r_{z} t_{z}, \\
m_{z} r_{z} t_{z}, m_{x} r_{z} t_{z}^{-1}, r_{z} t^{-1}\end{array}$ \\
\hline 2240 & $H_{690}\left\langle m_{x}\right\rangle$ & $r_{z}^{2}$ & $\begin{array}{l}m_{x} t_{x}, m_{x} t_{x}^{-1}, m_{x} t_{y}, m_{y} t_{y}^{-1}, m_{x} t_{y}^{-1}, r_{y}^{2} r_{z} t_{z}, \\
m_{z} r_{z}^{-1} t_{z}, r_{x}^{2} t_{z}^{-1}, r_{y}^{2} t_{z}^{-1}\end{array}$
\end{tabular}

$[10,35,76,132,204,292,396,516,652,804]$ $m_{z} r_{z}^{-1} t_{z}, r_{x}^{2} t_{z}^{-1}, r_{y}^{2} t_{z}^{-1}$

\begin{tabular}{|c|c|c|c|c|}
\hline 2241 & $H_{682}$ & $\left\langle m_{x}\right\rangle$ & $r_{z}^{2}$ & $\begin{array}{l}i t_{x}, r_{y}^{2} t_{x}, r_{x}^{2} t_{x}^{-1}, r_{y}^{2} t_{x}^{-1}, m_{x} t_{y}, m_{y} t_{y}^{-1} \\
m_{x} t_{y}^{-1}, r_{y}^{2} t_{z}, r_{x}^{2} t_{z}^{-1}\end{array}$ \\
\hline 2242 & $H_{680}$ & $\left\langle m_{x}\right\rangle$ & $r_{z}^{2}$ & $\begin{array}{l}r_{z}^{2} t_{x}, m_{x} t_{x}, m_{y} t_{x}^{-1}, m_{x} t_{x}^{-1}, m_{x} t_{y}, m_{y} t_{y}^{-1} \\
m_{x} t_{y}^{-1}, r_{y}^{2} t_{z}, r_{x}^{2} t_{z}^{-1}\end{array}$ \\
\hline 2243 & $H_{359}$ & 1 & $m_{z}$ & $\begin{array}{l}i t_{x}, r_{z}^{2} t_{x}, r_{y}^{2} t_{x}^{-1}, m_{x} t_{x}^{-1}, m_{y} t_{y}, r_{x}^{2} t_{y}^{-1} \\
t_{z}, m_{z} t_{z}^{-1}, t_{z}^{-1}\end{array}$ \\
\hline 2244 & $H_{452}$ & 1 & $r_{z}^{2}$ & $\begin{array}{l}t_{x}, r_{z}^{2} t_{x}^{-1}, t_{x}^{-1}, r_{z}^{2} t_{y}, t_{y}, r_{z}^{2} t_{y}^{-1} \\
t_{y}^{-1}, r_{y}^{2} r_{z} t_{z}, m_{z} t_{z}^{-1}\end{array}$ \\
\hline 2245 & $H_{503}$ & 1 & $r_{z}^{2}$ & $\begin{array}{l}t_{x}, r_{z}^{2} t_{x}^{-1}, t_{x}^{-1}, r_{z}^{2} t_{y}, t_{y}, r_{z}^{2} t_{y}^{-1} \\
t_{y}^{-1}, r_{y}^{2} r_{z} t_{z}, r_{x}^{2} t_{z}^{-1}\end{array}$ \\
\hline 2246 & $H_{541}$ & 1 & $r_{z}^{2}$ & $\begin{array}{l}r_{z}^{2} r_{x} t_{x}, r_{x} t_{x}, r_{y}^{2} r_{x} t_{x}^{-1}, r_{x}^{-1} t_{x}^{-1}, m_{x} t_{y}, m_{x} t_{y}^{-1}, \\
m_{y} t_{y}^{-1}, m_{z} t_{z}, i t_{z}^{-1}\end{array}$ \\
\hline 2247 & $H_{527}$ & 1 & $r_{z}^{2}$ & $\begin{array}{l}m_{x} t_{x}, m_{y} t_{x}, m_{x} t_{x}^{-1}, m_{y} t_{x}^{-1}, m_{x} t_{y}, m_{x} t_{y}^{-1} \\
m_{y} t_{y}^{-1}, r_{y}^{2} r_{z} t_{z}, m_{z} t_{z}^{-1}\end{array}$ \\
\hline 2248 & $H_{529}$ & 1 & $r_{z}^{2}$ & $\begin{array}{l}m_{x} t_{x}, m_{y} t_{x}, m_{x} t_{x}^{-1}, m_{y} t_{x}^{-1}, m_{x} t_{y}, m_{x} t_{y}^{-1}, \\
m_{y} t_{y}^{-1}, r_{y}^{2} r_{z} t_{z}, r_{y}^{2} t_{z}^{-1}\end{array}$ \\
\hline \multicolumn{5}{|c|}{$[10,36,76,132,204,292,396,516,652,804]$} \\
\hline 2249 & $H_{749}$ & $\left\langle m_{y}, r_{z}^{2}\right\rangle$ & $i$ & $\begin{array}{l}r_{y}^{2} t_{x}, r_{z}^{2} t_{x}, m_{z} t_{x}^{-1}, r_{z}^{2} t_{x}^{-1}, r_{x}^{2} t_{y}, m_{z} t_{y}^{-1} \\
r_{z}^{2} t_{z}, m_{z} t_{z}^{-1}, r_{z}^{2} t_{z}^{-1}\end{array}$ \\
\hline 2250 & $H_{604}$ & $\left\langle r_{y}^{2}\right\rangle$ & $r_{z}^{2}$ & $\begin{array}{l}i t_{x}, m_{x} t_{x}, m_{y} t_{x}^{-1}, m_{x} t_{x}^{-1}, m_{x} t_{y}, m_{y} t_{y}^{-1} \\
m_{x} t_{y}^{-1}, r_{x}^{2} r_{z} t_{z}, r_{z} t_{z}^{-1}\end{array}$ \\
\hline 2251 & $H_{750}$ & $\left\langle m_{y}, m_{z}\right\rangle$ & $r_{z}^{2}$ & $\begin{array}{l}m_{y} t_{x}, m_{x} t_{x}^{-1}, m_{y} t_{x}^{-1}, r_{z}^{2} t_{y}, m_{x} t_{y}^{-1}, r_{y}^{2} r_{z} t_{z} \\
m_{z} r_{z} t_{z}, m_{x} r_{z} t_{z}^{-1}, r_{z} t_{z}^{-1}\end{array}$ \\
\hline 2252 & $H_{750}$ & $\left\langle m_{y}, r_{z}^{2}\right\rangle$ & $i$ & $\begin{array}{l}r_{y}^{2} t_{x}, r_{z}^{2} t_{x}, m_{z} t_{x}^{-1}, r_{z}^{2} t_{x}^{-1}, r_{x}^{2} t_{y}, m_{z} t_{y}^{-1} \\
m_{x} r_{z} t_{z}, r_{x}^{2} r_{z} t_{z}^{-1}, m_{x} r_{z} t_{z}^{-1}\end{array}$ \\
\hline 2253 & $H_{750}$ & $\left\langle m_{y}, r_{z}^{2}\right\rangle$ & $i$ & $\begin{array}{l}r_{y}^{2} t_{x}, r_{z}^{2} t_{x}, m_{z} t_{x}^{-1}, r_{z}^{2} t_{x}^{-1}, r_{z}^{2} t_{y}, r_{z}^{2} t_{y}^{-1} \\
m_{x} r_{z} t_{z}, r_{x}^{2} r_{z} t_{z}^{-1}, m_{x} r_{z} t_{z}^{-1}\end{array}$ \\
\hline 2254 & $H_{598}$ & $\left\langle r_{y}^{2}\right\rangle$ & $r_{z}^{2}$ & $\begin{array}{l}r_{z}^{2} t_{x}, r_{y}^{2} t_{x}, r_{x}^{2} t_{x}^{-1}, r_{y}^{2} t_{x}^{-1}, r_{y}^{2} t_{y}, r_{x}^{2} t_{y}^{-1} \\
r_{y}^{2} t_{y}^{-1}, r_{x}^{2} r_{z} t_{z}, r_{z} t_{z}^{-1}\end{array}$ \\
\hline
\end{tabular}




\begin{tabular}{|c|c|c|c|c|c|}
\hline Nbr. & № & $H_{i}$ & $L$ & $m$ & $x$ \\
\hline & 2255 & $H_{611}$ & $\left\langle m_{z}\right\rangle$ & $r_{z}^{2}$ & $\begin{array}{l}t_{x}, r_{z}^{2} t_{x}^{-1}, t_{x}^{-1}, r_{z}^{2} t_{y}, t_{y}, r_{z}^{2} t_{y}^{-1} \\
t_{y}^{-1}, r_{y}^{2} r_{z} t_{z}, m_{x} r_{z} t_{z}^{-1}\end{array}$ \\
\hline & 2256 & $H_{622}$ & $\left\langle m_{z}\right\rangle$ & $r_{z}^{2}$ & $\begin{array}{l}m_{x} t_{x}, m_{y^{t}} t_{x}, m_{x} t_{x}^{-1}, m_{y} t_{x}^{-1}, m_{x} t_{y}, m_{x} t_{y}^{-1}, \\
m_{y} t_{y}^{-1}, r_{y}^{2} r_{z} t_{z}, m_{x} r_{z} t_{z}^{-1}\end{array}$ \\
\hline & 2257 & $H_{683}$ & $\left\langle m_{x}\right\rangle$ & $r_{z}^{2}$ & $\begin{array}{l}i t_{x}, r_{y}^{2} t_{x}, r_{x}^{2} t_{x}^{-1}, r_{y}^{2} t_{x}^{-1}, r_{y}^{2} t_{y}, r_{x}^{2} t_{y}^{-1} \\
r_{y}^{2} t_{y}^{-1}, r_{y}^{2} t_{z}, r_{x}^{2} t_{z}^{-1}\end{array}$ \\
\hline & 2258 & $H_{344}$ & 1 & $m_{z}$ & $\begin{array}{l}r_{z}^{2} t_{x}, i t_{x}^{-1}, i t_{y}, r_{z}^{2} t_{y}, i t_{y}^{-1}, r_{z}^{2} t_{y}^{-1} \\
m_{x} r_{z} t_{z}, r_{y}^{2} r_{z} t_{z}^{-1}, m_{x} r_{z} t_{z}^{-1}\end{array}$ \\
\hline & 2259 & $H_{682}$ & $\left\langle m_{y}\right\rangle$ & $m_{x}$ & $\begin{array}{l}m_{z} t_{x}, r_{y}^{2} t_{x}^{-1}, m_{z} t_{x}^{-1}, r_{z}^{2} t_{y}, m_{y} t_{y}, m_{x} t_{y}^{-1}, \\
t_{y}^{-1}, m_{z} t_{z}, r_{y}^{2} t_{z}^{-1}\end{array}$ \\
\hline & 2260 & $H_{370}$ & 1 & $m_{z}$ & $\begin{array}{l}i t_{x}, r_{z}^{2} t_{x}, r_{y}^{2} t_{x}^{-1}, m_{x} t_{x}^{-1}, m_{y} t_{y}, r_{x}^{2} t_{y}^{-1} \\
m_{y} t_{z}, r_{x}^{2} t_{z}^{-1}, m_{y} t_{z}^{-1}\end{array}$ \\
\hline & 2261 & $H_{378}$ & 1 & $m_{z}$ & $\begin{array}{l}r_{y}^{2} t_{x}, m_{x} t_{x}, r_{y}^{2} t_{x}^{-1}, m_{x} t_{x}^{-1}, m_{y} t_{y}, r_{x}^{2} t_{y}^{-1} \\
m_{x} r_{z} t_{z}, r_{y}^{2} r_{z} t_{z}^{-1}, m_{x} r_{z} t_{z}^{-1}\end{array}$ \\
\hline & 2262 & $H_{690}$ & $\left\langle m_{x}\right\rangle$ & $r_{z}^{2}$ & $\begin{array}{l}r_{z}^{2} t_{x}, m_{y} t_{x}^{-1}, m_{x} t_{y}, m_{y} t_{y}^{-1}, m_{x} t_{y}^{-1}, r_{y}^{2} r_{z} t_{z} \\
m_{z} r_{z}^{-1} t_{z}, r_{x}^{2} t_{z}^{-1}, r_{y}^{2} t_{z}^{-1}\end{array}$ \\
\hline & 2263 & $H_{452}$ & 1 & $r_{z}^{2}$ & $\begin{array}{l}t_{x}, r_{z}^{2} t_{x}^{-1}, t_{x}^{-1}, r_{z}^{2} t_{y}, t_{y}, r_{z}^{2} t_{y}^{-1} \\
t_{y}^{-1}, r_{y}^{2} r_{z} t_{z}, i t_{z}^{-1}\end{array}$ \\
\hline & 2264 & $H_{503}$ & 1 & $r_{z}^{2}$ & $\begin{array}{l}t_{x}, r_{z}^{2} t_{x}^{-1}, t_{x}^{-1}, r_{z}^{2} t_{y}, t_{y}, r_{z}^{2} t_{y}^{-1} \\
t_{y}^{-1}, r_{y}^{2} r_{z} t_{z}, r_{y}^{2} t_{z}^{-1}\end{array}$ \\
\hline & 2265 & $H_{511}$ & 1 & $r_{z}^{2}$ & $\begin{array}{l}r_{z}^{2} r_{x} t_{x}, r_{x} t_{x}, r_{y}^{2} r_{x} t_{x}^{-1}, r_{x}^{-1} t_{x}^{-1}, r_{y}^{2} t_{y}, r_{y}^{2} t_{y}^{-1}, \\
r_{x}^{2} t_{y}^{-1}, r_{x}^{2} t_{z}, r_{y}^{2} t_{z}^{-1}\end{array}$ \\
\hline & 2266 & $H_{511}$ & 1 & $r_{z}^{2}$ & $\begin{array}{l}r_{z}^{2} r_{x} t_{x}, r_{x} t_{x}, r_{x}^{-1} t_{x}^{-1}, r_{y}^{2} t_{y}, r_{x}^{2} t_{y}, r_{y}^{2} t_{y}^{-1} \\
r_{x}^{2} t_{y}^{-1}, r_{x}^{2} t_{z}, r_{y}^{2} t_{z}^{-1}\end{array}$ \\
\hline & 2267 & $H_{541}$ & 1 & $r_{z}^{2}$ & $\begin{array}{l}r_{z}^{2} r_{x} t_{x}, r_{x} t_{x}, r_{x}^{-1} t_{x}^{-1}, m_{x} t_{y}, m_{y} t_{y}, m_{x} t_{y}^{-1} \\
m_{y} t_{y}^{-1}, m_{z} t_{z}, i t_{z}^{-1}\end{array}$ \\
\hline & 2268 & $H_{527}$ & 1 & $r_{z}^{2}$ & $\begin{array}{l}m_{x} t_{x}, m_{y} t_{x}, m_{x} t_{x}^{-1}, m_{y} t_{x}^{-1}, m_{x} t_{y}, m_{x} t_{y}^{-1}, \\
m_{y} t_{y}^{-1}, r_{y}^{2} r_{z} t_{z}, i t_{z}^{-1}\end{array}$ \\
\hline & 2269 & $H_{529}$ & 1 & $r_{z}^{2}$ & $\begin{array}{l}m_{y} t_{x}, m_{x} t_{x}^{-1}, m_{y} t_{x}^{-1}, m_{x} t_{y}, m_{y} t_{y}, m_{x} t_{y}^{-1}, \\
m_{y} t_{y}^{-1}, r_{y}^{2} r_{z} t_{z}, r_{y}^{2} t_{z}^{-1}\end{array}$ \\
\hline
\end{tabular}

$[9,33,75,132,204,292,396,516,652,804]$ $2270^{*}, 2271^{*}$,

$[9,34,77,133,204,292,396,516,652,804]$

$2272^{*}, 2273^{*}, 2274^{*}, 2275^{*}, 2276^{*}, 2277^{*}, 2278^{*}, 2279^{*}, 2280^{*}, 2281^{*}$,

$[9,35,77,132,204,292,396,516,652,804]$

$2282^{*}, 2283^{*}, 2284^{*}, 2285^{*}, 2286^{*}, 2287^{*}, 2288^{*}, 2289^{*}, 2290^{*}, 2291^{*}, 2292^{*}, 2293^{*}, 2294^{*}$,

2295*, 2296*, 2297*, 2298*, 2299*, 2300*, 2301*,

$[10,35,76,132,204,292,396,516,652,804$ ]

\begin{tabular}{|c|c|c|c|c|}
\hline 2270 & $H_{648}$ & $\left\langle m_{x}\right\rangle$ & $r_{y}^{2} r_{x}$ & $\begin{array}{l}m_{x} t_{x}, m_{x} t_{x}^{-1}, r_{z}^{2} r_{x} t_{y}, r_{x}^{2} t_{y}^{-1}, r_{z}^{2} r_{x} t_{y}^{-1}, r_{x}^{2} t_{z} \\
r_{z}^{2} r_{x} t_{z}, r_{x}^{2} t_{z}^{-1}, r_{z}^{2} r_{x} t_{z}^{-1}\end{array}$ \\
\hline 2271 & $H_{650}$ & $\left\langle m_{x}\right\rangle$ & $r_{y}^{2} r_{x}$ & $\begin{array}{l}m_{x} t_{x}, m_{x} t_{x}^{-1}, m_{y} t_{y}, m_{x} r_{x} t_{y}, m_{y} t_{y}^{-1}, m_{x} r_{x} t_{y}^{-1}, \\
m_{x} r_{x}^{-1} t_{z}, m_{x} r_{x}^{-1} t_{z}^{-1}, r_{y}^{2} t_{z}^{-1}\end{array}$ \\
\hline
\end{tabular}

$[10,36,77,132,204,292,396,516,652,804]$

\begin{tabular}{|c|c|c|c|c|}
\hline 2272 & $H_{422}$ & 1 & $r_{z}^{2} r_{x}$ & $\begin{array}{l}r_{z}^{2} t_{x}, r_{y}^{2} t_{x}^{-1}, m_{x} r_{x}^{-1} t_{y}, m_{y} t_{y}, m_{x} r_{x}^{-1} t_{y}^{-1}, m_{y} t_{y}^{-1} \\
m_{x} r_{x} t_{z}, m_{x} r_{x} t_{z}^{-1}, m_{z} t_{z}^{-1}\end{array}$ \\
\hline 2273 & $H_{409}$ & 1 & $r_{z}^{2} r_{x}$ & $\begin{array}{l}r_{z}^{2} t_{x}, r_{y}^{2} t_{x}^{-1}, r_{y}^{2} r_{x} t_{y}, r_{y}^{2} r_{x} t_{y}^{-1}, r_{x}^{2} t_{y}^{-1}, r_{y}^{2} r_{x} t_{z} \\
r_{x}^{2} t_{z}, r_{y}^{2} r_{x} t_{z}^{-1}, r_{x}^{2} t_{z}^{-1}\end{array}$ \\
\hline 2274 & $H_{560}$ & 1 & $m_{z} r_{x}$ & $\begin{array}{l}i t_{x}, m_{x} t_{x}^{-1}, m_{z} r_{x}^{-1} t_{y}, r_{x}^{2} t_{y}^{-1}, m_{z} r_{x}^{-1} t_{y}^{-1}, r_{x}^{2} t_{z} \\
m_{z} r_{x}^{-1} t_{z}, r_{x}^{2} t_{z}^{-1}, m_{z} r_{x}^{-1} t_{z}^{-1}\end{array}$ \\
\hline 2275 & $H_{560}$ & 1 & $m_{z} r_{x}$ & $\begin{array}{l}r_{z}^{2} r_{x} t_{x}, m_{x} t_{x}^{-1}, m_{z} r_{x}^{-1} t_{y}, r_{x}^{2} t_{y}^{-1}, m_{z} r_{x}^{-1} t_{y}^{-1}, r_{x}^{2} t_{z} \\
m_{z} r_{x}^{-1} t_{z}, r_{x}^{2} t_{z}^{-1}, m_{z} r_{x}^{-1} t_{z}^{-1}\end{array}$ \\
\hline 2276 & $H_{580}$ & 1 & $m_{z} r_{x}$ & $\begin{array}{l}i t_{x}, m_{x} t_{x}^{-1}, m_{y} t_{y}, r_{x} t_{y}, m_{y} t_{y}^{-1}, r_{x} t_{y}^{-1} \\
r_{x}^{-1} t_{z}, r_{x}^{-1} t_{z}^{-1}, m_{z} t_{z}^{-1}\end{array}$ \\
\hline 2277 & $H_{580}$ & 1 & $m_{z} r_{x}$ & $\begin{array}{l}r_{z}^{2} r_{x} t_{x}, m_{x} t_{x}^{-1}, m_{y} t_{y}, r_{x} t_{y}, m_{y} t_{y}^{-1}, r_{x} t_{y}^{-1} \\
r_{x}^{-1} t_{z}, r_{x}^{-1} t_{z}^{-1}, m_{z} t_{z}^{-1}\end{array}$ \\
\hline 2278 & $H_{561}$ & 1 & $m_{z} r_{x}$ & $\begin{array}{l}m_{x} t_{x}, r_{y}^{2} r_{x} t_{x}^{-1}, m_{z} r_{x}^{-1} t_{y}, i t_{y}^{-1}, r_{z}^{2} r_{x} t_{y}^{-1}, r_{x}^{2} t_{z} \\
m_{z} r_{x}^{-1} t_{z}, i t_{z}^{-1}, r_{z}^{2} r_{x} t_{z}^{-1}\end{array}$ \\
\hline 2279 & $H_{571}$ & 1 & $m_{z} r_{x}$ & $\begin{array}{l}m_{x} t_{x}, r_{y}^{2} r_{x} t_{x}^{-1}, m_{y} t_{y}, r_{x} t_{y}, r_{z}^{2} t_{y}^{-1}, m_{x} r_{x} t_{y}^{-1} \\
r_{x}^{-1} t_{z}, m_{x} r_{x}^{-1} t_{z}^{-1}, r_{y}^{2} t_{z}^{-1}\end{array}$ \\
\hline 2280 & $H_{557}$ & 1 & $m_{z} r_{x}$ & $\begin{array}{l}m_{x} t_{x}, r_{y}^{2} r_{x} t_{x}^{-1}, m_{z} r_{x}^{-1} t_{y}, r_{x}^{2} t_{y}^{-1}, m_{z} r_{x}^{-1} t_{y}^{-1}, r_{x}^{2} t_{z}, \\
m_{z} r_{x}^{-1} t_{z}, r_{x}^{2} t_{z}^{-1}, m_{z} r_{x}^{-1} t_{z}^{-1}\end{array}$ \\
\hline 2281 & $H_{578}$ & 1 & $m_{z} r_{x}$ & $\begin{array}{l}m_{x} t_{x}, r_{y}^{2} r_{x} t_{x}^{-1}, m_{y} t_{y}, r_{x} t_{y}, m_{y} t_{y}^{-1}, r_{x} t_{y}^{-1} \\
r_{x}^{-1} t_{z}, r_{x}^{-1} t_{z}^{-1}, m_{z} t_{z}^{-1}\end{array}$ \\
\hline \multicolumn{5}{|r|}{20} \\
\hline 2282 & $H_{649}$ & $\left\langle m_{x}\right\rangle$ & $r_{y}^{2} r_{x}$ & $\begin{array}{l}i t_{x}, r_{x}^{2} t_{x}^{-1}, r_{z}^{2} r_{x} t_{y}, r_{x}^{2} t_{y}^{-1}, r_{z}^{2} r_{x} t_{y}^{-1}, r_{x}^{2} t_{z} \\
r_{z}^{2} r_{x} t_{z}, r_{x}^{2} t_{z}^{-1}, r_{z}^{2} r_{x} t_{z}^{-1}\end{array}$ \\
\hline 2283 & $H_{649}$ & $\left\langle m_{x}\right\rangle$ & $r_{y}^{2} r_{x}$ & $\begin{array}{l}r_{z}^{2} r_{x} t_{x}, r_{z}^{2} r_{x} t_{x}^{-1}, r_{z}^{2} r_{x} t_{y}, r_{x}^{2} t_{y}^{-1}, r_{z}^{2} r_{x} t_{y}^{-1}, r_{x}^{2} t_{z} \\
r_{z}^{2} r_{x} t_{z}, r_{x}^{2} t_{z}^{-1}, r_{z}^{2} r_{x} t_{z}^{-1}\end{array}$ \\
\hline
\end{tabular}




\begin{tabular}{|c|c|c|c|c|c|}
\hline Nbr. & № & $H_{i}$ & $L$ & $m$ & $x$ \\
\hline & 2284 & $H_{652}$ & $\left\langle m_{x}\right\rangle$ & $r_{y}^{2} r_{x}$ & $\begin{array}{l}i t_{x}, r_{x}^{2} t_{x}^{-1}, m_{y} t_{y}, m_{x} r_{x} t_{y}, m_{y} t_{y}^{-1}, m_{x} r_{x} t_{y}^{-1}, \\
m_{x} r_{x}^{-1} t_{z}, m_{x} r_{x}^{-1} t_{z}^{-1}, r_{y}^{2} t_{z}^{-1}\end{array}$ \\
\hline & 2285 & $H_{652}$ & $\left\langle m_{x}\right\rangle$ & $r_{y}^{2} r_{x}$ & $\begin{array}{l}r_{z}^{2} r_{x} t_{x}, r_{z}^{2} r_{x} t_{x}^{-1}, m_{y} t_{y}, m_{x} r_{x} t_{y}, m_{y} t_{y}^{-1}, m_{x} r_{x} t_{y}^{-1} \\
m_{x} r_{x}^{-1} t_{z}, m_{x} r_{x}^{-1} t_{z}^{-1}, r_{y}^{2} t_{z}^{-1}\end{array}$ \\
\hline & 2286 & $H_{422}$ & 1 & $r_{z}^{2} r_{x}$ & $\begin{array}{l}r_{x}^{-1} t_{x}, r_{x} t_{x}^{-1}, m_{x} r_{x}^{-1} t_{y}, m_{y} t_{y}, m_{x} r_{x}^{-1} t_{y}^{-1}, m_{y} t_{y}^{-1}, \\
m_{x} r_{x} t_{z}, m_{x} r_{x} t_{z}^{-1}, m_{z} t_{z}^{-1}\end{array}$ \\
\hline & 2287 & $H_{409}$ & 1 & $r_{z}^{2} r_{x}$ & $\begin{array}{l}r_{x}^{-1} t_{x}, r_{x} t_{x}^{-1}, r_{y}^{2} r_{x} t_{y}, r_{y}^{2} r_{x} t_{y}^{-1}, r_{x}^{2} t_{y}^{-1}, r_{y}^{2} r_{x} t_{z} \\
r_{x}^{2} t_{z}, r_{y}^{2} r_{x} t_{z}^{-1}, r_{x}^{2} t_{z}^{-1}\end{array}$ \\
\hline & 2288 & $H_{648}$ & $\left\langle m_{x}\right\rangle$ & $r_{y}^{2} r_{x}$ & $\begin{array}{l}r_{y}^{2} r_{x} t_{x}, m_{z} r_{x} t_{x}^{-1}, r_{z}^{2} r_{x} t_{y}, r_{x}^{2} t_{y}^{-1}, r_{z}^{2} r_{x} t_{y}^{-1}, r_{x}^{2} t_{z} \\
r_{z}^{2} r_{x} t_{z}, r_{x}^{2} t_{z}^{-1}, r_{z}^{2} r_{x} t_{z}^{-1}\end{array}$ \\
\hline & 2289 & $H_{650}$ & $\left\langle m_{x}\right\rangle$ & $r_{y}^{2} r_{x}$ & $\begin{array}{l}r_{y}^{2} r_{x} t_{x}, m_{z} r_{x} t_{x}^{-1}, m_{y} t_{y}, m_{x} r_{x} t_{y}, m_{y} t_{y}^{-1}, m_{x} r_{x} t_{y}^{-1}, \\
m_{x} r_{x}^{-1} t_{z}, m_{x} r_{x}^{-1} t_{z}^{-1}, r_{y}^{2} t_{z}^{-1}\end{array}$ \\
\hline & 2290 & $H_{551}$ & 1 & $m_{z} r_{x}$ & $\begin{array}{l}r_{z}^{2} r_{x} t_{x}, i t_{x}^{-1}, r_{z}^{2} r_{x} t_{y}, i t_{y}^{-1}, r_{z}^{2} r_{x} t_{y}^{-1}, i t_{z} \\
r_{z}^{2} r_{x} t_{z}, i t_{z}^{-1}, r_{z}^{2} r_{x} t_{z}^{-1}\end{array}$ \\
\hline & 2291 & $H_{572}$ & 1 & $m_{z} r_{x}$ & $\begin{array}{l}r_{z}^{2} r_{x} t_{x}, i t_{x}^{-1}, r_{z}^{2} t_{y}, m_{x} r_{x} t_{y}, r_{z}^{2} t_{y}^{-1}, m_{x} r_{x} t_{y}^{-1} \\
m_{x} r_{x}^{-1} t_{z}, m_{x} r_{x}^{-1} t_{z}^{-1}, r_{y}^{2} t_{z}^{-1}\end{array}$ \\
\hline & 2292 & $H_{555}$ & 1 & $m_{z} r_{x}$ & $\begin{array}{l}r_{z}^{2} r_{x} t_{x}, i t_{x}^{-1}, m_{z} r_{x}^{-1} t_{y}, r_{x}^{2} t_{y}^{-1}, m_{z} r_{x}^{-1} t_{y}^{-1}, r_{x}^{2} t_{z}, \\
m_{z} r_{x}^{-1} t_{z}, r_{x}^{2} t_{z}^{-1}, m_{z} r_{x}^{-1} t_{z}^{-1}\end{array}$ \\
\hline & 2293 & $H_{574}$ & 1 & $m_{z} r_{x}$ & $\begin{array}{l}r_{z}^{2} r_{x} t_{x}, i t_{x}^{-1}, m_{y} t_{y}, r_{x} t_{y}, m_{y} t_{y}^{-1}, r_{x} t_{y}^{-1} \\
r_{x}^{-1} t_{z}, r_{x}^{-1} t_{z}^{-1}, m_{z} t_{z}^{-1}\end{array}$ \\
\hline & 2294 & $H_{560}$ & 1 & $m_{z} r_{x}$ & $\begin{array}{l}i t_{x}, r_{y}^{2} r_{x} t_{x}^{-1}, m_{z} r_{x}^{-1} t_{y}, r_{x}^{2} t_{y}^{-1}, m_{z} r_{x}^{-1} t_{y}^{-1}, r_{x}^{2} t_{z} \\
m_{z} r_{x}^{-1} t_{z}, r_{x}^{2} t_{z}^{-1}, m_{z} r_{x}^{-1} t_{z}^{-1}\end{array}$ \\
\hline & 2295 & $H_{560}$ & 1 & $m_{z} r_{x}$ & $\begin{array}{l}r_{z}^{2} r_{x} t_{x}, r_{y}^{2} r_{x} t_{x}^{-1}, m_{z} r_{x}^{-1} t_{y}, r_{x}^{2} t_{y}^{-1}, m_{z} r_{x}^{-1} t_{y}^{-1}, r_{x}^{2} t_{z}, \\
m_{z} r_{x}^{-1} t_{z}, r_{x}^{2} t_{z}^{-1}, m_{z} r_{x}^{-1} t_{z}^{-1}\end{array}$ \\
\hline & 2296 & $H_{580}$ & 1 & $m_{z} r_{x}$ & $\begin{array}{l}i t_{x}, r_{y}^{2} r_{x} t_{x}^{-1}, m_{y} t_{y}, r_{x} t_{y}, m_{y} t_{y}^{-1}, r_{x} t_{y}^{-1} \\
r_{x}^{-1} t_{z}, r_{x}^{-1} t_{z}^{-1}, m_{z} t_{z}^{-1}\end{array}$ \\
\hline & 2297 & $H_{580}$ & 1 & $m_{z} r_{x}$ & $\begin{array}{l}r_{z}^{2} r_{x} t_{x}, r_{y}^{2} r_{x} t_{x}^{-1}, m_{y} t_{y}, r_{x} t_{y}, m_{y} t_{y}^{-1}, r_{x} t_{y}^{-1}, \\
r_{x}^{-1} t_{z}, r_{x}^{-1} t_{z}^{-1}, m_{z} t_{z}^{-1}\end{array}$ \\
\hline & 2298 & $H_{556}$ & 1 & $m_{z} r_{x}$ & $\begin{array}{l}m_{x} t_{x}, r_{y}^{2} r_{x} t_{x}^{-1}, r_{z}^{2} r_{x} t_{y}, i t_{y}^{-1}, r_{z}^{2} r_{x} t_{y}^{-1}, i t_{z} \\
r_{z}^{2} r_{x} t_{z}, i t_{z}^{-1}, r_{z}^{2} r_{x} t_{z}^{-1}\end{array}$ \\
\hline & 2299 & $H_{561}$ & 1 & $m_{z} r_{x}$ & $\begin{array}{l}m_{x} t_{x}, r_{y}^{2} r_{x} t_{x}^{-1}, r_{x}^{2} t_{y}, m_{z} r_{x}^{-1} t_{y}, i t_{y}^{-1}, r_{z}^{2} r_{x} t_{y}^{-1} \\
r_{x}^{2} t_{z}, m_{z} r_{x}^{-1} t_{z}, r_{z}^{2} r_{x} t_{z}^{-1}\end{array}$ \\
\hline & 2300 & $H_{576}$ & 1 & $m_{z} r_{x}$ & $\begin{array}{l}m_{x} t_{x}, r_{y}^{2} r_{x} t_{x}^{-1}, r_{z}^{2} t_{y}, m_{x} r_{x} t_{y}, r_{z}^{2} t_{y}^{-1}, m_{x} r_{x} t_{y}^{-1}, \\
m_{x} r_{x}^{-1} t_{z}, m_{x} r_{x}^{-1} t_{z}^{-1}, r_{y}^{2} t_{z}^{-1}\end{array}$ \\
\hline & 2301 & $H_{571}$ & 1 & $m_{z} r_{x}$ & $\begin{array}{l}m_{x} t_{x}, r_{y}^{2} r_{x} t_{x}^{-1}, m_{y} t_{y}, r_{x} t_{y}, r_{z}^{2} t_{y}^{-1}, m_{x} r_{x} t_{y}^{-1} \\
r_{x}^{-1} t_{z}, m_{z} t_{z}, m_{x} r_{x}^{-1} t_{z}^{-1}\end{array}$ \\
\hline
\end{tabular}

$42 \mathrm{~A}$

$[6,24,64,130,207,289,396,519,649,804]$ $2302^{*}$,

$[6,24,65,116,179,260,354,467,593,734]$ $2303 *$

$[7,29,70,134,205,289,398,517,649,806]$ $2302 \quad H_{393} \quad 1$ $r_{z}^{2} r_{x} \quad r_{y}^{2} r_{z} t_{x}, r_{y}^{-1} r_{z}^{-1} t_{x}^{-1}, r_{y}^{2} r_{z} t_{y}, r_{y} r_{x} t_{y}, r_{x}^{2} r_{y} t_{y}^{-1}, r_{y}^{2} r_{z} t_{z}$

$\left[\begin{array}{c}\text { 7, 29,71, 119, 188, 269,368, 481, 609,752] } \\ 2303 \quad H_{393} 1\end{array} r_{z}^{2} r_{x} r_{y}^{2} r_{z} t_{x}, r_{y}^{-1} r_{z}^{-1} t_{x}^{-1}, r_{y}^{2} r_{z} t_{y}, r_{y} r_{x} t_{y}, r_{y}^{-1} r_{z}^{-1} t_{y}^{-1}, r_{y} r_{x} t_{z}\right.$ $42 \mathrm{~B}$

$[6,24,56,98,153,222,303,396,503,622]$ $2304^{*}$

$[6,24,57,101,158,228,310,405,513,633]$ 2305*,

$[7,29,63,110,172,249,338,441,560,691]$ $2304 \quad H_{393} \quad 1$

$[7,29,64,112,175,252,342,446,565,697]$ $2305 \quad H_{393} \quad r_{z}^{2} r_{x} \quad r_{y}^{2} r_{z} t_{x}, r_{y} r_{x} t_{x}, r_{y}^{2} r_{z} t_{y}, r_{x}^{2} r_{y} t_{y}^{-1}, r_{y}^{2} r_{z} t_{z}, r_{y}^{-1} r_{z}^{-1} t_{z}^{-1}$

$[8,30,73,135,210,296,397,516,652,804]$ $2306^{*}, 2307^{*}$

$[8,31,73,129,200,288,392,512,648,800]$ $2308^{*}, 2309^{*}$,

$[8,31,75,134,205,292,396,516,652,804]$ $2310^{*}, 2311^{*}$,

$[8,31,75,136,209,294,396,516,652,804]$ $2312^{*}, 2313^{*}$

$[8,31,76,137,208,293,396,516,652,804]$ $2314^{*}, 2315^{*}, 2316^{*}, 2317^{*}$

$[8,32,73,128,200,288,392,512,648,800]$ $2318^{*}, 2319^{*}$,

$[8,32,76,132,202,290,394,514,650,802]$ $2320^{*}, 2321^{*}, 2322^{*}, 2323^{*}, 2324^{*}, 2325^{*}$

$[8,32,76,133,204,292,396,516,652,804]$ $2326^{*}, 2327 *$

$[8,32,77,135,205,292,396,516,652,804]$ $2328^{*}, 2329^{*}, 2330^{*}, 2331^{*}$,

$[8,32,77,136,207,293,396,516,652,804]$ $2332 *$,

$[8,32,78,135,204,292,396,516,652,804]$ 2333*, 2334*, 
$\begin{array}{ccccccc}\text { Nbr. } & \text { gr } & \text { № } & H_{i} & L & m & X\end{array}$

$32,78,137,206,292,396,516,6$

$[8,33,76,131,202,290,394,514,650,802]$ $2339 *$

$[8,33,77,135,207,293,396,516,652,804]$ $2340^{*}, 2341^{*}, 2342^{*}, 2343^{*}, 2344^{*}, 2345^{*}, 2346^{*}, 2347^{*}, 2348^{*}, 2349^{*}, 2350^{*}$,

$[8,33,78,134,204,292,396,516,652,804]$ $2351 *$

$[8,33,78,136,206,292,396,516,652,804]$ $2352 *$

$[8,34,76,130,202,290,394,514,650,802]$ $2353^{*}, 2354^{*}, 2355^{*}, 2356^{*}, 2357^{*}, 2358^{*}, 2359^{*}$

$[8,34,78,133,204,292,396,516,652,804]$ $2360^{*}, 2361^{*}, 2362^{*}, 2363^{*}, 2364^{*}, 2365^{*}, 2366^{*}$

$[8,34,78,135,206,292,396,516,652,804]$

$2367^{*}, 2368^{*}, 2369^{*}, 2370^{*}, 2371^{*}, 2372^{*}, 2373^{*}, 2374^{*}, 2375^{*}, 2376^{*}, 2377^{*}, 2378^{*}, 2379^{*}$

[ $9,33,76,135,207,293,396,516,652,804$ ]
$2306 \quad H_{580} \quad 1$
$m_{z} r_{x} \quad$ it $t_{x}, r_{z}^{2} r_{x} t_{x}, m_{x} t_{x}^{-1}, m_{y} t_{y}, m_{y} t_{y}^{-1}, r_{x} t_{y}^{-1}$,
$2307 \quad H_{578} \quad 1$
$m_{z} t_{z}, r_{x}^{-1} t_{z}^{-1}$
$m_{z} t_{z}, r_{x}^{-1} t_{z}^{-1} r_{y}^{2} r_{x}{ }_{x}^{-1}, m_{x} t_{x}^{-1}, m_{y} t_{y}, m_{y} t_{y}^{-1}, r_{x} t_{y}^{-1}$

$9,34,75,130,202,290,394,514,650,802]$

\begin{tabular}{|c|c|c|c|c|}
\hline 2308 & $H_{560}$ & 1 & $m_{z} r_{x}$ & $\begin{array}{l}i t_{x}, r_{z}^{2} r_{x} t_{x}, m_{x} t_{x}^{-1}, m_{z} r_{x}^{-1} t_{y}, r_{x}^{2} t_{y}^{-1}, m_{z} r_{x}^{-1} t_{y}^{-1} \\
m_{z} r_{x}^{-1} t_{z}, m_{z} r_{x}^{-1} t_{z}^{-1}\end{array}$ \\
\hline 2309 & $H_{557}$ & 1 & $m_{z} r_{x}$ & $\begin{array}{l}m_{x} t_{x}, r_{y}^{2} r_{x} t_{x}^{-1}, m_{x} t_{x}^{-1}, m_{z} r_{x}^{-1} t_{y}, r_{x}^{2} t_{y}^{-1}, m_{z} r_{x}^{-1} t_{y}^{-1} \\
m_{z} r_{x}^{-1} t_{z}, m_{z} r_{x}^{-1} t_{z}^{-1}\end{array}$ \\
\hline
\end{tabular}

$[9,34,77,133,204,292,396,516,652,804]$

$\begin{array}{lllll}2310 & H_{580} & 1 & m_{z} r_{x} & \begin{array}{l}i t_{x}, r_{z}^{2} r_{x} t_{x}, m_{x} t_{x}^{-1}, r_{x} t_{y}, m_{y} t_{y}^{-1}, r_{x} t_{y}^{-1}, \\ r_{x}^{-1} t_{z}, r_{x}^{-1} t_{z}^{-1}\end{array} \\ 2311 & H_{578} & 1 & m_{z} r_{x} & \begin{array}{l}m_{x} t_{x}, r_{y}^{2} r_{x} t_{x}^{-1}, m_{x} t_{x}^{-1}, r_{x} t_{y}, m_{y}{ }_{y}^{-1}, r_{x} t_{y}^{-1}, \\ r_{x}^{-1} t_{z}, r_{x}^{-1} t_{z}^{-1}\end{array}\end{array}$

$[9,34,77,135,206,292,396,516,652,804]$

\begin{tabular}{|c|c|c|c|c|}
\hline 2312 & $H_{560}$ & 1 & $m_{z} r_{x}$ & $\begin{array}{l}i t_{x}, r_{z}^{2} r_{x} t_{x}, m_{x} t_{x}^{-1}, m_{z} r_{x}^{-1} t_{y}, r_{x}^{2} t_{y}^{-1}, r_{x}^{2} t_{z} \\
m_{z} r_{x}^{-1} t_{z}, r_{x}^{2} t_{z}^{-1}\end{array}$ \\
\hline 2313 & $H_{557}$ & 1 & $m_{z} r_{x}$ & $\begin{array}{l}m_{x} t_{x}, r_{y}^{2} r_{x} t_{x}^{-1}, m_{x} t_{x}^{-1}, m_{z} r_{x}^{-1} t_{y}, r_{x}^{2} t_{y}^{-1}, r_{x}^{2} t_{z} \\
m_{z} r_{x}^{-1} t_{z}, r_{x}^{2} t_{z}^{-1}\end{array}$ \\
\hline \multicolumn{5}{|c|}{$[9,34,78,135,205,292,396,516,652,804]$} \\
\hline 2314 & $H_{580}$ & 1 & $m_{z} r_{x}$ & $\begin{array}{l}i t_{x}, r_{z}^{2} r_{x} t_{x}, r_{y}^{2} r_{x} t_{x}^{-1}, m_{y} t_{y}, m_{y} t_{y}^{-1}, r_{x} t_{y}^{-1} \\
m_{z} t_{z}, r_{x}^{-1} t_{z}^{-1}\end{array}$ \\
\hline 2315 & $H_{571}$ & 1 & $m_{z} r_{x}$ & $\begin{array}{l}r_{y}^{2} r_{x} t_{x}, r_{y}^{2} r_{x} t_{x}^{-1}, m_{x} t_{x}^{-1}, m_{y} t_{y}, r_{z}^{2} t_{y}^{-1}, m_{x} r_{x} t_{y}^{-1} \\
m_{z} t_{z}, m_{x} r_{x}^{-1} t_{z}^{-1}\end{array}$ \\
\hline 2316 & $H_{571}$ & 1 & $m_{z} r_{x}$ & $\begin{array}{l}m_{x} t_{x}, r_{y}^{2} r_{x} t_{x}^{-1}, m_{x} t_{x}^{-1}, m_{y} t_{y}, r_{z}^{2} t_{y}^{-1}, m_{x} r_{x} t_{y}^{-1} \\
m_{z} t_{z}, m_{x} r_{x}^{-1} t_{z}^{-1}\end{array}$ \\
\hline 2317 & $H_{578}$ & 1 & $m_{z} r_{x}$ & $\begin{array}{l}r_{y}^{2} r_{x} t_{x}, r_{y}^{2} r_{x} t_{x}^{-1}, m_{x} t_{x}^{-1}, m_{y} t_{y}, m_{y} t_{y}^{-1}, r_{x} t_{y}^{-1} \\
m_{z} t_{z}, r_{x}^{-1} t_{z}^{-1}\end{array}$ \\
\hline \multicolumn{5}{|c|}{$[9,35,74,130,202,290,394,514,650,802]$} \\
\hline 2318 & $H_{555}$ & 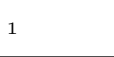 & $m_{z} r_{x}$ & $\begin{array}{l}r_{z}^{2} r_{x} t_{x}, i t_{x}^{-1}, r_{z}^{2} r_{x} t_{x}^{-1}, m_{z} r_{x}^{-1} t_{y}, r_{x}^{2} t_{y}^{-1}, m_{z} r_{x}^{-1} t_{y}^{-1} \\
m_{z} r_{x}^{-1} t_{z}, m_{z} r_{x}^{-1} t_{z}^{-1}\end{array}$ \\
\hline 2319 & $H_{560}$ & ${ }_{1}$ & $m_{z} r_{x}$ & $\begin{array}{l}r_{z}^{2} r_{x} t_{x}, r_{y}^{2} r_{x} t_{x}^{-1}, m_{x} t_{x}^{-1}, m_{z} r_{x}^{-1} t_{y}, r_{x}^{2} t_{y}^{-1}, m_{z} r_{x}^{-1} t_{y}^{-1}, \\
m_{z} r_{x}^{-1} t_{z}, m_{z} r_{x}^{-1} t_{z}^{-1}\end{array}$ \\
\hline \multicolumn{5}{|c|}{$[9,35,77,131,203,291,395,515,651,803]$} \\
\hline 2320 & $H_{555}$ & 1 & $m_{z} r_{x}$ & $\begin{array}{l}i t_{x}, i t_{x}^{-1}, r_{z}^{2} r_{x} t_{x}^{-1}, m_{z} r_{x}^{-1} t_{y}, r_{x}^{2} t_{y}^{-1}, m_{z} r_{x}^{-1} t_{y}^{-1} \\
m_{z} r_{x}^{-1} t_{z}, m_{z} r_{x}^{-1} t_{z}^{-1}\end{array}$ \\
\hline 2321 & $H_{560}$ & 1 & $m_{z} r_{x}$ & $\begin{array}{l}i t_{x}, r_{z}^{2} r_{x} t_{x}, r_{y}^{2} r_{x} t_{x}^{-1}, m_{z} r_{x}^{-1} t_{y}, r_{x}^{2} t_{y}^{-1}, m_{z} r_{x}^{-1} t_{y}^{-1}, \\
m_{z} r_{x}^{-1} t_{z}, m_{z} r_{x}^{-1} t_{z}^{-1}\end{array}$ \\
\hline 2322 & $H_{560}$ & 1 & $m_{z} r_{x}$ & $\begin{array}{l}i t_{x}, r_{y}^{2} r_{x} t_{x}^{-1}, m_{x} t_{x}^{-1}, m_{z} r_{x}^{-1} t_{y}, r_{x}^{2} t_{y}^{-1}, m_{z} r_{x}^{-1} t_{y}^{-1} \\
m_{z} r_{x}^{-1} t_{z}, m_{z} r_{x}^{-1} t_{z}^{-1}\end{array}$ \\
\hline 2323 & $H_{561}$ & 1 & $m_{z} r_{x}$ & $\begin{array}{l}r_{y}^{2} r_{x} t_{x}, r_{y}^{2} r_{x} t_{x}^{-1}, m_{x} t_{x}^{-1}, m_{z} r_{x}^{-1} t_{y}, i t_{y}^{-1}, r_{z}^{2} r_{x} t_{y}^{-1} \\
m_{z} r_{x}^{-1} t_{z}, r_{z}^{2} r_{x} t_{z}^{-1}\end{array}$ \\
\hline 2324 & $H_{561}$ & 1 & $m_{z} r_{x}$ & $\begin{array}{l}m_{x} t_{x}, r_{y}^{2} r_{x} t_{x}^{-1}, m_{x} t_{x}^{-1}, m_{z} r_{x}^{-1} t_{y}, i t_{y}^{-1}, r_{z}^{2} r_{x} t_{y}^{-1} \\
m_{z} r_{x}^{-1} t_{z}, r_{z}^{2} r_{x} t_{z}^{-1}\end{array}$ \\
\hline 2325 & $H_{557}$ & 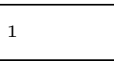 & $m_{z} r_{x}$ & $\begin{array}{l}r_{y}^{2} r_{x} t_{x}, r_{y}^{2} r_{x} t_{x}^{-1}, m_{x} t_{x}^{-1}, m_{z} r_{x}^{-1} t_{y}, r_{x}^{2} t_{y}^{-1}, m_{z} r_{x}^{-1} t_{y}^{-1}, \\
m_{z} r_{x}^{-1} t_{z}, m_{z} r_{x}^{-1} t_{z}^{-1}\end{array}$ \\
\hline \multicolumn{5}{|c|}{$[9,35,77,132,204,292,396,516,652,804]$} \\
\hline 2326 & $H_{574}$ & 1 & $m_{z} r_{x}$ & $\begin{array}{l}r_{z}^{2} r_{x} t_{x}, i t_{x}^{-1}, r_{z}^{2} r_{x} t_{x}^{-1}, r_{x} t_{y}, m_{y} t_{y}^{-1}, r_{x} t_{y}^{-1} \\
r_{x}^{-1} t_{z}, r_{x}^{-1} t_{z}^{-1}\end{array}$ \\
\hline 2327 & $H_{580}$ & ( & $m_{z} r_{x}$ & $\begin{array}{l}r_{z}^{2} r_{x} t_{x}, r_{y}^{2} r_{x} t_{x}^{-1}, m_{x} t_{x}^{-1}, r_{x} t_{y}, m_{y} t_{y}^{-1}, r_{x} t_{y}^{-1}, \\
r_{x}^{-1} t_{z}, r_{x}^{-1} t_{z}^{-1}\end{array}$ \\
\hline \multicolumn{5}{|c|}{$[9,35,78,133,204,292,396,516,652,804]$} \\
\hline 2328 & $H_{580}$ & 1 & $m_{z} r_{x}$ & $\begin{array}{l}i t_{x}, r_{z}^{2} r_{x} t_{x}, r_{y}^{2} r_{x} t_{x}^{-1}, r_{x} t_{y}, m_{y} t_{y}^{-1}, r_{x} t_{y}^{-1} \\
r_{x}^{-1} t_{z}, r_{x}^{-1} t_{z}^{-1}\end{array}$ \\
\hline 2329 & $H_{571}$ & 1 & $m_{z} r_{x}$ & $\begin{array}{l}r_{y}^{2} r_{x} t_{x}, r_{y}^{2} r_{x} t_{x}^{-1}, m_{x} t_{x}^{-1}, r_{x} t_{y}, r_{z}^{2} t_{y}^{-1}, m_{x} r_{x} t_{y}^{-1} \\
r_{x}^{-1} t_{z}, m_{x} r_{x}^{-1} t_{z}^{-1}\end{array}$ \\
\hline 2330 & $H_{571}$ & 1 & $m_{z} r_{x}$ & $\begin{array}{l}m_{x} t_{x}, r_{y}^{2} r_{x} t_{x}^{-1}, m_{x} t_{x}^{-1}, r_{x} t_{y}, r_{z}^{2} t_{y}^{-1}, m_{x} r_{x} t_{y}^{-1} \\
r_{x}^{-1} t_{z}, m_{x} r_{x}^{-1} t_{z}^{-1}\end{array}$ \\
\hline
\end{tabular}




\begin{tabular}{|c|c|c|c|c|c|c|}
\hline Nbr. & $\mathrm{gr}$ & № & $H_{i}$ & $L$ & $m$ & $x$ \\
\hline & & 2331 & $H_{578}$ & 1 & $m_{z} r_{x}$ & $\begin{array}{l}r_{y}^{2} r_{x} t_{x}, r_{y}^{2} r_{x} t_{x}^{-1}, m_{x} t_{x}^{-1}, r_{x} t_{y}, m_{y} t_{y}^{-1}, r_{x} t_{y}^{-1}, \\
r_{x}^{-1} t_{z}, r_{x}^{-1} t_{z}^{-1}\end{array}$ \\
\hline \multicolumn{7}{|c|}{$[9,35,78,134,205,292,396,516,652,804]$} \\
\hline & & 2332 & $H_{571}$ & 1 & $m_{z} r_{x}$ & $\begin{array}{l}m_{x} t_{x}, r_{y}^{2} r_{x} t_{x}^{-1}, m_{x} t_{x}^{-1}, m_{y} t_{y}, r_{x} t_{y}, r_{z}^{2} t_{y}^{-1} \\
r_{x}^{-1} t_{z}, r_{y}^{2} t_{z}^{-1}\end{array}$ \\
\hline \multicolumn{7}{|c|}{$[9,35,79,132,204,292,396,516,652,804]$} \\
\hline & & 2333 & $H_{574}$ & 1 & $m_{z} r_{x}$ & $\begin{array}{l}\text { it }_{x}, i t_{x}^{-1}, r_{z}^{2} r_{x} t_{x}^{-1}, r_{x} t_{y}, m_{y} t_{y}^{-1}, r_{x} t_{y}^{-1} \\
r_{x}^{-1} t_{z}, r_{x}^{-1} t_{z}^{-1}\end{array}$ \\
\hline & & 2334 & $H_{580}$ & 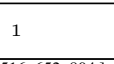 & $m_{z} r_{x}$ & $\begin{array}{l}i t_{x}, r_{y}^{2} r_{x} t_{x}^{-1}, m_{x} t_{x}^{-1}, r_{x} t_{y}, m_{y} t_{y}^{-1}, r_{x} t_{y}^{-1}, \\
r_{x}^{-1} t_{z}, r_{x}^{-1} t_{z}^{-1}\end{array}$ \\
\hline \multicolumn{7}{|c|}{$[9,35,79,134,204,292,396,516,652,804]$} \\
\hline & & 2335 & $H_{560}$ & 1 & $m_{z} r_{x}$ & $\begin{array}{l}i t_{x}, r_{z}^{2} r_{x} t_{x}, r_{y}^{2} r_{x} t_{x}^{1}, m_{z} r_{x} t_{y}, r_{x}^{2} t_{y}^{1}, r_{x}^{2} t_{z} \\
m_{z} r_{x}^{-1} t_{z}, r_{x}^{2} t_{z}^{-1}\end{array}$ \\
\hline & & 2336 & $H_{561}$ & 1 & $m_{z} r_{x}$ & $\begin{array}{l}r_{y}^{2} r_{x} t_{x}, r_{y}^{2} r_{x} t_{x}^{-1}, m_{x} t_{x}^{-1}, r_{x}^{2} t_{y}, i t_{y}^{-1}, r_{z}^{2} r_{x} t_{y}^{-1}, \\
r_{x}^{2} t_{z}, r_{z}^{2} r_{x} t_{z}^{-1}\end{array}$ \\
\hline & & 2337 & $H_{561}$ & 1 & $m_{z} r_{x}$ & $\begin{array}{l}m_{x} t_{x}, r_{y}^{2} r_{x} t_{x}^{-1}, m_{x} t_{x}^{-1}, r_{x}^{2} t_{y}, i t_{y}^{-1}, r_{z}^{2} r_{x} t_{y}^{-1} \\
r_{x}^{2} t_{z}, r_{z}^{2} r_{x} t_{z}^{-1}\end{array}$ \\
\hline & & 2338 & $H_{557}$ & 1 & $m_{z} r_{x}$ & $\begin{array}{l}r_{y}^{2} r_{x} t_{x}, r_{y}^{2} r_{x} t_{x}^{-1}, m_{x} t_{x}^{-1}, m_{z} r_{x}^{-1} t_{y}, r_{x}^{2} t_{y}^{-1}, r_{x}^{2} t_{z} \\
m_{z} r_{x}^{-1} t_{z}, r_{x}^{2} t_{z}^{-1}\end{array}$ \\
\hline \multicolumn{7}{|c|}{$[9,36,76,131,203,291,395,515,651,803]$} \\
\hline & & 2339 & $H_{561}$ & 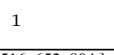 & $m_{z} r_{x}$ & $\begin{array}{l}m_{x} t_{x}, r_{y}^{2} r_{x} t_{x}^{-1}, m_{x} t_{x}^{-1}, m_{z} r_{x}^{-1} t_{y}, r_{z}^{2} r_{x} t_{y}^{-1}, r_{x}^{2} t_{z} \\
m_{z} r_{x}^{-1} t_{z}, r_{z}^{2} r_{x} t_{z}^{-1}\end{array}$ \\
\hline \multicolumn{7}{|c|}{$[9,36,77,134,205,292,396,516,652,804]$} \\
\hline & & 2340 & $H_{572}$ & 1 & $m_{z} r_{x}$ & $\begin{array}{l}i t_{x}, i t_{x}^{-1}, r_{z}^{2} r_{x} t_{x}^{-1}, r_{z}^{2} t_{y}, r_{z}^{2} t_{y}^{-1}, m_{x} r_{x} t_{y}^{-1} \\
r_{y}^{2} t_{z}, m_{x} r_{x}^{-1} t_{z}^{-1}\end{array}$ \\
\hline & & 2341 & $H_{572}$ & 1 & $m_{z} r_{x}$ & $\begin{array}{l}r_{z}^{2} r_{x} t_{x}, i t_{x}^{-1}, r_{z}^{2} r_{x} t_{x}^{-1}, r_{z}^{2} t_{y}, r_{z}^{2} t_{y}^{-1}, m_{x} r_{x} t_{y}^{-1} \\
r_{y}^{2} t_{z}, m_{x} r_{x}^{-1} t_{z}^{-1}\end{array}$ \\
\hline & & 2342 & $H_{574}$ & 1 & $m_{z} r_{x}$ & $\begin{array}{l}i t_{x}, i t_{x}^{-1}, r_{z}^{2} r_{x} t_{x}^{-1}, m_{y} t_{y}, m_{y} t_{y}^{-1}, r_{x} t_{y}^{-1} \\
m_{z} t_{z}, r_{x}^{-1} t_{z}^{-1}\end{array}$ \\
\hline & & 2343 & $H_{574}$ & 1 & $m_{z} r_{x}$ & $\begin{array}{l}r_{z}^{2} r_{x} t_{x}, i t_{x}^{-1}, r_{z}^{2} r_{x} t_{x}^{-1}, m_{y} t_{y}, m_{y} t_{y}^{-1}, r_{x} t_{y}^{-1}, \\
m_{z} t_{z}, r_{x}^{-1} t_{z}^{-1}\end{array}$ \\
\hline & & 2344 & $H_{580}$ & 1 & $m_{z} r_{x}$ & $\begin{array}{l}i t_{x}, r_{y}^{2} r_{x} t_{x}^{-1}, m_{x} t_{x}^{-1}, m_{y} t_{y}, m_{y} t_{y}^{-1}, r_{x} t_{y}^{-1} \\
m_{z} t_{z}, r_{x}^{-1} t_{z}^{-1}\end{array}$ \\
\hline & & 2345 & $H_{580}$ & 1 & $m_{z} r_{x}$ & $\begin{array}{l}r_{z}^{2} r_{x} t_{x}, r_{y}^{2} r_{x} t_{x}^{-1}, m_{x} t_{x}^{-1}, m_{y} t_{y}, m_{y} t_{y}^{-1}, r_{x} t_{y}^{-1}, \\
m_{z} t_{z}, r_{x}^{-1} t_{z}^{-1}\end{array}$ \\
\hline & & 2346 & $H_{576}$ & 1 & $m_{z} r_{x}$ & $\begin{array}{l}r_{y}^{2} r_{x} t_{x}, r_{y}^{2} r_{x} t_{x}^{-1}, m_{x} t_{x}^{-1}, r_{z}^{2} t_{y}, r_{z}^{2} t_{y}^{-1}, m_{x} r_{x} t_{y}^{-1} \\
r_{y}^{2} t_{z}, m_{x} r_{x}^{-1} t_{z}^{-1}\end{array}$ \\
\hline & & 2347 & $H_{576}$ & 1 & $m_{z} r_{x}$ & $\begin{array}{l}m_{x} t_{x}, r_{y}^{2} r_{x} t_{x}^{-1}, m_{x} t_{x}^{-1}, r_{z}^{2} t_{y}, r_{z}^{2} t_{y}^{-1}, m_{x} r_{x} t_{y}^{-1} \\
r_{y}^{2} t_{z}, m_{x} r_{x}^{-1} t_{z}^{-1}\end{array}$ \\
\hline & & 2348 & $H_{576}$ & 1 & $m_{z} r_{x}$ & $\begin{array}{l}m_{x} t_{x}, r_{y}^{2} r_{x} t_{x}^{-1}, m_{x} t_{x}^{-1}, r_{z}^{2} t_{y}, m_{x} r_{x} t_{y}, r_{z}^{2} t_{y}^{-1} \\
m_{x} r_{x}^{-1} t_{z}, r_{y}^{2} t_{z}^{-1}\end{array}$ \\
\hline & & 2349 & $H_{576}$ & 1 & $m_{z} r_{x}$ & $\begin{array}{l}r_{y}^{2} r_{x} t_{x}, r_{y}^{2} r_{x} t_{x}^{-1}, m_{x} t_{x}^{-1}, r_{z}^{2} t_{y}, m_{x} r_{x} t_{y}^{-1}, r_{y}^{2} t_{z}, \\
m_{x} r_{x}^{-1} t_{z}^{-1}, r_{y}^{2} t_{z}^{-1}\end{array}$ \\
\hline & & 2350 & $H_{571}$ & 1 & $m_{z} r_{x}$ & $\begin{array}{l}r_{y}^{2} r_{x} t_{x}, r_{y}^{2} r_{x} t_{x}^{-1}, m_{x} t_{x}^{-1}, m_{y} t_{y}, r_{x} t_{y}, r_{z}^{2} t_{y}^{-1} \\
r_{x}^{-1} t_{z}, r_{y}^{2} t_{z}^{-1}\end{array}$ \\
\hline \multicolumn{7}{|c|}{$[9,36,78,132,204,292,396,516,652,804]$} \\
\hline & & 2351 & $H_{571}$ & 5 & $m_{z} r_{x}$ & $\begin{array}{l}m_{x} t_{x}, r_{y}^{2} r_{x} t_{x}^{-1}, m_{x} t_{x}^{-1}, m_{y} t_{y}, r_{x} t_{y}, m_{x} r_{x} t_{y}^{-1} \\
r_{x}^{-1} t_{z}, m_{x} r_{x}^{-1} t_{z}^{-1}\end{array}$ \\
\hline \multicolumn{7}{|c|}{$[9,36,78,134,204,292,396,516,652,804]$} \\
\hline & & 2352 & $H_{561}$ & Fe 10 & $m_{z} r_{x}$ & $\begin{array}{l}m_{x} t_{x}, r_{y}^{2} r_{x} t_{x}^{-1}, m_{x} t_{x}^{-1}, m_{z} r_{x}^{-1} t_{y}, i t_{y}^{-1}, r_{x}^{2} t_{z}, \\
m_{z} r_{x}^{-1} t_{z}, i t_{z}^{-1}\end{array}$ \\
\hline \multicolumn{7}{|c|}{$[9,37,75,131,203,291,395,515,651,803]$} \\
\hline & & 2353 & $H_{551}$ & 1 & $m_{z} r_{x}$ & $\begin{array}{l}{ }^{t_{x}}, \imath t_{x}, r_{z}^{r_{x} r_{x}}, r_{z}^{r_{x} t_{y}, \imath t} y, r_{z}^{r_{x} t} y \\
r_{z}^{2} r_{x} t_{z}, r_{z}^{2} r_{x} t_{z}^{-1}\end{array}$ \\
\hline & & 2354 & $H_{551}$ & 1 & $m_{z} r_{x}$ & $\begin{array}{l}r_{z}^{2} r_{x} t_{x}, i t_{x}^{-1}, r_{z}^{2} r_{x} t_{x}^{-1}, r_{z}^{2} r_{x} t_{y}, i t_{y}^{-1}, r_{z}^{2} r_{x} t_{y}^{-1} \\
r_{z}^{2} r_{x} t_{z}, r_{z}^{2} r_{x} t_{z}^{-1}\end{array}$ \\
\hline & & 2355 & $H_{551}$ & 1 & $m_{z} r_{x}$ & $\begin{array}{l}i t_{x}, i t_{x}^{-1}, r_{z}^{2} r_{x} t_{x}^{-1}, r_{z}^{2} r_{x} t_{y}, r_{z}^{2} r_{x} t_{y}^{-1}, i t_{z} \\
r_{z}^{2} r_{x} t_{z}, r_{z}^{2} r_{x} t_{z}^{-1}\end{array}$ \\
\hline & & 2356 & $H_{556}$ & 1 & $m_{z} r_{x}$ & $\begin{array}{l}r_{y}^{2} r_{x} t_{x}, r_{y}^{2} r_{x} t_{x}^{-1}, m_{x} t_{x}^{-1}, r_{z}^{2} r_{x} t_{y}, i t_{y}^{-1}, r_{z}^{2} r_{x} t_{y}^{-1}, \\
r_{z}^{2} r_{x} t_{z}, r_{z}^{2} r_{x} t_{z}^{-1}\end{array}$ \\
\hline & & 2357 & $H_{556}$ & 1 & $m_{z} r_{x}$ & $\begin{array}{l}m_{x} t_{x}, r_{y}^{2} r_{x} t_{x}^{-1}, m_{x} t_{x}^{-1}, r_{z}^{2} r_{x} t_{y}, i t_{y}^{-1}, r_{z}^{2} r_{x} t_{y}^{-1} \\
r_{z}^{2} r_{x} t_{z}, r_{z}^{2} r_{x} t_{z}^{-1}\end{array}$ \\
\hline & & 2358 & $H_{556}$ & 1 & $m_{z} r_{x}$ & $\begin{array}{l}m_{x} t_{x}, r_{y}^{2} r_{x} t_{x}^{-1}, m_{x} t_{x}^{-1}, r_{z}^{2} r_{x} t_{y}, r_{z}^{2} r_{x} t_{y}^{-1}, i t_{z} \\
r_{z}^{2} r_{x} t_{z}, r_{z}^{2} r_{x} t_{z}^{-1}\end{array}$ \\
\hline & & 2359 & $H_{561}$ & 5 & $m_{z} r_{x}$ & $\begin{array}{l}r_{y}^{2} r_{x} t_{x}, r_{y}^{2} r_{x} t_{x}^{-1}, m_{x} t_{x}^{-1}, m_{z} r_{x}^{-1} t_{y}, r_{z}^{2} r_{x} t_{y}^{-1}, r_{x}^{2} t_{z}, \\
m_{z} r_{x}^{-1} t_{z}, r_{z}^{2} r_{x} t_{z}^{-1}\end{array}$ \\
\hline \multicolumn{7}{|c|}{$[9,37,77,132,204,292,396,516,652,804]$} \\
\hline & & 2360 & $H_{572}$ & 1 & $m_{z} r_{x}$ & $\begin{array}{l}{ }^{i t_{x}}, i t_{x}^{-1}, r_{z}^{2} r_{x} t_{x}^{-1}, m_{x} r_{x} t_{y}, r_{z}^{2} t_{y}^{-1}, m_{x} r_{x} t_{y}^{-1} \\
m_{x} r_{x}^{-1} t_{z}, m_{x} r_{x}^{-1} t_{z}^{-1}\end{array}$ \\
\hline
\end{tabular}




\begin{tabular}{|c|c|c|c|c|c|}
\hline Nbr. & № & $H_{i}$ & $L$ & $m$ & $x$ \\
\hline & 2361 & $H_{572}$ & 1 & $m_{z} r_{x}$ & $\begin{array}{l}r_{z}^{2} r_{x} t_{x}, i t_{x}^{-1}, r_{z}^{2} r_{x} t_{x}^{-1}, m_{x} r_{x} t_{y}, r_{z}^{2} t_{y}^{-1}, m_{x} r_{x} t_{y}^{-1} \\
m_{x} r_{x}^{-1} t_{z}, m_{x} r_{x}^{-1} t_{z}^{-1}\end{array}$ \\
\hline & 2362 & $H_{576}$ & 1 & $m_{z} r_{x}$ & $\begin{array}{l}r_{y}^{2} r_{x} t_{x}, r_{y}^{2} r_{x} t_{x}^{-1}, m_{x} t_{x}^{-1}, m_{x} r_{x} t_{y}, r_{z}^{2} t_{y}^{-1}, m_{x} r_{x} t_{y}^{-1} \\
m_{x} r_{x}^{-1} t_{z}, m_{x} r_{x}^{-1} t_{z}^{-1}\end{array}$ \\
\hline & 2363 & $H_{576}$ & 1 & $m_{z} r_{x}$ & $\begin{array}{l}m_{x} t_{x}, r_{y}^{2} r_{x} t_{x}^{-1}, m_{x} t_{x}^{-1}, m_{x} r_{x} t_{y}, r_{z}^{2} t_{y}^{-1}, m_{x} r_{x} t_{y}^{-1} \\
m_{x} r_{x}^{-1} t_{z}, m_{x} r_{x}^{-1} t_{z}^{-1}\end{array}$ \\
\hline & 2364 & $H_{576}$ & 1 & $m_{z} r_{x}$ & $\begin{array}{l}m_{x} t_{x}, r_{y}^{2} r_{x} t_{x}^{-1}, m_{x} t_{x}^{-1}, r_{z}^{2} t_{y}, m_{x} r_{x} t_{y}, m_{x} r_{x} t_{y}^{-1} \\
m_{x} r_{x}^{-1} t_{z}, m_{x} r_{x}^{-1} t_{z}^{-1}\end{array}$ \\
\hline & 2365 & $H_{576}$ & 1 & $m_{z} r_{x}$ & $\begin{array}{l}r_{y}^{2} r_{x} t_{x}, r_{y}^{2} r_{x} t_{x}^{-1}, m_{x} t_{x}^{-1}, m_{x} r_{x} t_{y}, m_{x} r_{x} t_{y}^{-1}, m_{x} r_{x}^{-1} t_{z}, \\
m_{x} r_{x}^{-1} t_{z}^{-1}, r_{y}^{2} t_{z}^{-1}\end{array}$ \\
\hline & 2366 & $H_{571}$ & 1 & $m_{z} r_{x}$ & $\begin{array}{l}r_{y}^{2} r_{x} t_{x}, r_{y}^{2} r_{x} t_{x}^{-1}, m_{x} t_{x}^{-1}, m_{y} t_{y}, r_{x} t_{y}, m_{x} r_{x} t_{y}^{-1} \\
r_{x}^{-1} t_{z}, m_{x} r_{x}^{-1} t_{z}^{-1}\end{array}$ \\
\hline \multicolumn{6}{|c|}{$[9,37,77,134,204,292,396,516,652,804]$} \\
\hline & 2367 & $H_{551}$ & 1 & $m_{z} r_{x}$ & $\begin{array}{l}i t_{x}, i t_{x}^{-1}, r_{z}^{2} r_{x} t_{x}^{-1}, r_{z}^{2} r_{x} t_{y}, i t_{y}^{-1}, i t_{z} \\
r_{z}^{2} r_{x} t_{z}, i t_{z}^{-1}\end{array}$ \\
\hline & 2368 & $H_{551}$ & 1 & $m_{z} r_{x}$ & $\begin{array}{l}r_{z}^{2} r_{x} t_{x}, i t_{x}^{-1}, r_{z}^{2} r_{x} t_{x}^{-1}, r_{z}^{2} r_{x} t_{y}, i t_{y}^{-1}, i t_{z} \\
r_{z}^{2} r_{x} t_{z}, i t_{z}^{-1}\end{array}$ \\
\hline & 2369 & $H_{551}$ & 1 & $m_{z} r_{x}$ & $\begin{array}{l}r_{z}^{2} r_{x} t_{x}, i t_{x}^{-1}, r_{z}^{2} r_{x} t_{x}^{-1}, i t_{y}, i t_{y}^{-1}, r_{z}^{2} r_{x} t_{y}^{-1} \\
i t_{z}, r_{z}^{2} r_{x} t_{z}^{-1}\end{array}$ \\
\hline & 2370 & $H_{555}$ & 1 & $m_{z} r_{x}$ & $\begin{array}{l}i t_{x}, i t_{x}^{-1}, r_{z}^{2} r_{x} t_{x}^{-1}, m_{z} r_{x}^{-1} t_{y}, r_{x}^{2} t_{y}^{-1}, r_{x}^{2} t_{z} \\
m_{z} r_{x}^{-1} t_{z}, r_{x}^{2} t_{z}^{-1}\end{array}$ \\
\hline & 2371 & $H_{555}$ & 1 & $m_{z} r_{x}$ & $\begin{array}{l}r_{z}^{2} r_{x} t_{x}, i t_{x}^{-1}, r_{z}^{2} r_{x} t_{x}^{-1}, m_{z} r_{x}^{-1} t_{y}, r_{x}^{2} t_{y}^{-1}, r_{x}^{2} t_{z} \\
m_{z} r_{x}^{-1} t_{z}, r_{x}^{2} t_{z}^{-1}\end{array}$ \\
\hline & 2372 & $H_{555}$ & 1 & $m_{z} r_{x}$ & $\begin{array}{l}r_{z}^{2} r_{x} t_{x}, i t_{x}^{-1}, r_{z}^{2} r_{x} t_{x}^{-1}, r_{x}^{2} t_{y}, r_{x}^{2} t_{y}^{-1}, m_{z} r_{x}^{-1} t_{y}^{-1} \\
r_{x}^{2} t_{z}, m_{z} r_{x}^{-1} t_{z}^{-1}\end{array}$ \\
\hline & 2373 & $H_{560}$ & 1 & $m_{z} r_{x}$ & $\begin{array}{l}i t_{x}, r_{y}^{2} r_{x} t_{x}^{-1}, m_{x} t_{x}^{-1}, m_{z} r_{x}^{-1} t_{y}, r_{x}^{2} t_{y}^{-1}, r_{x}^{2} t_{z} \\
m_{z} r_{x}^{-1} t_{z}, r_{x}^{2} t_{z}^{-1}\end{array}$ \\
\hline & 2374 & $H_{560}$ & 1 & $m_{z} r_{x}$ & $\begin{array}{l}r_{z}^{2} r_{x} t_{x}, r_{y}^{2} r_{x} t_{x}^{-1}, m_{x} t_{x}^{-1}, m_{z} r_{x}^{-1} t_{y}, r_{x}^{2} t_{y}^{-1}, r_{x}^{2} t_{z} \\
m_{z} r_{x}^{-1} t_{z}, r_{x}^{2} t_{z}^{-1}\end{array}$ \\
\hline & 2375 & $H_{560}$ & 1 & $m_{z} r_{x}$ & $\begin{array}{l}r_{z}^{2} r_{x} t_{x}, r_{y}^{2} r_{x} t_{x}^{-1}, m_{x} t_{x}^{-1}, r_{x}^{2} t_{y}, r_{x}^{2} t_{y}^{-1}, m_{z} r_{x}^{-1} t_{y}^{-1}, \\
r_{x}^{2} t_{z}, m_{z} r_{x}^{-1} t_{z}^{-1}\end{array}$ \\
\hline & 2376 & $H_{556}$ & 1 & $m_{z} r_{x}$ & $\begin{array}{l}r_{y}^{2} r_{x} t_{x}, r_{y}^{2} r_{x} t_{x}^{-1}, m_{x} t_{x}^{-1}, r_{z}^{2} r_{x} t_{y}, i t_{y}^{-1}, i t_{z} \\
r_{z}^{2} r_{x} t_{z}, i t_{z}^{-1}\end{array}$ \\
\hline & 2377 & $H_{556}$ & 1 & $m_{z} r_{x}$ & $\begin{array}{l}m_{x} t_{x}, r_{y}^{2} r_{x} t_{x}^{-1}, m_{x} t_{x}^{-1}, r_{z}^{2} r_{x} t_{y}, i t_{y}^{-1}, i t_{z} \\
r_{z}^{2} r_{x} t_{z}, i t_{z}^{-1}\end{array}$ \\
\hline & 2378 & $H_{556}$ & 1 & $m_{z} r_{x}$ & $\begin{array}{l}r_{y}^{2} r_{x} t_{x}, r_{y}^{2} r_{x} t_{x}^{-1}, m_{x} t_{x}^{-1}, i t_{y}, i t_{y}^{-1}, r_{z}^{2} r_{x} t_{y}^{-1} \\
i t_{z}, r_{z}^{2} r_{x} t_{z}^{-1}\end{array}$ \\
\hline & 2379 & $H_{561}$ & 1 & $m_{z} r_{x}$ & $\begin{array}{l}r_{y}^{2} r_{x} t_{x}, r_{y}^{2} r_{x} t_{x}^{-1}, m_{x} t_{x}^{-1}, m_{z} r_{x}^{-1} t_{y}, i t_{y}^{-1}, r_{x}^{2} t_{z} \\
m_{z} r_{x}^{-1} t_{z}, i t_{z}^{-1}\end{array}$ \\
\hline
\end{tabular}

44

$[8,29,69,128,203,292,396,516,652,804]$ $2380^{*}$

$[8,30,69,123,193,279,381,499,633,783]$ $2381^{*}$

$[8,30,71,128,200,288,392,512,648,800]$ $2382 *$

$[8,30,71,130,204,292,396,516,652,804]$ $2383^{*}$

$[8,30,73,134,207,293,396,516,652,804]$ $2384^{*}, 2385^{*}, 2386^{*}$,

$[8,31,70,123,193,279,381,499,633,783]$ $2387^{*}, 2388^{*}$,

$[8,31,73,129,200,288,392,512,648,800$ ] $2389^{*}$,

$[8,31,73,130,202,290,394,514,650,802]$ $2390^{*}, 2391^{*}, 2392^{*}, 2393^{*}$,

$[8,31,74,132,204,292,396,516,652,804]$ $2394^{*}, 2395^{*}, 2396^{*}$,

$[8,31,75,133,204,292,396,516,652,804$ ] $2397 *$,

$[8,31,75,135,206,292,396,516,652,804]$ $2398^{*}, 2399^{*}, 2400^{*}$,

$[8,31,75,135,207,293,396,516,652,804]$ $2401 *$

$[8,31,76,136,207,293,396,516,652,804]$ $2402 *, 2403^{*}$,

$[8,31,76,137,208,293,396,516,652,804]$ $2404^{*}$,

$[8,32,74,130,202,290,394,514,650,802]$ $2405^{*}$,

$[8,32,76,133,204,292,396,516,652,804]$ $2406^{*}$,

[ $8,32,76,135,206,292,396,516,652,804$ ] $2407^{*}$,

$[8,32,76,135,207,293,396,516,652,804]$ $2408^{*}, 2409^{*}, 2410^{*}, 2411^{*}, 2412^{*}, 2413^{*}, 2414^{*}$

$[8,32,77,136,206,292,396,516,652,804]$ $2415^{*}, 2416^{*}$,

$[8,33,75,130,202,290,394,514,650,802]$ 


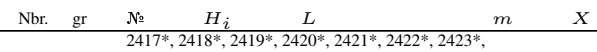

$[8,33,77,133,204,292,396,516,652,804]$

$2424^{*}, 2425^{*}, 2426^{*}, 2427^{*}, 2428^{*}, 2429^{*}, 2430^{*}, 2431^{*}$,

$[8,33,77,135,206,292,396,516,652,804]$

$2432^{*}, 2433^{*}, 2434^{*}, 2435^{*}, 2436^{*}, 2437^{*}, 2438^{*}$,

$[8,33,78,135,206,293,396,516,652,804]$

$2439^{*}$,

$[9,32,73,131,204,292,396,516,652,804]$
$2380 \quad H_{412} \quad 1$

$$
r_{z}^{2} r_{x} \quad t_{x}, r_{z}^{2} r_{x} t_{x}^{-1}, t_{x}^{-1}, m_{y} t_{y}, r_{z}^{2} t_{y}^{-1}, r_{x}^{-1} t_{y}^{-1}
$$

$[9,33,72,127,198,285,388,507,642,793]$

$2381 \quad H_{388}$

$[9,33,74,130,202,290,394,514,650,802]$

$2382 \quad H_{407} \quad 1$

$[9,33,74,132,204,292,396,516,652,804]$

$2383 \quad H_{394} \quad 1$

$[9,33,76,134,205,292,396,516,652,804]$

$r_{x} t_{z}, m_{z} t_{z}^{-1}$

$\begin{array}{llll}2384 & H_{402} & 1 & r_{z}^{2} r_{x} \\ 2385 & H_{413} & 1 & r_{z}^{2} r_{x} \\ 2386 & H_{419} & 1 & r_{z}^{2} r_{x}\end{array}$

$t_{x}, r_{z}^{2} r_{x} t_{x}^{-1}, t_{x}^{-1}, m_{z} r_{x} t_{y}, i t_{y}^{-1}, m_{z} r_{x} t_{y}^{-1}$,

$\begin{array}{ll}r_{z}^{2} r_{x} & t_{x}, r_{z}^{2} r_{x} t_{x}^{-1}, t_{x}^{-1}, m_{z} \\ m_{z} r_{x} t_{z}, m_{z} r_{x} t_{z}^{-1}\end{array}$

$4,72,127,198,285,388,507,642,793]$

$\begin{array}{lll}2387 & H_{389} & 1 \\ 2388 & H_{393} & 1\end{array}$

$[9,34,75,130,202,290,394,514,650,802]$

$2389 \quad H_{414} \quad 1$

$[9,34,75,131,203,291,395,515,651,803]$

$2390 \quad H_{394} \quad 1$

$\begin{array}{lll}2391 & H_{398} & 1 \\ 2392 & H_{400} & 1 \\ 2393 & H_{395} & 1\end{array}$

$[9,34,76,132,204,292,396,516,652,804]$

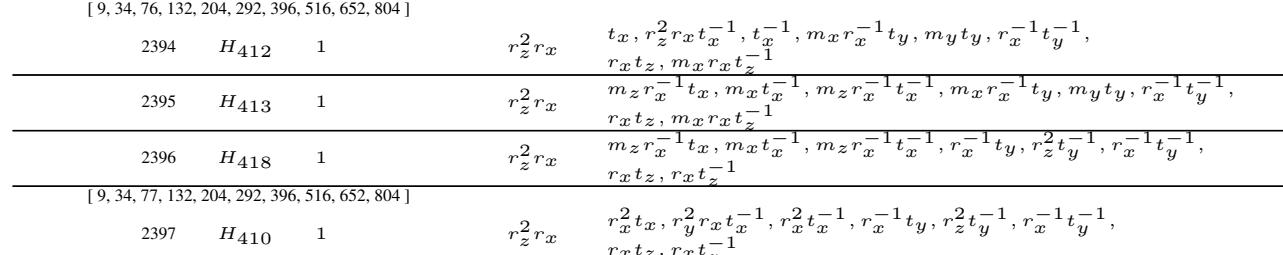

$[9,34,77,134,204,292,396,516,652,804]$

\begin{tabular}{lllll}
2398 & $H_{391}$ & 1 & $r_{z}^{2} r_{x}$ & $\begin{array}{l}t_{x}, r_{z}^{2} r_{x} t_{x}^{-1}, t_{x}^{-1}, r_{x}^{2} t_{y}, r_{y}^{2} r_{x} t_{y}^{-1}, r_{x}^{2} t_{y}^{-1}, \\
r_{y}^{2} r_{x} t_{z}^{2} r_{z}^{2}\end{array}$ \\
\hline 2399 & $H_{395}$ & 1 & $\begin{array}{l}r_{z}^{2} r_{x} \\
m_{z} r_{x}^{-1} t_{x}, m_{x} t_{x}^{-1}, m_{z} r_{x}^{-1} t_{x}^{-1}, r_{x}^{2} t_{y}, i t_{y}^{-1}, m_{z} r_{x} t_{y}^{-1}, \\
m_{z} r_{x} t_{z}, r_{x}^{2} t_{z}^{-1}\end{array}$ \\
\hline
\end{tabular}

$[9,34,77,134,205,292,396,516,652,804]$

$$
2401 \quad H_{407} \quad 1
$$

$[9,34,78,134,205,292,396,516,652,804]$

\begin{tabular}{ccc}
2402 & $H_{422}$ & 1 \\
\hline 2403 & $H_{422}$ & 1
\end{tabular}

$[9,34,78,135,205,292,396,516,652,804]$

$$
2404 \quad H_{393} \quad 1
$$

$[9,35,75,131,203,291,395,515,651,803]$

$$
2405 \quad H_{394} \quad 1
$$$$
r_{z}^{2} r_{x} \quad t_{x}, r_{z}^{2} r_{x} t_{x}^{-1}, t_{x}^{-1}, r_{z}^{2} t_{y}, r_{z}^{2} t_{y}^{-1}, r_{x}^{-1} t_{y}^{-1},
$$$$
r_{x} t_{z}, r_{y}^{2} t_{z}^{-1}
$$

$r_{z}^{2} t_{x}, r_{x}^{-1} t_{x}, r_{x} t_{x}^{-1}, m_{y} t_{y}, m_{x} r_{x}^{-1} t_{y}^{-1}, m_{y} t_{y}^{-1}$ $m_{x}^{2} r_{x} t_{z}, m_{z} r_{z}^{-1}{ }^{-1} t_{x}, r_{x} t_{x}^{-1}, m_{y}{ }^{t} y, m_{x} r_{x}^{-1} t_{y}^{-1}, m_{x} r_{x} t_{z}$ $m_{z} t_{z}, m_{z} t_{z}^{-1}$

$r_{z}^{2} r_{x} \quad m_{z} t_{z}, m_{z} t_{z}^{-1}$

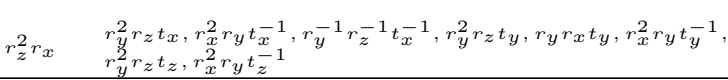

$r_{z}^{2} r_{x} \quad t_{x}, r_{z}^{2} r_{x} t_{x}^{-1}, t_{x}^{-1}, r_{y}^{2} r_{x} t_{y}, i t_{y}^{-1}, m_{z} r_{x} t_{y}^{-1}$, 
gr $\quad$ № $\quad H_{i} \quad L$
$[9,35,77,132,204,292,396,516,652,804]$

$2406 \quad H_{412} \quad 1$

$[9,35,77,134,204,292,396,516,652,804]$

$$
2407 \quad H_{388} \quad 1
$$

$[9,35,77,134,205,292,396,516,652,804]$

\begin{tabular}{ccc}
2408 & $H_{414}$ & 1 \\
\hline 2409 & $H_{415}$ & 1 \\
\hline 2410 & $H_{412}$ & 1 \\
\hline 2411 & $H_{410}$ & 1 \\
\hline 2412 & $H_{404}$ & 1 \\
\hline 2413 & $H_{413}$ & 1 \\
\hline 2414 & $H_{418}$ & 1
\end{tabular}

$[9,35,78,134,204,292,396,516,652,804]$

$\begin{array}{lll}2415 & H_{409} & 1 \\ 2416 & H_{409} & 1\end{array}$

$[9,36,75,131,203,291,395,515,651,803]$

\begin{tabular}{|c|c|c|c|c|}
\hline 2417 & $H_{396}$ & 1 & $r_{z}^{2} r_{x}$ & $\begin{array}{l}m_{z} r_{x} t_{x}, i t_{x}^{-1}, m_{z} r_{x} t_{x}^{-1}, r_{y}^{2} r_{x} t_{y}, r_{y}^{2} r_{x} t_{y}^{-1}, r_{x}^{2} t_{y}^{-1} \\
r_{y}^{2} r_{x} t_{z}, r_{y}^{2} r_{x} t_{z}^{-1}\end{array}$ \\
\hline 2418 & $H_{409}$ & 1 & $r_{z}^{2} r_{x}$ & $\begin{array}{l}r_{z}^{2} t_{x}, r_{x}^{-1} t_{x}, r_{x} t_{x}^{-1}, r_{y}^{2} r_{x} t_{y}, r_{y}^{2} r_{x} t_{y}^{-1}, r_{x}^{2} t_{y}^{-1} \\
r_{y}^{2} r_{x} t_{z}, r_{y}^{2} r_{x} t_{z}^{-1}\end{array}$ \\
\hline 2419 & $H_{409}$ & 1 & $r_{z}^{2} r_{x}$ & $\begin{array}{l}r_{z}^{2} t_{x}, r_{x}^{-1} t_{x}, r_{x} t_{x}^{-1}, r_{y}^{2} r_{x} t_{y}, r_{y}^{2} r_{x} t_{y}^{-1}, r_{y}^{2} r_{x} t_{z} \\
r_{y}^{2} r_{x} t_{z}^{-1}, r_{x}^{2} t_{z}^{-1}\end{array}$ \\
\hline 2420 & $H_{391}$ & 1 & $r_{z}^{2} r_{x}$ & $\begin{array}{l}t_{x}, r_{z}^{2} r_{x} t_{x}^{-1}, t_{x}^{-1}, r_{y}^{2} r_{x} t_{y}, r_{y}^{2} r_{x} t_{y}^{-1}, r_{x}^{2} t_{y}^{-1} \\
r_{y}^{2} r_{x} t_{z}, r_{y}^{2} r_{x} t_{z}^{-1}\end{array}$ \\
\hline 2421 & $H_{392}$ & 1 & $r_{z}^{2} r_{x}$ & $\begin{array}{l}r_{x}^{2} t_{x}, r_{y}^{2} r_{x} t_{x}^{-1}, r_{x}^{2} t_{x}^{-1}, r_{y}^{2} r_{x} t_{y}, r_{y}^{2} r_{x} t_{y}^{-1}, r_{x}^{2} t_{y}^{-1} \\
r_{y}^{2} r_{x} t_{z}, r_{y}^{2} r_{x} t_{z}^{-1}\end{array}$ \\
\hline 2422 & $H_{395}$ & 1 & $r_{z}^{2} r_{x}$ & $\begin{array}{l}m_{z} r_{x}^{-1} t_{x}, m_{x} t_{x}^{-1}, m_{z} r_{x}^{-1} t_{x}^{-1}, r_{y}^{2} r_{x} t_{y}, i t_{y}^{-1}, m_{z} r_{x} t_{y}^{-1} \\
m_{z} r_{x} t_{z}, r_{y}^{2} r_{x} t_{z}^{-1}\end{array}$ \\
\hline 2423 & $H_{401}$ & 506528044 & $r_{z}^{2} r_{x}$ & $\begin{array}{l}m_{z} r_{x}^{-1} t_{x}, m_{x} t_{x}^{-1}, m_{z} r_{x}^{-1} t_{x}^{-1}, r_{y}^{2} r_{x} t_{y}, r_{y}^{2} r_{x} t_{y}^{-1}, r_{x}^{2} t_{y}^{-1}, \\
r_{y}^{2} r_{x} t_{z}, r_{y}^{2} r_{x} t_{z}^{-1}\end{array}$ \\
\hline \multicolumn{5}{|c|}{$[9,36,77,132,204,292,396,516,652,804]$} \\
\hline 2424 & $H_{415}$ & 1 & $r_{z}^{2} r_{x}$ & $\begin{array}{l}m_{z} r_{x} t_{x}, i t_{x}^{-1}, m_{z} r_{x} t_{x}^{-1}, m_{x} r_{x}^{-1} t_{y}, m_{x} r_{x}^{-1} t_{y}^{-1}, m_{y} t_{y}^{-1} \\
m_{x} r_{x} t_{z}, m_{x} r_{x} t_{z}^{-1}\end{array}$ \\
\hline 2425 & $H_{422}$ & 1 & $r_{z}^{2} r_{x}$ & $\begin{array}{l}r_{z}^{2} t_{x}, r_{x}^{-1} t_{x}, r_{x} t_{x}^{-1}, m_{x} r_{x}^{-1} t_{y}, m_{x} r_{x}^{-1} t_{y}^{-1}, m_{y} t_{y}^{-1} \\
m_{x} r_{x} t_{z}, m_{x} r_{x} t_{z}^{-1}\end{array}$ \\
\hline 2426 & $H_{422}$ & 1 & $r_{z}^{2} r_{x}$ & $\begin{array}{l}r_{z}^{2} t_{x}, r_{x}^{-1} t_{x}, r_{x} t_{x}^{-1}, m_{x} r_{x}^{-1} t_{y}, m_{x} r_{x}^{-1} t_{y}^{-1}, m_{x} r_{x} t_{z} \\
m_{x} r_{x} t_{z}^{-1}, m_{z} t_{z}^{-1}\end{array}$ \\
\hline 2427 & $H_{402}$ & 1 & $r_{z}^{2} r_{x}$ & $\begin{array}{l}t_{x}, r_{z}^{2} r_{x} t_{x}^{-1}, t_{x}^{-1}, m_{x} r_{x}^{-1} t_{y}, m_{x} r_{x}^{-1} t_{y}^{-1}, m_{y} t_{y}^{-1} \\
m_{x} r_{x} t_{z}, m_{x} r_{x} t_{z}^{-1}\end{array}$ \\
\hline 2428 & $H_{404}$ & 1 & $r_{z}^{2} r_{x}$ & $\begin{array}{l}r_{x}^{2} t_{x}, r_{y}^{2} r_{x} t_{x}^{-1}, r_{x}^{2} t_{x}^{-1}, m_{x} r_{x}^{-1} t_{y}, m_{x} r_{x}^{-1} t_{y}^{-1}, m_{y} t_{y}^{-1} \\
m_{x} r_{x} t_{z}, m_{x} r_{x} t_{z}^{-1}\end{array}$ \\
\hline 2429 & $H_{413}$ & 1 & $r_{z}^{2} r_{x}$ & $\begin{array}{l}m_{z} r_{x}^{-1} t_{x}, m_{x} t_{x}^{-1}, m_{z} r_{x}^{-1} t_{x}^{-1}, m_{x} r_{x}^{-1} t_{y}, r_{z}^{2} t_{y}^{-1}, r_{x}^{-1} t_{y}^{-1} \\
r_{x} t_{z}, m_{x} r_{x} t_{z}^{-1}\end{array}$ \\
\hline 2430 & $H_{419}$ & 1 & $r_{z}^{2} r_{x}$ & $\begin{array}{l}m_{z} r_{x}^{-1} t_{x}, m_{x} t_{x}^{-1}, m_{z} r_{x}^{-1} t_{x}^{-1}, m_{x} r_{x}^{-1} t_{y}, m_{x} r_{x}^{-1} t_{y}^{-1}, m_{y} t_{y}^{-1}, \\
m_{x} r_{x} t_{z}, m_{x} r_{x} t_{z}^{-1}\end{array}$ \\
\hline 2431 & $H_{393}$ & $516<9281$ & $r_{z}^{2} r_{x}$ & $\begin{array}{l}r_{y}^{2} r_{z} t_{x}, r_{x}^{2} r_{y} t_{x}^{-1}, r_{y}^{-1} r_{z}^{-1} t_{x}^{-1}, r_{y}^{2} r_{z} t_{y}, r_{y} r_{x} t_{y}, r_{y}^{-1} r_{z}^{-1} t_{y}^{-1} \\
r_{y} r_{x} t_{z}, r_{x}^{2} r_{y} t_{z}^{-1}\end{array}$ \\
\hline \multicolumn{5}{|c|}{$[9,36,77,134,204,292,396,516,652,804]$} \\
\hline 2432 & $H_{389}$ & 1 & $r_{z}^{2} r_{x}$ & $\begin{array}{l}m_{z} r_{x} t_{x}, i t_{x}^{-1}, m_{z} r_{x} t_{x}^{-1}, m_{z} r_{x} t_{y}, i t_{y}^{-1}, i t_{z} \\
i t_{z}^{-1}, m_{z} r_{x} t_{z}^{-1}\end{array}$ \\
\hline 2433 & $H_{396}$ & 1 & $r_{z}^{2} r_{x}$ & $\begin{array}{l}m_{z} r_{x} t_{x}, i t_{x}^{-1}, m_{z} r_{x} t_{x}^{-1}, r_{x}^{2} t_{y}, r_{y}^{2} r_{x} t_{y}^{-1}, r_{x}^{2} t_{y}^{-1} \\
r_{y}^{2} r_{x} t_{z}, r_{x}^{2} t_{z}^{-1}\end{array}$ \\
\hline 2434 & $H_{394}$ & 1 & $r_{z}^{2} r_{x}$ & $\begin{array}{l}t_{x}, r_{z}^{2} r_{x} t_{x}^{-1}, t_{x}^{-1}, r_{y}^{2} r_{x} t_{y}, i t_{y}^{-1}, i t_{z} \\
r_{y}^{2} r_{x} t_{z}^{-1}, r_{x}^{2} t_{z}^{-1}\end{array}$ \\
\hline 2435 & $H_{398}$ & 1 & $r_{z}^{2} r_{x}$ & $\begin{array}{l}r_{x}^{2} t_{x}, r_{y}^{2} r_{x} t_{x}^{-1}, r_{x}^{2} t_{x}^{-1}, m_{z} r_{x} t_{y}, i t_{y}^{-1}, i t_{z} \\
i t_{z}^{-1}, m_{z} r_{x} t_{z}^{-1}\end{array}$ \\
\hline 2436 & $H_{392}$ & 1 & $r_{z}^{2} r_{x}$ & $\begin{array}{l}r_{x}^{2} t_{x}, r_{y}^{2} r_{x} t_{x}^{-1}, r_{x}^{2} t_{x}^{-1}, r_{x}^{2} t_{y}, r_{y}^{2} r_{x} t_{y}^{-1}, r_{x}^{2} t_{y}^{-1} \\
r_{y}^{2} r_{x} t_{z}, r_{x}^{2} t_{z}^{-1}\end{array}$ \\
\hline
\end{tabular}
$r_{x} t_{z}, m_{x} r_{x} t_{z}^{-1}$

$r_{z}^{2} r_{x} \quad t_{x}, r_{z}^{2} r_{x} t_{x}^{-1}, t_{x}^{-1}, m_{z} r_{x} t_{y}, i t_{y}^{-1}, i t_{z}$ $i t_{z}^{-1}, m_{z} r_{x} t_{z}^{-1}$

$r_{z}^{2} r_{x} \quad m_{z} r_{x} t_{x}, i t_{x}^{-1}, m_{z} r_{x} t_{x}^{-1}, r_{z}^{2} t_{y}, r_{z}^{2} t_{y}^{-1}, r_{x}^{-1} t_{y}^{-1}$, $r_{x} t_{z}, r_{y}^{2} t_{z}^{-1}$

$r_{z}^{2} r_{x} \quad m_{z} r_{x} t_{x}, i t_{x}^{-1}, m_{z} r_{x} t_{x}^{-1}, m_{y} t_{y}, m_{x} r_{x}^{-1} t_{y}^{-1}, m_{y} t_{y}^{-1}$, $m_{x} r_{x} t_{z}, m_{z} t_{z}^{-1}$

$\begin{array}{ll}r_{z}^{2} r_{x} & t_{x}, r_{z}^{2} r_{x} t_{x}^{-1}, t_{x}^{-1}, m_{x} r_{x}^{-1} t_{y}, m_{y} t_{y}, r_{z}^{2} t_{y}^{-1}, \\ & r_{y}^{2} t_{z}, m_{x} r_{x} t_{z}^{-1}\end{array}$

$\begin{array}{ll}r_{z}^{2} r_{x} & r_{x}^{2} t_{x}, r_{y}^{2} r_{x} t_{x}^{-1}, r_{x}^{2} t_{x}^{-1}, r_{z}^{2} t_{y}, r_{z}^{2} t_{y}^{-1}, r_{x}^{-1} t_{y}^{-1}, \\ r_{x} t_{z}, r^{2} t_{x}^{-1}\end{array}$

$r_{x} t_{z}, r_{y}^{2} t_{z}^{-1}$

$r_{z}^{2} r_{x} \quad r_{x}^{2} t_{x}, r_{y}^{2} r_{x} t_{x}^{-1}, r_{x}^{2} t_{x}^{-1}, m_{y} t_{y}, m_{x} r_{x}^{-1} t_{y}^{-1}, m_{y} t_{y}^{-1}$,

$m_{x} r_{x} t_{z}, m_{z} t_{z}^{-1}$

$\begin{array}{ll}r_{z}^{2} r_{x} & m_{z} r_{x}^{-1} t_{x}, m_{x} t_{x}^{-1}, m_{z} r_{x}^{-1} t_{x}^{-1}, m_{x} r_{x}^{-1} t_{y}, m_{y} t_{y}, r_{z}^{2} t_{y}^{-1}, \\ & r_{y}^{2} t_{z}, m_{x} r_{x} t_{z}^{-1}\end{array}$

$r_{z}^{2} r_{x} \quad m_{z} r_{x}^{-1} t_{x}, m_{x} t_{x}^{-1}, m_{z} r_{x}^{-1} t_{x}^{-1}, r_{z}^{2} t_{y}, r_{z}^{2} t_{y}^{-1}, r_{x}^{-1} t_{y}^{-1}$, $r_{x} t_{z}, r_{y}^{2} t_{z}^{-1}$ $\begin{array}{ll}r_{z}^{2} r_{x} & r_{z}^{2} t_{x}, r_{x}^{-1} t_{x}, r_{x} t_{x}^{-1}, r_{x}^{2} t_{y}, r_{y}^{2} r_{x} t_{y}^{-1}, r_{x}^{2} t_{y}^{-1}, \\ r_{y}^{2} r_{x} t_{z}, r_{x}^{2} t_{z}^{-1}\end{array}$ $r_{z}^{2} t_{x}, r_{x}^{-1} t_{x}, r_{x} t_{x}^{-1}, r_{x}^{2} t_{y}, r_{y}^{2} r_{x} t_{y}^{-1}, r_{y}^{2} r_{x} t_{z}$ $r_{x}^{2} t_{z}, r_{x}^{2} t_{z}^{-1}$

$r_{x} t_{x}^{-1}, r_{x}^{2} t_{y}, r_{y}^{2} r_{x} t_{y}^{-1}, r_{y}^{2} r_{x} t_{z}$

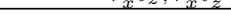

$r_{z}^{2} r_{x} \quad t_{x}, r_{z}^{2} r_{x} t_{x}^{-1}, t_{x}^{-1}, m_{x} r_{x}^{-1} t_{y}, r_{z}^{2} t_{y}^{-1}, r_{x}^{-1} t_{y}^{-1}$, 


\begin{tabular}{|c|c|c|c|c|c|c|}
\hline Nbr. & gr & № & $H_{i}$ & $L$ & $m$ & $X$ \\
\hline & & 2437 & $H_{400}$ & 1 & $r_{z}^{2} r_{x}$ & $\begin{array}{l}m_{z} r_{x}^{-1} t_{x}, m_{x} t_{x}^{-1}, m_{z} r_{x}^{-1} t_{x}^{-1}, m_{z} r_{x} t_{y}, i t_{y}^{-1}, i t_{z} \\
i t_{z}^{-1}, m_{z} r_{x} t_{z}^{-1}\end{array}$ \\
\hline & & 2438 & $H_{395}$ & 1 & $r_{z}^{2} r_{x}$ & $\begin{array}{l}m_{z} r_{x}^{-1} t_{x}, m_{x} t_{x}^{-1}, m_{z} r_{x}^{-1} t_{x}^{-1}, r_{y}^{2} r_{x} t_{y}, i t_{y}^{-1}, i t_{z} \\
r_{y}^{2} r_{x} t_{z}^{-1}, r_{x}^{2} t_{z}^{-1}\end{array}$ \\
\hline \multicolumn{7}{|c|}{$[9,36,78,133,205,292,396,516,652,804]$} \\
\hline & & 2439 & $H_{393}$ & 1 & $r_{z}^{2} r_{x}$ & $\begin{array}{l}r_{y}^{2} r_{z} t_{x}, r_{y} r_{x} t_{x}, r_{y}^{-1} r_{z}^{-1} t_{x}^{-1}, r_{y}^{2} r_{z} t_{y}, r_{y} r_{x} t_{y}, r_{x}^{2} r_{y} t_{y}^{-1}, \\
r_{y}^{2} r_{z} t_{z}, r_{y}^{-1} r_{z}^{-1} t_{z}-1\end{array}$ \\
\hline
\end{tabular}

45

$[9,33,76,135,207,293,396,516,652,804]$ $2440^{*}, 2441^{*}, 2442^{*}, 2443^{*}$

$[9,34,75,130,202,290,394,514,650,802]$ $2444^{*}, 2445^{*}, 2446^{*}, 2447^{*}$,

$[9,34,77,133,204,292,396,516,652,804]$ $2448^{*}, 2449^{*}, 2450^{*}, 2451^{*}$

$[9,34,77,135,206,292,396,516,652,804]$ $2452^{*}, 2453^{*}, 2454^{*}, 2455^{*}$

$[10,35,77,134,205,292,396,516,652,804]$

\begin{tabular}{|c|c|c|c|c|}
\hline 2440 & $H_{652}$ & $\left\langle m_{x}\right\rangle$ & $r_{y}^{2} r_{x}$ & $\begin{array}{l}i t_{x}, r_{z}^{2} r_{x} t_{x}, r_{x}^{2} t_{x}^{-1}, r_{z}^{2} r_{x} t_{x}^{-1}, m_{y} t_{y}, m_{y} t_{y}^{-1} \\
m_{x} r_{x} t_{y}^{-1}, r_{y}^{2} t_{z}, m_{x} r_{x}^{-1} t_{z}^{-1}\end{array}$ \\
\hline 2441 & $H_{422}$ & 1 & $r_{z}^{2} r_{x}$ & $\begin{array}{l}r_{z}^{2} t_{x}, r_{x}^{-1} t_{x}, r_{y}^{2} t_{x}^{-1}, r_{x} t_{x}^{-1}, m_{y} t_{y}, m_{x} r_{x}^{-1} t_{y}^{-1} \\
m_{y} t_{y}^{-1}, m_{x} r_{x} t_{z}, m_{z} t_{z}^{-1}\end{array}$ \\
\hline 2442 & $H_{650}$ & $\left\langle m_{x}\right\rangle$ & $r_{y}^{2} r_{x}$ & $\begin{array}{l}r_{y}^{2} r_{x} t_{x}, m_{x} t_{x}, m_{z} r_{x} t_{x}^{-1}, m_{x} t_{x}^{-1}, m_{y} t_{y}, m_{y} t_{y}^{-1} \\
m_{x} r_{x} t_{y}^{-1}, r_{y}^{2} t_{z}, m_{x} r_{x}^{-1} t_{z}^{-1}\end{array}$ \\
\hline 2443 & $H_{580}$ & 1 & $m_{z} r_{x}$ & $\begin{array}{l}i t_{x}, r_{z}^{2} r_{x} t_{x}, r_{y}^{2} r_{x} t_{x}^{-1}, m_{x} t_{x}^{-1}, m_{y} t_{y}, m_{y} t_{y}^{-1} \\
r_{x} t_{y}^{-1}, m_{z} t_{z}, r_{x}^{-1} t_{z}^{-1}\end{array}$ \\
\hline
\end{tabular}

$[10,36,75,131,203,291,395,515,651,803]$

\begin{tabular}{|c|c|c|c|c|}
\hline 2444 & $H_{649}$ & $\left\langle m_{x}\right\rangle$ & $r_{y}^{2} r_{x}$ & $\begin{array}{l}i t_{x}, r_{z}^{2} r_{x} t_{x}, r_{x}^{2} t_{x}^{-1}, r_{z}^{2} r_{x} t_{x}^{-1}, r_{z}^{2} r_{x} t_{y}, r_{x}^{2} t_{y}^{-1} \\
r_{z}^{2} r_{x} t_{y}^{-1}, r_{z}^{2} r_{x} t_{z}, r_{z}^{2} r_{x} t_{z}^{-1}\end{array}$ \\
\hline 2445 & $H_{409}$ & 1 & $r_{z}^{2} r_{x}$ & $\begin{array}{l}r_{z}^{2} t_{x}, r_{x}^{-1} t_{x}, r_{y}^{2} t_{x}^{-1}, r_{x} t_{x}^{-1}, r_{y}^{2} r_{x} t_{y}, r_{y}^{2} r_{x} t_{y}^{-1} \\
r_{x}^{2} t_{y}^{-1}, r_{y}^{2} r_{x} t_{z}, r_{y}^{2} r_{x} t_{z}^{-1}\end{array}$ \\
\hline 2446 & $H_{648}$ & $\left\langle m_{x}\right\rangle$ & $r_{y}^{2} r_{x}$ & $\begin{array}{l}r_{y}^{2} r_{x} t_{x}, m_{x} t_{x}, m_{z} r_{x} t_{x}^{-1}, m_{x} t_{x}^{-1}, r_{z}^{2} r_{x} t_{y}, r_{x}^{2} t_{y}^{-1}, \\
r_{z}^{2} r_{x} t_{y}^{-1}, r_{z}^{2} r_{x} t_{z}, r_{z}^{2} r_{x} t_{z}^{-1}\end{array}$ \\
\hline 2447 & $H_{560}$ & 1 & $m_{z} r_{x}$ & $\begin{array}{l}i t_{x}, r_{z}^{2} r_{x} t_{x}, r_{y}^{2} r_{x} t_{x}^{-1}, m_{x} t_{x}^{-1}, m_{z} r_{x}^{-1} t_{y}, r_{x}^{2} t_{y}^{-1} \\
m_{z} r_{x}^{-1} t_{y}^{-1}, m_{z} r_{x}^{-1} t_{z}, m_{z} r_{x}^{-1} t_{z}^{-1}\end{array}$ \\
\hline \multicolumn{5}{|c|}{$[10,36,77,132,204,292,396,516,652,804]$} \\
\hline 2448 & $H_{652}$ & $\left\langle m_{x}\right\rangle$ & $r_{y}^{2} r_{x}$ & $\begin{array}{l}i t_{x}, r_{z}^{2} r_{x} t_{x}, r_{x}^{2} t_{x}^{-1}, r_{z}^{2} r_{x} t_{x}^{-1}, m_{x} r_{x} t_{y}, m_{y} t_{y}^{-1} \\
m_{x} r_{x} t_{y}^{-1}, m_{x} r_{x}^{-1} t_{z}, m_{x} r_{x}^{-1} t_{z}^{-1}\end{array}$ \\
\hline 2449 & $H_{422}$ & 1 & $r_{z}^{2} r_{x}$ & $\begin{array}{l}r_{z}^{2} t_{x}, r_{x}^{-1} t_{x}, r_{y}^{2} t_{x}^{-1}, r_{x} t_{x}^{-1}, m_{x} r_{x}^{-1} t_{y}, m_{x} r_{x}^{-1} t_{y}^{-1}, \\
m_{y} t_{y}^{-1}, m_{x} r_{x} t_{z}, m_{x} r_{x} t_{z}^{-1}\end{array}$ \\
\hline 2450 & $H_{650}$ & $\left\langle m_{x}\right\rangle$ & $r_{y}^{2} r_{x}$ & $\begin{array}{l}r_{y}^{2} r_{x} t_{x}, m_{x} t_{x}, m_{z} r_{x} t_{x}^{-1}, m_{x} t_{x}^{-1}, m_{x} r_{x} t_{y}, m_{y} t_{y}^{-1}, \\
m_{x} r_{x} t_{y}^{-1}, m_{x} r_{x}^{-1} t_{z}, m_{x} r_{x}^{-1} t_{z}^{-1}\end{array}$ \\
\hline 2451 & $H_{580}$ & 1 & $m_{z} r_{x}$ & $\begin{array}{l}i t_{x}, r_{z}^{2} r_{x} t_{x}, r_{y}^{2} r_{x} t_{x}^{-1}, m_{x} t_{x}^{-1}, r_{x} t_{y}, m_{y} t_{y}^{-1} \\
r_{x} t_{y}^{-1}, r_{x}^{-1} t_{z}, r_{x}^{-1} t_{z}^{-1}\end{array}$ \\
\hline \multicolumn{5}{|c|}{$[10,36,77,134,204,292,396,516,652,804]$} \\
\hline 2452 & $H_{649}$ & $\left\langle m_{x}\right\rangle$ & $r_{y}^{2} r_{x}$ & $\begin{array}{l}i t_{x}, r_{z}^{2} r_{x} t_{x}, r_{x}^{2} t_{x}^{-1}, r_{z}^{2} r_{x} t_{x}^{-1}, r_{z}^{2} r_{x} t_{y}, r_{x}^{2} t_{y}^{-1} \\
r_{x}^{2} t_{z}, r_{z}^{2} r_{x} t_{z}, r_{x}^{2} t_{z}^{-1}\end{array}$ \\
\hline 2453 & $H_{409}$ & 1 & $r_{z}^{2} r_{x}$ & $\begin{array}{l}r_{z}^{2} t_{x}, r_{x}^{-1} t_{x}, r_{y}^{2} t_{x}^{-1}, r_{x} t_{x}^{-1}, r_{x}^{2} t_{y}, r_{y}^{2} r_{x} t_{y}^{-1} \\
r_{x}^{2} t_{y}^{-1}, r_{y}^{2} r_{x} t_{z}, r_{x}^{2} t_{z}^{-1}\end{array}$ \\
\hline 2454 & $H_{648}$ & $\left\langle m_{x}\right\rangle$ & $r_{y}^{2} r_{x}$ & 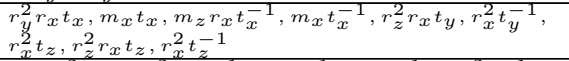 \\
\hline 2455 & $H_{560}$ & 1 & $m_{z} r_{x}$ & $\begin{array}{l}i t_{x}, r_{z}^{2} r_{x} t_{x}, r_{y}^{2} r_{x} t_{x}^{-1}, m_{x} t_{x}^{-1}, m_{z} r_{x}^{-1} t_{y}, r_{x}^{2} t_{y}^{-1} \\
r_{x}^{2} t_{z}, m_{z} r_{x}^{-1} t_{z}, r_{x}^{2} t_{z}^{-1}\end{array}$ \\
\hline
\end{tabular}

46

$[9,33,75,132,204,292,396,516,652,804]$ $2456^{*}, 2457^{*}$

$[9,34,76,132,204,292,396,516,652,804]$ $2458^{*}, 2459^{*}, 2460^{*}$

$[9,34,77,133,204,292,396,516,652,804]$ $2461^{*}, 2462^{*}, 2463^{*}, 2464^{*}, 2465^{*}, 2466^{*}, 2467^{*}, 2468^{*}$,

$[9,34,78,134,204,292,396,516,652,804]$ $2469^{*}$

$[9,35,77,132,204,292,396,516,652,804]$ $2470^{*}, 2471^{*}, 2472^{*}, 2473^{*}, 2474^{*}, 2475^{*}, 2476^{*}, 2477^{*}, 2478^{*}, 2479^{*}, 2480^{*}, 2481^{*}, 2482^{*}$ $2483^{*}$

$[9,35,78,133,204,292,396,516,652,804]$ $2484^{*}, 2485^{*}, 2486^{*}, 2487^{*}, 2488^{*}, 2489^{*}, 2490^{*}, 2491^{*}, 2492^{*}, 2493^{*}, 2494^{*}, 2495^{*}, 2496^{*}$, $2497^{*}, 2498^{*}$

$[9,36,78,132,204,292,396,516,652,804]$

$2499^{*}, 2500^{*}, 2501^{*}, 2502^{*}, 2503^{*}, 2504^{*}, 2505^{*}, 2506^{*}, 2507^{*}, 2508^{*}, 2509^{*}, 2510^{*}, 2511^{*}$ $2512^{*}, 2513^{*}, 2514^{*}, 2515^{*}, 2516^{*}, 2517^{*}, 2518^{*}, 2519^{*}, 2520^{*}$,

$[10,35,76,132,204,292,396,516,652,804]$

\begin{tabular}{|c|c|c|c|c|}
\hline 2456 & $H_{359}$ & 1 & $m_{z}$ & $\begin{array}{l}i t_{x}, r_{z}^{2} t_{x}, m_{x} t_{x}^{-1}, m_{y} t_{y}, r_{x}^{2} t_{y}^{-1}, m_{y} t_{y}^{-1} \\
t_{z}, m_{z} t_{z}^{-1}, t_{z}^{-1}\end{array}$ \\
\hline 2457 & $H_{650}$ & $\left\langle m_{z} r_{x}\right\rangle$ & $m_{x}$ & $\begin{array}{l}t_{x}, m_{x} t_{x}^{-1}, t_{x}^{-1}, m_{y} t_{y}, r_{z}^{2} t_{y}^{-1}, m_{y} t_{y}^{-1} \\
r_{x}^{-1} t_{z}, m_{x} r_{x}^{-1} t_{z}^{-1}, r_{x}^{-1} t_{z}^{-1}\end{array}$ \\
\hline
\end{tabular}




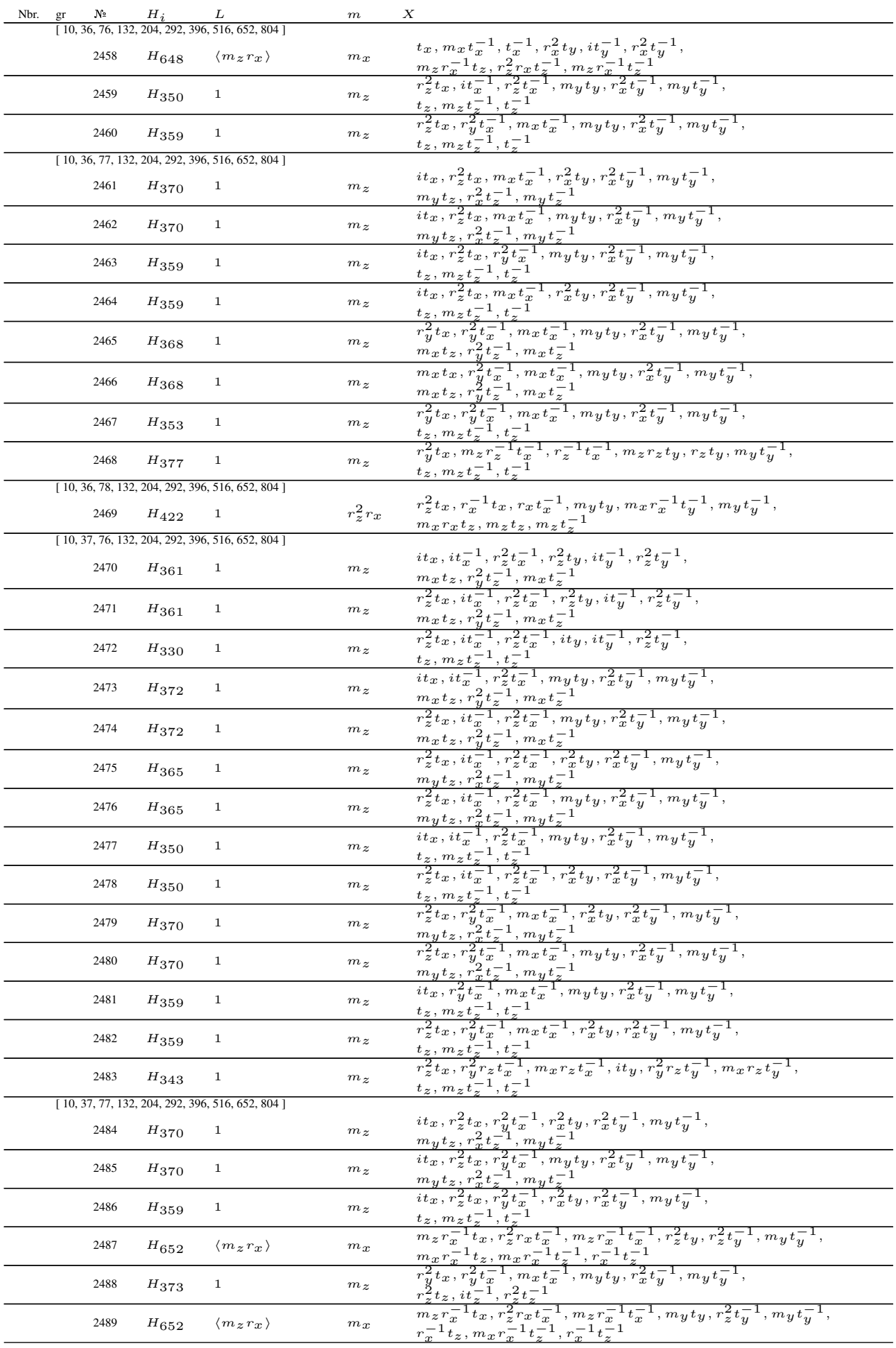




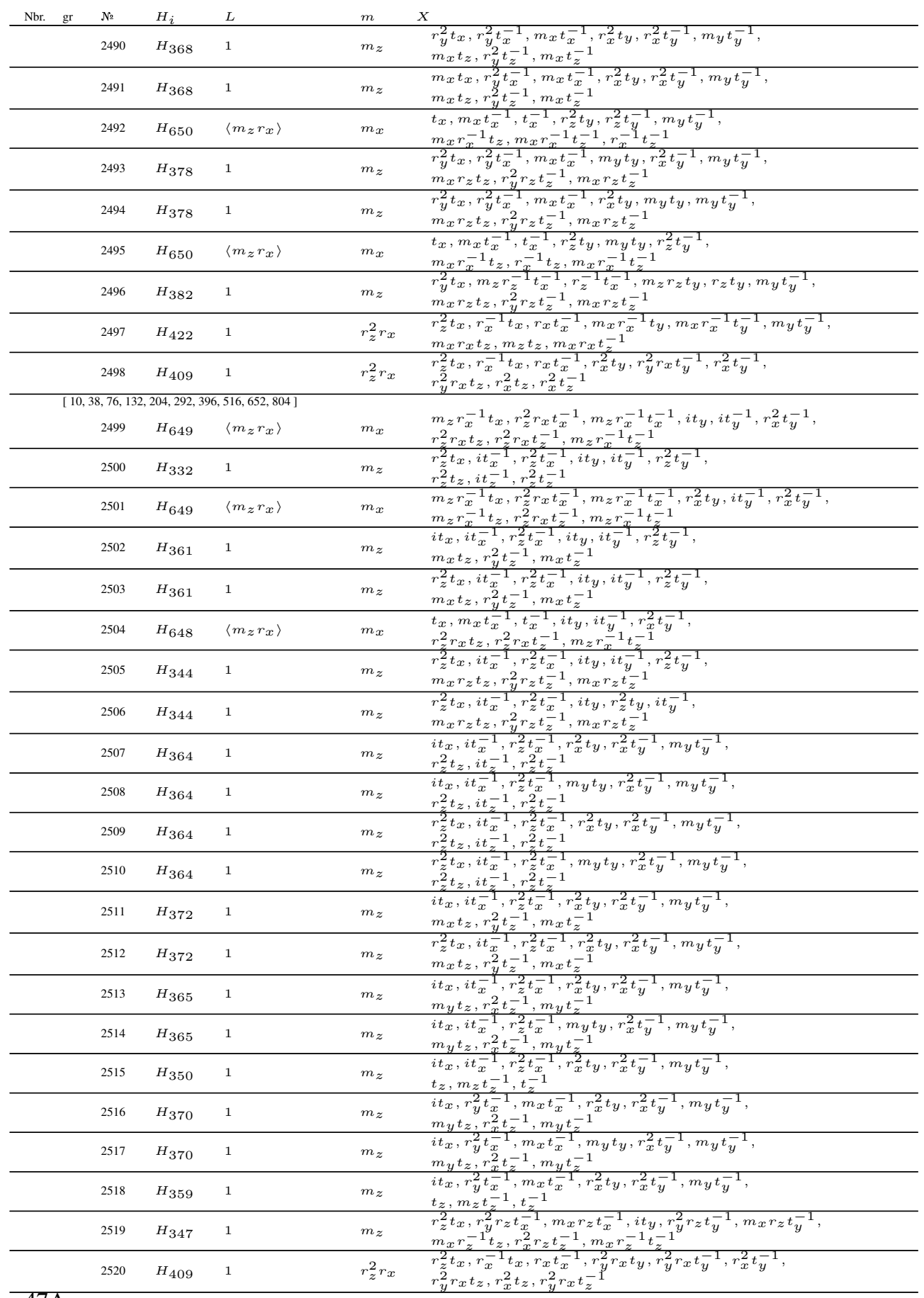




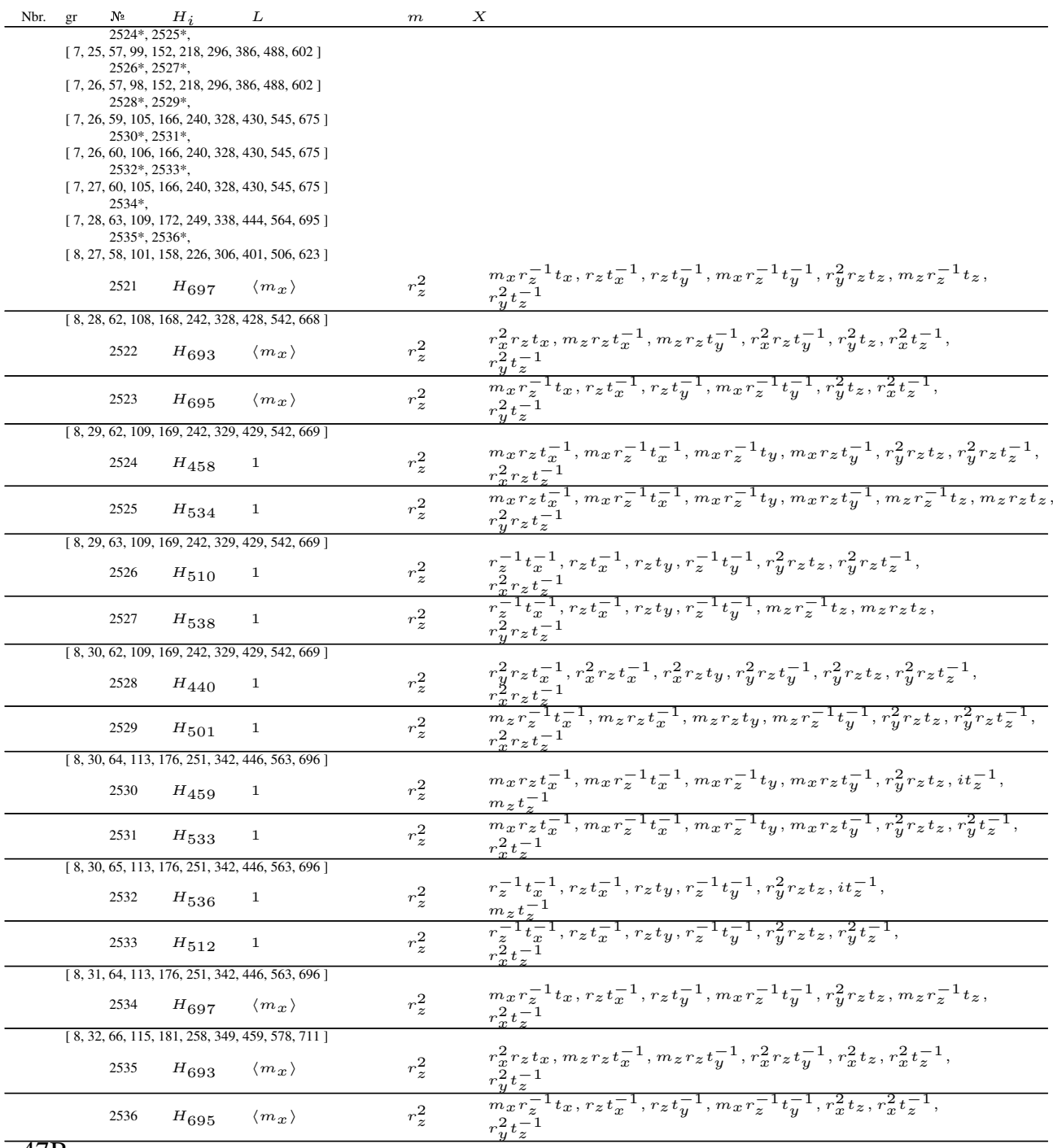

47B

[ $7,24,60,119,195,284,388,508,644,796$ ] $2537^{*}$

$[7,25,60,113,184,272,376,496,632,784]$ $2538^{*}$

$[7,25,63,122,196,284,388,508,644,796]$ $2539^{*}$

$[7,25,63,124,196,284,388,508,644,796]$ $2540^{*}$

$[7,26,64,120,192,280,384,504,640,792$ ] $2541^{*}$

$[7,26,66,124,196,284,388,508,644,796]$ $2542 *$

$[7,26,68,128,200,288,392,512,648,800$ ] $2543^{*}$,

$[7,26,69,129,200,288,392,512,648,800]$ $2544^{*}$,

$[7,27,66,122,194,282,386,506,642,794]$ $2545^{*}$

[ $7,27,67,123,194,282,386,506,642,794$ ] 2546*,

$[7,27,69,128,200,288,392,512,648,800]$ $2547^{*}, 2548^{*}, 2549^{*}$,

$[7,27,70,129,200,288,392,512,648,800]$ $2550^{*}$,

[ $7,28,67,122,194,282,386,506,642,794]$ $2551^{*}, 2552^{*}, 2553^{*}$, 


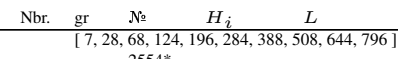
$2554 *$

$[7,28,69,125,196,284,388,508,644,796]$ $2555^{*}$

$[7,28,70,128,200,288,392,512,648,800]$ $2556^{*}$,

$[7,29,69,124,196,284,388,508,644,796]$ $2557 *$

$[7,29,71,128,200,288,392,512,648,800]$ $2558^{*}, 2559^{*}$

$[7,30,71,126,198,286,390,510,646,798]$ $2560^{*}, 2561^{*}$

[ $8,28,68,127,200,288,392,512,648,800]$

$$
2537 \quad H_{581} \quad 1
$$

$m_{z} r_{x} \quad{ }^{i t_{x}}, r_{z}^{2} r_{x} t_{x}, m_{x} t_{x}^{-1}, r_{x}^{-1} t_{y}^{-1}, m_{y} t_{z}, r_{x} t_{z}$,

$[8,29,67,122,194,282,386,506,642,794]$ $2538 \quad H_{552} \quad 1$

$[8,29,70,128,200,288,392,512,648,800]$ $2539 \quad H_{579} \quad 1$

$m_{z} r_{x} \quad i t_{x}, r_{z}^{2} r_{x} t_{x}, m_{x} t_{x}^{-1}, t_{y}, m_{z} r_{x} t_{y}^{-1}, t_{y}^{-1}$,

, 70, 130, 198, 290, 390, 514, 646, 802 ] $2540 \quad H_{577} \quad 1$

$[8,30,70,126,198,286,390,510,646,798]$ $2541 \quad H_{546} \quad 1$

$[8,30,72,128,200,288,392,512,648,800]$ $2542 \quad H_{548} \quad 1$

$[8,30,74,130,202,290,394,514,650,802]$ $2543 \quad H_{575} \quad 1$

$[8,30,75,130,202,290,394,514,650,802]$ $2544 \quad H_{575} \quad 1$

$[8,31,71,127,199,287,391,511,647,799]$ $2545 \quad H_{549} \quad 1$

$[8,31,72,127,199,287,391,511,647,799]$ $2546 \quad H_{549} \quad 1$

$m_{z} r_{x}$
$m_{z} r_{x} t_{z}$

$m_{x} t_{x}, r_{y}^{2} r_{x} t_{x}^{-1}, m_{x} t_{x}^{-1}, r_{x}^{-1} t_{y}^{-1}, m_{y} t_{z}, r_{x} t_{z}$, $m_{y} t_{z}^{-1}$

$m_{z} r_{x} \quad m_{x} t_{x}, r_{y}^{2} r_{x} t_{x}^{-1}, m_{x} t_{x}^{-1}, m_{x} r_{x}^{-1} t_{y}^{-1}, r_{z}^{2} t_{z}, m_{x} r_{x} t_{z}$, $r_{z}^{2} t_{z}^{-1}$

$8,31,74,130,202,290,394,514,650,802]$

$\begin{array}{lll}2547 & H_{573} & 1 \\ 2548 & H_{573} & 1 \\ 2549 & H_{581} & 1\end{array}$

[ $8,31,75,130,202,290,394,514,650,802]$ $2550 \quad H_{581} \quad 1$

$[8,32,71,127,199,287,391,511,647,799]$

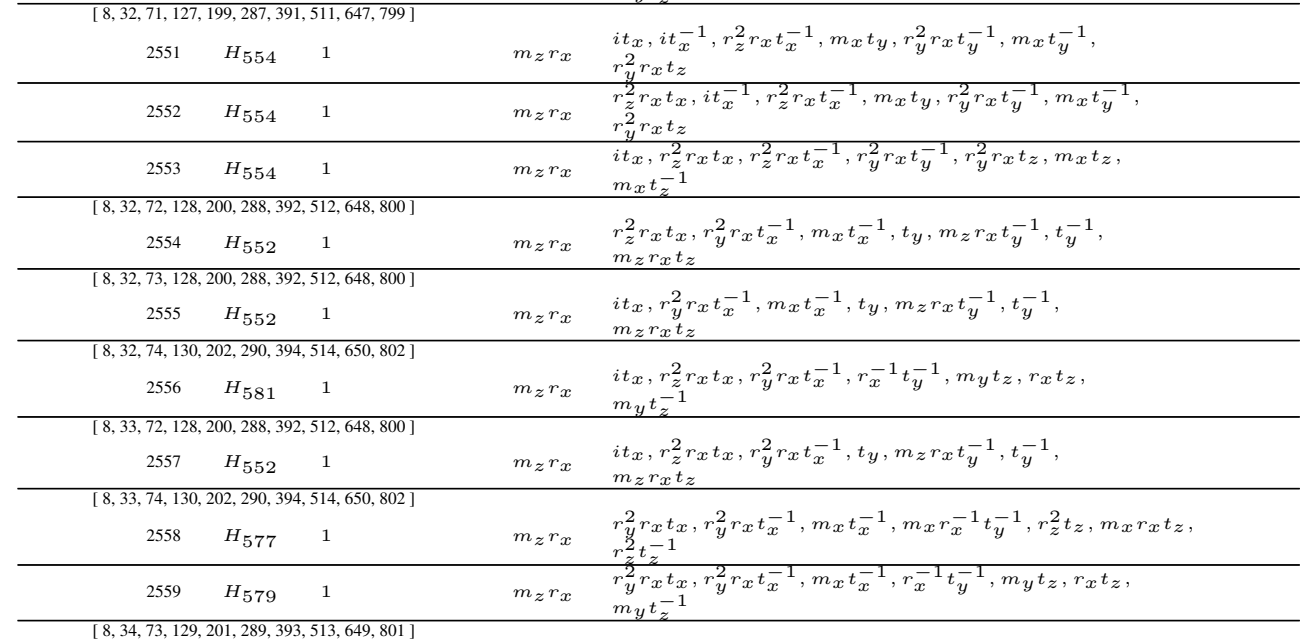




\begin{tabular}{|c|c|c|c|c|c|c|}
\hline Nbr. & gr & № & $H_{i}$ & $L$ & $m$ & $X$ \\
\hline & & 2560 & $H_{548}$ & 1 & $m_{z} r_{x}$ & $\begin{array}{l}r_{y}^{2} r_{x} t_{x}, r_{y}^{2} r_{x} t_{x}^{-1}, m_{x} t_{x}^{-1}, m_{x} t_{y}, r_{y}^{2} r_{x} t_{y}^{-1}, m_{x} t_{y}^{-1}, \\
r_{y}^{2} r_{x} t_{z}\end{array}$ \\
\hline & & 2561 & $H_{546}$ & 1 & $m_{z} r_{x}$ & $\begin{array}{l}r_{y}^{2} r_{x} t_{x}, r_{y}^{2} r_{x} t_{x}^{-1}, m_{x} t_{x}^{-1}, t_{y}, m_{z} r_{x} t_{y}^{-1}, t_{y}^{-1} \\
m_{z} r_{x} t_{z}\end{array}$ \\
\hline
\end{tabular}

$48 \mathrm{~A}$

$[9,32,72,128,200,288,392,512,648,800]$ $2562^{*}, 2563^{*}$

$[9,33,74,130,202,290,394,514,650,802]$

$2564^{*}, 2565^{*}, 2566^{*}, 2567^{*}, 2568^{*}, 2569^{*}$

$[9,33,75,131,202,290,394,514,650,802]$ $2570^{*}$

$[9,33,75,132,204,292,396,516,652,804]$ $2571^{*}, 2572^{*}, 2573^{*}, 2574^{*}$,

$[9,34,75,130,202,290,394,514,650,802]$

$2575^{*}, 2576^{*}, 2577^{*}, 2578^{*}, 2579^{*}, 2580^{*}, 2581^{*}, 2582^{*}, 2583^{*}, 2584^{*}, 2585^{*}, 2586^{*}, 2587^{*}$

$[9,34,76,132,204,292,396,516,652,804]$

$2588^{*}, 2589^{*}, 2590^{*}, 2591^{*}, 2592^{*}, 2593^{*}, 2594^{*}, 2595^{*}, 2596^{*}, 2597^{*}, 2598^{*}, 2599^{*}, 2600^{*}$, $2601 *, 2602^{*}, 2603^{*}$,

[ $9,34,77,133,204,292,396,516,652,804$ ] $2604 *$

$[9,35,77,132,204,292,396,516,652,804]$

$2605^{*}, 2606^{*}, 2607^{*}, 2608^{*}, 2609^{*}, 2610^{*}, 2611^{*}, 2612^{*}, 2613^{*}, 2614^{*}, 2615^{*}, 2616^{*}, 2617^{*}$

$2618^{*}, 2619^{*}, 2620^{*}, 2621^{*}, 2622^{*}, 2623^{*}, 2624^{*}, 2625^{*}, 2626^{*}, 2627^{*}, 2628^{*}, 2629^{*}, 2630^{*}$

$2631 * 2632 * 2633 * 2634 *, 2635 *$

$[10,34,74,130,202,290,394,514,650,802]$

\begin{tabular}{|c|c|c|c|c|}
\hline 2562 & $H_{629}$ & $\left\langle m_{z} r_{x}^{-1}\right\rangle$ & $r_{x}^{2}$ & $\begin{array}{l}r_{z}^{2} r_{x} t_{x}, r_{y}^{2} r_{x} t_{x}^{-1}, r_{z}^{2} r_{x} t_{x}^{-1}, t_{y}, m_{z} r_{x} t_{y}^{-1}, t_{y}^{-1} \\
r_{x}^{2} t_{z}, m_{z} r_{x}^{-1} t_{z}, m_{z} r_{x}^{-1} t_{z}^{-1}\end{array}$ \\
\hline 2563 & $H_{638}$ & $\left\langle m_{z} r_{x}^{-1}\right\rangle$ & $r_{x}^{2}$ & $\begin{array}{l}r_{z}^{2} r_{x} t_{x}, r_{z}^{2} t_{x}^{-1}, m_{x} r_{x} t_{x}^{-1}, t_{y}, m_{z} r_{x} t_{y}^{-1}, t_{y}^{-1} \\
r_{x}^{2} t_{z}, m_{z} r_{x}^{-1} t_{z}, m_{z} r_{x}^{-1} t_{z}^{-1}\end{array}$ \\
\hline \multicolumn{5}{|c|}{$[10,35,75,131,203,291,395,515,651,803]$} \\
\hline 2564 & $H_{433}$ & 1 & $r_{z}^{2}$ & $\begin{array}{l}m_{z} t_{x}, i t_{x}^{-1}, m_{z} t_{x}^{-1}, t_{y}, r_{z}^{2} t_{y}^{-1}, t_{y}^{-1} \\
m_{z} t_{z}, i t_{z}^{-1}, m_{z} t_{z}^{-1}\end{array}$ \\
\hline 2565 & $H_{462}$ & 1 & $r_{z}^{2}$ & $\begin{array}{l}m_{z} t_{x}, i t_{x}^{-1}, m_{z}{ }^{-1}{ }^{-1}, t_{y}, r_{z}^{2} t_{y}^{-1}, t_{y}^{-1}, \\
r_{y}^{2} t_{z}, r_{y}^{2} t_{z}^{-1}, r_{x}^{2} t_{z}^{-1}\end{array}$ \\
\hline 2566 & $H_{460}$ & 1 & $r_{z}^{2}$ & $\begin{array}{l}t_{x}, r_{z}^{2} t_{x}^{-1}, t_{x}^{-1}, t_{y}, r_{z}^{2} t_{y}^{-1}, t_{y}^{-1} \\
r_{y}^{2} t_{z}, i t_{z}^{-1}, m_{z} t_{z}^{-1}\end{array}$ \\
\hline 2567 & $H_{425}$ & 1 & $r_{z}^{2}$ & $\begin{array}{l}t_{x}, r_{z}^{2} t_{x}^{-1}, t_{x}^{-1}, t_{y}, r_{z}^{2} t_{y}^{-1}, t_{y}^{-1} \\
r_{y}^{2} t_{z}, r_{y}^{2} t_{z}^{-1}, r_{x}^{2} t_{z}^{-1}\end{array}$ \\
\hline 2568 & $H_{503}$ & 1 & $r_{z}^{2}$ & $\begin{array}{l}t_{x}, r_{z}^{2} t_{x}^{-1}, t_{x}^{-1}, t_{y}, r_{z}^{2} t_{y}^{-1}, t_{y}^{-1} \\
r_{y}^{2} r_{z} t_{z}, r_{x}^{2} r_{z} t_{z}, r_{y}^{2} t_{z}^{-1}\end{array}$ \\
\hline 2569 & $H_{519}$ & 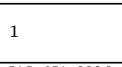 & $r_{z}^{2}$ & $\begin{array}{l}t_{x}, r_{z}^{2} t_{x}^{-1}, t_{x}^{-1}, t_{y}, r_{z}^{2} t_{y}^{-1}, t_{y}^{-1} \\
m_{z} r_{z}^{-1} t_{z}, m_{z} r_{z} t_{z}, r_{y}^{2} t_{z}^{-1}\end{array}$ \\
\hline \multicolumn{5}{|c|}{$[10,35,76,131,203,291,395,515,651,803]$} \\
\hline 2570 & $H_{561}$ & 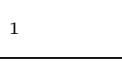 & $m_{z} r_{x}$ & $\begin{array}{l}m_{x} t_{x}, r_{y}^{2} r_{x} t_{x}^{-1}, m_{x} t_{x}^{-1}, m_{z} r_{x}^{-1} t_{y}, i t_{y}^{-1}, r_{z}^{2} r_{x} t_{y}^{-1} \\
r_{x}^{2} t_{z}, m_{z} r_{x}^{-1} t_{z}, r_{z}^{2} r_{x} t_{z}^{-1}\end{array}$ \\
\hline \multicolumn{5}{|c|}{$[10,35,76,132,204,292,396,516,652,804]$} \\
\hline 2571 & $H_{468}$ & 1 & $r_{z}^{2}$ & $\begin{array}{l}t_{x}, r_{z}^{2} t_{x}^{-1}, t_{x}^{-1}, m_{x} t_{y}, m_{x} t_{y}^{-1}, m_{y} t_{y}^{-1} \\
m_{z} t_{z}, i t_{z}^{-1}, m_{z} t_{z}^{-1}\end{array}$ \\
\hline 2572 & $H_{461}$ & 1 & $r_{z}^{2}$ & $\begin{array}{l}t_{x}, r_{z}^{2} t_{x}^{-1}, t_{x}^{-1}, m_{x} t_{y}, m_{x} t_{y}^{-1}, m_{y} t_{y}^{-1} \\
r_{y}^{2} t_{z}, r_{x}^{2} t_{z}, m_{z} t_{z}^{-1}\end{array}$ \\
\hline 2573 & $H_{640}$ & $\left\langle m_{z} r_{x}^{-1}\right\rangle$ & $r_{x}^{2}$ & $\begin{array}{l}r_{z}^{2} r_{x} t_{x}, r_{y}^{2} r_{x} t_{x}^{-1}, r_{z}^{2} r_{x} t_{x}^{-1}, m_{z} t_{y}, r_{x}^{-1} t_{y}^{-1}, m_{z} t_{y}^{-1}, \\
r_{x}^{-1} t_{z}, m_{z} t_{z}, r_{x}^{-1} t_{z}-1\end{array}$ \\
\hline 2574 & $H_{645}$ & $\left\langle m_{z} r_{x}^{-1}\right\rangle$ & $r_{x}^{2}$ & $\begin{array}{l}r_{z}^{2} r_{x} t_{x}, r_{z}^{2} t_{x}^{-1}, m_{x} r_{x} t_{x}^{-1}, m_{z} t_{y}, r_{x}^{-1} t_{y}^{-1}, m_{z} t_{y}^{-1} \\
r_{x}^{-1} t_{z}, m_{z} t_{z}, r_{x}^{-1} t_{z}^{-1}\end{array}$ \\
\hline \multicolumn{5}{|c|}{$[10,36,75,131,203,291,395,515,651,803]$} \\
\hline 2575 & $H_{631}$ & $\left\langle m_{z} r_{x}^{-1}\right\rangle$ & $r_{x}^{2}$ & $\begin{array}{l}r_{y}^{2} r_{x} t_{x}, r_{y}^{2} r_{x} t_{x}^{-1}, r_{z}^{2} r_{x} t_{x}^{-1}, m_{x} t_{y}, r_{y}^{2} r_{x} t_{y}^{-1}, m_{x} t_{y}^{-1}, \\
i t_{z}, r_{z}^{2} r_{x} t_{z}, r_{z}^{2} r_{x} t_{z}^{-1}\end{array}$ \\
\hline 2576 & $H_{631}$ & $\left\langle m_{z} r_{x}^{-1}\right\rangle$ & $r_{x}^{2}$ & $\begin{array}{l}r_{z}^{2} r_{x} t_{x}, r_{y}^{2} r_{x} t_{x}^{-1}, r_{z}^{2} r_{x} t_{x}^{-1}, m_{x} t_{y}, r_{y}^{2} r_{x} t_{y}^{-1}, m_{x} t_{y}^{-1} \\
i t_{z}, r_{z}^{2} r_{x} t_{z}, r_{z}^{2} r_{x} t_{z}^{-1}\end{array}$ \\
\hline 2577 & $H_{471}$ & 1 & $r_{z}^{2}$ & $\begin{array}{l}m_{z} t_{x}, i t_{x}^{-1}, m_{z} t_{x}^{-1}, m_{z} t_{y}, i t_{y}^{-1}, m_{z} t_{y}^{-1} \\
r_{y}^{2} t_{z}, r_{y}^{2} t_{z}^{-1}, r_{x}^{2} t_{z}^{-1}\end{array}$ \\
\hline 2578 & $H_{433}$ & 1 & $r_{z}^{2}$ & $\begin{array}{l}m_{z} t_{x}, i t_{x}^{-1}, m_{z} t_{x}^{-1}, t_{y}, r_{z}^{2} t_{y}^{-1}, t_{y}^{-1} \\
i t_{z}, i t_{z}^{-1}, m_{z} t_{z}^{-1}\end{array}$ \\
\hline 2579 & $H_{462}$ & 1 & $r_{z}^{2}$ & $\begin{array}{l}m_{z} t_{x}, i t_{x}^{-1}, m_{z} t_{x}^{-1}, t_{y}, r_{z}^{2} t_{y}^{-1}, t_{y}^{-1} \\
r_{x}^{2} t_{z}, r_{y}^{2} t_{z}^{-1}, r_{x}^{2} t_{z}^{-1}\end{array}$ \\
\hline 2580 & $H_{629}$ & $\left\langle m_{z} r_{x}^{-1}\right\rangle$ & $r_{x}^{2}$ & $\begin{array}{l}r_{y}^{2} r_{x} t_{x}, r_{y}^{2} r_{x} t_{x}^{-1}, r_{z}^{2} r_{x} t_{x}^{-1}, t_{y}, m_{z} r_{x} t_{y}^{-1}, t_{y}^{-1} \\
r_{x}^{2} t_{z}, m_{z} r_{x}^{-1} t_{z}, m_{z} r_{x}^{-1} t_{z}^{-1}\end{array}$ \\
\hline 2581 & $H_{638}$ & $\left\langle m_{z} r_{x}^{-1}\right\rangle$ & $r_{x}^{2}$ & $\begin{array}{l}r_{y}^{2} r_{x} t_{x}, r_{z}^{2} t_{x}^{-1}, m_{x} r_{x} t_{x}^{-1}, t_{y}, m_{z} r_{x} t_{y}^{-1}, t_{y}^{-1} \\
r_{x}^{2} t_{z}, m_{z} r_{x}^{-1} t_{z}, m_{z} r_{x}^{-1} t_{z}^{-1}\end{array}$ \\
\hline 2582 & $H_{507}$ & 1 & $r_{z}^{2}$ & $\begin{array}{l}r_{y}^{2} r_{z} t_{x}, r_{y}^{2} r_{z} t_{x}^{-1}, r_{x}^{2} r_{z} t_{x}^{-1}, r_{y}^{2} r_{z} t_{y}, r_{x}^{2} r_{z} t_{y}, r_{y}^{2} r_{z} t_{y}^{-1} \\
r_{y}^{2} t_{z}, r_{y}^{2} t_{z}^{1}, r_{x}^{2} t_{z}^{-1}\end{array}$ \\
\hline 2583 & $H_{531}$ & 1 & $r_{z}^{2}$ & $\begin{array}{l}m_{x} r_{z} t_{x}, m_{x} r_{z} t_{x}^{-1}, m_{x} r_{z}^{-1} t_{x}^{-1}, m_{x} r_{z} t_{y}, m_{x} r_{z}^{-1} t_{y}, m_{x} r_{z} t_{y}^{-1} \\
r_{y}^{2} t_{z}, i t_{z}^{-1}, m_{z} t_{z}^{-1}\end{array}$ \\
\hline
\end{tabular}




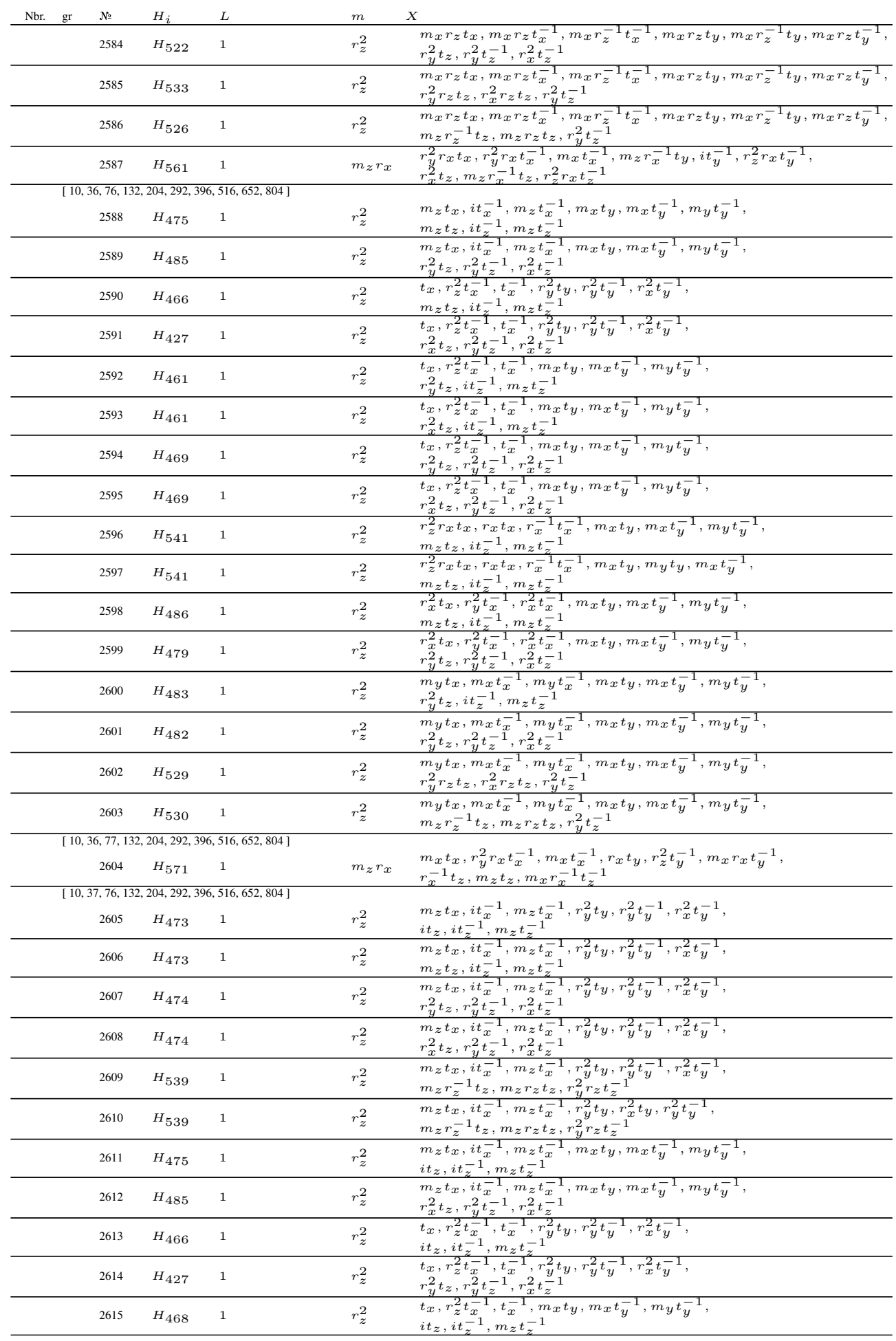




\begin{tabular}{|c|c|c|c|c|c|}
\hline Nbr. & № & $H_{i}$ & $L$ & $m$ & $X$ \\
\hline & 2616 & $H_{461}$ & 1 & $r_{z}^{2}$ & $\begin{array}{l}t_{x}, r_{z}^{2} t_{x}^{-1}, t_{x}^{-1}, m_{x} t_{y}, m_{x} t_{y}^{-1}, m_{y} t_{y}^{-1} \\
r_{y}^{2} t_{z}, r_{x}^{2} t_{z}, i t_{z}^{-1}\end{array}$ \\
\hline & 2617 & $H_{511}$ & 1 & $r_{z}^{2}$ & $\begin{array}{l}r_{z}^{2} r_{x} t_{x}, r_{x} t_{x}, r_{x}^{-1} t_{x}^{-1}, r_{y}^{2} t_{y}, r_{y}^{2} t_{y}^{-1}, r_{x}^{2} t_{y}^{-1} \\
r_{y}^{2} t_{z}, r_{y}^{2} t_{z}^{-1}, r_{x}^{2} t_{z}^{-1}\end{array}$ \\
\hline & 2618 & $H_{511}$ & 1 & $r_{z}^{2}$ & $\begin{array}{l}r_{z}^{2} r_{x} t_{x}, r_{x} t_{x}, r_{x}^{-1} t_{x}^{-1}, r_{y}^{2} t_{y}, r_{y}^{2} t_{y}^{-1}, r_{x}^{2} t_{y}^{-1} \\
r_{y}^{2} t_{z}, r_{x}^{2} t_{z}, r_{y}^{2} t_{z}^{-1}\end{array}$ \\
\hline & 2619 & $H_{511}$ & 1 & $r_{z}^{2}$ & $\begin{array}{l}r_{z}^{2} r_{x} t_{x}, r_{x} t_{x}, r_{x}^{-1} t_{x}^{-1}, r_{y}^{2} t_{y}, r_{y}^{2} t_{y}^{-1}, r_{x}^{2} t_{y}^{-1} \\
r_{x}^{2} t_{z}, r_{y}^{2} t_{z}^{-1}, r_{x}^{2} t_{z}^{-1}\end{array}$ \\
\hline & 2620 & $H_{511}$ & 1 & $r_{z}^{2}$ & $\begin{array}{l}r_{z}^{2} r_{x} t_{x}, r_{x} t_{x}, r_{x}^{-1} t_{x}^{-1}, r_{y}^{2} t_{y}, r_{x}^{2} t_{y}, r_{y}^{2} t_{y}^{-1} \\
r_{x}^{2} t_{z}, r_{y}^{2} t_{z}^{-1}, r_{x}^{2} t_{z}^{-1}\end{array}$ \\
\hline & 2621 & $H_{541}$ & 1 & $r_{z}^{2}$ & $\begin{array}{l}r_{z}^{2} r_{x} t_{x}, r_{x} t_{x}, r_{x}^{-1} t_{x}^{-1}, m_{x} t_{y}, m_{x} t_{y}^{-1}, m_{y} t_{y}^{-1} \\
i t_{z}, i t_{z}^{-1}, m_{z} t_{z}^{-1}\end{array}$ \\
\hline & 2622 & $H_{541}$ & 1 & $r_{z}^{2}$ & $\begin{array}{l}r_{z}^{2} r_{x} t_{x}, r_{x} t_{x}, r_{x}^{-1} t_{x}^{-1}, m_{x} t_{y}, m_{x} t_{y}^{-1}, m_{y} t_{y}^{-1} \\
i t_{z}, m_{z} t_{z}, i t_{z}^{-1}\end{array}$ \\
\hline & 2623 & $H_{639}$ & $\left\langle m_{z} r_{x}^{-1}\right\rangle$ & $r_{x}^{2}$ & $\begin{array}{l}r_{y}^{2} r_{x} t_{x}, r_{y}^{2} r_{x} t_{x}^{-1}, r_{z}^{2} r_{x} t_{x}^{-1}, r_{y}^{2} t_{y}, m_{x} r_{x}^{-1} t_{y}^{-1}, r_{y}^{2} t_{y}^{-1}, \\
m_{x} r_{x}^{-1} t_{z}, r_{y}^{2} t_{z}, m_{x} r_{x}^{-1} t_{z}^{-1}\end{array}$ \\
\hline & 2624 & $H_{639}$ & $\left\langle m_{z} r_{x}^{-1}\right\rangle$ & $r_{x}^{2}$ & $\begin{array}{l}r_{z}^{2} r_{x} t_{x}, r_{y}^{2} r_{x} t_{x}^{-1}, r_{z}^{2} r_{x} t_{x}^{-1}, r_{y}^{2} t_{y}, m_{x} r_{x}^{-1} t_{y}^{-1}, r_{y}^{2} t_{y}^{-1} \\
m_{x} r_{x}^{-1} t_{z}, r_{y}^{2} t_{z}, m_{x} r_{x}^{-1} t_{z}^{-1}\end{array}$ \\
\hline & 2625 & $H_{429}$ & 1 & $r_{z}^{2}$ & $\begin{array}{l}r_{x}^{2} t_{x}, r_{y}^{2} t_{x}^{-1}, r_{x}^{2} t_{x}^{-1}, r_{y}^{2} t_{y}, r_{y}^{2} t_{y}^{-1}, r_{x}^{2} t_{y}^{-1} \\
r_{y}^{2} t_{z}, r_{y}^{2} t_{z}^{-1}, r_{x}^{2} t_{z}^{-1}\end{array}$ \\
\hline & 2626 & $H_{486}$ & 1 & $r_{z}^{2}$ & $\begin{array}{l}r_{x}^{2} t_{x}, r_{y}^{2} t_{x}^{-1}, r_{x}^{2} t_{x}^{-1}, m_{x} t_{y}, m_{x} t_{y}^{-1}, m_{y} t_{y}^{-1} \\
i t_{z}, i t_{z}^{-1}, m_{z} t_{z}^{-1}\end{array}$ \\
\hline & 2627 & $H_{479}$ & 1 & $r_{z}^{2}$ & $\begin{array}{l}r_{x}^{2} t_{x}, r_{y}^{2} t_{x}^{-1}, r_{x}^{2} t_{x}^{-1}, m_{x} t_{y}, m_{x} t_{y}^{-1}, m_{y} t_{y}^{-1} \\
r_{x}^{2} t_{z}, r_{y}^{2} t_{z}^{-1}, r_{x}^{2} t_{z}^{-1}\end{array}$ \\
\hline & 2628 & $H_{640}$ & $\left\langle m_{z} r_{x}^{-1}\right\rangle$ & $r_{x}^{2}$ & $\begin{array}{l}r_{y}^{2} r_{x} t_{x}, r_{y}^{2} r_{x} t_{x}^{-1}, r_{z}^{2} r_{x} t_{x}^{-1}, m_{z} t_{y}, r_{x}^{-1} t_{y}^{-1}, m_{z} t_{y}^{-1}, \\
r_{x}^{-1} t_{z}, m_{z} t_{z}, r_{x}^{-1} t_{z}^{-1}\end{array}$ \\
\hline & 2629 & $H_{645}$ & $\left\langle m_{z} r_{x}^{-1}\right\rangle$ & $r_{x}^{2}$ & $\begin{array}{l}r_{y}^{2} r_{x} t_{x}, r_{z}^{2} t_{x}^{-1}, m_{x} r_{x} t_{x}^{-1}, m_{z} t_{y}, r_{x}^{-1} t_{y}^{-1}, m_{z} t_{y}^{-1} \\
r_{x}^{-1} t_{z}, m_{z} t_{z}, r_{x}^{-1} t_{z}^{-1}\end{array}$ \\
\hline & 2630 & $H_{524}$ & 1 & $r_{z}^{2}$ & $\begin{array}{l}m_{z} r_{z} t_{x}, m_{z} r_{z}^{-1} t_{x}^{-1}, m_{z} r_{z} t_{x}^{-1}, m_{z} r_{z}^{-1} t_{y}, m_{z} r_{z} t_{y}, m_{z} r_{z}^{-1} t_{y}^{-1} \\
r_{y}^{2} t_{z}, r_{y}^{2} t_{z}^{-1}, r_{x}^{2} t_{z}^{-1}\end{array}$ \\
\hline & 2631 & $H_{535}$ & 1 & $r_{z}^{2}$ & $\begin{array}{l}r_{z}^{t} t_{x}, r_{z}^{-1} t_{x}^{-1}, r_{z} t_{x}^{-1}, r_{z}^{-1} t_{y}, r_{z} t_{y}, r_{z}^{-1} t_{y}^{-1} \\
r_{y}^{2} t_{z}, i t_{z}^{-1}, m_{z} t_{z}^{-1}\end{array}$ \\
\hline & 2632 & $H_{509}$ & 1 & $r_{z}^{2}$ & $\begin{array}{l}r_{z} t_{x}, r_{z}^{-1} t_{x}^{-1}, r_{z} t_{x}^{-1}, r_{z}^{-1} t_{y}, r_{z} t_{y}, r_{z}^{-1} t_{y}^{-1} \\
r_{y}^{2} t_{z}, r_{y}^{2} t_{z}^{-1}, r_{x}^{2} t_{z}^{-1}\end{array}$ \\
\hline & 2633 & $H_{512}$ & 1 & $r_{z}^{2}$ & $\begin{array}{l}r_{z} t_{x}, r_{z}^{-1} t_{x}^{-1}, r_{z} t_{x}^{-1}, r_{z}^{-1} t_{y}, r_{z} t_{y}, r_{z}^{-1} t_{y}^{-1} \\
r_{y}^{2} r_{z} t_{z}, r_{x}^{2} r_{z} t_{z}, r_{y}^{2} t_{z}^{-1}\end{array}$ \\
\hline & 2634 & $H_{537}$ & 1 & $r_{z}^{2}$ & $\begin{array}{l}r_{z} t_{x}, r_{z}^{-1} t_{x}^{-1}, r_{z} t_{x}^{-1}, r_{z}^{-1} t_{y}, r_{z} t_{y}, r_{z}^{-1} t_{y}^{-1} \\
m_{z} r_{z}^{-1} t_{z}, m_{z} r_{z} t_{z}, r_{y}^{2} t_{z}^{-1}\end{array}$ \\
\hline & 2635 & $H_{571}$ & 1 & $m_{z} r_{x}$ & $\begin{array}{l}r_{y}^{2} r_{x} t_{x}, r_{y}^{2} r_{x} t_{x}^{-1}, m_{x} t_{x}^{-1}, r_{x} t_{y}, r_{z}^{2} t_{y}^{-1}, m_{x} r_{x} t_{y}^{-1} \\
r_{x}^{-1} t_{z}, m_{z} t_{z}, m_{x} r_{x}^{-1} t_{z}^{-1}\end{array}$ \\
\hline
\end{tabular}

$[9,35,79,134,204,292,396,516,652,804]$ $2636^{*}$,

$[9,36,79,133,204,292,396,516,652,804]$ $2637^{*}, 2638^{*}$,

$[9,37,79,132,204,292,396,516,652,804]$ $2639^{*}, 2640^{*}, 2641^{*}, 2642^{*}, 2643^{*}$,

$[10,37,78,132,204,292,396,516,652,804]$

$2636 \quad H_{766} \quad\left\langle m_{y}, r_{x}^{2}\right\rangle$

$[10,38,77,132,204,292,396,516,652,804]$

$\begin{array}{lll}2637 & H_{561} & 1 \\ 2638 & H_{571} & 1\end{array}$

$[10,39,76,132,204,292,396,516,652,804]$

\begin{tabular}{lll}
2639 & $H_{768}$ & $\left\langle m_{y}, r_{x}^{2}\right\rangle$ \\
\hline 2640 & $H_{768}$ & $\left\langle m_{y}, r_{x}^{2}\right\rangle$ \\
\hline 2641 & $H_{766}$ & $\left\langle m_{y}, r_{x}^{2}\right\rangle$ \\
\hline 2642 & $H_{561}$ & 1 \\
\hline 2643 & $H_{571}$ & 1
\end{tabular}

$m_{z} r_{x}^{-1} \quad m_{x} t_{x}, m_{x} t_{x}^{-1}, r_{z}^{2} r_{x} t_{x}^{-1}, m_{y} t_{y}, r_{x}^{-1} t_{y}, t_{y}^{-1}$, $m_{z} r_{x}^{-1} t_{y}^{-1}, m_{z} r_{x}^{-1} t_{z}, r_{x} t_{z}^{-1}$

$m_{x} t_{x}, r_{y}^{2} r_{x} t_{x}^{-1}, m_{x} t_{x}^{-1}, r_{x}^{2} t_{y}, m_{z} r_{x}^{-1} t_{y}, i t_{y}^{-1}$

$m_{z} r_{x} \quad m_{x} t_{x}, r_{y}^{2} r_{x} t_{x}^{-1}, m_{x} t_{x}^{-1}, r_{x}^{2} t_{y}$

$m_{z} r_{x} \quad m_{x} t_{x}, r_{y}^{2} r_{x} t_{x}^{-1}, m_{x} t_{x}^{-1}, m_{y} t_{y}, r_{x} t_{y}, r_{z}^{2} t_{y}^{-1}$,

$m_{x} r_{x} t_{y}^{-1}, r_{x}^{-1} t_{z}, m_{x} r_{x}^{-1} t_{z}^{-1}$

$m_{x} t_{x}, m_{x} t_{x}^{-1}, r_{z}^{2} r_{x} t_{x}^{-1}, r_{z}^{2} t_{y}, m_{x} r_{x}^{-1} t_{y}, m_{x} t_{y}^{-1}$,

$\begin{array}{ll}m_{z} r_{x}^{-1} & m_{x} t_{x}, m_{x} t_{x}^{-1}, r_{z}^{2} r_{x} t_{x}^{-1}, r_{z}^{2} t_{y} \\ & r_{z}^{2} r_{x} t_{y}^{-1}, r_{z}^{2} r_{x} t_{z}, m_{x} r_{x} t_{z}^{-1}\end{array}$

$r_{z}^{2} r_{x} t_{x}, m_{x} t_{x}^{-1}, r_{z}^{2} r_{x} t_{x}^{-1}, r_{z}^{2} t_{y}, m_{x} r_{x}^{-1} t_{y}, m_{x} t_{y}^{-1}$,

$\begin{array}{ll}m_{z} r_{x}^{-1} & r_{z}^{2} r_{x} t_{x}, m_{x} t_{x}, r_{z}^{2} r_{x} t_{x}, r_{z}^{2} t_{z}^{2} \\ & r_{z}^{2} r_{x} t_{y}^{-1}, r_{z}^{2} r_{x} t_{z}, m_{x} r_{x} t_{z}^{-1}\end{array}$

$m_{z} r_{x}^{-1} r_{z}^{2} r_{x} t_{x}, m_{x} t_{x}^{-1}, r_{z}^{2} r_{x} t_{x}^{-1}, m_{y} t_{y}, r_{x}^{-1} t_{y}, t_{y}^{-1}$,

$r_{z}^{2} r_{x} t_{x}, m_{x} t_{x}^{-1}, r_{z}^{2} r_{x} t_{x}^{-1}, m_{y}$
$m_{z} r_{x}^{-1} t_{y}^{-1}, m_{z} r_{x}^{-1} t_{z}, r_{x} t_{z}^{-1}$

$r_{y}^{2} r_{x} t_{x}, r_{y}^{2} r_{x} t_{x}^{-1}, m_{x} t_{x}^{-1}, r_{x}^{2} t_{y}, m_{z} r_{x}^{-1} t_{y}, i t_{y}^{-1}$,

$\begin{array}{ll}m_{z} r_{x} & r_{y}^{2} r_{x} t_{x}, r_{y}^{2} r_{x} t_{x}^{2}, m_{x} t_{x} \\ r_{z}^{2} r_{x} t_{y}^{-1}, m_{z} r_{x}^{-1} t_{z}, r_{z}^{2} r_{x} t_{z}^{-1}\end{array}$

$m_{z} r_{x} \quad r_{y}^{2} r_{x} t_{x}, r_{y}^{2} r_{x} t_{x}^{-1}, m_{x} t_{x}^{-1}, m_{y} t_{y}, r_{x} t_{y}, r_{z}^{2} t_{y}^{-1}$,

$m_{x} r_{x} t_{y}^{-1}, r_{x}^{-1} t_{z}, m_{x} r_{x}^{-1} t_{z}^{-1}$

$[10,36,77,132,204,292,396,516,652,804]$ 


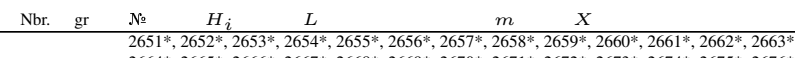

$2664^{*}, 2665^{*}, 2666^{*}, 2667^{*}, 2668^{*}, 269^{*}, 2670^{*}, 2671^{*}, 2672^{*}, 2673^{*}, 2674^{*}, 2675^{*}, 2676^{*}$,

$2677^{*}, 2678^{*}, 2679^{*}, 2680^{*}, 2681^{*}, 2682^{*}, 2683^{*}, 2684^{*}, 2685^{*}, 2686^{*}, 2687^{*}, 2688^{*}, 2689^{*}$

$2690 *, 2691 *, 2692^{*}, 2693^{*}, 2694^{*}$

$[11,36,76,132,204,292,396,516,652,804]$

\begin{tabular}{|c|c|c|c|c|}
\hline 2644 & $H_{682}$ & $\left\langle m_{x}\right\rangle$ & $r_{z}^{2}$ & $\begin{array}{l}i t_{x}, r_{y}^{2} t_{x}, r_{x}^{2} t_{x}^{-1}, r_{y}^{2} t_{x}^{-1}, m_{x} t_{y}, m_{y} t_{y}^{-1} \\
m_{x} t_{y}^{-1}, r_{y}^{2} t_{z}, r_{x}^{2} t_{z}^{-1}, r_{y}^{2} t_{z}^{-1}\end{array}$ \\
\hline 2645 & $H_{680}$ & $\left\langle m_{x}\right\rangle$ & $r_{z}^{2}$ & $\begin{array}{l}r_{z}^{2} t_{x}, m_{x} t_{x}, m_{y} t_{x}^{-1}, m_{x} t_{x}^{-1}, m_{x} t_{y}, m_{y} t_{y}^{-1}, \\
m_{x} t_{y}^{-1}, r_{y}^{2} t_{z}, r_{x}^{2} t_{z}^{-1}, r_{y}^{2} t_{z}^{-1}\end{array}$ \\
\hline 2646 & $H_{359}$ & 1 & $m_{z}$ & $\begin{array}{l}i t_{x}, r_{z}^{2} t_{x}, r_{y}^{2} t_{x}^{-1}, m_{x} t_{x}^{-1}, m_{y} t_{y}, r_{x}^{2} t_{y}^{-1} \\
m_{y} t_{y}^{-1}, t_{z}, m_{z} t_{z}^{-1}, t_{z}^{-1}\end{array}$ \\
\hline 2647 & $H_{343}$ & 1 & $m_{z}$ & $\begin{array}{l}r_{z}^{2} t_{x}, r_{y}^{2} r_{z} t_{x}^{-1}, m_{x} r_{z} t_{x}^{-1}, i t_{y}, r_{z}^{2} t_{y}, r_{y}^{2} r_{z} t_{y}^{-1} \\
m_{x} r_{z} t_{y}^{-1}, t_{z}, m_{z} t_{z}^{-1}, t_{z}^{-1}\end{array}$ \\
\hline 2648 & $H_{377}$ & 1 & $m_{z}$ & $\begin{array}{l}r_{y}^{2} t_{x}, m_{x} t_{x}, m_{z} r_{z}^{-1} t_{x}^{-1}, r_{z}^{-1} t_{x}^{-1}, m_{z} r_{z} t_{y}, r_{z} t_{y}, \\
m_{y} t_{y}^{-1}, t_{z}, m_{z} t_{z}^{-1}, t_{z}^{-1}\end{array}$ \\
\hline 2649 & $H_{690}$ & $\left\langle m_{x}\right\rangle$ & $r_{z}^{2}$ & $\begin{array}{l}r_{z}^{2} t_{x}, m_{x} t_{x}, m_{y} t_{x}^{-1}, m_{x} t_{x}^{-1}, m_{x} t_{y}, m_{y} t_{y}^{-1} \\
m_{x} t_{y}^{-1}, r_{y}^{2} r_{z} t_{z}, m_{z} r_{z}^{-1} t_{z}, r_{y}^{2} t_{z}^{-1}\end{array}$ \\
\hline 2650 & $H_{541}$ & 1 & $r_{z}^{2}$ & $\begin{array}{l}r_{z}^{2} r_{x} t_{x}, r_{x} t_{x}, r_{y}^{2} r_{x} t_{x}^{-1}, r_{x}^{-1} t_{x}^{-1}, m_{x} t_{y}, m_{x} t_{y}^{-1} \\
m_{y} t_{y}^{-1}, m_{z} t_{z}, i t_{z}^{-1}, m_{z} t_{z}^{-1}\end{array}$ \\
\hline \multicolumn{5}{|c|}{$[11,37,76,132,204,292,396,516,652,804]$} \\
\hline 2651 & $H_{683}$ & $\left\langle m_{x}\right\rangle$ & $r_{z}^{2}$ & $\begin{array}{l}i t_{x}, r_{y}^{2} t_{x}, r_{x}^{2} t_{x}^{-1}, r_{y}^{2} t_{x}^{-1}, r_{y}^{2} t_{y}, r_{x}^{2} t_{y}^{-1} \\
r_{y}^{2} t_{y}^{-1}, r_{x}^{2} t_{z}, r_{y}^{2} t_{z}, r_{x}^{2} t_{z}^{-1}\end{array}$ \\
\hline 2652 & $H_{683}$ & $\left\langle m_{x}\right\rangle$ & $r_{z}^{2}$ & $\begin{array}{l}i t_{x}, r_{y}^{2} t_{x}, r_{x}^{2} t_{x}^{-1}, r_{y}^{2} t_{x}^{-1}, r_{y}^{2} t_{y}, r_{x}^{2} t_{y}^{-1} \\
r_{y}^{2} t_{y}^{-1}, r_{x}^{2} t_{z}, r_{y}^{2} t_{z}, r_{y}^{2} t_{z}^{-1}\end{array}$ \\
\hline 2653 & $H_{682}$ & $\left\langle m_{x}\right\rangle$ & $r_{z}^{2}$ & $\begin{array}{l}i t_{x}, r_{y}^{2} t_{x}, r_{x}^{2} t_{x}^{-1}, r_{y}^{2} t_{x}^{-1}, m_{x} t_{y}, m_{y} t_{y}^{-1} \\
m_{x} t_{y}^{-1}, r_{x}^{2} t_{z}, r_{x}^{2} t_{z}^{-1}, r_{y}^{2} t_{z}^{-1}\end{array}$ \\
\hline 2654 & $H_{344}$ & 1 & $m_{z}$ & $\begin{array}{l}i t_{x}, i t_{x}^{-1}, r_{z}^{2} t_{x}^{-1}, i t_{y}, r_{z}^{2} t_{y}, i t_{y}^{-1} \\
r_{z}^{2} t_{y}^{-1}, m_{x} r_{z} t_{z}, r_{y}^{2} r_{z} t_{z}^{-1}, m_{x} r_{z} t_{z}^{-1}\end{array}$ \\
\hline 2655 & $H_{344}$ & 1 & $m_{z}$ & $\begin{array}{l}i t_{x}, r_{z}^{2} t_{x}, i t_{x}^{-1}, r_{z}^{2} t_{x}^{-1}, i t_{y}, r_{z}^{2} t_{y} \\
i t_{y}^{-1}, m_{x} r_{z} t_{z}, r_{y}^{2} r_{z} t_{z}^{-1}, m_{x} r_{z} t_{z}^{-1}\end{array}$ \\
\hline 2656 & $H_{344}$ & 1 & $m_{z}$ & $\begin{array}{l}r_{z}^{2} t_{x}, i t_{x}^{-1}, r_{z}^{2} t_{x}^{-1}, i t_{y}, r_{z}^{2} t_{y}, i t_{y}^{-1} \\
r_{z}^{2} t_{y}^{-1}, m_{x} r_{z} t_{z}, r_{y}^{2} r_{z} t_{z}^{-1}, m_{x} r_{z} t_{z}^{-1}\end{array}$ \\
\hline 2657 & $H_{344}$ & 1 & $m_{z}$ & $\begin{array}{l}i t_{x}, r_{z}^{2} t_{x}, i t_{x}^{-1}, r_{z}^{2} t_{x}^{-1}, i t_{y}, r_{z}^{2} t_{y} \\
r_{z}^{2} t_{y}^{-1}, m_{x} r_{z} t_{z}, r_{y}^{2} r_{z} t_{z}^{-1}, m_{x} r_{z} t_{z}^{-1}\end{array}$ \\
\hline 2658 & $H_{682}$ & $\left\langle m_{x}\right\rangle$ & $r_{z}^{2}$ & $\begin{array}{l}i t_{x}, r_{y}^{2} t_{x}, r_{x}^{2} t_{x}^{-1}, r_{y}^{2} t_{x}^{-1}, m_{x} t_{y}, m_{y} t_{y}^{-1} \\
m_{x} t_{y}^{-1}, r_{x}^{2} t_{z}, r_{y}^{2} t_{z}, r_{x}^{2} t_{z}^{-1}\end{array}$ \\
\hline 2659 & $H_{683}$ & $\left\langle m_{x}\right\rangle$ & $r_{z}^{2}$ & $\begin{array}{l}i t_{x}, r_{y}^{2} t_{x}, r_{x}^{2} t_{x}^{-1}, r_{y}^{2} t_{x}^{-1}, r_{y}^{2} t_{y}, r_{x}^{2} t_{y}^{-1} \\
r_{y}^{2} t_{y}^{-1}, r_{x}^{2} t_{z}, r_{x}^{2} t_{z}^{-1}, r_{y}^{2} t_{z}^{-1}\end{array}$ \\
\hline 2660 & $H_{683}$ & $\left\langle m_{x}\right\rangle$ & $r_{z}^{2}$ & $\begin{array}{l}i_{x}, r_{y}^{2} t_{x}, r_{x}^{2} t_{x}^{-1}, r_{y}^{2} t_{x}^{-1}, r_{y}^{2} t_{y}, r_{x}^{2} t_{y}^{-1} \\
r_{y}^{2} t_{y}^{-1}, r_{y}^{2} t_{z}, r_{x}^{2} t_{z}^{-1}, r_{y}^{2} t_{z}^{-1}\end{array}$ \\
\hline 2661 & $H_{682}$ & $\left\langle m_{y}\right\rangle$ & $m_{x}$ & $\begin{array}{l}m_{z} t_{x}, r_{y}^{2} t_{x}^{-1}, m_{z} t_{x}^{-1}, r_{z}^{2} t_{y}, m_{y} t_{y}, m_{x} t_{y}^{-1}, \\
t_{y}^{-1}, r_{y}^{2} t_{z}, r_{y}^{2} t_{z}^{-1}, m_{z} t_{z}^{-1}\end{array}$ \\
\hline 2662 & $H_{682}$ & $\left\langle m_{y}\right\rangle$ & $m_{x}$ & $\begin{array}{l}m_{z} t_{x}, r_{y}^{2} t_{x}^{-1}, m_{z} t_{x}^{-1}, r_{z}^{2} t_{y}, m_{y} t_{y}, m_{x} t_{y}^{-1} \\
t_{y}^{-1}, m_{z} t_{z}, r_{y}^{2} t_{z}^{-1}, m_{z} t_{z}^{-1}\end{array}$ \\
\hline 2663 & $H_{680}$ & $\left\langle m_{x}\right\rangle$ & $r_{z}^{2}$ & $\begin{array}{l}r_{z}^{2} t_{x}, m_{x} t_{x}, m_{y} t_{x}^{-1}, m_{x} t_{x}^{-1}, m_{x} t_{y}, m_{y} t_{y}^{-1}, \\
m_{x} t_{y}^{-1}, r_{x}^{2} t_{z}, r_{x}^{2} t_{z}^{-1}, r_{y}^{2} t_{z}^{-1}\end{array}$ \\
\hline 2664 & $H_{370}$ & 1 & $m_{z}$ & $\begin{array}{l}i t_{x}, r_{z}^{2} t_{x}, r_{y}^{2} t_{x}^{-1}, m_{x} t_{x}^{-1}, r_{x}^{2} t_{y}, r_{x}^{2} t_{y}^{-1} \\
m_{y} t_{y}^{-1}, m_{y} t_{z}, r_{x}^{2} t_{z}^{-1}, m_{y} t_{z}^{-1}\end{array}$ \\
\hline 2665 & $H_{370}$ & 1 & $m_{z}$ & $\begin{array}{l}i t_{x}, r_{z}^{2} t_{x}, r_{y}^{2} t_{x}^{-1}, m_{x} t_{x}^{-1}, m_{y} t_{y}, r_{x}^{2} t_{y}^{-1} \\
m_{y} t_{y}^{-1}, m_{y} t_{z}, r_{x}^{2} t_{z}^{-1}, m_{y} t_{z}^{-1}\end{array}$ \\
\hline 2666 & $H_{359}$ & 1 & $m_{z}$ & $\begin{array}{l}i t_{x}, r_{z}^{2} t_{x}, r_{y}^{2} t_{x}^{-1}, m_{x} t_{x}^{-1}, r_{x}^{2} t_{y}, r_{x}^{2} t_{y}^{-1} \\
m_{y} t_{y}^{-1}, t_{z}, m_{z} t_{z}^{-1}, t_{z}^{-1}\end{array}$ \\
\hline 2667 & $H_{347}$ & 1 & $m_{z}$ & $\begin{array}{l}i t_{x}, r_{y}^{2} r_{z} t_{x}^{-1}, m_{x} r_{z} t_{x}^{-1}, i t_{y}, r_{z}^{2} t_{y}, r_{y}^{2} r_{z} t_{y}^{-1} \\
m_{x} r_{z} t_{y}^{-1}, m_{x} r_{z}^{-1} t_{z}, r_{x}^{2} r_{z} t_{z}^{-1}, m_{x} r_{z}^{-1} t_{z}^{-1}\end{array}$ \\
\hline 2668 & $H_{347}$ & 1 & $m_{z}$ & $\begin{array}{l}r_{z}^{2} t_{x}, r_{y}^{2} r_{z} t_{x}^{-1}, m_{x} r_{z} t_{x}^{-1}, i t_{y}, r_{z}^{2} t_{y}, r_{y}^{2} r_{z} t_{y}^{-1}, \\
m_{x} r_{z} t_{y}^{-1}, m_{x} r_{z}^{-1} t_{z}, r_{x}^{2} r_{z} t_{z}^{-1}, m_{x} r_{z}^{-1} t_{z}^{-1}\end{array}$ \\
\hline 2669 & $H_{343}$ & 1 & $m_{z}$ & $\begin{array}{l}i t_{x}, r_{y}^{2} r_{z} t_{x}^{-1}, m_{x} r_{z} t_{x}^{-1}, i t_{y}, r_{z}^{2} t_{y}, r_{y}^{2} r_{z} t_{y}^{-1} \\
m_{x} r_{z} t_{y}^{-1}, t_{z}, m_{z} t_{z}^{-1}, t_{z}^{-1}\end{array}$ \\
\hline 2670 & $H_{378}$ & 1 & $m_{z}$ & $\begin{array}{l}r_{y}^{2} t_{x}, m_{x} t_{x}, r_{y}^{2} t_{x}^{-1}, m_{x} t_{x}^{-1}, r_{x}^{2} t_{y}, r_{x}^{2} t_{y}^{-1} \\
m_{y} t_{y}^{-1}, m_{x} r_{z} t_{z}, r_{y}^{2} r_{z} t_{z}^{-1}, m_{x} r_{z} t_{z}^{-1}\end{array}$ \\
\hline 2671 & $H_{378}$ & 1 & $m_{z}$ & $\begin{array}{l}r_{y}^{2} t_{x}, m_{x} t_{x}, r_{y}^{2} t_{x}^{-1}, m_{x} t_{x}^{-1}, m_{y} t_{y}, r_{x}^{2} t_{y}^{-1} \\
m_{y} t_{y}^{-1}, m_{x} r_{z} t_{z}, r_{y}^{2} r_{z} t_{z}^{-1}, m_{x} r_{z} t_{z}^{-1}\end{array}$ \\
\hline 2672 & $H_{382}$ & 1 & $m_{z}$ & $\begin{array}{l}r_{y}^{2} t_{x}, m_{x} t_{x}, m_{z} r_{z}^{-1} t_{x}^{-1}, r_{z}^{-1} t_{x}^{-1}, m_{z} r_{z} t_{y}, r_{z} t_{y}, \\
r_{x}^{2} t_{y}^{-1}, m_{x} r_{z} t_{z}, r_{y}^{2} r_{z} t^{-1}, m_{x} r_{z} t_{z}^{-1}\end{array}$ \\
\hline 2673 & $H_{382}$ & 1 & $m_{z}$ & $\begin{array}{l}r_{y}^{2} t_{x}, m_{x} t_{x}, m_{z} r_{z}^{-1} t_{x}^{-1}, r_{z}^{-1} t_{x}^{-1}, m_{z} r_{z} t_{y}, r_{z} t_{y}, \\
m_{y} t_{y}^{-1}, m_{x} r_{z} t_{z}, r_{y}^{2} r_{z} t_{z}^{-1}, m_{x} r_{z} t_{z}^{-1}\end{array}$ \\
\hline
\end{tabular}




\begin{tabular}{|c|c|c|c|c|c|}
\hline Nbr. gr & № & $H_{i}$ & $L$ & $m$ & $x$ \\
\hline & 2674 & $H_{377}$ & 1 & $m_{z}$ & $\begin{array}{l}r_{y}^{2} t_{x}, m_{x} t_{x}, m_{z} r_{z}^{-1} t_{x}^{-1}, r_{z}^{-1} t_{x}^{-1}, m_{z} r_{z} t_{y}, r_{z} t_{y} \\
r_{x}^{2} t_{y}^{-1}, t_{z}, m_{z} t_{z}^{-1}, t_{z}^{-1}\end{array}$ \\
\hline & 2675 & $H_{453}$ & 1 & $r_{z}^{2}$ & $\begin{array}{l}m_{z} t_{x}, i t_{x}^{-1}, m_{z} t_{x}^{-1}, i t_{y}, m_{z} t_{y}, i t_{y}^{-1} \\
m_{z} t_{y}^{-1}, r_{y}^{2} r_{z} t_{z}, r_{y}^{2} r_{z} t_{z}^{-1}, r_{x}^{2} r_{z} t_{z}^{-1}\end{array}$ \\
\hline & 2676 & $H_{539}$ & 1 & $r_{z}^{2}$ & $\begin{array}{l}i t_{x}, m_{z} t_{x}, i t_{x}^{-1}, m_{z} t_{x}^{-1}, r_{y}^{2} t_{y}, r_{y}^{2} t_{y}^{-1} \\
r_{x}^{2} t_{y}^{-1}, m_{z} r_{z}^{-1} t_{z}, m_{z} r_{z} t_{z}, r_{y}^{2} r_{z} t_{z}^{-1}\end{array}$ \\
\hline & 2677 & $H_{539}$ & 1 & $r_{z}^{2}$ & $\begin{array}{l}m_{z} t_{x}, i t_{x}^{-1}, m_{z} t_{x}^{-1}, r_{y}^{2} t_{y}, r_{x}^{2} t_{y}, r_{y}^{2} t_{y}^{-1} \\
r_{x}^{2} t_{y}^{-1}, m_{z} r_{z}^{-1} t_{z}, m_{z} r_{z} t_{z}, r_{y}^{2} r_{z} t_{z}^{-1}\end{array}$ \\
\hline & 2678 & $H_{690}$ & $\left\langle m_{x}\right\rangle$ & $r_{z}^{2}$ & $\begin{array}{l}r_{z}^{2} t_{x}, m_{x} t_{x}, m_{y} t_{x}^{-1}, m_{x} t_{x}^{-1}, m_{x} t_{y}, m_{y} t_{y}^{-1}, \\
m_{x} t_{y}^{-1}, r_{y}^{2} r_{z} t_{z}, m_{z} r_{z}^{-1} t_{z}, r_{x}^{2} t_{z}^{-1}\end{array}$ \\
\hline & 2679 & $H_{452}$ & 1 & $r_{z}^{2}$ & $\begin{array}{l}t_{x}, r_{z}^{2} t_{x}^{-1}, t_{x}^{-1}, r_{z}^{2} t_{y}, t_{y}, r_{z}^{2} t_{y}^{-1} \\
t_{y}^{-1}, r_{y}^{2} r_{z} t_{z}, i t_{z}^{-1}, m_{z} t_{z}^{-1}\end{array}$ \\
\hline & 2680 & $H_{503}$ & 1 & $r_{z}^{2}$ & $\begin{array}{l}t_{x}, r_{z}^{2} t_{x}^{-1}, t_{x}^{-1}, r_{z}^{2} t_{y}, t_{y}, r_{z}^{2} t_{y}^{-1} \\
t_{y}^{-1}, r_{y}^{2} r_{z} t_{z}, r_{y}^{2} t_{z}^{-1}, r_{x}^{2} t_{z}^{-1}\end{array}$ \\
\hline & 2681 & $H_{438}$ & 1 & $r_{z}^{2}$ & $\begin{array}{l}t_{x}, r_{z}^{2} t_{x}^{-1}, t_{x}^{-1}, r_{z}^{2} t_{y}, t_{y}, r_{z}^{2} t_{y}^{-1} \\
t_{y}^{-1}, r_{y}^{2} r_{z} t_{z}, r_{y}^{2} r_{z} t_{z}^{-1}, r_{x}^{2} r_{z} t_{z}^{-1}\end{array}$ \\
\hline & 2682 & $H_{495}$ & 1 & $r_{z}^{2}$ & $\begin{array}{l}t_{x}, r_{z}^{2} t_{x}^{-1}, t_{x}^{-1}, r_{z}^{2} t_{y}, t_{y}, r_{z}^{2} t_{y}^{-1} \\
t_{y}^{-1}, m_{z} r_{z}^{-1} t_{z}, m_{z} r_{z} t_{z}, r_{y}^{2} r_{z} t_{z}^{-1}\end{array}$ \\
\hline & 2683 & $H_{511}$ & 1 & $r_{z}^{2}$ & $\begin{array}{l}r_{z}^{2} r_{x} t_{x}, r_{x} t_{x}, r_{y}^{2} r_{x} t_{x}^{-1}, r_{x}^{-1} t_{x}^{-1}, r_{y}^{2} t_{y}, r_{y}^{2} t_{y}^{-1} \\
r_{x}^{2} t_{y}^{-1}, r_{y}^{2} t_{z}, r_{y}^{2} t_{z}^{-1}, r_{x}^{2} t_{z}^{-1}\end{array}$ \\
\hline & 2684 & $H_{511}$ & 1 & $r_{z}^{2}$ & $\begin{array}{l}r_{z}^{2} r_{x} t_{x}, r_{x} t_{x}, r_{y}^{2} r_{x} t_{x}^{-1}, r_{x}^{-1} t_{x}^{-1}, r_{y}^{2} t_{y}, r_{y}^{2} t_{y}^{-1} \\
r_{x}^{2} t_{y}^{-1}, r_{x}^{2} t_{z}, r_{y}^{2} t_{z}^{-1}, r_{x}^{2} t_{z}^{-1}\end{array}$ \\
\hline & 2685 & $H_{511}$ & 1 & $r_{z}^{2}$ & $\begin{array}{l}r_{z}^{2} r_{x} t_{x}, r_{x} t_{x}, r_{x}^{-1} t_{x}^{-1}, r_{y}^{2} t_{y}, r_{x}^{2} t_{y}, r_{y}^{2} t_{y}^{-1} \\
r_{x}^{2} t_{y}^{-1}, r_{y}^{2} t_{z}, r_{y}^{2} t_{z}^{-1}, r_{x}^{2} t_{z}^{-1}\end{array}$ \\
\hline & 2686 & $H_{511}$ & 1 & $r_{z}^{2}$ & $\begin{array}{l}r_{z}^{2} r_{x} t_{x}, r_{x} t_{x}, r_{x}^{-1} t_{x}^{-1}, r_{y}^{2} t_{y}, r_{x}^{2} t_{y}, r_{y}^{2} t_{y}^{-1} \\
r_{x}^{2} t_{y}^{-1}, r_{x}^{2} t_{z}, r_{y}^{2} t_{z}^{-1}, r_{x}^{2} t_{z}^{-1}\end{array}$ \\
\hline & 2687 & $H_{541}$ & 1 & $r_{z}^{2}$ & $\begin{array}{l}r_{z}^{2} r_{x} t_{x}, r_{x} t_{x}, r_{y}^{2} r_{x} t_{x}^{-1}, r_{x}^{-1} t_{x}^{-1}, m_{x} t_{y}, m_{x} t_{y}^{-1}, \\
m_{y} t_{y}^{-1}, i t_{z}, i t_{z}^{-1}, m_{z} t_{z}^{-1}\end{array}$ \\
\hline & 2688 & $H_{541}$ & 1 & $r_{z}^{2}$ & $\begin{array}{l}r_{z}^{2} r_{x} t_{x}, r_{x} t_{x}, r_{x}^{-1} t_{x}^{-1}, m_{x} t_{y}, m_{y} t_{y}, m_{x} t_{y}^{-1} \\
m_{y} t_{y}^{-1}, i t_{z}, i t_{z}^{-1}, m_{z} t_{z}^{-1}\end{array}$ \\
\hline & 2689 & $H_{541}$ & 1 & $r_{z}^{2}$ & $\begin{array}{l}r_{z}^{2} r_{x} t_{x}, r_{x} t_{x}, r_{x}^{-1} t_{x}^{-1}, m_{x} t_{y}, m_{y} t_{y}, m_{x} t_{y}^{-1}, \\
m_{y}{ }^{-1}, m_{z} t_{z}, i t_{z}^{-1}, m_{z} t_{z}^{-1}\end{array}$ \\
\hline & 2690 & $H_{505}$ & 1 & $r_{z}^{2}$ & $\begin{array}{l}r_{y}^{2} t_{x}, r_{x}^{2} t_{x}, r_{y}^{2} t_{x}^{-1}, r_{x}^{2} t_{x}^{-1}, r_{y}^{2} t_{y}, r_{y}^{2} t_{y}^{-1} \\
r_{x}^{2} t_{y}^{-1}, r_{y}^{2} r_{z} t_{z}, r_{y}^{2} r_{z} t_{z}^{-1}, r_{x}^{2} r_{z} t_{z}^{-1}\end{array}$ \\
\hline & 2691 & $H_{527}$ & 1 & $r_{z}^{2}$ & $\begin{array}{l}m_{x} t_{x}, m_{y} t_{x}, m_{x} t_{x}^{-1}, m_{y} t_{x}^{-1}, m_{x} t_{y}, m_{x} t_{y}^{-1}, \\
m_{y} t_{y}^{-1}, r_{y}^{2} r_{z} t_{z}, i t_{z}^{-1}, m_{z} t_{z}^{-1}\end{array}$ \\
\hline & 2692 & $H_{529}$ & 1 & $r_{z}^{2}$ & $\begin{array}{l}m_{x} t_{x}, m_{y} t_{x}, m_{x} t_{x}^{-1}, m_{y} t_{x}^{-1}, m_{x} t_{y}, m_{x} t_{y}^{-1}, \\
m_{y} t_{y}^{-1}, r_{y}^{2} r_{z} t_{z}, r_{y}^{2} t_{z}^{-1}, r_{x}^{2} t_{z}^{-1}\end{array}$ \\
\hline & 2693 & $H_{496}$ & 1 & $r_{z}^{2}$ & $\begin{array}{l}m_{x} t_{x}, m_{y} t_{x}, m_{x} t_{x}^{-1}, m_{y} t_{x}^{-1}, m_{x} t_{y}, m_{x} t_{y}^{-1}, \\
m_{y} t_{y}^{-1}, r_{y}^{2} r_{z} t_{z}, r_{y}^{2} r_{z} t_{z}^{-1}, r_{x}^{2} r_{z} t_{z}^{-1}\end{array}$ \\
\hline & 2694 & $H_{502}$ & 1 & $r_{z}^{2}$ & $\begin{array}{l}m_{x} t_{x}, m_{y} t_{x}, m_{x} t_{x}^{-1}, m_{y} t_{x}^{-1}, m_{x} t_{y}, m_{x} t_{y}^{-1}, \\
m_{y} t_{y}^{-1}, m_{z} r_{z}^{-1} t_{z}, m_{z} r_{z} t_{z}, r_{y}^{2} r_{z} t_{z}^{-1}\end{array}$ \\
\hline
\end{tabular}

[ 10, 36, 78, 133, 204, 292, 396, 516, 652, 804 ] $2695^{*}, 2696^{*}, 2697^{*}, 2698^{*}, 2699^{*}, 2700^{*}$

$[10,37,78,132,204,292,396,516,652,804]$

$2701^{*}, 2702^{*}, 2703^{*}, 2704^{*}, 2705^{*}, 2706^{*}, 2707^{*}, 2708^{*}, 2709^{*}, 2710^{*}, 2711^{*}, 2712^{*}, 2713^{*}$,

$2714^{*}, 2715^{*}, 2716^{*}, 2717^{*}, 2718^{*}, 2719^{*}, 2720^{*}, 2721^{*}, 2722^{*}, 2723^{*}, 2724^{*}, 2725^{*}, 2726^{*}$,

$2727^{*}, 2728^{*}, 2729^{*}, 2730^{*}, 2731^{*}$,

$[11,37,77,132,204,292,396,516,652,804$ ]

\begin{tabular}{|c|c|c|c|c|}
\hline 2695 & $H_{560}$ & 1 & $m_{z} r_{x}$ & $\begin{array}{l}i t_{x}, r_{z}^{2} r_{x} t_{x}, m_{x} t_{x}^{-1}, m_{z} r_{x}^{-1} t_{y}, r_{x}^{2} t_{y}^{-1}, m_{z} r_{x}^{-1} t_{y}^{-1} \\
r_{x}^{2} t_{z}, m_{z} r_{x}^{-1} t_{z}, r_{x}^{2} t_{z}^{-1}, m_{z} r_{x}^{-1} t_{z}^{-1}\end{array}$ \\
\hline 2696 & $H_{580}$ & 1 & $m_{z} r_{x}$ & $\begin{array}{l}i t_{x}, r_{z}^{2} r_{x} t_{x}, m_{x} t_{x}^{-1}, m_{y} t_{y}, r_{x} t_{y}, m_{y} t_{y}^{-1} \\
r_{x} t_{y}^{-1}, r_{x}^{-1} t_{z}, r_{x}^{-1} t_{z}^{-1}, m_{z} t_{z}^{-1}\end{array}$ \\
\hline 2697 & $H_{561}$ & 1 & $m_{z} r_{x}$ & $\begin{array}{l}m_{x} t_{x}, r_{y}^{2} r_{x} t_{x}^{-1}, m_{x} t_{x}^{-1}, m_{z} r_{x}^{-1} t_{y}, i t_{y}^{-1}, r_{z}^{2} r_{x} t_{y}^{-1}, \\
r_{x}^{2} t_{z}, m_{z} r_{x}^{-1} t_{z}, i t_{z}^{-1}, r_{z}^{2} r_{x} t_{z}^{-1}\end{array}$ \\
\hline 2698 & $H_{571}$ & 1 & $m_{z} r_{x}$ & $\begin{array}{l}m_{x} t_{x}, r_{y}^{2} r_{x} t_{x}^{-1}, m_{x} t_{x}^{-1}, m_{y} t_{y}, r_{x} t_{y}, r_{z}^{2} t_{y}^{-1} \\
m_{x} r_{x} t_{y}^{-1}, r_{x}^{-1} t_{z}, m_{x} r_{x}^{-1} t_{z}^{-1}, r_{y}^{2} t_{z}^{-1}\end{array}$ \\
\hline 2699 & $H_{557}$ & 1 & $m_{z} r_{x}$ & $\begin{array}{l}m_{x} t_{x}, r_{y}^{2} r_{x} t_{x}^{-1}, m_{x} t_{x}^{-1}, m_{z} r_{x}^{-1} t_{y}, r_{x}^{2} t_{y}^{-1}, m_{z} r_{x}^{-1} t_{y}^{-1}, \\
r_{x}^{2} t_{z}, m_{z} r_{x}^{-1} t_{z}, r_{x}^{2} t_{z}^{-1}, m_{z} r_{x}^{-1} t_{z}^{-1}\end{array}$ \\
\hline 2700 & $H_{578}$ & 1 & $m_{z} r_{x}$ & $\begin{array}{l}m_{x} t_{x}, r_{y}^{2} r_{x} t_{x}^{-1}, m_{x} t_{x}^{-1}, m_{y} t_{y}, r_{x} t_{y}, m_{y} t_{y}^{-1} \\
r_{x} t_{y}^{-1}, r_{x}^{-1} t_{z}, r_{x}^{-1} t_{z}^{-1}, m_{z} t_{z}^{-1}\end{array}$ \\
\hline \multicolumn{5}{|c|}{$[11,38,76,132,204,292,396,516,652,804]$} \\
\hline 2701 & $H_{551}$ & 1 & $m_{z} r_{x}$ & $\begin{array}{l}i t_{x}, i t_{x}^{-1}, r_{z}^{2} r_{x} t_{x}^{-1}, r_{z}^{2} r_{x} t_{y}, i t_{y}^{-1}, r_{z}^{2} r_{x} t_{y}^{-1} \\
i t_{z}, r_{z}^{2} r_{x} t_{z}, i t_{z}^{-1}, r_{z}^{2} r_{x} t_{z}^{-1}\end{array}$ \\
\hline 2702 & $H_{551}$ & 1 & $m_{z} r_{x}$ & $\begin{array}{l}r_{z}^{2} r_{x} t_{x}, i t_{x}^{-1}, r_{z}^{2} r_{x} t_{x}^{-1}, r_{z}^{2} r_{x} t_{y}, i t_{y}^{-1}, r_{z}^{2} r_{x} t_{y}^{-1}, \\
i t_{z}, r_{z}^{2} r_{x} t_{z}, i t_{z}^{-1}, r_{z}^{2} r_{x} t_{z}^{-1}\end{array}$ \\
\hline
\end{tabular}




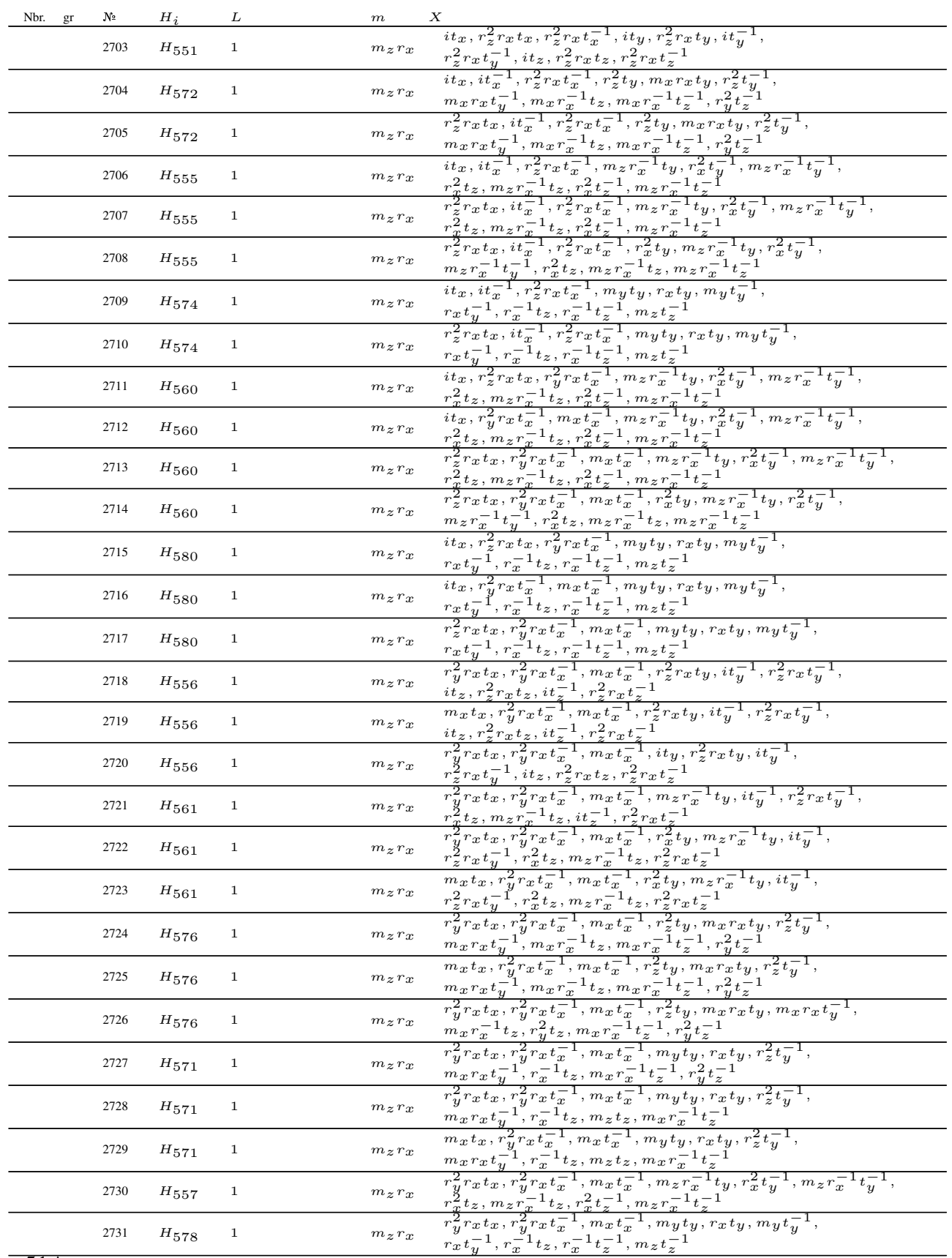

$51 \mathrm{~A}$

$[7,23,50,87,135,194,263,343,434,535]$ $2732 *$

$[7,23,52,97,158,233,325,431,547,682]$ $2733^{*}$

$[7,24,53,94,147,212,289,378,479,592]$

$2734 *$

$[7,24,54,96,150,216,294,384,486,600]$ $2735 *$

$[7,25,56,98,152,218,296,386,488,602]$ $2736^{*}, 2737^{*}$,

$[7,27,62,109,172,249,338,444,564,695]$ 


\begin{tabular}{|c|c|c|c|c|c|}
\hline Nbr. & gr $\quad$ № & $H_{i}$ & $L$ & $m$ & $X$ \\
\hline \multicolumn{6}{|c|}{$\begin{array}{c}2738^{*}, 2739^{*} \\
{[8,27,58,101,158,226,306,401,506,623]}\end{array}$} \\
\hline & 2732 & $H_{306}$ & 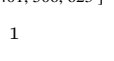 & $i$ & 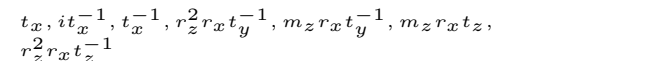 \\
\hline \multicolumn{6}{|c|}{$[8,27,60,109,172,246,338,446,564,701]$} \\
\hline & 2733 & $H_{321}$ & $+40,504,701]$ & $i$ & ${ }_{r_{x}-1 t_{t}-1}^{-1}, t_{x}^{-1}, m_{x} r_{x}^{-1} t_{y}^{-1}, r_{x} t_{y}^{-1}, m_{x} r_{x} t_{z}$ \\
\hline \multicolumn{6}{|c|}{$[8,28,60,107,166,239,325,425,537,664]$} \\
\hline & 2734 & $H_{307}$ & 1 & $i$ & $\begin{array}{l}m_{z} r_{x} t_{x}, r_{z}^{2} r_{x} t_{x}^{-1}, m_{z} r_{x} t_{x}^{-1}, r_{z}^{2} r_{x} t_{y}^{-1}, m_{z} r_{x} t_{y}^{-1}, m_{z} r_{x} t_{z}, \\
r_{z}^{2} r_{x} t_{z}^{-1}\end{array}$ \\
\hline \multicolumn{6}{|c|}{$[8,28,61,108,168,241,328,428,541,668]$} \\
\hline & 2735 & $H_{325}$ & 1 & $i$ & $\begin{array}{l}m_{z} r_{x} t_{x}, r_{z}^{2} r_{x} t_{x}^{-1}, m_{z} r_{x} t_{x}^{-1}, m_{x} r_{x} t_{y}^{-1}, r_{x}^{-1} t_{y}^{-1}, r_{x} t_{z} \\
m_{x} r_{x}^{-1} t_{z}^{-1}\end{array}$ \\
\hline \multicolumn{6}{|c|}{$[8,29,62,109,169,242,329,429,542,669]$} \\
\hline & 2736 & $H_{324}$ & 1 & $i$ & $\begin{array}{l}m_{z} r_{x} t_{x}, r_{z}^{2} r_{x} t_{x}^{-1}, m_{z} r_{x} t_{x}^{-1}, m_{x} r_{x}^{-1} t_{y}^{-1}, r_{x} t_{y}^{-1}, m_{x} r_{x} t_{z} \\
r_{x}^{-1} t_{z}^{-1}\end{array}$ \\
\hline & 2737 & $H_{309}$ & 1 & $i$ & $\begin{array}{l}m_{z} r_{x} t_{x}, r_{z}^{2} r_{x} t_{x}^{-1}, m_{z} r_{x} t_{x}^{-1}, r_{y}^{2} r_{x} t_{y}^{-1}, m_{z} r_{x}^{-1} t_{y}^{-1}, r_{y}^{2} r_{x} t_{z}, \\
m_{z} r_{x}^{-1} t_{z}^{-1}\end{array}$ \\
\hline \multicolumn{6}{|c|}{$[8,31,66,115,181,258,349,459,578,711]$} \\
\hline & 2738 & $H_{310}$ & 1 & $i$ & $\begin{array}{l}r_{x}^{2} t_{x}, m_{x} t_{x}^{-1}, r_{x}^{2} t_{x}^{-1}, r_{z}^{2} r_{x} t_{y}^{-1}, m_{z} r_{x} t_{y}^{-1}, m_{z} r_{x} t_{z} \\
r_{z}^{2} r_{x} t \bar{z}^{-1}\end{array}$ \\
\hline & 2739 & $H_{322}$ & 1 & $i$ & $\begin{array}{l}r_{x}^{2} t_{x}, m_{x} t_{x}^{-1}, r_{x}^{2} t_{x}^{-1}, m_{x} r_{x}^{-1} t_{y}^{-1}, r_{x} t_{y}^{-1}, m_{x} r_{x} t_{z}, \\
r_{x}^{-1} t_{z}^{-1}\end{array}$ \\
\hline
\end{tabular}

$51 \mathrm{~B}$

$[7,24,62,118,190,278,382,502,638,790]$ $2740^{*}$

[ $7,24,62,120,192,280,384,504,640,792$ ] $2741^{*}$

$[7,25,59,109,175,257,355,469,599,745]$ $2742 *$

$[7,25,61,116,188,276,380,500,636,788]$ $2743^{*}$

$[7,25,65,124,196,284,388,508,644,796]$ $2744^{*}$,

$[7,25,66,127,200,288,392,512,648,800]$ $2745^{*}$,

$[7,26,63,117,187,273,375,493,627,777]$ $2746^{*}$,

$[7,26,64,121,194,282,386,506,642,794]$ $2747^{*}$,

$[7,26,68,128,200,288,392,512,648,800]$ $2748^{*}, 2749^{*}, 2750^{*}$,

$[7,27,66,122,194,282,386,506,642,794]$ $2751^{*}, 2752^{*}$,

$[7,27,68,126,198,286,390,510,646,798]$ $2753^{*}$,

$[7,27,69,128,200,288,392,512,648,800]$ $2754^{*}$,

$[7,28,69,126,198,286,390,510,646,798]$ $2755^{*}$

[ $7,28,70,128,200,288,392,512,648,800$ ] 2756*, 2757*,

$[7,29,70,126,198,286,390,510,646,798]$ $2758^{*}, 2759^{*}$

$[8,28,70,124,198,284,390,508,646,796]$ $2740 \quad H_{408} \quad r_{z}^{2} r_{x} \quad t_{x}, r_{z}^{2} r_{x} t_{x}^{-1}, t_{x}^{-1}, r_{x} t_{y}^{-1}, r_{z}^{2} t_{z}, r_{z}^{2} t_{z}^{-1}$,

$[8,28,70,126,198,286,390,510,646,798]$ $2741 \quad H_{403} \quad 1$

$[8,29,66,119,188,273,374,491,624,773]$ $2742 \quad H_{383} \quad 1$

$[8,29,68,124,196,284,388,508,644,796]$ $2743 \quad H_{385} \quad 1$

$8,29,72,128,200,288,392,512,648,800]$ $2744 \quad H_{416} \quad 1$

$8,29,73,130,202,290,394,514,650,802]$ $2745 \quad H_{411} \quad 1$

$[8,30,69,124,195,282,385,504,639,790]$ $2746 \quad H_{387} \quad 1$

$[8,30,70,127,199,287,391,511,647,799]$ $2747 \quad H_{399} \quad 1$

$[8,30,74,130,202,290,394,514,650,802]$ $2748 \quad H_{417} \quad 1$ $r_{z}^{2} r_{x} \quad t_{x}, r_{z}^{2} r_{x} t_{x}^{-1}, t_{x}^{-1}, m_{x} r_{x} t_{y}^{-1}, m_{y} t_{z}, m_{x} r_{x}^{-1} t_{z}^{-1}$, $r_{z}^{2} r_{x} \quad t_{x}, r_{z}^{2} r_{x} t_{x}^{-1}, t_{x}^{-1}, t_{y}, r_{z}^{2} r_{x} t_{y}^{-1}, t_{y}^{-1}$, ${ }_{z}^{2} r_{x} \quad r_{z}^{2} r_{x} t_{z}^{-1}$ $r_{z}^{2} r_{x} \quad t_{x}, r_{z}^{2} r_{x} t_{x}^{-1}, t_{x}^{-1}, m_{x} t_{y}, m_{x} t_{y}^{-1}, m_{z} r_{x}^{-1} t_{y}^{-1}$, $n_{z} r_{x}^{-1} t_{z}^{-1}$ 


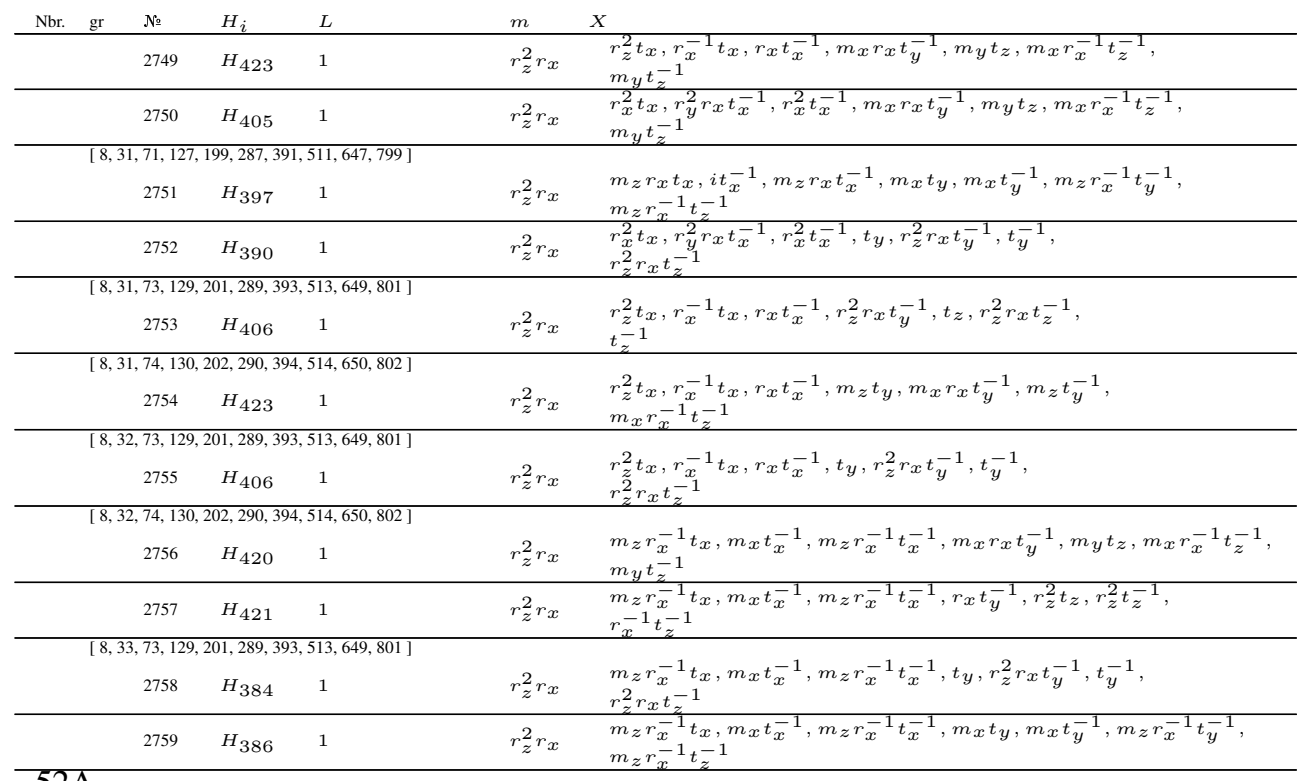

$52 \mathrm{~A}$

$[8,26,56,98,152,218,296,386,488,602]$ $2760^{*}$,

$[8,27,59,105,166,240,328,430,545,675]$ $2761^{*}$,

$[8,28,62,109,172,249,338,444,564,695]$ $2762 *$

[ $9,29,62,109,169,242,329,429,542,669$ ] $2760 \quad H_{752}\left\langle m_{y}, r_{z}^{2}\right\rangle$ $r_{z}^{2} r_{x} t_{x}, m_{x} r_{x}^{-1} t_{x}, m_{z} r_{x}^{-1} t_{x}^{-1}, r_{x}^{-1} t_{x}^{-1}, r_{y}^{2} r_{x} t_{y}, m_{x} r_{x} t_{y}^{-1}$ $m_{x} r_{x} t_{z}^{-1}, r_{y}^{2} r_{x} t_{z}^{-1}$

$[9,30,64,113,176,251,342,446,563,696]$

$2761 \quad H_{697} \quad\left\langle m_{x}\right\rangle$

$m_{x} r_{z}^{-1} t_{x}, r_{z} t_{x}^{-1}, r_{z}{ }^{-1}, m_{x} r_{z}^{-1} t_{y}^{-1}, r_{y}^{2} r_{z} t_{z}, m_{z} r_{z}^{-1} t_{z}$,

$2761 \quad H_{697} \quad\left\langle m_{x}\right\rangle$ $r_{x}^{2} t_{z}^{-1}, r_{y}^{2} t_{z}^{-1}$

$[9,31,66,115,181,258,349,459,578,711]$ $r_{y}^{2} t_{x}, r_{z}^{2} t_{x}, m_{z} t_{x}^{-1}, r_{z}^{2} t_{x}^{-1}, r_{y}^{2} r_{x} t_{y}, m_{x} r_{x} t_{y}^{-1}$ $2762 \quad H_{751} \quad\left\langle m_{y}, r_{z}^{2}\right\rangle$ $m_{x} r_{x} t_{z}^{-1}, r_{y}^{2} r_{x} t_{z}^{-1}$

\section{B}

$[8,27,68,128,200,288,392,512,648,800]$ $2763^{*}$,

$[8,28,66,122,194,282,386,506,642,794]$ $2764^{*}$

$[8,28,69,128,200,288,392,512,648,800$ ] $2765^{*}, 2766^{*}$

$[8,29,68,124,196,284,388,508,644,796$ ] $2767^{*}$

$[8,29,69,126,198,286,390,510,646,798]$ $2768^{*}$

$[8,29,70,128,200,288,392,512,648,800]$ $2769 *$,

$[8,30,70,126,198,286,390,510,646,798]$ $2770^{*}$

$[9,30,74,130,202,290,394,514,650,802]$ $2763 \quad H_{653} \quad\left\langle m_{x}\right\rangle \quad r_{y}^{2} r_{x} \quad{ }^{i t} t_{x}, r_{z}^{2} r_{x} t_{x}, r_{x}^{2} t_{x}^{-1}, r_{z}^{2} r_{x} t_{x}^{-1}, m_{x} r_{x}^{-1} t_{y}^{-1}, m_{y} t_{z}$,

$[9,31,71,127,199,287,391,511,647,799]$

$$
2764 \quad H_{647} \quad\left\langle m_{x}\right\rangle
$$
$m_{x} r_{x} t_{z}, m_{y} t_{z}^{-1}$

$[9,31,74,130,202,290,394,514,650,802]$

$$
2765 \quad H_{423} \quad 1
$$

$2766 \quad H_{581} \quad 1$

$[9,32,72,128,200,288,392,512,648,800]$

$$
2767 \quad H_{552} \quad 1
$$

$[9,32,73,129,201,289,393,513,649,801]$

$$
2768 \quad H_{406} \quad 1
$$$$
r_{y}^{2} r_{x} \quad i t_{x}, r_{z}^{2} r_{x} t_{x}, r_{x}^{2} t_{x}^{-1}, r_{z}^{2} r_{x} t_{x}^{-1}, m_{x} t_{y}, m_{z} r_{x} t_{y}^{-1} \text {, }
$$
$m_{x} t_{y}^{-1}, m_{z} r_{x} t_{z}$

$$
r_{z}^{2} t_{x}, r_{x}^{-1} t_{x}, r_{y}^{2} t_{x}^{-1}, r_{x} \bar{x}^{-1}, m_{x} r_{x} t_{y}^{-1}, m_{y} t_{z},
$$
$m_{x} r_{x}^{-1} t_{z}^{-1}, m_{y} t_{z}^{-1}$ $i t_{x}, r_{z}^{2} r_{x} t_{x}, r_{y}^{2} r_{x} t_{x}^{-1}, m_{x} t_{x}^{-1}, r_{x}^{-1} t_{y}^{-1}, m_{y} t_{z}$ $r_{x} t_{z}, m_{y} t_{z}^{-1}$

$m_{z} r_{x} \quad r_{x} t_{z}, m_{y} t_{z}^{-1}$

$[9,32,74,130,202,290,394,514,650,802]$

$i t_{x}, r_{z}^{2} r_{x} t_{x}, r_{y}^{2} r_{x} t_{x}^{-1}, m_{x} t_{x}^{-1}, t_{y}, m_{z} r_{x} t_{y}^{-1}$ $t_{y}^{-1}, m_{z} r_{x} t_{z}$

$r_{z}^{2} r_{x} \quad r_{z}^{2} t_{x}, r_{x}^{-1} t_{x}, r_{y}^{2} t_{x}^{-1}, r_{x} t_{x}^{-1}, t_{y}, r_{z}^{2} r_{x} t_{y}^{-1}$ 


\begin{tabular}{|c|c|c|c|c|c|c|}
\hline Nbr. & gr & № & $H_{i}$ & $L$ & $m$ & $X$ \\
\hline & & 2769 & $H_{651}$ & $\left\langle m_{x}\right\rangle$ & $r_{y}^{2} r_{x}$ & $\begin{array}{l}r_{y}^{2} r_{x} t_{x}, m_{x} t_{x}, m_{z} r_{x} t_{x}^{-1}, m_{x} t_{x}^{-1}, m_{x} r_{x}^{-1} t_{y}^{-1}, m_{y} t_{z} \\
m_{x} r_{x} t_{z}, m_{y} t_{z}^{-1}\end{array}$ \\
\hline \multicolumn{7}{|c|}{$[9,33,73,129,201,289,393,513,649,801]$} \\
\hline & & 2770 & $H_{646}$ & $\left\langle m_{x}\right\rangle$ & $r_{y}^{2} r_{x}$ & $\begin{array}{l}r_{y}^{2} r_{x} t_{x}, m_{x} t_{x}, m_{z} r_{x} t_{x}^{-1}, m_{x} t_{x}^{-1}, m_{x} t_{y}, m_{z} r_{x} t_{y}^{-1} \\
m_{x} t_{y}^{-1}, m_{z} r_{x} t_{z}\end{array}$ \\
\hline
\end{tabular}

$53 \mathrm{~A}$

[ 7, 27, 64, 113, 179, 260, 354, 467, 593, 734 ] $2771^{*}$

$[8,31,68,119,188,269,368,481,609,752]$

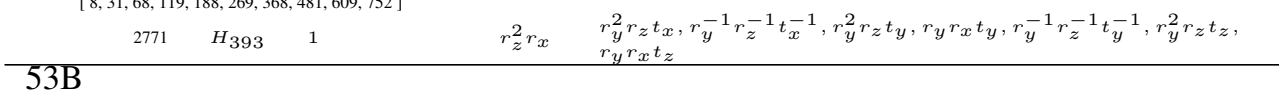

[ $7,24,55,98,153,222,303,396,503,622$ ]

$2772^{*}$,

$[7,27,60,103,160,230,312,407,515,635$ ] $2773^{*}$

$[8,28,63,110,172,249,338,441,560,691]$

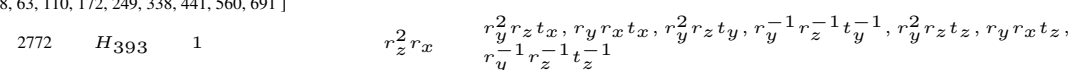

$[8,31,65,113,176,253,343,447,566,698]$ $2773 \quad H_{393} \quad 1$ $r_{z}^{2} r_{x} \quad r_{x}^{2} r_{y} t_{x}^{-1}, r_{y}^{-1} r_{z}^{-1} t_{x}^{-1}, r_{y} r_{x} t_{y}, r_{y}^{-1} r_{z}^{-1} t_{y}^{-1}, r_{y}^{2} r_{z} t_{z}, r_{y} r_{x} t_{z}$

$[7,29,77,134,203,294,395,515,654,803$ ] $2774 *$

$[8,33,79,131,205,292,395,517,652,803$ ]

$\mathrm{S4}_{[8,32,76,131,203,294,395,515,654,803]}$ $2775^{*}$,

$[9,35,76,131,205,292,395,517,652,803$ ]

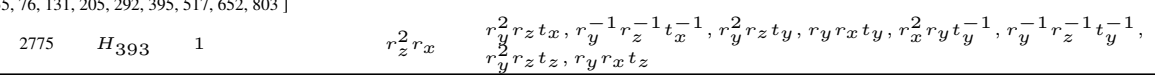

54B

[ $8,27,59,103,160,230,312,407,515,635$ ] $2776^{*}$

$[9,30,65,113,176,253,343,447,566,698]$
$2776 \quad H_{393} \quad 1$
$r_{z}^{2} r_{x}$
$r_{y}^{2} r_{z} t_{x}, r_{y} r_{x} t_{x}, r_{y}^{2} r_{z} t_{y}, r_{x}^{2} r_{y} t_{y}^{-1}, r_{y}^{-1} r_{z}^{-1} t_{y}^{-1}, r_{y}^{2} r_{z} t_{z}$, $r_{y} r_{x} t_{z}, r_{y}^{-1} r_{z}^{-1} t_{z} \frac{y}{1}$

\section{$55 \mathrm{~A}$}

$[9,31,69,123,193,279,381,499,633,783]$ $2777^{*}$,

$[9,32,72,128,200,288,392,512,648,800]$ $2778^{*}, 2779^{*}$

$[9,32,73,130,202,290,394,514,650,802]$ $2780^{*}, 2781^{*}$,

$[9,33,74,130,202,290,394,514,650,802]$ $2782^{*}, 2783^{*}, 2784^{*}, 2785^{*}, 2786^{*}, 2787^{*}, 2788^{*}, 2789^{*}$

[ $9,33,75,132,204,292,396,516,652,804$ ] $2790^{*}, 2791^{*}$

$[9,34,76,132,204,292,396,516,652,804]$ $2792^{*}, 2793^{*}, 2794^{*}, 2795^{*}, 2796^{*}, 2797^{*}, 2798^{*}, 2799^{*}, 2800^{*}, 2801^{*}$,

$[10,33,72,127,198,285,388,507,642,793]$

$\begin{array}{llll}H_{777} & \left\langle m_{z} r_{x}, r_{y} r_{x}\right\rangle & r_{z}^{2} r_{x} & t_{x}, r_{z}^{2} r_{x} t_{x}^{-1}, t_{x}^{-1}, m_{x} r_{z}^{-1} t_{y}, m_{x} r_{y}^{-1} r_{x}^{-1} t_{y}^{-1}, m_{x} r_{z}^{-1} t_{y}^{-1},\end{array}$

$[10,34,74,130,202,290,394,514,650,802]$

$\begin{array}{lllll}2778 & H_{301} & 1 & i & \begin{array}{l}t_{x}, i t_{x}^{-1}, t_{x}^{-1}, t_{y}, i t_{y}^{-1}, t_{y}^{-1}, \\ m_{y} t_{z}, r_{y}^{2} t_{z}^{-1}, m_{y} t_{z}^{-1}\end{array} \\ 2779 & H_{658} & \left\langle m_{z} r_{x}\right\rangle & r_{z}^{2} r_{x} & \begin{array}{l}t_{x}, r_{z}^{2} r_{x} t_{x}^{-1}, t_{x}^{-1}, r_{x}^{-1} t_{y}, r_{z}^{2} t_{y}^{-1}, m_{z} t_{y}^{-1}, \\ m_{y} t_{z}, m_{x} r_{x}^{-1} t_{z}^{-1}, r_{x} t_{z}^{-1}\end{array}\end{array}$

$[10,34,75,131,203,291,395,515,651,803]$

\begin{tabular}{rllll}
2780 & $H_{406}$ & 1 & $r_{z}^{2} r_{x}$ & $\begin{array}{l}r_{z}^{2} t_{x}, r_{x}^{-1} t_{x}, r_{x} t_{x}^{-1}, t_{y}, r_{z}^{2} r_{x} t_{y}^{-1}, t_{y}^{-1}, \\
t_{z}, r_{z}^{2} r_{x} t_{z}^{-1}, t_{z}^{-1}\end{array}$ \\
\hline 2781 & $H_{394} 1$ & $r_{z}^{2} r_{x}$ & $\begin{array}{l}t_{x}, r_{z}^{2} r_{x} t_{x}^{-1}, t_{x}^{-1}, r_{y}^{2} r_{x} t_{y}, i t_{y}^{-1}, m_{z} r_{x} \bar{y}^{-1} \\
m_{z} r_{x} t_{z}, r_{y}^{2} r_{x}{ }_{z}^{-1}, r_{x}^{2} t_{z}^{-1}\end{array}$ \\
\hline
\end{tabular}

\begin{tabular}{|c|c|c|c|c|}
\hline \multicolumn{5}{|c|}{$[10,35,75,131,203,291,395,515,651,803]$} \\
\hline 2782 & $H_{655}$ & $\left\langle m_{z} r_{x}\right\rangle$ & $r_{z}^{2} r_{x}$ & $\begin{array}{l}m_{z} r_{x}^{-1} t_{x}, m_{x} t_{x}^{-1}, m_{z} r_{x}^{-1} t_{x}^{-1}, m_{z} r_{x} t_{y}, i t_{y}^{-1}, t_{y}^{-1}, \\
t_{z}, r_{z}^{2} r_{x} t_{z}^{-1}, m_{z} r_{x} t_{z}^{-1}\end{array}$ \\
\hline 2783 & $H_{302}$ & 1 & $i$ & $\begin{array}{l}t_{x}, i t_{x}^{-1}, t_{x}^{-1}, m_{z} t_{y}, r_{z}^{2} t_{y}^{-1}, m_{z} t_{y}^{-1} \\
r_{z}^{2} t_{z}, r_{z}^{2} t_{z}^{-1}, m_{z} t_{z}^{-1}\end{array}$ \\
\hline 2784 & $H_{312}$ & 1 & $i$ & $\begin{array}{l}t_{x}, i t_{x}^{-1}, t_{x}^{-1}, m_{z}{ }^{t} y, r_{z}^{2} t_{y}^{-1}, m_{z} t_{y}^{-1} \\
m_{x} t_{z}, m_{x} t_{z}^{-1}, r_{x}^{2} t_{z}^{-1}\end{array}$ \\
\hline 2785 & $H_{656}$ & $\left\langle m_{z} r_{x}\right\rangle$ & $r_{z}^{2} r_{x}$ & $\begin{array}{l}t_{x}, r_{z}^{2} r_{x} t_{x}^{-1}, t_{x}^{-1}, r_{y}^{2} r_{x} t_{y}, m_{x} t_{y}^{-1}, r_{x}^{2} t_{y}^{-1}, \\
m_{x} t_{z}, r_{y}^{2} r_{x} \bar{z}_{z}^{-1}, m_{z} r_{x}^{-1} t_{z}^{-1}\end{array}$ \\
\hline
\end{tabular}




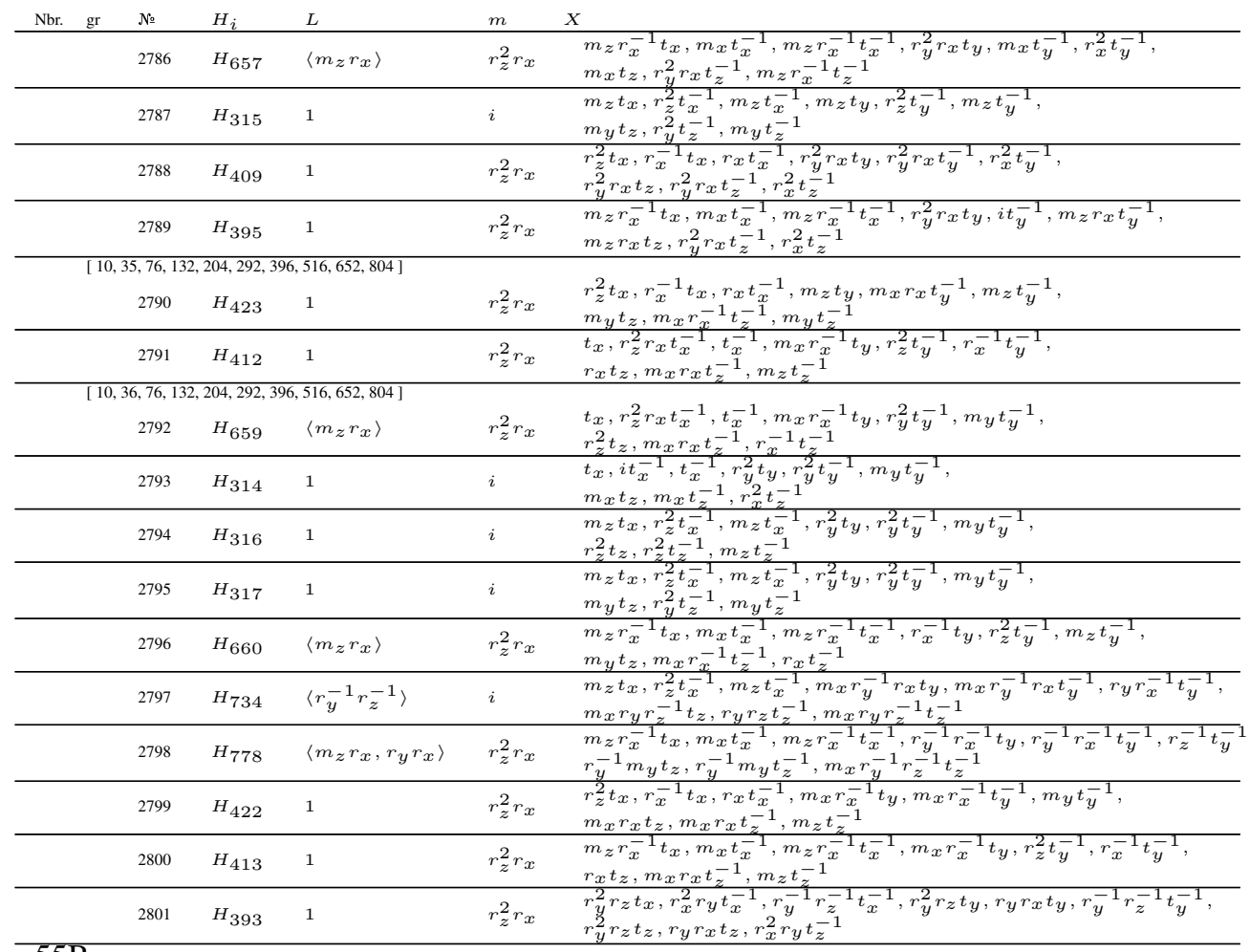

$55 \mathrm{~B}$

[ 9, 34, 76, 132, 204, 292, 396, 516, 652, 804 ] $2802 *$

$[9,35,77,132,204,292,396,516,652,804]$ $2803^{*}, 2804^{*}$,

$[9,35,79,134,204,292,396,516,652,804]$ $2805^{*}$,

$[9,36,78,132,204,292,396,516,652,804]$ $2806^{*}, 2807^{*}, 2808^{*}, 2809^{*}, 2810^{*}$,

$[9,36,79,133,204,292,396,516,652,804$ ] $2811^{*}$

$[10,36,76,132,204,292,396,516,652,804]$

$2802 \quad H_{759} \quad\left\langle m_{z}, r_{x}^{2}\right\rangle$

$[10,37,76,132,204,292,396,516,652,804]$

$\begin{array}{lll}2803 & H_{394} & 1 \\ 2804 & H_{412} & 1\end{array}$

$[10,37,78,132,204,292,396,516,652,804]$

$2805 \quad H_{393} \quad 1$

$[10,38,76,132,204,292,396,516,652,804]$

$2806 \quad H_{760}$

$2807 \quad H_{758}\left\langle m_{z}, r_{x}^{2}\right\rangle$

$2807 \quad H_{758}\left\langle m_{z}, r^{2}\right\rangle$

$2808 \quad H_{757} \quad\left\langle m_{z}, r_{x}^{2}\right\rangle$

$2809 \quad H_{395} \quad 1 \quad r_{z}^{2}$

$2810 \quad H_{413} \quad 1$

$[10,38,77,132,204,292,396,516,652,804]$

$2811 \quad H_{393} \quad 1$ $r_{z}^{2} r_{x} \quad r_{x}^{2} t_{x}, r_{y}^{2} r_{x} t_{x}^{-1}, r_{x}^{2} t_{x}^{-1}, r_{z}^{2} t_{y}, r_{x}^{-1} t_{y}, m_{x} t_{y}^{-1}$ $m_{z} r_{x}^{-1} t_{y}^{-1}, m_{z} r_{x}^{-1} t_{z}, r_{x} t_{z}^{-1}$

$t_{x}, r_{z}^{2} r_{x} t_{x}^{-1}, t_{x}^{-1}, r_{y}^{2} r_{x} t_{y}, r_{x}^{2} t_{y}, i t_{y}^{-1}$ $m_{z} r_{x} t_{y}^{-1}, m_{z} r_{x} t_{z}, r_{y}^{2} r_{x} t_{z}^{-1}$ $t_{x}, r_{z}^{2} r_{x} t_{x}^{-1}, t_{x}^{-1}, m_{x} r_{x}^{-1} t_{y}, m_{y} t_{y}, r_{z}^{2} t_{y}^{-1}$ $r_{x}^{-1} t_{y}^{-1}, r_{x} t_{z}, m_{x} r_{x} t_{z}^{-1}$

$r_{y}^{2} r_{z} t_{x}, r_{y} r_{x} t_{x}, r_{x}^{2} r_{y} t_{x}^{-1}, r_{y}^{2} r_{z} t_{y}, r_{y}^{-1} r_{z}^{-1} t_{y}^{-1}, r_{y}^{2} r_{z} t_{z}$ $r_{y} r_{x} t_{z}, r_{x}^{2} r_{y} t_{z}^{-1}, r_{y}^{-1} r_{z}^{-1} t_{z}^{-1}$

$m_{z} r_{x}^{-1} t_{x}, m_{x} t_{x}^{-1}, m_{z} r_{x}^{-1} t_{x}^{-1}, r_{z}^{2} t_{y}, r_{x}^{-1} t_{y}, m_{x} t_{y}^{-1}$, $m_{z} r_{x}^{-1} t_{y}^{-1}, m_{z} r_{x}^{-1} t_{z}, r_{x} t_{z}^{-1}$ $m_{z} r_{x}^{-1} t_{x}, m_{x} t_{x}^{-1}, m_{z} r_{x}^{-1} t_{x}^{-1}, m_{x} r_{x} t_{y}, r_{x}^{2} t_{y}, r_{y}^{2} r_{x} t_{y}^{-1}$ $r_{x}^{2} t_{y}^{-1}, m_{x} r_{x}^{-1} t_{z}, r_{y}^{2} r_{x} t_{z}^{-1}$

$r_{x}^{2} t_{x}, r_{y}^{2} r_{x} t_{x}^{-1}, r_{x}^{2} t_{x}^{-1}, m_{x} r_{x} t_{y}, r_{x}^{2} t_{y}, r_{y}^{2} r_{x} t_{y}^{-1}$ $r_{x}^{2} t_{y}^{-1}, m_{x} r_{x}^{-1} t_{z}, r_{y}^{2} r_{x} t_{z}^{-1}$ $m_{z} r_{x}^{-1} t_{x}, m_{x} t_{x}^{-1}, m_{z} r_{x}^{-1} t_{x}^{-1}, r_{y}^{2} r_{x} t_{y}, r_{x}^{2} t_{y}, i t_{y}^{-1}$ $m_{z} r_{x}^{-1} t_{x}, m_{x} t_{x}^{-1}, m_{z} r_{x}^{-1} t_{x}^{-1}$
$m_{z} r_{x} t_{y}^{-1}, m_{z} r_{x} t_{z}, r_{y}^{2} r_{x} t^{-1}$ $m_{z} r_{x}^{-1} t_{x}, m_{x} t_{x}^{-1}, m_{z} r_{x}^{-1} t_{x}^{-1}, m_{x} r_{x}^{-1} t_{y}, m_{y} t_{y}, r_{z}^{2} t_{y}^{-1}$ $r_{x}^{-1} t_{y}^{-1}, r_{x} t_{z}, m_{x} r_{x} t_{z}^{-1}$

$r_{y} r_{x} t_{x}, r_{x}^{2} r_{y} t_{x}^{-1}, r_{y}^{-1} r_{z}^{-1} t_{x}^{-1}, r_{y} r_{x} t_{y}, r_{y}^{-1} r_{z}^{-1} t_{y}^{-1}, r_{y}^{2} r_{z} t_{z}$ $r_{y} r_{x} t_{z}, r_{x}^{2} r_{y} t_{z}^{-1}, r_{y}{ }^{1} r_{z}^{-1} t_{z}^{-1}$ 
$\begin{array}{lllllll}\text { Nbr. } & \text { gr } & \text { № } & H_{i} & L & m & X\end{array}$

$[10,35,76,132,204,292,396,516,652,804]$

$2815^{*}, 2816^{*}, 2817^{*}, 2818^{*}, 2819^{*}, 2820^{*}, 2821^{*}, 2822^{*}, 2823^{*}, 2824^{*}, 2825^{*}, 2826^{*}, 2827^{*}$ $2828 *, 2829 *$

$[11,35,75,131,203,291,395,515,651,803$ ]

\begin{tabular}{|c|c|c|c|c|}
\hline 2812 & $H_{745}$ & $\left\langle m_{z} r_{x}^{-1}, r_{z}^{2} r_{x}\right\rangle$ & $i$ & $\begin{array}{l}r_{y}^{2} r_{x} t_{x}, r_{z}^{2} r_{x} t_{x}, m_{z} r_{x} t_{x}^{-1}, r_{z}^{2} r_{x} t_{x}^{-1}, m_{x} t_{y}, m_{z} r_{x} t_{y}^{-1}, \\
m_{x} t_{y}^{-1}, r_{x}^{2} t_{z}, r_{z}^{2} r_{x} t_{z}, r_{z}^{2} r_{x} t_{z}^{-1}\end{array}$ \\
\hline 2813 & $H_{746}$ & $\left\langle m_{z} r_{x}^{-1}, r_{z}^{2} r_{x}\right\rangle$ & $i$ & $\begin{array}{l}r_{z}^{2} t_{x}, m_{x} r_{x} t_{x}, m_{y} t_{x}^{-1}, r_{x} t_{x}^{-1}, m_{x} t_{y}, m_{z} r_{x} t_{y}^{-1} \\
m_{x} t_{y}^{-1}, r_{x}^{2} t_{z}, r_{z}^{2} r_{x} t_{z}, r_{z}^{2} r_{x} t_{z}^{-1}\end{array}$ \\
\hline 2814 & $H_{638}$ & $\left\langle m_{z} r_{x}^{-1}\right\rangle$ & $r_{x}^{2}$ & $\begin{array}{l}r_{y}^{2} r_{x} t_{x}, r_{z}^{2} r_{x} t_{x}, r_{z}^{2} t_{x}^{-1}, m_{x} r_{x} t_{x}^{-1}, t_{y}, m_{z} r_{x} t_{y}^{-1} \\
t_{y}^{1}, r_{x}^{2} t_{z}, m_{z} r_{x}^{-1} t_{z}, m_{z} r_{x}^{-1} t_{z}^{-1}\end{array}$ \\
\hline \multicolumn{5}{|c|}{$[11,36,76,132,204,292,396,516,652,804]$} \\
\hline 2815 & $H_{615}$ & $\left\langle m_{z}\right\rangle$ & $r_{z}^{2}$ & $\begin{array}{l}t_{x}, r_{z}^{2} t_{x}^{-1}, t_{x}^{-1}, m_{x} t_{y}, m_{x} t_{y}^{-1}, m_{y} t_{y}^{-1} \\
i t_{z}, m_{z} t_{z}, r_{z}^{2} t_{z}^{-1}, t_{z}^{-1}\end{array}$ \\
\hline 2816 & $H_{305}$ & 1 & $i$ & $\begin{array}{l}t_{x}, i t_{x}^{-1}, t_{x}^{-1}, i t_{y}, t_{y}, i t_{y}^{-1} \\
t_{y}^{-1}, m_{x} r_{z}^{-1} t_{z}, r_{y}^{2} r_{z} t_{z}^{-1}, m_{x} r_{z}^{-1} t_{z}^{-1}\end{array}$ \\
\hline 2817 & $H_{747}$ & $\left\langle m_{z} r_{x}^{-1}, r_{z}^{2} r_{x}\right\rangle$ & $i$ & $\begin{array}{l}r_{y}^{2} r_{x} t_{x}, r_{z}^{2} r_{x} t_{x}, m_{z} r_{x} t_{x}^{-1}, r_{z}^{2} r_{x} t_{x}^{-1}, r_{y}^{2} t_{y}, m_{x} r_{x}^{-1} t_{y}^{-1} \\
r_{y}^{2} t_{y}^{-1}, m_{x} r_{x}^{-1} t_{z}, r_{y}^{2} t_{z}, m_{x} r_{x}^{-1} t_{z}^{-1}\end{array}$ \\
\hline 2818 & $H_{616}$ & $\left\langle m_{z}\right\rangle$ & $r_{z}^{2}$ & $\begin{array}{l}t_{x}, r_{z}^{2} t_{x}^{-1}, t_{x}^{-1}, m_{x} t_{y}, m_{x} t_{y}^{-1}, m_{y} t_{y}^{-1} \\
r_{y}^{2} t_{z}, r_{x}^{2} t_{z}, m_{x} t_{z}^{-1}, m_{y} t_{z}^{-1}\end{array}$ \\
\hline 2819 & $H_{748}$ & $\left\langle m_{z} r_{x}^{-1}, r_{z}^{2} r_{x}\right\rangle$ & $i$ & $\begin{array}{l}r_{z}^{2} t_{x}, m_{x} r_{x} t_{x}, m_{y} t_{x}^{-1}, r_{x} t_{x}^{-1}, r_{y}^{2} t_{y}, m_{x} r_{x}^{-1} t_{y}^{-1}, \\
r_{y}^{2} t_{y}^{-1}, m_{x} r_{x}^{-1} t_{z}, r_{y}^{2} t_{z}, m_{x} r_{x}^{-1} t_{z}^{-1}\end{array}$ \\
\hline 2820 & $H_{308}$ & 1 & $i$ & $\begin{array}{l}m_{z} t_{x}, r_{z}^{2} t_{x}^{-1}, m_{z}{ }_{x}^{-1}, r_{z}^{2} t_{y}, m_{z}{ }^{t} y, r_{z}^{2} t_{y}^{-1} \\
m_{z}{ }^{-1}, m_{x} r_{z}^{-1} t_{z}, r_{y}^{2} r_{z}{ }_{z}^{-1}, m_{x} r_{z}^{-1} t_{z}^{-1}\end{array}$ \\
\hline 2821 & $H_{323}$ & 1 & $i$ & $\begin{array}{l}r_{z}^{2} t_{x}, m_{z} t_{x}, r_{z}^{2} t_{x}^{-1}, m_{z} t_{x}^{-1}, m_{x} r_{y} t_{y}, r_{x}^{2} r_{y}{ }^{t}{ }^{-1} \\
m_{x} r_{y} t_{y}^{-1}, m_{x}{ }_{z}, m_{x} t_{z}^{-1}, r_{x}^{2} t_{z}^{-1}\end{array}$ \\
\hline 2822 & $H_{326}$ & 1 & $i$ & $\begin{array}{l}m_{z} r_{x} t_{x}, r_{z}^{2} r_{x} t_{x}^{-1}, m_{z} r_{x} t_{x}^{-1}, r_{y}^{2} t_{y}, m_{y} t_{y}, r_{y}^{2} t_{y}^{-1} \\
m_{y}{ }^{-1}, r_{z}^{2} t_{z}, r_{z}^{2} t_{z}^{-1}, m_{z} t_{z}^{-1}\end{array}$ \\
\hline 2823 & $H_{539}$ & 1 & $r_{z}^{2}$ & $\begin{array}{l}m_{z} t_{x}, i t_{x}^{-1}, m_{z} t_{x}^{-1}, r_{y}^{2} t_{y}, r_{y}^{2} t_{y}^{-1}, r_{x}^{2} t_{y}^{-1} \\
m_{z} r_{z}^{-1} t_{z}, m_{z} r_{z} t_{z}, r_{y}^{2} r_{z} t_{z}^{-1}, r_{x}^{2} r_{z} t_{z}^{-1}\end{array}$ \\
\hline 2824 & $H_{540}$ & 1 & $r_{z}^{2}$ & $\begin{array}{l}m_{z} t_{x}, i t_{x}^{-1}, m_{z} t_{x}^{-1}, r_{y} t_{y}, r_{x}^{2} r_{y} t_{y}^{-1}, r_{y}^{-1} t_{y}^{-1} \\
m_{x} t_{z}, m_{y} t_{z}, m_{x} t_{z}^{-1}, m_{y} t_{z}^{-1}\end{array}$ \\
\hline 2825 & $H_{461}$ & 1 & $r_{z}^{2}$ & $\begin{array}{l}t_{x}, r_{z}^{2} t_{x}^{-1}, t_{x}^{-1}, m_{x} t_{y}, m_{x} t_{y}^{-1}, m_{y} t_{y}^{-1} \\
r_{y}^{2} t_{z}, r_{x}^{2} t_{z}, i t_{z}^{-1}, m_{z} t_{z}^{-1}\end{array}$ \\
\hline 2826 & $H_{504}$ & 1 & $r_{z}^{2}$ & $\begin{array}{l}t_{x}, r_{z}^{2} t_{x}^{-1}, t_{x}^{-1}, r_{y} t_{y}, r_{x}^{2} r_{y} t_{y}^{-1}, r_{y}^{-1} t_{y}^{-1} \\
r_{z}^{2} t_{z}, t_{z}, r_{z}^{2} t_{z}^{-1}, t_{z}^{-1}\end{array}$ \\
\hline 2827 & $H_{511}$ & 1 & $r_{z}^{2}$ & $\begin{array}{l}r_{z}^{2} r_{x} t_{x}, r_{x} t_{x}, r_{x}^{-1} t_{x}^{-1}, r_{y}^{2} t_{y}, r_{y}^{2} t_{y}^{-1}, r_{x}^{2} t_{y}^{-1} \\
r_{y}^{2} t_{z}, r_{x}^{2} t_{z}, r_{y}^{2} t_{z}^{-1}, r_{x}^{2} t_{z}^{-1}\end{array}$ \\
\hline 2828 & $H_{541}$ & 1 & $r_{z}^{2}$ & $\begin{array}{l}r_{z}^{2} r_{x} t_{x}, r_{x} t_{x}, r_{x}^{-1} t_{x}^{-1}, m_{x} t_{y}, m_{x} t_{y}^{-1}, m_{y} t_{y}^{-1} \\
i t_{z}, m_{z} t_{z}, i t_{z}^{-1}, m_{z} t_{z}^{-1}\end{array}$ \\
\hline 2829 & $H_{645}$ & $\left\langle m_{z} r_{x}^{-1}\right\rangle$ & $r_{x}^{2}$ & $\begin{array}{l}r_{y}^{2} r_{x} t_{x}, r_{z}^{2} r_{x} t_{x}, r_{z}^{2} t_{x}^{-1}, m_{x} r_{x} t_{x}^{-1}, m_{z} t_{y}, r_{x}^{-1} t_{y}^{-1}, \\
m_{z} t_{y}^{-1}, r_{x}^{-1} t_{z}, m_{z} t_{z}, r_{x}^{-1} t_{z}^{-1}\end{array}$ \\
\hline
\end{tabular}

$56 \mathrm{~B}$

[ $10,37,78,132,204,292,396,516,652,804$ ] $2830^{*}$,

[ $11,38,76,132,204,292,396,516,652,804$ ]
$2830 \quad H_{779} \quad\left\langle i, r_{x}^{2}, r_{z}^{2}\right\rangle$
$r_{z}^{2} r_{x} \quad m_{x} t_{x}, m_{z} r_{x}^{-1} t_{x}, t_{x}^{-1}, r_{z}^{2} r_{x} t_{x}^{-1}, m_{y} t_{y}, m_{x} r_{x}^{-1} t_{y}$,

57

[ $10,35,76,132,204,292,396,516,652,804]$ $2831^{*}, 2832^{*}, 2833^{*}, 2834^{*}$,

$[10,36,77,132,204,292,396,516,652,804]$

$2835^{*}, 2836^{*}, 2837^{*}, 2838^{*}, 2839^{*}, 2840^{*}, 2841^{*}, 2842^{*}, 2843^{*}, 2844^{*}, 2845^{*}, 2846^{*}, 2847^{*}$,

$2848^{*}, 2849^{*}, 2850^{*}, 2851^{*}, 2852^{*}, 2853^{*}, 2854^{*}, 2855^{*}, 2856^{*}, 2857^{*}, 2858^{*}$,

$[10,36,78,133,204,292,396,516,652,804]$

$2859^{*}, 2860^{*}$,

$[11,36,76,132,204,292,396,516,652,804]$

\begin{tabular}{|c|c|c|c|c|}
\hline 2831 & $H_{388}$ & 1 & $r_{z}^{2} r_{x}$ & $\begin{array}{l}t_{x}, r_{z}^{2} r_{x} t_{x}^{-1}, t_{x}^{-1}, m_{z} r_{x} t_{y}, i t_{y}^{-1}, m_{z} r_{x} t_{y}^{-1} \\
i t_{z}, m_{z} r_{x} t_{z}, i t_{z}^{-1}, m_{z} r_{x} t_{z}^{-1}\end{array}$ \\
\hline 2832 & $H_{394}$ & 1 & $r_{z}^{2} r_{x}$ & $\begin{array}{l}t_{x}, r_{z}^{2} r_{x} t_{x}^{-1}, t_{x}^{-1}, r_{y}^{2} r_{x} t_{y}, r_{x}^{2} t_{y}, i t_{y}^{-1} \\
m_{z} r_{x} t_{y}^{-1}, m_{z} r_{x} t_{z}, r_{y}^{2} r_{x} t_{z}^{-1}, r_{x}^{2} t_{z}^{-1}\end{array}$ \\
\hline 2833 & $H_{412}$ & 1 & $r_{z}^{2} r_{x}$ & $\begin{array}{l}t_{x}, r_{z}^{2} r_{x} t_{x}^{-1}, t_{x}^{-1}, m_{x} r_{x}^{-1} t_{y}, m_{y} t_{y}, r_{z}^{2} t_{y}^{-1}, \\
r_{x}^{-1} t_{y}^{-1}, r_{x} t_{z}, m_{x} r_{x} t_{z}^{-1}, m_{z} t_{z}^{-1}\end{array}$ \\
\hline 2834 & $H_{407}$ & 1 & $r_{z}^{2} r_{x}$ & $\begin{array}{l}t_{x}, r_{z}^{2} r_{x} t_{x}^{-1}, t_{x}^{-1}, r_{z}^{2} t_{y}, r_{x}^{-1} t_{y}, r_{z}^{2} t_{y}^{-1} \\
r_{x}^{-1} t_{y}^{-1}, r_{x} t_{z}, r_{y}^{2} t_{z}^{-1}, r_{x} t_{z}^{-1}\end{array}$ \\
\hline \multicolumn{5}{|c|}{$[11,37,76,132,204,292,396,516,652,804]$} \\
\hline 2835 & $H_{389}$ & 1 & $r_{z}^{2} r_{x}$ & $\begin{array}{l}m_{z} r_{x} t_{x}, i t_{x}^{-1}, m_{z} r_{x} t_{x}^{-1}, m_{z} r_{x} t_{y}, i t_{y}^{-1}, m_{z} r_{x} t_{y}^{-1}, \\
i t_{z}, m_{z} r_{x} t_{z}, i t_{z}^{-1}, m_{z} r_{x} t_{z}^{-1}\end{array}$ \\
\hline 2836 & $H_{414}$ & 1 & $r_{z}^{2} r_{x}$ & $\begin{array}{l}m_{z} r_{x} t_{x}, i t_{x}^{-1}, m_{z} r_{x} t_{x}^{-1}, r_{z}^{2} t_{y}, r_{x}^{-1} t_{y}, r_{z}^{2} t_{y}^{-1} \\
r_{x}^{-1} t_{y}^{-1}, r_{x} t_{z}, r_{y}^{2} t_{z}^{-1}, r_{x} t_{z}^{-1}\end{array}$ \\
\hline
\end{tabular}




\begin{tabular}{|c|c|c|c|c|c|}
\hline Nbr. & № & $H_{i}$ & $L$ & $m$ & $X$ \\
\hline & 2837 & $H_{415}$ & 1 & $r_{z}^{2} r_{x}$ & $\begin{array}{l}m_{z} r_{x} t_{x}, i t_{x}^{-1}, m_{z} r_{x} t_{x}^{-1}, m_{x} r_{x}^{-1} t_{y}, m_{y} t_{y}, m_{x} r_{x}^{-1} t_{y}^{-1}, \\
m_{y} t_{y}^{-1}, m_{x} r_{x} t_{z}, m_{x} r_{x} t_{z}^{-1}, m_{z} t_{z}^{-1}\end{array}$ \\
\hline & 2838 & $H_{396}$ & 1 & $r_{z}^{2} r_{x}$ & $\begin{array}{l}m_{z} r_{x} t_{x}, i t_{x}^{-1}, m_{z} r_{x} t_{x}^{-1}, r_{y}^{2} r_{x} t_{y}, r_{y}^{2} r_{x} t_{y}^{-1}, r_{x}^{2} t_{y}^{-1} \\
r_{y}^{2} r_{x} t_{z}, r_{x}^{2} t_{z}, r_{y}^{2} r_{x} t_{z}^{-1}, r_{x}^{2} t_{z}^{-1}\end{array}$ \\
\hline & 2839 & $H_{422}$ & 1 & $r_{z}^{2} r_{x}$ & $\begin{array}{l}r_{z}^{2} t_{x}, r_{x}^{-1} t_{x}, r_{x} t_{x}^{-1}, m_{x} r_{x}^{-1} t_{y}, m_{y} t_{y}, m_{x} r_{x}^{-1} t_{y}^{-1} \\
m_{y} t_{y}^{-1}, m_{x} r_{x} t_{z}, m_{x} r_{x} t_{z}^{-1}, m_{z} t_{z}^{-1}\end{array}$ \\
\hline & 2840 & $H_{422}$ & 1 & $r_{z}^{2} r_{x}$ & $\begin{array}{l}r_{z}^{2} t_{x}, r_{x}^{-1} t_{x}, r_{x} t_{x}^{-1}, m_{x} r_{x}^{-1} t_{y}, m_{x} r_{x}^{-1} t_{y}^{-1}, m_{y} t_{y}^{-1} \\
m_{x} r_{x} t_{z}, m_{z} t_{z}, m_{x} r_{x} t_{z}^{-1}, m_{z} t_{z}^{-1}\end{array}$ \\
\hline & 2841 & $H_{409}$ & 1 & $r_{z}^{2} r_{x}$ & $\begin{array}{l}r_{z}^{2} t_{x}, r_{x}^{-1} t_{x}, r_{x} t_{x}^{-1}, r_{y}^{2} r_{x} t_{y}, r_{y}^{2} r_{x} t_{y}^{-1}, r_{x}^{2} t_{y}^{-1} \\
r_{y}^{2} r_{x} t_{z}, r_{x}^{2} t_{z}, r_{y}^{2} r_{x} t_{z}^{-1}, r_{x}^{2} t_{z}^{-1}\end{array}$ \\
\hline & 2842 & $H_{409}$ & 1 & $r_{z}^{2} r_{x}$ & $\begin{array}{l}r_{z}^{2} t_{x}, r_{x}^{-1} t_{x}, r_{x} t_{x}^{-1}, r_{y}^{2} r_{x} t_{y}, r_{x}^{2} t_{y}, r_{y}^{2} r_{x} t_{y}^{-1} \\
r_{x}^{2} t_{y}^{-1}, r_{y}^{2} r_{x} t_{z}, r_{y}^{2} r_{x} t_{z}^{-1}, r_{x}^{2} t_{z}^{-1}\end{array}$ \\
\hline & 2843 & $H_{394}$ & 1 & $r_{z}^{2} r_{x}$ & $\begin{array}{l}t_{x}, r_{z}^{2} r_{x} t_{x}^{-1}, t_{x}^{-1}, r_{y}^{2} r_{x} t_{y}, i t_{y}^{-1}, m_{z} r_{x} t_{y}^{-1} \\
i t_{z}, m_{z} r_{x} t_{z}, r_{y}^{2} r_{x} t_{z}^{-1}, r_{x}^{2} t_{z}^{-1}\end{array}$ \\
\hline & 2844 & $H_{412}$ & 1 & $r_{z}^{2} r_{x}$ & $\begin{array}{l}t_{x}, r_{z}^{2} r_{x} t_{x}^{-1}, t_{x}^{-1}, m_{x} r_{x}^{-1} t_{y}, m_{y} t_{y}, r_{z}^{2} t_{y}^{-1} \\
r_{x}^{-1} t_{y}^{-1}, r_{y}^{2} t_{z}, r_{x} t_{z}, m_{x} r_{x} t_{z}^{-1}\end{array}$ \\
\hline & 2845 & $H_{402}$ & 1 & $r_{z}^{2} r_{x}$ & $\begin{array}{l}t_{x}, r_{z}^{2} r_{x} t_{x}^{-1}, t_{x}^{-1}, m_{x} r_{x}^{-1} t_{y}, m_{y} t_{y}, m_{x} r_{x}^{-1} t_{y}^{-1} \\
m_{y} t_{y}^{-1}, m_{x} r_{x} t_{z}, m_{x} r_{x} t_{z}^{-1}, m_{z} t_{z}^{-1}\end{array}$ \\
\hline & 2846 & $H_{391}$ & 1 & $r_{z}^{2} r_{x}$ & $\begin{array}{l}t_{x}, r_{z}^{2} r_{x} t_{x}^{-1}, t_{x}^{-1}, r_{y}^{2} r_{x} t_{y}, r_{y}^{2} r_{x} t_{y}^{-1}, r_{x}^{2} t_{y}^{-1} \\
r_{y}^{2} r_{x} t_{z}, r_{x}^{2} t_{z}, r_{y}^{2} r_{x} t_{z}^{-1}, r_{x}^{2} t_{z}^{-1}\end{array}$ \\
\hline & 2847 & $H_{398}$ & 1 & $r_{z}^{2} r_{x}$ & $\begin{array}{l}r_{x}^{2} t_{x}, r_{y}^{2} r_{x} t_{x}^{-1}, r_{x}^{2} t_{x}^{-1}, m_{z} r_{x} t_{y}, i t_{y}^{-1}, m_{z} r_{x} t_{y}^{-1} \\
i t_{z}, m_{z} r_{x} t_{z}, i t_{z}^{-1}, m_{z} r_{x} t_{z}^{-1}\end{array}$ \\
\hline & 2848 & $H_{410}$ & 1 & $r_{z}^{2} r_{x}$ & $\begin{array}{l}r_{x}^{2} t_{x}, r_{y}^{2} r_{x} t_{x}^{-1}, r_{x}^{2} t_{x}^{-1}, r_{z}^{2} t_{y}, r_{x}^{-1} t_{y}, r_{z}^{2} t_{y}^{-1} \\
r_{x}^{-1} t_{y}^{-1}, r_{x} t_{z}, r_{y}^{2} t_{z}^{-1}, r_{x} t_{z}^{-1}\end{array}$ \\
\hline & 2849 & $H_{404}$ & 1 & $r_{z}^{2} r_{x}$ & $\begin{array}{l}r_{x}^{2} t_{x}, r_{y}^{2} r_{x} t_{x}^{-1}, r_{x}^{2} t_{x}^{-1}, m_{x} r_{x}^{-1} t_{y}, m_{y} t_{y}, m_{x} r_{x}^{-1} t_{y}^{-1}, \\
m_{y} t_{y}^{-1}, m_{x} r_{x} t_{z}, m_{x} r_{x} t_{z}^{-1}, m_{z} t_{z}^{-1}\end{array}$ \\
\hline & 2850 & $H_{392}$ & 1 & $r_{z}^{2} r_{x}$ & $\begin{array}{l}r_{x}^{2} t_{x}, r_{y}^{2} r_{x} t_{x}^{-1}, r_{x}^{2} t_{x}^{-1}, r_{y}^{2} r_{x} t_{y}, r_{y}^{2} r_{x} t_{y}^{-1}, r_{x}^{2} t_{y}^{-1} \\
r_{y}^{2} r_{x} t_{z}, r_{x}^{2} t_{z}, r_{y}^{2} r_{x} t_{z}^{-1}, r_{x}^{2} t_{z}^{-1}\end{array}$ \\
\hline & 2851 & $H_{400}$ & 1 & $r_{z}^{2} r_{x}$ & $\begin{array}{l}m_{z} r_{x}^{-1} t_{x}, m_{x} t_{x}^{-1}, m_{z} r_{x}^{-1} t_{x}^{-1}, m_{z} r_{x} t_{y}, i t_{y}^{-1}, m_{z} r_{x} t_{y}^{-1}, \\
i t_{z}, m_{z} r_{x} t_{z}, i t_{z}^{-1}, m_{z} r_{x} t_{z}^{-1}\end{array}$ \\
\hline & 2852 & $H_{395}$ & 1 & $r_{z}^{2} r_{x}$ & $\begin{array}{l}m_{z} r_{x}^{-1} t_{x}, m_{x} t_{x}^{-1}, m_{z} r_{x}^{-1} t_{x}^{-1}, r_{y}^{2} r_{x} t_{y}, i t_{y}^{-1}, m_{z} r_{x} t_{y}^{-1} \\
i t_{z}, m_{z} r_{x} t_{z}, r_{y}^{2} r_{x} t_{z}^{-1}, r_{x}^{2} t_{z}^{-1}\end{array}$ \\
\hline & 2853 & $H_{395}$ & 1 & $r_{z}^{2} r_{x}$ & $\begin{array}{l}m_{z} r_{x}^{-1} t_{x}, m_{x} t_{x}^{-1}, m_{z} r_{x}^{-1} t_{x}^{-1}, r_{y}^{2} r_{x} t_{y}, r_{x}^{2} t_{y}, i t_{y}^{-1} \\
m_{z} r_{x} t_{y}^{-1}, m_{z} r_{x} t_{z}, r_{y}^{2} r_{x} t_{z}^{-1}, r_{x}^{2} t_{z}^{-1}\end{array}$ \\
\hline & 2854 & $H_{413}$ & 1 & $r_{z}^{2} r_{x}$ & $\begin{array}{l}m_{z} r_{x}^{-1} t_{x}, m_{x} t_{x}^{-1}, m_{z} r_{x}^{-1} t_{x}^{-1}, m_{x} r_{x}^{-1} t_{y}, m_{y} t_{y}, r_{z}^{2} t_{y}^{-1}, \\
r_{x}^{-1} t_{y}^{-1}, r_{y}^{2} t_{z}, r_{x} t_{z}, m_{x} r_{x} t_{z}^{-1}\end{array}$ \\
\hline & 2855 & $H_{413}$ & 1 & $r_{z}^{2} r_{x}$ & $\begin{array}{l}m_{z} r_{x}^{-1} t_{x}, m_{x} t_{x}^{-1}, m_{z} r_{x}^{-1} t_{x}^{-1}, m_{x} r_{x}^{-1} t_{y}, m_{y} t_{y}, r_{z}^{2} t_{y}^{-1}, \\
r_{x}^{-1} t_{y}^{-1}, r_{x} t_{z}, m_{x} r_{x} t_{z}^{-1}, m_{z} t_{z}^{-1}\end{array}$ \\
\hline & 2856 & $H_{418}$ & 1 & $r_{z}^{2} r_{x}$ & $\begin{array}{l}m_{z} r_{x}^{-1} t_{x}, m_{x} t_{x}^{-1}, m_{z} r_{x}^{-1} t_{x}^{-1}, r_{z}^{2} t_{y}, r_{x}^{-1} t_{y}, r_{z}^{2} t_{y}^{-1} \\
r_{x}^{-1} t_{y}^{-1}, r_{x} t_{z}, r_{y}^{2} t_{z}^{-1}, r_{x} t_{z}^{-1}\end{array}$ \\
\hline & 2857 & $H_{419}$ & 1 & $r_{z}^{2} r_{x}$ & $\begin{array}{l}m_{z} r_{x}^{-1} t_{x}, m_{x} t_{x}^{-1}, m_{z} r_{x}^{-1} t_{x}^{-1}, m_{x} r_{x}^{-1} t_{y}, m_{y} t_{y}, m_{x} r_{x}^{-1} t_{y}^{-1}, \\
m_{y} t_{y}^{-1}, m_{x} r_{x} t_{z}, m_{x} r_{x} t_{z}^{-1}, m_{z} t_{z}^{-1}\end{array}$ \\
\hline & 2858 & $H_{401}$ & 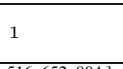 & $r_{z}^{2} r_{x}$ & $\begin{array}{l}m_{z} r_{x}^{-1} t_{x}, m_{x} t_{x}^{-1}, m_{z} r_{x}^{-1} t_{x}^{-1}, r_{y}^{2} r_{x} t_{y}, r_{y}^{2} r_{x} t_{y}^{-1}, r_{x}^{2} t_{y}^{-1} \\
r_{y}^{2} r_{x} t_{z}, r_{x}^{2} t_{z}, r_{y}^{2} r_{x} t_{z}^{-1}, r_{x}^{2} t_{z}^{-1}\end{array}$ \\
\hline & {$[11,37,77,1$} & $H_{393}$ & $\begin{array}{l}-516,652,804] \\
1\end{array}$ & $r_{z}^{2} r_{x}$ & $\begin{array}{l}r_{y}^{2} r_{z} t_{x}, r_{x}^{2} r_{y} t_{x}^{-1}, r_{y}^{-1} r_{z}^{-1} t_{x}^{-1}, r_{y}^{2} r_{z} t_{y}, r_{y} r_{x} t_{y}, r_{x}^{2} r_{y} t_{y}^{-1}, \\
r_{y}^{-1} r_{z}^{-1} t_{y}^{-1}, r_{y}^{2} r_{z} t_{z}, r_{y} r_{x} t_{z}, r_{x}^{2} r_{y} t_{z}^{-1}\end{array}$ \\
\hline & 2860 & $H_{393}$ & 1 & $r_{z}^{2} r_{x}$ & $\begin{array}{l}r_{y}^{2} r_{z} t_{x}, r_{y} r_{x} t_{x}, r_{y}^{-1} r_{z}^{-1} t_{x}^{-1}, r_{y}^{2} r_{z} t_{y}, r_{y} r_{x} t_{y}, r_{x}^{2} r_{y} t_{y}^{-1} \\
r_{y}^{-1} r_{z}^{-1} t_{y}^{-1}, r_{y}^{2} r_{z} t_{z}, r_{y} r_{x} t_{z}, r_{y}^{-1} r_{z}^{-1} t_{z}^{-1}\end{array}$ \\
\hline
\end{tabular}

58

$[11,36,76,132,204,292,396,516,652,804]$

$2861^{*}, 2862^{*}, 2863^{*}$

$[12,36,76,132,204,292,396,516,652,804]$

\begin{tabular}{|c|c|c|c|c|}
\hline 2861 & $H_{779}$ & $\left\langle m_{z}, m_{z} r_{x}, r_{x}^{2}\right\rangle$ & $r_{z}^{2} r_{x}$ & $\begin{array}{l}m_{z} r_{x}^{-1} t_{x}, m_{x} t_{x}^{-1}, m_{z} r_{x}^{-1} t_{x}^{-1}, r_{z}^{2} t_{y}, r_{x}^{2} t_{y}, m_{x} t_{y}^{-1} \\
m_{z} r_{x}^{-1} t_{y}^{-1}, m_{x} r_{x}^{-1} t_{z}, m_{z} r_{x}^{-1} t_{z}, r_{y}^{2} r_{x} t_{z}^{-1}, r_{x}^{2} t_{z}^{-1}\end{array}$ \\
\hline 2862 & $H_{750}$ & $\left\langle m_{y}, m_{z}\right\rangle$ & $r_{z}^{2}$ & $\begin{array}{l}m_{y} t_{x}, m_{x} t_{x}^{-1}, m_{y} t_{x}^{-1}, r_{z}^{2} t_{y}, m_{y} t_{y}, m_{x} t_{y}^{-1} \\
m_{y} t_{y}^{-1}, r_{y}^{2} r_{z} t_{z}, m_{z} r_{z} t_{z}, m_{x} r_{z}{ }_{z}^{-1}, r_{z}{ }_{z}^{-1}\end{array}$ \\
\hline 2863 & $H_{690}$ & $\left\langle m_{x}\right\rangle$ & $r_{z}^{2}$ & $\begin{array}{l}r_{z}^{2} t_{x}, m_{x} t_{x}, m_{y} t_{x}^{-1}, m_{x} t_{x}^{-1}, m_{x} t_{y}, m_{y} t_{y}^{-1} \\
m_{x} t_{y}^{-1}, r_{y}^{2} r_{z} t_{z}, m_{z} r_{z}^{-1} t_{z}, r_{x}^{2} t_{z}^{-1}, r_{y}^{2} t_{z}^{-1}\end{array}$ \\
\hline
\end{tabular}

59

$[11,37,77,132,204,292,396,516,652,804]$

$2864^{*}, 2865^{*}, 2866^{*}, 2867^{*}, 2868^{*}, 2869^{*}, 2870^{*}, 2871^{*}, 2872^{*}$

$[12,37,76,132,204,292,396,516,652,804]$

\begin{tabular}{|c|c|c|c|c|}
\hline 2864 & $H_{649}$ & $\left\langle m_{x}\right\rangle$ & $r_{y}^{2} r_{x}$ & $\begin{array}{l}i t_{x}, r_{z}^{2} r_{x} t_{x}, r_{x}^{2} t_{x}^{-1}, r_{z}^{2} r_{x} t_{x}^{-1}, r_{z}^{2} r_{x} t_{y}, r_{x}^{2} t_{y}^{-1} \\
r_{z}^{2} r_{x}{ }_{y}^{-1}, r_{x}^{2} t_{z}, r_{z}^{2} r_{x} t_{z}, r_{x}^{2} t_{z}^{-1}, r_{z}^{2} r_{x} t_{z}^{-1}\end{array}$ \\
\hline 2865 & $H_{652}$ & $\left\langle m_{x}\right\rangle$ & $r_{y}^{2} r_{x}$ & $\begin{array}{l}i t_{x}, r_{z}^{2} r_{x} t_{x}, r_{x}^{2} t_{x}^{-1}, r_{z}^{2} r_{x} t_{x}^{-1}, m_{y} t_{y}, m_{x} r_{x} t_{y}, \\
m_{y} t_{y}^{-1}, m_{x} r_{x}{ }_{y}^{-1}, m_{x} r_{x}^{-1} t_{z}, m_{x} r_{x}^{-1} t_{z}^{-1}, r_{y}^{2} t_{z}^{-1}\end{array}$ \\
\hline
\end{tabular}




\begin{tabular}{|c|c|c|c|c|c|c|}
\hline Nbr. & gr & № & $H_{i}$ & $L$ & $m$ & $X$ \\
\hline & & 2866 & $H_{422}$ & 1 & $r_{z}^{2} r_{x}$ & $\begin{array}{l}r_{z}^{2} t_{x}, r_{x}^{-1} t_{x}, r_{y}^{2} t_{x}^{-1}, r_{x} t_{x}^{-1}, m_{x} r_{x}^{-1} t_{y}, m_{y}{ }^{t} y \\
m_{x} r_{x}^{-1} t_{y}^{-1}, m_{y}{ }^{-1}, m_{x} r_{x} t_{z}, m_{x} r_{x}{ }_{z}^{-1}, m_{z}{ }_{z}^{-1}\end{array}$ \\
\hline & & 2867 & $H_{409}$ & 1 & $r_{z}^{2} r_{x}$ & $\begin{array}{l}r_{z}^{2} t_{x}, r_{x}^{-1} t_{x}, r_{y}^{2} t_{x}^{-1}, r_{x} t_{x}^{-1}, r_{y}^{2} r_{x} t_{y}, r_{y}^{2} r_{x} t_{y}^{-1} \\
r_{x}^{2} t_{y}^{-1}, r_{y}^{2} r_{x} t_{z}, r_{x}^{2} t_{z}, r_{y}^{2} r_{x} t_{z}^{-1}, r_{x}^{2} t_{z}^{-1}\end{array}$ \\
\hline & & 2868 & $H_{648}$ & $\left\langle m_{x}\right\rangle$ & $r_{y}^{2} r_{x}$ & $\begin{array}{l}r_{y}^{2} r_{x} t_{x}, m_{x} t_{x}, m_{z} r_{x} t_{x}^{-1}, m_{x} t_{x}^{-1}, r_{z}^{2} r_{x} t_{y}, r_{x}^{2} t_{y}^{-1} \\
r_{z}^{2} r_{x} t_{y}^{-1}, r_{x}^{2} t_{z}, r_{z}^{2} r_{x} t_{z}, r_{x}^{2} t_{z}^{-1}, r_{z}^{2} r_{x} t_{z}^{-1}\end{array}$ \\
\hline & & 2869 & $H_{650}$ & $\left\langle m_{x}\right\rangle$ & $r_{y}^{2} r_{x}$ & $\begin{array}{l}r_{y}^{2} r_{x} t_{x}, m_{x} t_{x}, m_{z} r_{x} t_{x}^{-1}, m_{x} t_{x}^{-1}, m_{y} t_{y}, m_{x} r_{x} t_{y} \\
m_{y} t_{y}^{-1}, m_{x} r_{x} t_{y}^{-1}, m_{x} r_{x}^{-1} t_{z}, m_{x} r_{x}^{-1} t_{z}^{-1}, r_{y}^{2} t_{z}^{-1}\end{array}$ \\
\hline & & 2870 & $H_{393}$ & 1 & $r_{z}^{2} r_{x}$ & $\begin{array}{l}r_{y}^{2} r_{z} t_{x}, r_{y} r_{x} t_{x}, r_{x}^{2} r_{y}{ }_{x}^{-1}, r_{y}^{-1} r_{z}^{-1} t_{x}^{-1}, r_{y}^{2} r_{z} t_{y}, r_{y} r_{x} t_{y} \\
r_{y}^{-1} r_{z}^{-1} t_{y}^{-1}, r_{y}^{2} r_{z} t_{z}, r_{y} r_{x} t_{z}, r_{x}^{2} r_{y} t_{z}^{-1}, r_{y}^{-1} r_{z}^{-1} t_{z}-1\end{array}$ \\
\hline & & 2871 & $H_{560}$ & 1 & $m_{z} r_{x}$ & $\begin{array}{l}i t_{x}, r_{z}^{2} r_{x} t_{x}, r_{y}^{2} r_{x} t_{x}^{-1}, m_{x} t_{x}^{-1}, m_{z} r_{x}^{-1} t_{y}, r_{x}^{2} t_{y}^{-1} \\
m_{z} r_{x}^{-1} t_{y}^{-1}, r_{x}^{2} t_{z}, m_{z} r_{x}^{-1} t_{z}, r_{x}^{2} t_{z}^{-1}, m_{z} r_{x}^{-1} t_{z}^{-1}\end{array}$ \\
\hline & & 2872 & $H_{580}$ & 1 & $m_{z} r_{x}$ & $\begin{array}{l}i t_{x}, r_{z}^{2} r_{x} t_{x}, r_{y}^{2} r_{x} t_{x}^{-1}, m_{x} t_{x}^{-1}, m_{y} t_{y}, r_{x} t_{y} \\
m_{y} t_{y}^{-1}, r_{x} \bar{y}^{-1}, r_{x}^{-1} t_{z}, r_{x}^{-1} t_{z}^{-1}, m_{z}{ }_{z}^{-1}\end{array}$ \\
\hline
\end{tabular}

\section{ORCID iDs}

Kirill V. Kostousov (iD https://orcid.org/0000-0002-8955-5912

\section{References}

[1] B. Eick, F. Gähler and W. Nickel, Cryst manual. a gap4 package, version 4.1.10: [e-resource], https://www.gap-system.org/Manuals/pkg/cryst/htm/chapters.htm.

[2] gap-system.org, Gap - groups, algorithms, programming - a system for computational discrete algebra, ver. 4.5.7: [e-resource], http: / /www.gap-system.org.

[3] E. A. Konovalchik and K. V. Kostousov, Symmetrical 2-extensions of the 2-dimensional grid. i, Trudy Inst. Mat. Mekh. UrO RAN 22 (2016), 159-179, In Russian, http: / / journal . imm. uran.ru/sites/default/files/archive/trudy_imm-2016-1.pdf.

[4] E. A. Konovalchik and K. V. Kostousov, Symmetrical 2-extensions of the 2-dimensional grid. ii, Trudy Inst. Mat. Mekh. UrO RAN 23 (2017), 192-211, doi:10.21538/ 0134-4889-2017-23-4-192-211, In Russian.

[5] E. A. Neganova and V. I. Trofimov, Symmetrical extensions of graphs, Izv. Math. 78 (2014), 809-835, doi:10.1070/IM2014v078n04ABEH002707.

[6] V. I. Trofimov, Symmetrical extensions of graphs and some other topics in graph theory related with group theory, Proc. Steklov Inst. Math. 279 (2012), 107-112, doi:10.1134/ S0081543812090088.

[7] V. I. Trofimov, The finiteness of the number of symmetrical 2-extensions of the $d$-dimensional grid and similar graphs, Proc. Steklov Inst. Math. 285 (2014), 169-182, doi:10.1134/ s0081543814050198. 